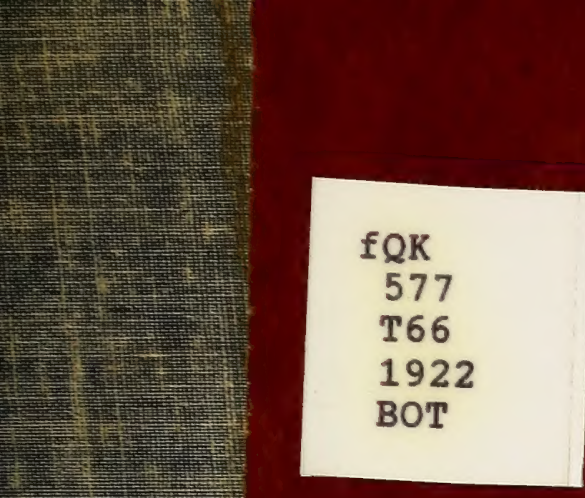

1) 


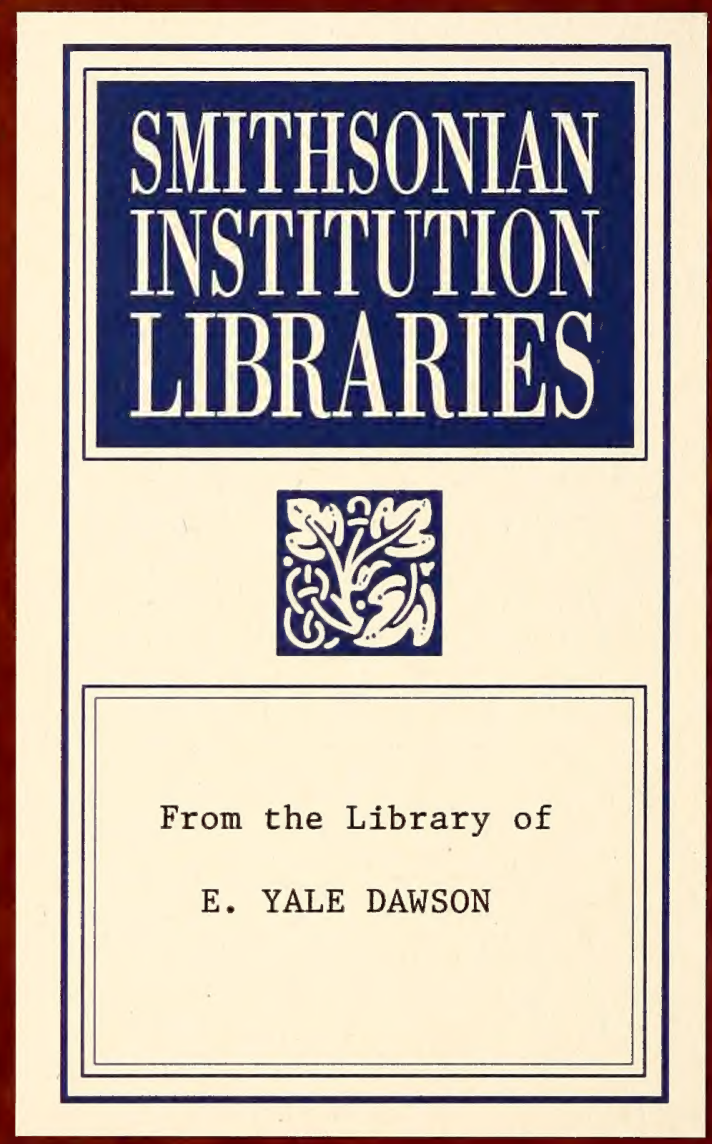


LIBRARY OF

E.Y. DAWSON 



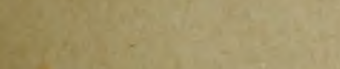



G. B. DE TONI e ACHILLE FORTI

\section{Alghe di Australia, Tasmania e Nuova Zelanda}

raccolte dal rev. dott. GIUSEPPE CAPRA

nel 1908-1909

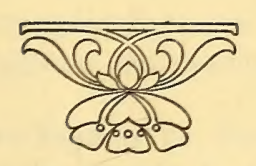

VENEZIA

Premiate Officine Grafiche Carlo Ferrari 1922. 
fQK

577

166

1922 BOT

Estratto dalle Memorie del $R$. Istituto Veneto di Scienze, Lettere ed Arti. 《Vol. XXIX. - N. 3. »

(presentata nell' adunanza ordinaria del 17 Febbraio 1921) 
Il rev. prof. dott. GiUSEPPE CAPRA, nell' occasione di un viaggio intrapreso per visitare gli emigrati italiani nel Nuovissimo Continente e in altre terre dell' Oceania ( ${ }^{(1)}$, raccolse nell' Australia, nella Tasmania e nella Nuova Zelanda un copioso materiale di Alghe, in prevalenza marine, le quali furono da lui poste a nostra piena disposizione per lo studio, porgendoci così modo di recare un contributo alla flora di quelle interessanti regioni.

Risalgono al primo ventennio del secolo scorso i contributi, degni di speciale menzione, sulle flore marine di Australia, Tasmania e Nuova Zelanda, dati in luce dal Labillardière $\left({ }^{2}\right)$, dal Turner $\left({ }^{3}\right)$, dal Mertens ( $\left.{ }^{4}\right)$, da C. Agardh ( ${ }^{5}$ ) e in modo particolare dal botanico francese LAmouroux $\left(^{6}\right)$, aumentati poi a

(I) Cfr. Capra Gruseppe, Emigrati italiani in Australasia. Appunti preparatori per una relazione sul viaggio compiuto ecc.; Milano, Igog, Tipografia Salesiana, $8^{\circ}$.

(2) La Billardiere J. J. H. (DE), Novae Hollandiae plantarum specimen, c. 265 tab.; Parisiis, 1804-1806, $4^{\circ}$.

(3) TURner Dawson, Fuci, sive plantarum Fucorum generi a botanicis adscriptarum icones, descriptiones et historia (Fuci, or colored figures and descriptions etc.) ; London, I808-1819, Arch, 4 vol. in $4^{\circ}, 25^{8} \mathrm{tab}$.

(4) Mertens, Mémoire sur plusieurs Espèces de Fucus, nouvelles ou peu connues, observées dans la collection du Muséum (Mémoires du Muséum T. 5, pag. I72-19o, tab. I4-16).

(5) AGARdH C. A., Species Algarum rite cognitae, cum synonymis, differentiis specificis et descriptionibus succinctis; Gryphiswaldiae, 1821, Sumptibus Ernesti Mauritii, 160.

(6) Lamouroux J. V. F., Essai sur les genres de la famille des Thalassiophytes non articulées, c. 7 tab. ; Paris, 1813, Dufour, $4^{\circ}$.

- - Histoire des polypiers coralligènes fléxibles, vulgairement nommées Zoophytes; Caen, 1816 , in $8^{\circ}, 19$ tab.

- - Exposition méthodique des genres de l'ordre des Polypiers; Paris, r821, Agasse, $4^{\circ}, 84$ tab.

In opere del secolo XVIII e de' primi anni del successivo trovansi scarse indicazioni su Alghe dell' Oceania sia in lavori trattanti di argomento botanico sia in lavori riferentisi a soggetti zoologici, in quest' ultimo caso soprattutto per quanto concerne Alghe calcaree (Halimeda, Jania, Corallina ecc.) allora ascritte agli Zoofiti. Per la storia dell' argomento ricordinsi :

Ellis J. AND Solander D, The Natural History of many curious and uncommon Zoophytes collected from various parts of the Globe, with sixty-two plates; London, 1786, Ben. White and Son, $4^{\circ}$; ESPER E. J. C., Icones Fucorum cum characteribus systematicis, synonymis auctorum et descri- 
merito dei viaggi d' esplorazione e di circumnavigazione compiuti dalle navi Uranie e Physicienne (1817 - 1820), Coquille (1822-1825), Astrolabe (1826-1829), Venus (1836-1839), Astrolabe e Zélée (1837-40), Erebus e Terror (1839-1843), Novara (1857 - 1859), Challenger (1873 - 1876), Gazelle (1874 - 1876), Vettor Pisani (1884 - 1885) ecc.

Prescindendo dai resultati dei grandi viaggi, nei successivi decennii del secolo XIX, e più precisamente fin verso il 1850, apparvero altri scritti su Alghe dell' Oceania quali si possono ricavare dall' elenco bibliografico che accompagna la presente Memoria; tra i più importanti si devono ricordare quelli di AREschoug, Montagne, Sonder e Kuetzing. Si trattava però di notizie desunte da osservazioni compiute su materiali raccolti senza lo scopo prefisso di fornire una vera e propria flora australasica marina; non per questo devonsi dimenticare le benemerenze di L. Preiss, di Binder, di Lenormand e di altri che raccolsero Alghe ovvero misero le loro cospicue collezioni a disposizione degli studiosi o ne distribuirono con generosità gli esemplari.

A vasti lavori di sintesi, riguardo in particolare alle Florideae, Fucoideae, Chlorophyceae e Myxophyceae, attesero J. D. Hooker e W. H. Harvey; le opere di questi due autori costituiscono la base fondamentale per lo studio della flora marina dell' Oceania; mentre il primo ci diede le mirabili flore antartica, tasmanica e neo-zelandese, il secondo pubblicò due lavori ragguardevolissimi: la $\mathrm{Ne}$ reis Australis considerando in questa le piante marine raccolte sulle coste del Capo di Buona Speranza (Africa meridionale), delle regioni australiane extratropicali, della Tasmania, della Nuova Zelanda e delle regioni antartiche, la Phycologia Australica riguardando le ficee della Nuova Galles del Sud, della Tasmania, della Victoria, dell' Australia meridionale e occidentale; per questa seconda importantissima opera W. H. HARVEY si giovò e di materiali da lui stesso raccolti durante un soggiorno in Australia (1854-1856) e di materiali comunicatigli da moltissime persone ch' egli aveva all' uopo interessate, specialmente G. CLIFton, R. Gunn e F. Mueller (1); in essa anzi l' autore ebbe pure il merito d'aver riunito in catalogo, in una sorte di sinossi, le specie fino allora (1863) conosciute per la Nuova Olanda e per la Tasmania, indicando per molte di esse i rapporti

ptionibus novarum specierum [es, tab. CXXXI Fucus flexuosus = Carpophyllum Phyllanthus (Turn.) H. et H.] ; Nürnberg, I798-I808, Raspesche Buchh., 4; LAMARCK J. B., Histoire naturelle des animaux sans vertèbres T. II; Paris, I8ro. Poche Alghe dell' Oceania (Acetabularia Calyculus, Jania compressa, Amphiroa crassa ecc.) trovansi descritte o indicate nella parte zoologica del Voyage autour du monde exécuté snr les corvettes de S. M. l' Uranie el la Physicienne pendant les années I817, I818, 1819 et 1820 per M. Lours DE Frevcinet; Paris, 1824, Fillet ainé, $4^{\circ}$.

(I) Meritano ricordo, a titolo d'onore, i più solerti raccoglitori di Alghe Australiane che comunicarono materiali di studio a W. H. Harvey ; essi sono Curdie, Watts, Rawlinson, Stuart, Moore, Wood, Woolhouse, Browne, Barker, Jeannerett, Fereday, Brown, Archer, Ewing, M' Kenzie, Taylor, Eddington, Mallard, Cox, Wilhelmi, Drummond, Smith, Baines, Henty, Mc William, Hannaford, Sheridan Wall, Oldfield, Milligan, Mylne; a non pochi di codesti raccoglitori l' autore della Phycologia Australica dedicò generi ovvero specie.

Oltre che nell' Erbario Harvey conservato al Trinity College di Dublino, si trovano Alghe Harveyane, nonchè di G. Clifton, R. Gunn, F. Mueller ecc. nel Museo Britannico di Storia Naturale; cfr. History of the Collections in the Natural History Department of the British Museum Vol. I (Alphabetical List of the more important Contributors to the Collections in the Department of Botany pag. I29 e seg.ti); London, 1904, W. Cloves and Sons, $8^{\circ}$. Alghe australiche, di provenienza Harvey, sono largamente rappresentate anche nell' Erbario ZaNARDINI, conservato nel Museo Civico di Venezia e in parecchi altri erbarii. 
fitogeografici con la Nuova Zelanda, l' isola di Ceylon, la Cina, il Giappone; il Mar Rosso, Madagascar, il Capo di Buona Speranza, l'America boreo - occidentale e meridionale; in quella sinossi l' illustre botanico fece un primo tentativo rivolto a indagare quali rapporti potessero sussistere tra le flora marine del Continente Australiano e della vicina Tasmania e le flore di altre regioni del Globo.

La Nereis Australis e la Phycologia Australica di W. H. Harvey, giova ripeterlo, costituirono, può dirsi, i principali caposaldi per lo studio delle flora dell' Oceania, vuoi per la qualità delle descrizioni, vuoi per la bellezza delle illustrazioni degli habitus delle specie più forse che per l' esattezza dei particolari anatomici; d'altra parte J. D. Hooker giovò grandemente alla conoscenza della flora marina neozelandese pubblicando per quest' ultima un' opera sintetica, il noto Handbook of the New Zealand Flora, pure accurata quanto alla disamina delle fonti.

A questi libri davvero magistrali tennero dietro scritti di minore mole, non ultimo per valore quello del SoNDER contenente molte novità floristiche descritte in base a materiali dell' Australia tropicale fornitigli da E. DAEMEL, F. Mueller e altri diligenti raccoglitori ; anche questo autore cercò di dare le indicazioni delle aree geografiche delle specie allo stato delle conoscenze scientifiche di allora.

Descrizioni e osservazioni critiche sulle Alghe dell' Oceania andava man mano elaborando e pubblicando, con maravigliosa attività, l' insigne ficologo J. G. AGARDH in numerosi lavori, traendo profitto del ricchissimo erbario paterno e personale, e dei materiali comunicatigli per le isole Chatham e per la Nuova Zelanda rispettivamente dal Travers e dal Berggren, per la Nuova Olanda e per la Tasmania e le piccole isole vicine in particolare dal solertissimo FERDINANdo Mueller (1), da J. Br. Wilson e da molti altri raccoglitori, com' era avvenuto per sua fortuna a W. H. Harvey (2). Quando si rifletta che J. G. AgardH iniziò i suoi contributi alla flora Australiana nel 1846 e li proseguì fino al 1901, ossia poco prima della sua morte $\left(^{3}\right)$ è facile comprendere com' egli, nel periodo d'oltre mezzo secolo di indefesso lavoro, abbia recato considerevoli aggiunte alla conoseenza della flora marina dell' Oceania.

In base al complesso degli studii sopra menzionati, contemporanea o susseguente ai quali si svolse, con pubblicazioni di minor mole, l' attività di altri botanici, come l' Areschoug, l' Askenasy, il Grunow, il Reinbold e il nostro G. ZanARDini, vennero, dietro l' esempio della sinossi già ricordata di W. H. HarveY e degli elenchi dati in luce dal Sonder $\left(^{(4)}\right.$ e dal WiLson ${ }^{(5)}$, redatti in questi

(I) L'abbondantissimo materiale comunicato dal barone Ferdinando Mueller a J. G. AgardH diede modo a quest' ultimo di fare larga distribuzione di specie nelle Alghe Muellevianae curante J. G. Agardh distributae, alla stessa maniera di W. H. HARvex con le Algae Australicae exsiccatae.

(2) Fra le altre persone che raccolsero Alghe Oceaniche per J. G. AGARDH meritano ricordo Lodder, Oakden, Elliot, Spalding, Meredith, Banclinet, Vahl, Cole, Hawker, Collie, Kilner, Brooke, Hussey, Robinson, Molineux, Desmond, Irvine, Johnson, Halloran, King, Ryai, Laing, Walcotr, Ramsay, Gale, Maxwell, Merrifield, Webi, Beal, Archer, Hodgzinson, Dall, Goodwin, Bauer, Engelhardt, Keys, Fairchild.

(3) Cfr. De Toni G. B., G. G. Agardh e la sua opera scientifica (La Nuova Notarisia serie XIII, pag. I-28; Padova 1902); ERIKsson J., Jakob Georg Agardh. Minnesteckning ( $K$. Svenska Vetenskapsakademiens Lefnadst. Bd. 5, I916, pag. I-136).

(4) Vedi nell' elenco bibliografico in fine al presente lavoro.

(5) Vedi nell' elenco bibliografico c. s. 
ultimi tempi due censimenti delle Alghe per l'Australia e Tasmania e per la Nuova Zelanda rispettivamente dal LuCAS ( ${ }^{1}$ ) e dal LAING ${ }^{(2}$, censimenti ai quali conviene ricorrere quando si voglia semplicemente conoscere i nomi delle specie segnalate per quelle terre dell' Oceania. Questi due accurati cataloghi del LucAs e del LaIng si riferiscono però alle Alghe Florideae, Fucoideae, Chlorophyceae e Myxophyceae dell' Oceania, mentre manca affatto un censimento delle Bacillavieae; su queste ultime Alghe microscopiche si posseggono soltanto notizie sparpagliate, spesso incidentali, in moltissime pubblicazioni sia illustrative delle forme planctoniche sia di quelle provenienti dai sondaggi a grandi profondità ; più nota, dal punto di vista diatomologico, è la Malesia per la quale, in base soprattutto agli scritti del Leuduger-Fortmorel, del Cleve e di altri, si ebbe un lavoro sintetico a cura del De Wildeman ( $\left.{ }^{3}\right)$, che non tralasciò di istituire opportuni raffronti fitogeografici. Si può dunque concludere che, se la flora marina quanto alle Alghe superiori è abbastanza bene studiata, scarsissimi sono i contributi diatomologici, tali da non permettere neppure lontani paragoni con le flore di altri mari; lo stesso valga in generale per le Alghe delle acque dolci, per quanto intorno ad esse non manchino pregevoli contributi di Borge, Maskell, Moebius, Nordstedt, Playfair, Schmidle da noi indicati nell' elenco bibliografico.

Da quanto precedentemente fu esposto, è ovvio comprendere che per la flora dell' Oceania riesce oltremodo difficile istituire raffronti ficogeografici, come pur sarebbe desiderabile e come venne tentato, già si disse, da W. H. Harvey e da qualche altro botanico ${ }^{(4)}$.

Le difficoltà che si oppongono allo studioso dipendono esse da cagioni impossibili a togliersi ? o derivano invece dalla qualità dell' ambiente di vegetazione o da altri motivi che occorra rimuovere per poter ottenere un quadro comparativo delle singole flore e investigare le leggi di distribuzione degli elementi costitutivi?

Ardue sono le risposte a entrambe queste domande. La prima troverebbe la sua base risolutiva nella completa conoscenza delle singole flore in guisa da essere in condizione di eseguire tra le flore stesse confronti così fatti da permettere l' indagine di eventuali rapporti e quindi la deduzione di collegamenti floristici. La seconda domanda riceve pur troppo la sua risposta dalla natura stessa dell' Oceania, che ci impedisce il necessario ordine di ricerche, inquantochè, eccezion fatta della Nuova Olanda, della Tasmania, e della Nuova Zelanda meglio investigate rispetto alle ficee marine, noi ci troviamo di fronte a un numero rivelantissimo di isole, di isolotti, di piccoli scogli remoti assai o aggruppati in arcipelaghi, le cui flore algologiche sono conosciute solo in modo imperfetto $o$, in parecchi casi, completamente ignote.

In realtà se, per le Alghe superiori, sono a sufficienza note le flore marine dell' Australia, della Tasmania e della Nuova Zelanda soprattutto in seguito alle

(I) Vedi nell' elenco bibliografico c. s.

(2) Vedi nell' elenco bibliografico c. s.

(3) De Wildeman Em., Prodrome de la Flore Algologique des Indes Néerlandaises. Supplement et tableaux statistiques; Batavia, I899, Impr. de 1' Etat, $8^{\circ}$.

(4) E. Askenasy, illustrando le Alghe raccolte dalla spedizione della "Gazelle 》 diede le aree di distribuzione geografica, ma il numero delle specie è poco rilevante; T. REINboLD, nelle due Memorie riguardanti le ficee dei viaggi della "Valdivia 》 e della «Gauss 》 segui lo stesso metodo; D. Cotton, A e E. S. GEPP fecero altrettanto nei loro pregevoli contributi. 
fondamentali opere già menzionate di Hooker e HARVEY e di altri botanici, come apparisce evidente dagli elenchi dati in luce dal LuCAS e dal LAING, pel rimanente dell'Oceania si posseggono soltanto notizie frammentarie, illustrative di materiali incidentalmente raccolti; basti ricordare che solo alcune isole ebbero notevoli ragguagli, così le isole Chatham da J. G. AGARDH e dal Cotton, le isole Figi, Samoa e Tonga dal Grunow, l' isola Norfolk dal Reinbold, le Kermadecs dai coniugi Gepp, le Hawaii dal Piccone e dalla Tilden, le isole Caroline e Marianne dall' OKamura ecc.; altre isole o arcipelaghi, come le isole Marchesi, quelle della Società, la Nuova Caledonia, l' isola Lord Howe, non escluso l' arcipelago della Sonda, ebbero scarsi contributi, dispersi nelle opere generali o in note particolari, senza poter ricavarne dati sicuri sulla facies della vegetazione.

Non va dimenticato che gli studii moderni (1) si sono a preferenza rivolti a porre in rilievo le condizioni (intensità e qualità delle radiazioni solari, composizione, densità, temperatura delle acque, correnti, maree, natura dei substrati ecc.) più o meno favorevoli allo sviluppo delle piante marine, riuscendo così a determinare la distribuzione dei Talassiofiti col criterio delle zone o regioni di profondità che, modificando le idee, a vero dire, troppo assolute che erano state messe innanzi da J. G. Agardh $\left({ }^{2}\right)$ e da A. S. Oersted $\left(^{(3)}\right.$ furono proposte dal KJellman $\left.{ }^{(}\right)$sotto i nomi di zona littorale (compresa fra i limiti dell' alta e bassa marea), sublitorale (tra il limite inferiore della marea e circa 36 metri di profondità) ed elittorale (al di sotto di 36 metri); alle quali, quantunque un po' artificiose, corrispondono press' a poco le tre zone ammesse dall' ARDissone (5) e dal PREDA $\left(^{6}\right)$ per il Mare Mediterraneo, sebbene le troppe eccezioni tolgano il valore assoluto di queste zone di vegetazione, come con ammirevole antiveggenza era stato affermato circa un secolo fa dal LyngBye (7).

La distribuzione zonale serve bene per le flore locali, ma di essa non potè in ogni caso tenere il debito conto W. H. Harvey nella sua Phycologia Australica, perchè molte delle specie da lui illustrate erano rappresentante da esemplari rejetti sulle spiaggie, anzichè raccolti in posto o dragati da una profondità conosciuta. Malgrado queste deficienze, non torna arduo riconoscere, anche nella flora australasica, una distribuzione batimetrica accordantesi con le leggi generali: infatti le Chlorophyceae (Caulerpa, Struvea, Penicillus, Polyphysa, Apjohnia ecc.) caratterizzano la zona o regione littorale, le Fucoideae (Seirococcus, Scylothalia, Phyllospora, Carpoglossum, Splachnidium, Hormo-

(1) Cfr. a questo proposito De Toni G. B., Alcune considerazioni sulla flora marina ( $L a$ Nuova Notarisia serie XXVII, pag. 6I-7I; Padova I916).

(2) Agardh J. G., Novitiae Florae Sueciae ex Algarum familia, quas in itineribus ad oras occidentales Sueciae annis $1832-35$ collegit et cum observationibus diagnosticis et geographicis etc.; Lundae, 1836 , C. Fr. Berling, $8^{\circ}$.

(3) OeRsted A. So, De regionibus marinis. Elementa topographiae historico-naturalis freti Oeresund; Hauniae 1844 .

(4) Kjellman F. R., The Algae of the Arctic Sea pag. 8 ( $K$. Svenska Vetensk. Akad. Handl. Band XX, N. 5, 1885 ).

(5) Ardissone FR., Phycologia Mediterranea parte I: Floridee pag. 2-6 (Memorie Soc. crittog. ital. vol. I; Varese 1883 ).

(6) PREDA A., Florideae pag. XXIII-XXIV (Flora italica cryptogama pars II: Algae; Rocca S. Casciano, I909, Cappelli, $80 \%$.

(7) Lyngrye H. C., Rariora Codana, opusculi posthumi pag. 6 (220) edit. Eug. Warming (Vidensk. Meddel. fra de nat. Foren. $i$ Kjöbenhavn 1879-80). 
sira ecc.) predominano nella zona sublittorale, la maggior parte delle Florideae costituisce la zona elittorale, non senza eccezione per talune forme a struttura delicata, come Claudea elegans (a 18 metri di profondità), Halodictyon arachnoideum (11-15 metri di profondità) nonchè alcune Fucoideae (ad es. Zonaria (Homoeostrichus) Sinclairii H. et H.) le quali, al pari di piccole Florideae dei generi Catenella, Hypnea, Gymnogongrus ecc.) crescono nella zona prettamente littorale. Non mancano singolari coincidenze biologiche le quali dimostrano uguali adattamenti nelle specie a una zona determinata anche in mari molto lontani come si verifica, per citare un esempio molto dimostrativo, in Catenella Opuntia Grev. che si riscontra nell' Australia in condizioni affatto identiche a quelle in cui essa vegeta nel Mare Mediterraneo e nell' Oceano Atlantico.

Il numero non rilevante di Alghe raccolte in posto in confronto di quelle rejette sulle spiaggie o inviategli dai suoi corrispondenti senza indicazione di sorta impedì a W. H. Harvey di presentare lo stato della distribuzione batimetrica delle specie ma all' insigne botanico non isfuggì, come abbiamo detto, la opportunità di tentare quello della distribuzione geografica, tanto più che la ficogeografia aveva avuto dei precursori ed egli stesso se n' era occupato prima di pubblicare la Phycologia Australica.

Il Lamouroux (') aveva osservato che la distribuzione geografica o topografica delle Alghe non avrebbe avuto per ciascheduna specie un centro con diffusione radiale, ma invece una estensione seguendo le linee delle coste in serie lineari, influenzate dalla comune profondità dell' acqua; aveva egli pure avvertito peculiari vegetazioni nell' Oceano Atlantico a partire dalle regioni verso il polo artico fino a $40^{\circ}$ di latitudine nord; nell' Oceano delle Indie occidentali compresovi il Golfo del Messico; nelle coste orientali dell' America del Sud; nell'Oceano Indiano e' suoi golfi; nei mari della Nuova Olanda; per il Mediterraneo egli aveva notato l' esistenza d' un sistema di vegetazione a esso proprio, esteso fino ai recessi del Mar Nero ; cosi per il Mar Rosso e per i Mari Cino Giapponesi.

In queste traccie progredì W. H. HARvey (2) osservando che le specie di struttura più semplice, le sue «Lower Tribes » hanno un carattere cosmopolita (Ulva, Enteromorpha, Codium tomentosum, molte Confervoideae) e che invece meno indifferenti si addimostrano le Feosporee e le Floridee per quanto tra esse pur non manchino quelle ad estesissima area di distribuzione geografica (Plocamium coccineum, Gelidium corneum, Ceramium varie specie ecc.).

Un fatto assai importante venne rilevato eziandio dallo stesso botanico cioè che le spiaggie dell' Africa meridionale fornite di molte specie ad esse peculiari mostrano una vegetazione per molti rispetti analoga a quella dell' Australia, ma con alcune considerevoli deficienze; mancano all' Africa del Sud affatto le Cystophora tanto largamente rappresentate nell' Oceano Pacifico, mentre non vi mancano lo Splachnidium rugosum (L.) Grev. e la Pterocladia lucida (R. Br.) J. Ag. Vi sono altre cause, indipendenti dal clima, le quali senza dubbio tendono

(I) Cfr. Lamouroux J. V. F., in Ann. Scienc. Nat. T. VII.

Ruprecht F. J., Die Vegetation des Rothen Meeres und ihre Beziehung zu den allgemeinen Sätzen der Pflanzen-Geographie (Mém. Sc. natur. T. VI, pag. 71, St. Petersbourg 1849).

(2) Cfr. Harvey W. H., Manual of the British Marine Algae pag. XXXIII-XXXIV; London I 84 I, J, van Voorst, $8^{\circ}$. 
a circoscrivere la distribuzione delle Alghe; lo stesso LAMOURoux, con molto acume, riteneva le grandi profondità marine costituire delle vere barriere analogamente alle altissime montagne per le flore terrestri, nelle quali però la disseminazione può usufruire di altri fattori ; cos̀̀ pure grandi estensioni di banchi sabbiosi poco propizii per alcune forme di vegetazioni sottomarine, correnti forti, capi prominenti, influenza delle acque dolci immesse nel mare dai grandi fiumi. W. H. Harvey però concluse col considerare sempre il clima quale fattore precipuo nella distribuzione geografica delle ficee marine.

Anche il DickIE $\left({ }^{1}\right)$ non mancò di accennare un fatto che ci può interessare cioè il carattere generale della florula dell' Isola Maurizio è misto, escludendo le specie cosmopolite e endemiche havvi larga percentuale di specie dell' Africa meridionale, delle Indie occidentali e dell' Australia.

In cosi fatta maniera venivano questi botanici a preludere a quei rapporti che poteva mostrare la flora dell' Oceania con quella dell' Africa australe, rapporti che furono più ampiamente estesi dalle ulteriori, sia pure incomplete, cognizioni man mano acquisite per le flore algologiche sia dell' Oceano Indiano che dell' Africa inferiore a partire dai lidi della Somalia, dell' isola di Madagascar, delle isole Riunione e Maurizio. La BARTon - Gepp (2) fino dal 1893 richiamò l' attenzione, con un accurato censimento delle Alghe del Capo di Buona Speranza, sulla comunanza di molte specie tra la flora Capense e quelle dell' Australia e della Terra di Kerguelen. Certo lacune considerevoli non mancano nei tentativi di questi allacciamenti, le quali derivano dal fatto fondamentale che le piccole flore, malgrado tante ricerche, sono ben lungi dall' essere ben conosciute, ma altrettanto certo è che ad ogni nuovo lavoro illustrativo delle flore stesse, nuove coincidenze rendono ognor più probabile il legame tra la flora dell' $\mathrm{Au}-$ stralia, della Tasmania e della Nuova Zelanda con quella dell' Africa meridionale attraverso l' Oceano Indiano, più che non i rapporti floristici con le Indie occidentali $\left(^{(3)}\right.$; a prescindere dalle forme note come cosmopolite ${ }^{(4)}$ che perciò non possono fornire alcuna dimostrazione rigorosa, non poche specie australiane hanno i loro riscontri anche nell' America meridionale, come risulta dalle osservazioni di P. HaRIOT ( $\left.{ }^{5}\right)$.

Si potrebbero scegliere moltissimi esempii di specie, di più sicura determinazione, atti a suffragare i concetti della BARTON - GEPP, senza che le particolari aree di tali specie possano confondersi con quelle delle forme sconosciute. L'Amansia Dietrichiana Grun. di Port Mackay (Nuova Olanda orientale) venne dal BoRnet indicata per l' isola di Madagascar e da F. Schmitz per l'A Africa orientale, la Martensia elegans Her. di Port Natal (Africa orientale) si collega alla flora della Nuova Olanda austro - occidentale mediante esemplari raccolti, secondo il Dickie, all' isola Maurizio: il comune Splachnidium rugosum Grev.

(1) Dickie G., On the Algae of Mauritius pag. 202 (Journ. Linn. Soc. Botany, vol. XIV, London 1874).

(2) Barton E. S., A Provisional List of the Marine Algae of the Cape of Good Hope (Journal of Botany vol. XXXI, 1893).

(3) Murray G., A Comparison of the Marine Floras of the Warm Atlantic, Indian Ocean, and the Cape of Good Hope (Phycological Memoirs, part II, 1893, pag. 65-69).

44) Cfr. Ardissone Fr., Le Alghe cosmopolite (Rendic. R. Istituto Lombardo di scienze e lettere, ser. II, vol. XXVII, I894).

(5) Cfr. Hariot P., Algues (Mission du Cap Horn, V, Cryptogamie; Paris 1889). 
dell' Australia, Tasmania e Nuova Zelanda non manca all' Africa meridionale ed, in una forma minore, trovasi, secondo il GRunow, presente all' isola di San Paolo; ad analoghe osservazioni si prestano Neurymenia fraxinifolia (Mert.) J. Ag., Champia compressa Harv. ecc. mentre non mancano Florideae, come Vanvoorstia spectabilis Harv. che segnano le relazioni tra l' isola di Ceylon, l' isola Maurizio e la costa occidentale dell' Africa.

Si aggiunga che alcuni generi sono rappresentati da una specie nell' Africa meridionale, da un' altra nell' Australia o nella Tasmania; valgono ad esempii Glaphyrhymenia pustulosa J. Ag. e Glaphyrhymenia porphyroidea Schm.; Meristotheca tasmanica J. Ag. e Meristotheca natalensis J. Ag., Heringia filiformis Harv. e Hevingia mirabilis (Ag.) J. Ag. E rappresentanti nell' una e nell' altra flora hanno, tra le Florideae, moltissimi generi come Thysanocladia Endl., Phacelocarpus E. et D., Sarcodia J. Ag., Corallopsis Grev., Rhodymenia Grev., Delisea Lamour., Aeodes J. Ag., Dasyphila Sond., Haloplegma Mont., Schizymenia J. Ag., Peyssonnelia Decne, Vidalia Lamour., Prionitis J. Ag., Pachymenia J. Ag., Euptilota Kuetz. e va' dicendo.

Pur accettando quest' insieme di collegamenti floristici, resta il fatto che parecchi generi sono esclusivamente proprii dell' Oceania e in questa talvolta dotati di aree molto circoscritte; così abbiamo specie o anche generi schiettamente australiani (Sonderia Bennettiana (Harv.) F. Müll., Asparagopsis Sanfordiana Harv., Nizymenia australis Sond., Acrotylus australis J. Ag., Hennedya crispa Harv., Gattya pinnella Harv., Dasyphila Preissii Sond., Ptilocladia pulchra Sond., Bindera splachnoides Harv., le Gelinaria, Cliftonaea, Amansia e Vidalia, gli Erythroclonium ecc.) o tasmanici (Martensia gigas Harv., Lenormandia marginata Hook. et Harv., Chiracanthia arborea (Harv.) Falk., Heterosiphonia Archeri (Harv.) De Toni, Wrangelia Jeannerettii Hook. et Harv., Gunniana J. Ag. e ballioides J. Ag., Meristotheca tasmanica J. Ag., Apoglossum tasmanicum (F. Müll.) J. Ag., i Chaetangium) o neozelandici (Euptilota formosissima (Mont.) Kuetz., Plumaria pellucida (Harv.) Schm., Pandorea Traversi J. Ag., Callophyllis Hombroniana Mont., calliblepharoides J. Ag.., decumbens J. Ag. e centrifuga J. Ag., Sarcodia Montagneana (Hook. et Harv.) J. Ag., Cladhymenia Lyallii Hook. et Harv. e oblongifolia Hook. et Harv., Metamorphe Colensoi (Hook. e Harv.) Falk., Dasyphloea insignis Mont., alcune specie di Euzoniella, Heterosiphonia, Microcladia, le Ectophora e Apophloea ecc.).

$\mathrm{Col}$ presente lavoro, risultato dallo studio d' un materiale copioso fornitoci dal CAPRA, viene maggiormente provata l' esistenza di rapporti floristici tra il Continente Australiano, la Tasmania e la Nuova Zelanda. Collegamenti nuovi tra la Nuova Zelanda e l'Australia sono forniti da Mychodea foliosa (Harv.) J. Ag., Herposiphonia Filipendula (Harv.) Falk., Sargassum lacerifolium (Turn.) J. Ag., Cystophora Cephalornithos (Labill.) J. Ag. e da altre meno rare specie, tra la Nuova Zelanda e la Tasmania da Polysiphonia mollis Hook. et Harv., Cladosiphon zostericola Harv., Codium Muelleri Kuetz. ecc.; di altre specie è estesa l'area di distribuzione, venendo esse indicate in località nuove sia dell' Australia e Tasmania che della Nuova Zelanda.

Abbiamo ritenuto opportuno limitarci ad accompagnare ciascuna specie con le indicazioni relative all' area di distribuzione più interessanti per l' Oceania, sembrandoci più conveniente rimandare, per quanto concerne la distribuzione geografica mondiale, alle opere generali di algologia descrittiva. 
Il materiale di cui forma oggetto la nostra memoria proviene dalle località seguenti: 1. Avon River, Australia occidentale; 2. Baia di Geroldton, id.; 3. Victor Harbour, Australia meridionale; 4. Porto di Adelaide, id. ; 5. Daylesford, Victoria; 6. Baron River, N. Queensland; 7. Burmie, Tasmania; 8. Baia di Latrobe, id.; 9. Hobart, id.; 10. Deloraine, id.; 11. Fiume Tamar, id.; 12. Bluff, Nuova Zelanda; 13. Lyttleton, id. ; 14. Port Charmers, id.; 15. Rotorua [Geyser] id.; 16. Rotorua (Ohinemuthu), id.; 17. Wakarevareva, id.

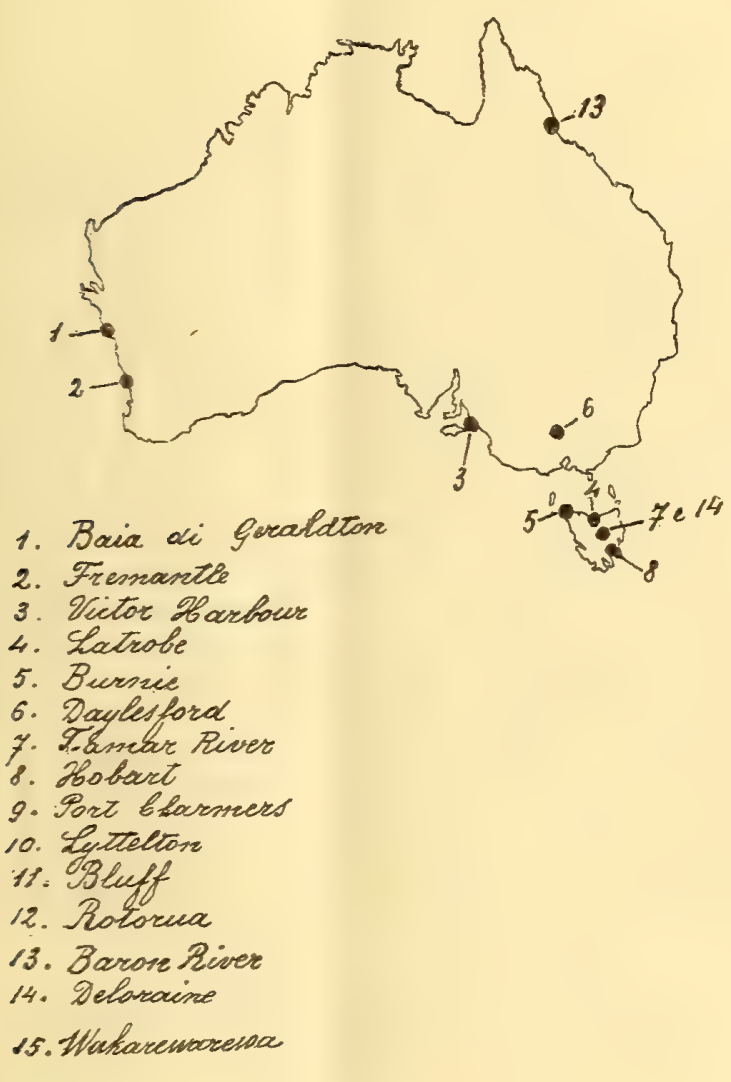

Scake $\frac{4}{72,000.600}$

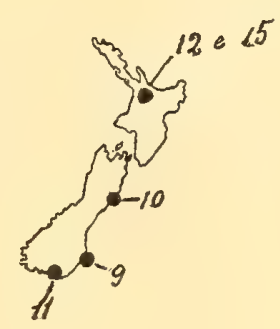





\title{
FLORIDEAE Lamour.
}

\section{Subclassis I. BANGiOIDEAE Schm. et Hauptfl.}

\section{Fam. BANGIACEAE (Zanard.) Berth.}

\author{
Porphyra C. Ag.
}

Porphyra nobilis J. Ag. Till Algernes Systematik VI (1882) pag. 62, tab. II, fig. 49-50; De Toni Sylloge Algarum IV (1897) pag. 15; Laing Revised List of New Zealand Seaweeds I (1899) pag. 61, n. 35.

Hab. Nuova Zelanda: Lyttleton; 6 febbraio 1909.

Esemplari di piccole dimensioni (due con fronda di $2-4 \mathrm{~cm}$.), a fronda monostromatica e di colore tendente al livido; essi ricordano alquanto la Porphyra crispata Kjellm. Japanska Arter af Slàgtet Porphyra (1897) pag. 15, tab. I, fig. 4-5, tab. III, fig. 5-7, tab. V, fig. 15. La specie venne per la prima volta descritta da J. Agardh su esemplari raccolti nei lidi della Nuova Zelanda da S. BERGgren; la presenza in quella regione fu confermata, essendo la $P$. nobilis $\mathrm{J}$. Ag. stata raccolta all' isola Campbell da J. Crosby Smith; cfr. LAing R. M., The marine Algae of the Subantarctic Islands of New Zealand (1909) pag. 504.

Piccoli esemplaretti raccolti dal CAPRA nella Baia di Geroldton (Australia occidentale) rappresentano primordii di sviluppo d' una Porphyra indeterminabile ma è assai probabile appartengano a Wildemania laciniata (Lightf.) D. T. J.D. Hooker (Handbook of the New Zealand Flora, 1867, pag. 715) aveva attribuito alla Nuova Zelanda tre specie: $P$. laciniata Ag., $P$. vulgaris Ag. e $P$. capensis Kuetz. (P. columbina Mont.) ; ma mentre dapprima J. AgardH (De Algis Novae Zelandiae marinis, 1877, pag. 1) aveva per la flora neozelandese accettato le due prime specie ommettendo la terza, più tardi nell' opera sopra citata restrinse l' area di distribuzione geografica all' Oceano Atlantico superiore per la Porphyra vulgaris Ag. e riferì la P. lacinıata Ag. attribuita alla Nuova Zelanda alla $P$. columbina Mont., mantenendo quest' ultima affatto distinta dalla $P$. capensis Kuetz esclusiva del Capo di Buona Speranza (Africa meridionale); la $P$. capensis di Hooker, come giustamente osservò il LAING, è sinonimo di $P$. columbina e siccome possiede tallo distromatico appartiene al genere Wildemania De Toni. 


\title{
SubcLAsSIS II. EUFLORIDEAE
}

Ordo I. Nemalioninae Schm.

\section{Fam. HeLMinthocla DIACEAE (Harv.) Schm.}

\author{
Subfam. Chantransieae (Kuetz.) Trev.
}

\author{
Chantransia (D. C.) Schmitz.
}

Chantransia corymbifera Thur. in Le Jolis Liste des Algues marines de Cherbourg (1863) pag. 107; De Toni Sylloge Algarum IV, pag. 69.

Hab. Nuova Zelanda: Bluff, su frondi di Stilophora rhizodes (Ehrh.).

Del genere Chantransia finora, per la Nuova Zelanda, erano state indicate: Chantransia Naumanni Asken. (Ch. interposita Heydr.), per la Nuova Olanda Ch. polyrhiza (Harv.), Ch. radicans (Harv.) e Ch.? botryocarpa (Harv.); per la Tasmania si trova un esemplare, determinato come Chantransia Daviesii, nell' Erbario Zanardini conservato al Museo Civico di Venezia. Esemplari di Ch. corymbifera Thur. epifiti su Helminthocladia purpurea (Harv.) J. Ag. vennero raccolti però nell' Oceano Pacifico a S. Pedro di Calitornia da H. D. Johnston e distribuiti al n. 1040 della Phycotheca Boreali-Americana così che non può ritenersi un elemento nuovo per codesto Oceano.

\section{Fam. CHAETANGIACEAE Schmitz}

\section{Galaxaura Lamour.}

Galaxaura dolicharthra J. Ag. in Kjellm. Om Floridé - Slägtet Galaxaura (1900) pag. 85, tab. 18, fig. $33-44$, tab. 20. fig. 55.

Galaxaura umbellata b. major J. Ag. Epicrisis (1876) pag. 526; De Toni Sylloge Algarum IV, pag. 111.

Galaxaura umbellata Harv. Phyc. Australica IV (1862) tab. 228 (non Lamouroux); Sonder Algen des tropischen Australiens (1871) pag. 61.

Galaxaura major Decaisne Classif. des Corall. (1842) pag. 103.

Hab. Australia occid: Baia di Geroldton; settembre 1908.

La sinonimia delle Galaxaura è assai intricata per i riferimenti fatti dagli autori a forme dapprima credute polipai, come si può scorgere nelle opere di Ellis e Solander, di Esper, di Lamarck, di Lamouroux e d' altri; così avvenne che sotto il nome di Galaxaura umbellata furono confuse più specie, che la moderna critica, soprattutto a merito di J. AgardH e di KJELlman, distinse benchè in maniera non di raro soverchia sottilizzando troppo su minuti particolari diagnostici e sostituendo nuovi nomi ai più antichi ; laonde, seguendo J. AGARDH, i nostri esemplari vanno ascritti alla Galaxaura dolicharthra, meglio descritta che non la Galaxaura major della quale il Decaisne fornì una frase diagnostica 
poco atta a discernerne le differenze dalle specie affini Gal. obtusata, Gal. umbellata e Gal. oblongata; solo nel DeCAISNE troviamo che di queste quattro specie riunite nella prima sezione Dichotomaria, la Gal. major (Gal. umbellata var. major Lamour. herb.) proviene dalla Nuova Olanda con i materiali raccolti nel viaggio compiuto dal FrEYcINET, laddove le altre appartengono alla flora dell' Oceano Atlantico. Com' è noto, il genere Galaxaura è costituito da specie proprie delle regioni calde e temperate, una sola tra esse, la Galaxaura adriatica Zanard., vegetando nel Mare Mediterraneo (1), un' altra, la Galaxaura Schimperi Decne., nel Mar Rosso (2).

Il SONDER nel suo censimento delle Alghe Australiane (Algae Ausiralianae hactenus cognitae ; Melbourne 1878 -1881) al n. 619 riassunse l' area di distribuzione geografica per la Galaxaura umbellata Lamour. var. oblongata Decne. e var. obtusata Harv.; per la Gal. dolicharthra però occorre riferirsi alla sinonimia sopra esposta e in particolare al riferimento di Gal. obtusata di HARveY il quale, pur confondendo la sua specie con quella del Lamouroux e con l'Alysium Holtingii (Mert.) Ag. e perciò dando a essa un' ampia distribuzione geografica nelle regioni oceaniche tropicali in tutte le longitudini, ricordò che $\mathrm{i}$ proprii esemplari vennero raccolti nell' Australia occidentale da G. Clifton e nell' isola Norfolk dal D.r Mc William; nel Synoptic Catalogue of Australian and Tasmanian Algae (Londra, 1863) egli aggiunse, in base a materiali dell' erbario GREville, la Tasmania. Nei nostri esemplari le articolazioni della fronda variano in lunghezza tra 8 e 13 millimetri e in larghezza tra 2 e 2,8 millimetri.

\section{Chaetangium Kuetz.}

Chaetangium variolosum (Mont.) J. Ag. Species Algarum II (1852) pag. 461; Epicrisis (1876) pag. 539; De Toni Sylloge Algarum IV, pag. 118.

Nothogenia variolosa Mont. in Ann. Sc. Nat., Botan., (1843) pag. 303; Voyage au Pole Sud (1845) pag. 109, tab. 10, fig. 3.

(1) Cfr. Bentrvoglio Trro, La Galaxaura adriatica Zanard. e la sua area di distribuzione nel Mediterraneo (La Nuova Notarisia serie XIV, 1903, pag. I09). La Galaxaura adriatica distribuita dalla TrLden nelle American Algae Cent. V n. 4I5 di Waianae, Oahu va ascritta alla Gal. fragilis (Lamk.) secondo F. K. Butters, Notes on the Species of Liagora and Galaxaura of the Central Pacific (rgir) pag. I79; cfr. sull' esemplare della Tilden le osservazioni in MAzzA A., Saggio di Algologia Oceanica n. 36 .

(2) A Galaxaura Schimperi Decne devesi attribuire Gal. dactyliophora Picc. et Grun. (Algol. Eritrea in N. Giorn. bot. ital. vol. XVI, I884, tab. VIII, fig. 7) differente forse soltanto per la fronda più sottile e slanciata. II KJELLMAN nella sua monografia non tenne affatto conto di questa specie. Per verità essa venne tenuta fin da principio come una varietà della Gal. cylindrica Lamour., almeno cosi è scritto nella scheda originale; del resto già nella Sylloge Algarum a proposito di Gal. cylindrica e Gal. fragilis si accennava a non ritenerle specie schiette e si tendeva a separarne gli esemplari eritrei; quello che fece il KJELLMAN con Gal. Schimperi Decne. Interessanti ancora sono gli isolati reperti nel Mar Rosso di Galaxaura lapidescens auct. rappresentati nell' erbario Piccone (ora ForTr) da due esemplari: 1' uno di Suez (FIGARI BEY) forse rimaneggiato, benchè il cartellino di mano del DE NOTARIS non ne accenni, ricorda assai gli esemplari dell' Oceano Indiano sulla costa dei Somali (Galaxaura clavigera Kjellm.), l'altro è detto Gal. lapidescens var. annuligera Picc, et Grun. (in PICcONE Algol. Eritrea pag. 3I2, n. 67) e parrebbe piuttosto avvicinarsi a Gal. lenta Kjellm., specie intermedia fra i due gruppi Microthoé e Brachycladia, nota dell' Oceano Indiano; venne raccolta da CESARE Marcaccr nella Baia di Assab (marzo 1880) in due frammenti; anche questa forma venne trascurata dal KJeLlman. 
Hab. Australia occid. : Baia di Geroldton; settembre 1908.

La specie venne descritta per la prima volta su esemplari raccolti da D' URville e Hombron sulle coste delle isole Auckland; più tardi essa fu segnalata per l'Australia a Port Phillip nel censimento delle Alghe del SoNDER al n. 630; il LAING indica il Chaetangium variolosum anche per l' isola Campbell.

Fra questa specie che dimostrerebbe piuttosto attitudine a ritrovarsi sulle coste dell' Oceano Pacifico meridionale o dell' Oceano Indiano o, in altri termini, la cui area d' espansione geografica si estende dall'isola di Kerguelen alla Nuova Zelanda e il Chaetangium fastigiatum (Bory) J. Ag. proprio delle Maluine, della Terra del Fuoco, dello Stretto di Magellano, ecc. corre diversità per la fronda saccato - tubolosa invece che complanata. Peraltro nel secco torna facile l' equivoco ed avvenne assai di frequente, fomentato altresì dall' avere J. AGARDH distribuito i primi esemplari di Ch. fastigiatum dell' is. Maluine orientali (gli stessi poi dati fuori nella raccolta di HoHENACKer) sotto il nome di Ch. variolosum ; così probabilmente avvenne a P. Hariot (Baie d' Orange, Terra del Fuoco) al Piccone e al Grunow (Stretto di Magellano [Vettor Pisani]) d' essere indotti nel medesimo errato apprezzamento.

Del Chaetangium corneum J. Ag. Analecla Algologica contin. V (1899) pag. 106 che forma cespugli subglobosi ed ha fronda «teretiuscula decompositodichotoma superne ob segmenta approximata subpolychotoma» e fu raccolto dalla sig. Hussey a Port Elliot (Australia meridionale) in mancanza di esemplari autentici non possiamo profferire alcun giudizio.

\section{Fam. GELIDIACEAE (Kuetz.) Schm.}

\section{Gelidium Lamour.}

Gelidium asperum (Mert.) Grev. Alg. Brit. Syn. (1830) pag. LVII; Kuetz. Tab. Phyc. XVIII (1868) tab. 43, fig. I ; De Toni Sylloge Algarum IV, pag. 154. Hab. Australia merid.: Victor Harbour; 12 novembre 1908.

La specie, già segnalata dal LABILLARDIEkE per l'Australia, vegeta soprattutto nella regione meridionale di questo Continente e nella Tasmania; gli esemplari indicati col nome di Gelidium asperum per la Nuova Zelanda da alcuni autori, vanno riferiti a altre specie, non figurando questo Gelidium nei censimenti pubblicati da J. Agardh e da R. M. Laing per la flora di quelle isole. La stazione classica o, per lo meno, donde proviene la maggior parte degli esemplari distribuiti è Port Phillip che si trova alquanto lontano da Victor Harbour; per tale maniera è lecito ritenere che la specie si trovi abbastanza di frequente lungo i lidi meridionali dell' Australia e che quivi sia il posto dove meglio si sviluppa. Gli esemplari raccolti dal CAPRA sono due con frondi sottili ed eleganti. 
Ordo II. Gigartininae Schmitz.

Fam. ACROTYlaCEAE Schm.

Hennedya Harv.

Hennedya cRISPa Harv. in Trans. R. Irish Academy vol. XXII (1852) pag. 552 ; Phyc. Australica II (1859) tab. 75; Kuetz. Tab. Phyc. XIX (1869) tab. 37; De Toni Sylloge Algarum IV, pag. 172; Mazza Aggiunte al Saggio di Algologia Oceanica n. 685. Icon. Tab. nostra I (IV), figg. 1, 2.

Hab. Australia occid. : Baia di Geroldton, con cistocarpi ; settembre 1908.

Abbonda questa specie, secondo Harvey, all' isola Rottnest e vegeta anche a Fremantle, donde egli l' ebbe da G. Clifton; giusta il censimento Sonder (Alg. Austral. n. 633) fu raccolta anche nella Geographe Bay ; l' erbario ZANARDINI al n. 597 ne contiene esemplari di provenienza australiana, senza precisa indicazione di località.

I due esemplari raccolti dal CAPRA sono così differenti fra loro nell' aspetto esterno della fronda da lasciar adito a credere di primo acchito non trattarsi di individui appartenenti alla stessa specie. L' uno più si assomiglia alla figura datane da W. H. Harvey, ha consistenza simile a quella del Chondrus crispris, superficie levigata, quasi lucida, lacinie ampie, poco frastagliate, non molto arricciate ai margini, translucide; la fronda per luce rifratta mostra colore piceo bruno, per trasparenza vermiglio scuro; l'altro invece, che forse rappresenta un individuo più tenero, pur conservando una notevole elasticità, non ha la consistenza del precedente; di più ha fronda arricciatissima ai margini e superficie sottilmente rugulosa così che la fronda, specialmente nei siti di maggior spessore, perde la trasparenza. Questo fenomeno di variabilità è noto peraltro anche in parecchie altre Florideae; così si scorge nel suaccennato Chondrus crispus (L.) Stackh., in Phyllophora Brodiaei (Turn.) J. Ag., Delesseria sanguinea (L.) Lamour. ecc.

\section{Fam. GIGARTINACEAE Schmitz.}

Subfam. Gigartineae $J$. Ag.

Iridaea Bory

IridaEA LAtissima (Hook. et Harv.) Grun. Alg. Exped. Novara (1867) pag. 69, tab. IX, fig. $3 a-d$; De Toni Sylloge Algarum IV, pag. 191 ; Lucas Revised List (1909) pag. 24. Mazza Aggiunte al Saggio di Algologia Oceanica n. 686.

Halymenia latissima Hook. et Harv. Flora Antarctica (1847) tab. 73, fig. 1 ; Handbook of N. Zeal. Flora (1867) pag. 703; Kuetz. Tab. Phyc. XVI (1866) tab. 92. Rhodoglossum latissimum J. Ag. Epicrisis (1876) pag. 187 partim, De Algis 
Novae Zelandiae marinis (1877) pag. 15, n. 123; Laing The mar. Algae of the Subantarctic Islands of New Zealand (1909) pag. 508.

Hab. Nuova Zelanda: Port Charmers, con sporangi; 30 gennaio 1919.

Dalla località sopra indicata il CAPRA ci riportò due esemplari; il più grande lungo 38 centimetri, largo nel sito della maggiore ampiezza 13 centimetri, costituito da una grossa lamina spessa, trifida, con lobi troncati, munito di tre proliferazioni più sottili, lanceolate laterali; l' altro ha forma ellittica ed è privo di proliferazioni, in complesso rammenta abbastanza bene la figura delle Tabulae Phycologicae. I sori sporangiferi sono puntiformi, immersi, subcorticali, sparsi senz' alcuna regola; la maggior parte dagli sporangi mostra un solo setto traversale, come si verifica non di raro negli sporangi crocipartiti e come appare anche da una delle figure esibite dal Grunow.

L'Iridaea latissima (Hook. et Harv.) Grun. fu già raccolta sui lidi neozelandesi da S. BERGgren, poscia nella Lyall 's Bay e ad Akaroa dal LAing; vegeta eziandio alle isole Auckland, Campbell e Enderby secondo Hooker, Grunow e LAING ; esemplari cistocarpiferi riferiti a questa specie vennero indicati per l' isola Whidbey (America occidentale) da Setchell e Gardner (Algae of North-western America pag. 300 ; Berkeley 1903); che l' area di distribuzione di questa Iridaea si estenda fino alla Tasmania fu posto in dubbio; cfr. Lucas A. H. S., Revised List pag. 24; anche J. Agardh Till Algernes Systematik VII (1885) pag. 27.

Subfam. Mychodeae Schmitz.

Mychodea Harv.

Mychodea foliosa (Harv.) J. Ag. Epicrisis (1876) pag. 573; De Toni Sylloge Algarum IV, pag. 266; Mazza Saggio di Algologia Oceanica n. 98.

Gymnogongrus foliosus Harv. Alg. Austral. exsicc. n. 396, Phyc. Australica IV (1862) tab. 194 ; Kuetz. Tab. Phyc. XIX (1869) tab. 62, fig. II.

Hab. Nuova Zelanda: Port Charmers, a poca profondità; 30 gennaio 1909.

La Mychodea foliosa (Harv.) J. Ag. era nota finora per l' Australia meridionale sulla scorta dei dati esibiti da Harvey, Sonder e Lucas; cosi si conosce di Port Phillip, Victoria (Grunow in Erb. PICCONe, ora ForTi) raccolta da F. Mueller. Vegeta essa per lo più epifita su Alghe maggiori (Perithalia, Sargassum ecc.) o sui cauli di Ruppia; però l' esemplare nostro è probabile vivesse attaccato alla roccia; è molto sviluppato in confronto di quelli distribuiti dal Mueller.

Il Reinbold (Die Algen der Lacépède und Guichen Bay n. 196; Padova 1898) ne distinse una varietà elata per esemplari comunicatigli dal dott. A. ENGELHART.

\section{Subfam. Dicranemeae Schmitz}

\section{Dicranema Sond.}

Dicranema Grevillei Sond. in Botanische Zeitung 1845, pag. 56; J. Ag. Florideernes Morphologi (1879) tab. 26, fig. 4-5; Harv. Phyc. Australica II (1859) 
tab. 120; De Toni Sylloge Algarum IV, pag. 269; Mazza Saggio di Algologia Oceanica n. 100.

Cystoclonium Grevillei Kuetz. Tab. Phyc. XVIII (1868) tab. 17.

Gracilaria pumila Grev. in Edinb. Journ. of Natur. and. Geogr. Sc. III (1831) pag. 328 cum icone.

Hab. Australia merid. : Victor Harbour.

Presenta 1' aspetto della Heringia mirabilis (Ag.) J. Ag. e fu raccolta questa specie lungo le spiaggie meridionali e occidentali dell Australia; forse non è a sufficienza distinta dal Dicranema revolutum (Ag.) J. Ag. se non per avere quest' ultimo gli apici delle frondi, situati oltre i cistocarpi, più circinati o revoluti e le estremità rigonfie sporangifere pur revolute in luogo di essere quasi rettilinee come nel Dicranema Grevillei; entrambe le specie crescono, secondo W. H. Harvey, sopra la stessa matrice; il $D$. Grevillei è di solito di maggiori dimensioni; le due specie sono in egual modo distribuite sui lidi meridionali e occidentali dell' Australia.

Dicranema revolutum (Ag.) J. Ag. Species Algarum II (1852) p. 634; Harv. Phyc. Australica II (1859) tab. 74; Kuetz. Tab. Phyc. XIX (1869) tab. 38; De Toni Sylloge Algarum IV, pag. 269; Mazza Saggio di Algologia oceanica n. 99.

Hab. Australia occidentale: Baia di Geroldton; settembre 1908.

A proposito di questa specie valgono le osservazioni fatte riguardo al $D i$ cranema Grevillei Sond.; come quello, trovasi attaccato ai cauli di Ruppia e anche d'altre Fanerogame marine.

\section{Fam. RHODOPHYLLIDACEAE Schmitz.}

\section{Subfam. RHoDophylLidEAE Schmitz.}

Rhodophyllis Kuetz.

Rhodophyllis acanthocarPa (Harv.) J. Ag. Epicrisis (1876) pag. 364, De Algis Novae Zelandiae marinis (1877) pag. 21, n. 177 ; Asken. Alg. Forschungsreise * Gazelle» (1888), pag. 53; De Toni Sylloge Algarum IV, pag. 343; Laing Revised List pag. 341, n. 224 ; Mazza Aggiunte al Saggio di Algologia oceanica n. 736. Icon: Tab. nostra I (IV), fig. 3, 4, 5.

Callophyllis acanthocarpa Harv. in Hook. Fl. N. Zel. II (1855) pag. 251, Handbook of New Zealand Flora (1867) pag. 697.

Hab. Nuova Zelanda: Port Charmers, acque basse, con cistocarpi ; 30 gennaio 1909 .

E specie più volte riscontrata nella regione neozelandese; raccolta dapprima al C. Cooper (coste orientali) da Lyall e Colenso secondo Hooker, venne poscia rinvenuta a Patterson's Inlet, Timaru, Lyttelton e Lyall 's Bay dal LAING, all' isola Chatam dal Travers secondo J. AgardH, all' isola Auckland dal Laing su esemplari identificati da A. GEPP. La Rhodophyllis acanthocarpa (Harv.) J. Ag. somiglia, giusta J. D. HooKer, a Callophyllis Hombroniana (Mont.) Kuetz; il paragone non sembra però dei meglio appostí sia perchè se ne differenzia bene per i concettacoli aculeati e per la forma delle pinnule che sono sempre 
a margine intiero sia perchè la fronda vi è molto più sottile e flessibile anche nel secco e la ramificazione meno slanciata.

Questa specie si dimostra anche alquanto incostante nelle proporzioni della fronda e nell' aspetto, frequenza e relativa grandezza delle pinnule; queste bene spesso possono assurgere a misure equivalenti alla fronda e come essa fruttificare. In esemplari tetrasporiferi raccolti dal LAING a Timaru nella Nuova Zelanda (che si trovano nell' erbario di ANGelo MAZZA) si scorgono i larghi sori invadere pure taluna delle pinnule maggiori. I cistocarpi eziandio sono ornati a volte di un solo aculeo, talvolta ne recano due o anche tre; sono, come è norma per il genere Rhodophyllis, del tutto inseriti sul margine del tallo e si osservano più o meno fitti relativamente fra di loro, talvolta quasi contigui, spesso anche di molto remoti, rimanendone pure intieri lobi completamente privi, malgrado che i lobi stessi siano del tutto sviluppati dal lato vegetativo.

Rhodophyllis multipartita Harv. Alg. Austral. exsicc. n. 370; Hook. Fl. Tasman. II (1860) pag. 318 ; De Toni Sylloge Algarum IV, pag. 346; Kuetz. Tab. Phyc. XIX (1869) tab. 51.

Hab. Tasmania: Baia di Latrobe.

Un solo piccolo frammento di fronda sterile ma corrispondente assai bene ad esemplari del Lenormand (Erb. Piccone, 1864) e del Sonder provenienti dall' Australia (Port Phillip). Le ramificazioni più sottili possono ricordare la figura delle Tabulae Phycologicae con più evidenza che non gli esemplari di Port Phillip; ma resta così dimostrato che l' una e gli altri debbono certamente corrispondersi senza ulteriore dubbio; anche l'esemplare del Kuetzing infatti proviene dalla Tasmania, forse che l'assottigliarsi della fronda sia proprio agli individui provenienti di colà.

Rhodophyllis ramentacea (Ag.) J. Ag. Epicrisis (1876) pag. 365 ; De Toni Sylloge Algarum IV, pag. 344 ; Lucas Revised List pag. 27 ; Mazza Saggio di Algologia oceanica n. 125. Icon: Tab. nostra I (IV) fig. 6.

Calliblepharis ramentacea J. Ag. Species II (1852) pag. 621; Harv. Phyc. Austral. Syn. (1863) pag. XXXIII, n. 415.

Delesseria ramentacea C. Ag. Species I (1821) pag. 177.

Hab. Australia merid.: Victor Harbour; 12 novembre 1908.

La specie venne descritta la prima volta da C. AgARDH su esemplari del Museo di Parigi provenienti dall' Australia meridionale dove essa pare abbia limitata la propria area. I nostri esemplari quadrano esattamente con uno raccolto a Encounter Bay e distribuito nelle Algae Muellerianae da J. AGardH.

Dell' Australia meridionale, oltre che dal Censimento del Sonder (Algae Austral. n. 473) figura indicata dal Reinbold il quale determinò materiali speditigli dal dott. Engelhart; cfr. Reinbold Th., Die Algen der Lacépède und Guichen Bay I, pag. 49, n. 48; Padova 1897. - Gli esemplari raccolti dal rev. G. CAPRA provengono tutti da Victor Harbour; il più grande raggiunge benissimo i $15 \mathrm{~cm}$. di fronda, non aderisce alla carta seccando e mostra il colore roseo sporco cangiante in verde olivaceo già rilevato nella descrizione originale di J. AGARDH ; sembra esservi parecchio frequente a giudicare anche dai trammenti di fronda numerosissimi che si trovano interpolati alle altre alghe del convoluto. 
Subfam. Solierieae Harv.

\section{Erythroclonium Harv.}

Erythroclonium Muelleri Sond. in Linnaea vol. 25 (1852) pag. 692; Harv. Phyc. Australica V (1863) tab. 298; Kuetz. Tab. Phyc. XVI (1866) tab. 72 ; De Toni Sylloge Algarum IV, pag. 355.

Hab. Australia occid. : Baia di Geroldton; settembre 1908.

A C. J. Connolly (Beiträge zur Kenntniss einiger Florideen pag. 156, fig. 17-20, in Flora vol. 103, 1901) devesi un' illustrazione di questo Erythroclonium quanto alla struttura e all' accrescimento secondario della fronda. Si tratta di specie non comune, raccolta in poche località dell' Australia, nella penisola Lefebre dal Mueller, nello Investigator Street e nelle Baie di Lacépède e Guichen (Australia meridionale) rispettivamente dalla sig. N. DAvey e dal dott. A. ENGELHART. L'Erythroclonium Muelleri Sond. fu con molta facilità confuso con la Rhabdonia verticillata; la località Port Phillip, per esso indicata dal SonDER (Algae Austral. hactenus cognitae n. 394) spetta in realtà a quest'ultima specie giusta l' esemplare distribuito da W. H. Harvey nelle Algae austral. exsicc. n. 390. Intorno queste somiglianze tra specie di generi differenti richiamarono l' attenzione J. AgaRdH (Analecta algologica contin. IV, pag. 37; Lundae 1897) e Th. Reinbold (Die Algen der Lacépède und Guichen Bay pag. 43, n. 204 ; Padova 1898).

Erythroclonium Sonderi Harv. Alg. Austral. exsicc. n. 391 ; Phyc. Australica II (1859) tab. 86 ; De Toni Sylloge Algarum IV, pag. 354.

Rhabdonia Sonderi Harv. in Trans. R. Irish Academy vol. XXII (1852) pag. 554 (non J. Agardh).

Hab. Australia occid.: Baia di Geroldton; settembre 1908.

La specie, raccolta dapprima a Fremantle da G. Clifton, venne poscia riscontrata da HaRvey a Garden Island (Australia occidentale) e più tardi dal Reinbold nell' Australia meridionale. Esemplari distribuiti sotto il nome di Erythroclonium Sonderi dalla Geographe Bay, stando al materiale da noi posseduto, corrispondono piuttosto alla Rhabdonia Sondevi J. Ag. ossia al Coeloclonium opuntioides (Harv.) J. Ag.

\section{Rhabdonia Harv.}

Rhabdonia charoides Harv. Phyc. Australica IV (1862) tab. 196; De Toni Sylloge Algarum IV, pag. 359; Lucas Revised List pag. 28.

Erythroclonium charoides Harv. Alg. Austral. exsicc. n. 393.

Hab. Australia merid.: Victor Harbour; Australia occid.: Baia di Geroldton, frammenti di fronda.

La stazione della Baia di Geroldton riuscirebbe di particolare importanza se potesse venir confermata con nuovi reperti perchè sarebbe la prima volta che la specie viene osservata sui lidi occidentali dell' Australia. Gli esemplari di Victor Harbour sono grandi e bene sviluppati. 
Rhabdonia Dendroides Harv. Phyc. Australica III (1860) tab. 152; De Toni Sylloge Algarum IV, pag. 3j̄; Lucas Revised List pag- 28.

Hab. Tasmania: Burmie, roccie battute dalle onde; 15 gennaio 1909.

E specie ad area di distribuzione geografica, stando alle attuali cognizioni, piuttosto ristretta, limitandosi al Capo Shank (BARKer), Western Port (HARVeY), e Garden Island (G. Clifton) secondo Harvey; anche il Reinbold n' ebbe frammenti sporangiferi dall' Australia meridionale, i quali egli rimase in dubbio di ascrivere a questa specie se non piuttosto alla Rhabdonia clavigera J. Ag.

Il presente esemplare fruttifero sarebbe dunque per la prima volta segnalato della Tasm ania.

Rhabdonia globifera (Lamour.) J. Ag. Species II (1852) pag. 355 (excl. synon. plur.); Harv. Phyc. Australica III (1860) tab. 129; De Toni Sylloge Algarum IV, pag. 360 ; Lucas Revised List pag. 28.

Hab. Australia occid.: Baia di Geroldton; settembre 1908.

La Rhabdonia globifera (Lamour.) J. Ag. è finora localizzata alla regione occidentale dell' Australia, essendo già stata raccolta presso Fremantle dal Clifron. Il Connolly, nell' opera poco addietro citata a proposito dell' Erythroclonium Muelleri, studiò esemplari determinati dal ReINBoLd illustrandone l' accrescimento della fronda e la continuità del citoplasma.

\section{Eucheuma J. Ag.}

Eucheuma speciosum (Sond.) J. Ag. Species II (1852) pag. 628, Analecta algologica (1892) pag. 126; Harv. Phyc. Australica II (1859) tab. 64 ; De Toni Sylloge Algarum IV, pag. 375 ; Lucas Revised List pag. 28.

Gigartina speciosa Sond. in Botanische Zeitung 1845, pag. 55.

Gigartina ornata Kuetz. Tab. Phyc. XVIII (1868) tab. 6.

$H a b$. Australia occid.: Baia di Geroldton.

Un solo frammento di questa bella specie venne raccolto dal rev. G. CAPRA.

L'Eucheuma speciosum (Sond.) J. Ag., la « Jelly plant » de' coloni dell' Australia occidentale, vegeta nelle acque profonde ed è casualmente rejetto sul littorale; fu descritto la prima volta su esemplari provenienti da Fremantle e dall' isola Rottnest. Il Sonder lo segnala eziandio nella Tasmania.

\section{Areschougia Harv.}

Areschougra Laurencia (Hook. et Harv.) Harv. in Hooker Flora Tasmaniae II (1860) pag. 321; J. Ag. Epicrisis (1876) pag. 282; De Toni Sylloge Algarum IV, pag. 376 ; Mazza Saggio di Algologia Oceanica n. 132.

Carpothammion Laurencia Kuetz. Tab. Phyc. XVIII (1868) tab. 14.

Hab. Australia merid. : Victor Harbour ; Australia occid. : Baia di Geroldton.

È specie distribuita con discreta frequenza lungo i lidi austro-occidentali della Nuova Olanda e quelli della Tasmania (Georgetown). La fronda varia molto nell' aspetto e nel colore; prima d' un bel rosso vivo, poi nereggiante nel secco, ma sempre con ramificazioni tereti-cilindriche e le piccole rigonfiature elevate alle estremità che rammentano l'aspetto di Rhabdonia clavigera J. Ag., con la 
quale specie non è difficile la confusione. Ma dopo ancora le ramificazioni più vecchie si consumano all' estremità prima che altrove ed assumono un colore grigio-violaceo, più che altro dovuto alla corrosione e al disfacimento.

Areschougia Stuartir Harv. in Hooker Flora Tasmaniae II (1860) pag. 321 ; Phyc. Australica V (1863) tab. 294; De Toni Sylloge Algarum IV, pag. 277; Lucas, Revised List pag. 28.

Hab. Australia merid.: Victor Harbour.

La specie è finora conosciuta per poche località : South Port in Tasmania (la prima volta ivi raccolta da C. STUART) e Warnamboul nell' Australia meridionale (H. WATts). Gli esemplari raccolti dal rev. G. CAPRA sono di considerevoli dimensioni e piuttosto numerosi di guisa che è verosimile a Victor Harbour questa specie sia tutt' altro che rara.

\section{Thysanocladia Endl.}

Thysanocladia coriacea (Sond.) Harv. Nereis Australis (1847) pag. 90, tab. 36, Phyc. Australica Syn. (1863) pag. XXXIII, n. 418; Kuetz. Tab. Phyc. XIX (1869) tab. 29; Sond. Algae Austral. hactenus cognitae (1881) n. 406; De Toni Sylloge Algarum IV, pag. 381.

Sphaerococcus coriaceus Sond. in Botanische Zeitung 1845, pag. 56.

Hab. Australia occid. : Baia di Geroldton; settembre 1908.

La specie, descritta la prima volta su esemplari provenienti dallo Swan River (Algae Muellevianae) venne poscia fatta conoscere per molte altre località dell'Australia occidentale.

Thysanocladia oppositifolia (Ag.) J. Ag. Species II (1852) pag. 617, Epicrisis (1876) pag. 288; Harv. Phyc. Australica IV (1862) tab. 187; Kuetz. Tab. Phyc. XIX (1869) tab. 30; De Toni Sylloge Algarum IV, pag. 383.

Hab. Australia occid. : Baia di Geroldton; settembre 1908.

È specie comune lungo i lidi occidentali e meridionali dell Australia; per la regione meridionale ne fu accertata la presenza dal Reinbold. Quattro esemplari raccolti dal rev. G. CAPRA sono bene sviluppati, uno di essi raggiungendo nel secco circa 23 centimetri di lunghezza.

\section{Ordo Rhodymeninae Schmitz}

\section{Fam. SPHAEROCOCCACEAE (Dum.) Schm.}

\section{Subfam. Phacelocarpeae ( $J$. Ag.) Schm.}

Phacelocarpus Endl. et Dies.

Phacelocarpus complanatus Harv. Alg. Austral. exsicc. n. 306, Phyc. Australica V (1863) tab. 252; De Toni Sylloge Algarum IV (1900) pag. 390 ; Lucas Revised List pag. 29.

Hab. Australia occid. : Baia di Geroldton; settembre 1908. 
Questa specie, appartenente al sottogenere Euctenodus comprendente specie tutte australasiche, è abbastanza comune nella Nuova Olanda e Tasmania, essendo stata indicata, secondo il Sonder (Alg. Austral. n. 532) di Port Phillip, Port Fairy, Warnamboul per l'Australia e di Southport per la Tasmania ; il REINBold la segnalò per la Nuova Olanda meridionale tra le Alghe raccolte dal D.r A. EngElHaRT. Il rev. CAPRA raccolse soltanto piccoli esemplari, per lo più epifiti su la Stenocladia Cliftoni, alcuni di essi tetrasporiferi.

Phacelocarpus Labillardieri (Mert.) J. Ag. Species II (1852) pag. 648; Harv. Phyc. Australica III (1860) tab. 162 ; Kuetz. Tab. Phyc. XVIII (1868) tab. 67; De Toni Sylloge Algarum IV, pag. 391; Lucas Revised List pag. 29 ; Mazza Saggio di Algologia oceanica n. 136.

Hab. Australia occid. : Baia di Geroldton; settembre 1908.

Il Phacelocarpus Labillardieri (Mert.) J. Ag. è assai comune lungo i lidi della Nuova Olanda occidentale e meridionale, della Tasmania e della Nuova Zelanda; tra le specie del genere è, si può dire, la più largamente rappresentata negli erbarii; ne ebbimo un solo frammento corroso, sterile e scolorito. Molto simile per l' aspetto esterno al Phacelocarpus Labillardieri (Mert.) J. Ag. è il Ph. echionotus J. Ag. (Analecta algologica contin. V (1899) pag. 92) ma se ne distingue per la struttura.

Phacelocarpus sessilis Harv. in J. Ag. Epicrisis (1876) pag. 400; De Toni Sylloge Algarum IV, pag. 392; Lucas Revised List pag. 29; Mazza Saggio di Algologia Oceanica n. 138.

$H a b$. Australia merid.: Victor Harbour.

E specie meno frequente della precedente. Il Sonder (Algae austral. n. 536) la indica per Port Phillip Heads, Mc Donnell Bay, Geographe Bay; il Reinbold ne confermò, su materiali raccolti dal Dott. A. EngelHART, la presenza nei lidi meridionali dell' Australia. Noi ne abbiamo esemplari provenienti dalla Israëliten Bay, ivi raccolti da F. Mueller.

\section{Subfam. Stenocladieae Schm.}

Stenocladia J. Ag.

Stenocladia Ci.iftoni J. Ag. Epicrisis (1876) pag. 440; De Toni Sylloge Algarum IV, pag. 404; Lucas Revised List pag. 29; Mazza Aggiunte al Saggio di Algologia Oceanica n. 741. Icon: Tab. nostra II (V), fig. 1.

Sienocladia conterta var. Cliftoni J. Ag. Bidrag till Florid. System. (1871) pag. 45.

Aveschougia conferla Harv. Phyc. Australica III (1860; tab. 166 [quoad specimen a G. Clifton lectum].

Hab. Australia occid. : Baia di Geroldton, con cistocarpi; settembre 1908.

Con il nome collettizio di Slenocladia conferta (Harv.) venne da J. AgardH nel 1871 ridotta al genere Stenocladia l'Aveschougia conferta Harv., distinguendone però due varietà : l' una Cliftoni per la Nuova Olanda occidentale, l'altra Harveyi per la Nuova Olanda meridionale; così per la Stenocladia Cliftoni J. Ag. rimangono per primi reperti le località Swan River e Fremantle. 
Presso Champion Bay il Dott. E. SPEncER raccolse nel giugno 1895 una Floridea con tetrasporangi evoluti entro agli apici intumescenti degli ultimi rametti, tali da simulare dei cistocarpi; per quest' Alga il DARBISHIRE (Spencerella australis, eine neue Florideen-Gattung und Art, Berichte der deutschen botanischen Gesellschaft XIV, 1896, pag. 195-200, T. XIV) istitù̀ un nuovo genere Spencerella il quale, riguardo alla struttura, si accosta molto alle Stenocladia, ma non ne può rappresentare uno stato sporangifero, come di primo acchito si sarebbe indotti a supporre, perchè nelle Stenocladia gli sporangi sono divisi a croce, in Spencerella a triangolo; si deve nondimeno avvertire che la figura esibita dal DARBISHIRE mostra, oltre a parecchi sporangi triangoli-partiti, uno sporangio con divisione perfettamente crociata.

Stenocladia Harveyana J. Ag. Epicrisis (1876) pag. 440; De Toni Sylloge Algarum IV, pag. 405; Lucas Revised List pag. 29. Icon: Tab. nostra II (V), figg. $2-5$ et III (VI) fig. 1.

Stenocladia conferta var. Harveyi J. Ag. Bidrag till Florid. System. (1871) pag. 45.

Areschougia conferta Harv. Phyc. Australica III (1860) tab. 166 lquod specimina ex Australia meridionali].

Hab. Australia occ. : Baia di Geroldton, con cistocarpi ; settembre 1908.

I nostri esemplari si accordano con quelli di Port Phillip esistenti nell' Erbario ZANARDini al n. 1148. Il Lucas dà come area di distribuzione l' Australia occidentale e meridionale.

\section{Nizymenia Sond.}

Nizymenia Australis Sond. in Linnaea vol. XXVI (1853) pag. 521 ; Harv. Phyc, Australica III (1860) tab. 165; J. Ag. Florideernes Morphologi (1879) tab. XXIII, fig. 9; De Toni Sylloge Algarum IV, pag. 408; Lucas Revised List pag. 29; Mazza Saggio di Algologia Oceanica n. 145.

Hab. Australia occid. : Baia di Geroldton; settembre 1908.

I nostri esemplari rappresentano una forma sterile, vetusta. Nizymenia australis Sond. appartiene a un genere monotipico finora esclusivo della Nuova Olanda austro-occidentale e della Tasmania. Tra gli esemplari distribuiti da W. H. Harvey nelle Algae Austral. exsicc. n. 385 sotto il nome di Areschougia conferta taluni vanno riferiti alla Nizymenia. J. AGARDH (Analecta Algologica contin. II, pag. 60; Lundae 1894) ridusse a questa specie un genere già da lui proposto col nome di Amylophora (Am. Coleae) che, con le cellule infarcite di materiali di riserva, rappresenta uno stato senile della Nizymenia australis Sond.

\section{Subfam. Melanthalieae $J$. Ag.}

\section{Sarcocladia Harv.}

Sarcocladia obesa Harv. Mar. Bot. of West Australia (1854) pag. 550, Phyc. Australica IV (1862) tab. 217; J. Ag. Epicrisis (1876) pag. 550 ; De Toni Sylloge Algarum IV, pag. 426; Lucas Revised List pag. 30. Icon: t. III (VI), fig. 4.

Hab. Australia occid. : Baia di Geroldton; settembre 1908. 
Di questa rara specie, propria della Nuova Olanda austro-occidentale, il Sonder (Algae Austral. hactenus cognitae N. 557) diede le sole località Geographe Bay e King George 's Sound. Haryey e Clifton la raccolsero anch' essi di quest' ultima località e di Rottnest Island.

Subfam. Gracilarieae (Naeg.) J. Ag.

Gracilaria Grev.

Gracilaria confervoides (L.) Grev. Alg. Brit. (1830) pag. 123; Hooker Fl. N. Zel. II (1855) pag. 243, Handbook (1867) pag. 687; J. Ag. De Algis Novae Zelandiae marinis (1877) pag. 23, n· 187; De Toni Sylloge Algarum IV, pag. 431 ; Okam. Icones Algar. Japon. IV (1916) tab. 151.

Hab. Australia occid.: Baia di Geroldton, con cistocarpi ; settembre 1908; Nuova Zelanda: Port Charmers, con cistocarpi e anche nella forma capillaris (Kuetz.); 30 gennaio 1909.

La Gracilaria confervoides (L.) Grev. è una specie quasi cosmopolita; anche nell' Oceania essa mostra una larga distribuzione geografica; secondo HaRveY (Phyc. Austral. Syn. 1863, pag. 428) vegeta lungo i lidi occidentali e meridionali dell' Australia e quelli della Tasmania e della Nuova Zelanda, spingendosi fino alle isole Kermadecs (A. e E. S. Gepp) e d'altra parte fino alle isole dell' Ammiragliato (GRunow). La specie è certo comune nelle spiaggie neozelandesi, lungo le quali ivenne raccolta o segnalata da Lyall, Colenso, Davies, Berggren, Grunow. Lo stesso Laing (List of New Zealand Seaweeds II, 1901, pag. 342, n. 236) la indica ovunque comune.

Gracilaria lichenoides (L.) Harv. in Lond. Journ. Bot. III (1844) pag. 445 ; J. Ag. Epicrisis (1876) pag, 412, Species III, 4 (1901) pag. 52; De Toni Sylloge Algarum IV, pag. 430; Lucas Revised List pag. 30 ; Mazza Aggiunte al Saggio di Algologia Oceanica n. 745.

Hab. Australia merid.: Victor Harbour; 12 novembre 1908.

Secondo J. Agardh la specie venne dapprima riportata da Giava (Batavia; HoHenacker n. 148) poi del Queensland, infine dalla Nuova Zelanda; piccoli esemplari, provenienti da Sumatra furono distribuiti nelle Reliquiae Brebissonianae da Roumegukere e Movgeot. Per l'Australia sembra sia essa più comune lungo le coste settentrionali e orientali, in particolare a Port Denison, Cleveland Bay e Capo York secondo il Sonder; a George Town in Tasmania la raccolse Rol. Gúnn; più di recente la Gracilaria lichenoides fu segnalata per la nuova Guinea dal Grunow e per lo Stretto di Torres da K. OKamura. Questa specie venne anche denominata Plocaria candida Nees perchè, secondo riferisce HoHENACKER, sulle rive dell' Oceano Indiano viene imbianchita e usata cruda o cotta per cibo. In farmacopea costituisce il Fucus o Lichen amylaceus (1) del commercio. L'imbianchimento, secondo J. AgardH (in scheda Alg. Muellerianae) succede spontaneamente col sole al limite estremo della marea. A. e

(I) Cfr. Oudemans C. A. J. A., Bijdrage tot de Kennis van het Agar-Agar vlugtige Opmerkingen omtrent Fucus amylaceus en Tjientjau (HaAxman, Tijdschrift voor Wetensch. Pharmacie, Tweede Serie, 3 Jaargang, pl. I, fig. 2). 
E. S. GEPP (Some marine Algae from New South Wales 1906, pag. 256) descrissero col nome di Gracilaria Lucasii una specie raccolta da A. H. S. Lucas a Farm Cove, Sydney la quale, rispetto alla struttura, è molto affine a Gracilaria lichenoides (I.) Harv.

Subfam. Hypneene (J. Ag.) Havv.

Hypnea Lamour.

Hypnea Cenomyce J. Ag. Species II (1852) pag. 452, Epicrisis (1876) pag. 564 ; De Toni Sylloge Algarum IV, pag. 481 ; Lucas Revised List pag. 31.

Hab. Tasmania: Baia di Latrobe.

La specie finora era nota per l' Australia occidentale a Holdfast Bay (SonDER Algae Austral. hactenus cognitae n. 652).

Hypnea episcopalis Hook. et Harv. Alg. Tasman. n. 64, Phyc. Australica I (1858) tab. 23; Kuetz. Tab. Phyc. XVIII (1868) tab. 30; Sond. Alg. Austral. hactenus cognitae (1881) n. 645; De Toni Sylloge Algarum IV, pag. 473 ; Mazza Saggio di Algologia Oceanica n. 179.

Hab. Australia occid. : Baia di Geroldton; settembre 1908.

Al pari della comunissima Hypnea musciformis (Wulf.) Lamour. del Mediterraneo e di qualche altra congenere ( $H$. ceramioides Kuetz., H. valida J. Ag., H. Eckloni Suhr) la Hypnea episcopalis Hook. et Harv. presenta parecchi rami uncinati all' apice a guisa di pastorale da vescovo, donde il nome specifico. L'area di distribuzione di questa specie va dalla Nuova Olanda occidentale e meridionale alla Tasmania (SoNDER) e alle isole Flinders (DE TONI). I nostri esemplari concordano con quelli tasmanici conservati nell'erbario ZANARDINI al n. 608 del rispettivo catalogo illustrativo.

Hypiea musciformis (Wulf.) Lamour. Essai (1813) pag. 43; Kuetz. Tab. Phyc. XIX (1869) tab. 19, fig. a-e ; Hooker Handbook (1867) pag. 689; J. Ag. De Algis novae Zelandiae marinis (1877) pag. 26, n. 224; De Toni Sylloge Algarum IV, pag. 472.

Hab. Tasmania: Baia di Latrobe; Nuova Zelanda: Port Charmers.

Sono frammenti di fronda con ramificazione sottile e non troppo densa, paragonabili a quelli di Hypnea musciformis pubblicati in RabenhoRst Algen Eurupas n. 760 e in De Toni-Levi Phycotheca italica n. 10. In particolare nell'unico esemplare raccolto dal rev. G. CAPRA nella Baia di Latrobe gli organi d'avvinghiamento sono bene sviluppati e dimostrano anzi un' ispessimento all' asse.

La specie fu segnalata per più località dell' Australia occidentale, meridionale e orientale; nella Nuova Zelanda J. Agardh la dice molto abbondante, anche sulla scorta di esemplari raccolti da S. BERGGREN. È importante avvertire che A. e E. S. GEPP (Some marine Algae from New South Wales 1906, pag. 257) ricordano la Hypnea musciformis raccolta dal LucAs a Sandringham e Botany Bay nel gennaio 1904 con sporangi e con fronda somigliante alla Hypnea Rissoana Kuetz. Tab. Phyc. XVIII (1868) tab. 19. 
Hypnea nidifica J. Ag. Species II (1852) pag. 451, Epicrisis (1876) pag. 564 ; Sond. Alg. Austral. hactenus cognitae (1881) n. 650; De Toni Sylloge Algarum IV, pag. 479; Mazza Saggio di Algologia Oceanica n. 183.

Hab. Australia occid. : Baia di Geroldton; settembre 1908.

La Hypnea nidifica J. Ag. fu descritta dall' illustre J. AgardH su esemplari provenienti dalle isole Hawaii comunicatigli dal BINDER; si deve però trattare di una specie di amplissima distribuzione geografica, se si tien conto dei molti dati d' erbario più o meno pubblicati o noti ; nell' erbario THURET esistono esemplari delle coste Somali avuti da F. Hauck; nella collezione Piccone (ora incorporata nell' Erbario ForTI) sonvi molti campioni provenienti dall' isola Mandola nel Mar Rosso ; così l' area di distribuzione di Hypnea nidifica viene ad essere estesa all' Oceano Indiano e perfino al Mar Rosso ; è vero che la forma eritrea differisce dalla pacifica per la ramificazione più densa quasi raccorciata e per non esservi così notevoli quei grovigli che contribuirono all' appellativo di nidifica; ma, d'altronde, nessun particolare anatomico può riconoscersi sempre distinto fra le due forme geografiche; di più essendo queste passibili di grande variazione, tanto la forma eritrea quanto l'oceanica mostrano esemplari estremi o per accorciamento o per allungamento nell' una o nell' altra, i quali riescono in tutto identici fra loro. Del resto polimorfa si dimostra anche la Hypnea musciformis (Wulf.) Lamour. del nostro mediterraneo e forse cosmopolita, salvo che questa non ha mai gli aggrovigliamenti di Hypnea nidifica J. Ag. Gli uncini sono deboli con asse non ispessito. In esemplari giapponesi (TANAKaI) l' estrema ramificazione è più sentita e richiama alla memoria quella di Hypnea seticulosa J. Ag.

Si deve poi notare che la Hypnea nidifica J. Ag. venne già segnalata per il Continente Australiano a Cookstown e Port Denison (SoNDER) malgrado il LuCAS la ommetta nella sua Revised List of the Fucoideae and Florideae of Australia.

Hypnea seticulosa J. Ag. Species II (1852) pag. 446; De Toni Sylloge Algarum IV, pag. 476 ; Lucas Revised List pag. 31.

Hypnea charoides Sond. Alg. Preiss. pag. 42; Kuetz. Tab. Phyc. XVIII (1868) pag. 8, tab. 22, fig. $a-b$ (non Lamouroux).

Hab. Australia occid. : Baia di Geroldton; settembre 1908; Australia merid.: Victor Harbour; 12 novembre 1908.

Nell'Oceano Pacifico questa specie è abbastanza comune lungo i lidi della Tasmania (J. D. Hooker) e dell'Australia occidentale e meridionale; essa non manca alla regione marina del Giappone (OKamura Algae Japonicae exsiccatae n. 62).

Fam. RHODYMENIACEAE (Nagg.) J. Ag.

Subfam. Rhodymenieae (J. Ag.) Schmitz

Hymenocladia J. Ag.

Hymenocladia divaricata (R. Br.) Harv. in Trans. R. Irish Academy vol. 22 (1855) pag. 553, Phyc. Australica I (1858) tab. 20; De Toni Sylloge Algarum IV pag. 502; Lucas Revised List pag. 32.

Hab. Australia occid.: Baia di Geroldton; settembre 1908. 
L'area di distribuzione di questa specie è finora limitata ai lidi occidentali e meridionali dell' Australia, essendo stata per la prima volta dragata nel King George's Sound; nelle Alghe Muellerianae, distribuite da J. AGaRDH, si hanno esemplari dell' is. di Flinders.

Hymenocladia polymorpha (Harv.) J. Ag. Om Chathamöarnes Alger (1870) pag. 453, Epicrisis (1876) pag. 315; De Toni Sylloge Aloarum IV, pag. 504; Lucas Revised List pag. 32 ; Mazza Saggio di Algologia oceanica n. 187. Icon: Tab. nostra III (VI), fig. 2-3.

Rhodymenia polymorpha Harv. Alg. Austral. exsicc. n. 383, Phyc. Australica III (1860) tab. $15 \%$.

Hab. Australia occid. : Baia di Geroldton, in esemplari cistocarpiferi e sporangiferi; settembre 1908 .

È specie soprattutto comune nei lidi meridionali dell' Australia e in quelli della Tasmania. La stazione della Baia di Geroldton è già nota (Algae Muelle. rianae: Champion Bay) cosicchè non può neanche ritenersi specie nuova per l' Australia occidentale. Il Grunow ne ebbe magnifici esemplari da Port Phillip e da Portland Bay (Victoria). La Hymenocladia polymorpha ha una fronda molto alterabile e deliquescente ma dore è frequentissimo riscontrare tanto i cistocarpi quanto i tetrasporangi.

Hyamenocladia Usnea (R. Br.) J. Ag. Species II (1852) pag. 772, Epicrisis (1876) pag. 313; Harv. Phyc. Australica Il (1859) tab. 118; De Toni Sylloge Algarum IV, pag. 502; Mazza Saggio di Algologia Oceanica n. 185.

Hab. Australia occid.: Baia di Geroldton; settembre 1908.

Anche questa Hymenocladia preferisce la regione meridionale dell'Australia nella quale è assai comune, al pari della specie precedente; ivi è comune nella Portland Bay.

\section{Rhodymenia Grev.}

RhodYMENia AUSTRalis Sond. in Botanische Zeitung 1845, pag. 54 (Rhodomenia); Harv. Phyc. Australica III (1860) tab. 146; J. Ag. Epicrisis (1876) pag. 332;

De Toni Sylloge Algarum IV, pag. 519; Lucas Revised List pag. 32.

Acropeltis australis J. Ag. Species II (1852) pag. 609; Kuetz. Tab. Phyc.

XIX (1869) tab. 34, f. c-e (tetraspore).

Hab. Australia occid. : Baia di Geroldton, con cistocarpi ; settembre 1908.

Gli esemplari della Baia di Geroldton, comunicatici dal rev. G. CAPra, collimano perfettamente con un esemplare dell' erbario DE 'ToNı proveniente da Fremantle, località nella quale la Rhodymenia australis Sond. è, secondo HaRVEY, assai abbondante; di detta località esistono esemplari anche nell' erbario ZaNARDINI al n. 1091 della rispettiva illustrazione. È una bella specie questa Rhodymenia, conosciuta per i lidi occidentali, meridionali e orientali dell'Australia.

Rhodymevia palmata (L.) Grev. Alg. Brit. (1830) pag. 93; De Toni Sylloge Algarum IV, pag. 512.

Hab. Nuova Zelanda: Port Charmers, acque basse; 30 gennaio 1909. 
Fronda sterile, scolorata. E con molto riserbo che viene enunciata questa specie la quale pur comparendo proteiforme in quasi tutti i mari, non escluso l' Oceano Pacifico su le coste dell' Alaska e degli Stati Uniti, sembra però non sia finora penetrata in Oceania. La Rhodymenia sanguinea Harv. potrebbe costituire la sua entità vicariante per la Nuova Zelanda e, in tal caso, la presente fronda sterile sarebbe meglio riunita a questa; ma essendo sterile e più piccola riproduce con migliore esattezza l' aspetto della Rhodymenia palmata Grev. La miglior distinzione tra le due specie, a quanto sembra, risiede nel fatto della disposizione dei tetrasporangi che nella palmata stanno riuniti in sciami irregolarmente limitati sull' intiera fronda, laddove nella sanguinea sone sparsi per tutto il tallo e stanno per lo più nelle ramificazioni più giovani non mai aggruppati in sciami; quando anche non s'abbia a considerare come una differenza il fatto che solo assai di raro e per lo più nella varietà sarniensis si rinvengono i cistocarpi (') per Rhodymenia palmata mentre sono frequentissimi, quasi costanti, in Rhodymenia sanguinea.

Non è escluso infine che la specie dell' Oceano Pacifico non abbia a considerarsi come un' entità ben distinta dall' altra e non sarebbe maraviglia che ciò avvenisse quando si pensi che anche SETCHELl e GARDNER proposero una forma mollis per gli esemplari provenienti dall' is. Whidbey (Wash.) distribuiti nella Phycotheca Boreali-Americana, troppo differenti da quello che s' intende per lo più come tipo di Rhodymenia palmata Grev. Rimane poi da dimostrare se questa forma mollis potrà assomigliarsi invece a Rhodymenia sanguinea Harv. o se debbano mantenersi separate anche queste due fra di loro.

Rhodymenia sanguinea Harv. in Hooker Fl. N. Zel. II (1855) pag. 248, Handbook of New Zealand Flora (1867) pag. 692; De Toni Sylloge Algarum IV, pag. 513. Icon. Tab. nostra IV (VII), figg. 1, 2, 3.

Hab. Nuova Zelanda: Port Charmers, acque basse, con cistocarpi e con tetrasporangi.

Ricevemmo dal rev. G. CAPRA un frammento fruttifero d' una fronda divelta fino dal callo radicale, lunghissima raggiungendo essa con due sole ramificazioni oltre 40 centimetri di lunghezza. La lacinia estrema membranosa presenta a tre quarti lunghezza una sinuosità rientrante da ambi i lati e continua più sottile $\mathrm{e}$ trasparente fino all' estremità bifida ; regge anche due prolificazioni laterali membranose sottilissime, annesse con una congiunzione filiforme; sono esse di contorno lanceolato diafane quantunque ricche di sporangi ma prive di cistocarpi. La ramificazione di base è più scura, di membrana più grossa e meno trasparente e prende origine da uno stipite brevissimo arrotondato come vuole la diagnosi specifica «stipite tereti brevi».

La Rhodymenia sanguinea Harv. finora è cognita per la Nuova Zelanda, essendo stata raccolta per la prima volta dal Lyall nel Foveaux Straits. I cistocarpi, nel nostro esemplare, sono puntiformi, emisferici, del diametro di $750-900 \mu$. e risaltano per il loro colore intensamente rosso.

(I) Gli esemplari di Plymouth determinati da Hooker ricevettero persino il nome di Rhodymenia polycarpa Hook. (v. Brit. Flora pag. 289; Wvatт Algae Danmonienses n. Io8!). 
Sebdenia Berth.

Sebdenia? kallymenioides (Harv.) De Toni Sylloge Algarum IV (1900) pag. 533. Icon: Tab. nostra III (V1), fig. 5.

Halymenia kallymenioides Harv. in Trans. Irish Acad. vol. 22 (1855) pag. 556 , n. 257; J. Ag. Analecta algologica (1892) pag. 53.

Halymenia? Cliftoni Harv. Phyc. Austral. II (1859) tab. 103 partim, Syn. Phyc: Austral. (1863) pag. XLVI, n. 593 partim.

Hab. Nuova Zelanda: Port Charmers, con cistocarpi.

Sono esemplaretti epifitici, di piccole dimensioni $(1-2,5 \mathrm{~cm}$.) che riferiamo a questa specie, corrispondendo alle piccole frondi rappresentate nella parte inferiore della figura 1 della tavola della Phycologia Australica sopra citata. La specie è nota per i lidi occidentali della Nuova Zelanda. Le piccole frondi ricordano molto, come avvertiva lo stesso Harvey, un Nitophyllum. Recentemente la sig. A. Weber van Bosse (Liste des Algues du Siboga II, 1921, pag. 230, fig. 7) riferì a questa specie esemplari raccolti da Tissot van РАтоT durante la spedizione della \&iboga a Bolik-Pappan, sulla costa orientale di Borneo.

\section{Chrysymenia J. Ag.}

Chrystmenia Brownit (J. Ag.) De Toni Sylloge Algarum IV (1900) pag. 545; Lucas Revised List pag. 33.

Gloiosaccion Browniii Harv. Phyc. Australica II (1859) tab. 83; J. Ag. Analecta Algologica (1892) pag. 86; J. Br. Wilson Catal. of Algae coll. at or near Port Phillip etc. (1892) pag. 179.

Halosaccion firmum Harv. Alg. Austral. exsicc. n. 420.

$H a b$. Australia occid. : Baia di Geroldton.

Esemplaretti giovanili di questa bella specie (quattro riuniti per il callo radicale appena pronunciato lunghi da 1,5 a 3 centimetri) che raggiunge, allo stato cistocarpifero, dimensioni assai considerevoli, fino a 2 decimetri di lunghezza per 3 a 4 centimetri di larghezza. La Chrysymenia Brownii (Harv.) De Toni è tra le Florideae, in certo qual modo, analoga a Adenocystis utricularis (Bory) Skottsb. fra le Fucoideae. J. AGARDH, mantenendo il genere Gloiosaccion Harv. distinse tre specie: Gl. pumilum J. Ag. di Port Phillip Heads (Australia meridionale), Gl. Hydrophora (Harv.) J. Ag. [= Gloiosaccion Brownii var. a membranaceum Harv. Phyc. Australica; Halosaccion Hydrophora Harv. Alg. Austral. exsicc. n. 419] di Port Phillip Heads e della Tasmania e Gl. Brownii (Harv.) della Tasmania e delle coste occidentali e meridionali della Nuova Olanda; l'illustre botanico però non si nascose il dubbio che per queste tre specie trattisi di differenze dovute all' età o a peculiari condizioni di vita. Il LucAs, conservando la sola Chrysymenia Brownii (Harv.) dà come area di distribuzione la Tasmania e l' Australia occidentale e meridionale. 
Subfam. Plocamieae (Reich.) Kuetz.

Plocamium Lamour.

Plocamium angustum (J. Ag.) Hook. et Harv. Alg. Tasman. p. 8 ; Kuetz. Tab. Phyc. XVI (1866) tab. 48, fig. $a-b$; De Toni Sylloge Algarum IV, pag. 596 ; Lucas Revised List pag. 34 ; Mazza Saggio di Algologia Oceanica n. 215.

Hab. Tasmania: Baia di Latrobe; Burmie: 15 gennaio 1909.

É specie largamente distribuita lungo i lidi dell' Australia, della Tasmania e della Nuova Zelanda come venne indicato da W. H. Harvey (Syn. Phyc. Austral. pag. XXXIX, n. 492) e dal Sonder (Algae Austral. hactenus cognitae n. 459); può dirsi essere uno dei Plocamium più comuni nell' Oceania. Ne ricevemmo dal CAPRA tre piccoli esemplari scoloriti.

Plocamium nidificum (Harv.) J. Ag. Epicrisis (1876) pag. 346; De Toni Sylloge Algarum IV, pag. 599; Lucas Revised List pag. 35. Icon: Tab. nostra III (VI), figg. 6-7.

Plocamium procerum nidificum Harv. Phyc. Austral. Syn. (1863) n. 491 b.

Thamnocarpus Mertensii Kuetz. Tab. Phyc. XVI (1866) tab. 55, fig. $d-h$ (non Species 1849).

Hab. Australia occid. : Baia di Geroldton, con sporangi; settembre 1908.

Gli esemplari raccolti dal CAPRA (uno grande con tetrasporangi assai bene sviluppati) concordano con quelli delle Algae Muellevianae curante J. G. Agardh distributae de' nostri erbarii, provenienti da più località (Moouta Bay, Waterloo Bay, Champion Bay, Fowler 's Bay, Port Phillip, Queenscliff, Geographe Bay). La specie regeta lungo i lidi meridionali e occidentali dell' Australia.

Plocamium Preissianum Sond. in Botanische Zeitung 1845, pag. 54 ; Harv. Phyc. Australica II (1859) tab. 63; De Toni Sylloge Algarum IV, pag. 594,

Plocamium Preissii Kuetz. Tab. Phyc. XVI (1866) tab. 53.

Hab. Australia occid. : Baia di Geroldton, con cistocarpi e con sporangi; settembre 1908.

Gli esemplari della Baia di Geroldton vennero da noi confrontati con quelli delle Algae Muellevianae provenienti da Champion Bay e Encounter Bay e con altri del Grunow e del Lenormand provenienti da Port Phillip, con i quali corrispondono egregiamente. La specie è distribuita lungo le coste meridionali e occidentali della Nuova Olanda.

\section{Fam. DELESSERIACEA (Naeg.) Schmitz}

\section{Subfam. NitophylleaE (Naeg.) Schmitz}

Nitophyllum AfFine Harv. in Hooker Lond. Journ. 1844, pag. 447, Nereis Australis (1847) pag. 119; De Toni Sylloge Algarum IV, pag. 652 ; Lucas Revised List pag. 35 ; Mazza Saggio di Algologia Oceanica n. 236.

Aglaophyllum affine Kuetz. Tab. Phyc. XIX (1869) tab. 8.

Hab. Tasmania: Baia di Latrobe. 
Gli esemplari raccolti dal rev. G. CAPRA corrispondono perfettamente, riguardo alla struttura, con saggi delle nostre collezioni ossia con uno distribuito da J. G. Agardh nelle Algae Muellerianae (provenienza: Tasmania), con un altro raccolto nel 1884 dal Grunow a Brighton (Victoria) e con altri ancora di Port Phillip comunicati da F. Mueller. Alcune volte però la fronda si sfrangia in lacinie molto più strette in maniera da simulare perfino l' aspetto del Nitophyllum multipartitum Hook. et Harv.; si tratta sempre di esemplari giovanili sterili. Anche il Sonder (Algae Austral. hactenus cognitae n. 576) indica il $N$. affine Harv. per Port Phillip (Nuova Olanda meridionale) e per Georgetown (Tasmania); il ReInBold enumera questa specie per l' Australia meridionale tra le alghe comunicategli dal dott. A. Engelhart.

Nitophyllum erosum Harv. Alg. Austral. exsicc. n. 293, Phyc. Australica II (1859) tab. 94; De Toni Sylloge Algarum IV, pag. 639; Lucas Revised List pag. 35.

Aglaophyllum erosum Kuetz. Tab. Phyc. XIX (1869) tab. 6, fig. c-d.

Nitophyllum fimbriatum Harv. in Trans. R. Irish Academy vol. XXII (1855) pag. 549 (non Greville).

Hab. Australia occid.: Baia di Geroldton; settembre 1908.

Quattro piccoli esemplari vennero raccolti dal CAPRA. Sembra trattarsi di specie piuttosto rara, essendo finora stata segnalata per pochissime località dell'Australia occidentale e meridionale; Garden Island (G. Clifton, Harvey), Port Fairy (Harvey), Mac Donnell Bay (Sonder), Lacépède e Guichen Bay (Reinbold).

Nitophyllum minus (Sond.) Harv. Nereis Australis (1847) pag. 119; De Toni Sylloge Algarum IV, pag. 651; Lucas Revised List pag. 35.

Cryptopleura minor Sond. in Botanische Zeitung 1845, pag. 57; Kuetz. Tab. Phyc. XVI (1866) tab. 26, fig. $a-b$ (juvenilis).

Hab. Australia occid. : Baia di Geroldton; settembre 1908.

I nostri esemplari s' accordano bene con la figura del KuETzing sopra citata.

Il Sonder (Algae Austral. hactenus cognitae n. 588) indica il Nitophyllum minus (Sond.) Harv. per Fremantle, Garden Island, Guichen Bay e Mac Donnell Bay; il Grunow (Erbario Piccone ora Forti) trovò che piccoli individui di questa specie rivestono le frondi di parecchie alghe a Port Phillip.

Nitophyllum multipartitum Hook. et Harv. Alg. Tasman. pag. 7, Nereis Australis (1847) pag. 121 ; De Toni Sylloge Aloarum IV, pag. 653; Lucas Revised List pag. 35.

Aglaophyllum multipartitum Kuetz. Tab. Phyc. XVI (1866) tab. 7, fig. $c-d$.

Hab. Australia occid. : Baia di Geroldton, sulla Dictymenia Sonderi; Australia merid.: Victor Harbour, su conchiglie frammisto a Rhodophyllis ramentacea (Ag.) J. Ag. con la quale di primo acchito si può confondere anche per il colore, qualora non si badi ai margini fimbriati della fronda di quest' ultima specie.

I nostri esemplari, piuttosto rappresentanti una forma giovanile, corrispondono egregiamente alla figura esibita dal KuETzing. La specie è già nota per le coste meridionali dell' Australia e per quelle della Tasmania. 


\section{Subfam. Delesserieae (Kuetz.) Schmitz \\ Paraglossum J. Ag.}

Paraglossum lancifolium (H. et H.) J. Ag. Species III, 3 (1898) pag. 217 (nomen nudum), De Toni Sylloge Algarum IV, pag. 703.

Delesseria lancifolia J. Ag. Bidrag till Florid. Systematik (1872) pag. 59.

Delessseria sanguinea var. lancifolia Hook. et Harv. in Hook. Fl. Antarct. II (1847) pag. 470.

Hab. Nuova Zelanda : Port Charmers.

Questa specie è segnalata del Capo Horn (America meridionale) ma una varietà minor descritta dal LAING (Transact. N. Zeal. Institute vol. XXIX, 1896, tab. 28) venne raccolta all' isola Macquarie che si trova fra il $54^{\circ}$ e il $55^{\circ}$ lat. S. in rispondenza alla Nuova Zelanda e non differisce se non per la minore lunghezza della lamina che non supera i 10 centimetri. 11 maggiore dei due esemplari di Port Charmers è lungo circa 22 centimetri; ambedue gli esemplari sono sterili.

\section{Schizoneura J. Ag.}

Schizoneura dichotoma (Hook. et Harv.) J. Ag. Species III, 3 (1898) pag. 168; De Toni Sylloge Algarum IV, pag. 725.

Delesseria dichotoma Hook. et Harv. Crypt. Antarct. pag. 72, tab. 71, fig. 2 ; Kuetz. Tab. Phyc. XVI (1866) tab. 24, fig. $c-f$.

Hab. Nuova Zelanda: Port Charmers.

La Schizoneura dichotoma (H. et H.) J. Ag. venne trovata alle isole Auckland e Campbell dal Lyale. Il Laing (Subantarct. Islands of Nero Zealand pag. 512) accenna però che fu anche raccolta al passaggio di Foveaux, all' isola di S. Paolo e sui lidi della Terra di Kerguelen. Gli esemplari di Port Charmers sono sterili e malandati.

\section{Subfam. Sarcomenieae Schmitz$$
\text { Caloglossa Harv. }
$$

Caloglossa Beccarir Zanard. Phycearum Indicarum Pugillus (1872) pag. 140, n. 29, tab. V, A, fig. 1-3 (sub Delesseria); De Toni Sylloge Algarum IV, pag. 730. lcon: Tab. nostra VII, figg. 4-6; VIII, fig. 1.

Hab. Tasmania: fiume Tamar, sponda orientale, sulle roccie bagnate, insieme a Calothrix aeruginea, con sporangi; 12 gennaio 1909.

La Caloglossa Leprieurii (Mont.) J. Ag., con la quale la specie Zanardiniana ha senza dubbio molta affinità e forse non ne è che una razza geografica, costituisce, può dirsi, il tipo del genere Caloglossa (1). J. AGardH (Epicrisis pag. 499) dubitando intorno l' identità degli esemplari americani con quelli austra-

(I) Si noti che il Sonder (Plantae Muellerianae 1853, pag. 522) indicò la Delesseria Leprieurii Mont. per il Yarra River, verso la foce di questo fiume; cfr. anche C. H. WRIGHT, Distribution of Caloglossa Leprieurii (Mont.) J. Ag., Journal of Botany vol. XXVII (1889) n. 3i3, pag. 22. 
lasici (sugli americani era stata istituita la Caloglossa Leprieurii) preluse alla distinzione di altre Caloglossa dalla genuina Leprieurii; gli esemplari di Cayenne (la autentica Caloglossa Leprieurii) mostrano le cellule sopra le ascelle della costa biforcuta raggianti verso i margini, quelli d' Australia invece parallele con le coste di due foglioline gemelle. Se realmente dalla Caloglossa Beccarii Zanard. siano a sufficienza distinte la Caloglossa amboinensis Karst. (dell' isola Amboina) la Cal. zanzibariensis Goeb. (di Kokotoni nella regione dello Zanzibar) è lecito dubitare (1) mostrandosi in uno stesso esemplare una certa variabilità nelle forme dei segmenti ne' quali è differenziata la fronda. La Cal. ogasawaraensis Okam. (dell' isola detta Ogasawarajima) costituisce il passaggio dalla Cal. Beccarii Zanard. alla Cal. muioides Harv. (della Nuova Guinea, della Nuova Caledonia e delle Isole degli Amici). Da tutte le specie del genere si discosta la Caloglossa adnata (Zanard.) D. T. di Borneo ().

\section{Sarcomenia Sond.}

Sarcomenia tenera (Harv.) J. Ag. Species II, 3 (1863) pag. 1264; De Toni Sylloge Algarum IV, pag. 740; Lucas Revised List pag. 37; Mazza Saggio di Algologia Oceanica n. 253. Icon: Tab. nostra V, fig. 2-4. n. 209.

Dasya tenera Harv. Mar. Bot. West Austral. n. 116, Alg. Austral. exsicc.

Hab. Australia occid. : Baia di Geroldton; settembre 1908, con tetrasporangi.

Gli esemplari raccolti dal CAPRA sono bene sviluppati con frondi lunghe fino ad oltre 20 centimetri; essi corrispondono benissimo con uno autentico distribuito da Harvey nella collezione sopra citata e conservato nell'erbario De TonI.

La Sarcomenia tenera (Harv.) J. Ag. vegeta lungo i lidi occidentali e me-

(I) Anzi K. Okamura (Algae Japonicae exsiccatae n. 68) la ritiene senz' altro identica alla sua Caloglossa ogasawaraensis che conserverebbe il nome per priorità.

(2) La Delesseria Beccarii Zanard, per quanto attesta il nostro grande botanico esploratore ODOARDO BECCARI (Vedi : « Nelle foreste di Borneo» II ediz. Firenze I92I, pag. 123), ricopriva di uno strato roseo la superficie degli scogli nel torrente di Sodomak che scende dal massiccio granitico del Gunong Gading a Borneo. Quivi la curiosa floridea venne osservata e raccolta la prima volta a piủ di dieci chilometri entro terra. A proposito poi dell' abituale convivenza dei vari tipi di Caloglossa con differenti specie di Bostrychia, oltre il caso presente di Caloglossa Beccarii trovata nel fume Tamar insieme con Bostrychia simpliciuscula Harv., si conosce quello di Caloglossa Leprieurii (Mont.) J. Ag. della Gujana, associata con Bostrychia Leprieurii Montagne e di Caloglossa adnata Zanard. di Borneo con Bostrychia fulcrata Zanard. Nè queste analogie erano anche sfuggite al BECCARI (op. cit. p. 334) che accennando a una Bostrychia bryophila Zanard. adattatasi alla vita aerobia tra le epatiche e i muschi; rammenta le osservazioni di Leprieur e Montagne su le congeneri americane e nota il caso di Caloglossa adnata con Bostrychia bryophila da lui scoperta nella medesima isola di Borneo.

Nei nostri esemplari osservammo sporangi perfettamente divisi a croce; questo dimostra ancora una volta come il modo di divisione non costituisca un sicuro dato per la sistematica. Va notato quanto al genere Caloglossa che il Kuetzing nelle Tab. Phyc. XVI (1866) tab. тo, fig. $g$ figurò i sori del suo Hypoglossum Leprieurii [ = Caloglossa Leprieurii (Mont.) J. Ag.] con sporangi divisi a triangolo (salvo qualcuno diviso solo da un setto trasversale); più precise e rispondenti al vero, sono le figure, per la Caloglossa Leprieurii, fornite dal CRAMER Ueber Calogl. Lepr. (1891) tab. 3, fig. 6-7, delle quali gli sporangi sono rappresentati divisi da un setto trasversale o crocipartiti.

ZANARDINI (1872) nella sua Cal. adnata riscontrò solo sporangi immaturi; lo stesso dicasi per la C. zanzibariensis descritta dal Goebel (1898) le altre specie, C. ogasavaraensis Okam. C. amboinensis Karst. C. mnioides Harv. si conoscono solo allo stato sterile. 
ridionali della Nuova Olanda; alle località enumerate dal Sonder (Algae Austral. hactenus cognitae n. 802: Fremantle, Port Phillip, Geographe Bay, King George's Sound) si può aggiungere l' Investigator Street (REINBoLD).

\section{Fam. Bonnemaisoniaceae (Trev.) Schmitz}

\section{Delisea Lamour.}

Delisea hypneojdes Harv. Alg. Austral. exsicc. n. 248, Phyc. Australica, III (1860) tab. 134 ; De Toni Sylloge Algarum IV, pag. 761; Lucas Revised List pag. 38.

Hab. Australia merid.: Victor Harbour; 12 novembre 1908.

Sono esemplari sterili, per lo più scoloriti, lunghi anche 20 centimetri. La specie ha, come area di distribuzione, l'Australia meridionale e la Tasmania. La consistenza della fronda varia con l' età della pianta e con lo stato di conservazione, come giustamente fu avvertito dal Reinbold (Die Algen der Lacépède und Guichen Bay pag. 48, n. 220). Ad eccezione della Delisea Suhrii J. Ag., propria dell' Africa meridionale (Capo di Buona Speranza) tutte le altre specie vegetano nell' Oceano Pacifico.

\section{Fam. RHodomelaceae (Reich.) Harv.}

\section{Subfam Laurencieae (Harv.) Zanard.}

\section{Laurencia Lamour.}

Laurencia botryoides (Turn.) Gaill. Résumé (1828) pag. 15; Harv. Phyc. Australica IV (1862) tab. 182; Kuetz. Tab. Phyc. XV (1865) tab. 71, fig. $\boldsymbol{a}-\boldsymbol{b}$; De Toni Sylloge Algarum IV (1903) pag. 802; Lucas Revised List pag. 39.

Fucus botryoides Turn. Hist. Fuc. III (1811) pag. 103, tab. 178.

Hab. Tasmania: Burmie.

Di questa specie, raccolta dapprima all' isola Kent (R. BRown) venne da ulteriori indagini allargata l' area di distribuzione geografica così che oggigiorno la Laurencia botryoides (Turn.) Gaill. è nota per il Golfo di Carpentaria (SoNDER), per l' is. Dick Hartog (Askenasy), per l' is. Flinders (De Toni) e Chatham (CotTON), nonchè per la regione meridionale dell' Australia da Port Fairy allo Stretto di Bass e per la Tasmania (Harvey, Sonder). Nell' erbario Zanardini sono conservati esemplari tasmanici spediti da Harvey, con i quali quelli di Burmie si attagliano perfettamente. Non sappiamo spiegarci per quale motivo il Lucas limiti all' Australia meridionale l' area di questa Laurencia.

Laurencia Forsteri (Mert.) Grev. Syn. Alg. Brit. (1830) pag. LII ; Kuetz. Tab. Phyc. XV (1865) tab. 46, fig. $c \cdot d$, tab. 47-48; De Toni Sylloge Algarum IV, pag. 779; Lucas Revised List pag. 38; Mazza Saggio di Algologia Oceanica n. 262. 
Hab. Australia occid. : Baia di Geroldton, con anteridi ; settembre 1908; Australia merid. : Victor Harbour; Tasmania: Burmie, con cistocarpi.

Specie assai comune lungo i lidi occidentali, meridionali e orientali dell'Australia e quelli della Tasmania; è tra le più polimorfe del genere.

Laurencia Gracilıs Hook. et Harv. Nereis Australis (1847-9) pag. 84 ; De Toni Sylloge Algarum IV, pag. 780.

Laurencia filiformis Hook. et Harv. in Lond. Journ. Bot. vol. VII (1848) pag. 444 (non alibi nec Montagne). Icon: Tab. nostra V, fig. 5; VI, figg. 1-3.

Hab. Australia merid. : Victor Harbour; 12 novembre 1908.

Riferiamo, non senza qualche esitazione, gli esemplari raccolti dal rev. G. CAPra a questa specie, finora indicata per Hawkes Bay (Nuova Zelanda) dove la raccolse il Colenso, per Cooktown (Queensland) secondo il Sonder (Algae Austral. hactenus cognitae n. 728) e per l' is. Chatham (Cotron).

$$
\text { Corynecladia J. Ag. }
$$

Corynecladia umbellata J. Ag. Epicrisis (1876) pag. 643; De Toni Sylloge Algarum IV, pag. 810.

Corallopsis australasica. Sond. in Linnaea vol. 25 (1852) pag. 687; Kuetz. Tab. Phyc. XIX (1869) tab. 28.

Hab. Australia merid.: Victor Harbour, 12 novembre 1908.

La specie ha diflusione sulle spiaggie occidentali e meridionali dell' Australia e venne fatta conoscere su esemplari della Encounter Bay dal Sonder e della vicina Guichen Bay dal Kuetzing. Victor Harbour è poco lontano sul medesimo littorale, segno che tutte queste località stanno nel tratto dove questa Floridea si rinviene più di frequente e che forse quindi è meno frequente sulla costa occidentale. La fronda disseccata è eminentemente fragile in maniera che le ramificazioni pur conservando buona parte della turgescenza del vivo si staccano al minimo urto.

\section{Subfain. Chondrieae (Kuetz.) Schmitz}

Chondria (Ag.) Harv.

Chondria Debilis Harv. Phyc. Australica Vv(1863) pag. XIX, n. 206; De Toni Sylloge Algarum IV, pag. 847; Lucas Revised List pag. 40.

Chondriopsis debilis J. Ag. Analecta Algologica (1890) pag. 155.

Laurencia uncinata Kuetz. Tab. Phyc. XV (1865) tab. 44, fig. $a-b$ (non Zanardini). Icon: Tab. nostra VI, figg. 4-6.

Hab. Australia merid.: Victor Harbour, con sporangi ; 12 novembre 1908; Nuova Zelanda: Port Charmers, acque basse; 30 gennaio 1909.

Questa Chondria, raffigurata dal Kuetzing allo stato sterile in modo da ricordare per i rametti circinati le figure esibite dallo stesso autore per la sua Hypnea denudata, è nei nostri esemplari di Victor Harbour diffusamente sporangifera; gli esemplari stessi raggiungono dimensioni molto più considerevoli di quelle assegnate da Harvey, in quanto i più evoluti sono lunghi fino a 20 centimetri. Harvey la ebbe dal King George's Sound, ma la specie vegeta eziandio lungo le coste della Tasmania a Georgetown e quelle occidentali dell' Australia. 
Subfam. Polysiphonieae (Kuetz.) S. et F.

Lophurella Schmitz.

Lophurella Periclados (Sond.) Schmitz in Engler-Prantl Die natürl. Pflanzenfam. I (1897) pag. 441 ; Falkenb. Die Rhodomelaceen (1901) pag. 154, tab. 19, fig. 24-26; De Toni Sylloge Algarum IV, pag. 855 ; Lucas Revised List pag. 40.

Rhodomela periclados Sond. in Linnaea XXVI (1853) pag. 523; Harv. Phyc. australica I (1858) tab. 28; Hooker Flora Tasman. II (1860) pag. 296.

Hab. Tasmania: Baia di Latrobe, con sporangi e cistocarpi.

L' area di distribuzione geografica, riassunta dal Lucas, è data dall'Australia meridionale e dalla Tasmania; la specie fu indicata dagli autori per Twofold Bay, Brighton, Port Phillip, località alle quali si può, da materiali dei nostri erbarii e dell' erbario ZANARDINI, aggiungere per l'Australia la Encounter Bay; in Tasmania, oltre che al Brown's River (R. Gunn) e a South Port (C. Stuart) venne raccolta in individui sporangiferi a Port Hobarton da K. Fuijta secondo $\mathrm{K}$. Okamura (List of Marine Algae collected in Caroline Islands and Australia pag. 14; Tokyo 1904). La fronda adulta col disseccamento si assottiglia e imbrunisce più della giovanile.

\section{Polysiphonia Grev.}

Polysiphonia abscissa Hook. et Harv. Alg. Antarct. (1845) n. 38 (in Lond. Journ. Bot. IV pag. 266), Crypt. Antarct. pag. 174, tab. 183, II, Fl. N. Zel. Il (1855) pag. 227, Handbook (1867) pag. 669; Kuetz. Tab. Phyc. XIII (1863) tab. 70, fig. $a-c$; De Toni Sylloge Algarum IV, pag. 879; Laing Revised List (1901) pag. 353, n. 326.

Polysiphonia microcarpa Hook. et Harv. Fl. Antarctica II (1847) pag. 479, tab. 182 III.

Hab. Nuova Zelanda: Bluff, con cistocarpi.

Specie propria dell'Oceano Pacifico, con distribuzione geografica molto estesa dal Capo Horn (America meridionale) e dalla Terra di Graham (KrLin e SкоттSBERG) fino alla Nuova Zelanda e ai lidi meridionali dell' Australia ed a quelli della Tasmania. L' Askenasy (Algen, Forschungsreise S. M. S. Gazelle 1888, pag. 49, tab. X, fig. 1-4) indica la Polysiphonia abscissa Hook. et Harv. per l' isola Kerguelen in numerosi individui e ne descrive lo sviluppo de' cistocarpi. Il Laing, sulla scorta di materiali raccolti da Raoul, Lyall, S. Berggren, nota parecchie località per la Nuova Zelanda.

Polysiphonia Blandi Harv. Phyc. Australica IV (1862) tab. 184; Kuetz. Tab. Phyc. XIV (1864) tab. 43, fig. a.c ; De Toni Sylloge Algarum IV, pag. 899; Lucas Revised List pag. 41.

Hab. Australia occid. : Baia di Geroldton; settembre 1908.

Cresce questa specie lungo i lidi meridionali dell' Australia soprattutto a Port Phillip e Geographe Bay. È bene avvertire che secondo il LAING (Revised List, pag. 355, n. 349) la stessa specie fu raccolta nella Nuova Zelanda a Petone e a S.t Clair, Otago Harbour, ciò che fa ammettere una estesa area anche per la Polysiphonia Blandi Harv. che si credeva limitata all' Australia meridionale. 
Polysiphonia cancellata Harv. in Lond. Journ. Bot. III (1844) pag. 440 ; Nereis Australis (1847) pag. 51, tab. 15; Hooker Fl. Tasman. II (1860) pag. 300; Kuetz. Tab. Phyc. XIII (1863) tab. 66, fig. a-c ; J. Ag. Florideernes Morphologi (1879) tab. 33, fig. 7-8; De Toni Sylloge Algarum IV, pag. 928; Lucas Revised List pag. 41.

Polysiphonia nigrita Sond. in Botanische Zeitung 1845, pag. 53 (excl. stichidiis); Kuetz. Tab. Phyc. XIII (1863) tab. 67, fig. $\boldsymbol{e}-\boldsymbol{h}$.

Hab. Australia occid.: Baia di Geroldton; settembre 1908: Tasmania: Baia di Latrobe, 9 gennaio 1909; Burmie, sulle roccie battute dalle onde, 15 gennaio 1909.

Questa Polysiphonia, a detta di W. H. Harvey estremamente comune ai lidi della Tasmania, in particolare epifita sulle Fucoideae maggiori e quivi raccolta da Gunn, Hooker, Ewing ecc., vegeta eziandio lungo le coste occidentali e meridionali della Nuova Olanda (Sonder. Algae Austral. hactenus cognitae n. 870-871) e della Nuova Zelanda (Hooker Handbook pag. 672).

La fronda modifica l' aspetto sensibilmente con l' età ; dapprima è rossobruna e aderisce tenacemente alla carta, poscia disseccando si assottiglia, è nera e non aderisce alla carta, i rami inferiori sono all' apice circinati come quelli della Hypnea. La terza fase dimostra frequenti fenomeni di corrosione all'estremità della fronda, i rami circinati non si trovano se non in via eccezionale e la fronda non aderisce più affatto alla carta.

Polysiphonia Frutex Harv. in Lond. Journ. III (1844) pag. 439, Nereis Australis (1847) pag. 52 ; Hooker. Fl. Tasman II (1860) pag. 301; Kuetz. Tab. Phyc. XIII (1863) tab. 66, fig. $d$-e ; De Toni Sylloge Algarum IV, pag. 925.

Polysiphonia decipiens Mont. Prodr. Phyc. Antarct. (1842) pag. 5, Voyage au Pole Sud (1845) pag. 131; Harv. Nereis Australis (1847) pag. 50, Kuetz. Tab. Phyc. XIII (1863) tab. 65, fig. c-e (non J. Agardh nec De Notaris).

Hab. Australia occid. : Baia di Geroldton, settembre 1908; Australia merid.: Victor Harbour, con sporangi; 12 novembre 1908; Tasmania: Burmie, con cistocarpi ; 15 gennaio 1909; Nuova Zelanda : Port Charmers, Bluff, 25 gennaio 1909.

Negli esemplari tasmanici, che costituiscono il tipo della Polysiphonia frutex Harv. i cistocarpi sono ovato-globosi, ottusissimi, sessili, somiglianti a quelli della specie affine Polysiphonia cancellata Harv. che forma un gruppo facile a riconoscersi dalle congeneri assieme a Pol. fuscescens Harv. e a Pol. frutex Harv. Gli esemplari sporangiferi di Victor Harbour concordano con la Pol. decipiens Mont. la quale, come già sospettò J. AgARdH (Species III, pag. 1047) a mala pena può separarsi dalla Pol. frutex Harv. I sifoni pericentrali sono sette come nella Pol. frutex Harv. così nella Pol. decipiens Mont., mentre nella Pol. cancellata Harv. variano da sette a dieci; tutt' al più la Pol. decipiens Mont. potrebbe considerarsi una semplice forma della Pol. frutex Harv. caratterizzata da rami principali più eretti e rametti più densi; a nostro vedere il conservare separate le due specie è troppo artificioso, anche tenuto calcolo che leggere differenze possono intercorrere, non sempre dagli autori con giudizio valutate, tra individui sterili, sporangiferi e cistocarpiferi; chè se la genuina Pol. cancellata Harv. possedesse sempre, come resulta dalla diagnosi esibitane da W. H. HARVEY (Nereis Australis pag. 51) sette soli sifoni pericentrali, non vi sarebbe motivo di distinguerla dalla Pol. frutex se non per la forma delle articolazioni, 
della quale però convien ricordare la variabilità secondo le regioni della fronda che vengano esaminate. Se il maggior numero dei sifoni o cellule pericentrali, attribuito da J. AgARDH e da altri e dovuto all' aver riferito alla genuina Pol. cancellata Harv. la Pol. nigrita Sond., deve servire di base per tenere disgiunta la Pol. cancellata Harv. dalla Pol. frutex Harv., meglio sarebbe districare la sinonimia fondandosi sulle diagnosi originali fornite dagli autori delle singole specie, in altre parole risalire alle prime e non alterate descrizioni; ciò che non è qui il momento opportuno di fare.

Dal confronto dei dati forniti riguardo al numero dei sifoni pericentrali dal Kuetzing (Species Algarum pag. 815: Polysiphonia sectio Platysiphonia, in cui colloca, malgrado il limite « articulis 5-8 siphoneis » specie con 4 soli ovvero con 12 cellule pericentrali), da Harvey (Nereis Australis pag. 52: Polysiphonia $\S$ 8. Cancellatae: siphones 7, raro 8-9; vi colloca 1' autore però la Pol.aterrima con 9-10 cellule pericentrali) e da J. Agardh (Species II, 3, pag. 1045: P'olysiphonia 18. Cancellatae: articulis ecorticatis, $7-8$ siphoneis; nondimeno vi si trova inclusa la Pol. atervima con 9-10 cellule pericentrali) è ovvio comprendere di fronte a quante incertezze si sono trovati i ficologi nella classificazione di questo importante gruppo di Polysiphonia oceaniche; nè deve disconoscersi che piccole modificazioni, forse arbitrarie, alle frasi diagnostiche originali, contribuirono ad aggrovigliare ognor più la matassa; valga l'esempio di Polysiphonia nigrila alla quale il SONDER attribuì nella descrizione prima data in luce (1845) 8 cellule pericentrali, W. H. Harvey (1847) ne assegnò 8-9, J. AgardH (1863) 8 a 10.

Noi riteniamo che le Polysiphonia australasiche e neozelandiche della sezione Cancellatae potranno, in seguito a ulteriori confronti di più abbondante materiale, venire disposte in due gruppi: l' uno costituito da Pol. cancellala Harv., P. frutex Harv., P. nigrita Sond., P. fuscescens Harv., P. decipiens Mont. e forse $P$. vamulosa Harv., l' altro formato dalla Pol. aterrima Hook. et Harv. mentre d'altro canto non si sa spiegarsi per quali motivi il KuEtzing nella sopra ricordata sezione Platysiphonia (cui dava come carattere l'avere 5-8 cellule pericentrali) abbia incluso le Pol. abscissa Hook. et Harv. e P. microcarpa Hook. et Harv. (identiche fra loro) e la P. incompla Harv. che mostrano soltanto 4 cellule pericentrali.

Polysiphonia Hystrix Hook. et Harv. Alg. Tasman. n. 10, Nereis Australis (1847) pag. 41, tab. 14, Kuetz. Tab. Phyc. XIV (1864) tab. 18, fig. a-c; De Toni Sylloge Algarum IV, pag. 906; Lucás Revised List pag. 41.

Hab. Australia occid. : Baia di Geroldton; Australia merid. : Victor Harbour.

I nostri esemplari vennero confrontati con quelli dei nostri erbarii, de' quali uno distribuito da J. AsaRDH nelle Algae Muellerianae con provenienza da Hopkins River, un altro raccolto da A. GRunow a Queenscliff (Victoria), un terzo del Sonder di Port Phillip, La Polysiphonia Hystrix Hook. et Harv., oltre che sui lidi occidentali e meridionali dell' Australia e su quelli della Tasmania, vegeta sulle coste della Nuova Zelanda (R. J. HARYEy-GiBson, On some marine Algae from New Zealand pag. 2; London 1893).

Polysiphonia mollis Hook. et Harv. in Harv. Nereis Australis (1847) pag. 43 ; Kuetz. Tab. Phyc. XIII (1863) tab. 88, fig. a-c; Askenasy Australische Meere- 
salgen (1894) pag. 13; De Toni Sylloge Algarum IV, pag. 877; Mazza Saggio di Algologia Oceanica n. 284.

Hab. Nuova Zelanda: Bluff.

Gli esemplari comunicatici dal rev. G. CAPRA corrispondono a quelli di $P O^{*}$ lysiphonia tongatensis Harv. raccolti a Honolulu durante il viaggio di circumnavigazione della «Vettor Pisani » da Cesare Marcacci e conservati ne' nostri erbarii. La Pol. tongatensis Harv. di Tongatabu a mala pena differisce, secondo il Grunow (Algen der Fïdschi, Tonga und Samoa Inseln pag. 48) e l' Askenasy (op. cit. pag. 13) dalla Pol. mollis Hook. et Harv.; l' Askenasy descrisse per la Pol. mollis H. et H. gli sporangi, i cistocarpi e gli anteridi. La Pol. mollis vegeta lungo le coste della Nuova Guinea, dell' Australia occidentale e meridionale e della Tasmania.

Alla specie in parola apparisce essere molto affine la Polysiphonia flavescens Zanard. finora conosciuta solo di Georgetown (Tasmania) che il compianto Antonio De Toni, Sopra alcune Polysiphonia inedile o vare n. 18 ; in Nuova Notarisia ser. XVIII, pag. 166-168; Padova 1907) controllò su esemplari autentici illustrandone i cistocarpi la cui esistenza era sfuggita a G. ZANARDin.

Digenea C. Ag.

Digenea simplex (Wulf.) Ag. Species I (1821) pag. 389; Harv. Nereis Boreali-Americana II (1852) pag. 30, tab. XIII D; Falkenberg Die Rhodomelaceen (1901) pag. 159, tab. 9, fig. 25-29; Okam. Illustr. Alg. Japon. 1901, tab. 9; De Toni Sylloge Algarum IV, pag. 963; Lucas Revised List pag. 41.

Digenea Wulfeni Kuetz. Tab. Phyc. XV (1865) tab. 28, fig 1.

Hab. Australia occid. : Baia di Geroldton, con sporangi; settembre 1908.

Questa specie, come osservò giustamente l' ARdissone (Le Alghe cosmopolite pag. 2; Milano 1894) ha un' area estesissima, vegetando nell' Oceano Atlantico boreale, nel Mare Mediterraneo e negli oceani tropicali. Nell' Oceano Pacifico, oltre che sulle coste del Giappone (OKamura Alg. Japon. exsicc. n. 24), essa vegeta lungo i lidi settentrionali orientali (Queensland a Cape York, Cape Flattery e Cape Upstart; isola Dunk) e occidentali (isola Dick Hartog) dell' Australia. I nostri esemplari vennero riscontrati, oltre che con le forme mediterranee, con quelle distribuite da J. AGardH nelle Alghe Muellevianae col nome di Digenea simplex var. Longiseta raccolti a Cape Flattery. Il carattere de' rametti semplici o divisi sui quali rispettivamente il Kuetzing mantenne distinte la Digenea Wulfeni Kuetz. dalla Digenea Vieillardi Kuetz. (della Nuova Caledonia) non ci sembra sufficiente a stabilire l' autonomia di due specie; HaRvey (op. cit.) a proposito degli esemplari americani della Digenea simplex affermò infatti che i rametti sono " filiform, simple or sligthy branched"; anche il SonDER (Algen der tropischen Australiens pag. 51) riferendo come varietà innominata la Dig. Vieillardi Kuetz. alla Dig. simplex Ag. con l' avvertenza « ramellis saepius divisis » non ommise di ricordare il fatto che negli esemplari di Digenea simplex $\mathrm{A}$. provenienti dalle Indie occidentali i rametti sono di regola semplici, ma taluni anche divisi. 


\section{Bryocladia Schmitz}

Bryocladia cervicornis (Kuetz.) Schmitz in Engler-Prantl Die natürl. Pflanzenfam. (1897) pag. 442; Falkenb. Die Rhodomelaceen (1901) pag. 170; De Toni Sylloge Algarum IV, pag. 966.

Polysiphonia cervicornis Kuetz. in Flora 1847, pag. 776, Species (1849) pag. 831, Tab. Phyc. XIV (1864) tab. 15, fig. $d$-g.

Hab. Australia merid.; Victor Harbour; 12 novembre 1908.

Questa specie era nota per le coste dell' isola di Giava dove a Malang nella regione austro-orientale la raccolse H. Zol.Linger. I nostri esemplari mostrano, nell' aspetto generale, grande rassomiglianza con la Bryocladia evicoides (Harv.) Schmitz ma la fronda in sezione lascia scorgere nettamente solo 6-9 cellule pericentrali, mentre la Bryocladia evicoides ne possiede 16 ; noi non osiamo affermare che la Bryocladia cervicornis rappresenti una semplice forma della Bryocladia ericoides ma ci sembra lecito il supporlo.

\section{Chiracanthia Falk.}

Chiracanthia Arborea (Harv.) Falk. Rhodomelaceen (1901) pag. 179, tab. 19, fig. 18-23; De Toni Sylloge Algarum IV, pag. 971.

Acanthophora arborea Harv. in Hook. Fl. Tasman. II (1860) pag. 296, Phyc. Australica III (1860) tab. 132; J. Ag. Species III, 3 (1863) pag. 822 (saltem pro parte).

Hab. Tasmania: Baia di Latrobe, attaccata alle rocce.

Specie endemica per la Tasmania, ove fu trovata da J. Fereday e Harvey alla foce del Tamar presso Georgetown; venne inoltre distribuita sotto il nome di Achanthophora tasmanica dallo stesso Harvey al n. 140 delle Algae Austral. exsicc. per quanto risulta dalla sinonimia originale recata accanto alla tavola della Phycologia Australica. Dalle relazioni degli autori successivi non riesce specificata nessun' altra località di raccolta, cosł che sembra non trattarsi di specie molto comune. Siccome poi la Baia di Latrobe non trovasi assai discosta dalle foci del Tamar ed ambedue le località convergono verso la costa settentrionale, è probabile che l' area di distribuzione debba ancora restringersi a questa sola parte del litorale della Tasmania spingendosi tutt' al più ad invadere le coste delle isole vicine. Gli esemplari raccolti dal rev. G. CAPra sono due, l'uno d' essi è intiero e munito di una base ramificato-divisa dove ciascuna estremità dimostra un callo di adesione; le ramificazioni vi sorgono a una notevole distanza da questa e lasciano scoperto un tratto abbastanza lungo il caule allungato e nudo quale venne descritto nella diagnosi; le prime ramificazioni risalendo sembrano abortite essendo prive di spine e di ramuli; forse non sono che residui di vegetazione come il caule e da principio non saranno state nude; $i$ sifoni o cellule pericentrali sono quattro, nelle Acanthophora genuine sono sempre cinque. 


\section{Subfam. Pterosiphonieae Falk.}

\section{Pollexfenia Harv.}

Pollexfenia Pedicellata Harv. in Lond. Journ. III (1844) pag. 431; Nereis Australis (1847) pag. 22, tab. V ; Kuetz. Tab. Phyc. XVI (1866) tab. 9, fig. a-c ; Falkenb. Rhodomelaceen (1901) pag. 291, tab. 4, fig. 14-19; De Toni Sylloge Algarum IV, pag. 979; Lucas Revised List pag. 42; Mazza Saggio di Algologia Oceanica n. 302.

Hab. Australia merid.: Victor Harbour; 12 novembre 1908.

Insieme a Pollexfenia lobata (Lamour.) Falk. costituisce nel genere una delle forme più comuni lungo i lidi occidentali e meridionali dell' Australia e quelli della Tasmania. Le fronde di questa specie mostrano una certa variabilità per l'aspetto più o meno allungato delle lacinie del tallo ed anche per la maggiore o minore densità dei sori sporangiferi che la rendono più o meno trasparente.

\section{Dictymenia Grev.}

Dictymenia Sonderi Harv. List Austral. Alg. exsicc. n. 122, Phyc. Australica I (1858) tab. 21 ; Kuetz. Tab. Phyc. XIV (1864) tab. 96 ; Falkenb. Die Rhodomelaceen (1901) pag. 285, tab. 19, fig. 13-16; De Toni Sylloge Algarum IV, pag. 986; Lucas Revised List pag. 42.

Dictymenia fimbriata Harv. Mar. Bot. of West Australia (1854) n. 58 (nec Greville).

Dictymenia tridens Sond. in Linnaea XXV (1852) pag. 697 (non Harvey nec Greville).

Hab. Australia occid. : Baia di Geroldton; settembre 1908.

Il genere Diclymenia comprende specie tutte proprie della regione oceanica; solo una, di dubbia appartenenza al genere, cioè la Diclymenia? ecostata J. Ag. vegeta nell' Oceano Indiano. La Dictymenia Sonderi Harv., della Nuova Olanda occidentale, venne finora segnalata dagli autori (Sonder, Harver) per la Penisola Lefebre, Cape Leeuwin, Busseltown, Fremantle e Garden Island.

Dictrumenia tridens (Mert.) Grev. Syn. Alg. Brit. (1830) pag. LI; Kuetz. Species (1849) pag. 848, Tab. Phyc. XIV '(1864) tab. 94, fig.f-g; De Toni Sylloge Algarum IV, pag. 983 (non Harvey).

Fucus tridens Mert. in Turn. Hist. Fuc. IV (1819) tab. 255.

Hab. Australia occid. : Baia di Geroldton; settembre 1908.

Questa specie sembra essere alquanto comune sulle coste occidentali dell'A Australia essendovi stata raccolta dai primi esploratori Péron, La Billardière ecc. e in parecchie località si trova promiscua con la precedente. Gli esemplari raccolti dal rev. G. CAPRA sono stichidiferi con fronda non più larga di due 0 tre millimetri. 
Subfam. Lophothalieae $S$. et $F$.

\section{Brongniartella Bory}

Brongniartella australis (Ag.) Schmitz Die Gattung Lophothalia (1893) pag. 218; Falk. Die Rhodomelaceen (1901) pag. 546, tab. 19, fig. 6-7; De Toni Sylloge Algarum IV, pag. 1010; Lucas Revised List pag. 43; Mazza Saggio di Algologia Oceanica n. 310.

Polysiphonia Cladostephus Mont. Voyage au Pole Sud (1845) pag. 132, tab. 13, fig. 4; Harvey Phyc. Australica III (1860) tab. 154 (excl. fig. 2); Kuetz. Tab. Phyc. XIV (1864) tab. 19, fig. a-c; Sond. Alg. Austral. hactenus cognitae (1881) n. 857.

Hab. Tasmania: Baia di Latrobe; Nuova Zelanda: Port Charmers, Bluff.

Gli esemplari speditici dal rev. G. CAPRA corrispondono perfettamente a quella specie che un tempo il Montagne descrisse col nome di Polysiphonia Cladostephus su materiali raccolti da Dumont D' URville sui lidi delle isole Auckland; già fino da allora il Montagne aveva con molta acutezza intravveduto ch' egli si trovava sott' occhio una Floridea da iscrivere in via provvisoria fra le Polysiphonia, degna di venire elevata a costituire un nuovo genere ( $\mathrm{He}$ teromitus) precorrendo così J. AGARDH che nel 1890 la incluse nel genere Lo phothalia e F. Sснмiтz (1893) il quale da quest' ultimo la tolse per ascriverla al genere Brongniarlella Bory da lui rimesso in vigore. Noi abbiamo confrontato le piante del CAPRA con un esemplare crescente sulla Cyslophora paniculata (Turn.) J. Ag. ad Adelaide (Australia) raccolto da Schomburgk e donato da A. Grunow e con un altro delle Algae Muellerianae proveniente dalla Nuova Zelanda. La Brongniartella australis (Ag.) Schmitz vegeta lungo i lidi occidentali e meridionali dell' Australia, quelli della Tasmania e della Nuova Zelanda; a Bluff fu già raccolta da S. Berggren e poscia dal LaIng (Revised List II, 1901, pag. 356, n. 367).

L'aspetto della fronda vecchia è alquanto differente da quello de' rami giovanili, esso ricorda lontanamente quello di Polysiphonia frutex Harv. e simili; non è così per le ramificazioni più giovani dove in particolare si scorge quella somiglianza col Cladostephus che dette origine al nome del Montagne ora abbandonato.

Brongniartella Sarcocaulon (Harv.) Schmitz Die Gattung Lophothalia (1893) pag. 218; De Toni Sylloge Algarum IV, pag. 1013 ; Lucas Revised List pag. 43.

Dasya Sarcocaulon Harv. Phyc. Australica V (1863) tab. 278.

Hab. Australia occid. : Baia di Geroldton; settembre 1908.

La Brongniartella Sarcocaulon (Harv.) Schm. vegeta alle coste occidentali dell' Australia (scoperta la prima volta a Fremantle nel 1858 da G. Clifton) e della Tasmania settentrionale. L' esemplare raccolto dal CAPRA è dei più grandi, misurando oltre 11 centimetri in lunghezza. 


\section{Subfam. Polyzonieae Schmitz.}

Euzoniella Falk.

Euzoniella incisa (J. Ag.) Falk. Die Rhodomelaceen (1901) pag. 361, tab.5, fig. 2-8, 11, tab. 14, fig. 28-32; De Toni Sylloge Algarum IV, pag. 1028; Lucas Revised List pag. 44.

Polyzonia incisa J. Ag. Symbolae (1841) pag. 24; Harv. Phyc. Australica I (1858) tab. 42 A; Kuetz. Tab. Phyc. XV (1865) tab. 5, fig. $e-f$.

Hab. Australia occid. : Baia di Geroldton, settembre 1908.

Questa elegante Floridea epifitica non è rara nell' Oceano Pacifico australe lungo i lidi occidentali e meridionali della Nuova Olanda, quelli della Tasmania, della Nuova Zelanda e delle is. Chatham, Kermadecs e Little Barrier; di essa si occuparono, dal punto di vista anatomico, il Connolly (Beitrage zur Kenntnis einiger Florideen pag. 150-152, fig. 10-11; Flora vol. 103, 1911) e la sig. ${ }^{\text {na }}$ LuISA Cuoghi-Costantini (Osservazioni critiche intorno la Euzoniella incisa (J. Ag.). Falk., con 2 tavole; Nuova Notarisia serie XXIII, 1912, pag. 183-194).

\section{Subfain. Herposiphonieae $S$. et $F$.}

\section{Herposiphonia Naeg.}

Herposiphonia Filipendula (Harv.) Falk. Die Rhodomelaceen (1901) pag. 317; De Toni Sylloge Algarum IV, pag. 1058.

Polysiphonia Filipendula Harv. Alg. Austral. exsicc. n. 193; Kuetz. Tab. Phyc. XIV (1864) tab. 38, fig. a-e.

Hab. Nuova Zelanda: Bluff, con anteridi e sporangi.

La specie finora era stata indicata per le coste occidentali e meridionali dell' Australia ; infatti Harvey (Phyc. Austral. Syn. 1863, pag. XXI, n. 244) e Sunder (Algae Auslral. hactenus cognilae n. 864) la segnalarono per Fremantle e Port Fairy.

Herposiphonia versicolor (Hook. et Harv.) Falk. Die Rhodomelaceen (1901) pag. 305; De Toni Sylloge Algarum IV, pag. 1056 ; Lucas Revised List pag. 44.

Polysiphonia versicolor Hook. et Harv. in Lond. Journal VI (1847) pag. 399, n. 15; Nereis Australis (1847) pag. 48, tab. 16; Kuetz. Tab. Phyc. XIII (1863) tab. 31, fig. a-c.

Hab. Tasmania: Baia di Latrobe, sulla Hypnea nidifica.

I nostri esemplari s' accordano con quelli tasmanici dell' Erbario ZaNARDINI, indicati al n. 1033 del Catalogo illustrativo di quella collezione. Oltre che nella Tasmania, la specie venne raccolta lungo le coste occidentali e meridionali dell'Australia, come risulta dalle pubblicazioni di Harvey, Sonber e Reinbold e dagli esemplari provenienti da Port Phillip distribuiti dal Lenormand. Di questa bella Herposiphonia furono esattamente descritti i cistocarpi, che hanno forma obovato-subglobosa, da J. AGARDH (Analecta algologica contin. III, 1896, pag. 111). 


\section{Subfam. Rytiphloeà (Decne) Kuetz.}

\section{Protokuetzingia Falk.}

Protokuetzingia australasica (Mont.) Falk. in Engler-Prantl Die natürl. Pflanzenfam. I (1897) pag. 469, Die Rhodomelaceen (1901) pag. 475, tab. 9, fig. 6 ; De Toni Sylloge Algarum IV, pag. 1076; Lucas Revised List pag. 45.

Rytiphloea australasica Endl., Harv. Phyc. Australica I (1858) tab. 27.

Halopithys australasica Kuetz. Tab. Phyc. XV (1865) tab. 27, fig. e-g.

Hab. Australia occid.: Baia di Geroldton; settembre 1908; Australia merid. : Victor Harbour; Tasmania: Baia di Latrobe, con stichidii.

Anche secondo Harvey questa specie è assai abbondante lungo i lidi occidentali e meridionali della Nuova Olanda e non manca alla Tasmania.

Il Kuetzing (Species, 1849, pag. 841) riferì questa specie al genere Halopithys (caratterizzato da sporangi biseriati entro gli stichidii) attribuendole, sulla fede del Montagne e forse di W. H. Harvey (Nereis Australis pag. 32) sporangi uniseriati, rettificando solo più tardi l' errore nel quale egli era incorso, figurando nelle Tabulae Phycologicae gli sporangi, come in realtà sono, biseriati. L'errore nel quale caddero gli autori nell' attribuire sporangi uniseriati derivò dalla posizione in cui si trovavano gli stichidii, i quali di faccia mostrano due serie, di fianco una sola serie di sporangi. Il Sonder (Plantae Muellevianae 1852, pag. 700) ne ebbe esemplari sterili dai lidi della penisola Lefebre.

\section{Kuetzingia Sond.}

Kuetzingia canaliculata (Grev.) Sond. in Botanische Zeitung 1845, pag. 54 ; Harv. Nereis Australis (1847) pag. 23, tab. 9; Phyc. Australica IV (1862) tab. 232 ; Kuetz. Tab. Phyc. XV (1865) tab. 10; Falk. Die Rhodomelaceen (1901) pag. 451, tab. 7, fig. 30-36 ; De Toni Sylloge Algarum IV, pag. 1078; Lucas Revised List pag. 45.

Rytiphloea canaliculata Grev. in Edinb. Journ. of Nat. and Geogr. Sc. n. ser., n. III (1831), tab. 4, fig. 1-4.

Hab. Australia occid.: Baia di Geroldton, con sporangi; settembre 1908.

È una specie abbastanza comune lungo le coste occidentali dell' Australia, spingendosi fino al King George's Sound. Esemplari sterili vennero già distribuiti nelle Algae Muellevianae di Champion Bay vicina alla Geroldton Bay. Vegeta anche, secondo il Sonder, a Fremantle e Geographe Bay.

\section{Amansia Lamour.}

Amansia Daemeliı (Sond.) J. Ag. Analecta algologica (1892) pag. 172; Falk. Die Rhodomelaceen (19)1) pag. 422, tab. 7, fig. 6-7; De Toni Sylloge Algarum IV, pag. 1088; Lucas Revised List pag. 45. Icon: Tab. nostra VI, fig. 7-8.

Vidalia Daemelii Sond. Alg. des trop. Australiens (1871) pag. 49, tab. I, fig. 1-10.

Hab. Australia merid.: Victor Harbour, con stichidi, novembre 1908, 
I nostri esemplari, di consistenza coriaceo-soverosa e di color bistro-bruno, rappresentano una forma senile di questa specie e che si tratti propriamente di fronda vecchia si desume dal contrasto che si ha tanto per la tinta delicata quanto per la tinta rosea di certe estremità che sembrano rigetti i quali bene aderiscono alla carta, ciò che non si verifica per il rimanente'

Amansia Daemelii (Sond.) J. Ag. fu raccolta per la prima volta dal DAEMEL al Capo York nell' Australia.

Amansia Kuetzingioides Harv. Phyc. Australica I (1858) tab. 51; Falk. Die Rhodomelaceen (1901) pag. 420, tab. 7, fig. 5; De Toni Sylloge Algarum IV, pag. 1085; Lucas Revised List pag. 45.

Amansia Hawkeri J. Ag. Analecta Algologica (1892) pag. 173.

Kuetzingia serrata Harv. in Trans. R. Irish Academy vol. XXIl (1855) pag. 583, Alg. Austral. exsicc. n. 132.

Hab. Australia occid. : Baia di Geroldton ; settembre 1908; Australia merid. : Victor Harbour, con sporangi; novembre 1908.

Gli esemplari raccolti da G. CAPRA in entrambe le località sopra citate vennero confrontati con quelli esistenti nelle nostre collezioni sotto il nome di Amansia Hawkeri J. Ag. da Sturt Bay, Investigator Street (Australia meridionale) raccolti da E. A. Davey e determinati da T. ReinBold; essi vi corrispondono bene quanto a struttura, cosi da non lasciare dubbii sul riferimento dei medesimi alla Amansia Kuetzingioides Harv. La specie, scoperta da G. Clifton nella Nuova Olanda occidentale all' isola Rottnest, venne poscia raccolta a Champion Bay; più tardi l' area di distribuzione fu estesa all' Australia meridionale, essendo la specie stata rinvenuta a Port Elliot e ad Adelaide rispettivamente dalla attivissima sig. ${ }^{\text {na }}$ Hussey e da G. C. Hawker.

\section{Vidalia Lamour.}

Vidalia Spiralis Lamour. in Dict. cl. V, pag. 387 ; J. Ag. Florideernes Morphologi (1879) tab. 33, fig. 28-29; De Toni Sylloge Algarum IV, pag. 1106; Lucas Revised List pag. 46.

Delesseria spiralis Lamour. Essai (1813) tab. 9, fig. 2.

Epineuron spirale Harv. Nereis Australis (1847) pag. 25, tab. 9; Kuetz. Tab. Phyc. XIV (1864) tab. 100, fig. a-c.

Hab. Australia occid.: Baia di Geroldton; settembre 1908.

Gli esemplari raccolti dal rev. G. CAPRA nella Baia di Geroldton corrispondono egregiamente a quelli distribuiti da J. AgARDH nelle Algae Muellerianae ed a quelli conservati nell' erbario ZANARDin indicati al n. 1164 della rispettiva illustrazione. L' area della specie, che il LucAs indicò nella sola Nuova Olanda occidentale deve però venire estesa alla regione meridionale di detto Continente, dopo che della specie stessa, dietro l' esame di materiali raccolti dal dott. A. ENGELHART, venne dal ReinBold affermata la presenza nello Investigator Street.

Vidalia GREgARIA Falk. Die Rhodomelaceen (1901) pag. 435, tab. 8, fig. 22-23; De Toni Sylloge Algarum IV, pag. 1108.

Polyphacum proliferum J. Ag. (juven.), Harv. Phyc. Australica IV (1862) tab. 188, fig. 2-4.

Hab. Australia occid. : Baia di Geroldton. 
Questa stranissima epifita che non s'aggrappa se non alle ramificazioni dell' Osmundaria prolifera Lamour. e che anzi con essa venne confusa perchè ritenuta una sua fase giovanile di sviluppo, si trovò in un esiguo numero di piccoli esemplari riuniti in gruppo sulla ramificazione di base dell' esemplare di Osmundaria indicato più oltre. Sono individui mal conservati in guisa da non potersi riconoscere che dalla struttura reticolato-striata delle lamine, ma i margini seghettati non si scorgono e mancano gli apici vegetativi. Così neanche in questa occasione torna possibile sciogliere il dubbio da uno di noi espresso nella Sylloge Algarum se l' intiero tallo della Vidalia gregaria Falk. s' intenda limitato a ciascun elemento fogliforme o se questi elementi, come nella figura esibita da HaRveY, si dispongano parte per parte di un asse come pinnule costituendo in tal modo un unico individuo. La specie sembra finora propria delle coste occidentali e meridionali dell' Australia. Gli esemplari originali provengono da King George's Sound.

\section{Osmundaria Lamour.}

Osmundaria prolifera Lamour. Essai (1813) pag. 23, tab. 7, fig. 4-6; Decaisne Bot. Voyage de la Venus tab. 3 ; Falk. Die Rhodomelaceen (1901) pag. 469, tab. 8, fig, 24-26; De Toni Sylloge Algarum IV, pag. 1109; Lucas Revised List pag. 46; Mazza Saggio di Algologia Oceanica n. 326.

Polyphacum proliferum C. Ag., Harv. Phyc. Australica IV (1862) tab. 188 (excl. fig. 2-4); Kuetz. Tab. Phyc. XVI (1860) tab. 7, fig. $g-l$.

$H a b$. Australia occid. : Baia di Geroldton; settembre 1908.

Specie caratteristica e finora esclusiva delle coste occidentali e meridionali dell' Australia. L' esemplare comunicatoci dal rev. G. CAPRA è largamente stichidifero e molto bene sviluppato oltrepassando i 25 centimetri di lunghezza; porta sulla ramificazione di base alcuni esemplari di Vidalia gregaria Falk. L'Osmundaria prolifera Lamour. è una specie con area geografica ristretta ma venne ripetutamente raccolta (Lamouroux, Preiss, Harvey, Clifton ecc.).

\section{Len orm andia Sond.}

Lenormandia angustifolia (Harv.) J. Ag. De Algis Novae Zelandiae marinis (1877) pag. 30, n. 269; Falk. Die Rhodomelaceen (1901) pag. 458, tab. 8, fig, 1-6; De Toni Sylloge Algarum IV, pag. 1120; Laing Revised List (1901) pag. 365, n. 356. Icon: Tab. nostra VII, fig. 4.

Lenormandia Chauvinii $\beta$ angustifolia Harv. in Hooker Fl. N. Zel. II (1855) pag. 222.

Hab. Nuova Zelanda: Bluff.

La specie, propria della Nuova Zelanda, fu già segnalata da J. AgardH per Bluff, località nella quale la raccolse S. BergGREN. Venne proposta da principio da W. H. HaRveY come varietà di Lenormandia Chauvinii Harv. e non è improbabile che quell' egregio botanico si sia apposto bene al vero. L'esemplare, comunicatoci dal CAPRA, reca alcuni scarsi stichidii.

Lenormandia Chauvini Harv. in Hooker Fl. N. Zel. II (1855), pag. 222, Handbook (1867) pag. 662; J. Ag. De Algis Novae Zelandiae marinis (1877) pag. 31, 
n. 270; De Toni Sylloge Algarum IV, pag. 1116; Laing Revised List, pag. 355,

n. 355. Icon: Tab. nostra VII, fig. 1.

Hab. Nuova Zelanda: Port Charmers, acque basse; 30 gennaio 1909.

Anche questa Lenormandia è propria della Flora neozelandese, essendo stata rinvenuta in più località da Lyall, Colenso, Berggren, J. C. Smith e Laing e non mancando alle isole Chatham (CотTON). E dubbio se essa in realtà vegeti sui lidi della Nuova Olanda. W. H. Harver nella sua Phycologia Australica non ne fa cenno e il Sonder (Algae Austral. hactenus cognitae n. 794) dà la semplice indicazione "Australia ». Il LucAs (Revised List pag. 46) ne riporta solo il nome, senza indicazione di area geografica. I nostri sono magnifici esemplari stichidiferi con stichidii allineati a frangie sul margine.

Lenormandia Muelleri Sond. in Linnaea vol. 25 (1852) pag. 696; Harv. Phyc. Australica I (1858) tab. 45; Kuetz. Tab. Phyc. XIX (1869) tab. 15, fig. $a-b$; Falk. Die Rhodomelaceen (1901) pag. 467, tab. 8, fig. 13-16; De Toni Sylloge Algarum IV, pag. 1116; Lucas Revised List pag. 46; Mazza Saggio di Algologia Oceanica n. 328.

Hab. Australia occid. : Baia di Geroldton; settembre 1908 ; Australia merid. Victor Harbour, con sporangi.

La Lenormandia Muelleri Sond. ha larga distribuzione geografica lungo i lidi soprattutto meridionali dell' Australia, come resulta dai lavori di HARVEY, SONDER e ReINBold; la prima volta venne raccolta a Rivoli-Bay.

\section{Subfam. Bostrychieae Falk.}

Bostrychia Mont.

Bostrychia mixta Hook, et Harv. in Hooker Fl. N. Zel. Il (1855) pag. 225, Handbook (1867) pag. 665; Harv. Phyc. Australica III (1860) tab. 176 A; Kuetz. Tab. Phyc. XV (1865) tab. 20, fig. d-f: De Toni Sylloge Algarum IV, pag. 1150; Lucas Revised List pag. 47.

Hab. N. Queensland, Baron River, 3 giugno 1909.

Mancano dati sicuri per controllo su materiali autentici rispetto all' area di distribuzione di questa Bostrychia per la quale si possono anche confrontare le osservazioni esposte dal Grunow (Alg. Novara pag. 90). Harvey (Neveis Australis pag. 70) indica la Bostrychia mixta $\mathrm{H}$. et $\mathrm{H}$. per la Nuova Zelanda e per Muysenberg (Capo di Buona Speranza, Africa meridionale); più tardi (Phycologia Australica cit. sopra) aggiunge la località Port Arthur in Tasmania. Il SONDER (Algae Austral. hactenus cognitae n. 835) nota per questa specie, oltre a Port Arthur, il S. Vincent Gulf (Australia meridionale). Più recentemente il Cotron (Marine Algae from the Chatham Islands 1907, pag. 42) enumera la specie tra quelle raccolte alle isole Chatham dalla sig. H. E. MaLtbY, assegnando come area l' Australia, la Nuova Zelanda e l' Africa meridionale (1).

(I) Il Kuntzing (Species Algarum pag. 840; Lipsiae 1849) indica il solo Capo di Buona Speranza come stazione per la Bostrychia mixta $\mathrm{H}$. et $\mathrm{H}$, avvertendo che un esemplare gli venne comunicato da HARveY; di detta località è figurata la specie nelle Tabulae Phycologicae dello stesso autore. W. Trson (South african marine Algat n. 82) distribui un bell' esemplare proveniente da Algoa Bay col quale il nostro esilissimo esemplare coincide allo scrupolo. 
Bostrychia simpliciuscula Harv. Alg. Friendly Isl. exsicc. n. 23; J. Ag. Species Il, 3 (1863) pag. 854 ; De Toni Sylloge Algarum IV, pag. 1155; Lucas Revised List pag. 47.

Bostrychia rivularis Harv. Phyc. Australica III (1860) tab. 176 B (non alibi). Zanard.

Hab. Tasmania: sponde del fiume Tamar, insieme a Caloglossa Beccarii

Descritta da Harvey su esemplari delle isole Tonga, venne questa Bostrychia segnalata più tardi del Moy River, Port Fairy, Victoria (Australia meridionale) e del Paramatta River (Australia orientale).

\section{Subfam. Dasyeae (Kuets.) S. et F.}

\section{Thuretia Decne.}

Thuretia Quercifolia Decne. in Ann. Sc. Nat. sér. 3, t. II, Botan. (1843) pag. 236, Voyage de la Venus tab. 2 A; Kuetz. Tab. Phyc. XII (1862) tab. 64, fig. e-g; Harv. Phyc. Australica I (1858) tab. 40 ; Falk. Die Rhodomelaceen (1901) pag. 668, tab. 17, fig. 1-9; De Toni Sylloge Algarum JV, pag. 1175 ; Lucas Supplementary List of the Marine Algae of Australia (1912) pag. 157; Mazza Saggio di Algologia Oceanica n. 342.

Hab. Australia occid.: Baia di Geroldton, su Mastophora Lamourouxir Decne.; settembre 1908.

Questa Thuretia ha larga distribuzione lungo i lidi occidentali e meridionali dell' Australia, come risulta in particolare dai contributi pubblicati da HarveY, SONDER e REINBOLD.

Thuretia teres Harv. Phyc. Australica IV (1862) tab. 192 ; De Toni Sylloge Algarum IV, pag. 1176; Lucas Supplementary List (1912) pag. 157.

Dictyurus australasicus Sond. in Linnaea vol. 26 (1853) pag. 527.

Dictyurus australis Sond. Alg. Austral. hactenus cognitae (1881) pag. 31, n. 762 ; Harv. Phyc. Australica Syn. (1863) pag. XV, n. 152.

Hab. Australia occid. : Baia di Geroldton, con anteridii; settembre 1908.

E specie abbastanza comune lungo le coste meridionali della Nuova Olanda; venne indicata per Port Fairy, Port Phillips, Cape Arid e Western Port. Gli anteridii corrispondono a quelli figurati da HARVEY come corpi pedicellati, liberi nelle maglie del reticolo tallino.

\section{Dasya C. Ag.}

Dasya hapalathrix Harv. Alg. Austral. exsicc."n. 201, Phyc. Australica II (1859) tab. 88; De Toni Sylloge Algarum IV, pag. 1191 ; Lucas Supplementary List (1912) pag. 157.

$H a b$. Tasmania : Burmie, con cistocarpi.

Meschini esemplari o piuttosto frammenti di fronda vecchia che portano ancora alcuni cistocarpi sessili attaccati. Sembra che l' area geografica di questa specie si estenda dai lidi meridionali dell' Australia alla Tasmania, anche secondo conferma del Lucas. 
Dasya villosa Harv. in Lond. Journ. III (1844) pag. 433, Nereis Australis (1847) pag 61, tab. 20 ; Hooker Fl. Tasman. II (1860) pag. 302 ; Kuetz. Tab. Phyc. XIV (1864) tab. 57; De Toni Sylloge Algarum IV, pag. 1203; Lucas Supplementary List (1912) pag. 158.

$H a b$. Tasmania: Baia di Latrobe.

Gli esemplari avuti dal rev. G. CAPRA s' accordano con un esemplare cistocarpifero delle nostre collezioni da Moonta Bay (Australia meridionale) raccolto da T. J. O' Halloran e con un altro, sterile, da Georgetown (Tasmania) di provenienza HaRvey (duplicati dell' erbario ZaNARDinI, in quest' ultimo recanti il n. 400 della rispettiva illustrazione). Il SonDER (Algae Austral. hactenus cognitae n. 884) dà per la Dasya villosa Harv. la seguente area di distribuzione: Australia occidentale e meridionale, Tasmania. Questa specie rappresenta nell' Oceano Pacifico la vicariante della nostra Dasya elegans (Mart.) Ag. Venne distribuita da J. Tilden (American Algae n. 403) di Oahu delle isole Hawaii ma gli esemplari sono così alterati da non essere riconoscibili, È da rilevarsi il mutevole colore della fronda che, roseo-vinoso nelle ramificazioni giovanili, subisce con l' invecchiare prima un imbrunimento, poi uno scoloramento.

\section{Heterosiphonia Mont.}

Heterosiphonia Callithamnion (Sond.) Falk. Die Rhodomelaceen (1901) pag. 647 ; De Toni Sylloge Algarum IV, pag. 1225; Lucas Supplementary List (1912) pag. 158.

Polysiphonia Callithamnion Sond. in Botanische Zeitung 1845, pag. 54.

Dasya Callithamnion Harv. Mar. Bot. of West Australia (1854) n. 115; Kuetz. Tab. Phyc. XIV (1864) tab. 77, fig. c-e.

Hab. Australia occid.: Baia di Geroldton; settembre 1908; Tasmania : Baia di Latrobe, sul Cladosiphon zostericola Harv.

A questa specie è assegnata dagli autori come area di distribuzione la regione austro-occidentale della Nuova Olanda; quivi W. H. Harvey (Phyc. Australica Syn. pag. XXIII, n. 282) la indica abbondante. Il Sonder (Algae $A u$ stral. hactenus cognitae n. 903) nota per essa le località Rottnest e Fremantle.

\section{Fam. CeRAMideEA (Bonn.) Naeg.}

\section{Subfam. GRIfFithsieAe Schm.}

$$
\text { Griffithsia C. Ag. }
$$

Griffithsia Flabelliformis Harv. in Lond. Journ. III (1844) pag. 450 ; J. Ag. Epicrisis (1876) pag. 61; Askenasy Alg. Exped. Gazelle (1888) pag. 36, tab. 9, fig. 2-3; De Toni Sylloge Algarum IV, pag. 1278 ; Lucas Revised List pag. 48. Griffithsia tasmanica Kuetz. Tab. Phyc. XII (1862) pag. 7, tab. 19, fig. c-e. Hab. Tasmania: Baia di Latrobe.

Sembra che l'area di distribuzione di questa specie non sia solamente ristretta alla Tasmania come avrebbe ritenuto il Lucas ma si estenda dall' isola 
di S. Paolo dove l' Askenasy la riscontrò frammezzo alle Alghe ivi raccolte dalla «Gazelle» alle coste neozelandesi (Grunow). Griffithsia flabelliformis Harv. ha una fronda molto lubrica, che aderisce assai tenacemente alla carta. Si fissa sui rami di Cymodocea frammista a colonie di molti Briozoarii.

\section{Bornetia Thur.}

Bornetia? antarctica (H. et H.) De Toni Sylloge Algarum IV (1903) pag. 1297 ; Mazza Saggio di Algologia Oceanica n. 366.

Griffithsia autarctica Hook. et Harv. Crypt. antarct. (1845) pag. 182; J. Ag. Epicrisis (1876) pag. 68; Kuetz. Tab. Phyc. XII (1862) tab. 23, fig. $a-b$; Laing New Zeal. Ceramiaceae (1905) pag. 390, tab. 25, fig. 2.

Hab. Nuova Zelanda: Port Charmers.

Anche in questa occasione la sterilità di tutti gli esemplari impedisce di giudicare se questa specie sia bene attribuita al genere Bornetia o debba ricollocarsi come da principio tra le genuine Griffithsia. Nel Mare Antartico sembra specie molto diffusa, arrivando alle Maluine, al Capo Horn e alle is. Kerguelen; ma già J. AGARDH ritenne che gli esemplari neozelandesi e tasmaniani dovessero essere tutt' uno con gli originali delle Maluine. La fronda sterile vuole assai cautamente distinguersi da quella di Monospora? elongata (Harv.) D. T. dell' Australia meridionale, dalla quale non è differente che per la forma degli articoli e per la dimensione diversa di questi ultimi.

\section{Subfam. Monosporeae Schm.}

\section{Monospora Solier}

Monospora australis (Harv.) J. Ag. Epicrisis (1876) pag. 610; De Toni Sylloge Algarum IV, pag. 1299; Lucas Revised List pag. 48.

Corynospora australis Harv. in Trans. R. Irish Academy vol. XXII (1855) pag. 559, Phyc. Australica V (1863) tab. 253.

Hab. Australia occid.: Baia di Geroldton; settembre 1908.

Questa Monospora (la quale deve cautamente distinguersi dal Callithamnion licmophorum Harv. che forse ne rappresenta lo stato sporangifero) vegeta lungo i lidi occidentali e meridionali della Nuova Olanda, indicata per Rottnest, Fremantle e Port Phillip (Sonder Algae Austral. hactenus cognitae n. 693). I nostri esemplari sono sterili ma corrispondono egregiamente alle figure esibite da W. H. Harvey.

Monospora? elongata (Harv.) De Toni Sylloge Algarum IV (1903) pag. 1302. Callithamnion elongatum Harv. in Hooker Fl. Tasman. II (1860) pag. 336, Phyc. Australica Syn. (1863) pag. LIII, n. 680.

Griffithsia elongata J. Ag. Epicrisis (1876) pag. 62.

Hab. Australia merid.: Victor Harbour; 12 novembre 1908.

Questa specie sembra molto diversa da Monospora? griffithsioides (Sond.) D. T. anche dimostrando una simile distribuzione geografica. Gli articoli sono però in questa molto più grandi e allungati e pare in tutto una forma assai 
lussureggiante di quella. Gli esemplari osservati sono sterili e li confrontammo con uno proveniente da Hopkins River distribuito da J. AgardH nelle Algae Muellevianae conservato nell' erbario DE TonI.

Monospora? GRIFfithsioides (Sond.) De Toni Sylloge Algarum IV (1903) pag. 1302; Mazza Saggio di Algologia Oceanica n. 368.

Griffithsia Sonderiana J. Ag. Epicrisis (1876) pag. 62.

Callithamnion griffithsioides Sond. in Linnaea vol. 26 (1853) pag. 512 ; Harv. Phyc. Australica III (1860) tab. 160 (non Solier in Kuetz. Tab. Phyc. nec alior.).

Hab. Australia merid.: Victor Harbour; 12 novembre 1908; Tasmania: Burmie.

Già nella Sylloge Algarum venne accennato che gli esemplari attribuiti da HaRvey a Griffithsia setacea (Ellis) Ag. devonsi ritenere appartenenti a Monospora? griffithsioides (Harv.) D. T. Infatti un esemplare raccolto in Tasmania da H. WATts di provenienza Harveyana collima perfettamente con altri della Nuova Zelanda distribuiti nelle Algae Muellerianae e con i nostri, sebbene questi ultimi sieno sterili. Alcuni esemplari raccolti dal rev. G. CAPRA a Port Charmers e a Bluff per la straordinaria lunghezza degli articoli e per la natura della fronda ricordano la Griffithsia? comosa Grun. (Alg. Novara (1867) pag. 62, tab. 10, fig. 2) che HochSTETter raccolse appunto sui lidi della Nuova Zelanda. Il Laing (Revised List of New Zealand Seaweeds II, 1901, pag. 329, n. 115) non menziona la specie Grunowiana ma senz' altro, a proposito di Griffithsia Sonderiana J. Ag. scrive: "This is probably the common species of Griffithsia, but the New Zealand forms are not sufficienty known» e in altro suo scritto (On the Nerw Zealand species of Ceramiaceae, 1904, pag. 389) ascrive addirittura la Griffithsia? comosa Grun. alla Griffithsia Sonderiana J. Ag. indicando tra le località anche Port Charmers.

\section{Subfam. Spongoclonieae Schmitz.}

\section{Haloplegma Mont.}

Haloplegma Preissi Sond. Pl. Preiss. II (1848) pag. 171, Plantae Muellerianae (185') pag. 676; Harv. Phyc. Australica II (1859) tab. 79; Kuetz. Tab. Phyc. XII (1862) tab. 62, fig. d-e; De Toni Sylloge Algarum IV, pag. 1366; Lucas Revised List pag. 50; Mazza Saggio di Algologia Oceanica n. 386.

Hab. Tasmania: Burmie; gennaio 1909.

É specie abbastanza comune lungo le coste occidentali e meridionali della Nuova Olanda e quelle della Tasmania, com' è accertato dai reperti di HARver, SONDER e REINBOLD. I nostri esemplari furono confrontati con quelli, di parecchie località, dell' erbario ZANARDINI, tra i quali se ne trova uno proveniente da Georgetown (Tasmania), nonchè con quelli distribuiti da J. AgardH Algae Muellevianae provenienti dall' Australia occidentale e meridionale e dalla Tasmania. La forma del tallo di questa specie è assai mutevole; un saggio nelle Algae Muellerianae raccolto a Streaky Bay (Australia meridionale) presenta singolare interesse dimostrando le successive fasi d' invecchiamento della fronda col relativo mutamento di colore che si osserva pure in altre Florideae (Amansia Dae- 
melii, Dasya villosa ecc.) dal roseo-porporino al bistro spugna. Cosi anche allo stato giovanile la fronda è molle e aderisce alla carta, poi s'ispessisce e non si attacca più. Gli esemplari di Tasmania pur essi delle Algae Muellevianae si distinguono inoltre per avere una fronda più piana e sottile a lacinie assai più larghe ma non sono molto diversi dai nostri di Burmie che sono vecchie frondi in tutto somiglianti a quelle d'Australia.

\section{Subjam. Ptiloteae Cram.}

\section{Euptilota Kuetz.}

Euptilota articulata (J. Ag.) Schmitz Kleinere Beiträge zur Kennt. der Florideen VI (1896) pag. 7; De Toni Sylloge Algarum IV, pag. 1370; Lucas Revised List pag. 50; Mazza Saggio di Algologia Oceanica n. 388.

Ptilota articulata J. Ag. Symbolae (1841) pag. 36; Kuetz. Tab. Phyc. XII (1862) tab. 56, fig. $d-e$.

Hab. Australia occid.: Baia di Geroldton; settembre 1908.

La specie è nota per l' Australia occidentale fino dai tempi del LA BiLlarDIĖRE, del celebre viaggiatore del secolo XVIII, il quale pubblicò poscia un importante contributo sulla flora della Nuova Olanda (1804-1806); il Sonder (Algae Austral. hactenus cognitae n. 270) indica la specie per l'Australia a Fremantle e King George 's Sound e per la Tasmania a Georgetown; nell' erbario ZanarDINI sono conservati esemplari provenienti da Port Phillip (1) e da Kangaroo Point. Il Reinbold scoperse la Euptilota articulala (J. Ag.) Schm. fra Alghe raccolte nello Investigator Street dal dott. A. Engelhart. Il CAPRA raccolse soltanto due piccoli frammenti di fronda.

Euptilota coralloidea (J. Ag.) Kuetz. Species (1849) pag. 672; De Toni Sylloge Algarum IV, pag. 1371; Lucas Revised List pag. 50.

Ptilota coralloidea J. Ag. Symbolae (1841) pag. 35; Epicrisis (1876) pag. 78. Icon: Tab. nostra 7, fig. 2.

Hab. Australia occid. : Baia di Geroldton; settembre 1908.

Per questa Euptilota viene data come area di distribuzione la Nuova Olanda occidentale (anzi fu distribuita di Champion Bay da J. Agardh nelle Algae Muellerianae) e meridionale; secondo il SONDER (Algae Austral. hactenus cognitae n. 269) la specie non manca alla Tasmania, ma non ci consta da quale fonte egli abbia tratta questa affermazione; il LucAs, sulla fede dell' ora menzionato autore, ripete la stessa cosa.

\section{Subfam. Crouanieae Schmitz.}

\section{Antithamnion Naeg.}

Antithamnion dispar (Harv.) J. Ag. Analecta Algologica (1892) pag. 20; De Toni Sylloge Algarum IV, pag. 1405; Lucas Revised List pag. 51.

(I) Sembra queșta la stazione preferita; di colà provengono gli esemplari delle Algae Muellerianae e quelli distribuiti nel i 864 dal Lenormand (Erbario Piccone, ora incorporato nell'Erbario Forti). 
Callithamnon dispar Harv. Alg. Austral. exsicc. n. 509, Phyc. Australica IV (1862) tab. 227.

Hab. Australia occid. : Baia di Geroldton; settembre 1908.

Questo Antithamnion, raccolto per la prima volta a Port Fairy (Australia meridionale) e poscia a Warnambool, non è raro lungo i lidi austro-orientali della Nuova Olanda e quelli orientali della Tasmania; esso ha fronda piccola, di solito epifitica, nel caso nostro sopra i rami della Hypnea episcopalis Hook. et Harv.

Antithamnion mucronatum (J. Ag.) Naeg. Ceramiaceen (1861) pag. 146; De Toni Sylloge Algarum IV, pag. 1410; Lucas Revised List pag. 51; Laing on the New Zealand Species of Ceramiaceae (1905) pag. 404.

Callithamnion mucronatum J. Ag. Species II (1851) pag. 29; Harv. Phyc. Austral. Syn. (1863) pag. LIII, n. 688.

Callithamnion cruciatum Harv. in Lond. Journ. III (1844) pag. 444 (non alior.).

Hab. Tasmania : Baia di Latrobe.

Gli esemplari raccolti dal dott. G. CAPRA coincidono esattamente con alcuni provenienti dalla Nuova Olanda e distribuiti da J. AGARDH; presentano essi cellule pleurogene a contenuto giallognolo, le quali non mostrano accenni a divisione come avviene negli sporangi ma all' incontro hanno un contenuto omogeneo, di aspetto quasi vitreo; riteniamo trạttarsi di quelle formazioni peculiari, conosciute per altre specie congeneri, illustrate in questi ultimi tempi dal NESTLER (1898) per l'Antithamnion Plumula (Ellis) Thur. e per l'Antithamnion cruciatum (Ag.) Naeg., dal Schussnig (1914) per queste due specie e per l'Antithamnion cladodermum (Zanard.) Hauck, da V. Schiffner (1915) per l' Antithamnion Spirographidis Schiffn. e per l'Ant. tenuissimum (Hauck) Sch., da Kylin e SkotTsBerg (1919) per l' Antithamnion ramulosum (Reinsch) Kyl. e per l' Ant. antarcticum Kyl. Tali formazioni cellulari, note per l' Antithamnion Plumula fino dall' epoca di C. AGardH (Species Algarum II, 1828, pag. 159) non mancano in altre Florideae, ad esempio Chondrococcus Hornemanni (Mert.) Schmitz [Desmia coccinea Zanard.], Nemastoma dichotoma J. Ag.

L'Antilhamnion mucronatum (J. Ag.) Naeg. ha un' estesa area di distribuzione geografica in quanto di esso venne riscontrata la presenza lungo i lidi occidentali e meridionali della Nuova Olanda, quelli della Tasmania e della Nuova Zelanda. Harvey (Phyc. Austral. Syn. 1963, pag. LIII, n. 688) cita come sinonimo il Callithamnion acanthocarpum Kuetz.; deve invece trattarsi del Callithamnion acanthophorum Kuetz. Species (1849) pag. 647, Tab. Phyc. XI (1861) tab. 80, fig. a-c, specie propria della Tasmania. Anche in Antithamnion mucronatum (J. Ag.) Naeg. la fronda nella prima età è d' un colore rosso mattone, invecchiando imbrunisce prima di scolorirsi.

Antithamnion Plumula (Ellis) Thur. in Le Jolis Liste des Algues de Cherbourg (1863) pag. 112; De Toni e Levi Frammenti Algologici I (1887) pag. 296; De Toni Sylloge Algarum IV, pag. 1400; Lucas Revised List pag. 51.

Callithamnion refraclum Kuetz. Tab. Phyc. XI (1861) tab. 84, fig. a-c.

Hab. Australia occid. : Baia di Geroldton; settembre 1908.

Come area di distribuzione nell' Oceano Pacifico gli autori assegnano le coste meridionali della Nuova Olanda, la Tasmania e la Nuova Zelanda. Grossi 
e bellissimi esemplari vennero raccolti a Port Phillips dal WiLhelmi ; quelli del CAPRa sono piccoli frammenti.

\section{Crouania J. Ag.}

Crouania vestita Harv. in Trans. R. Irish Academy vol. 22 (1855) pag. 558, Phyc. Australica III (1860) tab. 140 ; J. Ag. Florideernes Morphologi (1879) tab. 2, fig. 8-9; De Toni Sylloge Algarum IV, pag. 1419; Lucas Revised List pag. 51; Mazza Saggio di Algologia Oceanica n. 416.

Dasyphila Preissii $\beta$ minor Lenorm. (cistocarpifera; erb. Piccone-Forti).

Hab. Australia merid. : Victor Harbour, con sporangi.

Questa specie è indicata per le coste austro-occidentali delia Nuova Olanda, soprattutto nel King George 's Sound, all' isola Rottnest e a Fremantle. Esemplari australasici, distribuiti sotto il nome di Crouania attenuata (specie esclusiva dell' Oceano Atlantico e del Mare Mediterraneo) vanno riferiti alla Crouania vestita Harv. o alla Cronania australis (Harv.) J. Ag.

\section{Lasiothalia Harv.}

Lasiothalia hirsuta Harv. Mar. Bot. of West Australia (1854) n. 275; De Toni Sylloge Algarum IV, pag. 1420; Lucas Revised List pag. 52.

Callithamnion conspicuum Harv. in Hooker Fl. Tasman. II (1860) pag. 335.

Callithamnion tingens Harv. Alg. Austral. exsicc. n. 508.

Hab. Australia occid.: Baia di Geroldton, settembre 1908; Tasmania : Burmie, con cistocarpi.

Stando al Sonder (Algae Austral. hactenus cognitae n. 205) questa specie vegeta a Cape Liptrap, in Tasmania, a Cape Riche; nell' erbario Zanardini esistono esemplari provenienti dalla Tasmania. Il Lucas indica solo le coste austrooccidentali della Nuova Olanda.

\section{Subfam. Ceramieae (Dumort.) Schm.}

\section{Ceramium C. Ag.}

Ceramium aucklandicum Kuetz. Species Algarum (1849) pag. 686, Tab. Phyc. XIII (1863) tab. 7, fig. d-f; De Toni Sylloge Algarum IV, pag. 1472; Laing Mar. Algae of the subantarctic Islands of New Zealand (1909) pag. 520.

Hab. Nuova Zelanda : Bluff.

Questa specie fu di recente riveduta dal LAIng su materiale raccolto da Crosby Smith all' isola di Enderby, ma poco egli aggiunse a meglio dilucidarne la diagnosi, riproducendo per l'occasione la frase delle Species Algarum. Non è del tutto escluso che si tratti di una forma non autonoma. Due piccoli esemplari vennero osservati sostenere le frondi di Griffithsia comosa (Grun.) ma sono sterili. Gli articoli ovali che conferiscono un aspetto leggermente toruloso alle ramificazioni maggiori corrispondono bene a quelli della figura esibita dal KUETZING.

Ceramium nobile J. Ag. Analecta Algologica II (1894) pag. 41; De Toni Sylloge Algarum IV, pag. 1480; Lucas Revised List pag. 53. 
Hab. Australia occid.: Baia di Geroldton, settembre 1908; Tasmania: Baia di Latrobe.

Questo Ceramium, indicato dagli autori per la Nuova Olanda meridionale e per la Tasmania, è la specie vicariante del Ceramium rubrum (Huds.) Ag. Dal LAIng (Revised List II, 1901, pag. 331, n. 130) si afferma essere questa specie stata raccolta anche nella Nuova Zelanda a Brighton, Otago (Laing) e a St. Clair (J. Crosby Smith).

\title{
Ordo IV. Cryptoneminae Schmitz \\ Fam. GRATELOUPIACEAE Sehmitz
}

\author{
Grateloupia C. Ag.
}

Grateloupia prolifera J. Ag. Epicrisis (1876) pag. 150, Florideernes Morphologi (1879) tab. 7, fig. 1-8; De Toni Sylloge Algarum IV (1905) pag. 1556; Lucas Revised List pag. 54.

Hab. Australia occid. : Baia di Geroldton, esemplari con sporangi ed esemplari con cistocarpi, settembre 1908; Tasmania: Baia di Latrobe.

L' aspetto degli esemplari ricorda alquanto la Dumontia filiformis (Fl. Dan.) Grev. e infatti la specie venne dapprima designata da J. AgardH nel suo erbario col nome di Dumontia prolifera. E probabilissimo che esemplari oceanici, ascritti a Dumontia filiformis, vadano riferiti alla Grateloupia prolifera J. Ag.; cfr. LaInG R. M., The marine Algae of the Subantarctic Islands of New Zealand (1909) pag. 524.

\section{Carpopeltis Schmitz}

Carpopeltis elata (Harv.) Schmitz Marine Florideen von Deutsch-Ost-Afrika (1895) pag. 168, notula 1; De Toni Sylloge Algarum IV, pag. 1605; Mazza Saggio di Algologia Oceanica n. 512.

Cryptonemia elata J. Ag. Epicrisis (1876) pag. 164; Lucas Revised List pag. 54.

Acropellis elata Harv. Phyc. Australica III (1860) tab. 122; Kuetz. Tab. Phyc. XIX (1869) tab. 35, fig. II.

Hab. Australia occid. : Baia di Geroldton, con cistocarpi; settembre 1908.

Il colore dei nostri esemplari è purpureo, ma le frondi, segnatamente verso gli apici, sono qua e là decolorate. I cistocarpi erano ignoti prima che noi comunicassimo un esemplare all' egregio amico A. MAzzA, forse perchè confusi coi nematecii che si riuniscono come questi in un soro all' apice della fronda, sono facili a distinguersi praticando una sezione, gli sporangi e di conseguenza le tetraspore non si veggono infatti che negli strati corticali ; all' incontro le carpospore stanno in gruppi sferici sotto la corteccia e si distinguono di primo acchito risaltando in rosso sul tessuto midollare diafano. Tutte insieme però costituiscono un piccolo cistocarpo appena sporgente dalle due pagine della fronda, similmente ai cistocarpi già noti della Carpopeltis phyllophora (Hook. et Harv.) Schmitz già raffigurati dal Kuetzing (Tabulae Phycologicae XIX, 1869, tab. 35, fig. b). La Carpopeltis elata (Harv.) Schmitz venne per la prima volta rinvenuta da Harvey sui lidi meridionali dell' isola Rottnest, aggiungendo poi a questa lo- 
calità Garden Island (Phyc. Austral. Syn. 1863, pag. XLIV, n. 577). Il SondER (Alg. Austral. hactenus cognilae n. 332) la indica pure per Denial Bay e Geographe Bay.

\section{Cryptonemia J. Ag.}

Cryptonemia undulata Sond. in Linnaea vol.26 (1853) pag. 516; Harv. Phyc. Australica IV (1860) tab. 205; Kuetz. Tab. Phyc. XIX (1869) tab. 31 ; De Toni Sylloge Algarum IV, pag. 1610; Lucas Revised List pag. 54 ; Mazza Saggio di Algologia Oceanica n. 516.

Cryptonemia luxurians Harv. Alg. Austral. exsicc. n. 402 (non J. Agardh).

Hab. Australia occid.: Baia di Geroldton, settembre 1908.

A quanto si ricava dal Sonder (Algen des tropischen Australiens pag. 63) le prime località nelle quali venne scoperta questa Cryptonemia sono Cap York (Australia settentrionale) e Port Phillip (Australia meridionale); tali due reperti stanno a provare la larga distribuzione geografica della specie lungo le coste della Nuova Olanda; lo stesso Sonder (Alg. Austral. hactenus cognitae n. 333) aggiunse più tardi la località Geographe Bay; J. AGARDH distribuì nelle sue Algae Muellerianae esemplari provenienti dalla Encounter Bay; il ReinBold segnalò la stessa specie tra le Alghe raccolte dal Dr. A. Engelhart nei lidi meridionali (Lacépède e Guichen Bay).

\section{Fam. NeMASTOMACEAE (J. Ag.) Schmitz}

\section{Subfam. Schizymenieae Schmilz}

$$
\text { Schizymenia J. Ag. }
$$

Schizymenia Novae-Zelandiae J. Ag. Epicrisis (1876) pag. 677; De Toni Sylloge Algarum IV, pag. 1651; Laing Revised List (1901) pag. 332, n. 137; Mazza Saggio di Algologia Oceanica n. 557.

Hab. Nuova Zelanda: Bluff, 25 gennaio 1909.

Di questa specie ricevemmo dal rev. G. CAPRA un unico esemplare largo 15 centimetri, al massimo lungo 10 ; la fronda diffluentissima è di un colore carnicino violaceo. La Schizymenia Novae-Zelandiae J. Ag. fu già nella «Bay of Islands " raccolta dal BERGGREN; è molto affine a Schizymenia obovata J. Ag. del Capo di Buona Speranza (Africa meridionale) ed è da identificare con Schizymenia erosa var.? obliqua Grun. (Alg. Novara pag. 65, tab. 9, fig. 1) come venne già ritenuto da uno di noi nella Sylloge Algarum.

\section{Fam. CoRallinaceae (Gray) Harv.}

\section{Subfam. Choreonemeae Fosl.}

\section{Choreonema Schmitz}

Choreonema Thureti (Born.) Schmitz Systematische Uebersicht der bisher bekannten Gattungen der Florideen (1889) pag. 21; Engler-Prantl Die natürl. Pflanzenfam. I (1897) pag. 541, fig. 288 A ; De Toni Sylloge Algarum IV, pag. 1721. 
Melobesia Thureti Born. in Thur. Études Phycologiques (1878) pag. 96, tab. 50, fig. 1-8, Solms Corallinaceae (1881) pag. 12, tab. 3, fig. 1, 4-10.

Hab. Australia occid. : Baia di Geroldton; Australia merid. : Victor Hurbour.

Citiamo questa specie crescente sulle Coralline perchè essa venne segnalata su esemplari di Jania micrarthrodia Lamour. di Victor Harbour provenienti dalle raccolte fatte dal rev. G. CAPrA delle quali venne comunicato qualche esemplare all' amico A. Mazza che ne tenne parola nel suo Saggio di Algologia Oceanica al n. 606. Negli esemplari rimastici non potemmo riscontrare la presenza del Choreonema Thureti se non sulla Corallina Cuvierı della Baia di Geroldton. Del resto la presenza di questa Floridea endofita sulle Corallinaceae dell' Oceano Pacifico non deve recare maraviglia perchè già il THURET ne avvertì l' esistenza nell' Oceano Australe e nell' erbario di lui se ne hanno esemplari raccolti dal FiLHoL alla Lyall's Bay nella Nuova Zelanda (cfr. Lemoine, Catalogue des Mélobésiées de l'Herbier Thuret pag. LIII; Bull. Soc. Botan. de France T. LVIII, 1911); s' aggiunga che il Choreonema Thureti (Born.) Schm. venne riscontrato da K. Yendo a Misaki nel Mare del Giappone.

\section{Subfam. Lithothamnioneae Fosl.}

\section{Lithothamnion Phil.}

Lithothamnion Lichenoides (Ellis) Heydr. Melobesiae (1897) pag. 412 ; Lemoine Struct. anatomique des Mélobésiées (1911) pag. 127.

Var. Antarctica (Hook. et Harv.) Fosl. List of Species (1898) pag. 7; De Toni Sylloge Algarum IV, pag. 1752.

Melobesia antarctica Hook. et Harv. in Harv. Nereis Australis (1847) pag. 111; Rabenh. Alg. Eur. n. 2520.

Hab. Australia occid.: Baia di Geroldton, su Laurencia Forsteri, settembre 1908; Australia merid.: Victor Harbour, su Halopteris hordeacea.

Secondo la sig.ra LeMorne nell' erbario THURET questa varietà si trova in esemplari provenienti da Port Phillip, dalla Nuova Zelanda e dall' is. Auckland. Nelle Algae Muellerianae vennero da J. Agardh distribuite forme di Melobesia antarctica Harv, della Nuova Zelanda meridionale col dubbio che non si tratti d'una varietà di Melobesia Patena. E di fatti a ben considerare è molto difficile una chiara distinzione fra le due varietà in questione. Esemplari più antichi, pure provenienti dall' erbario AGARDH, dalle rive dello stretto di Magellano (America meridionale) stanno epifiti sulla Ballia callitricha (Ag.) Mont. come pure quelli delle Algae Muellerianae e ciò per confermare l' area geografica oltremodo estesa di questa Corallinacea che sembra spesso prescegliere la Ballia suddetta come supporto.

Var. PAtena (Hook. et Harv.) Fosl. New or critical calcareous Algae (1900) pag. 12; De Toni Sylloge Algarum IV, pag. 1752.

Melobesia Patena Hook. et Harv. in Harv. Nereis Australis (1847) pag. 111, tab. 40.

Lithothamnion Patena Heydr. Lith. Mus. Paris (1901) pag. 542; Mazza Saggio di Algologia Oceanica n. 602. 
Hab. Australia merid.: Victor Harbour; Tasmania: Baia di Latrobe, Burmie su Corallina Cuvieri, 15 gennaio 1909.

Questa varietà ha larga distribuzione nell' Oceano Pacifico in particolare lungo i lidi meridionali dell' Australia (F. Mueller secondo Grunow e LenorMAND), dell' is. Flinders, della Nuova Zelanda, delle is. Auckland e Chatham; sembra anch' essa prediligere la Ballia callitricha (Ag.) Mont. come più frequente supporto. Il precipuo carattere di distinzione dalla Var. antarctica si è l' essere affissa per il centro del tallo invece che per il margine.

Subfam. Melobesieae Fosl.

Melobesia Lamour.

Melobesia farinosa Lamour. Polyp. fléx. (1816) pag. 315, tab. 12, fig. 3 ; Kuetz. Tab. Phyc. XIX (1869) tab. 95, fig. c-e ; De Toni Sylloge - Algarum IV, pag. 1764; Lucas Revised List pag. 55; Mazza Saggio di Algologia Oceanica n. 607.

Hab. Australia occid. : Baia di Geroldton, settembre 1908; Australia merid.: Victor Harbour, 12 novembre 1908; Tasmania: Burmie.

Questa Melobesia ha una estesissima distribuzione geografica su matrici diverse; cfr. Lemoine, Mélob. Herb. Thuret pag. LXII; hanno contribuito a farne conoscere molte località dell' Oceania in particolare Harvey, Sonder, Grunow, Reinbold, Heydrich. L'Erbario Zanardini contiene esemplari provenienti da Port Phillip.

\section{Dermatolithon Fosl.}

Dermatolithon pustulatum (Lamour.) Fosl. Revised System. Survey of Melob. (1900) pag. 21 ; De Toni Sylloge Algarum IV, pag. 1771; Lucas Revised List pag. 55 ; Mazza Saggio di Algologia Oceanica n. 612.

Melobesia pustulata Lamour. Polyp. fléx. (1816) pag. 315, tab. 12, fig. c; Kuetz. Tab. Phyc. XIX (1869) tab. 94, fig. $a-b$; Harv. Nereis Australis (1847) pag. 110.

Hab. Australia occid. : Baia di Geroldton, sulla Cymodocea.

Sembra che questa specie trovisi finora segnalata soltanto per la Nuova Olanda orientale e per l' isola Norfolk. ma il saperla stata rinvenuta sia sulle coste dell' isola Formosa e del Giappone sia su quelle della California fa ammettere per essa una più larga distribuzione nell' Oceano Pacifico.

Subfam. Mastophoreae Fosl.

Mastophora Decaisne.

Mastophora Lamourouxin Decne in Ann. Scienc. Nat., Botan., sér. 2, T. XVI (1842) pag. 114 (sub Melobesia); Harv. Nereis Australis (1847) pag. 108, tab. 41 ; Kuetz. Tab. Phyc. XIX (1869) tab. 98, fig. h-l; De Toni Sylloge Algarum IV, pag. 1774 ; Lucas Revised List pag. 56; Mazza Saggio di Algologia Oceanica n. 624 . 
Melobesia (Mastophora) flabellata Sond. in Botanische Zeitung 1845, pag. 55.

Hab. Australia occid. : Baia di Geroldton, settembre 1908.

Questa specie fu segnalata finora su varie matrici provenienti dalle coste occidentali e meridionali della Nuova Olanda; cfr. oltre ai noti lavori di HARveY, Sonder, Reinbold ecc., Lemoine, Mélob. Herb. Thuret pag. LXV. Mastophora Lamourouxii Decne venne anche distribuita da J. AgARDH nelle Algae Muellerianae di Champion Bay e di Geographe Bay, in quest' ultima località raccolta dall' IRVINE.

Mastophora Plana (Sond.) Harv. Nereis Australis (1847) pag. 108; Kuetz. Tab. Phyc. VIII (1858) tab. 98, fig. a-g; De Toni Sylloge Algarum IV, pag. 1775.

Melobesia plana Sond. in Botanische Zeitung 1845, pag. 55.

Mastophora foliacea Kuetz. Tab. Phyc. VIII (1858) tab. 100, fig. e-h?

Hab. Australia occid. : Baia di Geroldton, cistocarpifera, sulla Cymodocea ; settembre 1908.

Questa specie venne già raccolta alla foce dello Swan River la prima volta dal SonDER e dev' essere con molta cautela distinta dalla Mastophora Lamourouxii Decne, cui in ogni modo devesi riguardare come assai affine. Si riconosce, oltre che per il colore roseo-bianchiccio, per le fronde più regolarmente laciniate e complanate. Mastophora foliacea Kuetz. è da considerarsi una forma giovanile non ancora caulescente.

\section{Subfam. Corallineae (Menegh.) Decne \\ Metagoniolithon Web. v. Bosse}

Metagoniolithon charoides (Lamour.) Web. v. Bosse in Web. et Fosl. Corallinaceae Siboga Exped. (1904) pag. 102, tab. 15, fig. 11 ; Mazza Saggio di Algologia Oceanica n. 636.

Amphiroa charoides Lamour. Polyp. fléx. (1816) pag. 301 ; Harv. Nereis Australis (1847) pag. 96, tab. 39; Kuetz. Tab. Phyc. VIIl (1858) tab. 52, fig. 1 ; De Toni Sylloge Algarum IV, pag. 1810 (excl. synon. nonnull.); Lucas Revised List pag. 56.

Hab. Australia occid.: Baia di Geroldton, settembre 1908; Tasmania: Baia di Latrobe, 9 gennaio 1909; Burmie, roccie battute dalle onde, 15 gennaio 1909.

Come area di distribuzione sono assegnati a questa specie i lidi occidentali e meridionali della Nuova Olanda e quelli della Tasmania; i nostri esemplari concordano con quelli provenienti da Port Phillip (Australia meridionale) dell' Erbario Zanardini e con altri di Sonder e di Lenormand.

Metagoniolithon stelligerum (Lamk.) Web. v. Bosse in Web, et Fosl. Corallinaceae Siboga Exped. (1904) pag. 103, tab. 15, fig. 10, 12 ; Mazza Saggio di Algologia Oceanica n. 638.

Amphiroa stelligera Decne Corall. (1842) pag. 124; Kuetz. Tab. Phyc. VIII (1858) tab. 53; Harvey Phyc. Australica IV (1862) tab. 230; De Toni Sylloge Algarum IV, pag. 1809; Lucas Revised List pag. 56.

Amphiroa elegans Sond. in Botanische Zeitung 1845, pag. 55 (non Hook. et Harv.). 
Corallina stelligera Lamarck in Mém. du Muséum II, pag. 239.

Hab. Australia occid. : Baia di Geroldton, settembre 1908 ; Australia merid. : Victor Harbour, 12 novembre 1908; Tasmania: Burmie, 15 gennaio 1909.

Questa specie ha la stessa area di distribuzione del Metagoniolithon charoides (Lamour.) Web. v. Bosse, cui è molto affine; fu di sovente distribuita anche con le Algae Muellevianae di Cape Leeuwin e C. Fenton in Tasmania, della Geographe Bay (Irvine) e di Victoria (Mueller ex Grunow) in Australia.

\section{Corallina Lamour.}

Corallina Cuvieri Lamour. Polyp. fléx. (1816) pag. 286 ; Harv. Nereis Australis (1847) pag. 106; Kuetz. Tab. Phyc. VIII (1858), tab. 70, fig. a-g; De Toni Sylloge Algarum IV, pag. 1848; Lucas Revised List pag. 56; Mazza Saggio di Algologia Oceanica n. 653.

Corallina crispata Lamour. Polyp. fléx. (1816) pag. 289, tab. 10, fig. 2 a B; Kuetz. Tab. Phyc. VIII (1858) tab. 71, fig. a-b.

Jania subulata Sond. Pl. Preiss. II, pag. 186; Harv. Nereis Australis (1847) pag. 106, tab. 40.

Hab. Australia occid.: Baia di Geroldton, settembre 1908; Tasmania : Baia di Latrobe, 9 gennaio 1909; Burmie, 15 gennaio 1909.

I nostri esemplari corrispondono alla forma descritta e figurata col nome di Corallina crispata Lamour. La specie ha un' estesa distribuzione nell' Oceania, come risulta dai contributi di Harvey, Sonder, Reinbold, Cotton, Gepp, OkaMURA, HARVEY-Gibson e altri algologi.

Oltre che sulle spiaggie della Nuova Olanda e Tasmania (Port Fenton, Algae Muellevianae) la Corallina Cuvieri Lamour. vegeta in quelle della Nuova Zelanda, delle is. Flinders, Norfolk, Chatham, Hawaii, Kermadecs; è specie assai polimorfa come la Corallina offlcinalis L. dell' Oceano Atlantico e del Mare Mediterraneo.

Corallina granifera Ell. et Soland. Zoophyt. (1786) pag. 120; De Toni Sylloge Algarum IV, pag. 1845.

Var. australis (Grun.) De Toni Sylloge Algarum (1905) pag. 1846.

Corallina virgata var. australis Grun. Alg. Fidschi etc. (1874) pag. 42.

Hab. Australia occid. : Baia di Geroldton; settembre 1908.

Il Sonder (Algae Austral. hactenus cognitae n. 526) indica la Corallina granifera E. et S. per Port Denison sulla scorta del GRunow che nell'opera sopra citata segnala per detta località la Corallina virgata var. australis raccolta da Amalia Dietrich. In alcune schede di A. Grunow nell' erbario Piccone (ora incorporato nell' erbario FORTI) trovasi menzionata una Corallina granifera Sond. Alg. Preiss. che non vuol essere assolutamente confusa con la presente specie, rappresentando anche, secondo il concetto dell' autore, una varietà di Corallina Cuvieri Lamour. a fronda piana e poco increspata che rammenta gli esemplari di Port Phillip distribuiti già dal Lenormand; questi esemplari non portano indicazione precisa di località e furono raccolti dal Sieber.

Corallina trichocarpa Kuetz. Tab. Phyc. VIII (1858), pag. 35, tab. 74, fig. $a \cdot b$; De Toni Sylloge Algarum IV, pag. 1854. 
Hab. Australia merid.: Victor Harbour, 12 novembre 1908.

Gli esemplari comunicatici dal rev. dott. G. CAPRA corrispondono egregiamente alle figure esibite dal KuETzing per la sua Corallina trichocarpa, specie già raccolta da F. MueleER nell' Australia (esemplari nell' erbario SónDER); data la variabilità di questa Corallina essa rientra, a nostro vedere, nel ciclo delle forme della Corallina Cuvieri Lamour.

\section{Jania Lamour.}

Jania Affinis Harv. Alg. Austral. exsicc. n. 449 ; Phyc. Australica Syn. (1863) pag. XIX, n. 349; De Toni Sylloge Algarum IV, pag. 1857 ; Lucas Revised List pag. 57.

Hab. Australia occid.: Baia di Geroldton, settembre 1908.

Questa specie, secondo lo stesso HARVEY di poco differente dalla Jania rubens (L.) Lamour., fu raccolta già nell' Australia occidentale all' isola Rottnest. Sotto il nome di Jania affinis Harv.? J. AgardH sembra aver distribuito nelle Algae Muellerianae una specie della Nuova Zelanda non dissimile da Corallina granifera var. australis proveniente dalla stessa regione.

Jania micrarthrodia Lamour. Polyp. fléx. (1816) pag. 271, tab. 9, fig. $5 a-b$; De Toni Sylloge Algarum IV, pag. 1855; Lucas Revised List pag. 56.

Jania tenuissima Sond. Pl. Preiss. II (1848) pag. 186.

Hab. Australia occid. : Baia di Geroldton, settembre 1908; Australia merid.: Victor Harbour, 12 novembre 1908; Tasmania : Baia di Latrobe, 9 gennaio 1909; Burmie, 15 gennaio 1909.

E una specie comune nell' Oceania, in particolare segnalata per i lidi austro-occidentali della Nuova Olanda, per la Tasmania, la Nuova Zelanda e le isole Chatham. Alcune forme di Jania in parte sterili provenienti da diversi punti delle coste australiane e neozelandesi vennero pubblicate nelle Algae Muellevianae senza poter essere con esattezza determinate; così vi troviamo Janiae micrarthrodiae sp. aff. della Nuova Olanda occidentale. E certo pertanto che sebbene Jania micrarthrodia Lamour. non riesca difficile a distinguersi dalle congeneri, non altrettanto può dirsi delle medesime fra di loro e, con le altre specie del gruppo al quale appartengono, anche Jania rubens (L.) Lamour., Jania fastigiata Harv., Jania Novae-Zelandiae Harv. e alcune specie, poco note, del Lamouroux.

FUCOIDEAE (Ag.) J. Ag.

Ordo Cyclosporinae Aresch.

Fam. SaRGaSsaCeaE (Decne) Kuetz.

Sargassum C. Ag.

Sargassum Carpophyllum J. Ag. Species I (1848) pag. 304 ; Species Sargassorum Australiae (1889) pag. 82, tab. 25, fig. II; De Toni Sylloge Algarum III (1895) pag. 39; Grunow Addit. ad cognit. Sargassorum (1913) pag. 366, n. 66. 
Hab. Nuova Zelanda: Bluff, 25 gennaio 1909.

Gli esemplari incompleti, raccolti dal rev. dott.' G. CAPRA, corrispondono alla forma pseudofilipendula Grun. di Noumea (Nuova Caledonia) della quale sono conservati alcuni pezzi di fronda nella collezione Grunowiana al $n .1590$ nell' Erbario del Museo di Storia Naturale di Vienna (1); come quasi tutti i Sargassi, il Sargassum carpophyllum J. Ag. presenta un notevole polimorfismo, quale apparisce dal confronto di individui raccolti da FulLagar e Lind all'isola Lord Howe, dal DAEMEL a Rockingham (Australia settentrionale), da E. A. MAN alle isole Andaman ecc. Tale è il polimorfismo di questa specie che il Grunow ne istituì alcune varietà come la var. leplophylla per esempları di Edgecombe Bay distribuiti da J. AgardH nelle Algae Muellevianae, var. subdivisa per individui raccolti a Rockingham (Queensland) dal DAEMEL e var. leptacantha per frammenti di fronda provenienti dallo stretto di Torres (Erb. LENORMAND).

Poco distinto dal Sargassum carpophyllum J. Ag. sembra essere il Sargassum macrophyllum Zanard. dell' isola Lord Howe, se tutt' al più non si voglia accordare valore al portamento più slanciato del tallo e alle foglie molto allungate; il Grunow non esitò a riferire la specie Zanardiniana, in seguito all'esame di materiale autentico, a semplice varietà macrophylla del Sargassum spinuligerum Sond., mostrando il Sargassum descritto da G. ZANARDIN! molta somiglianza con una var. stenophylla Grun. (dell' isolotto di Freycinet, Nuova Caledonia) dell' or menzionato Sargassum spinuligerum Sond. (cfr. Addit. ad cognitionem Sargassorum, 1913, pag. 163).

Sargassum decurrens (R. Br.) Ag. Species I (1821) pag. 42 ; Mont. Voyage au Pole Sud (1845) pag. 73, tab. 10, fig. 2; J. Ag. Species Sargassorum Australiae (1889) pag. 47, tab. 15, fig. II ; Harv. Phyc. Australica III (1860) tab. 145; De Toni Sylloge Algarum IlI, pag. 13; Lucas Revised List pag. 10.

Plerocaulon decurrens Kuetz. Tab. Phyc. X (1860) tab. 65, fig. II.

Hab. Australia occid. : Baia di Geroldton, settembre 1908.

Una sola fronda giovanile, priva di aerocisti, fu raccolta dal rev. G. CAPRA, la quale corrisponde bene ai caratteri assegnati al Sargassum decurrens (R. Br.) Ag. La specie è nota per i lidi occidentali e orientali della Nuova Olanda e per lo Stretto di Torres, secondo esemplari raccolti da R. BRown, Hombron, A. TAylor, Harvey, Sonder.

Il Kuntze (Revision von Sargassum und das sogenannte Sargasso-Meer pag. 215 ; Engler's Botan. Jahrb. I, 3; Leipzig 1880) sotto il nome di Sargassum Pterocaulon (con distribuzione geografica dal Capo di Buona Speranza fino al Giappone e alla Nuova Zelanda) dispose come forme estreme il Sargassum Peronii (Turn.) Ag., il S. Boryi Ag. e il S. decurrens (Turn.) Ag. seguendo in tali avvicinamenti le vedute già esposte dal Grunow il quale riscontrò, in esemplari raccolti da Amalia Dietrich presso Port Mackay (Nuova Olanda orientale) tutte le forme di passaggio in maniera da non poter indicare limiti sicuri fra dette tre specie (cfr. Grunow Algen der Fidschi-, Tonga-und Samoa-Inseln pag. 26; Hamburg 1873-74); in appoggio a queste affinità lo stesso SonDER, pur

(i) Per uno studio, al quale stiamo attendendo, intorno ai Sargassum ricevemmo dal Museo di Storia Naturale di Vienna 1' intiera collezione, con gli interessanti esemplari riveduti dal GRuNow; dî questo invio siamo debitori alla gentilezza del dott. A. ZaHLBRUCKNER. 
conservando distinto il Sargassum Peronii Ag. dal S. decurrens Ag. riferì al primo del Fucus Peronii Mert. Mém. nella tav. I la figura a destra e al secondo la figura a sinistra, ed in pari tempo ammise che il Sargassum Boryi Ag. non è separabile dal $S$. decurrens Ag. neppure a titolo di semplice varietà. Nell' erbario GRunow rispettivamente ai numeri 1900 e 1905 stanno un esemplare di Sargassum Peronii var. intermedia Grun. (costituente il materiale autentico raccolto a Port Mackay dalla Dietrich) e un' altro di S. Peronii var. lanceolata Grun. (pure di Port Mackay) che insieme a un esemplare, portante il n. 1920, di Sargassum Boryi var. angustissima forma neocaledonica raccolto dal Grunow a Noumea (Nuova Caledonia) mostrano la grande variabilità di questa specie ammessa come autonoma da alcuni autori.

Sargassum fallax Sond. in Botanische Zeitung 1845, pag. 52 ; J. Ag. Species Sargassorum Australiae (1889) pag. 68, tab. 20, fig. I; De Toni Sylloge Algarum III, pag. 29 ; Lucas Revised List pag. 10; Grunow Addit. ad cognit. Sargassorum pag. 356 , n. 45.

Blossevillea fallax Kuetz. Tab. Phyc. X (1860), tab. 87.

Hab. Australia occid. : Baia di Geroldton, settembre 1908.

Questa specie, che talora si presenta in individui privi di aerocisti, è assai comune lungo le coste della Nuova Olanda soprattutto occidentale. I nostri esemplari hanno aerocisti mucronate come quelli distribuiti da J. AGARDH nelle - Algae Muellerianae di Geographe Bay e di Cape Riche e quelli di Port Fairy conservati al n. 875 della collezione Grunow nell' erbario del Museo di Storia Naturale di Vienna; per questi ultimi il GRuNow distinse anzi una varietà aristifera.

Sargassum lacerifolium (Turn.) Ag. Species I (1821) pag. 15; Harv. Phyc. Australica IV (1862) tab. 208; J. Ag. Species Sargassorum Australiae (1889) pag. 74, tab. 23, fig. II ; De Toni Sylloge Algarum III, pag. 34. bona).

Carpacanthus lacerifolius Kuetz. Tab. Phyc. XI (1861), tab. 42, fig. II (non

Hab. Nuova Zelanda: Bluff, 25 gennaio 1909.

E specie segnalata per l'Australia meridionale e occidentale e per la Tasmania. Il Grunow (op. cit.) ne distinse alcune varietà, tenendo come forma tipo quella figurata dal Turner Hist. Fuc. (1811) tab. 167.

Sargassum lophocarpum J. Ag. Species Sargassorum Australiae (1889) pag. 93, tab. 27, fig. II ; De Toni Sylloge Algarum III, pag. 55; Lucas Revised List pag. 11.

Sargassum obovatum Sond. Algen des tropischen Australiens (1871) n. 13 (non Harvey).

Hab. Australia occid. : Baia di Geroldton, settembre 1908.

Il rev. G. CAPRA ci inviò un apice di fronda (della lunghezza di circa un decimetro) perfettamente concordante con la figura sopra citata esibita per il Sargassum lophocarpum da J. AGARDH; vi si osservano un' unica aerocisti picciuolata e parecchi ricettacoli ascellari, aggregati, di circa metà più brevi delle foglie contigue.

Il Lucas attribuisce come area geografica a questa specie le coste australi 
e orientali della Nuova Olanda, ciò che viene confermato dal Grunow (Additam. ad cognit. Sargass. pag. 402, n. 105).

Sargassum pachycarpum J. Ag. Species Sargassorum Australiae (1889) pag. 104, tab. 29, fig. 1; De Toni Sylloge Algarum III, pag. 79 ; Lucas Revised List pag. 11.

Hab. Australia occid. : Baia di Geroldton, settembre 1908.

I nostri esemplari rispondono alla diagnosi e alle figure datene da J. AGARDH così che, pur mancando di campioni autentici per il confronto (neppure il GRUNow potè esaminare gli esemplari Agardhiani) li riferiamo alla specie, che è nota per le coste della Tasmania dove la raccolse R. GunN.

Sargassum paradoxum (R. Br.) Harv. Alg. Tasman. n. 10; J. Ag. Species Sargassorum Australiae (1889) pag. 68, tab. 20, fig. II; De Toni Sylloge Algarum III, pag. 29; Lucas Revised List pag. 10; Grun. Addit. pag. 358, n. 46.

Blossevillea paradoxa Kuetz. Tab. Phyc. X (1860), tab. 88 (excl. receptac. quae aptius in Sarg. grande J. Ag. quadrant).

Hab. Australia occid.: Baia di Geroldton, settembre 1908.

La specie, secondo gli autori, vegeta lungo i lidi meridionali della Nuova Olanda e quelli della Tasmania; il Lucas (Notes on Australian marine Algae I. pag. 58 ; Proceed. of the Linnean Society of New South Vales vol. XXXVIII, 1913) indica il Sargassum paradoxum Harv. come nuovo per la Tasmania raccolto da L. Rodway nel maggio 1912 a Derwent e nel D'Entrecasteaux Channel, essendogli sfuggito che per la Tasmania la specie era stata segnalata già dal Kuetzing (Species 1849, pag. 630), da W. H. Harvey (Phyc. Austral. Syn. 1863, pag. VI, n. 13) e dal Sonder (Algae Austral. hactenus cognilae n. 13: Georgetown) e che di essa trovansi esemplari tasmatici nell' Erbario ZaNARDINI; in realtà il Sargassum grande J. Ag. istituito su questi esemplari tasmanici distribuiti da HARvey col nome di Sargassum paradoxum fem. non è altro che una pianta sporifera del genuino Sargassum paradoxum (R. Br.) Harv. che è una specie dioica.

Sargassum stenophyllum J. Ag. Species I (1848) pag. 335; Species Sargassorum Australiae (1889) pag. 104, tab. 29, fig. II ; De Toni Sylloge Algarum III, pag. 80 ; Lucas Revised List pag. 11.

Hab. Tasmania: Burmie, roccie battute dalle onde; 15 gennaio 1909.

La specie era nota per la regione costiera del Queensland (Rockingham's Bay, Edgecombe Bay, White Sunday Islands) e per l' isola Norfolk (cfr. ReinBoLD T., Meeresalgen von den Narfolk-Inseln pag. 149; Nuova Notarisia XI, 1900).

Sargassum tristichum (Grev. et Ag.) Sond. in Botanische Zeitung 1845, pag. 51; J. Ag. Species Sargassorum Australiae (1889) pag. 76, tab. 24, fig. 1; De Toni Sylloge Algarum III, pag. 35; Lucas Revised List pag. 10.

Carpacanthus oligophyllus Kuetz. Tab. Phyc. XI (1861) tab. 37, fig. 1.

Carpacanthus racemosus Kuetz. Tab. Phyc. XI (1861) tab. 45, fig. 1.

Hab. Australia occid. : Baia di Geroldton, settembre 1908; Tasmania, Burmie, 15 gennaio 1909.

Alla specie dagli autori fu assegnata come area la Nuova Olanda meridio- 
nale e austro-occidentale. Gli esemplari comunicatici dal rev. G. CAPRA della Baia di Geroldton possono più che tutto venire assomigliati alla var. comosa del Grunow distribuiti da J. AgardH nelle Algae Muellevianae dell' Israëliten Bay con foglie lineari. L' esemplare raccolto a Burmie corrisponde invece piuttosto alla figura che il Kuetzing nelle Tabulae Phycologicae esibisce per il suo Carpacanthus racemosus con foglie che ricordano per il contorno quelle del Sargassum lacerifolium (Turn.) Ag.

\section{Seirococcus Grev.}

Seirococcus axillaris (R. Br.) Grev. Alg. Brit. Syn. (1830) pag. XXXIV; Hook. et Harv. Alg. Tasman. n. 107 ; Harv. Phyc. Australica I (1858) tab. 4 ; A. L. Smith in G. Murray Phycological Memoirs vol. II, pag. 32, tab. X; De Toni Sylloge Algarum III, pag. 131 ; Lucas Revised List pag. 12.

Fucus axillaris R. Br. in Turn. Fuci III (1811) pag. 28, tab. 146.

Hab. Tasmania: Baia di Latrobe.

Il Seirococcus axillaris ( $\mathrm{R}$. Br.) Grev. è specie propria della Nuova Olanda e della Tasmania, come risulta dai reperti di Harvey, Sonder, J. AgardH, A. L. Smith, Reinbold e altri autori che trattarono della floristica dell' Australia e delle terre circonvicine. Venne questa specie di sovente distribuita nelle Algae Muelleviane in particolare della Tasmania e dell' is. Flinders.

\section{Scytothalia Grev.}

Scytothalia dorycarpa (Turn.) Grev. Alg. Brit. Syn. (1830) pag. XXXIV; Harv. Phyc. Australica I (1858) tab. 9; Kuetz. Tab. Phyc. X (1860) tab. 25, fig. III; Gruber Ueb. Bau u. Entw. Fuc. (1896) tab. IV, fig. 3; De Toni Sylloge Algarum III, pag. 132; Lucas Revised List pag. 12.

Fucus dorycarpus Turn. Fuc. III (1811) tab. 143.

Scytothalia rhyncocarpa Kuetz. Tab. Phyc. X (1860), tab. 25, fig. 1.

Scytothalia xiphocarpa Kuetz. Tab. Phyc. X (1860), tab. 25, fig. II.

Hab. Australia occ.: Baia di Geroldton, settembre 1908.

Questa specie (la quale non deve confondersi con la Scytothalia dorycarpa Kuetz. Phycologia generalis (1843) pag. 352 proveniente dal Capo di Buona Speranza [Africa meridionale] e ascritta al genere Axillaria) ha estesa distribuzione geografica nell' Oceano Pacifico, soprattutto lungo i lidi occidentali e meridionali della Nuova Olanda e quelli della Tasmania. Trovasi ripetutamente distribuita nelle Algae Muellevianae di solito delle coste occidentali dell'Australia. Gli esemplari di Port Fairy (Victoria) si distinguono per i ricettacoli ensiformi più allungati rispondenti alla Scytothalia xiphocarpa Kuetz. delle Tabulae Phycologicae.

\section{Scaberia Grev.}

Scaberia Agardhil Grev. Alg. Brit. Syn. (1830) pag. XXXVI; Harv. Phyc. Australica III (1860) tab. 164; De Toni Sylloge Algarum III, pag. 179; Lucas Revised List pag. 13.

Castraltia salicornioides A. Rich. Astrol. N. Zel. II (1834) pag. 143; Decaisne in Arch. du Muséum II, pag. 175, tab. 5, fig. 23-24 (non Martens). 
Hab. Australia occid.: Baia di Geroldton, settembre 1908.

Insieme alla Scaberia rugulosa J. Ag. questa specie rappresenta una delle piante caratteristiche della flora dell' Oceano Pacifico, trovandosi essa abbondante in particolare lungo i lidi meridionali della Nuova Olanda, frequentissima come attesta l' AREschoug (Phyceae extraeuropaeae pag. 331) nel porto di Adelaide; ma essa estende la sua area di vegetazione, con maggiore o minore frequenza, alle spiaggie occidentali della Nuova Olanda e a quelle della Tasmania e della Nuova Zelanda nonchè di parecchie isole come le Flinders, l'is Lord Howe, le Auckland. Così trovasi distribuita nelle Algae Muellerianae di Champion Bay, di Port Fairy e della Tasmania.

La Castraltia salicornoides che il MARTEns (Tange der Preussischen Expedition nach Ost-Asien pag. 117; Berlin 1866) indicò per Yokohama (Giappone) nulla ha a che fare con la omonima specie del RICHARD ma va riferita a una floridea, Acanthopeltis japonica Okam.; il Martens fu indotto in errore da una somiglianza superficiale degli esemplari giapponesi con la Castraltia salicornioides A. Rich. che è la genuina Scaberia Agardhii Grev.

$$
\text { Cystophora J. Ag. }
$$

Cystophora Cephalornithos (Labill.) J. Ag. Species I (1848) pag. 246; De Toni Sylloge Algarum III, pag. 138.

Fucus Cephalornithos Labill. Pl. N. Holland. (1806) tab. 261.

Blossevillea Cephalornithos Kuetz. Sp. (1849) pag. 631, Tab. Phyc. X (1860) tab. 83, fig. II.

Hab. Nuova Zelanda: Bluff, 25 gennaio 1909.

Scoperta dal La Billardière a Capo di Tasman dell' isola Melville lungo la costa dell' Australia settentrionale, poi ritrovata a Port Phillip dall' Areschoug (Erb. Zanardini) e dal Sonder (Plantae Muellevianae 1852, pag. 671) venne figurata dal Kuetzing su esemplari di quest' ultima località comunicatigli dal $\mathrm{Ru}$ CHINGER. Col ritrovamento fatto dal CAPRA l' area geografica si estende fino alla Nuova Zelanda, fatto che era presumibile quantunque finora ignorato; perchè era lecito supporre che questa fucoidea dovesse avere una distribuzione molto più estesa di quella conosciuta occupando le coste settentrionali e meridionali della Nuova Olanda che dimostrano caratteristiche fitogeografiche ben diverse e lasciano immaginare un' eccezionale capacità di adattamento per gli organismi trovantisi a prosperare da una parte e dall' altra.

Cystophora monilifera J. Ag. Species I (1848) pag. 241, Om Chatam-öarnes Alger (1870) pag. 447; Harv. Phyc. Australica V (1863) tab. 245; Hooker Fl. Tasman. II (1860) pag. 283; De Toni Sylloge Algarum III, pag. 146; Lucas Revised List pag. 13.

Fucus retroflexus Turn. Fuci (1811) tab. 155 (non Labillardière).

Blossevillea retroflexa Kuetz. Tab. Phyc. X (1860) tab. 76.

Hab. Tasmania: Baia di Latrobe, 9 gennaio 1909.

E specie propria dell' Australia occidentale, meridionale, orientale e della Tasmania. Secondo Hooker (Handbook of New Zealand Flora (1867) pag. 651) la Cystophora monilifera J. Ag. vegeta anche sulle coste della Nuova Zelanda, quivi raccolta dal Dumont D'URville; esemplari della Nuova Zelanda sono 
conservati nell' erbario ZANARDini cosi di questa come della Cystophora retroflexa (Labill.) J. Ag. Nelle Algae Muellerianae la specie venne distribuita di Champion Bay e di Geographe Bay; il Lenormand ne dispensò ai suoi corrispondenti esemplari provenienti da Port Phillip.

Cystophora paniculata (Turn.) J. Ag. Species I (1848) pag. 248, Om Chatham-öarnes Alger (1870) pag. 448; Harv. Phyc. Australica V (1863) tab. 247; De Toni Sylloge Algarum III, pag. 149; Lucas Revised List pag. 13.

Blossevillea paniculata Decne in Kuetz. Tab. Phyc. X (1860) tab. 75.

Hab. Tasmania: Baia di Latrobe, 9 gennaio 1909.

Questa Cystophora, raccolta dapprima all' isola Kent (R. BRown) venne poscia rinvenuta in parecchie località della Nuova Olanda meridionale e orientale e della Tasmania. Recentemente, secondo K Okamura, il sig. Fujita la raccolse a Bondai presso Sydney. Nelle Algae Muellerianae fu distribuita da J. AgardH con la semplice annotazione «Australia meridionale».

Cystophora racemosa Harv. Alg. Austral. exsicc. n. 5 ; J. Ag. Om Chathamöarnes Alger (1870) pag. 441; De Toni Sylloge Algarum III, pag. 140; Lucas Revised List pag. 12.

Blossevillea racemosa Harv. in Kuetz. Tab. Phyc. X (1860) tab. 85, fig. I.

Hab. Australia merid. : Victor Harbour, 11 novembre 1908.

È specie propria delle coste austro-occidentali e meridionali della Nuova Olanda. Secondo il Sonder (Algae Austral. hactenus cognitae n. 76) essa vegeta a Portland Bay, Lacépède Bay, Spencer's Gulf, Fowler's Bay e Geographe Bay ; il ReinBold la ebbe frammezzo alle alghe raccolte nello Investigator Street. Il Kuetzing reputa la Cystophora racemosa Harv. molto simile alla Cystophora pectinata (Grev. et Ag.) J. Ag. che cresce lungo i lidi austro-occidentali della Nuova Olanda. Giudicando dalle figure delle Tabulae Phycologicae l' aspetto del caule sarebbe ben diverso nell' una e nell' altra, in quest' ultima ottusangolo subcilindrico, nella presente acutangolo complanato.

Cystophora Retroflexa (Labill.) J. Ag. Species I (1848) pag. 242, Om Chatham-öarnes Alger (1870) pag. 443; De Toni Sylloge Algarum III, pag. 142; Lucas Revised List pag. 12.

Fucus retroflexus Labill. Pl. Nov. Holland. II (1806) pag. 113, tab. 260 (non Turner).

Blossevillea campylocoma Kuetz. Tab. Phyc. X (1860) tab. 81, fig. 1.

Blossevillea caudata Harv. Alg. Tasman. n. 119 ; Kuetz. Tab. Phyc. X (1860) tab. 76 .

Hab. Tasmania: Baia di Latrobe, 9 gennaio 1909.

Tra le Cystophora è, può dirsi, quella che possiede la più estesa area di distribuzione, essendo propria dei lidi occidentali, meridionali e orientali della Nuova Olanda, di quelli della Tasmania, della Nuova Zelanda, delle isole Aucklande Chatham e di numerosi isolotti dell' Oceania; è pure una delle più proteiformi tanto per l' aspetto del caule che muta da quasi cilindrico ad acutangolo quanto per la forma delle ramificazioni assai differente a seconda dell' età della pianta. Il Lenormand la distribuì di Port Phillip. 
Cystophora torulosa (R. Br.) J. Ag. Species I (1848) pag. 243; Om Chatham-öarnes Alger (1870) pag. 445; Harv. Phyc. Australica III (1860) tab. 123; De Toni Sylloge Algarum III, pag. 143; Lucas Revised List pag. 13.

Fucus torulosus R. Br. in Turn. Fuci (1811) t. 157.

Blossevillea torulosa Decne in Kuetz. Tab. Phyc. X (1860) tab. 72, fig. II.

Hab. Tasmania: Burmie, roccie battute dalle onde, 15 gennaio 1909.

Questa specie, descritta su esemplari raccolti nell' arcipelago delle isole Kent (R. BRown), vegeta lungo le coste meridionali della Nuova Olanda e della Tasmania; si trova pure comune alla Nuova Zelanda e in isolotti a questa vicini. I nostri esemplari corrispondono perfettamente, oltre che alle figure date da Harvey e Kuetzing, alla bellissima tavola originale del Turner nonchè ad esemplari di Georgetown (Tasmania) dell' erbario Zanardini. L' esemplare di Capo Fenton (Tasmania) distribuito da J. AgardH nelle Algae Muellerianae è meno denso di ramificazioni dei nostri di Burmie, che per essere tali sono privi delle aerocisti; questo perciò risponde meglio alla fisionomia delle tavole di Turner e di Harvey.

\section{Cystoseira C. Ag.}

Cystoseira abrotanifolia (Stackh.) Ag. Species I (1848) pag. 172; De Toni Sylloge Algarum III, pag. 172.

Var. Macrocarpa (Kuetz.) De Toni Sylloge Algarum III (1895) pag. 173.

Cystoseira macrocarpa Kuetz. Tab. Phyc. X (1860) pag. 18, tab. 48, fig. 3.

Hab. Australia merid. : Victor Harbour, 12 novembre 1908; Nuova Zelanda : Bluff, 25 gennaio 1909.

La figura delle Tabulae Phycologicae riproduce un semplice frammento di fronda apicale in cui risalterebbero la lunghezza dei ricettacoli e delle aerocisti ellissoidi in confronto ai medesimi organi della forma tipica atlantica e mediterranea. Il Kuetzing descrisse la sua Cystoseira macrocarpa su esemplari della Nuova Olanda provenienti dall' erbario SonDER, che ben corrispondono a quelli di Victor Harbour. Quelli di Bluff hanno struttura più esile ma sempre mantengono la caratteristica distanza fra ricettacolo e aerocisti, costituendo forse una forma intermedia. Importante sarebbe scoprire le frondi basilari tanto in Australia quanto nella Nuova Zelanda per completare il riconoscimento di questa varietà elegantissima e per confermarne se possibile l' affinità con la specie del nostro mare e dell' Oceano Atlantico, la quale in questi mari è così frequente.

$$
\text { Phyllospora C. Ag. }
$$

Phyllospora comosa (Labill.) Ag. Macrocystis (1839) pag. 311, tab. 28, fig. 11 ; Harv. Phyc. Australica III (1860) tab. 153; Hooker Fl. Tasman. II (1860) pag. 283; Kuetz. Táb. Phyc. X (1860) tab. 24, fig. I; De Toni Sylloge Algarum III, pag. 181 ; Gruber Ueb. Bau u. Entwick. einig. Fucaceen (1896) pag. 15, tab. IV, fig. 4; Lucas Revised List pag. 13.

Fucus comosus Labill. Pl. Nov. Holland. II (1806) pag. 112, tab. 258.

Hab. Tasmania: Baia di Latrobe, 9 gennaio 1909.

La Phyllospora comosa (Labill.) Ag. raccolta oltre un secolo fa nelle spiaggie della Terra di van Diemen, fu poscia rinvenuta dal Sieber nella Nuova 
Olanda ed ora si conosce per parecchie località dell' Australia occidentale, meridionale e orientale ed, oltre che per la Tasmania, per alcune piccole isole e per la Nuova Zelanda. Essa venne distribuita al n. 26 delle Reliquiae Brebissonianae da Mougeot, Dupray e Roumeguère raccolta a Port Phillip e dell' Australia meridionale, senza ulteriore specificazione, nelle Algae Muellerianae.

\section{Carpoglossum Kuetz.}

Carpoglossum quercifolium (R. Br.) J. Ag. Species I (1848) pag. 193; Harv. Phyc. Australica I (1858) tab. 43; De Toni Sylloge Algarum III, pag. 182 ; Lucas Revised List pag. 13.

Fucus quercifolius R. Br. in Turn. Fuci III (1811) tab. 151.

Platythalia quercifolia Sond. in Botanische Zeitung 1845, pag. 51; Gruber Ueber Bau u. Entwick. einig. Fucaceen (1896) tab. 7, fig. 6-7.

Myriodesma quercifolium Kuetz. Tab. Phyc. X (1860) tab. 9, fig. I.

Hab. Australia occid. : Baia di Geroldton, settembre 1908.

Questa specie va con cautela distinta, per il modo di sviluppo della fronda, dalla Landsburgia quercifolia (Hook. et Harv.) Harv. la quale è specie propria della Nuova Zelanda; così che rimane da confermare se sia vero che il Carpoglossum quercifolium appartenga alla flora neozelandese, come fu ammesso da qualche autore, includendo nella sinonimia di quest' ultima specie la Phyllospora quercifolia Hook. et Harv. che è affatto diversa dal Fucus quercifolius Turn, e va ascritta alla Landsburgia.

Il Carpoglossum quercifolium (R. Br.) J. Ag. è frequente lungo i lidi austrooccidentali della Nuova Olanda, secondo il Sonder (Algae Austral. hactenus cognitae n. 105) specialmente a Fremantle, is. Rottnest, Busseltown, Cape Leeuwin.

Il GRUBER (Ueber Aufbau und Entwickelung einiger Fucaceen pag. 23-24; Bibliotheca Botanica fasc. 38, 1896) mantenne il genere Carpoglossum Kuetz. per il solo Carpoglossum confluens ( $\mathrm{R}$. Br.) Kuetz., rimettendo in vigore, per il Carpoglossum quercifolium (R. Br.) J. Ag. e per il Carp. angustifolium (Sond.) J. Ag. le omonime specie del genere Platythalia Sond.

\section{Fam. FUCACEAE (Lamour.) Kjellm.}

Hormosira Endl.

Hormosira Banksil (Turn.) Decne in Ann. Sc. Nat., Botan., 2 sér., T. XVII (1842) pag. 330; Harv. Phyc. Australica III (1860) tab. 135; Kuetz. Tab. Phyc. X (1860) tab. 3, fig. I; De Toni Sylloge Algarum III, pag. 187; Lucas Revised List pag. 13.

Fucus Banksii Turn. Fuci I (1808) tab. I.

Hab. Australia merid.: Victor Harbour, 12 novembre 1908; Tasmania : Burmie, 15 gennaio 1909; Nuova Zelanda: Bluff, 25 gennaio 1909.

Specie estremamente variabile, così che venne distinta, a seconda del maggiore o minore valore dei caratteri desunti dalla forma delle aerocisti, in specie - varietà, quali la Sieberi, la Labillardieri, la gracilis, sebbene HARVEY opini doversi queste specie o varietà alle condizioni ambientali in cui vegeta la Fucoidea; anche il Grunow (Alg. Novara pag. 53) e tra gli autori più recenti il 
Lucas e il Latng (Revised List pag. 66, n. 77) ammettono implicitamente il considerevole polimorfismo della Hormosira Banksii (Turn.) Decne, studiata nella sua forma Labillardieri da T. A. Molnet (Transactions of New Zealand Institute vol. XIII, 1880, pag. 318-323, tab. XIII-XIV; Wellington, 1881).

La specie è comunissima nei littorali dell' Australia, della Tasmania, delI' isola Lord Howe, della Nuova Zelanda, delle isole Chatham e Kermadecs; venne raccolta presso Sydney durante il viaggio di circumnavigazione della * Magenta dal Biasi (Erb. Piccone-Forti).

\section{Myriodesma Decne}

Myriodesma integrifolium Harv. Alg. Austral. exsicc. n. 43; Hooker Fl. Tasman. II (1860) pag. 286, tab. 186, fig. 1.4 ; Kuetz. Tab. Phyc. X (1860) tab. 10, fig. II ; De Toni Sylloge Algarum III, pag. 191 ; Lucas Revised List pag. 14.

Myriodesma pinnatifidum J. Ag. in schedula.

Hab. Tasmania: Baia di Latrobe, 9 gennaio 1909.

La specie vegeta lungo le spiaggie della Tasmania, soprattutto a Georgetown, e in parecchi siti (Holdfast Bay, Port Phillip, Western Port, Geelong) dell' Australia meridionale; è polimorfa nel suo complesso; gli esemplari della Baia di Latrobe si avvicinano a quelli della forma più contratta, a lacinie più addensate enervi e scafidii sparsi numerosissimi su tutta la superficie della fronda fuorchè ai margini.

Ordo II Tetrasporinae D. T.

Fam. DICTY 0TACEAE (Lamour.) Zanard.

Zonaria (Draparn.) J. Ag.

Zonaria Crenata J. Ag. Till Algernes Systematik Il (1872) pag. 48, Analecta Algologica I (1894) pag. 13; De Toni Sylloge Algarum III, pag. 230; Lucas Revised List pag. 14.

Zonaria flava var. tenuior Sond. mscr.

Zonaria flava Harv. Alg. Austral. exsicc. n. 77 (non C. Agardh).

Icon: Tab. nostra IX, fig. 1.

Hab. Tasmania: Burmie.

La Zonaria crenata J. Ag. è dal Sonder (Algae Austral hactemus cognitae n. 147) indicata per Fremantle e Port Phillip; il Lucas assegna come area le coste occidentali e meridionali della Nuova Olanda; il ReInbold riscontrò questa specie nei materiali provenienti dallo Investigator Street.

Zonaria Diesingiana J. Ag. Symbolae (1841) pag. 443, Species I (1848) pag. 109, Analecta algologica I (1894) pag. 13; Harv. Phyc. Australica Syn. (1863) pag. XI, n. 101; De Toni Sylloge Algarum III, pag. 229; Lucas Revised List pag. 14. Icon: Tab. nostra IX, fig. 2.

Hab. Australia merid.: Victor Harbour.

La specie fu indicata per parecchie località della Nuova Olanda, più spesso rinvenuta lungo i lidi meridionali del Continente e alle isole Norfolk. I nostri 
esemplari concordano pienamente con quelli di Lake Macquarie, N. S. Wales, delle Algae Muellevianae di J. Agardh e con quelli distribuiti da K. OKamura nelle Algae Japonicae exsiccatae n. 84.

Zonaria Turneriana J. Ag. Om Chatham-öarnes Alger (1870) pag. 438, Analecta Algologica I (1894) pag. 14; De Toni Sylloge Algarum III, pag. 232; Lucas Revised List pag. 14.

Phycopteris interrupta Kuetz. Tab. Phyc. IX (1859) tab. 67, fig. 1.

Phycopteris angustata Kuetz. Tab. Phyc. IX (1859) tab. 69, fig. 1.

Zonaria interrupta Ag., Harv. Phyc. Australica IV (1862) tab. 190 ; Hooker Fl. Tasman. II (1860) pag. 290.

Hab. Australia merid.: Victor Harbour, 12 novembre 1908; Tasmania: Baia di Latrobe, 9 gennaio 1909; Burmie, rocce battute dalle onde, 15 gennaio 1909.

Questa specie venne da taluni autori confusa con la Zonaria interrupta (Lamour.) J. Ag. del Madagascar e dell'Africa meridionale; così fecero C. AGARDH descrivendo la Zonaria interrupta e il Kuetzing la Phycopteris intermpta; quest' ultimo diede come stazioni Teneriffa, Capo di Buona Speranza, l' isola di Madagascar e la Nuova Olanda. Anche il Lenormand distribuì erroneamente una Zonaria interrupta di Western Port e una Zonaria interrupta var. stricta di Port Phillip, ambedue senz' altro individui giovanili di Zonaria Turneviana J. Ag. ; l' equivoco, d'altronde, a solo considerare l'aspetto esteriore del tallo era facilissimo perchè esaminando taluni esemplari di Tasmania e di Port Phillip delle Algae Muellevianae non riesce certo ovvia la distinzione di primo acchito; si tratta con ogni probabilità di specie geografiche vicarianti. Torna a merito di J. Agardh (Om Chatham-öarnes Alger pag. 436-438) l' aver districato la sinonimia della Zonaria Turneriana e della Zonaria interrupta assegnando come area di distribuzione alla prima l' Oceano Pacifico (Nuova Zelanda, Tasmania, Australia meridionale e occidentale) alla seconda l' Oceano Indiano (is. Madagascar, Port Natal e Capo di Buona Speranza). La Zonaria Turneriana J. Ag. è in realtà localizzata nell' Oceano Pacifico sui lidi australi e occidentali della Nuova Olanda, su quelli della Tasmania, della Nuova Zelanda e d'alcune isole come le Flinders, le Chatham, le Little Barrier e venne infatti distribuita nelle Algae Muellerianae di Tasmania e di Australia; ma senza dubbio ulteriori esplorazioni nelle numerosissime isole dell' Oceania ne estenderanno ancor più 1 area di distribuzione geografica.

$$
\text { Pachydictyon J. Ag. }
$$

Pachydictyon paniculatum J. Ag. Analecta Algologica I (1894) pag. 84; De Toni Sylloge Algarum III, pag. 283; Lucas Revised List pag. 16.

Diclyota paniculata J. Ag. Symbolae (1841) pag. 5; Species I (1848) pag. 91 ; Sonder in Linnaea vol. 25 (1852) pag. 663. Icon: Tab. nostra 8, fig. 8.

Hab. Tasmania: Baia di Latrobe; Burmie, 15 gennaio 1909.

All' area di distribuzione di questo Pachydictyon (coste meridionali e austrooccidentali della Nuova Olanda, Tasmania) assegnata dal LucAs, va aggiunta la regione littoranea della Nuova Guinea secondo il Grunow. Gli esemplari raccolti dal rev. G. CAPRA non sono certamente tra i meglio sviluppati, anzi, più 
che tutto, si devono ritenere solo frammenti di fronda. Esemplari d' erbario, nei quali si scorge il piede stopposo, per lo più provengono da Port Phillip e vennero distribuiti dal Lenormand e da Ferd. Mueller.

\section{Dilophus J. Ag.}

Dilophus fasciculatus J. Ag. Analecta Algologica I (1894) pag. 87 ; De Toni Sylloge Algarum III, pag. 285 ; 'Lucas Revised List pag. 16. Icon: Tab. nostra 9 , fig. $5 ; 10$, fig. 1 .

Hab. Tasmania: Baia di Latrobe, 9 gennaio 1909.

Questa specie, affine al Dilophus Gunnianus J. Ag., vegeta insieme ad esso lungo le coste meridionali della Nuova Olanda e quelle della Tasmania. Delle diciotto specie descritte finora tutte, tranne il Dilophus repens J. Ag. del Mare Mediterraneo, il Dil. Furcula Woron. del Mar Nero, il Dil. guineensis (Kuetz.) J. Ag. e il Dil. alternans J. Ag. dell'Oceano Atlantico, il Dil. radicans Okam. del Giappone e il Dil. flabellatus Collins della California, appartengono alla flora della Nuova Olanda e della Tasmania; ma è probabile che una revisione di più copiosi materiali potrà ridurre in modo considerevole il numero delle specie. I nostri esemplari hanno fronda circinata a rami convoluti a spirale, ramificazioni principali di 2 millim. di larghezza, secondarie di 1 millim. avendo notevole lunghezza fino a superare anche i 20 centimetri.

\section{Glossophora J. Ag.}

Glossophora Harveyi J. Ag. Till Algernes Systematik V (1880) pag. 111; De Toni Sylloge Algarum III, pag. 291.

Dictyota Kunthii Harv. Phyc. Australica Syn. (1863) pag. XI, n. 110 (excl. specim. Peruv.) ; Kuetz. Tab. Phyc, IX (1859) Tab. 30, fig. I (non Zonaria Kunthii C. Ag.).

Hab. Tasmania: Burmie.

Questa specie comune secondo il LaIng (Revised List I, pag. 65, n. 69) sulle coste della Nuova Zelanda e delle isole Chatham, non manca ai lidi della Nuova Olanda (Western Port, Victoria, Geographe Bay); essa venne in passato confusa con la Glossophora Kunthii (Ag.) J. Ag. e perciò le erano state assegnate come area di distribuzione le spiaggie del Perù e della California; ciò spiega come il Grunow (Alg. Novara pag. 49) abbia per la Dictyota Kunthii Ag. segnalato il Chilì e la Nuova Zelanda; la medesima confusione fu compiuta da HOOKER (Handbook of the New Zealand Flora, 1867, pag. 657).

\section{Lobospira Aresch.}

Lobospira bicuspidata Aresch. Phyceae novae et minus cognitae (1854) pag. 364 ; Harv. Phyc. Australica I (1858); De Toni Sylloge Algarum III, pag. 292; Lucas Revised List pag. 16.

Metachroma thuyoides Harv. in Trans. R. Irish Academy vol. 22 (1854) pag. 535 ; Alg. Austral. exsicc. n. 75 ; Kuetz. Tab. Phyc. IX (1859) tab. 88.

Hab. Australia merid. : Victor Harbour.

La Lobospira bicuspidata Aresch. è specie caratteristica dei lidi meridionali 
ed austro-occidentali della Nuova Olanda, scoperta per la prima volta a Porto Adelaide; merita ricordare che tanto AREschoug quanto HaRvey descrivendo, a pochi mesi di distanza, con nomi generici diversi quest' elegante Dictyotacea, rimasero titubanti di fronte all' aspetto da essa presentato, di guisa che il primo dei due autori la paragonò a un Plocamium, il secondo a una piccola Cystoseira. Esemplari provenienti da Port Phillip (Australia meridionale) vennero distribuiti da J. Agardh nelle Algae Muellerianae. Il rev. dott. G. CAPRA non raccolse che alcuni frammenti di fronda nei quali però non mancano i ramuli ricurvi uncinati avvinghianti simili alle estreme fronde di molte specie di Hypnea.

Ordo III. Phaeozoosporinae (Thur.)

Fan. LAMINARIACEAE (Bory) Rostaf.

Adenocystis Hook. et Harv.

AdeNocystis utricularis (Bory) Skottsb. Subantarkt. und antarktische Meeresalgen I (1907) pag. 39.

Asperococcus utricularis Bory in D' Urville Flore des Malouines (1825) pagina 594. fig. 2.

Asperococcus Lessonii Bory Voyage de la Coquille (1828) pag. 199, tab. 11,

Asperococcus Lessonii minor Kuetz. Tab. Phyc. VIII (1858) tab. 16, fig. a-d.

Adenocystis Lessonii Hook. et Harv. in Hook. Fl. Antarctica I (1844) pag. 179, tab. 69, fig. 2 ; Harv. Phyc. Australica I (1858) tab. 48; Hariot Alg. C. Horn pag. 47, tab. 5, fig. 1-3; Laing Observ. on the Fucoideae of Banks Peninsula (1885) pag. 305, tab. 10, fig. 1-2 ; Subantarctic Islands of N. Zealand pag. 498; Kjellm. Undersökn. Slägt. Adenoc. (1889) pag. 8, fig. 9-23; De Toni Sylloge Algarum III, pag. 324 ; Gain Flore Algol. des régions antarct. et subantarct. pag. 43, fig. 46-49; Lucas Revised List pag. 17; G. Murray Phycological Memoirs II (1893) tab. 16, fig. 1-2.

Hab. Australia occid. : Baia di Geroldton, settembre 1908.

Pochi esemplari ricevemmo dal rev. G. CAPRA, de' quali taluni presentano la fronda divisa profondamente nella parte superiore, come furono figurati dal Gain op. cit. fig. 46 ; corrispondono essi molto bene con esemplari delle nostre collezioni raccolti a Wellington Head (Nuova Zelanda) da R. M. LAING nel settembre 1906, con l' avvertimento che in quel mese dell' anno sono rari a rinvenirsi.

L'Adenocystis utricularis, di cui SkotTsBerg e Gain nelle loro opere dànno l'area generale di distribuzione geografica è, per quanto riguarda l' Oceania, stata indicata per la Tasmania e per la Nuova Zelanda. Il Sónder Algae Austral. hactenus cognitae n. 174) la segnala soltanto per Port Arthur (Tasmania) e di questa località, nonchè delle isole Auckland, sono conservati campioni nell' erbario ZANARDINI (1) Bellissimi esemplari furono raccolti nello stretto di Magellano durante la circumnavigazione della «Vettor Pisani » da G. Chierchia (Erb. PICCONE-FORTI).

(1) Cfr. anche HARIOT P., Algues pag. 5 (Expédition antarctique française (1903-1905) commandee par le D.r JEAN CHARCOT, scienc. natur, : docum. scientifiques). 


\section{Ecklonia Hornem.}

Ecklonia Radiata (Turn.) J. Ag. Species I (1848) pag. 146; Suringar Illustr. Alg. Japon. II (1870) pag. 1, cum tabulis; De Toni Intorno al genere Ecklonia (1889) pag. 785 ; Sylloge Algarum III, pag. 354; Lucas Revised List pag. 17.

Hab. Australia occid.: Baia di Geroldton, settembre 1908; Tasmania: Baia di Latrobe, 9 gennaio 1909.

- Questa specie ha larga distribuzione in Oceania, soprattutto lungo le coste dell' Australia, della Tasmania, della Nuova Zelanda e delle isole Chatham ; frammenti di lamina scoloriti quelli raccolti dal CAPRA in Tasmania, un piccolo esemplare intiero quello di Australia.

Macrocystis Ag.

Macrocystis pyrifera (L.) C. Ag., De Toni Sylloge Algarum JIl (1895) pag. 372.

Var. DuebeniI (Aresch.) Harv. Phyc. Australica IV (1862) tab. 202; Hooker Handbook N. Zeal. Flora (1867) pag. 656.

Macrocystis Duebenii Aresch. Icon. Phyc. I (1847) pag. 5, tab. X, fig. 5, Phyceae novae (1854) pag. 32 ; Laing Revised List (1899) pag. 64, n. 62.

Hab. Tasmania: Baia di Latrobe, 9 gennaio 1909; Nuova Zelanda: Port Charmers, acque basse, 30 gennaio 1909.

Considerando la Macrocystis pyrifera Ag. come lo fu da C. SkotTsBerg (Subantarkt. und antarkt. Meeresalgen I, pag. 108 e seg.; Stockholm 1907) i nostri esemplari, per i caratteri presentati dalle aereocisti, rientrano nella serie di quelle forme che comprendono la Macrocystis Dübenii Aresch. e la Macr. Orbignyana Mont. Lo stesso autore, seguendo i concetti di W. H. HaRvey, riuni sotto il nome di Macrocystis pyrifera (L.) Ag. tutte le specie ch' erano state distinte da C. Agardh, Bory, Harvey, Montagne, Areschoug, Postels e Ruprecht, di modo che la specie, con tali riferimenti, venne ad acquistare una vasta area di distribuzione geografica, in cui C. SkotTsBeRg mostra i legami mediante i reperti nelle florule insulari tra l' America, l' Africa e l' Oceania. Per quanto concerne l' Australasia la specie, nella sua var. Dïbenii, è in alcune regioni assai abbondante, come a Rivoli Bay (Sonder) nonchè a Port Phillip nella Nuova Olanda meridionale e in parecchie località della Nuova Zelanda e delle isole circostanti Chatham, Campbell e Auckland.

\section{Fam. SP0RochnaCeat (Reichb.) Decne}

\section{Bellotia Harv.}

Bellotia Eriophorum Harv. Short Characters of some new genera and species of Algae discovered on the coast of the Colony of Victoria, Australia (1855) pag. 1, Phyc. Australica II (1859) tab. 69; Mont. Communication relative à une plante marine de 1' Australie (1855) pag. 1; Hooker Flora Tasman. II (1860) pag. 288, tab. 187, fig. 1-3; De Toni Sylloge Algarum III, pag. 377 ; Lucas Revised List pag. 17. Icon: Tab. nostra 9, fig. 4.

Hab. Tasmania: Baia di Latrobe, 9 gennaio 1909. 
La diffusione geografica della Bellotia Eriophorum Harv., specie elegantissima, finora sembra limitata ai lidi meridionali della Nuova Olanda e alla Tasmania. Una sola ombrella renne raccolta dal rev. G. CAPRA munita delle caratteristiche chiome erioforoidi ed anche questa si dimostra costituita di elementi giovanili forse appena prolificati se si giudica dalle dimensioni assai minori delle ordinarie. Ma interessanti oltre modo sono due altri individui da lui raccolti che sembrerebbero rappresentare la fase più avanzata dello sviluppo se non forse il residuo del tallo dopo l'ultima propagazione, corrispondendo il periodo iemale nell' emisfero australe al periodo più caldo dell' annata. I cauli in questi esemplari mantengono la loro caratteristica forma setacea e così pure i calli radicali sono rivestiti dalla breve stoppa vellutata di tinta bistro-olivastra; si ramificano successivamente due o tre volte ad ombrella ; ma nessuna traccia della caratteristica chioma ravvolge più l' estremità libera delle ultime ramificazioni; questa chioma è caduta e non persiste che un ricettacolo nudo il quale forse l' anno successivo potrà divenir centro d' una nuova proliferazione. Così pure la regione mediana dei rami sporangifera, dopo aver subito una contorsione longitudinale a spira, rimane sottile come il rimanente dei medesimi perchè costretti e stirati nel ravvolgimento gli scafidii poterono rimanere vuoti del volume delle spore.

Nell' erbario DE TONi trovansi piccoli esemplari autentici di HARveY, i quali corrispondono al portamento dei nostri raccolti dal CAPRA, mancando anch' essi dei ciuffi filamentosi.

\section{Perithalia J. Ag.}

Perithalia ineruis (R. Br.) J. Ag. Till Algernes Systematik IX (1890) pag. 2 ; De Toni Sylloge Algarum III, pag. 378; Lucas Revised List pag. 17.

Carpomitra inermis Kuetz., Harv. Phyc. Australica IV (1862) tab. 238.

Fucus inermis R. Br. in Turn. Fuci III (1811) tab. 186.

Hab. Tasmania: Baia di Latrobe.

Questa specie, non nuova per la Tasmania essendo stata segnalata da HARveY per il fiume Tamar, vegeta eziandio lungo i lidi meridionali e austro-orientali della Nuova Olanda e alle isole Flinders. Esemplari ne vennero distribuiti nelle Algae Muellerianae col nome di Carpomitra caudata Harv. e si contraddistinguono per la ramificazione più breve che non si vegga nel genuino tipo di Port Phillip, distribuito per opera di Alberto Grunow. Si tratta però di caratteri troppo evanescenti per ritenerli di vera e propria importanza sistematica.

\section{Sporochnus C. Ag.}

Sporochnus comosus C. Ag. Systema Algarum (1824) pag. 259 ; Harv. Phyc. Australica II (1859) tab. 104; Kuetz. Tab. Phyc. IX (1859) tab. 80, fig. Il ; Hooker Fl. Tasman. Il (1860) pag. 287; De Toni Sylloge Algarum III, pag. 381; Lucas Revised List pag. 17.

Hab. Tasmania: Burmie.

Gli esemplari raccolti dal rev. dott. G. CAPRA furono confrontati, e trovati corrispondere, con quelli provenienti dal King George 's Sound e dalla Tasmania, conservati nelle nostre collezioni. La specie è finora limitata ai lidi occidentali e meridionali della Nuova Olanda e a quelli della Tasmania. 
Fam. STILOPHORACEAE (Naeg.) D. T. et $\mathbf{L}$.

Stilophora J. Ag.

Stilophora RHIzodes (Ehrh.) J. Ag. Symbolae I (1841) pag. 6; Harv. Phyc. Brit. (1851) tab. 70; De Toni Sylloge Algarum III, pag. 390.

Sporochnus rhizodes Ag. Species I (1821) pag. 156.

Spermatochus rhizodes Kuetz. Tab. Phyc. VIII (1858) tab. 17.

Hab. Nuova Zelanda: Bluff, con epifite la Chantransia corymbifera e la Calothrix confervicola.

J. D. Hooker ebbe già a segnalare questa specie per l' Oceano Pacifico, anzi meglio per i lidi della Tasmania, dove poscia fu raccolta da R. Gunn (Harvey Phyc. Australica Syn. 1863, pag. XII, n. 117); la Stilophora rhizodes (Ehrh.) J. Ag. è eziandio indicata per l'Australia (Lefebre Penins. e Holdfast Bay; F. Mueller; Sonder Plantae Muellevianae 1852, pag. 663). Una forma assai affine sembrerebbe essere quella Stilophora australis Harv. pure di Tasmania (cfr. Lucas Revised List pag. 18). Pochi e meschini sono gli esemplari raccolti dal rev. G. CAPRA, soriferi, di grande interesse perchè finora sconosciuti per la regione neo-zelandese.

Fam. CHORDARIACEAE (Ag.) Zanard.

$$
\text { Myrionema Grev. }
$$

Myrionema strangulans Grev. Cryptog. Flora (1827) tab. 300 ; Kuetz. Tab. Phyc. VII (1857) tab. 93, fig. I; De Toni Sylloge Algarum III, pag. 399.

Myrionema Leclancherii Harv. Phyc. Australica Syn. (1863) pag. XIII, n. 134 (in Ulva) [non alibi].

Hab. Nuova Zelanda: Bluff, sulla Enteromorpha Linza var. crispata.

Sotto il nome più antico, Myrionema strangulans Grev., vanno, stando alle accurate osservazioni del Sauvageau (Sur quelques Myrionémacées pag. 25; Paris, 1897) riunite parecchie forme di Myrionema distinte dagli autori come specie differenti, in base alle matrici e ad uno studio non completo dei talli; col nome di Myrionema vulgare Thur. (1864) il Sauvageau infatti, ampliando le vedute del Thuret, comprende, oltre al Myrionema strangulans Grev. (1827) delle $\mathrm{Ul}$ vaceae, il Myr. punctiforme Harv. (1833) dei Ceramium e di altre Florideae a fronda filiforme, il Myr. maculiforme Kuetz. (1845) delle Ulvaceae, il Myr. ocellatum Kuetz. (1849) delle Laminaria, il Myr. Leclancheri Harv. (1833) della Rhodymenia palmata, il Myr. intermedium Fosl. (1894) dell' Enteromorpha compressa. Il Myrionema strangulans Grev. venne raccolto fino dal 1850 dal LyalL sull Ulva latissima ad Otago distribuito in esemplari delle Algae Muellerianae e sull Ulva rigida nei lidi occidentali della Nuova Zelanda, secondo il Corton (Notes on New Zealand marine Algae pag. 239; Bull. of miscell. Inform. Kew, 1909, n. 6). Siccome resulta dal sinonimo sopra citato la specie fu trovata anche a Georgetown in Tasmania; con ogni probabilità, esaminando gli esemplari di Ulvaceae dell' Oceania, l' area di distribuzione del Myvionema verrà considerevolmente estesa anche in considerazione che la specie venne trovata fino in $\mathrm{Ca}$ lifornia sopra il Nitophyllum Ruprechtianum J. Ag. (cfr. Collins, Holden ET Setchell Phyc. Bor. Amer. n. 1795). 
Cladosiphon Kuetz.

Cladosiphon zostericola Harv. Alg. Austral. exsicc. n. 98, Phyc. Australica Syn. (1863) pag. XIII, n. 130; Kuetz. Tab. Phyc. IX (1859) tab. I, fig, 1; De Toni Sylloge Algarum III, pag. 416; Lucas Revised List pag. 18.

Hab. Tasmania: Baia di Latrobe, 9 gennaio 1909; Burmie, 15 gennaio 1909; Nuova Zelanda: Bluff.

La specie venne riscontrata per la prima volta nel King George's Sound (Harvey) e a Port Phillip nella Nuova Olanda meridionale (F. Mueller), di quest' ultima località confrontammo esemplari esistenti nell' Erbario ZANARDINI e distribuiti dal Lenormand. Che cosa sia il Cladosiphon zostericola che fu da Maze e Schramm (Essai de classification des Algues de la Guadeloupe pag. 114; Basse-Terre 1870-1877) indicato per la Guadalupa, in mancanza di esemplari autentici, nulla possiamo dire; di questi esemplari guadalupensi che dai CrouAN furono classificati come Cladosiphon zostericola Harv. non si trova alcuna indicazione nelle opere moderne che trattano delle florule algologiche delle Indie occidentali, così che la detta stazione atlantica del Cladosiphon zostericola necessita di conferma (1). Gli esemplari raccolti dal rev. G. CAPRA nella Baia di Latrobe sono singolarmente bene sviluppati in maniera da poter simulare di primo acchito l' aspetto di talune forme di Chorda e trovansi in piena fruttificazione.

\section{Leathesia Gray.}

Leathesia difformis (L.) Aresch. Phyceae Scandin. (1846) pag. 376; De Toni Sylloge Algarum III, pag. 422 ; Lucas Revised List pag. 18.

Hab. Australia merid. : Victor Harbour.

Questa specie fu con grande facilità confusa col Cylindrocarpus (Petrospongium) Berkeleyi (Grev.) Crouan e con individui giovanili di Colpomenia sinuosa (Roth) Derb. et Sol, come dimostrò, dietro l' esame di materiale autentico raccolto nelle coste neozelandesi e conservato nell' erbario di Kew, il Cotton (Notes on New Zealand marine Algae pag. 239; Bull. of Miscell. Inform. Kew 1909, n. 6); infatti l' Asperococcus sinuosus quanto agli esemplari raccolti a Otago dal Lyall e citati da J. D. Hooker (Fl. N. Zel. II, pag. 219, Handbook pag. 658) va riferito alla Leathesia difformis (L.) Aresch., laddove la Leathesia Berkeleyi, ricordata nelle opere sopra citate di HooKeR e raccolta dal Colenso a Cape Kidnapper, è una specie forse nuova di Cylindrocarpus (Petrospongium); tali osservazioni del CotTon escludono affatto l' avviso espresso dal LAING (Revised List of New Zealand Seaweeds I, pag. 63, n. 52; Wellington, 1899) il quale riteneva che la Leathesia Berkeleyi della Nuova Zelanda fosse con ogni probabilità null' altro che la Leathesia difformis, specie riscontrata propria della flora neozelandese anche dal REInBoLD.

Il Sonder (Algae Austral. hactenus cognitae n. 185: Leathesia tuberiformis Gray) assegna per la specie in parola le località Western Port (Victoria) e Georgetown (Tasmania); la diffusione della Leathesia difformis (L.) Aresch. nel-

(i) Il BorRgesen (The marine Algae of the Danish West Indies parts, Phaeophyceae pag. 184; Copenhagen, I9I4) cita solo per le Indie occidentali la Castagnea Zosterae. 
l' Oceano Pacifico deve essere nulla di meno assai considerevole qualora si pensi che la specie venne raccolta sui lidi della California (DE Alton Saunders in Phyc. Bor. Amer. n. 829) e a Shirahama in Giappone (Okamura Alg. Japon. exsicc. n. 89).

\section{Fam. DESMARESTIACEAE (Thur.) Kjellm.}

\section{Desmarestia Lamour.}

Desmarestia chordalis Hook. et Harv. Alg. Antarct. (1845) pag. 249 ; Kuetz. Tab. Phyc. IX (1859) tab. 97, fig. I; De Toni Sylloge Algarum III, pag. 457.

Trinitaria confervoides Bory Voyage de la Coquille (1829) pag. 216, tab. 24, fig. 2?

Hab. Tasmania: Baia di Latrobe; Burmie.

I nostri esemplari si accostano, quanto allo habitus generale della fronda, a un campione di Desmarestia intermedia Post. et Rupr. proveniente dal Mare di Ochotsk; e non si può negare ch' essi somigliano alla Desmarestia media Grev. qual' è delineata in Kuetzing Tabulae Phycologicae IX (1859) tab. 95, fig. II di su un esemplare proveniente dall' Oceano Pacifico meridionale e comunicato dallo HoOKER.

Oltre che della Terra di Kerguelen, località classica, la Desmarestia chordalis Hook. et Harv. è nota per l' isola San Paolo nell' Oceano Australe secondo il Grunow (Alg. Novara pag. 50) il quale però la ritiene diversa dalla Desmarestia Menziesii (Ag.) J. Ag. della Nuova Setlandia e di Concepcion; con la Desm. chordalis Hook. et Harv. va confrontata la Desm. compressa (Reinsch) Skottsb. (Subantarkt. und antarkt. Meeresalgen I, pag. 19, tab. II ; Stockholm, 1907) della Georgia meridionale e delle Terre Graham e Victoria. Il LAING dà come raccolta in Australasia la Desmarestia ligulata Lamour. oltre che nelle isole Auckland e Enderby e la Desmarestia Willii Reinsch pure nelle isole Auckland; sono queste le uniche specie finora osservate sulle coste del Continente Australiano o delle isole floristicamente collegate con la Nuova Zelanda.

\section{Fam. ENCOELIACEAE (Kuetz.) Kjellm.}

Chnoospora J. Ag.

Chnoospora fastigiata J. Ag. Species I (1848) pag. 171; Sond. Algae Au stral. hactenus cognitae (1881) pag. 105; Barton On the fruit of Chnoosp. fastigiata (1898) pag. 507, tab. 28; De Toni Sylloge Algarum III, pag. 464.

Chnoospora pacifica J. Ag., Kuetz. Tab. Phyc. IX (1859) tab. 86, fig. 1.

Chnoospora atlantica J. Ag., Kuetz. Tab. Phyc. IX (1859) tab. 86, fig. II.

Hab. Tasmania: Baia di Latrobe.

Un solo esemplare di questa specie fu raccolto dal rev. G. CAPRA nella località sopra menzionata; esso ha una lunghezza di 17 centimetri e colore olivaceo chiaro.

La Chnoospora fastigiata J. Ag. sembra godere d' una estesa area di distribuzione, per quanto sia stata segnalata solo di poche località, ma queste sono tra loro assai remote. Secondo la BARTon se ne hanno esemplari raccolti 
da Elliot a S. Vincenzo (Indie occidentali) e da Harvey all' isola Ceylon. J. AGARDH assegna alla forma pacifica il littorale messicano (cui Mazè e Schramm aggiungono l' isola Guadalupa), all' atlantica le coste del Venezuela; sulla fede del SoNDER infine vuolsi ricordata la Nuova Galles del Sud. È verosimile che a questa specie sarà da attribuirsi la Chnoospora implexa var. gracilis raccolta da Amalia Dietrich a Boeven sulle coste nord-est dell' Australia (Grunow in schedula, Erb. Piccone-Forti) corrispondendo molto bene con la figura delle Tabulae Phycologicae. Del resto il Grunow riscontrò la Chnoospora fastigiata var. pacifica nei materiali raccolti da IDA Pfeiffer a Tahiti (Alg. Novara pag. 50) ; Chnoospora implexa (Her.) J. Ag. sembra vegetare piuttosto nel Mar Rosso.

\section{Colpomenia Derb. et Sol.}

Colpomenia sinuosa (Roth) Derb. et Sol. Mémoire physiol. Alg. (1856) pag. 11, tab. 22, fig. 18-20; De Toni Sylloge Algarum III, pag. 489; Lucas Revised List pag. 19.

Hydroclathrus sinuosus Zanard. Iconographia I (1863) pag. 277; Murray Phycological Memoirs II (1893) pag. 53, tab. XIV, fig. 1-6, tab. XV, fig. 1. Asperococcus sinuosus Kuetz. Tab. Phyc. IX (1859) tab. 8.

Hab. Tasmania: Burmie, rocce battute dalle onde; 15 gennaio 1909.

Per gli esemplari di questa specie raccolti alla Nuova Zelanda si veggano le osservazioni da noi esposte a proposito della Leathesia difformis (L.) Aresch.

Pochi individui di Colpomenia sinuosa (Roth.) D. et S. vennero a noi spediti dal rev. dott. G. CAPRA, i quali non lasciano dubbio sulla loro determinazione. La specie, quanto all' Oceania, è già conosciuta per il littorale della Nuova Olanda in parecchie località (Sonder, Algae Austral. hactenus cognitae n. 171: Asperococcus sinuosus) e dello stesso Continente la ricordano il Grunow, il Reinbold e l' Okamura. Nell' erbario Zanardini trovasi conservato un esemplare, comunicato da HaRvey, raccolto in Tasmania. Nell' Oceano Pacifico del resto la Colpomenia è diffusissima dalle coste della California (Phyc. Bor. Amer. n. 278 e 825) al Giappone (Okamura, Alg. Japon. exsicc. n. 42); il Sonder (Plantae Muellerianae 1852, pag. 663) cita la specie per la Holdfast Bay.

\section{Fam. SPHACELARIACEAE (Decne) Kuetz.}

$$
\text { Cladostephus C. Ag. }
$$

Cladostephus verticillatus (Lightf.) Lyngb. Hydrophyt. Dan. (1819) pag. 102, tab. 30 B; De Toni Sylloge Algarum III, pag. 513; Sauvageau, Remarques sur les Sphacélariacées (1914) pag. 601.

Agardh).

Cladostephus spongiosus Kuetz. Tab. Phyc. VI (1856) tab. 7, fig. II (non C.

Cladostephus tomentosus Kuetz. Tab. Phyc. VI (1856) tab. 10, fig. 1.

Hab. Tasmania: Baia di Latrobe, 9 gennaio 1909; Burmie; Nuova Zelanda: Bluff, 25 gennaio 1909.

Gli autori di flore australiane riferirono i Cladostephus dell' Oceania ora al Cladostephus spongiosus Ag. ora al Clad. verticillatus Lyngb., apportando in tal modo una grande confusione riguardo all' area fitogeografica delle due spe- 
cie. I nostri esemplari di Burmie soprattutto corrispondono con un esemplare proveniente dalla Tasmania e distribuito da J. AGARDH nelle Algae Muellerianae col nome di Cladostephus australis $\left({ }^{1}\right)$, specie che ha tutto il portamento del Cladostephus verticillatus Lyngb., di cui al più rappresenta una forma. Il LuCAS (Revised List pag. 20) ammette l' esistenza dei due Cladostephus spongiosus Ag. e verticillatus Lyngb., assegnando al primo le coste meridionali e orientali della Nuova Olanda e quelle della Tasmania, al secondo le coste meridionali della Nuova Olanda; il Sauvageau però, il quale ebbe occasione di esaminare un copioso materiale di queste speeie, considera dubbia l' esistenza del Cladostephus spongiosus Ag. nell' emisfero australe e, ricevuto da noi un esemplare tasmanico di Burmie, confermò trattarsi del Cladostephus verticillatus Lyngb. A questa specie saranno pure da attribuirsi gli esemplari di IRvINE distıibuiti nelle Algae Muellerianae col nome di Cladostephus verticillatus australis di Geographe Bay, nonchè quelli di Portland, Victoria, raccolti da F. Mueller e determinati dal Grunow col nome di Cladostephus Myriophyllum Ag. forma australiensis.

\section{Halopteris Kuetz.}

Halopteris HoRdeacea (Harv.) Sauv. Remarques sur les Sphacélariacées (1904) pag. 432.

Sphacelaria hordeacea Harv. in Hooker Icon. Plant. tab. 614.

Stypocaulon paniculatum Kuetz., Reinke Vergl. Anat. Morph. Sphacel. (1891) pag. 27, tab. 7, fig. 6-9; De Toni Sylloge Algarum III, pag. 516 partim; Lucas Revised List pag. 20.

Stypocaulon filare Kuetz. Tab. Phyc. V (1855) tab. 98.

Slypocaulon hordeaceum Kuetz. Tab. Phyc. V (1855) tab. 99.

Hab. Australia merid.: Victor Harbour, 12 novembre 1908; Tasmania: Baia di Latrobe, 9 gennaio 1919; Burmie, 15 gennaio 1909.

È specie largamente distribuita lungo i lidi dell' Australia, della Tasmania e della Nuova Zelanda, nonchè di parecchie piccole isole dell' Oceania; il SAuvaGEAU nella sua importante revisione critica delle Sphacelariaceae, limita la $\mathrm{Ha}$ lopteris hordeacea all' Australia e alla Nuova Zelanda. Gli esemplari africani che alcuni autori, compreso W. H. Harvey (Phyc. Austral. Syn. 1863, pag. XIII, n. 137: Sphacelaria paniculata Suhr) ascrissero a questa specie vanno invece riferiti alla Halopteris funicularis (Mont.) Sauv. che presenta una grande diffusione nei mari australi. La Halopteris hordeacea (Harv.) Sauv. fu più volte distribuita nelle Algae Muellerianae e prima ancora dal Grunow e dal LENoRMAND; si tratta di una delle specie più comuni nell' Oceano che bagna i lidi meridionali dell' Australia e della Tasmania; ad essa deve attribuirsi la Sphacelaria spicigera Aresch. (Phyceae extraeuropaeae pag. 39) proveniente da Port Adelaide.

(I) Notisi per incidenza che Cladostephus australis C. Ag. (Syst. 1824, pag. I69) e Cladostephus australis Kuetz. (Tab. Phyc. VI, 1856, pag. 5, tab. 9, fig. II) sono due entità affatto diverse; il primo è una Floridea cioè la Brongniartella australis (Ag.) Schmitz (cfr. DE Toni Sylloge Algarum IV, pag. roro), il secondo è un vero Cladostephus; entrambe le specie furono da C. AGARDH comunicate, col nome Griffithsia australis, ai di lui corrispondenti. 


\title{
Fam. ECTOCARPACEAE (Ag.) Kuetz.
}

\author{
Ectocarpus Lyngb.
}

Ectocarpus siliculosus (Dillw.) Lyngb., De Toni Sylloge Algarum III, pag. 549.

Forma ARctus (Kuetz.) Kuck. Ectocarp. der Kieler Föhrde (1891) pag. 18;

De Toni op. cit. pag. 550.

Corticularia arcta Kuetz. Tab. Phyc. V (1855) tab. 80, fig. 1.

Corticularia fuscata Kuetz. Tab. Phyc. V (1855) tab. 90, fig. II.

Hab. Australia occid. : Baia di Geroldton, settembre 1908; Australia merid. : Victor Harbour, 12 novembre 1908; Nuova Zelanda: Bluff, con la Calothrix confervicola, 25 gennaio 1909.

L'Ectocarpus siliculosus (Dillw.) Lyngb. è specie quasi cosmopolita. I nostri esemplari vanno riferiti alla forma sopra indicata, corrispondendo bene alle figure Kuetzingiane. L'Ardissone, avendo unito insieme nella sua Phycologia Mediterranea l'Ectocarpus siliculosus e l'Ectocarpus confervoides, attribuil in altro suo lavoro a quest' ultima specie il cosmopolitismo, laddove il genuino Ectocarpus confervoides (Roth) de Jol. ha un' area di distribuzione geografica molto più ristretta.

Il Lucas (Revised List pag. 20) indica l'Ectocarpus siliculosus (Dillw.) Lyngb. raccolto sulle coste meridionali e occidentali della Nuova Olanda e su quelle della Tasmania; non mancano dati espliciti per il ritrovamento di detta specie nella Nuova Zelanda, dove anzi essa pare abbastanza frequente, ivi raccolta da Hooker, Lyall, Davies, Colenso, J. Crosby Sitth (cfr. Laing, Revised List I, 1899, pag. 62, n. 41).

La molto maggiore scarsezza di casi in cui negli erbarii si trovano esemplari provenienti dall' Oceano Pacifico in confronto che da altri mari, lascia supporre una minore frequenza effettiva dell'Ectocarpus siliculosus (Dillw.) Lyngb. in codesto Oceano piuttosto che altrove; certo si è che le forme più sviluppate provengono dall' Oceano Atlantico e dal Mare Baltico in particolare e che tutto il materiale raccolto dal CAPRA rispecchia all' evidenza una fisonomia di vegetazione depauperata, sebbene spesso gli individui si trovino anche decisamente sporangiferi.

\section{CHLOROPHYCEAE (Kuetz.) Wittr.}

Ordo I. Confervoideae (Ag.) Falk.

\section{Fam. U L V A C E A E (Lamour.) Rabenh.}

Ulva (L.) J. Ag.

Ulva Lactuca (L.) Le Jolis List. Alg. Cherb. (1863) pag. 38 ; De Toni Sylloge Algarum I (1889) pag. 111.

Forma Myriotrema (Lenorm.) J. Ag. Till Algernes Systematik VI (1882) pag. 169; De Toni op. cit. pag. 112.

Phycoseris myriotrema Lenorm. in Kuetz. Tab. Phyc. VI (1856) tab. 23, fig. I.

Hab. Tasmania: Baia di Latrobe, 9 gennaio 1909. 
Esemplari in cattivo stato di conservazione affissi su conchiglie di bivalvi e su frondi di Perithalia inermis, corrispondenti agli esemplari peruviani e cileni (1). Finora questa forma, a nostro sapere, non apparisce segnalata per la Tasmania, probabilmente confusa col tipo cosmopolita o con la forma rigida (Kuetz.).

Forma RIGIDA (Kuetz.) De Toni Sylloge Algarum I (1889) pag. 111.

Phycoseris rigida Kuetz. Tab. Phyc. VI (1856) tab. 23, fig. 2.

$H a b$. Australia merid.: Victor Harbour.

Questa forma caratteristica per avere la fronda membranacea più solida delle sue simili possiede una estesissima area di distribuzione geografica sia nell' Oceano Pacifico che negli altri mari. Il Lucas (Revised List pag. 165) sulla scorta di W. H. HaRvey la dice raccoita sui lidi dell' Australia occidentale e della Nuova Zelanda; il LAING ne conferma l'abbondanza nelle spiaggie neozelandesi, nonchè per le isole Auckland e Enderby, ove la raccolse Crosby Sмith; sembra dunque anche per quanto da ultimo ha rilevato C. Skotrsberg che questa sia la forma più comune in tutte le regioni antartiche $\left({ }^{2}\right)$.

Ulva fasciata Del. Fl. Égypte (1824) pag. 153, tab. 58, fig. 5; J. Ag. Till Algernes Systematik VI (1882) pag. 173; Mont. Fl. d' Algérie (1849) pag. 151, tab. 14, fig. 1-2; De Toni Sylloge Algarum I, pag. 114.

Hab. Australia occid. : Baia di Geroldton, settembre 1908.

L' area di 'distribuzione di questa Ulva è molto estesa; la specie non manca all' Oceano Pacifico, ove fu già osservata dai lidi della California fino al Chili; di recente venne raccolta nelle isole Hawaii dalla TiLDEN (Amer. Algae n. 559) che anzi ne descrisse una forma minor; sembra però che bene spesso la specie australiana sia stata confusa con l' Ulva Lactuca (L.) Le Jol., almeno cosi lascierebbe supporre una scheda del GRunow esistente nell'Erbario PICCONE-ForTI, nella quale si dice essere Phycoseris Ulza Sond. uguale ad una varietà di Ulva fasciata e per verità si tratta d' un esemplare di quest' ultima specie raccolto dal Daemel sui lidi settentrionali della Nuova Olanda. Gli esemplari della Baia di Geroldton si assomigliano più che altro a quello delle isole Hawaii distribuito dalla TILDEN; possono pure ricordare, quand' anche non debbano esservi addirittura attribuiti, talune forme di Ulva Lactuca forma rigida (Kuetz.) De Toni che sembra, come s' è detto, molto comune nell'Oceano Pacifico e in tutta l'Antartide ${ }^{(3)}$.

Ulva Ligula Mont. in Kuetz. Species (1849) pag. 476 (sub Phycoseride), Tab. Phyc, VI (1856) tab. 18, fig. II ; De Toni Sylloge Algarum I, pag. 116.

Ulva reticulata Mont. Voyage au Pole Sud (1845) pag. 33 (non Forskaal).

Hab. Nuova Zelanda: Port Charmers, 30 gennaio 1909.

(1) Cfr. De Toni G. B. e Forti Ach., Contributo alla conoscenza della flora marina del Chili pag. 699 (Atti del Reale Istituto Veneto di scienze, lettere ed arti tomo LXXIX, Venezia I920).

(2) Della struttura della forma rigida si occupò recentemente D. E. HyLmö (Zur Kenntnis der subantarkt. und antarkt. Meeresalgen III, Chlorophyceen 1919, pag. 2, fig. 1-3). Dalle forme della comune Ulva Lactuca (L.) Le Jol. alcuni autori giudicano opportuno separare l' Ulva australis Aresch. (Phyceae extraeuropaeae, 1854, p. 44) proveniente da Porto Adelaide, la quale non ha nulla a che fare con la Phycoseris australis Kuetz. Tab. Phyc. VI (1856) tab. 24, fig. II.

(3) Notisi che W. H. Harvey (Phyc. Australica Syn. 1863, pag. LIX, n. 757) a proposito di Ulva rigida Ag. lascio scritto: «I venture to refer Sonder's Phycoseris Ulva to this plant ». 
E una tra le specie minori del genere Ulva, con le frondi a margine irregolarmente crenato; si accordano assai i nostri esemplari più gracili all' Ulva uncialis Suhr del Capo di Buona Speranza (Atrica meridionale) che è figurata sotto il nome di Phycoseris uncialis in Kuetzing Tab. Phyc. VI (1856) tab. 16, fig. II ; gli esemplari più grandi invece segnano, a motivo dell' ampiezza dei lobi, il passaggio graduale alle forme di Ulva figurate in Kuetzing Tab. Phyc. VI (1856) tab. 23, fig. II e tab. 24, fig. II rispettivamente come Phycoseris rigida e Phycoseris australis (benchè per quest' ultima l' autore dia come ambiente il Mare Adriatico); queste notevoli somiglianze vennero già avvertite dal GRUNow (Alg. Novara pag. 43) a proposito dell' Ulva capensis Aresch., sinonimo di Ulva uncialis Suhr; così rendesi probabile che una revisione di esemplari autentici condurrà a semplificare la sistemazione delle Ulva, riferendo queste forme assai controverse alla forma rigida dell' Ulva Lactuca, tanto più che quest' ultima forma appartiene al gruppo delle Alghe riconosciute cosmopolite (cfr. Ardissone, Le Alghe cosmopolite, 1894, pag. 4); d' altronde bisogna avvertire che la forma rigida fu segnalata comune lungo le spiaggie della Nuova Zelanda da J. AGJRDH e LAIng. L'Ulva Ligula fu elencata tra le specie dubbie per non essere stata prima d'ora ritrovata se non sulle coste dell' isola Toud e descritta dal MonTAGNE. I campioni raccolti dal rev. dott. G. CAPRA a Port Charmers corrispondono assai bene alla figura sopra citata delle Tabulae Phycologicae e ne dimostrano eziandio la caratteristica increspatura e i margini lacerati irregolarmente ed arricciati. Forse essa è da ritenersi una semplice varietà australe di Ulva fasciata Del. da cui non differisce se non per le suddette fimbrie e increspature marginali.

\section{Enteromorpha Link}

Enteromorpha CHLOROTICA J. Ag. Till Algernes Systematik VI (1882) pag. 136; De Toni Sylloge Algarum I, pag. 125; Lucas Supplement. List (1912) pag. 165 .

Hab. Queensland settentr. : Baron River, nell'acqua stagnante poco profonda, 3 giugno 1909 .

È questa una specie affine all' Enteromorpha compressa (L.) Grev., alla quale, riguardata cosmopolita, vanno con ogni probabilità riferiti gli esemplari che con quest' ultimo nome vennero da alcuni autori determinati per la Nuova Zelanda, benchè per la stessa J. Agardh nell' opera De Algis Novae Zelandice marinis pag. 2 , n. 9 ricordi essere abbondante lungo le spiaggie, mentre nelle Till Algernes Systematik ammette per l' Enteromorpha compressa la indicazione della Nuova Zelanda. Il LAing (Subantarct. Isl. of New Zealand pag. 494) cita per le isole Auckland esemplari di Enteromorpha compressa identificati da A. GEPP.

Enteromorpha flexuosa (Wulf.) J. Ag. Till Algernes Systematik VI (1882) pag. 126; De Toni Sylloge Algarum I, pag. 121.

Hab. Australia occid.: Avon River, 11 ottobre 1908; Baia di Geroldton, settembre 1908; Australia merid.: Victor Harbour, 12 novembre 1908.

Secondo il Lucas (Supplem. List 1912, pag. 165) questa Enteromorpha della sezione Intestinales fu raccolta in Tasmania; infatti J. AgARDH ve la indica co- 
municatagli da R. Gunn; fu distribuita delle Hawaii dalla Tilden (Amer. Algae n. 558) dove pure venne raccolta da CESARE MARCACCI durante il viaggio della «Vettor Pisani »; nello stesso viaggio fu riportata da Porto S. Giacinto di Ticao nelle Filippine. Gli esemplari del CAPRA sono numerosi e bene sviluppati di guisa che è verosimile trattarsi di una Cloroficea abbastanza comune su tutte le coste dell' Australia.

Enteromorpha Gunniana J. Ag. Till Algernes Systematik VI (1882) pag. 122; De Toni Sylloge Algarum I, pag. 119; Lucas Supplem. List (1912) pag. 165.

Jcon. Tab. nostra X, fig. 2, 3.

Hab. Tasmania: Burmie, roccie battute dalle onde, 15 gennaio 1909.

La specie venne per la prima volta comunicata a J. Agardh da R. GunN che ne raccolse esemplari a Georgetown; finora sembra sia stata rinvenuta soltanto sui lidi della Tasmania.

Enteromorpha Linza (L.) J. Ag. Till Algernes Systematik VI (1882) pag. 134, tab. 4, fig. 10-12 (1); De Toni Sylloge Algarum I, pag. 124; Laing Revised List of New Zealand Seaweeds pag. 61, n. 30.

Hab. Nuova Zelanda: Bluff, 25 gennaio 1909.

I nostri esemplari corrispondono alla forma crispata J. Ag. La specie venne già segnalata per le isole Chatham, sui lidi delle quali la raccolse il dott. ScHAUINSLAND.

Enteromorpha Ramulosa (Engl. Bot.) Hook. Brit. Flora II (1833) pag. 319; Kuetz. Tab. Phyc. VI (1856) tab. 33; J. Ag. Till Algernes Systematik VI (1882) pag. 154, tab. 4, fig. 117-118; De Toni Sylloge Algarum I, pag. 134; Laing Revised List (1899) pag. 61, n. 32.

Hab. Nuova Zelanda: Port Charmers, 30 gennaio 1909, Bluff, 25 gennaio 1909.

Secondo gli autori è specie abbastanza comune nelle coste dell'Australia, della Tasmania e della Nuova Zelanda. Trattasi del resto d' una specie quasi cosmopolita, con distribuzione geografica estesa dall' Oceano Atlantico settentrionale all' Antartide.

\section{Fam. CHAETOPELTidaCEAE Wille}

\section{Chaetosphaeridium Klebh.}

Chaetosphaeridium globosum (Nordst.) Klebh. Zur Kritik einiger Algengattungen (1893) pag. 306, tab. 14, fig. 5-10.

Aphanochaete globosa Wolle, De Toni Sylloge Algarum I, pag. 180.

Herposteiron globosum Nordst. De Algis et Çharaceis sandvic. (1878) pag. 23, tab. 2, fig. 22-23 (setarum vaginâ inconspicuâ).

Hab. Nuova Zelanda: Rotorua, sorgenti calde, marzo 1909.

Il Chaetosphaeridium globosum (Nordst.) Klebh., da alcuni a torto confuso

(1) L'opuscolo De Algis Novae Zelandiae marinis è anteriore all' opera Till Algernes Systematik vol. VI ; gli esemplari neozelandici dell' antica specie collettizia Ulva compressa C. Ag. vennero da J. Agardh nelle Till Algernes System. VI, pag. I 44 riferiti alla Enteromorpha lingulata J. Ag. 
con la Nordstedtia globosa Borzì (Studi algologici fasc. II, 1894, pag. 349, 377) venne finora segnalato per le isole Havaii, per la Nuova Zelanda ad Omatangi presso Taupo e nel Lago Pearson dal NoRdstedt; il Wolle indica la specie stessa per l'America boreale, il Wille per l'America meridionale, il Borge infine per la Nuova Galles del Sud. Il CAPRA raccolse il Chaetosphaeridium nelle sorgenti calde di Ohinemuthu dove riveste di una patina nero-olivacea crostosa i cauli secchi delle piante che vi stanno sommerse.

\section{Fam. CHAETOPHORACEAE (Harv.) Wille}

\section{Draparnaldia Bory}

Draparnaldia glomerata (Vauch.) Ag. Systema Algarum (1824) pag. 59; Kuetz. Tab. Phyc. III (1853) tab. 12 ; Cooke Brit. Freshw. Algae (1884) pag. 191, tab. 75, fig. 1; De Toni Sylloge Algarum I, pag. 192.

Hab. Australia occid.: Avon River, 11 ottobre 1908.

Secondo il SoNDER (Algae Austral. hactenus cognitae pag. 105) questa Draparnaldia fu rinvenuta nella regione della Victoria e della Nuova Galles del Sud nella varietà acuta. Tanto la forma tipica che la varietà ora menzionata furono raccolte anche nella Nuova Zelanda (cfr. NoRDstedt, Freshwater Algae collected by D.r S. Berggren in New Zealand and Australia pag. 14 ; Stockholm 1888). L' esemplare dell' Avon River è assai grande, ma deteriorato e scolorito.

\section{Fam. ULOTRICHIACEAE (Kuetz.) Wille}

\section{Conferva (L.) Lagerh.}

Conferva Martialis Hanst. Ueber gepanzerte Confervafäden (1878) pag. 78 ; De Toni Sylloge Algarum I, pag. 220.

Psichohormii sp. plures Kuetz. Species Algarum (1849) pag. 374, Tab. Phyc. III (1853) tab. 48, fig. II-V.

Hab. Nuova Zelanda: Rotorua, geyser.

La Conferva martialis di HANSTEIN sembra piuttosto essere una specie biologica che non una genuina specie sistematica; parecchie Alghe filamentose mostrano la tendenza, in ambienti appropriati, di rivestirsi di straterelli o di cingoli ocracei e di assumere lo stato che taluni autori preferiscono chiamare $\mathrm{col}$ nome Kuetzingiano di Psichohormium (cfr. Galdukov, Ueber die Eisenalgen Conferva und die Eisenorganismen des Süsswassers im allgemeinen; Ber. der deutschen botan. Gesellsch. XXIII, 1905, pag. 250-253).

La Conferva martialis Hanst. rappresenta forme da ascriversi ai generi Conferva (Tribonema) o Microspora. Gli esemplari distribuiti dal KuETZING col nome di Conferva rufescens (Algarum aquae dulcis Germanicarum Decas XV n. 149; Halis Saxonum 1836) riferibili allo stato di Psichohorminu presentano tutto 1' aspetto della Conferva bombycina, fatta astrazione dalle incrostazioni ferruginose. Gli esemplari, da noi esaminati nelle collezioni essiccate di Conferva (Psichohormium) Naveana Rabenh. Algen Europa's n. 2111, Psichohormium inaequale Kuetz. in Rabenh. n. 1968, Conferva (Psichohormium) globulifera Kuetz. in Rabenh. n. 2094, Conferva martialis Hanst. in Hauck \& Richter Phykotheka 
Universalis n. 491 ci lasciarono l'impressione trattarsi di specie riferibili piuttosto alle Microspora che alle Conferva. Sull' argomento controverso dei Psichohormium in riguardo alla relazione d' essi con le Conferve si può consultare W. Heering, Die Süsswasseralgen Schleswig-Holstein I, Heterokontae, pag. 129-132.

\section{Microspora Thur.}

Microspora Stagnorum (Kuetz.) Lagerh. Zur Entwick. einiger Confervaceen (1887) pag. 417; De Toni Sylloge Algarum I, pag. 229.

Conferva stagnorum Kuetz. Tab. Phyc. II (1852) tab. 87, fig. $d$.

Hab. Nuova Zelanda: Rotorua, geyser, marzo 1909; Ohinemuthu, sorgenti calde, marzo 1909.

Questa Microspora venne già indicata per Ohaeawai nella Nuova Zelanda dal Nordstedt.

\section{Fam. CLADOPHORACEAE (Hass.) Wittr.}

\section{Urospora Aresch.}

Urospora PENICILliformis (Roth.) Aresch. Observationes Phycologicae I (1866) pag. 15; De Toni Sylloge Algarum I, pag. 232.

Hormotrichi sp. plures Kuetz. Tab. Phyc. III (1853) tab. 63-65.

Hab. Nuova Zelanda: Lyttleton, 6 febbraio 1909.

Questa specie viene per la prima volta segnalata nella Nuova Zelanda; essa, che ha ampia distribuzione geografica, fu egregiamente illustrata con materiali provenienti dalle regioni antartiche sud-americane dal GaIN nelI'opera La Flore algologique des régions antarctiques et subantarctiques (Deuxième. Expédition française (1908-10) pag. 32-35, fig. 36-42.

\section{Rhizoclonium Kuetz.}

Rhizoclonium hieroglyphicum (Ag.) Kuetz. Phycologia generalis (1843) pag. 205 ; De Toni Sylloge Algarum I, pag. 281 ; Stockmayer Ueber die Algengattung Rhizoclonium (1890) pag. 578, forma cellulis majoribus.

Hab. Victoria : Daylesford, 5 decembre 1908.

Il Moebius (Australische Sïsswasseralgen pag. 327) indicò il Rhizoclonium hieroglyphicum (Ag.) Kuetz. per Burpengary, Queensland, indicazione riportata poscia da F. M. BaIley Contribution to the Queensland Flora pag. 25; Brisbane 1895.

Var. CRISPum (Kuetz.) Rabenh. Flora Europ. Algarum III (1868) pag. 330 ; De Toni op. cit. pag. 282.

Hab. Tasmania : torrente alla "Cascade » di Hobart, 22 gennaio 1909.

Var. Waikitense Hauck in Nordstedt Freshw. Alg. of N. Zeal. and Australia (1888) pag. 17; De Toni op. cit. pag. 282.

Hab. Nuova Zelanda: Wakarevareva, fonte d' acqua caldissima, con molte Diatomee, in particolare Eunotia Eruca, marzo 1909. 
Clad ophora Kuetz.

Cladophora Bainesi F. Muell. et Harv. Alg. Austral. exsicc. n. 579; Phyc. Australica II (1859) tab. 112; Hooker Fl. Tasman. II (1860) pag. 340; De Toni Sylloge Algarum I, pag. 319; Lucas Supplement. List pag. 166.

Hab. Tasmania: Baia di Latrobe.

La specie era stata raccolta a Port Phillip, Australia (Baines, Harvey) e a Georgetown, Tasmania (Gunn, Harvey); per queste due stazioni trovasi segnalata anche dal Sonder (Algae Austral. hactemus cognitae n. 1009). Bellissimi esemplari di Port Phillip, cui furono paragonati i nostri, vennero distribuiti dal Lenormand.

Cladophora callicoma Kuetz. Phyc. gener. (1843) pag. 257, Tab. Phyc. IV (1854) tab. 37, fig. 1; De Toni Sylloge Algarum I, pag. 297.

Hab. Australia occid.: Avon River, 4 ottobre 1908.

Il Sonder (Algae Austral. haclenus cognitae pag. 106) segnala la Cladophora callicoma Kuetz. per l' Australia meridionale, ciò che viene riportato dal Lucas (Supplementary List pag. 166).

Cladophora crispata (Roth) Kuetz. Phyc. gener. (1843) pag. 246, Species (1849) pag. 407, Tab. Phyc. IV (1854) tab. 40, fig. 1 ; De Toni Sylloge Algarum I, pag. 291.

Hab. Nuova Zelanda; Rotorua; Ohinemuthu, sorgenti calde, con Anomoeoneis sphaerophora, marzo 1909.

Il Nordstedt (Freshw. Algae of N. Zeal. and Australia 1888, pag. 16) ricorda per il Waikato River una forma waikatensis Hauck di questa specie, pubblicata inoltre al n. 943 delle Algae exsiccatae di WitTrock e NordsTEDT raccolta dal D.r S. BeRGgRen (n. 319); egli non indica nessuna Cladophora per le sorgenti calde di Ohinemuthu, donde pure ricevette materiali.

\section{Fam. VALONIACEAE (Zanard.) Wille}

\section{Siphonocladus Schmitz}

Siphonocladus valonioides (Sond.) Reinb. Meeresalgen Invest. Street (1899) pag. 41, n. 10.

Cladophora valonioides Sond. in Botanische Zeitung 1845, pag. 49; Kuetz. Species (1849) pag. 391, Tab. Phyc. III (1853) tab. 85, fig. I; Harv. Phyc. Australica II (1859) tab. 78; De Toni Sylloge Algarum I, pag. 308; Lucas Supplement. List pag. 166.

Hab. Australia occid. : Baia di Geroldton, settembre 1908; Tasmania : Burmie.

Di questa specie, ritenuta dapprima una Cladophora vicariante della Cladophora laetevirens Kuetz. europea, W. H. HARveY aveva già acutamente con le parole «its swollen, blunt cells reminds us of a Valonia; but the resemblance is one of analogy only " avvertito qualche rapporto strutturale con le Valonia, ciò che giustifica il successivo riferimento fattone dal REINBoLD al genere Siphonocladus, per la particolare maniera di origine delle proliferazioni cellulari. Il Siphonocladus valonioides (Sond.) Reinb. è abbastanza comune nel- 
1' Oceano Pacifico; fu segnalato dapprima allo Swan River (PreIsS) e di questa località, dove lo raccolsero poi G. Clifton, Harvey ed altri distribuito da J. AgardH nelle Algae Muellerianae; Harvey lo trovò anche nel King George's Sound. Il Sonder (Algae Austral. hactenus cognitae pag. 40, n. 1015) lo segnala per Fremantle e Champion Bay; nell'erbario ZaNARDINI trovansi esemplari con la semplice annotazione «Australia»; il REINBoLD ebbe la specie dallo Investigator Street. Il Siphonocladus valonioides (Sond.) Reinb. non manca neppure alla flora della Nuova Zelanda stando a una determinazione fatta dal KJELLMAN su materiale comunicato a R. J. Harvey-Gibson dal prof. Jeffrey Parker (cfr. Harvey Gibson, On some marinae Algae from New Zealand 1893, pag. 1). Gli esemplari della Baia di Geroldton sono scoloriti e deteriorati, non così quello di Burmie epifita sopra un frammento di Fucacea.

\section{Fam. GoMonTiACEAE Born. et Flah.}

Gomontia Born. et Flah.

Gomontia polyrhiza (Lagerh.) Born. et Flah. Sur deux nouv. genres d' Algues perforantes (1888) pag. 164; De Toni Sylloge Algarum I, pag. 389.

Codiolum polyrhizum Lagerh. Ett Bidrag till Känn. om Slägtet Codiolum (1885) pag. 22, tab. 28, fig. 1-6.

Hab. Nuova Zelanda: Port Charmers, acque basse.

Cresce questa specie su vecchie conchiglie abbandonate di Lamellibranchi e altri Molluschi e può ritenersi cosmopolita.

Ordo II. Siphoneae (Grev.)

\section{Fam. BRYOPSIDACEAE (Bory) Thur.}

Bry opsis Lamour.

Bryopsis plumosa (Huds.) Ag. Species Algarum I (1822) pag. 448; De Toni Sylloge Algarum I, pag. 431; Lucas Supplement. List pag. 168.

Hab. Australia occid.: Baia di Geroldton, settembre 1908.

Il Sonder (Algae Austral. hactenus cognitae n. 967) indica la Bryopsis plumosa Ag. per l'Australia e la Tasmania e ne distribuì taluni esemplari provenienti da Port Phillip; il Reinbold ne confermò la presenza lungo i lidi della Nuova Olanda meridionale. Qualcuno degli esemplari comunicatici dal rev. G. CAPRA ricorda la Bryopsis australis Sond. quale trovasi figurata in KuETzing Tabulae Phycologicae VI (1856) tab. 81, fig. I. La specie fu distribuita pure, delle isole Hawaii, da J. E. Tilden (Amer. Algae n. 453).

\section{Fam. CAULerPaCEAE Reichb.}

Caulerpa Lamour.

Caulerpa cactoides (Turn.) C. Ag. Species Algarum I (1822) pag. 439; Harv. Phyc. Australica I (1858) tab. 26 ; De Toni Sylloge Algarum I, pag. 485; Reinke Ueber Caulerpa (1899) pag. 43; Weber van Bosse Monogr. des Caulerp. (1898) pag. 390 ; Lucas Supplement. List pag. 170. 
Hab. Australia merid.: Victor Harbour, 12 novembre 1908.

La Caulerpa cactoides (Turn.) Ag. è abbastanza frequente lungo i lidi del Continente Australiano giusta le indicazioni fornite da Harvey, Sonder, Reinbold e altri ficologi. G. Murray (On new Species of Caulerpa pag. 211, tab. 52, fig. 8) descrisse una varietà gracilis della Nuova Olanda, riferita senz' altro dalla WEBER van Bosse alla forma tipica. I nostri esemplari corrispondono esattamente con quelli distribuiti da J. AGARDH nelle Algae Muellerianae.

Caulerpa hypnoides (R. Br.) C. Ag. Species Algarum I (1822) pag. 443; Harv. Phyc. Australica II (1859) tab. 84 ; De Toni Sylloge Algarum I, pag. 470 ; Reinke Ueber Caulerpa (1899) pag. 30, fig. 46-49; Weber van Bosse Monogr. des Caulerp. (1898) pag. 343, tab. 29; Lucas Supplement. List pag. 169.

Chauvinia hypnoides Kuetz. Tab. Phyc. VII (1857) tab. 18, fig. 1.

Hab. Tasmania: Baia di Latrobe, 9 gennaio 1909; Burmie, rocce battute dalle onde, 15 gennaio 1909 .

W. H. Harvey indica la specie comune per i lidi occidentali e meridionali dell' Australia, nonchè per la Tasmania e per la Nuova Zelanda; tale distribuzione geografica venne confermata e accresciuta per nuovi reperti di località da Sonder, Reinbold, Cotton, A. e E. S. Gepp e altri botanici. La Caulerpa hypnoides ( $\mathrm{R} . \mathrm{Br}$.) Ag. fu distribuita in bellissimi esemplari di Port Fairy nelle Algae Muellerianae.

Caulerpa Muelleri Sond. in Linnaea vol. 25 (1852) pag. 661 ; Harv. Phyc. Australica I (1858) tab. 2; De Toni Sylloge Algarum I pag. 470; Lucas Supplement. List pag. 169.

Hab. Australia occid. : Baia di Geroldton, settembre 1908.

L' unico esemplare frammentario e privo di caulidio o surculo, comunicatoci dal rev. dott. G. CAPra, s' avvicina al tipo della Caulerpa hypnoides $(\mathrm{R}$. Br.) Ag. per avere talvolta i rametti forcellati al di sotto se vuolsi del tipo di detta Caulerpa ma spesso assai distintamente; così il carattere della doppia forcella apicale dei ramenti non è costante. Del resto giova osservare che la Caulerpa Muelleri Sond. secondo qualche autore è niente più che una semplice varietà o forma della Caulerpa hypnoides (R. Br.) Ag. ; l' esemplare distribuito da HARvEY nelle Algae Austral. exsicc. n. 551 è, secondo la Weber van Bosse (Monogr. des Caulerp. 1898, pag. 349) da ridurre a varietà di quest' ultima specie; dello stesso parere, circa la specie Sonderiana, si mostra il ReINke (Ueber Caulerpa, 1899, pag. 33, fig. 50-51); si potrebbe notare che la Caulerpa Muelleri Sond. presenta in generale un aspetto alquanto licopodioide; del resto il nostro esemplare, raffrontato con esemplari di Port Phillip distribuiti dal Grunow, vi corrisponde assai bene; fu raccolta per la prima volta a Rivoli Bay (Sonder).

\section{Fam. SPONGODIACEAE Lamonr.}

\section{Codium Stackh.}

Codium galeatum J. Ag. Till Algernes Systematik VIII (1886) pag. 42, tab. 1, fig. 1; De Toni Sylloge Algarum I, pag. 494 ; Lucas Supplement. List (1912) pag. 171. Icon: Tab. nostra X, fig. 4-6. 
Codinm tomentosum var. $\gamma$ australasicum Aresch. Phyceae novae (1854) pag. 42 partim.

Hab. Australia occid. : Baia di Geroldton, settembre 1908.

Questa specie, secondo J. AGARDH, vegeta lungo le coste occidentali e meridionali della Nuova Olanda, ivi raccolta da Ferdinando Mueller e da altri e occupa un posto vicino al Codium Muelleri Kuetz. del quale, con molta probabilità, costituisce una forma di maggiore sviluppo. Il LucAs indica il Codium galeatum J. Ag. anche per la regione austro-orientale della Nuova Olanda. Un solo grande esemplare, di oltre 30 centimetri di lunghezza, venne raccolto dal rev. G. CAPRA e divelto sopra il callo di adesione. Piccoli esemplari comunicati dal MuelLer a diversi algologi (Erb. MAzzA) recano soltanto l'indicazione «Australia .

Codium Muelleri Kuetz. Tab. Phyc. VI (1856) pag. 34, tab. 95, fig. II ; J. Ag. Till Algernes Systematik VIII (1886) pag. 42; De Toni Sylloge Algarum I, pag. 493 ; Lucas Supplement. List p. 171.

Codium tomentosum var. $\gamma$ australasicum Aresch. Phyceae novae (1854) pag. 42 partim.

Codium tomentosum auct. nonnull. partim (quoad specimina australasica); Laing Revised List of New Zealand Seaweeds I (1899) pag. 59, n. 11.

Hab. Tasmania: Baia di Latrobe, 9 gennaio 1909; Nuova Zelanda: Port Charmers, acque basse, 30 gennaio 1909.

Questa specie sostituisce nell' Oceano Pacifico il Codium tomentosum (Huds.) Stackh. tanto comune nell' Oceano Atlantico e nel Mare Mediterraneo; fu raccolta dapprima all' isola Lefebre da F. Mueller pel 1852 secondo il KuETzING, al quale, sotto il nome di Codium tomentosum, la comunicò il SoNDER; a detta località, quest' ultimo botanico (Algae Austral. hactenus cognitae n. 958) aggiunse l' indicazione Champion Bay.

Il Codium Muelleri Kuetz. è largamente diffuso nelle coste australi, austrooccidentali e austro-meridionali della Nuova Olanda, nonchè in quelle della vicina Tasmania. Se a questa specie vada riferito il Codium tomentosum enumerato tra le Alghe neozelandesi spedite dal prof. Jeffrey-Parker a Harvey-Gibson (On some marine Algae from New Zealand 1893, pag. 1) non possiamo affermare mancandoci l' esame diretto del materiale quivi raccolto; lo stesso dicasi del Codium tomentosum elencato dai coniugi GEPP (Marine Algae from the Kermadecs 1911, pag. 20) tra le Alghe delle isole Kermadecs (Meyer Island.). Si vegga anche LAING (Subantarct. Isl. of New Zealand 1909, pag. 496) il quale usa Codium tomentosum in un significato di limite assai generale, riferendo però i suoi esemplari a Codium tomentosum $\beta$ Novae-Zelandiae.

Codium mucronatum J. Ag. Till Algernes Systematik VIII (1886) pag. 43 De Toni Sylloge Algarum I, pag. 494; Lucas Supplement. List (1912) pag. 171; A. M. Hurd in Public. Puget Sound Marine Station I (1916) pag. 109, c. tab.; Hylmö Zur Kenntn. der antarkt. und subantarkt. Meeresalgen III (1919) pag. 14, fig. 28.

Var. tasmanicum J. Ag. op. cit. pag. 44, tab. I, fig. 2.

Hab. Tasmania: Baia di Latrobe, 9 gennaio 1909.

Questa varietà fu indicata da J. AGARDH come propria delle coste della Ta smania (R. Gunn, Goodwin) e della Nuova Olanda occidentale e meridionale; 
secondo il Lucas essa vegeta anche sui lidi orientali; il Reinbold (Ergebnisse einer Reise nach dem Pacific 1899, pag. 288) enumera il Codium mucronatum J. Ag. tra le Alghe delle isole Chatham. Bellissimi esemplari di questa specie, nella forma tipica, vennero distribuiti da K. OKamURa provenienti da Enoshima (Algae Japon. exsicc. n. 50).

\section{Fam. PhyllosiphonaCEAE Frank}

Ostreobium Born. et Flah.

Ostreobium Quekettin Born. et Flah. Sur quelques plantes viv. dans le test calcaire des Mollusques (1889) pag. 15, tab. 9, fig. 5-8; Wille in Engler-Prantl Die natürl. Pflanzenfam. Nachtr. I, 2 (1911) pag. 133.

Hab. Australia occ. : Baia di Geroldton, settembre 1908.

11 Wille (op. cit.) indica per il genere Ostreobium una seconda specie, $O$. Reineckei Born. endozoico nei polipai delle isole Samoa e della Nuova Zelanda. Che l' Ostreobium Quekettii Born. et Flah. costituisca una condizione particolare della Conchocelis rosea Batters, come sostenne G. Nadson (Die perforierenden (Kalkbohrenden) Algen; Scripta Botanica Horti Univ. Petropol. Fasc. XVIII, 1900) fu posto in dubbio da osservatori più recenti, tra altri, nel 1910, dal RoSENvinge (cfr. De Toni G. B., Alcune considerazioni sulla flora marina pag. 65; Padova 1916).

MYXOPHYCEAE (Wallr.) Stiz.

I. Ordo Coccogoneae (Thur.) Kirchn.

Fam. CHRO0COCCACEAE Naeg.

Chroococcus Naeg.

Chroococcus cohaerens (Bréb.) Naeg. Gattungen einzell. Algen (1849) pag. 46; Forti, Myxophyceae in De Toni Sylloge Algarum V (1907) pag. 21.

Pleurococcus cohaerens Bréb. in Menegh. Monographia Nostochin. ital. (1846) pag. 35 , tab. 4 , fig. 3 .

Hab. Nuova Zelanda: Rotorua, Ohinemuthu, sorgenti termali, marzo 1909.

Il Nordstedt (Freshw. Algae of New Zealand and Australia 1888, pag. 77) segnala con dubbio il Chroococcus turgidus (Bréb.) Naeg. a Orakeikorako e altre specie (senza nome specifico) in diversi luoghi non indicati. Come la maggior parte delle Missoficee questa specie si dimostra cosmopolita o quasi. Essa venne infatti descritta dal NAEgeli al n. 446 delle Algen Europa's del RABENHORST su materiali raccolti nelle serre calde dell' Orto botanico di Friburgo in Brisgovia ma è nota e fu raccolta anche nelle acque termali di Ungheria, Russia, Italia, India e America settentrionale. 


\section{Fam. CHAMAESIPHONACEAE Borzì}

Hyella Born. et Flah.

Hyella caespitosa Born. et Flah. Note sur deux nouv. genres d'Algues perforantes (1888), pag. 163; Forti Myxophyceae in De Toni Sylloge Algarum $\mathrm{V}$, pag. 125.

Hab. Australia occid.: Baia di Geroldton, settembre 1908.

$\mathrm{Su}$ frammenti irriconoscibili di conchiglie corrose, insieme con Ostreobium Quekettii Born. et Flah. Questa Camesifonacea sembra doversi inscrivere anch' essa tra le specie cosmopolite. Si conosce infatti raccolta nei più lontani paesi, dalle coste del Mare Ligustico a Trayas (LAGERHEIm in WitTrock et NoRdSTEDT Alg. exsicc. n. 1199) all' Oceano atlantico settentrionale sulle coste del Maine (Coldins in Collins, Holden \& Setchell Phyc. Bor. Amer. n. 302) nonchè al Mare Caraibico a Colon in Columbia (Lagerheim in Wittrock et NordSTEDT Alg. exsicc. n. 1610) ; nè manca nell' Oceano Pacifico sui lidi della California, dove però il SETchell ritiene sia stata introdotta con le conchiglie di Ostrea virginiana (cfr. Phyc. Bor. Amer. LI).

\section{Xenococcus Thur.}

Xenococcus Schousboei Thur. in Born. et Thur. Notes algologiques II (1880) pag. 74, tab. 26, fig. 1-2; Forti Myxophyceae in De Toni Sylloge Algarum $\mathrm{V}$, pag. 133.

Dermocarpa Schousboei Born. in Batters Mar. Algae of Berwich-on-Tweed (1889) pag. 11, tab. 7, fig. 26.

Hab. Nuova Zelanda: Bluff, insieme a Lyngbya aestuarii Liebm., 25 gennaio 1909.

Xenococcus Schousboei Thur. ha spesso per matrice le Lyngbya ma fu talvolta riscontrato sopra altre Missoficee, su Cloroficee e Floridee filamentose.

Ordo Il. Hormogoneae (Thur.) Kirchn.

\section{Fam. OSCILLATORIACEAE (Gray) Gomont}

Phormidium Kuetz.

Phormidium laminosum (Ag.) Gomont in Journal de Botanique IV (1890) pag. 355, Monogr. des Oscillariées (1893) pag. 167, tab. 4, fig. 21-22; Forti Myxophyceae in De Toni Sylloge Algarum V, pag. 225.

Hab. Nuova Zelanda: Rotorua, Ohinemuthu, geyser, marzo 1909.

Il Nordstedt (Freshw. Alg. of New Zealand and Australia 1888, pag. 75 Oscillaria limosa Ag.) segnalò questa specie nelle acque termali a Tokano nella Nuova Zelanda (1); è una delle Alghe più copiosamente raccolte nelle sorgenti

(1) Notiamo per incidenza che il rev, dott. G. CAPRA raccolse, durante il suo viaggio, nelle Vasche di Salomone presso Aden (18 luglio 1909) il Phormidium Corium (Ag.) Gomont e il Phormidium Retzii (Ag.) Gomont. 
termali, nei geysers sotto forma di incrostazioni lucide, verdi-olivastre, scure, stratificate, più o meno screpolate e concrezionate di sostanza calcarea, macchiate di chiazze ocracee. Nel geyser si trova il Phormidium laminosum (Ag.) Gom. frammisto specialmente ad Alghe e Batterii ocracei, donde le diversità di colore dello strato.

Phormidium Retzil (Ag.) Gomont in Journal de Botanique IV (1890) pag. 355, Monogr. des Oscillariées (1893) pag. 195; Forti Myxophyceae in De Toni Sylloge Algarum V, pag. 241.

Hab. Australia occid. : Avon River, 11 ottobre 1908; Nuova Zelanda: Rotorua, Ohinemuthu, marzo 1909.

Secondo il Gomont questo Phormidium venne già raccolto nella Nuova Zelanda dal dott. S. Berggren. A Rotorua venne osservato frammisto al Phormidium laminosum (Ag.) Gom. in uno strato olivaceo-ceruleo; nell' Avon River fu raccolto in uno strato caratteristico sottile, fragile, verde-olivastro.

Phormidium uncinatum (Ag.) Gomont in Journal de Botanique IV (1890) pag. 355, Monogr. des Oscillariées (1893) pag. 204, tab. 5, fig. 21-22 ; Forti Myxophyceae in De Toni Sylloge Algarum V, pag. 250.

Hab. Australia merid. : Porto di Adelaide, sulle pareti della vaschetta d'acqua potabile nella « Jetty » del detto porto, novembre 1908.

E una specie cosmopolita, delle acque dolci e termali e del terreno umido.

$$
\text { Lyngbya C. Ag. }
$$

Lyngbya Aestuarir (Mert.) Liebm. Bemerkn. og Tilläg til Danske Algflora (1841) pag. 492 ; Born. et Thur. Notes algologiques II (1880) pag. 132, tab. 32 ; Forti Myxophyceae in De Toni Sylloge Algarum V, pag. 262.

Hab. Nuova Zelanda: Bluff, su Confervoideae, 25 gennaio 1909.

Una specie innominata di Lyngbya trovasi ricordata per Auckland e Bay of Island tra le raccolte fatte dal dott. S. Berggren (cfr. J. Agardh, De Algis Novae Zelandiae marinis pag. 1; LAING Revised List of New Zealand Seaweeds pag. 58, n. 3). La Lyngbya ferruginea C. Ag. di Port Fairy e della Tasmania enumerata dal SONDER (Alg. Austral. hactenus cognitae pag. 42, n. 1046) va riferita alla Lyngbya aestuarii (Mert.) Liebm., specie abbastanza frequente nell' Oceano Pacifico sulle coste della Nuova Olanda, della Papuasia e di alcune isole dell' Oceania.

\section{Sym ploca Kuetz.}

Srmploca Muscorum (Ag.) Gomont in Journal de Botanique IV (1890) pag. 354, Monogr. des Oscillariées (1893) pag. 110, tab. 2, fig. 9; Forti Myxophyceae in De Toni Sylloge Algarum V, pag. 303.

Hab. Nuova Zelanda: Rotorua, Ohinemuthu, sorgenti calde, marzo 1909.

L' aspetto dello strato è abbastanza caratteristico quantunque l' Alga sia per lo più frammischiata alla Hypheothrix calcicola (Ag.) Rabenh, incrostata sui Muschi. I fascicoli dei filamenti sono fosco-eruginei e costituiscono una superficie zigrinata. 


\section{Hypheothrix Kuetz.}

Hypheothrix calcicola (Ag.) Rabenh. Flora Europaea Algar. II (1865) pag. 78; Forti Myxophyceae in De Toni Sylloge Algarum V, pag. 327; Tilden Minnesota Algae I (1910) pag. 139, tab. 6, fig. 1-4.

Schizothrix calcicola Gomont Monogr. des Oscillariées (1893) pag. 307, tab. 8, fig. 1-3; Tilden Om some Alg. Stalactites (1897) pag. 197, tab. 8, fig. 3-4.

Hab. Nuova Zelanda: Rotorua, Ohinemuthu, sorgenti calde, marzo 1909.

Forma strati sottili pellicolari prasino-sporchi o verdognoli, piuttosto fragili, che si stendono sulla sabbia siliceo-argillosa. Il Beccari raccolse questa specie a Borneo.

\section{Fam. NosTochaCEAE (C. Ag.)}

\section{Nostoc Vauch.}

Nostoc entophytum Born. et Flah. Revis. des Nostoc. hétéroc. IV (1888) pag. 190; Forti Myxophyceae in De Toni Sylloge Algarum V, pag. 389.

Nostoc tenuissimum Born. et Thur. Notes algologiques (1880) pag. 86 et 130, tab. 21 (non Rabenhorst nec Wolle).

Hab. Nuova Zelanda: Bluff, insieme a Lyngbya aestuarii (Mart.) Liebm., 25 gennaio 1909.

Questa specie, della sezione Paludosae Born. et Flah., è affine al Nostoc Sergiamum Borzì della Papuasia ed è una delle pochissime forme del genere, la quale si sviluppi nelle acque salmastre o marine.

\section{Anabaena Bory}

Anabaena torulosa (Carm.) Lagerh. Bidrag till Sveriges Algflora (1883) pag. 47; Forti Myxophyceae in De Toni Sylloge Algarum V, pag. 455 [cum ampla synonymia]; Tilden Minnesota Algae I (1910) pag. 193, tab. 9, fig. 19.

Hab. Nuova Zelanda: Bluff, insieme a Lyngbya aestuarii (Mert.) Liebm., 25 gennaio 1909.

Per la Nuova Zelanda, nelle acque dolci, era nota finora una sola varietà Novae Zelandiae Lemmerm. (1899) della Anabaena oscillarioides Bory. Le Anabaena torulosa (Carm.) Lagerh. e Anab. baltica J. Schm. sono le sole specie che vegetano nelle acque marine.

\section{Fam. STigONEMACEAE (Hass.) Kirehn.}

\section{Hapalosiphon Naeg.}

Hapalosiphon laminosus (Kuetz.) Hansg. Ueber den Polymorphismus der Algen (1885) pag. 48; Born. et Flah. Revis. Nostoc. hétéroc. III (1887) pag. 55; Buscalioni in Malpighia vol. IX (1895) pag. 158, tab. 10 e vol. XXVIII (1917) pag. 83; Forti Myxophyceae in De Toni Sylloge Algarum V, pag. 565; Tilden Minnesota Algae I (1910) pag. 240, tab. 14, fig. 14-15. 
Anabaena bullosa Menegh. Consp. (1837) pag. 8; Kuetz. Tab. Phyc. I (1849) tab. 93, fig. II ; Grunow Alg. Novara (1867) pag. 31.

Hab. Nuova Zelanda: Rotorua, geyser, marzo 1909.

Questa specie venne già notata dal GRunow per la Nuova Zelanda nella sorgente calda * Te Rapa \& al lago Taupo, dove la raccolse HochstetTer. Negli esemplari raccolti dal rev. G. CAPRA si trova associata a Microspora stagnorum (Kuetz.) Lagerh. e a una Diatomea, Nitzschia scalpelliformis var. nana. Tutti e tre sono elementi cosmopoliti.

Fam. RivUlariaceae (Menegh.) Kirchn.

\section{Calothrix C. Ag.}

Calothrix aeruginea (Kuetz.) Thur. Essai classif. Nostoch. (1875) pag. 382 ; Born. et Thur. Notes algologiques (1880) pag. 157, tab. 37 ; Born. et Flah. Revis. Nostoc. hétéroc. I (1886) pag. 358; Forti Myxophyceae in De Toni Sylloge Algarum V, pag. 612 ; Tilden Minnesota Algae 1 (1910) pag. 261, tab. 17, fig. 1.

Hab. Tasmania: fiume Tamar, sulle roccie alternativamente irrorate da acqua dolce e salsa, epifita su Caloglossa Beccarii Zanard., 12 gennaio 1909.

La specie è già conosciuta per l' Oceania, raccolta a Tongatabu (GRunow), a Port Denison (Amalia Dietrich), a Capo York e altri siti della Nuova Olanda (Sonder); i coniugi Gepp (Some mar. Algae from New South Wales 1906, pag. 249) citano la Calothrix aeruginea (Kuetz.) Thur. raccolta nel 1903 da A. H. S. LucAs a Barwon Heads, Victoria. Si presenta in esemplari veramente caratteristici.

Calothrix confervicola (Roth) Ag. Systema Algarum (1824) pag. 70; Born. et Thur. Notes algologiques (1876) pag. 8, tab. 3; Born. et Flah. Revis. Nostoc. hetéroc. I (1886) pag. 349; Forti Myxophyeae in De Toni Sylloge Algarum V, pag. 606; Tilden Minnesota Algae I (1910) pag. 256, tab. 16, fig. 6-8.

Hab. Nuova Zelanda: Bluff, su Ectocarpus e Stilophora, 25 gennaio 1909.

Per l'Australia senza indicazioni precise di località la Calothrix confervicola (Roth) Ag. è enumerata da HARveY (Phyc. Australica Syn. pag. LXII, n. 794); il SONDER (Algae Austral. hactenus cognitae pag. 106) la segnala per la Victoria, la regione occidentale e meridionale della Nuova Olanda; l' Askenasy ne avvertì l' esistenza all' isola Dick Hartog; il Schauinsland, secondo il Lemmermann, la raccolse a Laysan delle Hawaii.

Calothrix parietina (Naeg.) Thur. Essai Classif. Nostoch. (1875) pag. 381 ; Born. et Flah. Revis. Nostoc. hétéroc. I (1886) pag. 366; Forti Myxophyceae in De Toni Sylloge Algarum V, pag. 621; Tilden Minnesota Algae I (1910) pag. 269, tab. 18, fig. 12 .

Hab Tasmania: fiume Tamar, sui legnami della sponda orientale

A quanto ci consta, la specie resulta nuova per la Tasmania; nell' Oceania finora venne segnalata per le isole Hawaii (Нітскоск) e per la Nuova Zelanda (NORDSTEDT). 


\title{
BACILLARIEAE Nitzsch
}

\section{Ordo I. Immoвiles ( $\left.{ }^{(}\right)$}

\section{Fan. M E L O S I R A C E A E (Kuetz.)}

\author{
Melosira C. Ag.
}

Melosira Crenulata Kuetz. Kieselschal. Bacillar. (1844) pag. 35, tab. 2, fig. 8; Van Heurck Synopsis (1885) pag. 199, tab. 88.

Var. Ambigua Grun. in V. H. op. cit. tab. 88, fig. 12-15; De Toni Sylloge Algarum II (1894) pag. 1335.

Hab. Nuova Zelanda: Wakarevareva.

O. Mueller (Bacill. aus d. Nyassalande II, 1904, pag. 283) ritiene che questa varietà corrisponda forse ad una nuova specie che denomina e raffigura col nome di Melosira ambigua frequentissima nel plancton lacustre dei bacini della Germania settentrionale nonchè dei laghi Africani; fossile a Klieken e nella terra di Berlino. Questa specie dimostrerebbe anche fenomeni di mutazione alternandosi nello stesso filamento e persino nello stesso individuo delle valve a punteggiatura di diverso rilievo e grandezza. La varietà sopra indicata non è nuova per la Nuova Zelanda essendo stata trovata fossile a Karawara da M. PeraGALLO. La Melosira crenulata Kuetz. tipica sembra però alquanto più frequente in tutta l' Oceania, tanto fossile che vivente. In Australia venne segnalata fossile nel deposito di Talbot presso Melbourne ma più che altro pare frequente nella Nuova Zelanda nel lago di Rotoreja o Rotorua dove confluiscono acque termali caldissime e fossile a Pakaraka, Waitangi e ad Auckland, dove fu trovata mista alla var. valida Grun.

Melosira granulata (Ehr.) Ralfs in Pritchard Infus. (1861) pag. 820; De Toni Sylloge Algarum II, pag. 1334.

Var. angustissima O. Muell. Bacill. der Natronth. von El Kab (1899) pag. 316, tab. 12, fig. 28, Bacill. aus d. Nyassalande II (1904) pag. 282, tab. 4, fig. 12.

Hab. Nuova Zelanda: Rotorua, Ohinemuthu.

Il Mueller rinvenne questa varietà solamente in acque salmastre, così ad El Kab nell' alto Egitto come nel lago di Rukwa nella regione del Nyassa; è una varietà affine assai alla var. procera. Tempère e Peragallo (Diat. du Monde entier n. 273 e 918), segnalano la forma tipica nel lago di Rotorua e fossile a Wangarei nella Nuova Zelanda.

Var. Decussata (Ehr.) Grun. in V. H. Synopsis tab. 87, fig. 17.

Melosira decussata Kuetz. Bacill. (1844) pag. 56, tab. 3, fig. 17, 1-6; De Toni Sylloge Algarum II, pag. 1344.

(1) Seguiamo l' ordinamento proposto da uno di noi in un lavoro di sistematica diatomologica; cfr. FORTI ACH., Contribuzioni diatomologiche XII. Metodo di classificazione delle Bacillariee Immobili fondate sull'affinità morfologica dei frustoli ed in relazione con l'evoluzione delle auxospore (Atti del Reale Istituto Veneto di scienze, lettere ed arti Tomo LXXI, parte seconda, pag. 677-731, Venezia 1912). Le idee fondamentali di questo ordinamento, tenuto conto anche della disposizone delle Bacillarieae in Centricae e Pennatae sostenuto da F. Schütt (I896) furono accolte da G. S. West (Algae; vol. I. pag. I22-I23; Cambridge, IgI6, University Press, $8^{\circ}$ ). 
Hab. Nuova Zelanda: Rotorua, Ohinemuthu.

L'EHRENBERg descrisse questa forma col nome di Gallionella decussata e la trovò fossile a Klieken presso il fiume Elba. La presente varietà del resto s'accosta molto alla forma tipica da cui non si distingue che per la misura e per la disposizione delle granulazioni. Una forma australiensis proveniente dal fiume Richmond (Australia meridionale) venne figurata dal Grunow (cfr. V. H. Synopsis tab. 87, fig. 13-16) e si differenzia dalla var. decussata soprattutto per la scultura assai più sottile.

Melosira lyrata (Ehr. ?) Kuetz. Species Algarum (1849) pag. 31 ; Grun. Diat. Franz-Josefs Land (1884) pag. 49, tab. 5, fig. 43; V. H. Atlas tab. 87, fig. 1-2;

De Toni Sylloge Algarum II, pag. 1333.

Hab. Nuova Zelanda: Wakarevareva.

Questa specie, dapprima descritta col nome di Gallionella lyrata dall' EHRENBERG su materiali provenienti dall' America settentrionale, venne poi riscontrata a Pudasjärvi e nella Terra di Francesco Giuseppe dal Grunow che ne diede una figura atta a meglio riconoscerla.

\section{Gallionella Bory}

Gallionella nummuloides Bory Dict. class. (1825); De Toni Sylloge Algarum II, pag. 1331.

Melosira nummuloides Ag. Syst. (1924) pag. 8; Kuetz. Bacillarien (1844), tab. 3 III, fig. 1.2 ; V. H. Synopsis tab. 85, fig. 1-2.

Hab. Australia occid. : Baia di Geroldton; Nuova Zelanda: Port Charmers.

Negli esemplari d' Australia osservammo megafrustoli del diametro di 60-70 $\mu$. Il Mereschkowsky (On Polynesian Diatoms 1902, pag. 17 e seg.) segnalò questa specie per le isole Samoa (Tempère e Peragallo Diat. du Monde entier n. 648), per Tahiti, per le is. Sandwich; così pure venne rinvenuta fossile a Waitangi nella Nuova Zelanda.

\section{Lysigonium Link.}

Lysigonium varians De Toni Alg. Abyss. (1891) pag. 268, n. 18, Sylloge Algarum II, pag. 1329.

Melosira varians Ag. Conspectus diatom. (1830) pag. 64 ; V. H. Synopsis pag. 198, tab. 85, fig. 10-11, 14-15, Traité pag. 641, tab. 18, fig. 611.

Hab. Nuova Zelanda: Wakarevareva; Rotorua, geyser (con megafrustoli).

La specie venne già segnalata per la Nuova Zelanda nel distretto di Otago (cfr. W. Lauder Lindsay, On the Diatomaceae of Otago, New Zealand, 1864, pag. 133) e più di recente fossile a Waitangi.

\section{Stephanopyxis Ehr.}

Stephanopyxis TurRis (Grev.) Ralfs in Pritchard History of Infus. (1861) pag. 826 , tab. 5 , fig. 74 ; V. H. Synopsis tab. 88 ter, fig. 12 ; De Toni Sylloge Algarum II, pag. 1138. 
Creswellia Turris Grev. in Trans. R. Soc. of Edinburgh vol. XXI (1857) pag. 358, tab. 14, fig. 109 .

Hab. Australia occid.: Baia di Geroldton; Nuova Zelanda: Port Charmers.

La specie venne segnalata per la Nuova Zelanda nel deposito di Oamaru (Grove e Sturt) e ripetutamente distribuita nelle Diatomées du Monde entier di Tempére e Peragallo con provenienza diversa : di Jackson's Paddock (n. 1, 2), di Bain's Farm (n. 139-141), di Cormack's Top (n. 415-416) ecc. Vivente si conosce delle isole Thursday (n. 592-593) e dello Stretto di Malacca; il CAstraCANE (Diat. of Challenger pag. 88) la indicò per il Mare di Arafura. La grande variabilità della Stephanopyxis turris (Grev.) Ralfs venne già posta in evidenza dal Grunow (Diat. Franz-Josefs Land, 1884, pag. 35-37); la maggior parte delle specie fossili e delle poche tuttora viventi proposte come nuove meriterebbe una revisione su materiali autentici, revisione che apporterebbe fuor di dubbio una notevole riduzione nel numero delle specie genuine. Gli esemplari nostri si avvicinano alla forma poco convessa con piccolissime spine raffigurata da H. e M. Peragallo (Diat. mar. de France tab. 119, fig. 7).

Paralia Heib.

Paralia sulcata (Ehr.) Cleve, On Diatoms from the Arctic Sea (1873) pag. 7 ; Grun. Diat Franz-Josefs Land (1884) pag. 41, fig. 35 ; De Toni Sylloge Algarum II, pag. 1349.

Melosira sulcata Kuetz. Bacill. (1844) pag. 55, tab. 2, fig. 7; V. H. Traité pag. 444, tab. 19, fig. 624 .

Hab. Australia occid.: Baia di Geroldton; Australia merid.: Victor Harbour; Nuova Zelanda: Port Charmers, Bluff.

La specie non è rara nell' Oceano Pacifico; quanto alla Nuova Zelanda essa venne segnalata allo stato vivente nella Lyall's Bay, nello stretto di Foveaux e nell' isola Campbell da P. Petit (Dzat. Campb. et N. Zél. pag. 40); allo stato fossile è abbondante nel deposito di Oamaru (Grove e STURT); venne largamente distribuita nelle Diatomées du. monde entier di Tempére e Peragallo (Bain 's Farm, Forrester Rocks, Cormack's Top, William's Bluff etc.) e nella stessa collezione se ne hanno esemplari dello stretto di Torres (n. 946), di Port Stevens (398-399) e di Albany, Australia meridionale (n. 160); si ha pure delle Samoa (n. 647-648), delle is. Thursday (n. 561-562, 591-592), dell' is. Mc Donald (n. 634) ecc.

\section{Hyalodiscus Ehr.}

Hyalodiscus scoticus (Kuetz.) Grun. in Journ. R. Microscopical Soc. 1879, pag. 690, tab. 21, fig. 5; Van Heurck Synopsis tab. 84, fig. 15-18; De Toni Sylloge Algarum II, pag. 1366.

Hab. Australia merid.: Victor Harbour; Tasmania : Baia di Latrobe; Nuova Zelanda: Bluff.

Questa specie fu indicata dal Lemmermann per le Lagune di Chatham, dal Peragallo per le isole Samoa, dal Mereschoowsky per le Hawaii; essa, secondo l'opinione di alcuni, rappresenta una semplice forma di Hyalodiscus subtilıs Bail. (cfr. V. H. Traité 1899, pag. 449, tab. 35, fig. 917). Tempère e Pera- 
GaLlo la segnalano per le isole Campbell (Diat. du monde entier n. 357 : var. minor), per l' is. d' Amsterdam (n. 443) e anch' essi per le is. Samoa (n. 648).

Hyalodiscus stelliger Bail. Notes on new Species and Localities of microsc. organisms (1853) pag. 10; V. H. Synopsis pag. 213, tab. 84, fig. 1-2, Traité pag. 449, tab. 22, fig. 650; De Toni Sylloge Algarum II, pag. 1367.

Podosira maculata W. Sm. Brit. Diat. II (1856) pag. 54, tab. 49, fig. 328.

Hab. Australia occid. : Baia di Geroldton; Nuova Zelanda: Port Charmers.

Non è specie nuova per l' Oceano Pacifico, essendo già stata segnalata dal Petit per la Nuova Zelanda e l' is. Campbell e dal Peragallo per le isole Samoa e Salomone; fu distribuita da Tempère e Peragallo nelle Diatomées $d u$ Monde entier da Albany, Stretto del Re Giorgio, Nuove Ebridi, is. Thursday e Samoa, Stretto di Torres e altre località oceaniche.

Hyalodiscus subtilis Bail. Notes on new Species and Localities of microsc. Organisms (1853) pag. 10, fig. 12; V. H. Traité pag. 449; De Toni Sylloge Algarum II, pag. 1366.

Hab. Australia occid.: Baia di Geroldton; Australia merid.: Victor Harbour; Tasmania: Baia di Latrobe, Burmie; Nuova Zelanda: Bluff.

La specie, che si trova anche allo stato fossile nel deposito di Oamaru (Grove, Sturt) fu indicata per la Nuova Zelanda dal Lauder e dal Petit, per le isole Sandwich dal Mereschkowsky; fu distribuita da alcune località oceaniche e più propriamente dall' Australia da Tempère e Peragallo nelle Diatomérs du monde entier.

\section{Fam. COSCINODISCACEAE (Kuetz).}

\section{Coscinodiscus Ehr.}

Coscinodiscus lineatus Ehr. in Abhandl. Berl. Akad. 1838, pag. 129, Mikrogeologie (1856) tab. 18, fig. 33; V. H. Synopsis pag. 217, tab. 131, fig. 3, Traité pag. 532, tab. 23, fig. 665; De Toni Sylloge Algarum II, pag. 1216.

Hab. Australia occid. : Baia di Geroldton; Australia merid.: Victor Harbour; Nuova Zelanda: Port Charmers.

Questo Coscinudiscus figura tra le specie enumerate dal Petit (Diat. Campbell et $N$. Zél. pag. 38) ; noi l'osservammo in un piccolo esemplare tra le raccolte della R. Nave "Liguria » di recente studiate (cfr. De Toni G. B. e ForTI A., Analisi microscopica di alcuni saggi di fitoplancton raccolti dalla $R . N$. - Liguria > pag. 15, Venezia 1916); il Coscinodiscus lineatus Ehr. fu distribuito da Tempere e Peragallo in più saggi delle loro Diatomées du monde entier, tra altro dello Stretto di Torres (n. 946), di Port Stevens (n. 398-399), delle is. Thursday (n. 561-562, 592-593), del porto di Oamaru (n. 774), da pesche fatte a $77^{\circ} 7$ lat. S. e $166^{\circ}$ long. E. sugli Idrarii raccolti dalla spedizione Scort al polo antartico. La specie fu pure indicata nel fitoplancton dell' Antartico in alcune località dal Mangin (Phytoplancton de l'antarctique 1915 pag. 5 ecc.) e nei sondaggi dal Van Heurck (Diat. Voy. du S. Y. Belgica 1909 pag. 48). 
Coscinodiscus nitidus Greg. On new Forms of Mar. Diat. (1857) pag. 499, n. 45, tab. 10, fig. 45; V. H. Traité pag. 532, tab. 23, fig. 667; De Toni Sylloge Algarum II, pag. 1221

Hab. Tasmania: Burmie.

Quanto all' Oceania il Coscinodiscus nitidus Greg. venne finora indicato per lo Stretto di Foveaux (Petit), per le is. Samoa e Hawaii (Peragallo), per Tahiti (Mereschkowsky), per la Nuova Caledonia (Kinker), per le Nuove Ebridi e per Albany (Tempère e Peragallo), per le is. Thursday, per lo stretto di Malacca e le coste della Nuova Zelanda (Tempère e Peragallo), per le Filippine a Manilla (Greville) ecc.; fossile fu scoperto nel deposito neo-zelandese di Oamaru (Grove e Sturt).

Coscinodiscus Perforatus Ehr. in Berichte Berl. Akad. 1844, pag. 78, Mikrogeologie (1856) tab. 18, fig. 46; A. Schmidt Atlas (1878), tab. 64, fig. 12-14; De Toni Sylloge Algarum II, pag. 1283.

$H a b$. Australia occid. : Baia di Geroldton.

Questa specie fu distribuita per l' isola d'Amsterdam da Tempere e PerAgallo (Diatomées du monde entier n. 443) e fossile di Oamaru nella Nuova Zelanda (n. 810-22).

Coscinodiscus Sol Wall. On the siliceous Organisms (1860) pag. 38, tab. 2, fig. 1-2; De Toni Sylloge Algarum II, pag. 1212; De Toni e Forti Analisi microsc. fitoplancton "Liguria » (1916) pag. 11, tab. 2, fig. 4.

Hab. Nuova Zelanda: Port Charmers.

Il RatrRay (Revision of Coscinodiscus 1890, pag. 18) tra le stazioni di questa bellissima Diatomea indicò il mare australe dall' esame, compiuto dal WeIssFLOG, di individui di Salpa spinosa, ma trattasi d' una indicazione troppo vaga; la specie fu trovata nel mare intorno all' isola di Giava ( $\mathrm{O}^{\prime}$ Meara, Cleve e Moeller) e nel mare di Arafura (De Toni e Forti).

\section{Thalassiosira Cleve}

Thalassiosira Decipiens (Grun.) Joerg. Protist. Plankton (1905) pag. 96, tab. 6, fig. 3.

Coscinodiscus decipiens Grun. Diat. Franz-Josefs Land (1884) pag. 85; V. H. Synopsis tab. 91 fig. 10; De Toni Sylloge Algarum II, pag. 1211 (non A. Schmidt Atlas (1878) tab. 59, fig. 18-19).

Orthosira angulata Greg. in Trans. R. Soc. Edinb. 1857, pag. 498, tab. 10, fig. 43 e $43 b$.

Hab. Nuova Zelanda: Bluff.

A questa specie, secondo Joergensen, andrebbero riferite le forme ascritte dagli autori alla Thalassiosira gelatinosa Hensen e secondo il GRAN (Nordisches Plankton XIX, 17, fig. 10) il Coscinodiscus excentricus var. catenata Gran La Thalassiosira decipiens (Grun.) Joerg. ci risulta nuova per l' Oceania.

Stephanodiscus Ehr.

Stephanodiscus Novae-Zelandiae Cleve Om some new or little known Diatoms (1881) pag. 21, tab. 5, fig. 62 ; De Toni Sylloge Algarum II, pag. 1153. 
Hab. Nuova Zelanda: Rotorua, Ohinemuthu, Wakarevareva.

La specie fu dapprima comunicata al ÇLEVE dal D.r S. BERGgREN che la raccolse nelle acque dolci o leggermente salate del Lago Rotorua; noi, tra altri frustoli, ne osservammo di assai minuti, del diametro di $15 \mu$, che si accostano allo Stephanodiscus carconensis Grun. (cfr. in questo proposito anche la figura in VAN Heurck Synopsis tab. 95, fig. 12, nella quale si scorge chiara l' affinità di Stephanodiscus Novae-Zelandiae con Steph. carconensis). Le figure date da F. Fricke (in A. Schmidt Atlas (1901) tab. 225) rappresentano nelle fig. 33-35 esemplari recenti della Nuova Zelanda, nella fig. 36 un esemplare fossile dell'isola Chiloë, il che lascia supporre che lo stephanodiscus Novae-Zelandiae Cleve non debbasi considerare un endemismo della Nuova Zelanda, ma forse come una forma quivi accantonata e che ebbe un tempo una diffusione su molto maggiore area così da raggiungere le isole vicine al Continente Americano.

\section{Cyclotella Kuetz.}

Cyclotella stelligera Cleve et Grun. On some new or little known Diatoms (1881) pag. 22, tab. 5, fig. 63; V. H. Synopsis tab. 94, fig. 22-27; De Toni Sylloge Algarum II; pag. 1355.

Hab. Nuova Zelanda: Wakarevareva.

La specie era già conosciuta per la Nuova Zelanda, come dalla figura 27 della tavola del VAN HEURck sopra citata che rappresenta un esemplare del Lago Taupo; il Cleve figurò una valva proveniente dalle raccolte fatte da $\mathrm{S}$. BERGGREn nel lago Rotorua o Rotoreja; di quest' ultimo lago la Cyclotella stelligera $\mathrm{Cl}$. et $\mathrm{Gr}$. fu distribuita da Tempère e Peragallo al n. 273 delle Diatomées du monde entier.

\section{Fam. HeLIOPELTACEAE H. L. Smith.}

Actinoptychus Ehr.

Actinoptychus splendens (Ehr.) Ralfs in Pritchard Hist. of Infus. (1861) pag. 840 ; V. H. Synopsis tab. 119, fig. 1-2, 4, Traité pag. 497, tab. 22, fig. 649; De Toni Sylloge Algarum II, pag. 1385. fig. 18.

Actinophaenia splendens Shadb. in Micr. Journal 1860, pag. 94, tab. 6,

Hab. Nuova Zelanda: Bluff.

Il Petit (Diat. Campb. et N. Zél. pag. 40) indicò l'Actinoplychus splendens (Ehr.) Ralfs per la Lyall's Bay; trovasi anche fossile nel deposito di Oamaru (Grove e Sturt; Tempere e Peragallo Diat. du monde entier n. 810-822); da varii siti della Nuova Zelanda la specie venne distribuita da Tempère e PeraGALLo ed oltre a ciò da parecchie località dell' Oceano Pacifico nel quale risulta abbastanza frequente.

Actinoptychus undulatus (Bail.) Ralfs in Pritchard Hist. of Infus. (1861) pag. 839 , tab. 5 , fig. 88 ; V. H. Synopsis tab. 22 bis, fig. 14, tab. 122, fig. 1-3; De Toni Sylloge Algarum II, pag. 1372. 
Hab. Tasmania: Burmie ; Nuova Zelanda: Port Charmers.

Nella regione oceanica questa specie non è rara; il Petit la segnalò per la Nuova Zelanda (Lyall's Bay), Tempère e Peragallo (Diatomées du monde entier n. 357) distribuirono materiali provenienti da sondaggi fatti all' is. Campbell, nonchè da altre località (isole Caroline, Thursday, Mc Donald ecc.) ; il LAUder LindSay la indicò per Otago, il Peragallo per le is. Samoa, il Mereschkowsky per Tahiti; si spinge fino alle regioni antartiche (VAn Heurck, Mangin); trovasi pure allo stato fossile nel deposito di Oamaru (Grove e SturT) e di Bain 's Farm (Temperre e Peragallo).

Come fu da noi avvertito in altro lavoro (Analisi microsc. fitoplancton * Liguria » 1916, pag. 10) l' Actinoplychus undulatus (Bail.) Ralfs è una specie ubiquitaria e assai diffusa in tutti i mari attuali, dove si dimostra alquanto polimorfa, molto meno però di quanto avvenisse nel mare terziario nel quale, probabilmente, questa Diatomea e le sue congeneri affini raggiunsero il massimo della diffusione e della variabilità.

Actinoptychus vulgaris Schum. Die Preuss. Diatomeen (1867) pag. 64 ; De Toni Sylloge Algarum II, pag. 1384.

Actinoptychus sp. Ehr. Mikrogeologie (1856) tab. 18, fig. 22-30 et aliae.

$H a b$. Australia occid. : Baia di Geroldton.

A proposito di questo Actinoptychus possiamo ricordare che il Grunow ne istitui, su materiali provenienti dalla Nuova Olanda settentrionale, una varietà australis figurandola nel VAN HEURCK Synopsis tab. 121, fig. 8 (distribuita al n. 592 delle Diatomées du monde entier di Tempère e Peragallo dall'is. Thursday). Il tipo venne poi segnalato nella stessa collezione di Tempère e PeraGALLO insieme ad altre specie raccolte ad Albany Australia occid. (n. 160), nello Stretto di Torres (n. 946), delle is. Thursday (n. 561) e Samoa (n. 647-648), dello Stretto di Malacca (n. 622), mentre la varietà doljensis Pant. è segnalata per il mare sulle coste dell' is. di Amsterdam (n. 443); infine ai n. 139 e 250 i due autori danno la var. maculata Gr. et St. (Actinoptychus maculatus Gr. et St. in A. Schmid Atlas, tab. 132, fig. 18-20) fossile a Farresters Rock, a Bain's Farm e William's Bluff.

\section{Fam. EUPODISCACEAE (Kuetz.)}

\section{A uliscus (Ehr.) Bail.}

Auliscus caelatus Bail. in Smithsonian Contributions 1853, pag. 6, fig. 3-4 ; Grev. in Trans. Micr. Soc. 1863, pag. 44, tab. 2, fig. 7; De Toni Sylloge Algarum II, pag. 1049.

Hab. Australia occid. : Baia di Geroldton.

Oltre che fossile nel deposito di Oamaru (Grove e Sturt) l'Auliscus caelatus Bail. venne indicato da Petit, Greville, Rattray, Tempère e Peragallo ecc. per l'Australia e per la Nuova Zelanda in diverse località. Il VAN Heurck (Traité 1899, pag. 482) considera la specie del BAILEY una semplice varietà dell'Auliscus sculptus (W. Sm.) Ralfs; le numerose varietà, nelle quali venne distinto l'Auliscus caelatus Bail. segnano certo un graduale passaggio alla sud- 
detta specie e spiegano le incertezze dei diatomologi riguardo all' autonomia di queste due specie strettamente affini.

Auliscus Reticulatus Grev. in Trans. Micr. Soc. 1863, pag. 46, tab. 2, fig. 10 ; A. Schm. Atlas (1875) tab. 30, fig. 1-3; De Toni Sylloge Algarum II, pag. 1060. $H a b$. Australia merid.: Victor Harbour.

La specie venne raccolta nello Stretto di Bass da Johnson secondo il RATTRAY (Revision of Auliscus 1888, pag. 34); del King George's Sound, delle is. Thursday e della Terra di Kerguelen la distribuirono Tempère e Peragallo nelle Diatomées du monde entier rispettivamente ai numeri 276, 561-562 e 651-652.

Auliscus Rhipis A. Schmidt Atlas (1875) tab. 32, fig. 10-11; De Toni Sylloge Algarum II, pag. 1048.

Hab. Australia merid. : Victor Harbour; Nuova Zelanda: Bluff.

Questo Auliscus, affine esso pure all'Auliscus sculptus (W. Sm.) Ralfs venne segnalato già per il King George's Sound dal RATTRAY, dietro l' esame di preparati microscopici del Grove; dalla stessa regione (Albany) trovasi distribuito al n. 160 delle Diatomées du monde entier di Tempère e Peragallo.

Auliscus sculptus (W. Sm.) Ralfs in Pritchard Hist. of Infus. (1861) pag. 845, tab. 6, fig. 3; Grev. in Trans. Micr. Soc. 1863, pag. 43, tab. 2, fig. 1-3; V. H. Synopsis tab. 117, fig. 1-2, Traité pag. 482, fig. 215 e tab. 21, fig. 646; De Toni Sylloge Algarum II, pag. 1047.

Hab. Australia occid.: Baia di Geroldton; Nuova Zelanda: Bluff.

Fu distribuito delle isole Thursday al n. 561-562 delle Diatomées du monde entier di Tempère e Peragallo.

\section{Actinocyclus Ehr.}

Actinocyclus samoenssis (Grun.) n. comb.

Actinocyclus Ralfsii var. samoënsis Grun in Van Heurck Synopsis tab. 124, fig. 1; De Toni Sylloge Algarum II, pag. 1170.

$H a b$. Australia occid. : Baia di Geroldton.

È noto originariamente per le isole Samoa (Grunow); i Peragallo (Botan. und Zool. Ergebnisse einer wiss. Forschungsreise nach den Samoa-Inseln, dem Neu-Guinea-Archipel und den Salomoninseln 1911, pag. 4) proposero una varietà inermis.

Actinocyclus samoënsis (Grun.) venne distribuito nelle Diatomées du monde entier di Tempère e Peragallo al n. 642 delle isole Samoa e al n. 622 dello stretto di Malacca.

Actinocyclus tenuissimus Cleve Diat. fr. West Ind. Archip. (1878) pag. 21, tab. 5, fig. 34 ; De Toni Sylloge Algarum II, pag. 1181.

Var. Australiensis Grun. in Van Heurck Synopsis tab. 125, fig. 3.

Hab. Australia Occid.: Baia di Geroldton; Tasmania : Baia di Latrobe, Burmie; Nuova Zelanda: Port Charmers.

Il tipo venne nell' Oceano Pacifico riscontrato alle isole Samoa (Tempère e Peragallo, Diatomées du monde entier n. 647-648). 


\section{Fam. BIDdUlPhIACEAE (Kuetz.) Heib.}

\section{Biddulphia Gray}

Biddulphia aurita (Lyngb.) Bréb. Consid. sur les Diatomées (1838) pag. 12 ; V. H. Synopsis tab. 98, fig. 4-9; Traité pag. 471, tab. 20, fig. 631.

Odontella aurita C. Ag. Consp. Diat. (1832) pag. 56; De Toni Sylloge Algarum II, pag. 862.

Hab. Australia occid. : Baia di Geroldton; Australia merid. : Victor Harbour.

E una specie largamente diffusa negli Oceani. Tempère e Peragallo la distribuirono nelle Diatomées du monde entier di Albany, Australia occidentale (n. 276-277), dal Yarra River, Melbourne (n. 241-243), del porto di Oamaru, Nuova Zelanda (n. 744), dello Stretto di Malacca (n. 622-623), dell' is. Thursday (n. 561-562, 591-593) delle is. Samoa (n. 647-648), dell' isola di S. Paolo (n. 432) ed anche del Royal Sound nella Terra di Kerguelen (n. 651-652).

Biddulphia pulchella Gray Arrang. of Brit. Plants (1831) pag. 294; V. H. Synopsis tab. 97, fig. 1-3, Traité tab. 20, fig. 630; De Toni Sylloge Algarum II, pag. 870.

Hab. Nuova Zelanda: Port Charmers.

11 Petit (Dial. Campb. et N. Zél. pag. 35) enumerò già questa Biddulphia tra le Diatomee raccolte nello stretto di Foveaux; la specie ha, al pari della precedente, un' estesa distribuzione geografica anche nell' Oceano Pacifico (isole Samoa, Sandwich, Tahiti, stretto del Re Giorgio e stretto di Torres, Yarra River, is. Thursday, Nuova Zelanda ecc.).

Biddulphia Reticulata Roper in Microsc. Journal VII (1858) tab. 2, fig. 14-17; V. H. Synopsis tab. 102, fig. 1-2.

Odontella? reticulata De Toni Sylloge Algarum II, pag. 868.

Hab. Tasmania: Baia di Latrobe.

Questa bellissima specie fu già dallo stesso Roper segnalata per la Nuova Zelanda; il Petrit (Diat. Campb. et N. Zél. pag. 36) ne confermò la presenza nello stretto di Foveaux; la Biddulphia reticulata Roper fu riscontrata pure alle isole Samoa (Peragallo, Mereschkowsky), ad Hatzfeldhafen (Grunow), alle isole Sandwich, Caroline, Tahiti (Mereschoowsky), alle isole degli Amici, alle Nuove Ebridi, a Port Stevens, al River Yarra (Tempère e Peragallo).

Var. Trigona Grun. in Van Heurck Synopsis tab. 102, fig. 3.

Hab. Tasmania: Burmie.

Corrisponde al n. 284 deile Diat. di Cleve e Moeller (dell' isola Ceylon) e forse non differisce dal Triceratium Pardus A. Schm. Atlas (1876) tab. 79, fig. 15 (di Celebes). La Biddulphia reticulata Roper ha la tendenza di assumere forme triceratioidi ; così in individui (provenienti dall' isola Giava) figurati in A. Schmid Atlas tab. 84, fig. 15-16 e in altri figurati in VAN Heurck Synopsis tab. 102, fig. 3. La forma trigona Grun. fu riscontrata fossile nel deposito neozelandese di Oamaru (Grove e Sturt) e distribuita da Tempère e Peragallo nelle Diatomées du monde entier di questo stesso deposito (n. 810-822) delle isole degli Amici (n. 590-591) e dello Stretto di Malacca (n. 622-623). 
Biddulphia Roperiana Grev. in Microsc. Journal VII (1859) pag. 163, tab. 8, fig. 11-13; V. H. Synopsis tab. 99, fig. 4-6.

Odontella Roperiana De Toni Sylloge Algarum II, pag. 868.

$H a b$. Australia occid. : Baia di Geroldton.

Recentemente i Peragallo indicarono questa specie per le isole Samoa (Tempère e Peragallo Diatomées $d u$ monde entier n. 647-648); in quest'ultima collezione fu distribuita la Biddulphia Roperiana Grev. del porto neozelandese di Oamaru (n. 774), dell' isola Rodrigue (n. 54) e della Terra di Kerguelen (in più volte).

\section{Cerataulus Ehr.}

Cerataulus turgidus Ehr. in Abhandl. Berl. Akad. 1843, pag. 270; Castr. Diat. of Challenger (1886) pag. 101, tab. 26, fig. 6, 8; Forti Contribuzioni diatomologiche IX (1910) tab. 2, fig. 14.

Odontella turgida V. H. Synopsis tab. 104, fig. 1-2; De Toni Sylloge Algarum II, pag. 864.

Biddulphia turgida W. Sm. Brit. Diat. II (1856) pag. 50, tab. 62, fig. 38; V. H. Traité (1909) pag. 473, tab. 21, fig. 638.

Hab. Nuova Zelanda: Bluff.

Il nostro esemplare rappresenta una forma trigona; per essa è da confrontare la specie fossile nel deposito di Oamaru descritta col nome di Cerataulus subangulatus da Grove e Sturt (On a Fossil Marine Diatomaceous Deposit from Oamaru, Otago, New Zealand, part I, 1886, pag. 325, tab. 19, fig. 12). TemPÈre e Peragallo distribuirono il Cerataulus turgidus Ehr. di Albany (Australia occidentale) ai n. 276-277 delle Diatomées du monde entier, nonchè delle isole degli Amici (n. 561-562) e dell' isola Maurizio (n. 444).

\section{Triceratium Ehr.}

Triceratium alternans Bail. Microsc. Observat. (1850) pag. 40, fig. 55-56; A. Schmidt Atlas (1876) tab. 78, fig. 9-10; De Toni Sylloge Algarum II, pagina 941.

Biddulphia alternans Van Heurck Synopsis tab. 113, fig. 4.7, Traité pag. 475, tab. 21, fig. 644 .

$H a b$. Nuova Zelanda: Bluff.

Questa specie venne già segnalata per la Lyall's Bay dal Petrt (Diat. Campb. et N. Zél. pag. 36).

Triceratium Frauenfeldi Grun. in V. H. Synopsis tab. 110, fig. 10; A. Schmidt Atlas tab. 94, fig. 13; De Toni Sylloge Algarum II, pag. 945.

Hab. Nuova Zelanda: Port Charmers.

La specie venne per la prima volta figurata su esemplari provenienti dal1 ' isola San Paolo nell' Oceano australe.

Triceratium grande Brightw. in Microsc. Journal I (1853) pag. 249, tab. 4, fig. 8; A. Schmidt Atlas (1885) tab. 82, fig. 5.

Forma QUADRATA Temp. in schedula (collez. Forti). 
Hab. Nuova Zelanda: Bluff.

Questa specie, appartenente al gruppo del Triceratium Favus Ehr., va distinta dal Triceratium Robertsianum Grev. proveniente dall' Oceano Pacifico meridionale (Is. Woodlark) benchè ad essa la riferisca dubitativamente il GREville (Trans. Micr. Soc. 1866, pag. 7). Del Triceratium grande Brightw. il PaNTOCSEK (Foss. Bacill. Ungarns I, pag. 52, tab. 4, fig. 33) descrisse una forma pentagonia fossile nei depositi di Kékkö e Szent-Péter. Gli esempii di polimeria nelle Biddulphiaceae sono abbastanza comuni, rappresentati nelle specie descritte nei generi Amphitetras, Amphipentas ecc. cosi da avere indotto il V AN Heurck (Traité pag. 466) a semplificare tanto la sistematica da accogliere solo il genere Biddulphia con limiti ampliati in maniera da includere in esso i generi Triceratium, Amphitetras, Amphipentas, Cerataulus ed altri.

La specie del Brightwell nella sua forma quadrata fu rinvenuta copiosa nel deposito neozelandese di Oamaru da Grove e Sturt i quali sono propensi a riguardarla corrispondente alla var. maxima Grun. (in VAN Heurck Synopsis tab. 107, fig. 5) del comune Triceratium Favus Ehr. Una var. heptagona è nota per l' isola Maurizio distribuita ai n. 445-447 delle Diatomées du monde entier di Tempère e Peragallo.

Triceratium Pentacrinus Wall. On Triceratium (1858) pag. 249, tab. 12, fig. 10-14; A. Schmidt Atlas (1886) tab. 98, fig. 7-10, 13.

Hab. Nuova Zelanda: Port Charmers; Australia occid.: Baia di Geroldton (individui dalla forma valvare quadrangolare).

Anche questo Triceratium può, secondo il Wallich, presentare la polimeria (forme quadrangolari ed esagonali) oltre la forma comune pentagonale. Il Triceratium Pentacrinus Wall. venne già per le coste della Nuova Zelanda indicato anche nella sua forma quadrangolare (TEMPÈre e Peragallo Diatomées du monde entier n. 478-479) e raccolto pure in Australia (WeIsSFLoG), alle isole Samoa (Peragallo), alle Nuove Ebridi, allo Stretto di Torres e in altri luoghi dell' Oceania (Tempère e Peragallo) donde risulta che la specie ha estesa distribuzione nell' Oceano Pacifico.

Triceratium punctatum Brightw. in Microsc. Journal IV (1856) pag. 275, tab. 17, fig. 18; A. Schmidt Atlas (1876) tab. 76, fig. 19-20; De Toni Sylloge Algarum II, pag. 944.

Hab. Australia occid.: Baia di Geroldton.

Il Petit (Diat. Campb. et N. Zél. pag. 36) indicò questa specie per la Nuova Zelanda nella Lyall's Bay. Non sembra specie comune nell' Oceano Pacifico (Honolulu ecc.) ma è rappresentata in mari molto remoti ed anche nel nostro Mediterraneo.

Triceratium spinosum (Ehr.) Bail. in Pritchard Hist. of. Infus. (1861) pag. 853, tab. 6, fig. 10; A. Schmidt Atlas (1885) tab. 87, fig. plur.

Hab. Nuova Zelanda: Bluff.

Anche di questo Triceratium nèll' Allas di A. Schмidt è figurata una forma quadrangolare. La specie fu distribuita del porto neozelandese di Oamaru da Tempère e Peragallo nelle Diatomées du monde entier n. 774. 


\title{
Fam. STRIATELLACEAE (Kuetz.) Heib.
}

\author{
Grammatophora Ehr.
}

Grammatophora angulosa Ehr. Kreidethiere (1840) pag. 73, n. 44; V. H. Traité pag. 355, tab. 31, fig. 862; De Toni Sylloge Algarum II (1892) pag. 757.

$H a b$. Australia occid. : Baia di Geroldton; Tasmania : Baia di Latrobe, Burmie; Nuova Zelanda: Port Charmers.

La Grammatophora angulosa Ehr. è comunissima in tutti i mari; non mancano segnalazioni di essa nell' Oceano Pacifico; il Petıt (Diat. Campb. et N. Zél. pag. 33) ne indicò la esistenza nella Lyall's Bay, Nuova Zelanda : il MereschkowSKY la enumera tra le specie trovate alle isole Sandwich. È una specie che è pure diffusa nei giacimenti fossiliferi, ad esempio nella Stiria e a Grotte (Sicilia).

Var. Hamulifera (Kuetz.) Grun. Die Oesterr. Diatom. (1862) pag. 419; V. H. Synopsis tab. 53, fig. 4, Traité pag. 355, tab. 11, fig. $481 a$; De Toni Sylloge Algarum II, pag. 757.

Hab. Tasmania: Baia di Latrobe, Burmie.

Già il Kuetzing (Kieselsch. Bacillarien 1844, pag. 128) avvertì di aver riscontrato questa diatomea tra le Alghe provenienti dalle coste del Chil e della Nuova Olanda; la Grammatophora angulosa var. hamulifera non manca alla florula delle isole Samoa (MereschKowsky).

Grammatophora arcuata Ehr. Mikrogeologie (1856) tab. 35 A, XXIII, fig. 11-12; Grun. Die Oesterr. Diatom. (1862) pag. 420, tab. 2, fig. 7 a-d; De Toni Sylloge Algarum II, pag. 758.

Grammatophora curvata Ehr. Mikrogeologie (1856) tab. 35 A, XXII, fig. 13.

Hab. Tasmania: Baia di Latrobe, Burmie; Nuova Zelanda: Bluff.

Questa specie che, secondo il Grunow, troppo poco e indecisamente si differenzia dalla Grammatophora serpentina (Ralfs) W. Sm. non è la prima volta che viene indicata per la Nuova Zelanda. Di là provengono gli esemplari che l' Autore ora menzionato trovò sulla Halopteris hordeacea (Harv.) Sauv. Sembra però avere un' amplissima distribuzione geografica, provenendo i campioni originali dell' Ehrenberg l' uno dalla 'Assistance Bay » nell' Oceano Artico, l'altro dall' Antartide. Così ancora, più di recẹnte, il CLEve la raccolse sulle coste dello Spitzberg, Tempere e Peragallo la distribuirono dell' isola Mac Donald (Diatomées du monde entier n. 634) e della terra di Kerguelen a Royal Sound (n. 652) e P. Klavsen di Kitsigsut nella Groenlandia occidentale. Cleve e Moeller (Diat. n. 294) distribuirono una Grammatophora arcuata Ehr. var. arctica Grun. di Pitlekay che non differisce se non per la struttura più tozza e abbreviata delle valve che la fa somigliare più a Gramm. angulosa Ehr. che a Gramm. serpentina Ehr.

Grammatophora marina (Lyngb.) Kuetz. Die Kieselsch. Bacill. (1844) pag. 128, tab. 17, fig. XXVI, 1-6; De Toni Sylloge Algarum II, pag. 752.

Var. TRopICA (Kuetz.) Grun. Diat. Franz-Josefs Land (1884) pag. 5 ; V. H. Synopsis tab. 53, fig. 9; Perag. Diat. Mar. de France tab. 87, fig. 4-5. 
Grammalophora tropica Kuetz. Die Kieselsch. Bacillarien (1844) pag. 128, tab. 20, fig. 71 .

Hab. Nuova Zelanda: Port Charmers, Bluff.

Questa varietà in tutto simile alla forma tipica (già nota per la Nuova Zelanda e per altre regioni dell' Oceano. Pacifico : is. Campbell, Port Stevens, Nuove Ebridi), ne differisce soltanto per la striatura più marcata, 13-14 strie in $10 \mu$ (anzichè 18-20 strie in $10 \mu$ ).

Grammatophora oceanica Ehr. Mikrogeologie (1856) tab. XIX, fig. $36 a$ et pl. al. icon.; De Toni Sylloge Algarum II, pag. 755.

$H a b$. Australia occid. : Baia di Geroldton [la forma tipica e la var. communis (Grun.), macilenta (W. Sm.) Grun. con la forma subtilis Grun.]; Australia merid. : Victor Harbour [la forma tipica e la var. macilenta (W.Sm.) Grun.]; Tasmania: Baia di Latrobe [le var. communis (Grun.) e macilenta (W. Sm.) Grun. e la forma vulgaris Grun.]; Burmie [la forma vulgaris Grun. e la var. macilenta (W. Sm.) Grun.; Nuova Zelanda: Port Charmers [la forma vulgaris e la var. macilenta (W. Sm.) Grun.]; Bluff [la var. macilenta (W. Sm.) Grun. e la forma minuscula $\mathrm{H}$. Perag.).

Questa specie, come la precedente della quale, secondo il Van Heurck, costituisce un complesso di forme, è essa pure comune in tutti i mari; si trova fossile nel deposito neozelandese di Oamaru (Grove e STURT). La var. macilenta (W. Sm.) Grun. venne distribuita da Tempère e Peragallo nelle Diatomées du monde entier delle is. Thursday (n. 590), del King George' s Sound (n. 276-277), di Yarra River (241-243), di Port Stevens (n. 398-399), delle isole degli Amici (n. 590-591).

Grammatophora serpentina Ehr. in Berichte Berl. Akad. 1844; V. H. Synopsis tab. 53, fig. 1-3, Traité pag. 355, tab. 11, fig. $482 a$; De Toni Sylloge Algarum II, pag. 757 .

Hab. Tasmania: Baia di Latrobe (forma minor); Nuova Zelanda: Port Charmers (forma minor).

Specie, come le precedenti, comune in tutti i mari, già segnalata per la Lyall's Bay e le isole Campbell dal Petit (Diat. Campb. et N. Zél. pag. 34).

\section{Rhabdonema Kuetz.}

Rhabdonema adriaticum Kuetz. Die Kieselsch. Bacill. (1844) pag. 126, tab. 18, fig. 7 ; V. H. Synopsis tab. 54, fig. 11-13, Traité pag. 360, tab. 12, fig. $486 a$; De Toni Sylloge Algarum II, pag. 764.

Hab. Australia occid.: Baia di Geroldton; Tasmania: Baia di Latrobe, Burmie.

Specie assai comune in tutti i mari, segnalata per la regione dell' Oceania dal Petit, dal Grunow, dai Peragallo e dal Mereschkowsky ; frustoli da Albany, Australia occidentale furono distribuiti da Tempère e Peragallo (Diatomées $d u$ monde entier n. 160, 278 e 279), altri dell' is. Campbell (n. 357), delle is. Samoa (n. 647-648), Caroline (n. 621), Thursday (n. 561-562, 590-591), dello Stretto di Malacca (n. 622-623). 
Rhabdonema minutum Kuetz. Die Kieselsch. Bacill. (1844) pag. 126, tab. 21, fig. II 4 ; V. H. Synopsis tab. 54, fig. 17-21, Traité pag. 361, tab. 12, fig. $488 a$;

De Toni Sylloge Algarum II, pag. 763.

$H a b$. Tasmania: Burmie; Nuova Zelanda; Port Charmers.

Il Petit (Diat. Campb. et N. Zél. pag. 34) indicò questo Rhabdonema per l' is. Campbell.

Rhabdonema robustum Grun. Die Oesterr. Diatom. (1862) pag. 422, tab. 8. fig. 1; De Toni Sylloge Algarum II, pag. 761 ; Mereschk. On Polynesian Diatoms (1902) pag. 26, tab. 6, fig. 10.

Hab. Tasmania: Burmie.

La specie venne descritta dal GRunow tra le Diatomee riscontrate frammezzo ad Alghe provenienti dalla regione settentrionale dell' Oceano Pacifico, insieme al Rhabdonema Crozierii (Ehr.) Grun, che il PeTrT enumera tra le specie raccolte nella Nuova Zelanda alla Lyall's Bay.

\section{Striatella C. Ag.}

Striatella unipunctata (Lyngb.) Ag. Consp. crit. Diatom. (1832) pag. 61 ; V. H. Synopsis tab. 54, fig. 9-10, Traité pag. 363, tab. 12, fig. $485 a$; De Toni Sylloge Algarum II, pag. 766.

Hab. Australia occid. : Baia di Geroldton.

E specie abbastanza frequente negli Oceani e nei mari interni; per la leggerezza dei suoi frustoli aumentata dalla presenza di numerose zone intercalari comparisce non di raro anche nella flora pelagica come elemento accidentale.

\section{Tessella Ehr.}

Tessella interRupta Ehr. Die Infusionsthierchen (1838) pag. 202; Kuetz. Die Kieselsch. Bacillarien (1844) pag. 125, tab. 18, fig. IV, 1-2.

Striatella interrupta Heib. Conspectus criticus Diat. Danic. (1864) pag. 73, tab. 5, fig. 15; V. H. Synopsis tab. 54, fig. 8, Traité pag. 363, tab. 12, fig. $484 a$; De Toni Sylloge Algarum II, pag. 768.

Hab. Nuova Zelanda: Port Charmers.

E una specie abbastanza frequente nelle acque marine, con estesa distribuzione geografica. Se può essere discutibile l' autonomia del genere Tessella Ehr. separato dalle Striatella genuine per il fatto che i setti sono interrotti anzichè continui, ci sembra invece esagerata la concezione sistematica di F. SchUETT (in Engler-Prantl Die Natürl. Pflanzenfamilien, Bacillarieen, pag. 103-105) che ammette il solo genere Tabellaria Ehr., includendo in esso tanto le Striatella quanto le Tessella. Avesse pure ragione F. SchuEtT con le sue vedute tassonomiche, non si può spiegare in nessun modo la preferenza data al genere Tabellaria, proposto dall' EHRENBERG nel 1839, in confronto del genere Striatella fondato da C. AgardH nel 1832 o di Tessella istituito dallo stesso EHRENBERG nel 1838. 


\section{Fam. LICMOPHORACEAE Kuetz.}

$$
\text { Licmophora C. Ag. }
$$

Licmophora Juergensil C. Ag. Consp. crit. Diatom. (1831) pag. 42; De Toni Sylloge Algarum II, pag. 7'32.

Var. Constricta Grun. in Van Heurck Synopsis tab. 47, fig. 6; De Toni op. cit. pag. 732 ; Licmophora constricta (Grun.) Meresch. On Polynesian Diatoms (1902) pag. 14, tab. VI, fig. 6-9.

Hab. Nuova Zelanda: Port Charmers.

La varietà venne proposta dal GRUNow per esemplari provenienti dalle isole Samoa, dove la sua presenza fu poscia confermata dal Mereschrowsky ; la specie è divulgatissima, anzi può dirsi, con le sue diverse varietà, rappresentata in tutti i mari.

Licmophora paradoxa (Lyngb.) C. Ag. Icones Algarum (1829); V. H. Synopsis tab. 48, fig. 10-12, Traité pag. 344, tab. 31, fig. 854; De Toni Sylloge Algarum II, pag. 736.

Hab. Tasmania: Baia di Latrobe; Nuova Zelanda: Port Charmers.

È specie nota per l' Oceano Atlantico, il mare Mediterraneo e vi si trova spesso epifita su alghe diverse.

Licmophora tincta (Ag.) Grun. in Hedwigia VI (1867) pag. 35; V. H. Synopsis tab. 48, fig. 13-15, Traité pag. 344, fig. 586; De Toni Sylloge Algarum II, pag. 736.

Hab. Tasmania: Baia di Latrobe.

Per l' Oceano Pacifico, questa specie tu già indicata dal Petit per la Lyall's Bay nella Nuova Zelanda (Diat. Campb. et N. Zél. pag. 33).

\section{Climacosphenia Ehr.}

Climacosphenia elongata Bail. Notes on new Species and Localities of microsc. Organisms (1853) pag. 8, tab. 1, fig. 10-11; Grun. Die Oesterr. Diatom. (1862) pag. 351, tab. 6, fig. 22 a-d; De Toni Sylloge Algarum II, pag. 739 ; Mereschk. On Polynesian Diatoms (1902) pag. 22, tab. 5, fig. 1-2.

Hab. Australia occid.: Baia di Geroldton; Tasmania: Burmie; Nuova Zelanda: Port Charmers.

Fu distribuita da Tempère e Peragallo fra i lavaggi di conchiglie di $\mathrm{Ha}$ liotis provenienti dalle coste della Nuova Zelanda analizzati da P. BERgon (Diatomées du monde entier $\mathrm{n}$. 478-479) e in campioni diatomiferi delle isole Thursday (n. 590-591 e 561-562).

La Climacosphenıa australis Kuetz., della quale con ogni probabilità secondo il Grunow (Alg. Novara pag. 5) la specie Baileyana è una semplice varietà fornita di pedicelli più lunghi e maggiormente ramificati, fu riscontrata epifita su parecchie Alghe provenienti dall' Oceano Pacifico soprattutto dalla Nuova Olanda e da isole della Polinesia. 


\title{
Fam. ENTOPYLaCeae Grun.
}

\author{
Entopyla Ehr.
}

Entopyla Australis Ehr. in Abhandl. Berl. Akad. 1841, tab. I, fig. $9 b$; De: Toni Sylloge Algarum II, pag. 773.

Var. Gigantea (Grev.) Fricke in A. Schmidt Atlas (1902) tab. 230, fig. 1-11.

Gephyria gigantea Grev. in Trans. Micr. Soc. 1866, pag. 122, tab. 11, fig. 7-8; Castrac. Diat. of Challenger (1886) pag. 42, tab. 15, fig. 10.

Hab. Nuova Zelanda: Bluff.

La forma tipica di questa Entopyla venne già segnalata dal Petri (Diat. Campb. et $N$. Zél. pag. 15) per la Lyall 's Bay, Nuova Zelanda; Tempère e PERAGALLo ne distribuirono pure esemplari da materiali sondati alle is. Campbell (Diatomées du monde entier $\mathrm{n}$. 357) e provenienti dai lidi della Nuova Zelanda. (n. 478-479), così pure dalla Terra di Kerguelen (n. 651-652) e dall' is. San Paolo (n. 432); fu anche riscontrata nei sondaggi nelle regioni antartiche (VAN HEURCK).

Entopyla ocellata (Arn.) Grun. Die Oesterr. Diatom. (1862) pag. 428; A. Schmidt Atlas (1902) tab. 231, fig. 1-6; De Toni Sylloge Algarum II, pag. 774.

$H a b$. Australia occid. : Baia di Geroldton.

Questa bella specie fu già segnalata sulla Ballia callitricha (Ag.) Mont. della Nuova Zelanda dal Walker-Arnott (On Rhabdonema, and a new allied Genus 1858, pag. 90); il Petit (Diat. Campb. et N. Zél. pag. 15) ne confermò la presenza nella Lyall's Bay e anche P. BERGon la riconobbe nei lavaggi di conchiglie provenienti dalla Nuova Zelanda distribuiti da Tempére e Peragallo ai n. 478 e 479 delle Diatomées du monde entier. Frustoli derivanti da raccolte fatte nell' Australia meridionale sono figurati da A. Schмid nell' opera sopra citata ed anche il GRUNow (Alg. Novara pag. 8) enumerò la Entopyla australis (Arn.) Grun. per la Nuova Olanda. Nelle Diatomées du monde entier la specie trovasi rappresentata ai n. 651-652 (Royal Sound, Terra di Kerguelen), ai n. 474.475 (isole Nicobar) e, in una varietà pulchella, al n. 634 (is. Mc Donald); non manca alle regioni antartiche (VAN HEURCK).

\section{Gephyria Arnott}

Gephyria media Arnott in Microsc. Journal VIII (1860) pag. 20 ; Pritchard Hist. of Infus. (1861) pag. 809, tab. 4, fig. 49; A. Schmidt Atlas (1902), tab. 231, fig. 18-21; De Toni Sylloge Algarum II, pag. 775.

Hab. Nuova Zelanda: Port Charmers.

La maggior parte delle Gephyria è rappresentata da specie fossili e la stessa Gephyria media Arnott fu riscontrata nel guano di California (ARNotT) e nel deposito di Jérémie, Hayti (Truan, Witt). Il Mereschkowsky (On Polynesiam Diatoms 1902, pag. 19, 23) segnalò questa specie per Tahiti e Hawaii; TemPÉRe e Peragallo nelle Diatomées du monde entier la distribuirono delle Nuove: Ebridi (n. 61, 63), dell' isola Rodrigue (n. 54) e Maurizio (n. 445-447). 


\title{
Fam. FRAGILARIACEAE (Kuetz.)
}

\author{
Subfam. Synedreae (Rabenh.)
}

Synedra Ehr.

Synedra Acus Kuetz. Die Kieselschal. Bacillarien (1844) pag. 68, tab. 15, fig. 7; V. H. Synopsis tab. 39, fig. 4, Traité pag. 311, tab. 10, fig. 420; Hustedt in A. Schmidt Atlas tab. 303, fig. 7-9; De Toni Sylloge Algarum II, pag. 656.

Hab. Tasmania: Hobart; Nuova Zelanda: Rotorua, Geyser a.

E una tra le specie abbastanza comuni nelle acque dolci; fu trovata anche allo stato fossile nel deposito di Bilin (Tempère e Peragallo, Diatomées du monde entier n. 564-565).

Synedra Gallionil (Bory) Ehr. in Abhandl. Berl. Akad. 1830, pag. 129; Kuetz. Die Kieselsch. Bacillarien (1844) pag. 68, tab. 30, fig. 42; V. H. Synopsis tab. 39 fig. 18, Traité pag. 312, tab. 10, fig. 424; De Toni Sylloge Algarum II, pag. 658 .

Hab. Tasmania: Baia di Latrobe; Nuova Zelanda: Port Charmers, Bluff.

Questa specie venne già segnalata nell' Oceano Pacifico dal PetrT (Diat. Compb. et N. Zél. pag. 31) per la Lyall 's Bay della Nuova Zelanda. Gli esemplari della Baia di Latrobe e di Bluff vanno riferiti alla var. elongata Perag. (Diat. mar. de France pag. 315, tab. 80, fig. 6) e quelli di Port Charmers alla var. macilenta Grun. in Peragallo (op. cit. pag. 315, tab. 80, fig. 8).

La Synedra Gallionii (Bory) Ehr. ha estesa distribuzione nei mari sulle coste Europee; non manca neppure verso i lidi africani al Capo di Buona Speranza (Grunow) e all' Oceano Indiano presso l' isola Maurizio (Tempere e Peragallo, Diatomées du monde entier n. 445-447).

Synedra Goulardi Bréb. in H. L. Smith Typ. n. 588 !; Cleve et Grun. Arct. Diat. (1880, pag. 107, tab. 6, fig. 119; De Toni Sylloge Algarum II, pag. 655.

Hab. Nuova Zelanda: Wakarevareva.

Frustuli lunghi $58 \mu$, bene corrispondenti al tipo della collezione di H. L. Sмітн. La specie sembra avere un' area di distribuzione geografica estesissima conoscendosi anche per l'America boreale, il Paraguay, il Victoria Nyanza, l' isola Riunione, il Giappone ecc.

Synedra Laevigata Grun. in Trans. R. Micr. Soc. 1877, tab. 193, fig. 3 ; De Toni Sylloge Algarum II, pag. 655.

$H a b$. Nuova Zelanda: Bluff.

Il GRunow indicò la Synedra laevigata per le isole Samoa e la Terra dell' imp. Guglielmo a Finschhafen; ma la specie fu pure trovata in altri luoghi (Honduras ; is. Maurizio ; isola di Francia: var. hyalina Grun.).

Synedra Ulna (Nitzsch) Ehr. Die Infusionsthierchen (1838) pag. 211, tab. 17., fig. 1 ; V. H. Synopsis tab. 38, fig. 7, Traité pag. 310, tab. 10, fig. 409; De Toni Sylloge Algarum II, pag. 653. 
Hab. Nuova Zelanda: Rotorua, Ohinemuthu, Wakarevareva.

L'EHRENBERG (Mikrogeologie 1856, pag. 4, 6, 7) indicò la Synedra Ulna per parecchie località dell' Australia occidentale (Swan River, Avon River, Cunning River, Adams River); per la Nuova Zelanda la specie fu segnalata nel distretto. di Otago dal Lauder Lindsay e per il lago Wakatipu dal Lemmermann; essa venne pure rinvenuta da quest' ultimo autore tra le Diatomee provenienti dalle lagune di Chatham. Tempère e Peragallo la distribuirono nelle Diatomées du monde entier del Lago di Rotorua (n. 273) e anche fossile di Wangarei (n. 918). e di Waitangi (n. 922); la diedero eziandio di Brunswick, Victoria (n. 921) e fossile di Talbot, Australia (n. 981-983) ove si trovano le var. aequalis e splendens.

Var. Lanceolata (Kuetz.) Grun. Die Oesterr. Diatom. (1862) pag. 397, V. H. Synopsis tab. 38, fig. 10, Traité pag. 311, tab. 10, fig. 416; De Toni Sylloge Algarum II, pag. 654.

Hab. Nuova Zelanda: Wakarevareva.

Var. oxyrhynchus (Kuetz.) Grun. in Van Heurck Synopsis tab. 39, fig. $1 a$, Traité pag. 311, tab. 10, fig. 418; De Toni Sylloge Algarum II, pag. 654.

Hab. Tasmania: Hobart.

Di questa varietà riscontrammo anche alcuni casi teratologici; le deformazioni nei frustoli delle Synedra sono abbastanza frequenti, come le osservarono non pochi autori (Macchiati, De Gasparis e Mastrostefano, Forti).

Forma medioconstricta Forti in Tempère et Peragallo Diatomées du monde entier pag. 252 , n. $480-481$.

$H a b$. Nuova Zelanda: Wakarevareva.

Esemplari di $58-60 \mu$ di lunghezza, in tutto somiglianti agli originali fossili delle Colline di Soddo in Etiopia ; si distinguono dalla var. oxyrhynchus, cosmopolita, per una lieve costrizione laterale nel punto corrispondente al pseudostauro.

\section{Ardissonia De Not.}

Ardissonia CRystallina (Ag.) Grun. in Cleve et Grun. Arkt. Diat. (1880) pag. 108; V. H. Synopsis tab. 42, fig. 10, Traité pag. 315, tab. 10, fig. 435 (sub Synedra); De Toni Sylloge Algarum II, pag. 673.

Hab. Australia occid.: Baia di Geroldton; Tasmania: Baia di Latrobe.

Per la regione dell' Oceania, l' Ardissonia crystallina (Ag.) Grun. venne segnalata alle isole Salomone dai Peragallo; la specie fu riscontrata fossile nel deposito neozelandese di Oamaru (Grove e Sturt); nelle isole degli Amici ne venne segnalata una varietà conspicua (Tempère e Peragallo, Diatomées du monde entier n. 590).

Ardissonia fulgens (Grev.) Grun. in Cleve et Grun. Arkt. Diat. (1880) pag. 108 ; V. H. Synopsis tab. 43, fig. 1-2, Traité pag. 316, tab. 10, fig. 436 (sub Sy. nedra); De Toni Sylloge Algarum II, pag. 674.

Hab. Australia occid. : Baia di Geroldton.

Venne distribuita nelle Diatomées du monde entier di Tempère e Peragallo dell' isola Mc Donald (n. 634), dello Stretto di Malacca (n. 622-623), del Royal Sound di Kerguelen (n. 651-652) e dell' isola Rodrigue (n. 54). 


\section{Toxarium Bail.}

Toxarium undulatum Bail. Notes on new Species and Localities of microsc. Organisms (1853), pag. 15, fig. 24-25; De Toni Sylloge Algarum II, pag. 677.

Synedra undulata Bail. in W. Sm. Brit. Diat. II (1856) pag. 97; Greg. On new Forms of marine Diatomaceae (1857) pag. 531, tab. 14, fig. 107; V. H. Synopsis tab. 42, fig. 2, Traité pag. 317, tab. 10, fig. 437.

Synedra undulans Greg. On a post-tertiary Sand containing Diatomaceous Exuviae from Glenshira (1854) pag. 13, tab. 4, fig. 23!

Hab. Tasmania: Baia di Latrobe.

Questa specie, abbastanza frequente negli Oceani e nei mari interni, venne segnalata nell' Oceano Pacifico per le isole Samoa dai Peragallo, per le isole Sandwich dal Mereschkowsky, per l' Australia occidentale e per lo stretto del Re Giorgio da Tempère e Peragallo ; abbiamo colto l'occasione per ripresentare qui rettificate la nomenclatura e la sinonimia di questa elegante Sinedrea (1) che neppure il Grunow (Die Oesterr. Diatom. 1862, pag. 405) fornì con esattezza.

\section{Fam. RAPHONEIDACEAE Van Heurck}

\section{Rhaphoneis Ehr.}

Rhaphoneis amphiceros Ehr. in Berichte Berl. Akad. 1844, pag. 87; V. H. Synopsis tab. 36, fig. 22-23, Traité pag. 330, tab. 10, fig. 394; De Toni Sylloge Algarum II, pag. 699.

Hab. Nuova Zelanda: Bluff.

I nostri esemplari corrispondono alla varietà gemmifera (Ehr.) [ = Rhaphoneis gemmifera Ehr. loc. cit. pag. 87] che il RABENHORST (Flora Europaea Algarum I, pag. 126) riferisce senz' altro alla Rhaphoneis amphiceros Ehr. Questa specie nell' Oceano Pacifico fu segnalata per Finschhafen (Grunow), per le isole Samoa (Peragallo; Tempère e Peragallo Diatomées du monde entier n. 647-648: var. gemmifera); di quest' ultima collezione si hanno anche esemplari da Albany nell' Australia occidentale.

Rhaphoneis Surirella (Ehr.) Grun. in Cleve et Moeller Diat. n. 208; V. H. Synopsis pag. 36, fig. 26.27 a, Traité pag. 330, tab. 10, fig. 397.

Dimerogramma Surirella Grun. in Cleve et Grun. Diat. from West Indian Archip. (1878) pag. 11, n. 89; De Toni Sylloge Algarum II, pag. 712.

$H a b$. Nuova Zelanda: Bluff.

Solo l' esame di campioni autentici potrà decidere la questione se Rhaphoneis Rhombus Ehr. e Rhaphoneis Surirella (Ehr.) Grun. costituiscano un'unica entità specifica. La varietà australis Grun. venne distribuita da Albany, Australia occidentale da Tempère e Peragallo nelle Diatomées du monde entier n. $276-277$.

(1) Cfr. De Ton G. B. e Forti AcH., Contribution à la flore algologique de la Tripolitaine et de la Cyrenaïque pag. 31, n. 103 (Annales de l'Institut Océanographique [fondation Albert Ier Prince de Monaco] T. V, fasc. 7). 


\section{Trachysphenia Petit}

Trachysphenia australis Petit Catalogue des Diatomées de l' île Campbell et de la Nouvelle Zélande (1878) pag. 32, tab. 5, fig. 19; De Toni Sylloge Algarum II, pag. 645.

Hab. Tasmania: Baia di Latrobe, Burmie; Nuova Zelanda: Bluff.

Questa bella specie venne dal PETIT segnalata per la Lyall's Bay e per I' isola Campbell ; lo stesso autore riscontrò più tardi la sua Trachysphenia australis al Capo Horn nell' America meridionale; secondo i Peragallo la stessa specie fu raccolta alle isole Hawaii.

\section{Fam. Platiogramaceae P. Petit}

Dimeregramma Ralfs

Dimeregrama nanum (Greg.) Ralfs in Pritchard Hist. of Infus. (1861) pag. 790 , tab. 4, fig. 33; V. H. Synopsis tab. 36, fig. $11 b$; De Toni Sylloge Algarum II, pag. 711 . 849.

Dimeregramma minor var. nana V. H. Traité (1899) pag. 336, tab. 30, fig.

Hab. Tasmania: Burmie.

Il Castracane (Diat. of Challenger 1886, pag. 46, tab. 19, fig. 5) descrisse una varietà tahitense la quale, essendo un po' più robusta, forma il passaggio al Dimeregramma minus (Greg.) Ralfs.

\section{G1yphodesmis Grev.}

Glyphodesmis Willtamsonit (W. Sm.) Grun. in Van Heurck Synopsis tab. 36, fig. 14, Traité pag. 334, fig. 91, tab. 30, fig. 847; De Toni Sylloge Algarum II, pag. 715 .

Himantidium? Williamsonii W. Sm. Brit. Diat. II (1856) pag. 14, tab. 33, fig. 287 !

Diadesmis? Williamsonii Greg. Diat. of Clyde (1857) pag. 497, tab. 10, fig. 40 !

Hab. Australia occid. : Baia di Geroldton.

La prima descrizione di questa elegante Plagiogrammacea spetta a W. Sмiтн il quale, non avendo potuto osservarne la faccia valvare, riferi gli esemplari comunicatigli dal prof. Williamson al genere Himantidium, accompagnando tuttavia il riferimento con un segno interrogativo; un anno dopo, il GREGORY, avuto modo di riconoscere le valve, passò la specie, pure con dubbio, al genere Diadesmis; il Grunow, nel 1862, pur non avendo veduto la fäccia valvare, si avvicinò di più al riconoscimento della specie proposta da W. Sмгтн ascrivendola allora al genere Dimeregramma. 


\section{Fam. EUNOTiACEAE (Kuetz.)}

\section{Eunotia Ehr.}

Eunotia SERPENTina Ehr. Mikrogeologie (1854) pag. 12 (nomen) sententiâ clar. Hustedt in A. Schmidt Atlas (1913) tab. 293, fig. 14-19 (Amphicampae Erucae).

Eunotia Eruca Brightw. On some of the rarer or undescribed Species of Diatomaceae p. 1 in Quarterly Journ. of Microsc. Science vol. VII (1859) pag. 179, tab. IX, fig. 1 et $1 a$ [non Ehrenberg, nec Amphicampa Eruca Ehr. quae genus proprium sistit] (1), Reichelt in A. Schmidt Atlas (1913) tab. 290, fig. 8.

Hab. Nuova Zelanda: Wakarevareva.

Il Grunow Die Oesterreichischen Diatomaceen 1862, pag. 343) nota erroneamente che Eunotia Eruca osservata da EHRENBERG in materiali fossili provenienti da Tisar nel Messico venne indicata, allo stato vivente, in una laguna di acqua dolce presso Melbourne (Australia); allude con ciò al reperto del BRIGHTWELL che dette origine alla confusione in seguito sempre accresciuta fra due specie diversissime per struttura e aspetto e che potevano rimaner distinte anche per il fatto che se l' una si rinviene fossile nel Messico, l' altra vive esclusivamente nelle acque salmastre e termali di Australia, Tasmania e Nuova Zelanda e tutt' al più si trova fossile nei depositi diatomiferi neozelandesi di origine analoga agli ambienti dove oggi si riscontra prosperare. Più tardi il Grunow ( $\mathrm{Alg}$. Novara 1867, pag. 94) la segnala nel "Kieselguhr » presso Auckland, ivi raccolta da Hochstetter e del medesimo deposito la distribuirono Tempère e PeRAGALlo nelle Diatomées du monde entier n. 919, nonchè di Yarra River, nelle acque salmastre, forse della medesima stazione, ove fu riscontrata dal BRIGHTWELL. Eunotia serpentina non è che soltanto accennata di nome dall'EHRENBERG che la indica raccolta nei fiumi Avon, Canning e Adams dell' Australia ed è

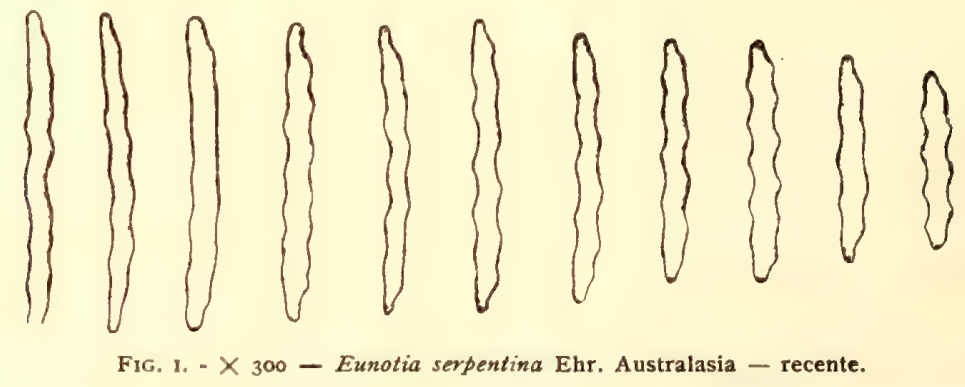

semplicemente sull' autorità dello HustedT (loc. cit.) che riposa l'identificazione con Eunotia Eruca. Ma il cambiamento di nome fu opportunissimo a togliere di mezzo finalmente ogni equivoco fra la specie messicana e l'australiano-neoze-

(1) Amphicampa Eruca Ehr. Mikrogeologie (1854) Inhalt des Atlas pag. 7, tab. 33, VII fig. I ; Hustedt in A. Schmidt Atlas (1913) tab. 293, fig. 14-19; Eunotia Eruca Ehr. in Berichte Berlin. Akad. (1844) pag. 340; Amphicampa mirabilis Ehr. Mikrogeologie (1854) tab. 33, VII, fig. 2.

A proposito dell' autonomia del genere Amphicampa torna utile riferire quanto asserisce il sig. HUSTEDT nella didascalia alla figura sopra citata * Endknoten an Raphe fehlen vollständig, eine Vereinigung mit Eunotia ist also ausgeschlossen $»$ 
landese. Si allegano (vedi le figure 1 e 2) i profili dei contorni dell' una e dell'altra specie in molti esemplari per dimostrarne la spiccata diversità delle serie, nonchè la variabilità notevolissima nelle dimensioni, nell'aspetto, nell'aggruppamento e nel numero delle sinuosità per ciascuno degli individui, fermi rimanendo i caratteri principali che si possono riassumere come segue: Valve vedute di fianco bacilliformi, con pseudonoduli evidenti nell' Eunotia serpentina Ehr., vivente o subfossile in Australasia; valve di fianco falcato-lunate o più o meno incurve, prive di pseudonoduli nell' Amphicampa Eruca Ehr., fossile nei depositi d'acqua dolce del Messico.

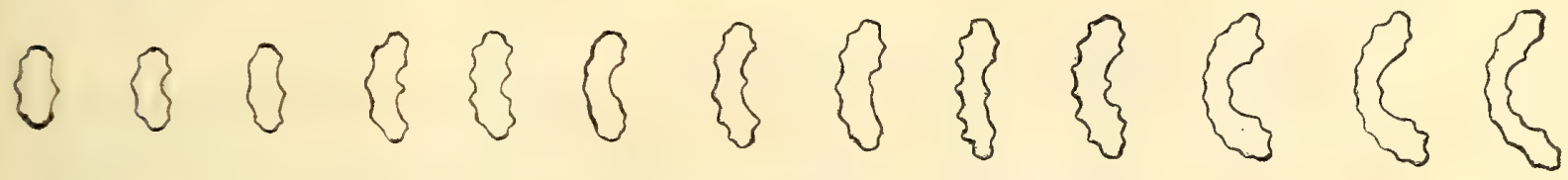

F1G. 2. - $\times 300-$ Amphicampa Eruca Ehr. Messico - Fossile d' acqua dolce.

Eunotia pectinalis (O. F. Muell.) Rabenh. Flora Europaea Algarum I (1864) pag. 793 ; V. H. Synopsis tab. 33, fig. 15-16, Traité pag. 300, tab. 9, fig. 370-371 ; De Toni Sylloge Algarum II, pag. 793.

Hab. Nuova Zelanda: Wakarevareva.

Il Lauder Lindsay (Diat. of Otago pag. 131) indicò già questa Eunotia per il distretto di Otago nella Nuova Zelanda; l' Eunotia pectinalis trovasi pure distribuita del Lago Rotorua in Tempère e Peragallo Diatomées du monde entier n. 273 e fossile di Talbot, Melbourne. A giudicare dalle figure e dalle opinioni successivamente espresse da Ralfs, Kuetzing ecc, ma più che tutto dall' ispezione fatta da W. Smith degli esemplari autentici del Dillwyn conservati nell' erbario Greville, la specie venne per la prima volta figurata dal Dillwyn nelle British Confervae (1809) pag. 51, tab. 24, mentre il nome specifico pectinalis e una breve descrizione erano stati dati da O. F. Mueller fino dal 1785, laonde a rigore la specie va indicata col nome Eınotia pectinalis (Muell.) Rabenh.

Eunotia Veneris Kuetz. Die Kieselsch. Bacillarien (1844) pag. 40, tab. 30, fig. 7 (sub Himantidio) ; V. H. Traité pag. 301, tab. 30, fig. 826; De Toni Sylloge Algarum II, pag. 794.

Eunotia incisa Greg. in Microsc. Journal II (1864) pag. 96, tab. 4, fig. 4.

Hab. Nuova Zelanda: Rotorua, Ohinemuthu. Zelanda.

Non si hanno indicazioni riguardo alla presenza di questa specie nella Nuova

\section{Epithemia Bréb.}

Epithemia Sorex Kuetz. Die Kieselsch. Bacillarien (1844) pag. 33, tab. 5, fig. XII 5 a-c; V. H. Synopsis tab. 32, fig. 6-10, Traité pag. 295, tab. 9, fig. 351. Cystopleura Sorex Kuntze Revisio II (1891) pag. 891 ; De Toni Sylloge Algarum II, pag. 780 .

Hab. Nuova Zelanda: Bluff, Wakarevareva.

Il Lauder Lindsay (Diat. of Otago pag. 131) segnalò l' Epithemia Sorex per il distretto di Otago; il Lemmermann la enumerò tra le specie nelle lagune di 
Chatham e nel lago Huro. La specie fu distribuita da Tempère e Peragallo nelle Diatomées du monde entier del Lago Kotorua (n. 273) e del * Lake Mori s d'Australia (n. 930); fossile di Brunswick, Victoria (n. 921) e di Wangarei, Nuova Zelanda (n. 918).

Eprthemia turgida (Ehr,) Kuetz. Die Kieselsch. Bacillarien (1844) pag. 34, tab. 5, fig. $14 ;$ V. H. Synopsis tab. 31, fig. 1-2.

Var. Westermanni (Ehr.) Grun. Die Oesterr. Diatom. (1862) pag. 325, tab. 6, fig. 8; V. H. Synopsis tab. 31, fig. 8; Traité pag. 295, tab. 9, fig. 347.

Epithemia Westermanni Kuetz. Die Kieselsch. Bacillarien (1844) pag. 32 r tab. 5, fig. XII 1-4.

Hab. Nuova Zelanda: Wakarevareva.

Anche questa varietà non è nuova per la flora neozelandese, essendo stata segnalata per il distretto di Otago dal LaUder Lindsay (Diat. of Otago pag. 131). Il GRunow (op. cit. pag. 326) dimostrò come la Epithemia turgida (Ehr.) Kuetz. con le sue varietà presenti adattamenti alle acque dolci, salmastre e persino marine.

Epithemia Zebra (Ehr.) Kuetz. Die Kieselsch. Bacillarien (1844) pag. 34, tab. 5, fig. 12 ; tab. 30, fig. 5 ; V. H. Synopsis tab. 31, fig. 9, 11-14, Traité pag. 296, tab. 9, fig. 357.

Cystopleura Zebra Kuntze Revisio II (1891) pag. 891; De Toni Sylloge Algarum II, pag. 784.

Hab. Nuova Zelanda: Port Charmers.

Il Lauder Lindsay (Diat. of Otago pag. 131) indicò già questa specie nella Nuova Zelanda per il distretto di Otago. Tempère e Peragallo distribuirono la var. Porcellus nelle Diatomées du monde entier del Lake Mori, Australia (n. 930). e la forma tipica fossile a Talbot, Melbourne (n. 981-983).

\section{Rhopalodia O. Müll.}

Rhopalodia GibBa (Ehr.) O. Müll. Rhopalodia (1895) pag. 65, tab. 1, fig. 15-17; A. Schmidt Atlas (1905) tab. 253, fig. 1.11.

Epithemia gibba Kuetz. Die Kieselsch. Bacillarien (1844) pag. 35, tab. 4, fig. XXII; V. H. Synopsis tab. 32, fig. 1-2, Traité pag. 296, tab. 9, fig. $352 a-b$.

Hab. Nuova Zelanda: Rotorua, Ohinemuthu.

Segnalata già nella Nuova Zelanda per il distretto di Otago dal LAUDER Lindsay (Diat. of Otago pag. 131), per Glenorchi e il lago Huro di Chatham dal Lemmermann (Ergebn. einer Reise nach dem Pacific 1899, pag. 340). TemPÈre e Peragallo nelle Diatomées $d u$ monde entier distribuirono questa specie di Yarra River, Melbourne (n. 241-243) e di Lake Mori (n. 930) e fossile di Talbot, Melbourne (n. 981-983).

Rhopalodia Gibberula (Ehr.) O. Müll. Rhopalodia (1895) pag. 58; De Toni e Forti Alghe del Ruwenzori pag. 29.

Epithemia gibberula Kuetz. Die Kieselsch. Bacillarien (1844) pag. 35, tab. 30, fig. 3 ; V. H. Traité pag. 297 , tab. 30 , fig. 825 ?

Hab. Nuova Zelanda: Rotorua, Ohinemuthu, Geyser b. 
Di questa Rhopalodia riscontrammo frustoli riferibili alle varietà producta (Grun.), sphaerula O. Müll. e Van-Heurckii O. Müll. Non è la prima volta che questa specie si trova nell' Oceania. Tempère e Peragallo nelle Diatomées $d u$ monde entier distribuirono la var. protracta di Yarra River (n. 241-243), la forma tipica delle lagune di West Melbourne, Victoria (n. 947) nonchè del Lake Mori d'Australia (n. 930).

Fu pure distribuita del deposito di Waitangi, Nuova Zelanda (n. 922).

Rhopalodia Musculus (Kuetz.) O. Müll. Bacillariaceen aus den Natronthälern von El Kab (1899) pag. 294.

Epithemia Musculus Kuetz. Die Kieselsch. Bacillarien (1844) pag. 33, tab. 30, fig. 6; V. H. Synopsis tab. 32, fig. 14-15, Traité pag. 297, tab. 9, fig. 359.

Hab. Australia occid. : Baia di Geroldton.

Per quanto riguarda la flora marina dell' Oceania, questa Rhopalodia fu segnalata per la Nuova Zelanda (Lauder Lindsay, Petit) e per le isole Samoa e Hawaii (Peragallo); delle isole Sandwich fu pure distribuita ai n. 61-63 delle Diatomées du monde entier di Tempère e Peragallo.

Rhopalodia ventricosa (Ehr.) O. Müll. Rhopalodia (1895) pag. 65, tab. 1, fig. 20-21.

Epithemia gibba var. ventricosa Grun. Die Oesterr. Diatom. (1862) pag. 327 ; V. H. Synopsis tab. 32, fig. 4-5, Traité pag. 296, tab. 9, fig. 354.

Hab. Nuova Zelanda: Wakarevareva.

Fu trovata nel Lake Mori, Australia e distribuita nelle Diatomées du monde entier di Tempère e Peragallo al n. 930.

\section{Ordo II. MoBILES}

\section{Fam. NITZCHIACEAE Grun.}

\section{Bacillaria Gmel.}

Bacillaria Paradoxa Gmel. in Linn. Syst. Nat. (1788) I, pag. 3903; Kuetz. Die Kieselsch. Bacillarien (1844) pag. 63, tab. 21, fig. 18; Grun. Die Oesterr. Diatom. (1862) pag. 583; De Toni Sylloge Algarum II, pag. 493.

Nitzschia paradoxa Grun. in Cleve et Grun. Arct. Diat. (1880) pag. 85; V. H. Synopsis tab. 61, fig. 6-7, Traité pag. 392, tab. 16, fig. 518.

Hab. Tasmania: Baia di Latrobe; Nuova Zelanda: Port Charmers.

P. A. C. HeIBerg (Kritisk Oversigt over de Danske Diatomeer 1863, pag. 113) riferendo a questa entità specifica il Vibrio paxillifer di O. F. Mueller (Animalc. Infus. 1786, pag. 54, tab. 7, fig. 3.7) indica la specie col nome Nitzschia paxillifer (O. F. Mueller). Se l'autonomia del genere Bacillaria Gmel. deva essere mantenuta o no in confronto di Nitzschia Hassall non è qui il luogo per discutere; però in caso negativo tutte le Nitzschia dovrebbero, per legge di priorità, venire incluse nel genere Bacillaria Gmel. ( $\left.{ }^{1}\right)$.

(I) De TonI, Sylloge Algarum II, pag. 492-493. 


\section{Homoeocladia C. Ag.}

Homoeocladia Martiana C. Ag. Consp. crit. Diatom. (1830) pag. 25 ; Kuetz. Die Kieselsch. Bacillarien (1844) pag. 110, tab. 30, fig. 83; De Toni Sylloge Algarum II, pag. 554.

Nitzschia $($ H.) Martiana Van Heurck Traité des Diatomées (1899) pag. 406, tab. 33, fig. 881 .

Hab. Nuova Zelanda: Bluff.

La sistematica delle Nitzschiaceae s' avvia ad essere molto tentennante, a causa dei criterii soggettivi dei diatomologi, de' quali alcuni sono troppo analitici, altri troppo sintetici; così avviene che si verificano pentimenti continui, non senza grave intralcio per gli studiosi nè senza ingombri per le sinonimie e le citazioni. Ci sembra, ad esempio, strano che il Van Heurck nel suo Traité des Diatomées a pag. 380 tenga distinti per le Nitzschiaceae i tre generi Nitzschia, Hantzschia e Homoeocladia e poi, con tutta disinvoltura, a pag. 406, non accetti più il genere Homoeocladia.

\section{Nitzschia (Hass.) Grun. (1)}

Nitzschia amphibia Grun. Die Oesterr. Diatom. (1862) pag. 574, tab. 12, fig. 23 ; V. H. Synopsis pag. 184, tab. 68, fig. 15-17; De Toni Sylloge Algarum II, pag. 543.

Hab. Nuova Zelanda: Rotorua, Ohinemuthu.

Specie già nota per questa località e per altre vicine, distribuita da TEMpère e Peragallo nelle Diatomées du monde entier (n. 273: lago Rotoreja; n. 920: Pakaraka, deposito fossile d' acqua dolce).

Nitzschia distans Greg. Diat. of Clyde (1857) pag. 58, tab. 6, fig. 103; V. H. Synopsis tab. 62, fig. 10, Traité pag. 394, tab. 33, fig. 878; De Toni Sylloge Algarum II, pag. 524.

Hab. Nuova Zelanda: Port Charmers.

Varietà di questa specie sono note dell' Oceano Pacifico per le coste dell'Australia e di parecchie isole; di queste ultime la si trova distribuita da Tempère e Peragallo nelle Diatomées du monde entier ai n. 647-648 (isole Samoa).

(I) Il genere Sigmatella istituito dal KUeTzIng nel 1833 (Algarum aquae dulcis Germanicarum Decades I, n. 2) venne 1' anno successivo dallo stesso autore (Synopsis Diatomearum pag. 26) incluso nel genere Frustulia C. Ag. accolto con limiti così stravaganti da permettere di riferirvi Diatomee di generi diversissimi (Navicula, Cymbella, Encyonema, Amphora, Gomphonema, Amphipleura, Cymatopleura, Synedra, Epithemia, Nitzschia, Pleurosigma, e persino Cyclotella'). Se si rifletta che del suo genere il Kuetzing non aveva un chiaro concetto in quanto che nel 1832 (in litteris) denominava Sigmatella acuta un vero e proprio Pleurosigma (il Pl.acuminatum) e nel 1833 (nelle Alg. Dec. I.) distribuiva col nome di Sigmatella Nitzschii una genuina specie di Nitzschia, si capisce come il genere Sigmatella cosi mal limitato nella mente dello stesso suo autore (nel I844 il KUETzıng implicitamente lo incluse nelle Synedra) non abbia motivo di venire preferito al nome Nitzschia proposto nel 1845 da Hassall. 
Nitzschia dubia W. Sm. Brit. Diat. I (1853) pag. 41, tab. 13, fig. 112 ; V. H. Synopsis tab. 59, fig. 9-12, Traité pag. 389, tab. 15, fig. 508; De Toni Sylloge Algarum II, pag. 511.

Hab. Tasmania: Burmie; Nuova Zelanda: Rotorua, Geyser.

E una specie che fu trovata promiscua, vegetando così nelle acque dolci, come nelle salse.

Nitzschia FrauenfeldiI Grun. in Cleve et Grun. Arct. Diat. (1880) pag. 98; V. H. Synopsis tab. 68, fig. 18; De Toni Sylloge Algarum II, pag. 545.

Bacillaria Frauenfeldii Grun. Die Oesterr. Diatom. (1862) pag. 584, tab. 12, fig. 1.

Hab. Nuova Zelanda: Rotorua, Ohinemuthu, geyser.

La specie venne per la prima volta indicata per il lago Wahiria (is. Tahiti); una sua var. major venne distribuita da Tempère e Peragallo nelle Diatomées du monde entier n. 44, del limo nella foce della Riviera Saint-Denis (isola Riunione).

Nitzschia GRacilis Hantzsch in Hedwigia II (1859) tab. 6, fig. 8; V. H. Synopsis tab. 68, fig. 11-12; De Toni Sylloge Algarum II, pag. 540.

Hab. Tasmania: Hobart. nerale.

Questa specie è nuova per la flora tasmanica e anche dell' Oceania in ge-

Nitzschia linearis (Ag.) W. Sm. Brit. Diat. 1 (1853) pag. 39, tab. 13, fig. 10 ; V. H. Synopsis tab. 67, fig. 13-15, Traité pag. 399, tab. 16, fig. 542; De Toni Sylloge Algarum II, pag. 535.

Hab. Nuova Zelanda: Rotorua, Ohinemuthu.

Var. TenuIs Grun. in Cleve et Grun. Arct. Diat. (1880) pag. 93; V. H. Synopsis tab. 67, fig. 16, Traité pag. 399, tab. 16, fig. 543; De Toni Sylloge Algarum II, pag. 536.

Hab. Tasmania: Hobart.

Tanto la specie quanto la varietà sono da aggiungere alle flore della Tasmania e della Nuova Zelanda.

Nitzschia marginulata Grun. in Cleve et Grun. Arct. Diat. (1880) pag. 72, tab. 5, fig. 93; V. H. Synopsis tab. 58, fig. 13; De Toni Sylloge Algarum II, pag. 503.

Hab. Tasmania: Burmie.

La Nitzschia marginulata Grun. ha estesa distribuzione geografica e non manca all' Oceano Pacifico. Si trova nei preparati delle Diatomées du monde entier di Tempère e Peragallo dello stretto del Re Giorgio (n. 276-277) e delle isole Thursday (n. 592-593).

Nitzschia obtusa W. Sm. Brit. Diat. I (1853) pag. 39, tab. 13, fig. 109; De Toni Sylloge Algarum II, pag. 533.

Var. scalpelliformis Grun. in Cleve et Grun. Arct. Diat. (1880) pag. 92 ; V. H. Synopsis tab. 67, fig. 2, Traité pag. 397, tab. 16, fig. 538; De Toni Sylloge Algarum II, pag. 534. 
Hab. Australia merid.: Victor Harbour; Nuova Zelanda: Rotorua, geyser $a$ e $b$, Ohinemuthu. Nei geyser anche la forma nana Grun. (cfr. Cleve et Moeller Diat. Typ. n. 293).

Nitzschia obtusa W. Sm. è un elemento assai comune nelle saline e nelle acque dolci termali e nelle submarine; come tale ha un'estesissima distribuzione geografica, può dirsi anzi cosmopolita (1). E tra Nitzschia obtusa W. Sm. tipica e la var. scalpelliformis Grun. più che tutto intercede la differenza nella lunghezza dei frustoli e si ha una lieve differenza nel rilievo delle sculture un poco più sottili nella seconda. Entrambe vennero usate come preparato di prova per i microscopii.

Nitzschia Palea (Kuetz.) W. Sm. Brit. Diat. II (1856; De Toni Sylloge Algarum II, pag. 540.

Var. TEnUIRostris Grun. in V. H. Synopsis pag. 183, tab. 69, fig. 31, Traité pag. 402, tab. 17, fig. 556; De Toni Sylloge Algarum II, pag. 541.

$H a b$. Nuova Zelanda: Rotorua, geyser $b$.

E una varietà della specie la quale ultima trovasi talora nelle sorgenti minerali, come ne offre un esempio quella preparazione di Saint-Floret (Puy-deDome) distribuita al n. 887 delle Diatomées $d u$ monde entier di Tempére e PeRAGALLO.

Nitzschia panduriformis Greg. Diat. of Clyde (1857) pag. 57, tab. 6, fig. 102 ; Hantzsch Diat. aus dem Ostindischen Archipel (1862) pag. 20, fig. 7 ; Cleve et Grun. Arct. Diat. (1880) pag. 71, tab. 5, fig. 92; V. H. Synopsis tab. 58, fig. 1-3, Traité pag. 386, tab: 15, fig. 500; De Toni Sylloge Algarum II, pag. 501.

Hab. Australia occid. : Baia di Geroldton; Nuova Zelanda: Port Charmers.

Il Petit (Diat. Campb. et .V. Zél. pag. 29) indicò questa Nitzschia per l'isola Campbell, lo stretto di Foveaux e la Lyall's Bay dov' essa vegeta in grandissima copia. I Peragallo ed il Mereschkowsky ricordano la stessa specie per le isole Samoa e Hawaii ; di parecchie località dell' Oceania la distribuirono TEMpère e Peragallo nelle Diatomées du monde entier: di Albany, Australia occid. (n. 160, 276-277), dello stretto di Torres (n. 946) delle Nuove Ebridi (n. 61, 63), di Yarra River (n. 241-243), delle isole Thursday (n. 561-562, 590-593), dell' is. Campbell (n. 393) e Mc Donald (n. 634), di lavaggi di conchiglie provenienti dai lidi della Nuova Zelanda (n. 478-479) ecc. La specie ha del resto una larga distribuzione geografica e può quasi considerarsi cosmopolita.

Nitzschia punctata (W. Sm.) Grun. in Cleve et Grun. Arct. Diat. (1880) pag. 68 ; De Toni Sylloge Algarum II, pag. 496.

Tryblionella punctata W. Sm. Brit. Diat. I (1853) pag. 36, tab. 10 fig. 76 a e tab. 30, fig. 261.

Var. Elongata Grun. in Cleve et Grun. op. cit. pag. 68; V. H. Synopsis tab. 57, fig. 3, Traité pag. 385, tab. 15, fig. 492; De Toni Sylloge Algarum II, pag. 496.

(I) Cfr. a questo proposito ForTI ACH., Enumerazione di alcune Alghe rinvenute nelle acque termali marine dell' isola Vulcano (Eolie) raccolte dal dott. Ottorino de Fiore (Bullettino della Soc. botan. ital. 1920, $n, 2-6$ ). 
Hab. Nuova Zelanda: Port Charmers.

A quanto ci consta, è nuova per la flora dell' Oceano Pacifico.

Nitzschia Rigida (Kuetz.) Perag. Diat. mar. de France (1900) pag. 291, tab. 74 , fig. 8-9.

Amphipleura rigida Kuetz. Die Kieselschal. Bacillarien (1844) pag. 104, tab. 4, fig. 30.

Nitzschia Sigma var. rigida Grun. Kasp. Alg. (1878) pag. 119; V. H. Synopsis tab. 66, fig. 2, Traité pag. 396, tab. 16, fig. 533; De Toni Sylloge Algarum II, pag. 531.

Hab. Nuova Zelanda: Bluff.

E specie, al pari della seguente, assai comune tanto negli Oceani quanto nei mari interni.

Nitzschia Sigma (Kuetz.) W. Sm. Brit. Diat. I (1853) pag. 39, tab. 13, fig. 108; V. H. Synopsis tab. 65, fig. 7-8, Traité pag. 396, tab. 16, fig. 531; De Toni Sylloge Algarum II, pag. 530.

Hab. Australia occid.: Baia di Geroldton; Tasmania: Baia di Latrobe; Nuova Zelanda: Port Charmers, Bluff.

Come la precedente, è specie con larga distribuzione geografica; una sua varietà indeterminata, proveniente dal porto neozelandese di Oamaru, venne distribuita da Tempère e Peragallo nelle Diatomées du Monde entier n. 774. Per l'Oceania fu segnalata delle isole Samoa dai Peragallo, oltre ai dati (Auckland, isole Nicobar, Tahiti) forniti dal Grunow dall' esame dei campioni raccolti durante la spedizione della "Novara ; è nota anche per le isole degli Amici (Diatomées du monde entier n. 591).

Var. Sigmatella (Greg.) Grun. Kasp. Alg. (1878) pag. 119; V. H. Synopsis tab. 66, fig. 6-7, Traité pag. 397, tab. 16, fig. 535.

Hab. Australia occid. : Baia di Geroldton.

Tempère e Peragallo nelle Diatomées du monde entier la distribuirono di Albany, Australia occidentale (n. 160) e dello stretto di Malacca (n. 622-623).

Nitzschia thermalis (Ehr.) Auersw. in Rabenh. Algen n. 1064 et 1266; Grun. Die Oesterr. Diatom. (1862) pag. 568, tab. 12, fig. 22; V. H. Synopsis tab. 59, fig. 20, Traité pag. 389, tab. 15, fig. 509; De Toni Sylloge Algarum II, pag. 512.

Hab. Nuova Zelanda: Rotorua, Ohinemuthu. saline.

E una specie non rara, come lo indica il nome, nelle acque termali e nelle

Nitzschia vitrea Norm. in Microsc. Journal IX (1861) pag. 7, tab. 2, fig, 4 ;

De Toni Sylloge Algarum II, pag. 536.

Var. RECTA (Hantzsch) Van Heurck Synopsis tab. 67, fig. 17-18, Traité pag.

400, tab. 16, fig. 547; De Toni Sylloge Algarum II, pag. 536.

Nitzchia recta Hantzsch in Rabenh. Algen n. 1283.

$H a b$. Tasmania: Baia di Latrobe.

Finora questa varietà non era segnalata per la Tasmania. 


\section{Hantzschia Grun.}

Hantzschia amphioxys (Ehr.) Grun. in Cleve et Grun. Arct. Diat. (1880) pag. 103 ; V. H. Synopsis tab. 56, fig. 1-2, Traité pag. 381, tab. 15, fig. $483 b$; De Toni Sylloge Algarum II, pag. 561.

Hab. Nuova Zelanda: Rotorua, Ohinemuthu.

L'EHRENBERg (Mikrogeologie 1854, pag. 2-7) segnalò già questa specie per la Tasmania e parecchie località dell' Australia occidentale; quanto alla Nuova Zelanda la Hantzschia amphioxys (Ehr.) Grun. trovasi indicata per il distretto di Otago dal Lauder Lindsay (Diat. of Otago pag. 132) e venne distribuita fossile nelle Diatomées du monde entier di Tempère e Peragallo di Cabbage Tree Swamp, Auckland (n. 223), di Auckland (n. 919), di Pakaraka (n. 920) e di Waitangi (n. 922).

Var. intermedia Grun. in Van Heurck Synopsis tab. 56, fig. 4, Traité pag. 381, tab. 15, fig. $485 b$; De Toni Sylloge Algarum II, pag. 563.

Hab. Tasmania: Burmie; Nuova Zelanda: Bluff.

Hantzschia marina (Donk.) Grun. in Cleve et Grun. Arct. Diat. (1880) pag. 105; V. H. Traité pag. 382, tab. 15, fig. $489 b$; Perag. Diat. mar. de France tab. 71, fig. 19-20; De Toni Sylloge Algarum II, pag. 564.

Hab. Tasmania: Burmie; Nuova Zelanda: Bluff.

Questa specie è diffusa a tutti gli oceani e mari interni ed è prettamente neritica.

Hantzschia virgata (Rop.j Grun. in Cleve et Grun. Arct. Diat. (1880) pag. 104 ; V. H. Synopsis pag. 169, tab. 56, fig. 12-13; De Toni Sylloge Algarum II, p. 563.

Nitzschia virgata Roper in Microsc. Journ. Vl (1858) pag. 23, tab. 3, fig. 6.

$H a b$. Australia occid.: Baia di Geroldton; Nuova Zelanda: Bluff.

Sembra che questa specie non sia stata finora segnalata per l' Oceano $\mathrm{Pa}$ cifico.

Var. SUBTILIOR n. var.

Hab. Nuova Zelanda: Bluff.

L' esemplare di Bluff non supera $75 * 6 \mu$; è ancora più esiguo di quelli della forma tipica; anche un' altra forma che si avvicina alla tipica vi si osserva frammischiata ma non supera $125 \mu$ di lunghezza per $13 \mu$ di larghezza. Gli

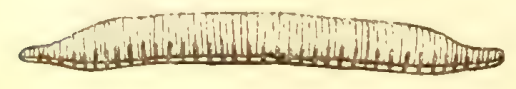

I

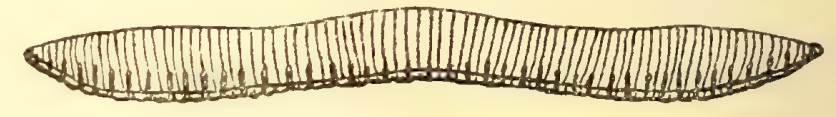

2

1. Hantzschia virgata (Rop.) Grun, var. subtilior.

2. Hantzschia virgata for. in typicam accedens. $-\times 640$.

esemplari tipici francesi di Tempère e Peragallo hanno le dimensioni $140 * 17 \mu$, altri distribuiti da H. L. Sмiтн (Typ. n. 375 di Villerville) misurano $100-120 *$ $12-16 \mu$, altri infine di un preparato di Тнuм del Mare del Nord hanno 115-180 $\mu$ di lunghezza per 16-18 $\mu$ di larghezza. 


\title{
Fam. SURIRELLACEAE (Knetz.) Grun.
}

\author{
Surirella Turp.
}

Surirella contorta Kitton in Microsc. Journ. XII (1874) pag. 220, tab. 81, fig. 4 ; A. Schmidt Atlas tab. 56, fig. 2; De Toni Sylloge Algarum II, pag. 576.

Hab. Nuova Zelanda: Wakarevareva.

F. Kitron descrisse la sua specie su materiali provenienti dall' is. Auckland; da un esemplare autentico è tratta la figura sopra citata di A. SchmidT; corrispondono bene i frustoli di Wakarevareva col n. 290 delle Diat. di Cleve e Moeleer. La Surirella contorta Kitt. trovasi in Tempère e Peragallo (Diatomées du monde entier n. 478-479) tra Diatomee provenienti da lavaggi di conchiglie di Haliotis della Nuova Zelanda. Il Campylodiscus contortus Pantoc. fossile in Ungheria, ridescritto da uno di noi ${ }^{(1)}$, quantunque sia molto affine alla Surirella spiralis Kuetz. e sotto questo punto di vista somigli assai ad una Suvirella è una specie ben diversa dalla presente del KitTon, interessantissima perchè invece di essere affine al Campylodiscus rammenta la Cymatopleura.

Surirella fastuosa Ehr. in Abhandl. Berl. Akad. 1841, pag. 19; Grun. Die Oesterr. Diatom. (1862) pag. 461, tab. 9, fig. 11-12; V. H. Synopsis tab. 73, fig. 18; Traité pag. 372, tab. 13, fig. 583; De Toni Sylloge Algarum II, pag. 582.

Hab. Tasmania: Burmie; Nuova Zelanda: Port Charmers, Bluff.

Il Petit (Diat. Campb. et N. Zél. pag. 30) segnalò questa specie per la Lyall's Bay e lo stretto di Foveaux; i Peragallo la indicarono per le isole Hawaii, Samoa e Salomone, il Mereschkowsky per Tahiti. Tempère e Peragallo la distribuirono nelle Diatomées du monde entier di parecchie località : Nuove Ebridi (n. 61, 63), Albany, Australia occidentale (n. 160, 276-277), Yarra River, Melbourne (n. 241-243), is. Campbell (n. 357, 445-447), is. Thursday (n. 561-562, 590-593), stretto di Malacca (n. 622-623) is. Caroline (n. 621) ecc.

SuRIRElla Gracilis Grun. Die Oesterr. Diatom. (1862) pag. 450, tab. 10, fig. 11 ; V. H. Synopsis tab. 76, fig. 16.

Hab. Nuova Zelanda: Wakarevareva.

E da aggiungersi questa specie alla flora neozelandese.

Surirella inducta A. Schmidt Atlas (1885) tab. 20, fig. 10, tab. 24, fig. 15-25; De Toni Sylloge Algarum II, pag. 596.

Hab. Nuova Zelanda: Rotorua, Ohinemuthu, Geyser a.

È specie nuova per la flora neozelandese. Tempére e Peragallo (Diatomées du monde entier) la distribuirono di Yarra River (n. 241-243) e fossile di Talbot, Melbourne (n. 981-983: var. intermedia M. Perag.).

Surirella intercedens Grun. in A. Schmidt Atlas (1885) tab. 19, fig. 5-6.

(1) Cfr. Forti ACr., Contribuzioni Diatomologiche X. Diatomacee quaternarie e subfossili d'acqua dolce d'Etiopia raccolte dal dott. Giovanni Negri pag. 1280 e 1293 (Atti del Reale Istituto Veneto di scienze lettere ed arti Tomo LXIX, parte seconda; Venezia 1910). 
Var. ABLudens Grun. in Perag. Diat. mar. de France (1900) pagina 250, tab. 60, fig. 3-4.

Surivella fastuosa var. abludens Grun. in Castrac. Diat. of Challenger (1886) pag. 59.

Hab. Nuova Zelanda: Port Charmers.

È specie da aggiungere alla flora marina della Nuova Zelanda.

Surirella ovalis Bréb. Considérations sur les Diatomées (1838) pag. 17; Kuetz. Die Kieselsch. Bacillarien (1844) pag. 61, tab. 30, fig. 64; V. H. Synopsis tab. 73, fig. 2, Traité pag. 373, tab. 13, fig. 585 ; De Toni Sylloge Algarum II, pag. 579.

Hab. Nuova Zelanda: Rotorua, Ohinemuthu.

Tempère e Peragallo la distribuirono nelle Diatomées du monde entier fossile di Waitangi (n. 922), nonchè dello stretto di Malacca (n. 622-623: var. Kotschyana).

Var.? angusta (Kuetz.) V. H. Synopsis tab. 73, fig. 12, Traité pag. 373, tab. 13, fig. 590 ; De Toni Sylloge Algarum II, pag. 580.

Hab. Tasmania: Hobart; Nuova Zelanda: Wakarevareva, Rotorua, Ohinemuthu.

La diversità perimetrica non fu ritenuta dal VAN HEURCK carattere sufficiente a convalidare la distinzione specifica proposta dal Kuetzing.

Var. Excelsa O. Muell. Bacill. aus den Natronth. von El Kab (1899) pag. 315, tab. 10, fig. 1 .

$H a b$. Nuova Zelanda: Rotorua, Ohinemuthu.

Esemplari in tutto corrispondenti alla figura originale di O. Mueller. La varietà è nuova per la Nuova Zelanda.

Surirella Robusta Ehr. in Berichte Berl. Akad. 1840, pag. 215; V. H. Synopsis tab. 71, fig. 1-2, Traité pag. 371, tab. 12, fig. 577; De Toni Sylloge Algarum II, pag. 571.

Hab. Tasmania: Hobart.

È specie da aggiungere alla flora tasmanica.

SuriRella splendida (Ehr.) Kuetz. Die Kieselsch. Bacillarien (1844) pag. 62, tab. 12, fig. 9; De Toni Sylloge Algarum II, pag. 571.

Surivella robusta var. splendida V. H. Synopsis tab. 72, fig. 4, Traité pag. 371, tab. 12, fig. 578 .

Hab. Nuova Zelanda: Wakarevareva.

Il LaUder Lindsay (Diat. of Olago pag. 132) segnalò già questa specie per il distretto di Otago, come la specie seguente.

Surirella tenera Greg. in Microsc. Journ. IV (1856) pag. 10, tab. 1, fig. 38; De Toni Sylloge Algarum II, pag. 582.

Surivella robusta var. tenera Van Heurck Traité (1899) pag. 371, tab. 12, fig. 579.

Hab. Tasmania: Burmie.

Venne da Tempère e Peragallo distribuita nelle Diatomées du monde entier. Questa specie e la precedente sono tanto affini alla Surivella robusta Ehr. che secondo il VAN HEURCK ne rappresentano semplici varietà. 


\section{Campylodiscus Ehr.}

Campylodiscus Lorenzianus Grun. Die Oesterr. Diatom. (1862) pag. 442, tab. 11, fig. 1.2; A. Schmidt Atlas tab. 14, fig. 24, tab. 18, fig. 4.

Hab. Tasmania: Baia di Latrobe.

Questa specie ci risulta nuova per l'Oceania.

Campylodiscus samoënsis Grun. in A. Schmidt Atlas (1874) tab. 15, fig. 19-20; De Toni Sylloge Algarum II, pag. 609.

Hab. Tasmania: Baia di Latrobe; Nuova Zelanda: Port Charmers, Bluff.

Il Petit (Diat. Campb. et N. Zél. pag. 31) segnalò questo Campylodiscus per la Lyall's Bay, lo Stretto di Foveaux e l'is. di Campbell; da sondaggi compiuti presso quest' isola provengono i materiali distribuiti da TemPère e Peragallo al n. 357 delle Diatomées du monde entier. È specie comune in Oceania perchè ne fu constatata la presenza dai Peragallo alle isole Samoa, Hawaii e Salomone, dal Mereschkowsky a Tahiti; si aggiunga che Tempère e Peragallo la distribuirono nelle Diatomées $d u$ monde entier anch' essi dell' is. Samoa (n. 647-648), nonchè dell' is. Thursday (n. 591-593), della Nuova Zelanda da lavaggi di conchiglie di Haliotis (n. 479-479) e di Albany, Australia occidentale (n. 276-277).

Campylodiscus Thureti Bréb. Diat. Cherbourg (1867) pag. 13, tab. 1, fig. 3; V. H. Synopsis tab. 77, fig. 1, Traité pag. 378, tab. 14, fig. 595; Deby Campylodiscus tab. 7, fig. 37 ; De Toni Sylloge Algarum II, pag. 622.

Hab. Australia merid. : Victor Harbour; Nuova Zelanda: Bluff.

Tempère e Peragallo distribuirono questo Campylodiscus nelle Diatomées du monde entier del porto di Oamaru (n. 774) e dei lavaggi di conchiglie di Haliotis provenienti dalla Nuova Zelanda (n. 478-479).

\section{Stenopterobia Bréb.}

Stenopterobia anceps (Lew.) Bréb. in litt. ad Kitton (1866); Héribaud Diat. d'Auvergne (1893) pag. 182, tab. 4, fig. 4.

Surirella anceps Lewis Note on new or rare species of Diat. of the U. S. Seabord (1861) pag. 342, tab. 1, fig. 3; De Toni Sylloge Algarum II, pag. 590.

Hab. Nuova Zelanda: Rotorua, Ohinemuthu.

Dopo molte perplessità questo genere sembra definitivamente separato da Surivella Turp.

La Stenopterobia anceps (Lew.) Bréb. è una specie la quale pare adattabile a vivere nelle acque dolci e subsalse e trovasi anche allo stato fossile; pur non mancando all' Europa, la specie è assai largamente rappresentata nell'America settentrionale (Cạnadà, Maine, ecc.). 


\section{Fam. ACHNANTHACEAE (Kuetz.) Grun.}

\section{Achnanthes Bory}

Achnanthes brevipes C. Ag. Systema Algarum (1824) pag. 1 ; V. H. Synopsis tab. 26, fig. 10, 12 ; De Toni Sylloge Algarum II (1891) pag. 471.

Hab. Australia occid.: Baia di Geroldton; Australia merid.: Victor Harbour; Nuova Zelanda: Port Charmers, Bluff.

Il Grunow (Alg. Novara pag. 7) descrisse come varietà l'Achnanthes capensis Kuetz. (Die Kieselsch. Bacull. 1844, pag. 76, tab. 21, fig. 1) raccolta durante il viaggio della « Novara al Capo di Buona Speranza, ad Auckland e all' isola di S. Paolo, questa sembrerebbe piuttosto una forma vicariante dei mari Australi che non una genuina varietà morfologicamente ben definita. Achnanthes brevipes Ag. è conosciuta anche per Port Stevens (Tempère e Peragallo, Diatomées du monde entier n. 398, 399) delle isole Samoa (n. 647-648) e del porto neozelandese di Oamaru (n. 774).

Var. Angustata Meresch. On Polynesian Diatoms (1902) pag. 11, tab. 4, fig. 21 (sub Achnanthidio).

Hab. Australia occid.: Baia di Geroldton; Nuova Zelanda: Bluff.

Questa varietà più sottile della forma tipica venne originariamente scoperta alle isole Samoa. Achnanthes glabrata Grun. delle Nuove Ebridi è molto affine a questa ma ancora più sottile.

Achnanthes inflata (Kuetz.) Grun. Alg. Novara (1867) pag. 98; De Toni Sylloge Algarum II (1891) pag. 475. fig. 22 ?

Stauroneis inflata Kuetz. Die Kieselsch. Bacillarien (1844) pag. 105, tab. 30,

Hab. Nuova Zelanda: Wakarevareva.

Questa specie venne raccolta nel " Kieselguhr» di Auckland (HochsteTter) e trovata nella terra edule dell' isola di Giava secondo il GRUnow; poi venne riportata dal Frauenfeld come raccolta nel lago * Wahiria » dell' is. Tahiti. Fu inoltre distribuita da Tempère e Peragallo nelle Diatomées du monde entier di parecchie località dell' Oceania: di Pakaraka, fossile in deposito d'acqua dolce (n. 920), di Cabbage Tree Swamp, Auckland pure fossile (n. 223), degli stagni di West Melbourne, Victoria (n. 947), dell' is. Mc. Donald (n. 634) e di Talbot, Melbourne, allo stato fossile (n. 981-983).

Achnanthes lanceolata (Bréb.) Grun. in Cleve et Grun. Arct. Diat. (1880) pag. 23 ; V. H. Synopsis tab. 27, fig. 8-11; De Toni Sylloge Algarum II, pag. 486. Achnanthidium lanceolatum Bréb. in Kuetz. Species Algarum (1849) pag. 54.

Hab. Nuova Zelanda: Wakarevareva.

Rinvenuta fossile e come tale distribuita da Tempère e Peragallo nelle Diatomées du monde entıer di Pakaraka (n. 920), di Cabbage Tree Swamp, Auckland (n. 223) e di Brunswick, Australia (n. 921).

Var. Dubia Grun. in Cleve et Grun. Arct. Diat. (1880) pag. 23; V. H. Synopsis tab. 27, fig. 12-13; De Toni Sylloge Algarum II, pag. 486.

Hab. Nuova Zelanda: Wakarevareva. 
Var. Inflata A. Mayer Die Bacill. des Regensburger Gewaesser (1917) pag. 81, tab. 14, fig. 35 (sub Achnanthidio, non Achnanthes inflata Grun).

Hab. Nuova Zelanda: Wakarevareva.

Achnanthes longipes C. Ag. Systema Algarum (1824) pag. 1; V. H. Synopsis tab. 26, fig. 13-16, Traité pag. 279, tab. 8, fig. 323; De Toni Sylloge Algarum II, pag. 470.

Hab. Australia occid.: Baia di Geroldton.

Il Petit (Diat. Campb. et N. Zél. pag. 16) indicò l'Achnanthes longipes Ag. per la Nuova Zelanda nella Lyall's Bay; i Peragallo la riscontrarono tra le Diatomee provenienti dalle isole Samoa, delle quali fu distribuita ai n. 647-648 delle Diatomées du monde entier di Tempère e Peragallo; in questa collezione trovansi anche saggi provenienti dall' isola S. Paolo (n. 432) e delle isole Thursday (n. 592-593).

Forma Lata H. Perag. in H. et M. Peragallo, Diat. mar. de France (1900) tab. 1, fig. 10-12.

Hab. Australia occid.: Baia di Geroldton.

Questa forma venne soltanto figurata su materiali provenienti da Agde (Hérault) sulle sponde del Golfo del Leone, si distingue subito per le valve e i frustoli più tozzi.

Achnanthes subsessilis Kuetz. Alg. Dec. (1833) n. 42, Die Kieselsch. Bacillarien (1844) pag. 76, tab. 20, fig. 4; V. H. Synopsis tab. 26, fig. 21-24-, Traité pag. 279, tab. 8, fig. 325; De Toni Sylloge Algarum. II, pag. 473.

Achnanthes brevipes var. intermedia Cleve Syn. Navic. Diatoms II (1895) pag. 193.

Hab. Nuova Zelanda: Port Charmers, Bluff.

Il Petit (Diat. Campb. et N. Zél. pag. 16) enumerò questa Achnanthes per la Nuova Zelanda alla Lyall's Bay, così che dagli esemplari provenienti dalle raccolte di alghe del rev. G. CAPRA risulta confermata la presenza della specie ai lidi neozelandesi.

\section{Fam. CoCCONEIDACEAE (Kuetz.) Grun.}

\section{Campyloneis Grun.}

Campyloneis Argus Grun. Die Oesterr. Diatom. (1862) pag. 429, tab. 10, fig. 9; De Toni Sylloge Algarum II, pag. 441.

Campyloneis Grevillei var. Argus V. H. Synopsis tab. 28, fig. 15-16, Traité pag. 285, tab. 8, fig. 345 ; Cleve Syn. Navic. Diatoms II (1895) pag. 167.

Hab. Australia occid. : Baia di Geroldton.

Il Petit (Diat. Campb. et N. Zél. pag. 13) indicò questa specie per la Lyall's Bay della Nuova Zelanda; notisi che la Campyloneis Argus Grun. fu osservata anche fossile nel deposito di Oamaru (GROVE e STURT) e che accompagna la precedente al n. 160 (provenienza King George's Sound) delle Diatomées du monde entier di Temperre e Peragallo. Il Grunow trattando nuovamente del genere Campyloneis (Alg. Novara 1867, pag. 10-12) fece tipo del genere la Cocconeis Grevillei W. Sm. e ascrisse alla Campyloneis Grevillei come sottospecie la 
Campyloneis Argus Grun. e la Campyloneis regalis (Grev.); per entrambe queste sottospecie il Grunow descrisse varietà nuove, per quella una var. reticulata, per questa una var. obliqua. Il Cleve (Syn. Navic. Diatoms II, 1895, pag. 167) semplificò le cose, riducendo la Campyloneis Argus Grun. a varietà della Camp. Grevillei e cosi pure la Camp. regalis, in certo qual modo seguendo lo schema proposto nel 1867 dal Grunow, ma accordando un valore di sottordine ai caratteri che quest' ultimo diatomologo aveva giudicato bastevoli a mantenere le sottospecie. Non è improbabile che la varietà reticulata Grun. altro non sia che la Cocconeis caelata Arn. (Campyloneis caelata Chase) la quale viene dal Cleve (op. cit. I, 1894, pag. 99) riferita alla Diploneis Campylodiscus (Grun.),

Campyloneis Grevillei (W. Sm.) Grun. et Eul. Alg. Novara (1867) pag. 10, 98 ; V. H. Synopsis tab. 28, fig. 10-12 ; Traité pag. 285, tab. 8, fig. 344; De Toni Sylloge Algarum II, pag. 439.

Hab. Australia occid. : Baia di Geroldton ; Australia merid. : Victor Harbour; Tasmania: Baia di Latrobe; Nuova Zelanda: Port Charmers.

La Campyloneis Grevillei (W. Sm.) Gr. et Eul. è specie abbastanza frequente nell' oceano Pacifico; dalla Nuova Zelanda vennero riportati materiali contenenti questa specie dalla spedizione della "Novara»; essa fu indicata per Tahiti, le isole Samoa, Hawaii ecc. Tempère e Peragallo nelle Diatomées du monde entier ne fecero distribuzione di alcune interessanti località: Yarra River, Melbourne (n. 241-243), costa della Nuova Zelanda (n. 478-479), Stretto di Malacca, (n. 622-623), isola S. Paolo (n. 432) ecc.

\section{Cocconeis Ehr.}

Cocconeis dirupta Greg. Diat. of Clyde (1857) pag. 19, tab. 1, fig. 25; V. H. Synopsis tab. 20, fig. 13-15, Traité pag. 290, tab. 8, fig. 343; Cleve Syn. Navic. Diat. II (1895) pag. 175; De Toni Sylloge Algarum II, pag. 453.

$H a b$. Tasmania: Baia di Latrobe.

La Cocconeis dirupta Greg., con le sue numerose varietà, ha estesa distribuzione geografica, anche nell' oceano Pacifico.

Cocconeis heteroidea Hantzsch in Rabenh. Beiträge 1 (1862) pag. 21, tab. 6 A, fig. 10; Cleve Syn. Navic. Diat. II (1885) pag. 178; De Toni Sylloge Algarum II, pag. 456.

Hab. Tasmania: Burmie; Nuova Zelanda: Port Charmers.

Anche questa Cocconeis ha larga distribuzione soprattutto nei mari caldi. Il Petit (Diat. Camp. et N. Zél. pag. 10) la indicò alla Lyall's Bay per la Nuova Zelanda; Tempère e Peragallo la distribuirono di numerose località dell'Oceania nelle Diatomées du monde entier come di Albany, Australia occidentale (n. 160, 276-277), delle Nuove Ebridi (n. 61-63), di Yarra River (n. 241-243) delle isole Thursday (n. 590-91), delle is. Caroline (n. 621) delle isole Samoa (n. 647-648), dello stretto di Malacca (n. 623) ecc.

Che la Cocconeis flexella Jan. et Rabenh. (Diat. Honduras 1862, pag. 7, tab. I, fig. 11) vada riferita alla valva inferiore della Cocconeis heteroidea Hantzsch come fu sostenuto dal Grunow (Alg. Novara 1867, pag. 12) non si può affermare con assoluta sicurezza; il CLEVE (op. cit. pag. 175) la giudica invece una varietà della Cocconeis dirupta Greg. 
Cocconets molesta Kuetz. Die Kieselsch. Bacillarien (1844) pag. 71, tab. 5, fig. VII, $1-2$; V. H. Synopsis tab. 30, fig. 18-23, Traité pag. 290, tab. 29, fig. 823; Cleve Syn. Navic. Diat. II (1895) pag. 174; De Toni Sylloge Algarum II, pag. 459.

Hab. Tasmania: Burmie.

Var. amygdalina (Bréb.) Grun. in V. H. Synopsis tab. 30, fig. 5, Traité pag. 291, tab. 29, fig. 823 bis; Cleve Syn. Navic. Diat. II (1895) pag. 174.

Hab. Tasmania: Burmie.

Forma Minor H. Perag.

Hab. Tasmania : Burmie.

Epifita gregaria ricopre densamente con i suoi frustoli le alghe maggiori.

Cocconeis Pediculus Ehr. Die Infusionsthierchen (1838) pag. 194, tab. 21, fig. 11 ; V. H. Synopsis tab. 30, fig. 28-30, Traité pag. 288, tab. 8, fig. 340; Cleve Syn. Navic. Diat. II (1895) pag. 169; De Toni Sylloge Algarum II, pag. 452.

Hab. Tasmania: Hobart.

Questa specie, assai comune nelle acque d'Europa e d'America, non manca alla flora dell' Oceania, essendo stata segnalata per la Nuova Zelanda dal LAUDER Lindsay (Diat. of Otago pag. 133) e al lago Huro per le isole Chatham dal LEMMERMANN.

Cocconeis Placentula Ehr. Die Infusionsthierchen (1838) pag. 194; V. H. Synopsis tab. 30, tig. 26-27, Traité pag. 288, tab. 8, fig. 341; Cleve Syn. Navic. Diat. II (1885) pag. 169; De Toni Sylloge Algarum II, pag. 454.

Hab. Tasmania: Hobart; Nuova Zelanda: Wakarevareva, Rotorua, Ohinemuthu.

E specie comunissima e promiscua alle acque dolci e subsalse. L'EHRENBERG (Mikrogeologie 1854, pag. 6) ne avverti la presenza in materiali provenienti dal Cunning River, Australia occidentale; il LAUDER LindSAy (Diat. of Otago pag. 133) la indicó per il distretto di Otago nella Nuova Zelanda, il CLEve anche per la Tasmania. Per la Nuova Zelanda la Cocconeis Placentula Ehr. fu segnalata pure dal Grunow (Alg. Novara 1867, pag. 14) sulle Cladophora del lago Taupo e distribuita di Rotorua da Tempère e Peragallo al n. 273 delle Diatomées du monde entier ; in questa ultima collezione è pubblicata in campioni di depositi fossili di Wangarei (n. 918) e Waitangi nella Nuova Zelanda (n. 922) e di Talbot in Australia (n. 981-983).

Var. Lineata (Ehr.) Van Heurck Synopsis tab. 30, fig. 31-32, Traité pag. 288, tab. 8, fig. 342 ; Cleve Syn. Navic. Diat. II (1885) pag. 169; De Toni Sylloge Algarum II, pag. 454.

Hab. Tasmania: Baia di Latrobe; Nuova Zelanda: Wakarevareva.

La Cocconeis lineata Ehr. fu segnalata per la Nuova Olanda (Swan River, Avon River) fino dai tempi dell' EhrenBerg (Mikrogeologie 1854, pag. 4, 6); una sua forma venne distribuita dello Yarra River da Tempère e Peragallo nelle Diatomées du monde entier n. 241-243. Per la Nuova Zelanda trovasi indicata nella Lyall's Bay dal Petit (Diat. Campb. et N. Zél. pag. 11).

Cocconeis Pellucida Grun. in Hantzsch Diat. Ostind. Archip. (1862) pag. 21, tab. 6, fig. 11; Cleve Synopsis Navic. Diat. II (1895) pag. 178; De Toni Sylloge Algarum II, pag. 455. 
Hab. Tasmania: Baia di Latrobe; Nuova Zelanda: Port Charmers.

Per la Nuova Zelanda la specie era già nota a merito del Grunow ( $\mathrm{Alg}$. Novara pag. 12) e del Petit (Diat. Campb. et N. Zél. pag. 11); fu distribuita da Tempère e Peragallo nelle Diatomées du monde entier dello Stretto di Torres (n. 946), dell' is. Campbell (n. 357), di Yarra River (n. 241-243), delle isole Sandwich (n. 62-63), S. Paolo (n. 432), Thursday (n. 590-593) ecc.

Il GRunow ingenerò alquanta confusione rispetto alla Cocconeis pellucida inquantochè essa venne da lui unita con la Cocconeis pseudomarginata Greg. alla quale in realtà appartiene la Cocconeis pellucida Grun. descritta e figurata nella Memoria Ueber einige neue Diatomaceen (1863) pag. 145, tab. 13, fig. 6.

Cocconeis Pinnata Greg. in Microsc. Journal VII (1859) pag. 79, tab. 6, fig. 1; Cleve Syn. Navic. Diat. II (1895) pag. 181 ; V. H. Synopsis tab. 30, fig. 6-7, Traité pag. 288, tab. 29, fig. 818 .

Hab. Tasmania: Burmie.

Costituisce un' aggiunta alla flora tasmanica. Il GRunow (Alg. Novara 1867, pag. 10) ascrisse questa specie con un segno dubitativo alla Cocconeis brundusiaca Rabenh., nota per il Mare Adriatico e per l' Oceano Atlantico.

Cocconers Scutellum Ehr. Die Infusionsthierchen (1838) pag. 194, tab. 14, fig. 8; V. H. Synopsis tab. 29, fig. 3, 'Traité pag. 287, tab. 8, fig. 338; Cleve Syn. Navic. Diat. II (1895) pag. 170 ; De Toni Sylloge Algarum II, pag. 444.

Hab. Australia occid.: Baia di Geroldton; Australia merid.: Victor Harbour; Tasmania: Baia di Latrobe, Burmie; Nuova Zelanda: Port Charmers, Bluff.

É, può dirsi, la specie marina del genere Cocconeis la più largamente distribuita nei mari; con ogni probabilità la Cocconeis Scutellum Ehr. del Cunning River (indicata in EHRENBERG Mikrogeologie 1854, pag. 6) va ascritta a Cocconeis Pediculus Ehr. od a Cocconeis Placentula Ehr.; si trova distribuita di Australia e Nuova Zelanda nelle Diatomées du monde entier di Tempère e Peragallo.

Var. Maxima (Grun.) Cleve Syn. Navic. Diat. II (1895) pag. 171.

Mastogloia maxima Grun. Ueber neue Diatom. (1863) pag. 156, tab. 13, fig. 1 (valva infer.).

Cocconeis maxima Perag. Diat. mar. de France (1899) pag. 18, tab. 3, fig. 1-4. Hab. Tasmania: Baia di Latrobe.

Var. Minuta Grun. Alg. Novara (1867) pag. 12; De Toni Sylloge Algarum II, pag. 445.

Cocconeis Scutellum var. parva Grun. in V. H. Synopsis tab. 29, fig. 8-9, Traité, pag. 287, tab. 8, fig. 339; Cleve Syn. Navic. Diat. II (1895) pag. 170.

Hab. Australia occid.: Baia di Geroldton; Australia merid.: Victor Harbour; Tasmania: Baia di Latrobe.

Nella Baia di Latrobe questa varietà è frequentissima.

Var. STAuroneiformis Rabenh. Fl. Eur. Algar. I (1864) pag. 101 ; Grun. Alg. Novara (1867) pag. 12 ; V. H. Traité, pag. 287 ; Cleve Syn. Navic. Diat. II (1895) pag. 170; De Toni Sylloge Algarum II, pag. 445.

Cocconeis Scutellum $\beta$ W. Sm. Brit. Diat. I (1853) pag. 22, tab. 30, fig. 34. 
Hab. Australia merid.: Victor Harbour; Tasmania: Baia di Latrobe; Nuova Zelanda: Bluff.

Questa varietà venne già segnalata dal Grunow (Alg. Novara pag. 12) sulla Halopteris hordeacea proveniente dalla Nuova Zelanda.

\section{Orthoneis Grun.}

Orthoneis binotata Grun. Alg. Novara (1867) pag. 15; V. H. Synopsis tab. 28, fig. 7, Traité pag. 284, tab. 29, fig. 815; De Toni Sylloge Algarum II, pag. 466.

Mastogloia binotata Cleve Syn. Navic. Diat. II (1895) pag. 148.

Cocconeis binotata Grun. Ueber neue Diatom. (1863) pag. 145, tab. 13, fig. 13.

Hab. Tasmania: Baia di Latrobe.

La specie era già nota per l'Australia a merito del Grunow che la indicò pure per le isole Tahiti e Nicobar. Nelle Diatomées du monde entier di TEMPÈRE e Peragallo trovasi distribuita di Port Stevens (n. 398.399) e di Albany, Australia occidentale (n. 160).

Il WRIGHT (On Mastogloia fimbriata and M. binotata, 1902, pag. 161-165) riscontrò la specie fra i materiali provenienti dallo Stretto di Torres e fece in

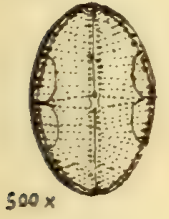

Orthoneis binotata Gr. var. quadrilocularis n. $\times 500$. proposito interessanti osservazioni sugli inviluppi mucosi e sui processi tentacoliformi di essa, completando le notizie fornite dal BuFFHAM (Nole on the Conjugation of Orthoneis binotata, 1892) sulla così detta periglea e sui rispettivi tentacoloidi.

Var. QUADRILOCULARIS n. var.: differt a forma typica loculis binis in utroque latere valvarum.

Hab. Tasmania: Baia di Latrobe.

tab. 7, fig. $10 c$ (non $a, b, d$ ).

Orthoneis CRIBrosa Grun. Alg. Novara (1867) pag. 16; De Toni Sylloge Algarum II, pag. 466.

Mastogloia cribrosa Grun. Ueb. neue Diatom. (1860) pag. 577,

Hab. Australia occid.: Baia di Geroldton; Tasmania: Baia di Latrobe; Nuova Zelanda: Port Charmers.

Questa specie venne ripetutamente raccolta dalla spedizione della « Novara * alle isole Nicobar, sulle coste dell'Australia e di Tahiti; sembra dunque alquanto frequente nei mari dell' Oceania.

Orthoneis fimbriata (Ehr.) Grun. Alg. Novara (1867) pag. 15; V. H. Synopsis tab. 28, fig. 3; De Toni Sylloge Algarum II, pag. 465.

Hab. Australia occid.: Baia di Geroldton; Tasmania: Baia di Latróbe; Nuova Zelanda: Port Charmers.

Specie già segnalata per la Nuova Olanda dal Grunow e dal Cleve; fu pure riscontrata fra le Diatomee di raccolte fatte presso le isole Samoa, Tahiti, Hawaii (Peragallo, Mereschkowsky). Tempere e Peragallo la distribuirono nelle Diatomées du monde entier, oltre che delle Samoa (n. 647.648) delle Nuove Ebridi (n. 61, 63), di Albany, Australia occidentale (n. 160, 276, 277), delle isole Thursday (n. 590-593), di Port Stevens (n. 398-399). 
Orthoneis? ovata Grun. Alg. Novara (1867) pag. 10 et 98; Cleve Syn. Navic. Diat. II (1895) pag. 156.

Mastogloia ovata Grun. Ueber neue Diat. (1860) pag. 578, tab. 5, fig. 12; Cleve et Grun. Arct. Diat. (1880) pag. 17, tab. 1, fig. 2; De Toni Sylloge Algarum II, pag. 318.

Hab. Tasmania: Baia di Latrobe.

Questa Orthoneis venne finora per l'Oceania segnalata di Tahiti (GRunow) e delle isole Samoa (Peragallo).

Orthoneis splendida (Greg.) Grun. Alg. Novara (1867) pag. 15; V. H. Synopsis tab. 28, fig. 1-2, Traité pag. 284, tab. 29, fig. 314.

Cocconeis splendida Greg. Diat. of Clyde (1857) pag. 21, tab. 1, fig. 29.

Hab. Australia occid.: Baia di Geroldton.

Il Cleve (Syn. Navic. Diat. II, 1895, pag. 148), seguendo l' opinione del GruNow, riferì a questa specie la Cocconeis punctatissima Grev. descritta dal GREVILLE nello stesso anno 1857 in cui il GREGORY aveva proposto la sua Cocconeis splendida, di guisa che il LAGERstedt (Diat.fr. Bohuslän 1876, pag. 57) chiamò la specie Orthoneis punctatissima (Grev.) Lagerst. dando la priorità alla denominazione specifica Grevilleana; il Mereschkowsky (On Polynesian Diatoms 1902, pag. 10) avendo studiato esemplari provenienti dalle isole Samoa e della Nuova Guinea ristabilì la specie Grevilleana come varietà punctatissima della Orthoneis splendida (Greg.) Grun.; la var. punctatissima ha 7-8 strie in $10 \mu \mathrm{e}$ $71 / 2-8$ punti in $10 \mu$, laddove la Orthoneis splendida tipica ne ha rispettivamente $4-5$ e $5-6^{1 / 2}$.

La Orthoneis splendida (Greg.) Grun. fu enumerata da Grove e Sturt tra la Diatomee fossili del deposito neozelandese di Oamaru; essa trovasi distribuita per l'Australia di Albany nelle Diatomées du monde entier di Tempère e PeraGALLO ai n. $160,276-277$.

\section{Fam. AMPHITROPIDACEAE (Pfitz.).}

\section{Tropidoneis Cleve.}

Tropidoneis Lepidoptera (Greg.) Cleve Syn. Navic. Diat. I (1894) pag. 25.

Amphiprora lepidoptera Greg. Diat. of Clyde (1857) pag. 33, tab. 4, fig. 59 (excl. fig. c); V. H. Synopsis tab. 22, fig. 23, Traité pag. 263, tab. 5, fig. 287; De Toni Sylloge Algarum II, pag. 328.

Hab. Nuova Zelanda: Bluff.

Questa specie non manca all' Oceano Pacifico, essendovi stata indicata dal Cleve per Port Jackson e per lo Stretto del Re Giorgio e dai Peragallo per le Hawaii, dal Mereschkowsky per le Samoa; nelle Diatomées du monde entier di Temperre e Peragallo trovasi per queste ultime isole ai n. 647-648, nonchè delle isole Thursday ai n. 590-591 e dello Stretto di Malacca ai n. 622-623. La varietà intermedia M. Perag. di Albany, Australia occidentale, ai n. 160, 276 e 277 della stessa collezione, differisce per il numero delle strie che sono 17 in $10 \mu$. 


\title{
Fam. NAViCULACEAE (Kuetz.).
}

\author{
Navicula (Bory) Cleve.
}

Navicula cancellata Donk. Brit. Diat. (1873) pag. 55, tab. 8, fig. 4; V. H. Synopsis, suppl. fig. 16, Traité pag. 183, tab. 3, fig. 128; Cleve Syn. Navic. Diat. 11 (1895) pag. 30; De Toni Sylloge Algarum II, pag. 48.

Var. APICUlata (Greg).

Pinnularia apiculata Greg. in Microsc. Journ. III (1855) pag. 41, tab. 1, fig. 21.

Hab. Australia merid.: Victor Harbour; Tasmania: Baia di Latrobe; Nuova Zelanda ; Port Charmers.

Var. subapiculata Grun. in Cleve et Grun. Arct. Diat. (1880) pag. 37; Cleve Syn. Navic. Diat. II (1895) pag. 30; De Toni Sylloge Algarum II, pag. 49.

Hab. Australia merid.: Victor Harbour.

La forma tipica della Navicula cancellata Donk. venne segnalata allo Stretto di Foveaux per la Nuova Zelanda dal Petrt (Diat. Campb. et N. Zél. pag. 22).

Navicula CRYPTOCePhala Kuetz. Die Kieselsch. Bacillarien (1844) pag. 95, tab. 3, fig. 26; V. H. Synopsis tab. 8, fig. 1-5, Traité pag. 180, tab. 3, fig. 122; Cleve Syn. Navic. Diat. II (1895) pag. 14; De Toni Sylloge Algarum II, pag. 46.

$H a b$. Tasmania: Deloraine, Hobart, in un fosso d' acqua piovana, 8 gennaio 1909.

Il LAUDER Lindsay (Diat. of Otago pag. 134) segnalò questa specie nel Distretto di Otago della Nuova Zelanda, il Lemmermann la riscontrò tra le Diatomee del Lago Huro, Chatham,

Navicula digito-Radiata (Greg.) Ralfs in Pritchard Hist. of Infus. (1861) pag. 904; V. H. Synopsis tab. 7, fig. 4, Traité pag. 184, tab. 3, fig. 130 ; Cleve Syn. Navic. Diat. II (1895) pag. 20 ; De Toni Sylloge Algarum II, pag. 51.

Hab. Tasmania: Baia di Latrobé.

Tempere e Peragallo nelle Diatomées $d u$ monde entier distribuirono ai n. 61, 63 questa specie delle Nuove Ebridi.

Var. Cyprinus (Ehr.?) Van Heurck Synopsis tab. 7, fig. 3, Traité pag. 185, tab. 3, fig. 131 ; Cleve Syn. Navic. Diat. II (1895) pag. 20; De Toni Sylloge A1garum II, pag. 51.

Hab. Tasmania : Baia di Latrobe; Nuova Zelanda: Bluff.

Navicula DIRECTA W. (Sm.) Ralfs in Pritchard Hist. of Infus. (1861) pag. 906; Cleve Syn. Navic. Diat. II (1895) pag. 27; De Toni Sylloge Algarum II, pag. 22. Hab. Australia merid.: Victor Harbour.

Nelle Diatomées du monde entier di Tempère e Peragallo trovasi questa specie distribuita di Albany, Australia occidentale (n. 160) e dell' isola d'Amsterdam (n. 443).

Var. Incus (Grun.) Cleve Syn. Navic. Diat. II (1895) pag. 27. 
Navicula Incus Grun. in A. Schmidt Atlas (1876) tab. 47, fig. 7; De Toni Sylloge Algarum II, pag. 62.

Hab. Nuova Zelanda: Bluff.

Navicula distans var. heterostriata Meresch. di California è da ritenersi un sinonimo della presente varietà (cfr. On Polynesian Diatoms 1902, pag. 24, tab. 5, fig. 6-8).

Navicula forcipata Grev. in Microsc. Journ. VI (1859) pag. 83, tab. 6, fig. 10.11 ; V. H. Synopsis tab. 10, fig. 3; Cleve Syn. Navic. Diat. II (1895) pag. 65;

De Toni Sylloge Algarum II, pag. 97.

Hab. Nuova Zelanda: Bluff.

Questa specie ha un' estesa distribuzione geografica anche nei mari australi e nell' Oceano Pacifico venne riscontrata in più luoghi; trovasi anche distribuita, da Albany, Australia occidentale ai n. 276-277 delle Diatomées du monde entier di Tempère e Peragallo.

Var. nummularia (Grev.) Cleve Syn. Navic. Diat. II (1895) pag. 66.

Navicula nummularia Grev. in Edinb. New Phil. Journ. X (1859) pag. 29, tab. 4, fig. 6 .

Navicula forcipata var. nummularioides Grun. in A. Schmidt Atlas tab. 70, fig. 30-31.

Hab. Tasmania: Burmie.

Navicula granulata Bail. in Smithson. Contrib. VII (1854) fig. 16; Cleve Syn. Navic. Diat. II (1895) pag. 48.

Navicula Baileyana Grun. in A. Schmidt Nords. Diat. (1874) tab. 1, fig. 31. Atlas (1874) tab. 6, fig. 26-27.

Navicula polysticta Grev. in Edinb. New Phil. Journ. X (1859) pag. 28, tab. 4, fig. 12.

Hab. Australia merid. : Victor Harbour.

Sotto il nome di Navicula granulata vennero descritte specie differenti cosi da dover fissare la sinonimia nel modo sovra esposto. Il BRÉBIsson descrisse nel 1858 come Navicula granulata una specie che il CLEVE riferì alla Nav. monilifera $\mathrm{Cl}$., laddove il VAN Heurck (Iraité 1899, pag. 211) la vuole conservata malgrado che il nome del BAiLey abbia la precedenza; A. Schmidt (Atlas tab. 6, fig. 15-16) figurò col nome Nav. granulata una forma corrispondente alla varietà heterosticha $\mathrm{Cl}$. della Nav. monilifera; strane coincidenze di nomenclatura: I' Ehrenberg nel 1836 con il nome di Nav. granulata descrisse una specie del genere Epithemia!

Il Cleve indicò la Navicula granulata Bail. per Sydney.

Var. Peragallorum n. var.

Navicula Baileyana var. Perag. Diat. mar. de France tab. 27, fig. 8.

A typica forma differt valvis elliptico-acuminatis, nunquam humerosis, tenuius granulatis quam in typo.

Hab. Nuova Zelanda: Bluff.

L' esemplare raffigurato nelle Diat. mar. de France è lungo $46 \mu$ e proviene da New Haven, Conn., Stati Uniti d'America. Si tratta di una forma assai variabile. 
Navicula humerosa Bréb. in W. Sm. Brit. Diat. II (1856) pag. 93; Grun. Ueb. neue Diat. (1860) pag. 536, tab. 1, fig. 26; V. H. Synopsis tab. 11, fig. 20, Traité pag. 210, tab. 4, fig. 182; Cleve Syn. Navic. Diat. II (1895) pag. 43; De Toni Sylloge Algarum II, pag. 127.

$H a b$. Nuova Zelanda: Bluff.

Per l' Oceania la Navicula humerosa Bréb. venne già segnalata dal CLEvE a Sydney; è una specie con estesissima distribuzione negli Oceani e nei mari interni.

Navicula hungarica Grun. Ueb. neue Diat. (1860) pag. 539, tab. 1, fig. 30 ; De Toni Sylloge Algarum II, pag. 47.

Var. Humilis (Donk.) Grun. in Cleve et Grun. Arct. Diat. (1880) pag. 27 ; V. H. Synopsis tab. 11, fig. 23; De Toni Sylloge Algarum II, pag. 47.

Navicula humilis Donk. Brit. Diat. (1872) pag. 67, tab. 10, fig. $7 ;$ V. H. Traité pag. 182, tab. 3, fig. 127.

Hab. Tasmania: Hobart.

Navicula laevissima Kuetz. Die Kieselsch. Bacillarien (1844) pag. 96, tab. 21, fig. 14; Van Heurck Traité pag. 225, tab. 27, fig. 715 (non Donkin); De Toni Sylloge Algarum II, pag. 164.

Hab. Nuova Zelanda: Rotorua, Ohinemuthu.

Il Cleve (Syn. Navic. Diat. II, 1895, pag. 137) ascrisse con dubbio questa specie alla Navicula Pseudobacillum Grun., la quale venne già indicata per la Nuova Zelanda.

Navicula Lanceolata Kuetz. Die Kieselsch. Bacillarien (1844) pag. 96, tab. 28, fig. $60,62$.

Var. ARenaria (Donk.) Van Heurck Synopsis tab. 8, fig. 18, Traité (1899) pag. 186, tab. 3, fig. 142; De Toni Sylloge Algarum II, pag. 58.

Navicula arenaria Donk. in Microsc. Journ.. vol. I (1861) pag. 10, tab. 1, fig. 9; A. Schmidt Atlas tab. 47, fig. 38-41.

Hab. Nuova Zelanda: Port Charmers, Bluff.

Non abbiamo trovato indicazioni di questa varietà per la Nuova Zelanda.

Navicula Lyra Ehr. Amer. (1843) pag. 131, tab. I, 1, fig. 9; V. H. Traité pag. 202, tab. 4, fig. 161 ; Cleve Syn. Navic. Diat. II (1895) pag. 63 (var. Ehrenbergii $\mathrm{Cl}$.); De Toni Sylloge Algarum II, pag. 95.

Hab. Australia occid.: Baia di Geroldton; Nuova Zelanda: Bluff.

Questa Navicula ha un' estesa distribuzione geografica tanto negli Oceani quanto nei mari interni e ne riscontrammo anche alcune varietà e forme più sotto indicate. Per l' Oceano Pacifico trovasi distribuita in Tempère e Peragallo di parecchie località nelle Diatomées $d u$ monde entier: Nuove Ebridi (n. 61-63), coste della Nuova Zelanda (n. 445-447), isole Thursday (n. 561-562, 590-593), stretto di Malacca (n. 622-623), isole Samoa (n. 647-648), Stretto di Torres da lavaggi di Ostriche perlifere (n. 946).

- Var. australica A. Schmidt Atlas (1874) tab. 2, fig. 37.

Hab. Australia merid.: Victor Harbour. 
Var. constricta H. Perag. Diat. mar. de France (1899) pag. 135, tab. 23, fig. 3.

Hab. Australia occid. : Baia di Geroldton.

Var. dilatata A. Schmidt Atlas (1874) tab. 2, fig. 26.

Hab. Nuova Zelanda: Bluff.

Distribuita delle Nuove Ebridi in Tempère e Peragallo Diatomées du monde entier n. 61-63. fig. 6-11.

Var. intermedia H. Perag. Diat. mar. de France (1899) pag. 135, tab. 23,

Hab. Australia merid. : Victor Harbour.

Distribuita da Tempère e Peragallo nelle Diatomées du monde entier delle isole Thursday (n. 561-562, 592-593), dello stretto di Malacca (n. 622-623).

Var. subtypica H. Perag. Diat. mar. de France (1899) pag. 135, tab. 22, fig. 2.

Hab. Nuova Zelanda: Bluff.

Navicula pennata A. Schmidt Atlas (1876) tab. 48, fig. 41-45.

Var. Maior H. Perag. in T. et P. Diat. du monde entier 2 éd. (1912) pag. $314,323$.

Hab. Nuova Zelanda: Port Charmers.

Conosciuta per l' is. Macdonald e per il Royal Sound, di Kerguelen, distribuita rispettivamente ai numeri 634 e 651-652 delle Diatomées du monde entier di Tempère e Peragallo.

Var. maxima Cleve Syn. Navic. Diat. II (1895) pag. 33, tab. 1, fig. 36.

Hab. Australia occid.: Baia di Geroldton.

Nella collezione diatomologica del Deby si trovano, secondo il Cleve, esemplari di Sumatra e dell' Oceano Indiano; ma la varietà trovasi anche nel mare Mediterraneo e nell' Adriatico.

Navicula Placentula (Ehr.) Kuetz. Die Kieselsch. Bacillarien (1844) pag. 94, tab. 28, fig. 37; Cleve Syn. Navic. Diat. II (1895) pag. 23; De Toni Sylloge Algarum II, pag. 55 .

Navicula Gastrum var. Placentula V. H. Synopsis tab. 8, fig. 26, Traité pag. 187, tab. 3, fig. 135.

Hab. Nuova Zelanda: Wakarevareva.

Per la Nuova Zelanda questa specie fu già segnalata dal Cleve e venne anche distribuita fossile di Wangarei al n. 918 delle Diatomées du monde entier di Tempere e Peragallo.

Navicula Pupula Kuetz. Die Kieselsch. Bacillarien (1844) pag. 93, tab. 30, fig. 40 ; V. H. Synopsis tab. 13, fig. 15-16, Traité pag: 225, tab. 5, fig. 226 ; Cleve Syn. Navic. Diat. I (1894) pag. 131; De Toni Sylloge Algarum II, pag. 162.

Hab. Nuova Zelanda: Rotorua, Ohinemuthu.

La specie venne già dal CLeve indicata per l'Australia e per la Nuova Zelanda; da Tempère e Peragallo fu distribuita nelle Diatomées du monde entier di Yarra River, Melbourne (n. 241-243) e fossile di Wangarei, Nuova Zelanda (n. 918) ; una var. minuta si trova fossile a Borries, Oamaru (n. 821).

Navicula Rhyncocephala Kuetz. Die Kieselsch. Bacillarien (1844) pag. 152, tab. 30, fig. 33; De Toni Sylloge Algarum II, pag. 44. 
Var. Amphiceros (Kuetz.) Grun. in Cleve et Grun. Arct. Diat. (1880) pag. 33 ; V. H. Synopsis tab. 7, fig. 30, Traité pag. 181, tab. 3, fig. 120 ; Cleve Syn. Navic. Diat. II (1895) pag. 15; De Toni Sylloge Algarum II. pag. 45.

Navicula amphiceros Kuetz. Die Kieselsch. Bacillarien (1844) pag. 95, tab. 3 , fig. 39 .

Hab. Tasmania: Hobart; Nuova Zelanda: Wakarevareva.

Il tipo venne distribuito da Tempère e Peragallo nelle Diatomées du monde entier n. 273 del Lago Rotorua, Nuova Zelanda. Il Cleve indica, quanto al1'Oceania, la presente varietà per le isole Sandwich.

Navicula Scoliopleura A. Schmidt Atlas (1876) tab. 46, fig. 63; Cleve Syn. Navic. Diat. II (1895) pag. 32.

Hab. Australia occid. : Baia di Geroldton.

La specie è nuova per l'Oceania. Fu descritta da A. Schmidt su esemplari dell' Oceano Atlantico (Messico).

Navicula Zostereti Grun. Ueb. neue Diatom. (1860) pag. 528, tab. 2, fig. 23 ; Cleve Syn. Navic. Diat. II (1895) pag. 31; De Toni Sylloge Algarum II, pag. 43.

Hab. Australia merid.: Victor Harbour; Nuova Zelanda : Port Charmers.

Riguardo alla distribuzione geografica nell' Oceano Pacifico è noto che questa specie venne dal CLEVE indicata delle isole Sandwich, e per le isole Nuove Ebridi (is. Vaté) distribuita da Tempère e Peragallo ai n. 61-63 delle Diatomées du monde entier; ma essa è pure comune lungo i lidi della Cina, del Giappone e delle isole dell' Arcipelago della Sonda.

\section{Libellus Cleve}

Libellus hamuliferus (Grun.) De Toni Int. al gen. Brachysira (1890) pag. 971, Sylloge Algarum II, pag. 202; Cleve Syn. Navic. Diat. I (1894) pag. 154, tab. 3, fig. 16-18.

Navicula hamulifera Grun. in Cleve et Grun. Arct. Diat. (1880) pag. 44.

Hab. Tasmania: Baia di Latrobe; Burmie.

Gli esemplari di Burmie sono piccoli in confronto al tipo, raggiungendo 30 $\mu$ di lunghezza.

Libellus plicatus (Donk.) De Toni Int. al gen. Brachysira (1890) pag. 971, Sylloge Algarum II, pag. 202; Cleve Syn. Navic. Diat. 1 (1894) pag. 154.

Navicula plicata Donk. Brit. Diat. (1873) pag. 59, tab. 9, fig. 2 (an Ehrenberg?).

Hab. Tasmania: Baia di Latrobe.

Questa specie è rappresentata nell' Arcipelago della Sonda.

\section{Caloneis Cleve}

Caloneis Liber W. Sm. Brit. Diat. 1 (1853) pag. 48, tab. 16, fig. 133; V. H. Synopsis tab. 12, fig. 36, Traité pag. 222, tab. 5, fig. 219; De Toni Sylloge A1garum II, pag. 157.

Hab. Australia merid.: Victor Harbour; Nuova Zelanda: Port Charmers. 
Questa specie, di assai larga distribuzione geografica, fu già indicata per parecchie stazioni nell' Oceania ; il Cleve la notò per Sydney, Port Jackson e la Tasmania; il Petrt (Diat. Camp. et N. Zél. pag. 21) la segnalò nella Lyall's Bay, Nuova Zelanda; i Peragallo la enumerarono tra le Diatomee provenienti dalle isole Sandwich. Per lo Stretto di Malacca trovasi distribuita ai n. 622-623 delle Diatomées du monde entier di Tempère e Peragallo.

Var. Elongata (Grun.) in Cleve Syn. Navic. Diat. I (1894) pag. 55.

Navicula elongata Grun. in A. Schmidt Nords. Diat. (1874) pag. 91, tab. 2. fig. 42.

Hab. Australia merid.: Victor Harbour; Nuova Zelanda: Port Charmers.

Var. Linearis (Grun.) Van Heurck Synopsis tab. 12 fig. 35, Traité pag. 222, tab. 5, fig. 220 ; Cleve Syn. Navic. Diat. I (1894) pag. 54; De Toni Sylloge Algarum II, pag. 158 .

$H a b$. Nuova Zelanda: Bluff.

Il Petit (Diat. Campb. et N. Zél. pag. 21) segnalò questa varietà per lo Stretto di Foveaux.

\section{Diploneis Ehr.}

Diploneis advena (A. Schm.) Cleve Syn. Navic. Diat. 1 (1894) pag. 81.

Var. PARCa (A. Schm.) Cleve op. cit. pag. 81.

Navicula parca A. Schmidt Atlas (1885) tab. 8, fig. 20-22.

Hab. Tasmania: Baia di Latrobe.

Nell' Oceano Pacifico questa varietà fu raccolta alle isole Samoa; TEMPÈRE e Peragallo nelle Diatomées du monde entier ai n. 61-63 distribuirono la forma tipica dalle Nuove Ebridi.

Diploneis Bomboides (A. Schm.) Çleve Syn. Navic. Diat. I (1894) pag. 88.

Navicula bomboides A. Schmidt Nords. Diat. (1874) pag. 85, tab. 1, fig. 2, Atlas (1874) tab. 13, fig. 36-40; De Toni Sylloge Algarum II, pag. 71.

Hab. Nuova Zelanda: Bluff.

Per l' Oceania fu questa Diploneis segnalata a Sydney, Australia dal CLeve, alle isole Salomone dai Peragallo.

Diploneis Bombus in Berichte Berl. Akad. 1844, pag. 84, Mikrogeologie (1854) tab. 19, fig. 31 ; Cleve Syn. Navic. Diat. I (1894) pag. 90.

Navicula Bombus Kuetz. Species (1849) pag. 83; V. H. Synopsis tab. B, fig. 22, Traité pag. 194, tab. 3, fig. 149; De Toni Sylloge Algarum II, pag. 75.

Hab. Tasmania: Baia di Latrobe; Nuova Zelanda: Port Charmers.

Questa specie ha estesa distribuzione negli Oceani e nei mari interni. Per l' Oceania fu distribuita da Tempère e Peragallo nelle Diatomées du monde entier di più luoghi: Albany, Australia occidentale (n. 276-277), isole Thursday (n. 592-593), is. Campbell (n. 357); il Cleve la indicò per le isole Samoa e per il Giappone, e nella varietà che segue per le Filippine, la Cina e il Giappone.

Var. Egena (A. Schm.) Cleve Syn. Navic. Diat. 1 (1894) pag. 90.

Navicula gemina var. egena. A. Schmidt Atlas (1874) tab. 13, fig. 10.

$H a b$. Australia occid. : Baia di Geroldton. 
Diploneis didyma (Ehr.) Cleve Syn. Navic. Diat. 1 (1894) pag. 90.

Navicula didyma Ehr. Kreideth. (1840) pag. 75; V. H. Synopsis tab. 9 fig. 5-6 et tab. B fig. 20, Traité pag. 193, tab. 3, fig. 147; De Toni Sylloge Algarum II, pag. 71 .

Hab. Tasmania: Baia di Latrobe, Burmie; Nuova Zelanda: Bluff.

Non manca all' Oceania questa specie, affine alla precedente; il CLEVE ne diede alcune indicazioni (Tahiti, Giappone); i Peragallo la enumerarono tra le Diatomee raccolte alle isole Samoa e Salomone; delle Samoa trovasi distribuita da Tempere e Peragallo ai n. 647-648 delle Diatomées du monde entier, nella quale collezione al n. 774 si hanno frustoli provenienti dal porto neozelandese di Oamaru.

Diṕloneis elliptica (Kuetz.) Cleve Syn. Navic. Diat. 1 (1894) pag. 92.

Navicula elliptica Kuetz. Die Kieselsch. Bacillarien (1844) pag. 98, tab. 30, fig. 55 ; V. H. Synopsis tab. 10, fig. 10, Traité pag. 201, tab. 4, fig. 156'; De Toni Sylloge Algarum II, pag. 89.

Hab. Nuova Zelanda: Wakarevareva.

Questa Diploneis venne già indicata dal LAUDER Lindsay (Diat. of Otago pag. 134) per la Nuova Zelanda nel Distretto di Otago e fu riscontrata dal LEMmermann nelle Lagune di Chatham. Tempère e Peragallo la distribuirono nelle Diatomées du monde entier delle Nuove Ebridi (n. 61, 63), di Yarra River, Australia (n. 241-243).

Diploneis Fusca (Greg.) Cleve Syn. Navic. Diat. 1 (1894) pag. 93.)

Navicula fusca Ralfs in Pritchard Hist. of Infus. (1861) pag. 898; V. H. Synopsis tab. B, fig. 24, Traité pag. 198, tab. 4, fig. 153; De Toni Sylloge Algarum II, pag. 87.

Hab. Australia merid.: Victor Harbour; Nuova Zelanda: Bluff.

E una specie che con le sue numerose varietà può venir considerata pressochè cosmopolita.

Il Petit (Diat. Campb. et N. Zél. pag. 25) la segnalò per lo Stretto di Foveaux; i Peragallo la elencarono tra le Diatomee delle isole Samoa e Sandwich. Tempère e Peragallo nelle Diatomées du monde entier la distribuirono delle isole Samoa (n. 647-648) e dell' isola Vaté delle Nuove Ebridi (n. 61-63).

Diploneis nitescens (Greg.) Cleve Syn. Navic. Diat. I (1894) pag. 97.

Navicula nitescens Ralfs in Pritchard Hist. of Infus. (1861) pag. 896; V. H. Traité pag. 198, tab. 26, fig. 747; De Toni Sylloge Algarum II, pag. 91.

Hab. Tasmania : Burmie.

Questa specie è già nota per l'Australia (Cleve), la Nuova Zelanda (PetiT), le isole Sandwich (Cleve, Peragallo); essa fu riscontrata anche fossile nel deposito neozelandese di Oamaru (Grove e Sturt).

Diploneis Suborbicularis (Greg.) Cleve Syn. Navic. Diat. I (1894) pag. 81.

Navicula Smithii var. suborbicularis Greg. Diat. of Clyde (1857) pag. 487, tab. 9, fig. 17 ; V. H. Traité pag. 199, tab. 26, fig. 748.

Hab. Nuova Zelanda: Bluff. 
La Diploneis suborbicularis (Greg.) Cleve ha un' estesa distribuzione geografica tanto negli Oceani quanto nei mari interni. I Peragallo la indicarono tra le Diatomee delle isole Salomone.

Diploners Weissflogir (A. Schm.) Cleve Syn. Navic. Diat. I (1894) pag. 91.

Navicula Weissflogii A. Schmidt in Zeitschr. für ges. Naturw. vol. 41 (1873) pag. 406, tab. 6, fig. 3-4, Atlas (1885) tab. 12, fig. 26-32; V. H. Synopsis tab. B, fig. 21; Traité pag. 194, tab. 3, fig. 148; De Toni Sylloge Algarum II, pag. 75.

Hab. Tasmania: Baia di Latrobe.

Questa specie non è nuova per l'Oceano Pacifico, essendo statã già indicata per la Nuova Zelanda (Petit), le isole Samoa (Grunow, Cleve), le Sandwich (A. Schmidt), le Filippine e Tahiti (Cleve); Tempere e Peragallo nelle Diatomées du monde entier la distribuirono di Albany, Australia occidentale (n. 276277), delle Nuove Ebridi (n. 61-63) e dello Stretto di Torres (n. 946).

\section{Pinnularia Ehr.}

Pinnularia appendiculata (Ag.) Cleve Syn. Navic. Diat. Il (1895) pag. 75.

Navicula appendiculata Kuetz. Die Kieselsch. Bacillarien (1844) pag. 93, tab. 3, fig. 28, tab. 4, fig. 1-2; V. H. Synopsis tab. 6, fig. 18, 20, Traité pag. 173, tab. 2, fig. 93; De Toni Sylloge Algarum II, pag. 28.

Hab. Nuova Zelanda: Wakarevareva.

La forma tipica è nuova per la flora neozelandese ma venne già segnalata per l'Australia (CLEVE).

Var. Budensis Grun. in V. H. Synopsis (1880) tab. 6, fig. 27.28; Cleve op. cit. pag. 75 .

Hab. Nuova Zelanda: Rotorua, geyser a.

Questa varietà venne finora segnalata soltanto per le acque termali dell' Ungheria (Grunow) e della Nuova Zelanda (Cleve).

Pinnularia Dactylus Ehr. Amer. (1843) pag. 132, tab. 4, I, fig. 3; Cleve Syn. Navic. Diat. II (1895) pag. 90.

Navicula nobilis var. Dactylus V. H. Synopsis tab. 5, fig. 1, Traité pag. 165, tab. 3, fig. 68; De Toni Sylloge Algarum II, pag. 9.

Hab. Nuova Zelanda: Wakarevareva.

Pinnularia divergens W. Sm. Brit. Diat. I (1853) pag. 57, tab. 18, fig. 177 ; Cleve Syn. Navic. Diat. II (1895) pag. 79.

Navicula divergens Ralfs in Pritchard Hist. of Infus. (1861) pag. 896; V. H. Traité pag. 170, tab. 25, fig. 706; De Toni Sylloge Algarum II, pag. 17.

Hab. Nuova Zelanda: Wakarevareva.

La specie venne già indicata per la Nuova Zelanda dal CLEVE, nonchè per le isole Sandwich. Fu distribuita fossile di Cabbage Tree Swamp, Auckland da Tempère e Peragallo nelle Diatomées du monde entier n. 223.

Pinnularia major (Kuetz.) Rabenh. Die Süssw. Diatom. (1851) pag. 42, tab. 6, fig. 5; W. Sm. Brit. Diat. I (1853) pag. 54, tab. 18, fig. 162; Cleve Syn. Navic. Diat. II (1895) pag. 89. 
Navicula major Kuetz. Die Kieselsch. Bacillarien (1844) pag. 97, tab. 4, fig. 19; V. H. Synopsis tab. 5, fig. 3-4, Traité pag. 165, tab. 2, fig. 69; De Toni Sylloge Algarum II, pag. 10.

Hab. Nuova Zelanda: Wakarevareva.

Il Lauder Lindsay (Diat. of Otago pag. 134) assegnò questa specie alla Nuova Zelanda per il Distretto di Otago, ciò che venne confermato dal CLEvE che nota la Pinnularia stessa per la Nuova Zelanda. Tempère e Peragallo la distribuirono nelle Diatomées du monde entier del Lago Rotorua (n. 273), nonchè di Yarra River, Melbourne (n. 241-243).

Pinvularia mesolepta (Ehr.) W. Sm. Brit. Diat. 1 (1853) pag. 58, tab. 19, fig. 182; Cleve Syn. Navic. Diat. II (1895) pag. 76.

Navicula mesolepta Ehr. Amer. (1843) tab. 4, II, fig. 4; V. H. Synopsis tab. 6, fig. 10-11, Traité pag. 174, tab. 2, fig. 96 ; De Toni Sylloge Algarum II, pag. 32. Hab. Tasmania: Deloraine, Hobart.

Questa specie è, quanto all' Oceania, nota per la Nuova Zelanda (LAUDER Lindsay, Cleve).

Pinnularia Parva (Ehr.) Greg. in Microsc. Journ. II (1854) tab. 4, fig. 11 ; Cleve Syn. Navic. Diat. II (1895) pag. 87.

Navicula gibba var. brevistriata Grun. in V. H. Synopsis tab. 6, fig. 5, Traité pag. 172, tab. 2, fig. 89.

Hab. Nuova Zelanda: Wakarevareva.

Questa specie ha una larga distribuzione geografica e fu gia indicata per l'Australia e per la Nuova Zelanda (CLEVE). Non si rinviene però quasi mai di frequente; in via eccezionale si trovò comune nel deposito fossile di Aberdeen (Scozia) come risulta dal n. 854 delle Diatomées du monde entier di Tempére e Peragallo.

Pinnularia Hartleyana Greville in Trans. Mier. Soc. (1865) p. 57, tab. 6, fig. 30 ; Cleve Syn. (1895) p. 80; A. Schmidt Atlas (1914) tab. 313, fig. 2.

Navicula Hartleyana H. L. Sm. Diat. Sp. Typ. n.o 280! De Toni Syll. II p. 13.

Hab. Nuova Zelanda: Wakarevareva.

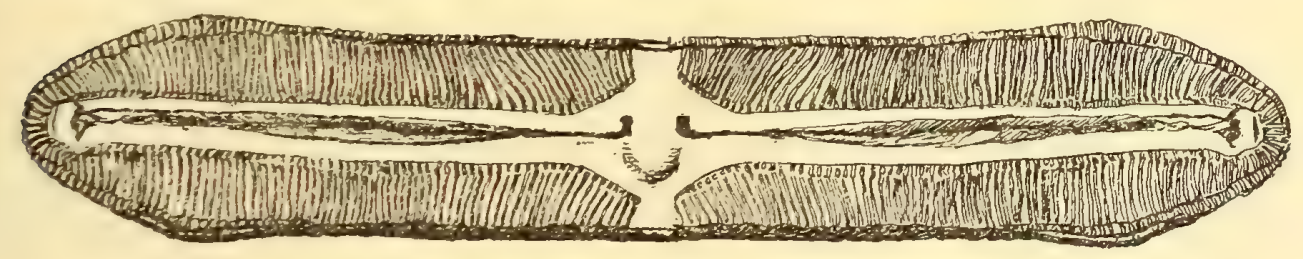

Fig. 6. - Pinnularia Hartleyana Greville $\times 300$

E una tra le più grandi Pinnularie; infatti l' unico esemplare che finora fu osservato oltrepassa i $400 \mu$ di lunghezza; la figura dello Hustedt dell' Atlas di A. Schmid corrisponde, tenuto conto dell'ingrandimento, a $320 \mu$. Forse non si tratta che di megafrustoli di Pinnularia Trevelyana (Donk.) Rabenh. la quale fu pure rinvenuta fossile nel medesimo deposito di Kalahari in Africa dal REIchelt.

La Pinnularia Trevelyana (Donk.) Rabenh. ha un' area di distribuzione abbastanza estesa. 
Pinnularia Rectangulata (Greg.) Rabenh. Fl. Eur. Algar. I (1864) pag. 215; Cleve Syn. Navic. Diat. Il (1895) pag. 98.

Navicula rectangulata Greg. Diat. of Clyde (1857) pag. 479, tab. 9, fig. 7 ; V. H. Synopsis tab. A, fig. 7, Traité pag. 166, tab. 2, fig. 74; De Toni Sylloge Algarum II, pag. 13.

Hab. Tasmania: Burmie.

Questa specie marina è soprattutto limitata all' Oceano Atlantico e al Mare Mediterraneo; fu però riscontrata a Labuan, Borneo (CLEVE).

Pinnularia stauroptera (Grun.) Cleve Syn. Navic. Diat. II (1895) pag. 82.

Navicula stauroptera Grun. Ueb. neue Diatom. (1860) pag. 516, tab. 2, fig. 18; V. H. Synopsis tab. 6, fig. 7 ; Traité pag. 171, tab. 2, fig. 85; De Toni Sylloge Algarum II, pag. 25.

Hab. Nuova Zelanda: Wakarevareva, Rotorua geyser, a.

La specie fu distribuita del Lago Rotorua al n. 273 delle Diatomées $d u$ monde entier di Tempère e Peragallo; essa è nota, secondo il Cleve, anche per l' Australia (Murray River, Blue Mountains).

Pinnularia Trevelyana (Donk.) Rabenh. Fl. Eur. Algar. I (1864) pag. 210 ; Cleve Syn. Navic. Diat. II (1895) pag. 98.

Navicula Trevelyana Donk. in Microsc. Journ. I (1861) pag. 8, tab. 1, fig. 2; V. H. Synopsis tab. A, fig. 5-6, Traité pag. 166, tab. 2, fig. 73; De Toni Sylloge Algarum II, pag. 13.

Hab. Australia merid. : Victor Harbour.

È specie da aggiungere alla ftora australasica.

Pinnularia viridis (Nitzsch) Ehr. Die Infusionsthierchen (1838) pag. 182 ; Cleve Syn. Navic.-Diat II (1895) pag. 91.

Navicula viridis Kuetz. Die Kieselsch. Bacillarien (1844) pag. 97, tab. 4, fig. 18; V. H. Synopsis tab. 5, fig. 5, Traité pag. 165, tab. 2, fig. 70; De Toni Sylloge Algarum II, pag. 11.

Hab. Tasmania: Deloraine, Hobart; Nuova Zelanda: Wakarevareva, Rotorua, Ohinemuthu.

E questa una tra le Pinnularia le più largamente distribuite nelle acque dolci. L'Ehrenberg (Mikrogeologie 1854, pag. 2, 4, 7, 8) la indicò per la Tasmania e per parecchie località dell' Australia occidentale e meridionale; il LAUDER LindsaY (Diat. of Otago pag. 134) segnalò la Pinnularia stessa nella Nuova Zelanda per il Distretto di Otago ; nell' Australia e nella Tasmania ne è confermata l'esistenza dal Cleve; essa trovasi anche nel Lago Wahiria a Tahiti (Grunow) e venne distribuita di Yarra River al n. 242 delle Diatomées du monde entier di Tempère e Peragallo.

\section{Neidium Pfitz.}

Neidium dubium (Ehr.) Cleve Syn. Navic. Diat. I (1894) pag. 70. Navicula dubia Ehr. Amer. (1843) pag. 130, tab. II, 2, fig. 8. 
Navicula Iridis var. dubia V. H. Synopsis tab. B, fig. 32, Traité pag. 221, tab. 5, fig. 215; De Toni Sylloge Algarum II, pag. 154.

Hab. Nuova Zelanda: Wakarevareva, Rotorua, Ohinemuthu.

Secondo il Cleve questa specie venne già riscontrata nella Nuova Zelanda e si trova pure in Australia (Blue Mountains).

\section{Anomoeoneis Pfitz.}

Anomoeoneis sphaerophora (Kuetz.) Pfitz. Bacillarien (1871) pag. 77, tab. 3, fig. 10; Cleve Syn. Navic. Diat. II (1895) pag. 6.

Navicula sphaerophora Kuetz. Alg. Dec. (1834) n. 84, Die Kieselsch. Bacillarien (1844) pag. 95, tab. 4, fig. 17; V. H. Synopsis tab. 12, fig. 2-3, Traité pag. 216, tab. 4, fig. 195; De Toni Sylloge Algarum II, pag. 140.

$H a b$. Nuova Zelanda: Rotorua, Ohinemuthu, sorgenti calde.

La specie venne già segnalata per la Nuova Zelanda dal Cleve.

Forma Güntheri O. Muell. Bacill. von El Kab (1899) pag. 302, tab. 12, fig. 6-7.

Hab. Nuova Zelanda: Rotorua, Ohinemuthu.

Forma rostrata O. Muell. Bacill. von El Kab (1899) pag. 303, tab. 12, fig. 3-5.

Hab. Nuova Zelanda: Rotorua, Ohinemuthu.

\section{Stauroneis Ehr.}

Stauroneis anceps Ehr. Amer. (1843) pag. 134, tab. 2, I, fig. 18; V. H. Synopsis tab. 4, fig. 4-5, Traité pag. 160, tab. 1 fig. 55; Cleve Syn. Navic. Diat. 1 (1894) pag. 147; De Toni Sylloge Algarum II, pag. 211.

Hab. Nuova Zelanda: Wakarevareva.

Questa specie non è nuova per la flora neozelandese essendo stata già segnalata dal LaUder Lindsay (Diat. of Otago pag. 134) per il Distretto di Otago ; una sua varietà innominata figura distribuita del Lago Rotorua da TEMPÉRE e Peragallo nelle Diatomées du monde entier al n. 273; allo stato fossile fu distribuita di Waitangi, Nuova Zelanda (n. 922) e di Talbot, Australia (n. 981-983).

Var. Gracilis (Rabenh.) Brun Diat. des Alpes et du Jura (1880) pag. 89, tab. 9, fig. 1; De Toni Sylloge Algarum II, pag. 211.

Stauroneis anceps forma gracilis Rabenh. Fl. Eur. Algar I (1864) pag. 247.

Hab. Nuova Zelanda: Wakarevareva.

Stanroners gracilis Ehr. distribuita di Yarra River, Melbourne da TempĖRE e Peragallo ai n. 241-242 delle Diatomées du monde entier è piuttosto da ritenersi uguale a Stauroneis Phoenicenteron (Nitzsch) Ehr. var. amphilepta (Ehr.).

\section{Pleurostauron Rabenh.}

Pleurostauron acutum (W. Sm.) Rabenh. in Hedwigia II (1859) n. 3, tab. I, fig. B, tab. II, fig. 13; Cleve Syn. Navic. Diat. I (1894) pag. 150; De Toni Sylloge Algarum II, pag. 222.

Slauroneis acuta W. Sm. Brit. Diat. I (1853) pag. 59, tab. 19, fig. 187 ; V. H. Synopsis tab. 4, fig. 3, Traité pag. 159, tab. 1, fig. 51.

Hab. Nuova Zelanda: Wakarevareva. 
Alcune altre specie di questo genere vennero segnalate per la Nuova Zelanda sia viventi che fossili (Pleurostauron Fulmen (Brightw.) Grun., Pleur. Frauenfeldianum Grun., Pleurostauron scaphulaeforme (Grev.) Grun. Il Pleurostauron acutum (W. Sm.) Rabenh. fu già indicato dal Cleve per l'Australia e per la Nuova Zelanda.

Pleurostauron inflatum (Heid.) n. comb.

Stauroneis inflata Heid. in A. Schmidt Atlas (1903) tab. 24l, fig. 2, 8, 11 (non Kuetzing).

Hab. Nuova Zelanda: Wakarevareva.

Questa specie è molto affine al Pleurostauron acutum (W. Sm.) Rabenh., da cui si differenzia per le due sporgenze laterali della valva prominentissime allo stauro, così da formare due angoli quasi retti, simmetricamente al centro. Si conosce di Talbot (fossile) e di Yarra River. Il Cleve la disse Stauroneis acuta W. Sm. var.

\section{Trachyneis Cleve}

Trachyneis aspera (Ehr.) Cleve Syn. Navic. Diat. I (1894) pag. 191 ; Peragallo in Micrographe préparateur VII (1890) pag. 240, tab. 30, fig. 1.

Navicula aspera Ehr. Mikrogeologie (1854) tab. 38 A fig. 5; V. H. Traité pag. 205, tab. 4, fig. 165; De Toni Sylloge Algarum II, pag. 109.

Hab. Australia merid.: Victor Harbour; Tasmania: Baia di Latrobe; Burmie; Nuova Zelanda: Port Charmers, Bluff.

Questa specie non è rara nell' Oceania ; la conosciamo segnalata per Sydney (Cleve), per le isole Salomone, Samoa, Hawaii, Tahiti (Peragallo, MereschkowSKY); venne pure raccolta dalla spedizione Scott nell' Antartide (Tempère e PERAGALlo, Diatomées du monde entier n. 935); in questa ultima collezione trovasi distribuita di Albany (n. 160) e di Port Stevens (n. 398-399), della Terra di Kerguelen (n. 3, 651-652), delle is. Nuove Ebridi (61-63), Campbell (n. 357), Thursday (n. 561-562, 590-593), S. Paolo (n. 432), Mc Donald (n. 634), Samoa (n. 647-648), Stretto di Torres (n. 946), porto di Oamaru (n. 774), Stretto di Malacca (n. (622-623) ecc.

Var. vulgaris Cleve Syn. Navic. Diat. I (1894) pag. 191.

Navicula aspera A. Schmidt Atlas (1876) tab. 48, fig. 2-6.

Hab. Australia occid. : Baia di Geroldton.

Questa varietà fu dal Cleve indicata per la Nuova Zelanda; la fig. 2 della tavola 48 dell' Atlas sopra citato rappresenta una valva di tale provenienza; la var. vulgaris fu distribuita da Tempère e Peragal.lo nelle Diatomées du monde entier delle isole Thursday (n. 561-562, 590-593), dello stretto di Malacca (n. 622-623) e delle isole Samoa (n. 647-648).

\section{Frustulia C. Ag.}

Frustulia Rhomboides (Ehr.) De Toni Sylloge Algarum II (1891) pag. 277. Var. Saxonica (Rabenh.) De Toni op. cit. pag. 277; Cleve Syn. Navic. Diat. I (1894) pag. 123. 
Frustulia saxonica Rabenh. Bacill. exsicc. (1851!) n. 42.

Navicula crassinervia Bréb. in W. Sm. Brit. Diat. I (1853!) pag. 47, tab. 31, fig. 271.

Vanheurckia rhomboides var. crassinervia Van Heurck Synopsis tab. 17, fig. 4-5, Traité pag. 240, tab. 5, fig. 250.

Hab. Nuova Zelanda: Wakarevareva.

Per la Nuova Zelanda e per l' Australia la presente varietà fu segnalata già dal Cleve; essa venne anche distribuita del lago Rotorua da Tempère e Peragallo al n. 273 delle Diatomées du monde entier. Le ragioni per le quali è preferibile il nome generico Frustulia C. Ag. in luogo di Vanheurckia Bréb. vennero da noi esposte in altro lavoro; cfr. De 'Toni G. B. e Forti A., Terza contribuzione alla flora algologica della Libia, 1914, pag. 1526-1527.

Frustulia vulgaris (Thwait.) De Toni Sylloge Algarum II (1891) pag. 280 ; Cleve Syn. Navic. Diat. I (1894) pag. 122.

Vanheurckia vulgaris Van Heurck Synopsis tab. 17, fig. 6, Traité pag. 240, tab. 5, fig. 252 .

Hab. Tasmania: Hobart; Nuova Zelanda: Wakarevareva.

Il Cleve indica questa specie per la Tasmania e l' Australia (Orara River, Blue Mountains ecc.). Tempère e Peragallo la distribuirono fossile di Waitangi (Diatomées du monde entier n. 922). Noi osservammo anche un megafrustolo di Wakarevareva con le dimensioni di $115 \mu$ di lunghezza e di 16 di larghezza fra i noduli centrali della rafe.

\section{Pleurosigma W. Sm.}

Pleurosigma formosum W. Sm. in Ann. and Magaz. of Nat. Hist. 2 ser., IX (1852) pag. 5, tab. 1, fig. 1, Brit. Diat. I (1853) pag. 63, tab. 20, fig. 195; V. H. Synopsis tab. 19, fig. 4, Traité pag. 254, tab. 6, fig. 268; Perag. Monogr. des Pleuros. tab. 1, fig. 3-5; Cleve Syn. Navic. Diat. I (1894) pag. 45; De Toni Sylloge Algarum II, pag. 243.

$H a b$. Nuova Zelanda: Bluff.

Il Petit (Diat. Campb. el N. Zél. pag. 28) indicò già questa specie per l'is. Campbell, donde fu poi distribuita al n. 357 delle Diatomées $d u$ monde entier da Tempére e Peragallo; il Cleve la segnalò per le isole Sandwich, il Mereschowsky confermandola per queste isole vi aggiunse Tahiti; il Pleurosigma formosum W. Sm. fu enumerato dal Lemmermann tra le Diatomee raccolte nel French Pass. Il CLeve riferisce alla presente specie il Pleurosigma australicum Witt e il Pleur. tahitense Witt dell' Oceania. Nella collezione sopra citata di TEMPÉRE e PeRagallo la specie è distribuita di parecchie località : Albany, Australia occidentale (n. 276-277), lavaggi di conchiglie di Haliotis della Nuova Zelanda (n. 478-479), isole Thursday (n. 561-562, 590-591), Stretto di Malacca (n. 622-623), lavaggi di conchiglie dello Stretto di Torres (n. 946).

Pleurosigma marinum Donk. in Trans. R. Micr. Soc. VI (1858) pag. 22, tab. 3, fig. 3 ; V. H. Traité pag. 254, tab. 28, fig. 794; Perag. Monogr. des Pleuros. 
tab. 3, fig. 11 ; Cleve Syn. Navic. Diat. 1 (1894) pag. 37; De Toni Sylloge Algarum II, pag. 236.

Hab. Nuova Zelanda: Port Charmers.

La specie non è nuova per la regione neozelandese, avendola indicata il PETIT (Diat. Camp. et N. Zél. pag. 28). Il Cleve la indica per Port Jackson, Australia.

Pleurosigma naviculaceum Bréb. Diat. de Cherbourg (1867) pag. 17, fig. 7 ; V. H. Synopsis Suppl. fig. 35, Traité pag. 252, tab. 6, fig. 266; Perag. Monogr. des Pleuros. tab. 4, fig. 12, 20-22 ; Cleve Syn. Navic. Diat. I (1894) pag. 36; De Toni Sylloge Algarum II, pag. 234.

Hab. Nuova Zelanda: Port Charmers.

\section{Gyrosigma Hass.}

Gyrosigma acuminatum (Kuetz.) Rabenh. Die Suessw. Diatom. (1853) pag. 47 (Gyrosigma acuminata).

Var. Brebissonir (Grun.) Cleve Syn. Navic. Diat. I (1894) pag. 114.

Pleurosigma balticum var. Brebissonii V. H. Synopsis tab. 21, fig. 6, Traité pag. 256, tab. 6, fig. 273; De Toni Sylloge Algarum II, pag. 250.

Pleurosigma Brebissonii Grun. in Cleve et Grun. Arct. Diat. (1880) pag. 56 ; Perag. Monogr. des Pleuros. tab. 7, fig. 29-30.

Hab. Nuova Zelanda: Wakarevareva.

Il genere Gyrosigma Hassall, istituito nel 1845, col tipo Gyr. Hippocampus Hass., venne lentamente surrogato dal nome Pleurosigma proposto da W. SMith, malgrado che il RABEnHorst avesse tentato di mantenerlo nello stesso anno 1853 (Die Suesswasser - Diatomaceen pag. 46); torna a merito del Cleve l' aver rimesso in vigore il genere Hassalliano ascrivendo al medesimo le specie di Pleurosigma mancanti dalle strie oblique (decussate) e conservando il genere Pleurosigma per le specie caratterizzate della presenza delle strie oblique, come aveva nettamente intravveduto lo stesso RABENHORST nella sua disposizione dei Pleurosigma, dandone anche due figure schematiche (Flora Europaea Algarum I, 1864, pag. 231 e 235).

\section{Mastogloia Thwait.}

Mastogloia angulata Lewis New and rare Species (1861) pag. 65, tab. 2, fig. 4 ; A. Schmidt Atlas (1893) tab. 187, fig. 4-11; Cleve Syn. Navic. Diat. II (1895) pag. 147.

Mastogloia apiculata Grun. Ueb. neue Diatom. (1860) pag. 577, tab. 7, fig. 9 (non W. Smith).

$H a b$. Australia occid. : Baia di Geroldton.

Questa specie per l'Australia era nota di Port Lincoln (A. SchmidT), venne poi distribuita delio Stretto di Malacca ai n. 622-6́23 delle Diatomées du monde entier di Tempère e Peragallo.

Mastogloia? Pernotata Meresch. On Polynesian Diatoms (1902) pag. 9, tab. 4, fig. 1-2.

Hab. Australia occid. : Baia di Geroldton; Nuova Zelanda: Port Charmers. 
Questa specie che, astraendo dal numero maggiore dei loculi marginali, ricorda assai la Orthoneis binotata Grun. fu descritta dal Mereschkowsky tra le Diatomee provenienti dalle isole Samoa.

Mastoglora Smithin Thwait. in W. Sm. Brit. Diat. II (1856) pag. 65, tab. 54, fig. 341 ; V. H. Synopsis tab. 4, fig. 13, Traité pag. 154, tab. 2, fig. 60 ; Cleve Syn. Navic. Diat. II (1895) pag. 152 ; De Toni Sylloge Algarum II, pag. 313.

Hab. Tasmania: Burmie.

La Mastogloia Smithii Thwait. era già conosciuta per l' Australia e per la Tasmania (CLEVE).

Var. Conifera Br. in Perag. Diat. pag. 37, tab. 6, fig. 41.

Hab. Tasmania: Burmie.

\section{Fam. GOMPHONEMACEAE (Kuetz.) Grun.}

\section{Gomphonema C. Ag.}

Gomphonema constrictum Ehr. in Abhandl. Berl. Akad. 1831? pag. 63; V. H. Synopsis tab. 23, fig. 6, Traité pag. 270, tab. 7, fig. 296; Cleve Syn. Navic. Diat. I (1894) pag. 186; De Toni Sylloge Algarum II, pag. 421.

Hab. Tasmania: Hobart.

Per l'Oceania in generale il CLEVE indica riguardo al Gomphonema constrictum Ehr. l'Australia e la Tasmania: la specie fu segnalata anche nella Nuova Zelanda per il Distretto di Otago (Lauder Lindsay) e nelle is. Chatham (LEMMERMANN).

Gomphonema parvulum (Kuetz.) Rabenh. Fl. Eur. Algar. I (1864) pag. 291 ; V. H. Synopsis tab. 25, fig. 9, Traité pag. 272, tab. 7, fig. 306 ; Cleve Syn. Navic. Diat. I (1894) pag. 180; De Toni Sylloge Algarum 1I, pag. 429. fig. 63.

Sphenella parvula Kuetz. Die Kieselsch. Bacillarien (1844) pag. 83, tab. 30,

Hab. Nuova Zelanda: Wakarevareva.

La specie venne già indicata per la Nuova Zelanda dal CLeve e poi distriY buita fossile di Pakaraka al n. 919 delle Diatomées du monde entier da Temperre e Peragallo.

\section{Rhoicosphenia Grun.}

Rhoicosphenia curvata (Kuetz.) Grun. Ueb. neue Diatom. (1860) pag. 511, Alg. Novara (1867) pag. 8; V. H. Synopsis tab. 26, fig. 1-3, Traité pag. 275, tab. 7, fig. 319; Cleve Syn. Navic. Diat. II (1895) pag. 165; De Toni Sylloge Algarum II, pag. 437.

Hab. Tasmania: Hobart; Nuova Zelanda: Wakarevareva.

La forma tipica era già nota per la Nuova Zelanda (Cleve) e le is. Chatham (LEMMERMANN).

Var. MaRina (W. Sm.) Rabenh. Fl. Eur. Algar. I (1864) pag. 113 ; V. H. Sy- 
nopsis tab. 26, fig. 4, Traité pag. 276, tab. 7, fig. 320; De Toni Sylloge Algarum II, pag. 438.

Hab. Tasmania: Baia di Latrobe: Nuova Zelanda: Port Charmers.

Il Grunow (Alg. Novara pag. 8) indicò già questa varietà epifita sulle Sphacelarieae della Nuova Zelanda; il Petit (Diat. Camp. et N. Zél. pag. 16) la enu. merò tra le Diatomee della Lyall's Bay e dell' isola Campbell. La varietà marina è appena differente dalla forma tipica per $\mathrm{i}$ frustoli alquanto più grandi $\mathrm{e}$ per la stazione marina.

\section{Fam. CYMBellaceae (Kuetz.) Grun.}

$$
\text { Cymbella C. Ag. }
$$

Cymbella amphicephala Naeg. in Kuetz. Species Algarum (1849) pag. 890 ; V. H. Synopsis tab. 2, fig. 6, Traité pag. 142, tab. 1, fig. 25; Cleve Syn. Navic. Diat. I (1894) pag. 164; De Toni Sylloge Algarum II, pag. 350.

Hab. Nuova Zelanda: Wakarevareva.

Come area di distribuzione nell' Oceania il Cleve dà la Tasmania e la Nuova Zelanda.

Cymbella australica (A. Schm.) Cleve Syn. Navic. Diat. I (1894) pag. 176.

Cocconema australicum A. Schmidt Atlas (1885) tab. 10, fig. 34-35; Cleve et Moeller Diat. n. 295 !; De Toni Sylloge Algarum II, pag. 368.

Hab. Nuova Zelanda: Wakarevareva.

La specie venne già segnalata per l'Australia e per la Nuova Zelanda; di quest' ultima trovansi distribuiti esemplari nella collezione sopra citata di CLEVE e Moeller.

In Australia di Yarra River fu distribuita ai n. 241-243 delle Diatomées du monde entier da Tempère e Peragallo. La Cymbella australica (A. Schm.) Cleve fu riscontrata anche fossile da uno di noi nel deposito delle Colline di Soddo in Etiopia.

Cymbella Cesatil (Rabenh.) Grun. in A. Schmidt Atlas (1876) tab. 71, fig. 48-49; Cleve Syn. Navic. Diat. I (1894) pag. 160.

Navicula Cesatii Rabenh. Die Süssw. Diatomaceen (1853) pag. 39, tab. 6, fig. 89: V. H. Synopsis tab. 8, fig. 35; De Toni Sylloge Algarum II, pag. 59.

Hab. Nuova Zelanda: Wakarevareva.

Considerazioni particolari su questa Cymbella furono esposte recentemente da N. Yermoloff (1).

Cymbella naviculiformis Auersw. in Kirchn. Alg. Schles. (1878) pag. 187; Cleve Syn. Navic. Diat. I (1894) pag. 166 (non Héribaud).

Cymbella cuspidata var. naviculiformis Auersw. in Rabenh. Alg. Eur. (1861)

(1) Cfr. Yermoloff Nich., Notes on some intermediate Forms of the genera Navicula and Cymbella (Jou'n. of the Quekett Microsc. Club ser. II, vol. XIII, 1918, n, 83). 
n. 1065 ; V. H. Synopsis tab. 2, fig. 5, Traite pag. 142, tab. 1, fig. 24 ; De Toni Sylloge Algarum II, pag. 350.

Hab. Tasmania: Hobart.

Il Cleve indicò questa Cymbella per l' Australia e per la Nuova Zelanda.

Cymbella Parva (W. Sm.) Cleve Syn. Navic. Diat. I (1894) pag. 172.

Cocconema parvum W. Sm. Brit. Diat. I (1853) pag. 76, tab. 23, fig. 222 (tab. 24, fig. 222?).

Cymbella cymbiformis var. parva V. H. Synopsis tab. 2, fig. 14, Traité pag. 147, tab. 1, fig. 39; De Toni Sylloge Algarum II, pag. 364.

Hab. Nuova Zelanda: Wakarevareva.

Crmbella sinuata Greg. in Microsc. Journ. IV (1856) tab. 1, fig. 17; Cleve Syn. Navic. Diat. I (1894) pag. 170; De Toni Sylloge Algarum II, pag. 361.

Hab. Tasmania : Hobart.

Questa specie è indicata dal Cleve per la Nuova Zelanda.

\section{Encyonema Kuetz.}

Encyonema ventricosum (Ag. ?) Grun. in Kirchn. Alg. Schles. (1878) pag. 189; V. H. Synopsis tab. 3, fig. 17, Traité pag. 150, tab. 1, fig. 49; Cleve Syn. Navic. Diat. I (1894) pag. 168; De Toni Sylloge Algarum II, pag. 373.

Hab. Nuova Zelanda: Rotorua, geyser, a.

Questa specie ha un'ampia distribuzione geografica; il CLEVE la segnalò per l'Australia, la Tasmania e la Nuova Zelanda; del lago Rotorua trovasi al n. 273 delle Diatomées du monde entier di Tempère e Peragallo; in questa medesima importante collezione si ha fossile di Cabbage Tree Swamp, Auckland (n. 223) e di Waitangi (n. 922) per la Nuova Zelanda, nonchè di Talbot, Melbourne per l' Australia (n. 981-983).

\section{Amphora Ehr.}

Amphora Arcus Greg. in Trans. Micr. Soc. V (1854) pag. 75, tab. 1, fig. 37, Diat. of Clyde (1857) pag. 522, tab. 13, fig. 88 ; Cleve Syn. Navic. Diat. II (1895) pag. 127, tab. 4, fig. 4; Perag. Diat. mar. de France tab. 50, fig. 6.

Hab. Tasmania: Baia di Latrobe.

Non è nuova questa specie per l' Oceania, essendo stata dai Peragallo segnalata per le isole Salomone.

Amphora ARenicola (Grun.) Cleve Syn. Navic. Diat. II (1895) pag. 104.

Var. stibaequalis Cleve op. cit. pag. 104, tab. 4, fig. 22.

Hab. Australia occid. : Baia di Geroldton.

Questa varietà era conosciuta del mare della China, secondo esemplari della collezione Deby studiati dal Cleve; fu poi distribuita da P. BERgon per Albany, Australia occidentale nelle Diatomées du monde entier n. 276-277 di TeMPÈre e Peragallo. 
Amphora costata W. Sm. Brit. Diat. I (1853) pag. 20, tab. 30, fig. 253; Cleve Syn. Navic. Diat. II (1895) pag. 122.

Hab. Tasmania: Baia di Latrobe; Nuova Zelanda: Port Charmers.

Questa Amphora riguardo all' Oceania venne indicata per le isole Salomone e Samoa (Peragallo) e di queste ultime distribuita al n. 648 delle Diatomées du monde entier da Tempere e Peragallo.

Amphora formosa Cleve in A. Schmidt Atlas (1875) tab. 28, fig. 34, tab. 39, fig. 2 ; Syn. Navic. Diat. II (1895) pag. 138; De Toni Sylloge Algarum II, pag. 397.

Hab. Tasmania: Burmie.

Per la regione australasica è nota di Albany, Australia occidentale, donde venne distribuita ai n. 276-277 delle Diatomées $d u$ monde entier di TeMpère e Peragallo.

Amphora gigantea Grun. in A. Schmidt Atlas (1875) tab. 27, fig. 46; Cleve Syn. Navic. Diat. II (1895) pag. 105; De Toni Sylloge Algarum II, pag. 409.

$H a b$. Australia occid. : Baia di Geroldton.

I Peragallo la indicarono per l' isola Salomone.

Amphora obtusa Greg. in Microsc. Journ. V (1857) tab. 1, fig. 34 ; H. L. Smith in The Lens II (1873) pag. 70, tab. 1, fig. 5; V. H. Traité pag. 137, tab. 24, fig. 688 ; Cleve Syn. Navic. Diat. II (1895) pag. 131; De Toni Sylloge Algarum II, pag. 404.

Hab. Tasmania : Burmie.

Questa specie ha un' estesa distribuzione geografica; i Peragallo la indicarono per le isole Samoa (cfr. anche Diatomées $d u$ monde entier n. 647-648). Nella collezione or menzionata è distribuita di Albany, Australia occidentale (n. 160, 276-277), dello Stretto di Malacca (n. 622-623) ed anche fossile del deposito neozelandese di Oamaru (n. 810).

Amphora ovalis (Brèb.) Kuetz. Die Kieselsch. Bacillarien (1844) pag. 107, tab. 5, fig. 35-37.

Var. AfFinis (Kuetz.) V. H. Synopsis pag. 59, tab. 1, fig. 2 ; De Toni Sylloge Algarum II. pag. 412.

Hab. Nuova Zelanda: Wakarevareva.

L'Amphora ovalis (Brèb.) Kuetz. con le sue varietà rappresenta. un tipo cosmopolita.

Amphora Proteus Greg. Diat. of Clyde (1857) pag. 518, tab. 13, fig. $81 ; \mathrm{H}$. L. Smith in the Lens II (1873) pag. 79, tab. 3, fig. 1; V. H. Traité pag. 129, tab. 24, fig. 671; Cleve Syn. Navic. Diat. II (1895) pag. 103; De Toni Sylloge Algarum II, pag. 403.

Hab. Australia occid.: Baia di Geroldton; Australia merid.: Victor Harbour; Tasmania: Baia di Latrobe, Burmie; Nuova Zelanda: Port Charmers.

Questa Amphora ha un' estesa distribuzione geografica. Per quanto concerne l' Oceania si ricordi che la specie venne segnalata dal Petri (Diat. Campb. et N. Zél. pag. 17) alla Lyall's Bay della Nuova Zelanda, dai Peragallo per le isole Salomone; da Tempère e Peragallo nelle Diatomées du monde entier fu 
distribuita di Albany, Australia occidentale (n. 160, 276-277) e di Yarra River (n. 241-243) ; una varietà di essa dell' isola Campbell (n. 357).

Amphora spectabilis Greg. Diat. of Clyde (1857) pag. 44, tab. 5, fig. 80 ; Cleve Syn. Navic. Diat. II (1895) pag. 132 ; De Toni Sylloge Algarum II, pag. 408 (non A. Schmidt Atlas (1876) tab. 40, fig. 20)

$H a b$. Australia occid.: Baia di Geroldton; Nuova Zelanda : Bluff.

La specie venne già segnalata dal Petit (Diat. Campb. et $N$. Zél. pag. 17) per lo Stretto di Foveaux e dai Peragallo per le isole Samoa, Salomone e Hawaii; Tempère e Peragallo la distribuirono nelle Diatomées du monde entier dello Stretto del Re Giorgio da Albany (n. 160, 276-277), dello Stretto di Torres (n. 946 ?) e delle isole Samoa (n. 647-648).

\section{Okedenia Eulenst.}

Okedenia inflexa (Bréb.) Eulenst. in Van Heurck Typ. (1868) n. 167; De Toni Sylloge Algarum II, pag. 229.

Amphipleura implexa Bréb. in Kuetz. Species Algarum (1849) pag. 88; Pritchard Hist. of Infus. (1861) pag. 783, tab. 4, fig. 31.

Amphora implexa H. L. Smith in The Lens II (1873) pag. 78, tab. 2, fig. 16 ; Cleve Syn. Navic. Diat. II (1895) pag. 131 ; V. H. Traité pag. 134, fig. 25 ; W. West in Journ. Quekett Micr. Club ser. 2, vol. XIV, 1919, pag. 35, tab. II.

Hab. Tasmania: Burmie.

Var. SYNEDROIDES $\mathrm{n}$. var. : Frustulis brevioribus quam in forma typica, $48-60 \mu$ longis; valvis fere rectis.

Hab. Nuova Zelanda: Port Charmers.

11 Peragallo (Diat. mar. de France tab. 49, fig. 1-2) dà per la Okedenia inflexa (Bréb.) Eulenst. le dimensioni in lunghezza corrispondenti a 90-150 $\mu$ per una larghezza di 5-10 $\mu$; il VAN HeURCK per la stessa specie fornisce i dati seguenti: Valve lunghe $100-120 \mu$, larghe, nella parte mediana, meno di $10 \mu$. Frustoli larghi $7-15 \mu$.

\section{APPENDICE (1)}

\section{Fam. CHARACEAE Rich.}

\section{Nitella A. Br.}

Nitella myriotricha A. Br. in Kuetz. Tab. Phyc. VII (1857) pag. 15, tab. 39, fig. II ; Fragmente einer Monogr. der Characeen [edit. O. Nordstedt] (1882) pag. 80, tab. 6, fig. 158-163; Nordst. Einige Characeenbestimmungen (1888) pag. 185, n. 13, De Algis et Characeis (1889) pag. 28.

Hab. Australia occid.: Avon River, o' e 9,11 ottobre 1908.

(1) Fra i materiali riportati dal suo viaggio in Australia il rev. G. CAPra riportò due Characeae che reputiamo conveniente inserire alla fine dell' illustrazione delle Alghe. 
Questa Nitella venne per la prima volta spedita, nel 1854, da Ferdinando Mueller al Sonder dalla Nuova Olanda e fu conservata nell'erbario di quest'ultimo botanico con l' indicazione di origine «Australia Felix "; il Sonder poi ( $\mathrm{Al}$ gae Austral. hactenus cognitae pag. 44, n. 21) diede la precisa località * Mc Ivor, Moreton Bay " su raccolte compiute dallo stesso Mueller nel 1867 ('). Aggiungendo che la specie fu poscia segnalata presso Port Phillip dove, secondo il NoRDSTEDT, la raccolsero rispettivamente nel 1883 e nel 1887 J. BRACEBRIDGE Wilson e C. French e che fu pure trovata al Golfo di Carpentaria, è ovvio comprendere trattarsi di una Nitella largamente distribuita nella Nuova Olanda. Gli esemplari raccolti dal Capra, pur non essendo dei più sviluppati, dimostrano evidente ogni loro caratteristica e tra l'altro la lucentezza sericea delle ramificazioni più tenui.

Nitella Sonderi A. Br. in Linnaea vol. 25 (1852) pag, 704 ; Kuetz. Tab. Phyc. VII (1857) pag. 17, tab. 42, fig. 1 (non bona); A. Br. Fragmente einer Monogr. der Characeen [edit. O. Nordstedt] (1882) pag. 47, tab. 5, fig. 111-113; Nordst. Einige Characeenbestimmungen (1888) pag. 182, n. 4; De Algis et Characeis (1889) pag. 24.

Hab. Australia orient.: Baron River, N. Queensland, 3 giugno 1909.

Per questa Nitella il Sonder (Algae Austral. hactenus cognitae pag. 44, n. 25) aveva indicato in base al dato del Braun (Plantae Muellerianae, Characeae, 1852, pag. 704) la località Melbourne; la stessa specie fu segnalata dal NoRDSTEDT per Avenal, Goulbourne River e per il Golfo di Carpentaria; ciò che lascia com. prendere come la Nitella Sondevi A. Br. abbia, al pari della precedente, una larga distribuzione geografica nella Nuova Olanda. I nostri materiali sono rappresentati da talli spezzettati frammisti a sabbia e tanghiglia più che tutto riconoscibili dalle forme degli oogonii maturi.

(1) A Nitella myriotricha A. Br. va ascritto, secondo l'opinione espressa dal NoRDSTEDT (Einige Characeenbestimmungen I888, pag. 185) un esemplare raccolto nel 1867 da FERDINANDO MUELLER con 1' indicazione «Murray River 》. Resta incerto se trattisi del "Murray River 》 della Nuova Olanda occidentale ovvero dell' omonimo "Murray River" della Nuova Olanda meridionale (Victoria); però con maggiore probabilità è a questo secondo fiume che va riferito il reperto Muelleriano. 


\section{BIBLIOGRAFIA (}

AgARdh C. A. - Species Algarum rite cognitae, Volumen primum, pars prima. Fucoideae; Gryphiswaldiae, I821, sumptibus Ernesti Mauritii, 160.

- Species Algarum rite cognitae, Voluminis primi pars posterior; Lundae, 1822, Ex officina Berlingiana, $16^{\circ}$.

- Species Algarum rite cognitae, voluminis secundi sectio prior; Gryphiae, 1828, sumptibus Ernesti Mauritii, $16^{\circ}$.

- Systema Algarum; Lundae, I824, literis Berlingianis, 160.

- Icones Algarum ineditae; Lundae, I820-22.

- Revision der Algengattung Macrocystis (Nova Acta Acad. Caesar. Leopold. Vol. XIX, I839, pag. 283-3I6, Tab, XXVI-XXVIII).

Agardh J. G. - In historiam Algarum Symbolae (Linnaea vol. XV, I84I, pag. 443-447).

- In Systemata Algarum hodierna adversaria; Lundae, I844, typis Berlingianis, $8^{\circ}$.

- Caroli Ad. Agardh Icones Algarum ineditae. Fasciculi qui extant duo. Editio nova; Lundae, 1846, ex officina Berlingiana, $4^{\circ}, 20$ tab.

- Algologiska Bidrag (Stockholm's Oefvers. VI, x849, pag. I-II).

- Species, genera et ordines Algarum, volumen primum: Algas Fucoideas complectens; Lundae, I 848 , typis Berlingianis, $8^{\circ}$.

- Species, genera et ordines Algarum, volumen secundum: Algas Florideas complectens; pars prior, pars secunda, pars tertia; Lundae, 1851, 1852, 1863, typis Berlingianis, $8^{\circ}$.

- Species, genera et ordines Algarum, volumen tertium. De Florideis curae posteriores [Epicrisis Systematis Floridearum]; Lundae, I 876, typis Berlingianis, $8^{\circ}$.

- Species, genera et ordines Algarum, voluminis tertii, pars secunda [Morphologia Floridearum]; Lundae, 1880, typis Berlingianis, $8^{\circ}$.

- Species, genera et ordines Algarum, voluminis tertii, pars tertia: De dispositione Delesseriearum curae posteriores; Lundae, I898, typ. E. Malmström, $8^{\circ}$.

- Species, genera et ordines Algarum, voluminis tertii, pars quarta: Supplementa ulteriora et indices sistens; Lundae, Igor, typ. E. Malmström, $8^{\circ}$.

- Om Chatham-ōarnes Alger (Oefvers, K. Vet, Akad. Förhandl. Stockholm, 187o, n. 5, pag. 435-455).

- Bidrag till Florideernes Systematik (Lunds Univers. Aarsskrift T. VIII, Lundae 187I).

- List of the Algae of the Chatham Islands, collected by H. H. Travers Esq. (New Zealand Institute Transact. VI, 1874, pag. 208-210).

- Om Bladets Udvikling hos Algerne, 1 pl.; Kjoebenhavn, I874, J. H. Schultz, $8^{\circ}$.

- De Algis Novae Zelandiae marinis (Lunds Univers. Aarsskrift T. XIV, Lundae 1877).

- Florideernes Morphologi, med. 33 Tafl. (K. Svenska Vet. Akad. Handl. Bandet 15, n. 6, Stockholm 1879).

- Till Algernes Systematik I. Caulerpa; II. Zonaria; III. Sargassum (Lunds Univers. Aarsskrift T. IX, Lundae 1872).

- Till Algernes Systematik IV. Chordarieae; V. Dictyoteae, 3 Tab. (Lunds Univers. Aarsskrift T. XVII, Lundae 1880 ).

- Till Algernes Systematik VI. Ulvaceae, cum 4 tab, color. (Lunds Univers. Aarsskrift T. XIX, Lundae 1882).

- Till Algernes Systematik VII. Florideae (Lunds Univers. Aarsskift T. XXII, Lundae 1885).

- Till Algernes Systematik VIII. Siphoneae (Lunds Univers. Aarsskrift T. XXIII, Lundae I886).

- Till Algernes Systematik IX. Sporochnoideae; X. Fucaceae; XI. Florideae, cum 3 tab. color. (Lunds Univers. Aarsskrift T. XXVI, Lundae r8go).

(x) Utili indicazioni bibliografiche intorno alcune opere di J. D. Hooker e W. H. HARVEY ci ventrero gentilmente fornite dal chiar,mo amico Astowo Gepp del Bratish Museum of Nalural History di Londra. 
Agardh J. G. - Species Sargassorum Australiae descriptae et dispositae, cum $3 \mathbf{I}$ tab. ( $K$. Svenska Vet. Akad. Handl. Bandet 23, N. 3; Stockholm 1889).

- Analecta algologica (Lunds Univers. Aarsskrift T. XXVIII, Lundae I892).

- Analecta algologica. Continuatio I, cum 2 tab. (Lunds Univers. Aarsskrift T. XXIX, Lundae 1894).

- Analecta algologica. Continuatio II, cum I tab. (Lunds Univers. Aarsskrift T. XXX, Lundae 1894).

- Analecta algologica. Continuatio III, cum I tab. (Acta Reg. Soc. Physiogr. Lund. T. VII, Lundae 1896 ).

- Analecta algologica. Continuatio IV, cum 2 tab. (Acta Reg. Soc. Physiogr. Lund. T. VIII, Lundae 1897).

- Analecta algologica. Continuatio V, cum 3 tab. (Acta Reg. Soc. Physiogr. Lund. T. X, Lundae 1899).

Archer W. - On the minute Structure and Mode of Growth of Ballia callitricha Ag. (Transact. Linn. Soc. Bot., ser, 2, vol. I, 1876, pag. 211-232, tab. 28-29).

- New Zealand Desmidieae (Grevillea vol. IX, 188ז, pag. 29).

Ardissone F. - Le Alghe cosmopolite (Rend. R. Istituto Lombardo di scienze e lettere ser. II, vol. XXVII, fasc. XIX, Milano I894).

AReschovg J. E. - Iconographia phycologica, seu Phycearum novarum et rariorum icones atque descriptiones, Decas I; Gothoburgi, 1847, N. J. Gumpert, $4^{\circ}, 6$ pp., Io tab.

- Phyceae novae et minus cognitae in maribus extraeuropaeis collectae (Acta Reg. Soc. Scient. ser. III, vol. I, Upsaliae I854).

- Observationes phycologicae, particula quinta: De Laminariaceis nonnullis (Acta Reg. Soc. Scient. ser. III, vol. XII, fasc. II, Upsaliae I884).

Askenasy E. - Algen [mit Unterstützung der Herren E. Bornet, A. Grunow, P. Hariot, M. MoeBIUS, O. NordSTEDT] (Forschungsreise $S_{*} M . S_{0}$ «Gazelle 》 IV. Teil, Botanik, pp. 58, T. I-XII, Berlin 1888).

- _. Ueber einige australische Meeresalgen. (Flora oder allgemeine botanische Zeitung 1894, Heft I, mit Taf.).

Bailey F. Manson - Contributions to the Queensland Flora (Departm. of Agriculture, Botany Bull. n. VI, pp. 58 VIII, plates I9, Brisbane 1893).

- Contributions to the Queensland Flora (Departm. of Agriculture, Botany Bull. n. XI, pp. 69, pl. I-XVII, Brisbane 1895).

- Contributions to the Queensland Flora (Departm. of Agriculture, Botany Bull. n. XV, pp. 38, pl. I-XVII, Brisbane I898).

- Contributions to the Flora of Queensland Algae (The Queensland Agric. Journ. XXVI, part 3 [191I] pag. 128-129, XXVII [1911] pag. 252).

Bailey J. W, v. HaRvey W. H.

Barton E. S. - A Systematic and structural account of the Genus Turbinaria, Lamx. (Transact. Linn. Soc., 2 Ser. Botany, vol. III, part 5, pag. 215-226, plates LIV-LV, London 1891).

- Xiphophora Billardieri Mont. (Phycological Memoirs by G. MURray p. VI, pag. 7-8, plate XI, fig. 1-4, London 1893).

- On Notheia anomala, Harv. et Bail. (Journ. Linn. Soc., Botany, vol. XXXIV, pag. 417-425, plates 12-I4, London I899).

- On the Forms, with a New Species, of Halimeda from Funafuti (Journ. Linn. Soc., Botany, vol. XXXIV, pag. 479-482, pl. I8, London I900).

- The Genus Halimeda (Siboga-Expeditie, Monogr. LX, pp. 32, pl. I-IV, Leiden I9or).

- v, Gepp A, et E. S.

BERNARD $\mathrm{CH}_{0}$ - Sur quelques Algues unicellulaires d'eau douce récoltées dans le domaine malais [con bibliografia relativa all' algologia malese]; Buitenzorg, 1909, Impr. du Département de 1'Agriculture, $8^{\circ}$.

- Algues d'eau douce [id.] (Nova Guinea VIII, pag. 253-27o, T. LXI-LXII).

Boodle L. A. v. Murray G.

Borge O. - Australische Süsswasserchlorophyceen (Bihang till K. Svenska Vet. Akad. Handl. Band 22, Afd. III, N. 9, pp. 32, T. I-IV, Stockholm I896).

Bornet E. - Deux Chantransia corymbifera Thuret. Acrochaetium et Chantransia (Bull. Soc. Botan. de France T. 5I, pag. XIV-XXIII, pl. I, Paris 1904).

Bornet E。 et Flahault $\mathrm{CH}_{\text {. }}$ - Revision des Nostocacées hétérocystées contenues dans les principaux herbiers de France (Annales des Sciences Natur. VII série, Botanique, tomes III, IV, V et VII ; Paris $1886-1888$ ). 
Borzì A. - Alghe d' acqua dolce della Papuasia raccolte su cranii umani dissepolti (La Nuova Notarisia ser. III, pag. $35-53$, Padova 1892).

- Studi sulle Mixoficee (Nuovo Giom. bofan. ital. Nuova Serie, vol. XXI, n. 4, vol. XXIV, n. 1-4, pp. 166, tav. VI-X, Firenze 1914-1917).

Bory de Saint-Vincent J. B. M. - Histoire des Hydrophytes ou Plantes agames des eaux récoltées par MM. D' Urville et Lesson dans leur voyage autour du monde sur la Corvette de Sa Majesté, la Coquille; Paris, 1829, Firmin Didot, fol., pp. 240, pl. i-24.

Brand F. - Ueber die Anheftung der Cladophoraceen und über verschiedene polynesische Formen dieser Familie (Beihefte zum Botan. Centralblatt Bd. XVIII, Abt. I, Heft 2, 1904, pag. 165-193, T. V-VI).

- Ueber einige neue Grünalgen aus Neuseeland und Tahiti (Ber. der deutschen botan. Gesellsch. Band XXIX, 1911, pag. I38-145, T. VII).

- Ueber die Siphoneengattung Chlorodesmis (Ber. der deutschen boton. Gesellsch. Band XXIX, IgII, pag. 606-61I, Fig. 1-7).

Braun AL. - Plantae Muellerianae. Characeae (Linnaea vol. 25, 1852, pag. 704-709).

- Characeae Australianae hactenus cognitae (In Mueller Ferd., Fragmenta Phytographiae Australiae vol. XI, Suppl. pag. 43-44; Melbourne, 1878-188I, ex off. J. Ferres, $8^{\circ}$ ).

- Fragmente einer Monographie der Characeen. Nach den hinterlassenen Manuscripten A. Braun's herausgegeben von D.r Otто NoRdstedt (Abhandl. K. Akad. der Wissenschaftên zu Berlin 1882, pp. 2 II, T. I-VII).

BRIGHTWELL T. - On the genus Triceratium, with Description and figures of the Species (Quart. Journ. Micr. Sc, vol. IV, 1856, pag. 245-252, plate IV).

- Further Observations on the Genus Triceratium, with Description and figures of new Species (Quart. Journ. Micr. Sc. vol. IV, 1856, pag. 272-276, plate XVII).

- On some of the rarer or undescribed Species of Diatomaceae, p. I (Quart.Journ. Micr. Sc, vol. VII, 1859, pag. I79, plate IX).

Brun J. - Diatomées. Espèces nouvelles marines, fossiles et pélagiques (Mèm. Soc. de phys, et d'hist. natur. de Genève T. XXXI, seconde partie, n. 1, 1891, pp. 48, pl. XI-XXII).

- Note sur quelques espèces nouvelles (Le Diatomiste I, 1890-93, pag. 173-177, pl. XXIV).

- Diatomées. Espèces nouvelles (Le Diatomiste II, 1893-96, pag. 72-78, 86-88, pl. V-VI).

- Diatomées lacustres, marines ou fossiles. Espèces nouvelles ou insuffisamment connues ( $\mathrm{Le}$ Diatomiste II, 1893-96, pl XIV-XVII).

Buchanan - Notes on the Flora of the Province of Wellington with a List of Plants collected therein (Transac. New Zealand Institute 1873, pag 210.235).

Butters F. K. - Observations on Trichogloea lubrica (Minnesota Botanical Studies Third Series, part I, pag. II-21, plates V-VI, Minneapolis 1903).

- Notes on the Species of Liagora and Galaxaura of the Central Pacific (Minnesota Botanical Studies vol. IV, part II, pag. I6I-184, plate XXIV, Minneapolis I911).

CARD G. W. and Dun W. S. - The Diatomaceous Earth Deposits of New South Wales (Rec. Geol. Survey of $N$. S. Wales vol, V, pag. 128-148, plates XII-XV).

Castracane F. - Report on the Diatomaceae collected by H. M. S. Challenger during te years $1873-76$; London, $1886,4^{\circ}, \mathrm{pp} .178$, 30 plates.

- Sul deposito di Jackson's Paddock Oamaru nella Nuova Zelanda. Osservazioni biologiche (Atti Accad. Pontif. N. Lincei vol. XLIII, pag. 79; Roma 189o).

CLEVE P. T. - Enumeration of Diatoms found in the Surface of the Sea of Java (Bihang till $K$. Svenska Vet. Akad. Handl. Band I, n. 11, pp. 13, pl. I-III, Stockholm 1873).

- On some new or little known Diatoms (K. Svenska Vet. Akad. Handl. Bandet 18, n. 5, pp. 28, pl. I-VI, Stockholm I881).

- Dictyoneis Cleve n. g. (Le Diatomiste vol. I, 1890, pag. 14-17).

- Sur quelques espèces nouvelles ou peu connues (Le Diatomiste vol. I1, I893, pag. I2-16 etc.).

- Synopsis of the Naviculoid Diatoms part I (K. Svenska Vet. Akad. Handl. Bandet 26, n. 2, pp. 194, pl. I-V, Stockholm 1894).

- Synopsis of the Naviculoid Diatoms part II (K. Suenska Vet. Akad. Handl. Bandet 27, n. 3, Pp. 220, pl. I-IV, Stockholm 1895).

- Plankton from the Indian Ocean and the Malay Archipelago (K. Svenska Vet. Akad. Handl. Bandet 35, n. 5, pp. 58, pl. I-VIII, Stockholm 1901). 
Cleve P. T. et Grunow A. - Beiträge zur Kenntniss der arctischen Diatomeen ( $K$. Svenska Vet. Akad. Handl. Bandet 17, n. 2, pp. I21, pl. I.VII, Stockholm 1880).

Connolly C. J. - Beiträge sur Kenntnis einiger Florideen (Flora 103. Band, 1911, pag. 125-170, T. I-II). Coppinger. - Oceanic Phenomenon (Nature vol. XXIlI, 1880, pag. 482-483, Fig.).

Cotton A. D. - Marine Algae from the Chatham Islands (R. Bot. Gardens, Kew, Bull. of misc. inform. 1907, n. 2, pag. 37-43).

- The New Zealand Species of Rhodophyllis (R. Bot. Gardens, Kerw, Bull. of misc. inform. 1908, pag. 97-102).

- Notes on New Zealand Algae (R. Bot. Gardens, Kew, Bull. of misc. inform. 1909, n. 6, pag. 239-243).

- Marine Algae from North of New Zealand and the Kermadecs (R. Bot. Gardens, Kew, Bull. of misc. inform. 1912, n. 6, pag. 256-264).

- Notes on Queensland Florideae (R. Bot. Gardens, Kew, Bull. of misc. inform. 1913, n. 7, pag. 252-255).

Cramer C. - Ueber die verticillirten Siphoneen, besonders Neomeris und Cymopolia (Denkschriften der Schweiz. naturf. Gesellsch. in Zürich Band XXX, 1887, pp. 50, T. I-V).

- Ueber die verticillirten Siphoneen, besonders Neomeris und Bornetella (Denkschriften der Schweizer. naturf. Gesellsch. in Zürîch Band XXXII, 1890, pp. 48, T. I-IV).

- Die Siphoneen (In Keller, Das Leben des Meeres pp. 28, fig. 241-255; Leipzig, 1895, Tauchnitz, 80).

Crosby C. M. - Observations on Dictyosphaeria (Minnesota Botanical Studies Third Series, part I, pag. 6I-70, plate XV, Minneapolis 1903).

Cuoghi-Costantini L. - Osservazioni critiche intorno l' Euzoniella incisa (J. Ag.) Falk. (La Nuova Notarisia ser. XXIII, pag. 183-194, T. I-II, Padova 1912).

Darbishire O. V. - Spencerella australis, eine neue Florideen-Gattung und Art (Ber. der deutschen botan. Gesellsch Band XIV, 1896, pag. 195-200, T. XIV).

Decaisne J. - Essai sur une classification des Algues et des Polypiers calcifères; Paris, 1842, P. Renouard, $8^{\circ}$, pp. 84, pl. 14-17.

- Mémoire sur les Corallines (Annales des sciences Natur.. XVIII, 1842, pag. 96-128).

De Toni A. - Sopra alcune Polysiphonia inedite o rare (La Nuova Notarisia ser. XVIII, pag. 153-168, Padova 1907).

De Toni G. B. - Boodlea Murray et De Toni, nuovo genere di Alghe a fronda reticolata (Malpighia vol. III, 1889, pag. 14-17).

- Intorno al genere Ecklonia Hornem. Nota (Notarisia vol. IV, n. 15, pag. 782-79o, Venezia 1889).

- Miscellanea phycologica, series altera IV. Il Nostoc punctiforme (Kuetz.) Har. nella Nuova Guinea; V. Una nuova specie di Porphyrosiphon [P. Kaernbachii (Henn.) mihi] (Atti del R. Ist. Veneto di scienze, lettere ed arti ser. VII, T. III pag. 84I-845, Venezia 1892).

- Pugillo di Alghe australiane raccolte all' isola di Flinders (Bull. Soc. botan. ital. 1896, n. 8, pag. 224-231).

- Sylloge Algarum omnium hucusque cognitarum Vol. I (Chlorophyceae), vol. II (Bacillarieae), vol. III (Fucoideae), vol. IV (Florideae), vol. V (Myxophyceae, curante A. Forti); Patavii, 1889-1907, typis Seminarii, 80.

De Toni G. B, e Forti A. - Pugillo di Diatomee bentoniche del Lago Ngebel (Bull, Soc. botan. ital. 1903, pag. 133-I41).

- Analisi microscopica di alcuni saggi di fitoplancton raccolti dalla R. N. «Liguria * (Mem. R. Ist. Veneto di scienze, lettere ed arti vol. XXIX, n. 1, pp. 33, Tav. 4, Venezia 1916).

De Toni G. B. e Levi D. - Frammenti algologici I-II (Notarisia vol. II, n. 5, pag. 298-30I, Venezia 1887 ).

- L'Algarium Zanardini ; Venezia. 1888, M. Fontana, $8^{\circ}$.

De Toni G. B. e SAcCardo F. - Revisione di alcuni generi di Cloroficee epifite (La Nuova Notarisia I, pag. 3-20, T. I-III, Padova 1890).

De Wildeman EM. - Prodrome de la Flore algologique des Indes néerlandaises. Supplément et tableaux statistiques; Batavia, 1899 , Impr. de 1' Ėtat, $8^{\circ}$.

Dickie G. - Notes on Algae from the Island of Mangaia, South Pacific (Journ. Linn. Soc. Bot. vol. XV, I875-76, pag, 30-33).

- Contributions to the Botany of the Expedition of H. M. S. "Challenger », Algae, chiefly Polynesian (Jour. Linn. Soc. Bot. vol, XV, 1875-76, pag. 235-246). 
DuN W. S. v. CARD G. W., RANDS W. H.

Ehrenberg C. G. - Die Infusionsthierchen als vollkommene Organismen; Leipzig, I838, L. Voss, fol., 64 Taf.

- Ueber die Verbreitung der mikroskopischen Organismen in Asien und Australien (Monatsberichte der Berl. Akad. 1842, pag. 269).

- Beobachtungen über die Verbreitung der jetzt wirkenden kleinsten organischen Lebens in Asien, Australien und Afrika (Monatsberichte der Berl. Akad. 1843, pag. 100, 105).

- Mikrogeologie: Das Erden und Felsen schaffende Wirken des unsichtbar kleinen selbständigen Lebens auf der Erde; Bd. I Text, Bd. II Atlas. Ein und vierzig Tafeln mit über viertausend grossentheilscolorirten Figuren gez. v. Verf. Leipz. und Berl,, I857, Leop. Voss, folio.

- Ueber Grundproben des Stillen Oceans, aus vermeindlich 19.80o Fuss Tiefe (Monatsberichte der Berl. Akad. 1869, pag. 466, 489, 588, 712, 765).

- Ueber den Tiefgrund des Stillen Oceans zwischen Californien und den Sandwich Inseln aus 15.600 Fuss Tiefe (Monatsberichte der Berl. Akad. 1860, pag. 819).

Esper E. J. C. - Icones Fucorum, cum I84 tab. col. - Norimb. 1797-1799.

EWART A. J. - Notes on a Collection of Marine Algae from King Island (Victorian Naturalist XXIII, 1906, pag. 90-91).

Falkenberg P. - Die Rhodomelaceen, m. 24 T. (Fauna und Flora des Golfes won Neapel T. XXVI, Berlin IgO1).

Flahault Ch. v. Bornet E.

Forti A. - Studii per una Monografia del genere Pyxilla (Diatomeae) e dei generi affini (La Nuova Notarisia serie XX, pag. 19-36, T. I.II, Padova 1909).

- Contribuzioni diatomologiche IX-XI (Atti del R. Ist. Veneto di scienze, lettere ed arti T. LXIX, 1910, pag. 1249-1317, Tav. I-IX).

- Sylloge Myxophycearum (In DE TONI G. B., Sylloge Algarum omnium hucusque cognitarum vol. V; Patavii, 1907, typis Seminarii, $8^{\circ}$ ).

- v. De Toni G. B.

Fosule M. - Some new or critical Lithothamnia (K. Norske Vidensk. Selskabs Skrift. N. 6, Trondhjem 1898).

- Notes on two Lithothamnia from Funafuti (K. Norske Vidensk. Selskabs Skrift. 1899, n. 5, Trondhjem 1900).

- New or critical calcareous Algae (K. Norske Vidensk. Selskabs Skrift. I899, n. 5, Trondhjem 1900).

- New Melobesieae (K. Norske Vidensk. Selskabs Skrift. Igoo, N. 6, Trondhjem I9or).

- Calcareous Algae from Funafuti (K. Norske Vidensk. Selskabs Skrift. 1900, n. 1, Trondhjem 1900).

- Three new Lithothamnia (K. Norske Vidensk. Selskabs Skrift. I901, N. 1, Trondhjem 1901).

- New Species or Forms of Melobesieae (K. Norske Vidensk. Selskabs Skrift. 1902, N. 2, Tron. dhjem IgO2).

- Two new Lithothamnia ( $K$. Norske Vidensk. Selskabs Skrift. 1903, N. 2, Trondhjem 1903).

- New Lithothamnia and systematical Remarks (K. Norske Vidensk. Selskabs Skrift. 1905, n. 5, Trondhjem 1905).

- Algologiske Notiser II (K. Norske Vidensk. Selskabs Skrift. 1906, N. 2, Trondhjem 1906).

- Algologiske Notiser III (K. Norske Vidensk. Selskabs Skrift. 1906, N. 8, Trondhjem 1907).

- Algologiske Notiser IV (K. Norske Vidensk. Selskabs Skrift. I907, N. 6, Trondhjem I907).

- Pliostroma, a new subgenus of Melobesia (K. Norske Vidensk. Selskabs Skrift. I908, N. II, Trondhjem 1908).

- Corallinaceae (In Rechinger, Botan. und Zool. Ergebnisse einer wissensch. Reise nach den Samoa und Salomonsinseln; Denkschr. Akad. Wien LXXXI, 1907, pag. 209-210).

- Algologiske Notiser V (K. Norske Vidensk. Selskabs Skrift. 1908, N. 7, Trondhjem 1908).

- Nye Kalkalger (K. Norske Vidensk. Selskabs Skrift. 1908, N. 12, Trondhjem 1908).

- Algologiske Notiser VI (K. Norske Vidensk. Selskabs Skrift. 1909, n. 2, Trondhjem 1909).

- v. Weber van Bosse A.

Francis GeORGE - Poisonous Australian Lake (Nature vol. XVIII, 1878, pag. II).

GAIN L. - La flore algologique des régions antarctiques et subantarctiques (Deuxiême Expêdition antarctique française (1908-19ro) commandée par le d.r JEAN CHARcot, Scienc. Nat.,'Docum. scientifiques, Paris, Masson et C., $4^{\circ}$, pp. 218, pl. I-VIII).

GRPP A. and E. S. - Rhipidosiphon and Callipsygma (Journal of Botany vol. XLII, 1904, pag. 363-366, plate 467 ). 
GepP A. and E. S. - Some Cryptogams from the Christmas Island (Journal of Botany vol. XLIII, 1905, pag. 337-344).

- Some marine Algae from New South Wales (Journal of Bolany vol. XLIV, 1906, pag. 249-26r, plate 481 ).

- Marine Algae from the Kermadecs (Journal of Botany vol. XLIX, 1911, pag. 17-23.

- The Codiaceae of the Siboga Expedition including a Monograph of Flabellariae and Udoteae, with 22 plates (Siboga Expeditiê Monogr. LXII, Leiden, 1911, E. J. Brill, 40).

- Marine Algae (British Antarctic "Terra Noz'a « Expedition, rgro, Nat. Hist, Report, Botany, part II, pag. 17-22, London 1917).

Gepp E. S, - Marine Algae (Journ. Linn. Soc, Bot., XLII, pag. 213-239).

Geyler T. - Zur Kenntniss der Sphacelariaceen (Jahrbücher für wissensch. Botanik IV, I865, pag. I-57, T. XXXIV-XXXVI).

Gibis Lilian S. - A contribution to the montane Flora of Fiji (Journ. Linn. Soc. Bot. vol. XXXIX, 1909, pag. 200-202),

Gibson Harvey R. J. - On some marine Algae from New Zealand (Journal of Botany vol. XXXI, 1893, pag. 161-167, plate 335).

- Dumontia filiformis var. (Journal of Botany vol. XXXI, 1893, pag. 250-251).

Gomont M. - Monographie des Oscillariées [Nostocacées homocystées] (Annales des scienc. natur. Botanique, 7 sér., T. XV-XVI, pp. 302, pl. 6-14 et 1-8, Paris 1893).

Greville R. K. - Descriptiones novarum specierum ex Algarum ordine (Nova acta Acad. Leopold. T. XIV, p. II, pag, 423-424, tab. XXVI, Bonn I 829)

- Algae Britannicae; Edinburgh, 1830, Maclaghlan \& Stewart, $8^{\circ}$.

- Notice of a new Species of Caulerpa (Trans. Bot. Soc. Edinb. vol. V, I856, pag. II, pl. III; Ann. and Magaz. of Nat. Hist. s. 2, vol. 14, pl. X).

- On Campylodiscus (Trans. Micr. Soc. vol. VIII, 1860, pag. 29, plate LXI).

- A Monograph of the genus Auliscus (Trans. Micr. Soc. vol. XI, 1863, pag. 36, plates II-III).

- Descriptions of New Genera and Species of Diatoms from the South Pacific I-III (Trans. Botan. Soc. Edinb. vol. VII-VIII, 2 pl., Edinburgh 1863-65).

- Descriptions of New and Rare Diatoms from the Tropics and Southern Hemisphere (Trans. Botan. Soc. Edinb. vol. VIII, pag. 436, 1 pl., Edinburgh 186.5).

- Descriptions of New and Rare Diatoms (Trans. Micr. Soc, vol. IX-XIV, London 186I-66).

Grove E. - v. Grunow A, Karop G. C.

Grove E, and Sturt G. - On a fossil marine Diatomaceous Deposit from Oamaru, Otago, New Zealand I-IV (Journ. Quekett. Micr. Club ser. 2, vol, II, n. I6, pag. 32I, vol. III, n. 17, pag. 7, n. 18, pag. 63, n. 19, pag. 131, 2 plates, London 1886-87).

Grunow A. - Ueber neue oder ungenügend gekannte Algen. Erste Folge. Diatomaceen, Familie Naviculaceen (Verhandl. der K. K. Zool. bot. Gesellsch. X, pag. 503-582, T. I-5, Wien I86o).

- Die Oesterreichischen Diatomaceen, nebst Anschluss einiger neuen Arten von andern Lokalitäten und einer kritischen Uebersicht der bisher bekannten Gattungen und Arten. Erste Folge. Epithemieae, Meridioneae, Diatomeae, Entopyleae, Surirelleae, Amphipleureae (Verhandl. der $K$. $K$. Zool. bot. Gesellsch. XII, pag. 315-472, T, 6-1I, Wien 1862).

- Die Oesterreichischen Diatomaceen. Zweite Folge. Familie Nitzschieae (Verhandl. der $K$. $K$. Zool. bot. Gesellsch. XII, pag. 545-588, T. 12, Wien 1862).

- Ueber einige neue und ungenügend bekannte Arten und Gattungen von Diatomaceen (Verhandl. der K. K. Zool. bot. Gesellsch. XIII, pag. 137-162, T. 13-14, Wien 1863).

- Algen, m. 12 Taf. (Reise S. M. Fregatte Novara um die Erde, Botan. Theil, Bd. I, Wien 1867).

- Algen der Fidschi- Tonga- und Samoa-Inseln (Journ. des Mus. Godeffroy Heft XVI, pag. 23-50; Hamburg 1874).

- Vorläufige Bemerkungen zu einer systematischen Anordnung der Schizonema- und BerkeleyaArten, mit Bezug auf die in Van Heurck's Diatomeen-Flora von Belgien veröffentlichen $\mathrm{Ab}$ bildungen der Frusteln auf Taf. XV, XVI, XVII (Botan. Centralblatt Bd. IV, pag. 1506, 1585, Cassel 1880).

- Ueber die Arten der Gattung Grammatophora mit Bezug auf die Tafeln LIII. und LIII. B in Van Heurck 's Synopsis der belgischen Diatomeen (Botan. Centralblatt Bd. VII, Beilage pag. 1-15, Cassel 1881).

- Some critical Remarks on the Oamaru Diatom Papers of Messrs. Grove and Sturt [translated by G. C. KAROP, with annotations by E. GRove] (Journ. Quekett Micr. Chub ser. 2, vol. III, n. 24, pag. 387 , London 1889). 
Grunow A. - Additamenta ad cognitionem Sargassorum. Opus posthumum (Verhandl。der, zool.bot. Gesellsch. 65. Band, pag. 329-448, 66. Band, pag. I-185, Wien I9I6)

- v. Cleve P. T.; Lauterbach \& Schumann; Schumann \& Hollrung.

Hampe v. Mueller (de) Ferd.

Hardy A. D. - Association of Alga and Fungus in Salmon Disease (Proceed of the R. Soc. Victoria N. S., vol. XXIII, part 1, 1910, pag. 27-32).

- Some Algae of the Zoological Gardens, Victoria (Victorian Naturalist XXX, 1913, pag. 89-95, I plate).

HaRiot P. - Note sur le genre Trentepohlia Martius (Journal de Botanique t. II nov-déc., t. III 1 févr., I mars, I et 16 mai, pp. 56, 24 Fìg., Paris I889-90)

Harvey W. H. - Algae of Tasmania. Description of Algae gathered at George Town, Van Diemen 's Land, and communicated to Sir W. J. Hooker by Ronald Gunn, Esq. (London Journ. of Botany vol. III, 1844, pag. 428-454).

- Nereis Australis or Algae of the Southern Ocean, being figures and descriptions of marine plants collected on the shores of the Cape of good hope, the extratropical australian colonies, Tasmania, New Zealand, and the antartic regions, deposited in the Herbarium of the Dublin University. Part I. 2; London, I847-49, Reeve brothers, 80, VIII-124 pp., 50 tab. color.

- Short Characters of some new Genera and Species of Algae discovered on the coast of the Colony of Victoria, Australia (Annals and Magaz. of Nat. Hist. f. May 1855, pp. 5, pl. VIII, C, fig, I-2).

- Some Account of the Marine Botany of the Colony of Western Australia (Transact. of the R. Irish Academy vol. XXII, 1855, pag. 525-566).

- List of Dr. Harvey 's Duplicate Australian Algae [1857].

- Phycologia Australica: or a History of Australian Seaweeds vol. I-V [with Synopsis and Indexes]; London, 1858-63, Reeve and Co., 8º, 300 col. plates.

- v. Hooker J. D.

Harvey W. H. and Bailey J. W. - New Species of Diatomaceae, collected by te United States Exploring Expedition, under the Command of Capt. Wilkes, U. S. N. (Proceed. Acad. Nat. Sc. Philadelphia 1853, pp. 3).

- Diatomaceae (U.S. Exploring Expedition under Capt. Wilkes in 1838-42 vol. XVII, Washington I862-74).

Hauck F. - Ueber einige von J. M. Hildebrandt im Rothen Meere und Indischen Ocean gesammelte Algen IV. Padina Adans. (Hedwigia XXVI, pag. 41-45, Dresden 1887).

Hennings P. - Algae novo-guineenses (Botan. Jahrb. fïr Syst., Pfanzengesch. und Pfanzengeogr. $\mathrm{XV}, 1893$, Beibl, n, 33, pag. 8).

HEydRICH F. - Beitrãge zur Kenntniss der Algenflora von Kaiser-Wilhelms-Land [Deutsch NeuGuinea] (Ber, der deutschen botan. Gesellsch. X, I892, pag. 458-485, T. XXIV-XXVI).

- Pleurostichidium, ein neues Genus der Rhodomelaceen (Ber. der dentschen botan. Gesellsch. XI, Generalversamml.-Heft, 1893, pag. 75-79, T. XXII).

- Corallinaceae, insbesondere Melobesieae (Ber. der deutschen botan. Gesellsch. XV, I897, pag. 34-70, T. III).

- Neue Kalkalgen von Deutsch-Neu-Guinea (Bibliotheca Botanica Heft 4r; Stuttgart, 1897, E. Nägele, $4^{0}$, pp. II, I Taf. \& I Textfigur).

- Eine neue Kalkalge von Kaiser-Wilhelmsland (Ber. der deutschen botan. Gesellsch. XIX, Igor, pag. 55-7I, t. III).

- Die Lithothamnien des Museum d'Histoire naturelle in Paris (Botan. Jahrb. für syst. Botanik XXVIII, 1901, pag. 529-545, T. VI).

- v. Lauterbach \& Schumany.

HIRN K. E. - Monographie und Iconographie der Oedogoniaceen (Acta Soc, Scient. Fennicae T. XXVIII, n 1, pp. 394, T. 1-64, Helsingsfors 1900).

Hollirung M. v. SchumanN $\mathrm{K}$.

Holtz L. - Ueber Characeen gesammelt in Australien und auf Sizilien (Mittheil, des Naturw. Ver. für Nen-Vorpommern und Rügen zu Greifswald 37. Jahrg. (1905) 1906, pp. 8).

HookeR J. D. - Flora antarctica part I-2; London, 1844-47, Reeve brothers, $4^{\circ}, 574$ pp., 198 tab. col.

- Flora Novae Zelandiae part 1-2, London, I853-55, Reeve brothers, $4^{\circ}$, XXXIX-31 2-378 pp., I30 tab. col.

- Flora Tasmaniae part I-2; London, 386o, Reeve brothers, $4^{\circ}$, CXXVIII-359-422 pp., 200 tab. col. 
Hooker J. D. - Cryptogamia Antarctica, or Cryptogamic Botany of the Antarctic Voyage of H. M. Ships Erebus and Terror during the years $1839-1843$, under the command of Captain Sir James Clark Ross; [London] 1847 , Reeve brothers, $4^{\circ}$, pp. 258, plates 74 . (E da riguardare, come ci scrisse il chiar. sig. A. GEPP, come una ristampa, con nuova paginatura, della parte crittogamica della Flora Antarctíca, mantenuta essendo però la stessa numerazione nelle tavole).

- Handbook of the New Zealand Flora: a systematic description of the native plants of New Zealand and the Chatham, Kermadec's, Lord Aucklands, Campbell's and Macquarries Islands; London, I867, Reeve and Co., $8^{\circ}$.

Hooker J. D. and Harvey W. H. - Algae Antarcticae, being characters and descriptions of the hitherto unpublished species of Algae, discovered in Lord Auckland 's Group, Campbell 's Island, Kerguelen's Land, Falkland Islands, Cape Horn and other southern circumpolar regions, during the voyage of H. M. discovery Ships «Erebus" and "Terror" (London Journ. of Botany IV, 1845 , pag. 249-276, 293-298).

- Algae Novae Zelandiae; being a Catalogne of all the species of Algae yet recorded as inhabiting the shores of New Zealand, with characters and brief descriptions of the new species discovered during the Voyage of H. M. discovery ships "Erebus" and "Terror", and of others communicated to Sir. W. Hooker by Dr. Sinclair, the Rev. W. Colenso, and M. Raoul (London Journ. of Botany vol. IV, 1845, pag. 521-551).

- Algae Tasmanicae: being a Catalogue of the Species of Algae collected on the shores of Tasmania by Ronald Gunn, Esq., Dr. Jeannerett, Mrs Smith, Dr. Lyall, and Dr. J. D. Hooker; with characters of the new species (London Journ. of Botany vol. VI, 1847, pag. 397-417).

- Algae Novae Zelandiae [Supplementum primum] (London Journal of Botany VII, 1848, pag. 443-445\%.

Howe M. A. - The Building of "Coral 》 Reefs (Science N. S. vol XXV, n. 909, 1912, pag. 837-842).

- Calcareous Algae from Murray Island, Australia, 'and Cocos-Keeling Islands (Carnegie Institution of Washington N. 213, 1918, pag. 291-296, plates 97-98).

Inglis John. - On new Species of New Zeland Diatoms (Trans. N. Zeal. Institute vol. XIV, I88o, pag. $357-359$, pl. XXII).

- Notes on some of the Diatomaceous Deposits of New Zealand (Ibidem vol. XV, I882, pag. 340345, pl. XXIX).

Jennings A. Vaughan. - Note on the Occurrence in New Zealand of two Forms of peltoid Trentepohliaceae and their relation to the Lichen Strigula; London, I895, Spottiswoode \& Co., $8^{\circ}$. 2 plates.

- The two new Species of Phycopeltis from New Zealand (Proceed. R. Irish Acad. 3 ser., vol. IIl, ก. 5, dec, I885, pag. $753^{-766}$, plates XXVIII-XXIX, Dublin 1896).

Karop G. C. - Some critical Remarks by Herr A. Grunow on the Oamaru Diatom Papers of Messrs Grove and Sturt; with annotations by E. Grove (Journ. Quekett Micr. Chub ser. 2, vol. III, n. 24, pag. $387-391$, London 1889).

KIRK T. - Description of a new Species of Cladophora (Transact. and Proceed. of the New Zealand Institule vol. XII, 1879 .

Kitton F. - Notes sur quelques Diatomées (Bull. de la Soc. belge de Microscopie T. V, 2 fig., Bruxelles I 879).

- New Species of Biddulphia from Fiji (Journ. R. Micr. Soc. 1888, pag. 466).

KLebahn H. - Zur Kritik einiger Algengattungen (Jahrbücher für wiss. Botanik XXV, 1893, pag. 278-321, T. XIV.

KJELLman F. R. - Om Floridé-Slägtet Galaxaura, dess Organografi och Systematik, m. 20 T. ( $K$. Sivenska Vetensk. Akad. Handl. Bandet 33, N. I, Stockholm 1900).

Kuetzing F. T. - Die Kieselschaligen Bacillarien oder Diatomeen, mit 30 Taf.; Nordhausen, I844, Fr. Fritsch, $4^{\circ}$

- Diagnosen und Bemerkungen zu neuen oder kritischen Algen (Botanische Zeitung V, 1847, pag. 1)

- Species Algarum; Lipsiae, 1849, Brockhaus, $8^{\circ}$.

- Tabulae Phycologicae oder Abbildungen der Tange -XIX, m. 1900 Taf.; Nordhausen, 18451871, Förstemann, $8^{\circ}$.

Kuntze O. - Revision von Sargassum und das sogenannte Sargasso-Meer (Jahrbiicher für syst. Botanik I, pag. 191-239, I Phototypie und I Karte, Leipzig I881).

la Billardière J. J. H. (de) - Novae Hollandiae plantarum specimen, c. 265 tab.; Parisiis, $1804-06,4^{\circ}$. 
Lajng R. M. - Observations on the Fucoideae of Banks Peninsula (Trans. New Zealand Institute vol. XIIII, I855, pag. 303-3II, plate X).

- Note on Splachnidium rugosum, Grev. (Trans, and Proceed. Nerv Zealand Institute vol. XXV, I892, pag. 288-289).

- On Lessonia variegata J. Ag. mscr. (Trans, and Proceed. Nerw Zealand Institute vol. XXVI, I893, pag. 304-310, plate XXXIX).

- The Algae of New Zealand; their Characteristics and Distribution (Trans. New Zealand Institute vol. XXVII, I894 [Wellington 1895] pag. 297-318).

- Notes on Several Species of Delesseria, One being New (Trans. New Zealand Institute vol. XXIX, I896, pag. 446-450, plates XXVII-XXVIII).

- Revised List of New Zealand Seaweeds, Part I (Trans. New Zealand Institute vol, XXXII, 1899, pag. $57-70$, plates $\mathrm{V}-\mathrm{VII}$ ).

- A List of Seaweeds of Norfolk Island (Trans. New Zealand Institute vol. XXXIII, I9oo, pag. 299-30r).

- Revised List of New Zealand Seaweeds, Part II (Trans. New Zealand Institute vol. XXXIV, 1901, pag. 327-359).

- On the New Zealand Species of Ceramiaceae (Trans. New Zealand Institute vol. XXXVII, 1904, pag, 384-408, plates XXIV-XXXI).

- Appendix to the List of Seaweeds of Norfolk Island (Trans. and Proceed. Nerw Zealand Institute vol. XXXVIII, I905, pag. 424).

- Note on the Occurrence of Phyllitis Fascia (Muell.) Kuetz. in New Zealand (Trans, and Proceed. Nere Zealand Institute vol. XXXIX, I906, pag. 220-22I).

- The Marine Algae of the Subantarctic Islands of New Zealand (The Subantarctic Isl. New Zealand vol. II, I909, pag. 503-527, plates XXI-XXIV).

- Scientific Results of the New Zealand government trawling Expedition, 1907, Algae (Records Canterbury Mus. N. Z. I, 2, 1909, pag. 65-70).

Lamourotx J. V. F. - Essai sur les genres de la famille des Thalassiophytes non articulées; Paris, I8r3, Dufour, $4^{\circ}, 84$ pp., 7 Tab.

- Histoire des Polypiers coralligènes fléxibles, vulgairement nommées Zoophytes; Caen, 1816, $8^{\circ}$, LXXXIV-559 pp., 19 Tab.

- Exposition méthodique des genres de 1'ordre des Polypiers; Paris, 1821, Agasse, $4^{\circ}$, VIII-115 pp., $84 \mathrm{Tab}$.

Lauterbach K. und Schumann $K$, Die Flora der Deutschen Schutzgebiete in der Südsee (Algen bearb, von Grunow, Hevdrich und Schmidle); Leipzig, igoo, Bornträger, $8^{\circ}$.

- Nachtrāge zur Flora der deutschen Schutzgebiete in der Südsee; Leipzig, 1905, $8^{\circ}$.

Lehmann J. G. C. - Plantae Preissianae vol, II; Hamburgi, 1846-48, Meissner, $8^{\circ}$.

Lemmermann E. - Ergebnisse einer Reise nach dem Pacific (H. Schauinsland 1896-97). Planktonalgen (Abhandl. Nat. Ver. Bremen XVI, I899, pag. 313-398, T. I-III).

- Beitrāge zur Kenntniss der Planktonalgen. VIII. Peridiniales aquae dulcis et submarinae (Hedwigia XXXIX, Beibl. n. 4, pag. II5-12I, Dresden 1900).

- Die Algenflora der Sandwich-Inseln (Jahrbiucher fiur syst. Botanik XXXIV, pag. 607-663, Tat. VII-VIII, Leipzig I905).

- Das Phytoplankton des Meeres, III. Beitrag (Beihefte zum Botan. Centralbl. Band XIX, Abt. II, I905, pag. 1-74).

- Die Algenflora der Chatham Islands (Jahrbiicher fiur syst. Botanik XXXVIII, pag. 343-382, T. V-VI, Leipzig 1907).

Lemorne P. M.me - Catalogue des Mélobésiées de 1'herbier Thuret (Bull. Soc. Botan. de France T. LVIII, IgII, pag. LI-LXV).

- Structure anatomique des Mélobésiées. Application à la classification (Annales de l'Instîtut Océanographique [Fondation Albert Ier, Prince de Monaco] T. II, fasc, 2, 191I, pp. 2I3, pl. I-V, Fig. 105 dans le text).

- Melobesieae (British Antarct. «Terra Nova» Expedition, 1910, Natur. Hist. Report. Botany par. II, pag. 23-25, Fig. I-4, London 1917).

LeUduger-FortMorel G. - Diatomées de 'Ia Malaisie (Ann, du Jard. Botan de Buitenzorg XI, I; Leiden, 1892, Brill, 80 , $60 \mathrm{pp} ., 7 \mathrm{pl}$.).

Levi D. v. De TONi G. B. 
Lindsay Lauder W. - On the Diatomaceae of Otago, New Zealand (Journ. Linn. Soc. Bot., vol, IX, I867, pag. I29).

- On the Protophyta (Diatomaceae) of New Zealand (Quart. Journ. Micr. Sc. vol. VII, I867, pag. 97).

Luc As A. H. S. - Revised List of the Fucoideae and Florideae of Australia (Proceed. Linn. Soc. of N. S. Wales vol. XXXIV, 1909, pag. I-60).

- Supplementary List of the Marine Algae of Australia (Proceed. Linn. Soc. of N. S. Wales vol. XXXVII, 1912, pag. 157-171 $)^{\circ}$

- Notes on Australian marine Algae (Proceed. Linn. Soc. of $N . S$. Wales vol. XXXVIII, r913, pag. 49-6o, plates I-V).

- Marine Algae (Brit. Assoc. Handbook N. S. Wales 19r4, pag. 459-463).

- An efflorescence on some New Zealand Kelps (Proceed. Linn. Soc. of N. S. Wales vol. XLI, 1916, pag. 676-679).

- Notes on Australian marine Algae, II (Proceed. Limn. Soc. of N. S. Wales vol. XLIV, I919, pag. I74-179, plate VI).

- The Algae of Commonwealth Bay (Australasian Antarctic Expedition 1911-14, Scientific Papers, Series C, Zoology and Botany vol. VII, part 2; Sydney 1919).

- v. MAIDEN J. H.

Mac Caughey Vaughan. - Algae of the Hawaiian Archipelago (Botanical Gazette vol. LV, 19r3, pag. 42-57, 121-149).

- The Seaweeds of Hawaii (Amer. Journ. of Botany vol. III, 1916, pag. 474-479).

- A Survey of the Hawaiian Coral Reefs (Amevican Naturalist vol. LII, 1918, pag. 409-438, 9 Fig.).

Mc Naв. - Cliftonaea pectinata, Harv., from Port Phillip (Dublin Microsc. Club vol. IV, part I, 1882, pag. 32 ).

Maiden J. H. - The Flora of Norfolk Island, part I (Proceed. Linn. Soc. of N. S. Wales vol. XXVIII, 1903, pag. 745-746 [Algae determ. A. H, S, LucAs]).

MANGIN L. - Phytoplancton de l'Antarctique (Deuxième Expédition antarctique française (rgo8-19ro) commandée par le Dr. Jean Charcot, Scienc. Nat., docum. scientifiques; Paris, I9I5, Masson et Cie, $4^{0}, 95 \mathrm{pp} ., \mathrm{pl}$. I-III).

MANN A. - Report on the Diatoms of the Albatross Voyages in the Pacific Ocean, I888-I904 (Contrib. from the Un. Stat. Herbarium vol. X, part 5, 1907, pag, 221-419, plates XLIV-LIV).

Martens (v.) G. - Die Tange (Die Preussische Expédition nach Ost-Asien, Botan. Theil, pp. I52, T. I-VIII, Berlin, I866, R. V. Decker, $8^{\circ}$ ).

Maskell W. M. - Contribution toward a List of the New Zealand Desmidieae (Trans. a. Proceed. New Zealand Institute vol. XIII, 1880 [1881], pag. 297-317, plates II-12).

- On the New Zealand Desmidieae. Additions to Catalogue and Notes on various Species (Trans. a. Proceed. New Zealand Institute vol XV, I883, pag. 237-259, plates 24-25).

- On a new Variety of Desmid (Trans. a. Proceed. New Zealand Institute vol. XVIII, I885, pag. 325).

- Further Note on the Desmidieae of New Zealand, with Descriptions of New Specics (Trans. New Zealand Institute vol. XXI, 1888, pag. I-32, plates I-6).

Mazza A. - Saggio di Algologia Oceanica (La Nuova Notarisia ser. XVI et seg.; Padova roo5 e seg.).

Mereschkowsky C. - On Polynesian Diatoms (Scripta Botan. Horti Univ. Petropolit. Fasc. XVIII, 1902, pp, 30, pl. IV-VI).

Merlin A. A. - On the Minute Structure of some Diatomaceae from Corica Bay, Melbourne (Journ. Quekett Micr. Club ser. 2, vol. VII, pag. 295-298, plate 17, London 1900).

- Note on New Diatom Structure (Journ. Quekett Micr. Club ser. 2, vol. X, 1907, pag. 83-86).

Mertens. - Mémoire sur plusieurs Espèces de Fucus, nouvelles ou peu connues, observées dans la collection du Muséum (Mémoir. du Mruséum T. 5, pag. 172-19o, pl. 14-16).

Míchrll M. O. - Notheia anomala Bail. et Harv. (Phycological Memoirs edit. G. MurRAY part II, I893, pag. 36-37, plate XI).

Mitchell M. O. and Whitting F. G. - On Splachnidium rugosum, Grev., the type of a new Order of Algae (Phycological Memorrs edit. G. Murray part I, 1892, pag. I-1o, pl. I-III).

Moebius M. - Conspectus Algarum endophytarum (La Notarisia vol. VI, pag. 1221-1236, 1279-1286, 1291-1304, Venezia 1891).

- Australische Süsswasseralgen (Flora 1892, pag. 421-450, Fig. I-22).

- Australische Süsswasseralgen, II (Abhandl. der Senckenb. naturf. Gesellsch. XVIII, 1894, pag. 309350, T. I-II). 
Mollet T. A. - On the Structure of Hormosira Billardieri (Trans. and Proceed. New Zealand Institute vol. XIII, I880, pag. 318-323, plates XIII-XIV).

Montagne C. - Du genre Xiphophora, et, à son occasion, Recherches sur cette question: Trouvet-on dans les Fucacées les deux modes de propagation qu'on observe chez les Floridées? (Ann. Scienc. Nat. octobre 1842, pp. 7 ).

Montagne C. -- Quelques Observations touchant la structure et la fructification des genre Ctenodus, Delisea et Lenormandia, de la famille des Floridées (Ann. Scienc. Nat., Botan., 3 sér., T. I, I844, pag. I5i-I6r, pl. 10-Ir).

- Plantes cellulaires du Voyage au Pole Sud et dans l'Océanie sur les Corvettes l'Astrolabe et la Zélée, sous le commandement de M. J. Dumont D'Urville 1837-1840; Paris, 1845, Gide, 80, XIV-349 pp., 20 tab.

- Communication relative à une plante marine de l'Australie constituant un nouveau genre que M. Harvey dédie à la mémoire du lieutenant de vaisseau Bellot, de la marine française (Compt. rend. Acad. Sciences T. XL, séance du 9 avril i 855,4 pp.).

- Sylloge generum specierumque cryptogamarum quas in variis operibus descriptas iconibusque illustratas, nunc ad diagnosin reductas, multasque novas interjectas, ordine systematico disposuit; Parisiis, I 856 , Baillière, $8^{\circ}, \mathrm{XXIV}-498 \mathrm{pp}$.

Morris G. C. - Fossil Diatoms from South Australia (Amer. Naturalist jun. 1877 et Monthly Micr. Journ. XVIII, pag. 46)

Moseley H. N. - Pelagic Life (Adress at the Southampton Meeting of the Brit. Assoc, 1882),

Mueller \& HaMpe. - Plantae Muellerianae Australasicae (Limnaea 1854).

Mueller (de) Ferd. - v. Sonder W.

Murray G. - On Boodlea, a new Genus of Siphonocladiaceae (Limn. Soc. Journ. Bot. vol. XXV, I889, pag. 243-245, plate 49).

- The Distribution of Marine Algae in Space and in Time (Trans. of the Biolog. Soc. Liverpool 189I; cfr. Journal of Botany vol. XXIX, I89I, pag. 253-255).

- On the Cryptostomata of Adenocystis, Alaria and Saccorhiza (Phycological Memoirs part II, I893, pag. 6I-64, plate XVI).

MURRay G, and Boodle L. A. - On the Structure of Spongocladia, Aresch. (Spongodendron Zanard.), with an Account of new Forms (Annals of Botany vol. II, N. VI, I888, pag. 169-175, Fig. 8-11).

- Further note on Spongocladia (Annals of Botany vol. III, I889).

- A structural and systematical Account of the genus Struvea (Annals of Botany vol. II, n. VII, I888, pag. 265-282, plate XVI).

- A systematic and structural account of the genus Avrainvillea Decne (Journal of Botany vol. XXVII, pag. 67-72, plates 288-289, London I889).

Nordstedt O. - De Algis et Characeis ex insulis Sandvicensibus a Sv. Berggren 1875 reportatis (Minnesskrift utg. af $K$. Fysiogr. sällsk. $i$ Lund 1878,24 pp., 2 tab.),

- De Algis et Characeis. I. De Algis nonnullis, praecipue Desmidieis inter Utricularias Musei Lugduno-Batavi; 2. Characeae Novae Zelandiae (Acta Univers. Lund. T. XVI, i88o, 20 pp., I tab.).

- Characeae Australianae hactenus cognitae a scriptis Alexandri Braun enumeratae (Supplem. ad vol. undecimum Fragment. Phytogr. Australiae pag. 43-44; Melbourne i 880).

- Algologiska Smaasaker. 4. Utdrag ur ett arbete öfver de af Dr. S. Berggren paa Nya Seland och in Australien samlade Sötvattensalgerna (Botaniska Notiser 1887, pag. 153-164).

- Ueber die von Prof. S. Berggren auf Neu-Seeland gesammelten Süsswasseralgen (Botan. Verein in Lund III. Sitzung am 18. April 1887, pag. I4-I5; Botanisches Centralblatt XXXI, n. 10, I887, pag. 321-322).

- Einige Characeenbestimmungen. (Hedwigia vol. XXVII, pag, I8I-196, T. VI, Dresden I888).

- Conjugatae, Characeae (Forschungsreise S. M. S. «Gazette» IV. Theil Botanik, 4 pp., I tab., Berlin 1888).

- Freshwater Algae, collected by Dr. S. Berggren in New Zealand and Australia (K. Suenska Vetensk. Akad. Handl. Bandet 22, N. 8, 1888, 97 pp., 7 tab.).

- De Algis et Characeis 3-6 (Aarsskr. Lunds Univers. T. XXV, I889, pp. 4I, Tab, 1).

- Australasian Characeae (Proceed. R. Soc. Victoria n. s, vol. XXXI, pag. I-6).

- v. Braun A., Wittrock V.

ORAMURA K. - List of Marine Algae collected in Caroline Islands and Australia (The Botanical Magazine vol. XVIII, pag. 77-96, Fig. I-4, Tokyo 1904). 
OKAmura K. - List of Marine Algae collected in Caroline and Mariana Islands (The Botanical Magazine vol. XXX, pag. I-I4, fig. 9, plate I, Tokyo 1916).

O' Meara E. - A new Gephyria (G. Harveyi) from Port Fairy, Victoria (Quart. Journ. Micr. Sc. vol. VI, I866, pag. 185).

- On Striatella curviseptata n. sp., found on Haloplegma Preissii from Australia (Quart. Journ. Micr. Sc. vol. VI, I866, pag. I89),

- On Navicula Chimmoana and N. spiralis (Quart. Journ. Nicr. Sc. vol. XII, 1872, pag. 90, 93).

- On some peculiar forms of Navicula from the Sulu Archipelago (Quart. Journ. Micr. Sc. vol. XII, I 872 , pag. 283 , plate XIII).

- Coscinodiscus Sol Wallich from the Sea of Java (Quart. Tourn. Micr. Sc. vol. XX, I88o, pag. III).

Peragallo H. - Monographie du genre Pleurosigma et des genres alliés (Le Diatomiste I, 1890-9r, pp. 35, planches I-X).

- Monographie du genre Rhizosolenia et de quelques genres voisins (Le Diatomiste I, I890-93, pag. 79-82, 99-117).

Peragallo H. et M. - Diatomaceae marinae (Denkschriften d. math. nat. Kl. der Kais. Akad. der Wissensch. LXXXVIII, pag. 3-II, T. I-II, Wien I9II).

- v. TEMPÉre J.

Petit P. - Catalogue des Diatomées de l'ile Campbell et de la Nouvelle Zélande (Fonds de la mer vol. III, 40 pp., 2 pl.; Paris 1877 ).

Piccone A. - Alghe del viaggio di circumnavigazione della Vettor Pisani, Genova, 1886, Tip. R. Ist. Sordo-muti, $8^{\circ}, 9^{8}$ pp., 2 Tav.

- Nuove Alghe del viaggio di circumnavigazione della Vettor Pisani ( $R$. Accad. dei Lincei, Memorie delle $\mathrm{cl}$, di sc. fis. matem. e natur. ser, 4, vol, VI, pag. Io-63, Roma I889).

Picquenabd C. A. - Études sur les collections botaniques des frères Crouan, II, Fucoideae (Trav. scientif. du Labor. de Concarneau T. III, fasc. 6, I9Ir, pp. 44).

- Etudes sur les collections botaniques des frères Crouan, III, Florideae (Trav. scientif. du Labor. de Concarneau T. IV, fasc. 4, I9r2, pp. I05).

Pigram F. - Queensland Spirogyra (Queensland Naturalist I, 1909, pag. 96-ro3, I plate).

Playfair G. I. - Some new or less Known Desmids found in New South Wales (Proceed. Linn. Soc. of N. S, Wales vol, XXXII, 1907, pag. 160-201, plates II-V).

- Some Sydney Desmids (Proceed, Linn. Soc. of N. S. Wales vol. XXXIII, 1908, pag. 603-628, plates XI-XIII).

- Polymorphism and Life-History in the Desmidiaceae Algae [Conjugatae] (Proceed. Linn. Soc, of N. S. Wales vol. XXXV, 1910, pag. 459-495, plates XI-XIV).

- Growth, Development and Life-History in the Desmidiaceae (Australasian Assoc. for Advanc. of Science vol. XIII, I9I2, pag. 278-298).

- Plankton of the Sydney Water-Supply (Proceed. Linn. Soc. of N. S. Wales vol. XXXVII, I9I2, pag. 512-552, plates LIII-LVII).

- Contributions to a Knowledge of the Biology of the Richmond River (Abstr. of Proceed. Linn. Soc. of N. S. Wales n. 318, I914, pag. IV).

- Contributions to a Knowledge of the Biology of the Richimond River (Proceed. Linn. Soc. of N. S. Wales vol, XXXIX, 1914, pag. 93-151).

- Freshwater Algae of the Lismore District, with an Appendix on the Algal Fungi and Schizonuycetes (Proceed. Linn. Soc. of N. S. Wales vol. XI, I915, pag. 310-362, ro Fig., plates XLI-XLVI).

- Oocystis and Eremosphaera (Proceed. Limn. Soc. of N. S. Wales vol. XLI, 1916, pag. I07-I47, plates VII-IX).

- Australian Freshwater Phytoplankton (Proceed. Linn. Soc. of N. S. Wales vol. XLI, I916, pag. $823-852$, plates LVI-LIX).

- Census of new South Wales Freshwater Algae (in Maiden J. H. \& Betche Ernst, a Census of New South Wales Plants pag. 219-263) Sydney, 1917, W. Applegate Gullick, 80.

- New and rare Freshwater Algae (Proceed. Linn. Soc. of N. S. Wales vol. XLIII, Igr8, pag. 497-543, Fig. I-II, plates LIV-LVIII).

PRITChARD A. - History of Infusoria, including the Desmidiaceae and Diatomaceae british and foreign; London, 186r, 80.

RABenhorst L. - Beitrag zur Meeresalgenflora von Aucklandinseln (Hedwigia XVII, pag. 65; Dresden 18781 . 
Rabevhorst L. - Flora Europea Algarum aquae dulcis et submarinae sect. I-III; Lipsiae, 1864-68, E. Kummer, so.

RAciborski M. - Desmidya zebrane przez Dr. E. Ciastona w podrózy c. i. k. Korweta Saida na okoto ziemi (Anzeiger der Akad. der Wissensch. in Krakau I892, pag. I12-114).

- Parasitische Algen und Pilze Java 's, I-II; Batavia, I90o, Staatsdruckerei, $8^{\circ}$.

Rands W. H., David \& Dun W. S. - Note on the occurrence of Diatoms, Radiolaria and Infusoria in the Rolling Downs Formation (Lower Cretaceous) Queensland (Proceed. Linn. Soc. N. S. Wales n. I02, r9or, pag. 299-309, plates XVII-XIX).

Rattray J, - A Revision of the Genus Aulacodiscus, Ehrb. (Journ. R. Micr. Soc. I888, pag. 337-382, plates V-VII).

- A Revision of the Genus Auliscus Ehrb. and of some allied Genera (Journ. R. Micr. Soc. 1888 pag. 86I-920, plates XII-XVI).

- A Revision of the Genus Coscinodiscus Ehrb. "and of some allied Genera (Proceed. R. Soc. Edinb. vol. XVI, ISgo, pag. $449 \cdot 692$, plates I-III).

- A Revision of the Genus Actinocyclus, Ehrb. (Journ. Quekett Aficr. Chub ser. II, vol. IV, n. 27, I890, pag. I37-2I2, plate XI).

Reed M. - The economic Seaweeds of Hawaii and their Food Value (Ann. Rep. Harv. Agr. Exper, Station rgo6, pag. 6I-88, plates 4).

Reinbold Th. - Meeresalgen [Schizophyceae, Chlorophyceae, Rhodophyceae] (In REINEcke F., Die Flora der Samoa-Inseln I896, pag. 266-275).

- Die Algen der Lacépède und Guichen Bay und deren näherer Umgebung [Süd-Australien] gesammelt von Dr. A. Engelhardt ( La Nuova Notarisia ser. VIII, pag. 4I-62, Padova 1897).

- Die Algen der Lacépède und Guichen Bay und deren näherer Umgebung [Süd-Australien] gesammelt von Dr. Engelhardt (La Nuova Notarisia ser. IX, pag. 33-54, Padova 1898).

- Ergebnisse einer Reise nach dem Pacific (Prof. Dr. Schauinsland 1896-97). Meeresalgen ( $A b h a n d l$. Nat. Ver. Bremen XVI, I899, pag. 287-302).

- Meeresalgen von Investigator Street [Süd-Australien] gesammelt von Miss Nellie Davey (Hedwigia XXXVIII, pag. 39-5I, Dresden 1899).

- Meeresalgen von den Norfolk Inseln (La Nuova Notarisia ser. XI, pag. I47-I53, Padova 1900),

- Meeresalgen [Phycochromophyceae, Chlorophyceae, Phaeophyceae, Rhodophyceae exklusive der nicht artikulirten Corallinaceae] (In Rechinger, Botan. u. zool. Ergebn. einer wissensch. Reise nach den Samoa-und Salomons-Inseln; Wiener Denkschr. LXXXI, 1907, pag. 200-208; Nachtrag, ibidem LXXXIX, I9I3, pag. 702).

REINKE.J. - Uebersicht der bisher bekannten Sphacelariaceen (Ber. der deutschen botan. Gesellsch, VIII, I890, pag. 20I-2I5).

- Beitrāge zur vergleichenden Anatomie und Morphologie der Sphacelariaceen (Bibliotheca Botanica Heft XVIII, I89I, pp. 40, T. I-I3)

- Ueber Caulerpa. Ein Beitrag zur Biologie der Meeres-Organismen (Wissensch. Meeresuntersuchungen N. F., Band 5 [Abt. Kiel] Heft I, I899, 98 pp., 87 Fig.).

Rosanoff S. - Recherches anatomiques sur les Mélobésiées (Mém. Soc. sc. natur. de Cherbourg T. XII, I866, pag, 6-II2, pl. I-VII).

Saccardo F. v. De Toni G. B.

Sauvagead C. - Sur les Sphacelaria d'Australasie (Notes from the Botan. School of Trinity Coll. Dublin n. 5, 1902, pag. 196-200).

- Remarques sur les Sphacélariacées (Journ. de Botanique vol. XIV, I9oo, pag. I-5I, vol. XV, Igor, pag. 5I-I67, vol. XVI, I902, pag. 167-228, vol. XVII, I903, pag. 228-332, vol. XVIII, I904, pag. 332-348, éd. à part [Bordeaux 1914] pp. XII-48I-634, Fig. I-I28).

SchмideE W. - Süsswasseralgen aus Australien (Flora 82. Band, 1896, pag. 297-313).

- Entwickelung von Sphaerozyga oscillarioides (Bory) Kuetz. (Ber, der deutschen botan. Gesellsch. XIV, 1896, pag. 422-43I, T. XXIV).

- Süsswasseralgen [Schizophyceae, Conjugatae und Chlorophyceae]. (In ReINEcke F., Die Flora der Samoa Inseln pag. 253-265, fig. I-7, Leipzig 1896).

- Algologische Notizen II-IV, VII (Algemeine botan. Zeitschrift III, 1897, pag. 3-4, pag. 37-4I, pag. 57-58, pag. 73-74, pag. ro8-ro9).

- Epiphylle Algen nebst einer Pitophora und Dasya aus Neu-Guinea (Flora 83. Band, I897, pag. 304-326, Fig. A-D).

- Einige Baumalgen aus Samoa (Hedwigia XXXVI, pag. 277-287, T. VI-VIII, Dresden r897). 
Schmidee W. - Zur Entwickelung einer Zygnema und Calothrix (Flora 84. Band, I897, pag, I67-173, T. V).

- Einige Algen, welche Prof. Dr. Volkens auf den Carolinen gesammelt hat (Hedwigia XL, pag. 343-349, T. XII, Dresden 1901).

- Neue Süsswasseralgen aus den Samoa-Inseln (Hedwigia LIII, pag. 144-I47, Dresden I9I3).

- v. Lauterbach K. \& Schumann K.

Schмidt AD. - Atlas der Diatomaceen-Kunde H. I-87, T. I-348; Aschersleben-Leipzig, 1874-1922, fol. Sснмiтz F. - Die Gattung Lophothalia J. Ag. (Ber. der deutschen botan. Gesellsch. IX, 1893, pag. 212-232).

- Kleinere Beiträge zur Kenntniss der Florideen, IV. (La Nuova Nolarisia ser. V, pag. 608-635, Padova 1894).

Schumann K. und Hollrung M. - Die Flora von Kaiser Wilhelms Land [Algae bearbeitet v. A. GRUNow]; Berlin, 1889, $8^{\circ}$.

Schumann K, v. Lauterbach K.

Setchell W. A. - On the Classification and geographical Distribution of the Laminariaceae (Trans. of the Connecticut Acad. vol. IX, 1893, pag. 333-375).

- Limu. Algae for eating or ceremonial uses of the Hawaiians (Univers. of California Public. Botany vol. 2, 1905, pag. 9r-II3).

- Algae novae et minus cognitae, I (Univers, of California Public. Botany vol. 4, I9r2, pag. 229268, plates 25-3I).

- The Scinaia Assemblage (Univers. of California Public. Botany vol. 6, 1914, pag. 79-152, plates 10-16).

- Parasitic Florideae, I (Univers, of California Public. Botany vol. 6, pag. 1-34, plates I-6).

Sкотtsberg C. - Zur Kenntnis der subantarktischen und antarktischen Meeresalgen I. Phaeophyceen (Wissensch. Ergebn. der schwed. Sïdpolar-Exped. 1901-1903, Band IV, Lief, 6; Stockholm 1907).

- Zur Kenntnis der subantarktischen und antarktischen Meeresalgen II. Rhodophyceen (Ibidem Band IV, Lief 15, Stockholm 1919).

- Remarks on Splachnidium rugosum (L.) Grev. (Svensk Botan. Tidsskrift B. I4, H. 2-3, 1920).

Smith A. Lorrain - Seirococcus axillaris (Phycological Memoirs ed. G. Murray part II, I893, pag. $4-6$, plate X).

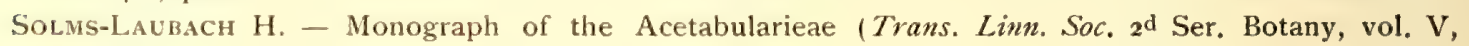
part I, I895, pag. I-39, plates I-IV).

Sonder W. - Nova Algarum genera et species, quas in itinere ad oras occidentales Novae Hollandiae, collegit L. Preiss (Botanische Zeilung III, I845, pag. 49-57).

- Plantae Muellerianae (Linnaea XXV, 1852, pag. 657-703, XXVI, I853, pag. 506-528).

- Die Algen des tropischen Australiens (Abhandl. des Naturw. Ver. zu Hamburg Band V, I87I, Pp. 42, T. I-6).

- Algae Australianae hactenus cognitae, I880. Algae. E manuscriptis praecipue Sonderi (In MUElLER (de) Ferd., Fragmenta Phytographiae Australiae vol. XI, suppl. pag. I-42, 105-107, Melbourne 1878-188I, I. Ferres, $8^{0} \%$

- Algae of the New Hebrides (Proceed. of the Royal Soc. of Tasmania 1880-8I).

- v. Lehmann J. G. C.

Spencer W. J. - On the Freshwater Algae of New Zealand (Trans. and Proceed. New Zealand Institule 1882 ).

- Notes on Freshwater Algae (Transact. and Proceed. New Zealand Institute I883).

Stockmayer S. - Die Algengattung Rhizoclonium, m. 27 Fig. (Verhandl. K. K. Zool. botan. Gesellschaft in Wien 1890, pag. 571-580).

Stroem K. M. - Algological Notes. I. Some Desmids from North Australia (Nyt Magazin for Naturvidensk. vol. LIX, Kristiania I921).

Sturt G. v. Grove E.

Svedelius N. - Om likhetem mellan Västindiens samt Indiska och Stilla Oceanens marina vegetation (Bolaniska Notiser 1906, pag. 49-57).

- Ecological and Systematic Studies of the Ceylon Species of Caulerpa (Ceylon Mar. Biol. Report part. II, I906, pag. 87-I44, Fig. 1-5r).

- Ueber den Bau und die Entwicklung der Florideengattung Martensia (K. Svenska Vet. Akad. Handl. Band. 43, rgo8, N. 7, pp. Ior, Fig. I-62, T. 4). 
Temperre et Peragallo. - Diatomées du monde entier, 2.e édition, Fasc, I-3o et tables; Grez-surLoing, 1907-I9I5, $8^{\circ}$.

TrevisAN V. - De Dictyoteis adumbratio (Linnaea XXII, IS49, pag. 42Y-464).

- Caulerpearum sciagraphia (Linnaea XXII, I849, pag. I29-I44).

TURner D. - Fuci, sive plantarum Fucorum generi a botanicis adscriptarum icones, descriptiones et historia; London, I808-1819, Arch, 258 tab.

Van Heurck H. - Synopsis des Diatomées de Belgique; Atlas de I4I planches; Anvers I8So-85, in $8^{\circ}$.

- Types du Synopsis des Diatomées de Belgique sér. I-XXII. Déterminations, notes et diagnoses par M. A. Gkunow; Anvers I884-88 (cfr. Botanisches Centralblatt XVII, I884, pag. 20I-202, XXXIII, I888, pag. 323-325).

- Traité des Diatomées, avec env, 2000 figures; Anvers, 1899, J. E. Buschmann, $4^{0}$.

Velley T. - Description of Conferva umbilicata, a new Plant, from New South Wales (Linn. Trans. V, I799, pag. I69-170, tab. VII).

Voyage autour du monde sur la Vénus pendant les années I836-I839 (Botanique par M. J. DEcAISNE) Paris 1846 (Atlas), I864 (Text.), $8^{\circ}$ et fol.

Walker-Arnotr G. A. - On Rhabdonema, and a new allied Genus (Quart. Journ. Micr. Sc. vol. VI, I 858 , pag. 87-93).

Weber van Bosse A. - Études sur des Algues de l'Archipel Malaisien (Ann. Jard. botan, de Buitenzorg vol. VIII, I890, pag. 79-94, 165-I88, pl. XVI-XVII, XXIV-XXVI, 2 sér, vol. II, I90r, pag. I26-I41, pl, XVII-XIX).

-- Monographie des Caulerpes (Ann. Jard. botan. de Buitenzorg vol. XV, I898, pag. 243-40r, plates XX-XXXIV).

- Note sur le genre Dictyosphaeria Dec. (La Nuova Notarisia ser. XVI, pag. I42-I44, Padova I905)

- Sur deux nouveaux cas de symbiose entre algues et éponges (Ann. Jard. botan. de Buitenzorg 2 sér., suppl. III, I9ro, pag. 587-593, plates XVI-XVII).

- Note sur deux Algues de l'Archipel Malaisien (Recueil des Trav. botan. néerland. N. I).

- Notice sur quelques genres nouveaux d'Algues de l'Archipel Malaisien (Ann. Jard. botan. de Buitenzorg 2 sér., vol. IX, I9lo, pag. 25-33).

- Note sur les Caulerpa de l'île Taïti et sur un nouveau Caulerpa de la Nouvelle Hollande (Ann. de l'Inst. océanographique [Fondation Albert Ie, prince de Monaco] vol. II, fasc. I, I9Io, pag. I-8, 5 Fig., pl. I-II).

- Liste des Algues du Siboga I. Myxophyceae, Chlorophyceae, Phaeophyceae (Siboga-Expeditie Monogr. LIX $a$; Leiden, 1913, E. J. Brill, 4, 5 pl. et 52 fig. dans le texte).

- Liste des Algues du Siboga II. Rhodophyceae [Protoflorideae, Nemalionales, Cryptonemiales] (Siboga-Expeditie Monogr. LIX b; Leiden, I92I, E. J. Brill, $4^{0}, 3$ pl. et 57 fig. dans le texte).

Weber van Bosse A. and Foslie M. - The Corallinaceae of the Siboga Expedition, with XVI plates and 34 Textfigures (Siboga-Expeditie Monogr. LVI; Leiden, 1904, E. J. Brill, $4^{0}$ ).

West G. S. - The Algae of the Yan Yan Reservoir, Victoria, a biological and oecological Study (Jour. Linn. Soc. vol. XXXIX, I909, n. 269, pag. I-88, plates I-6, to textfigures).

- Algological Notes V. A Diatomaceous Earth from Rhodesia; VI Some African Algae; VII. Algae from Queensland; VIII. Selenastrum acuminatum Lagerheim; IX. A new Species of Euastrum. - (Journal of Botany vol. 50, I9I2, pag. 79-89, fig. I-6).

West W. and West G. S. - A Monograph of the British Desmidiaceae, vol. I-IV, with 128 plates; London, I904-I9I2, Ray Society, $8^{\circ}$

White C. J. - Analysis of the Ash of a N. S. Wales Sea Weed [Ecklonia] (Proceed. R. Soc. N. S. Wales 1906, pag. 3).

Whitting F. G. - Sarcophycus potatorum Kütz. (Phycological Memoirs ed. G. MurRAy part II, 1893, pag, 38-39, plate XII, fig. I-3).

- On Chlorocystis Sarcophyci, a new endophytic Alga (Phycological Memoirs ed. G. Murray part II, 1893, pag. 4I-45, plate XII, fig. 4-II).

- v. Mitchell M. O.

Wille N. - Neue Süsswasseralgen von den Samoainseln (Hedwigia LIII, pag. 144-147, Dresden I9I3).

- Süsswasseralgen von den Samoainseln, Hawaii, den Salomoninseln und Ceylon (Denkschr. math. nat. Kl. der Akad. der Wissensch. in Wien Band 92, pag. 139-162, T. I-III).

Wilson J. Bracebridge - Catalogue of Algae collected at or near Port Phillip Heads and Western Port (Proceed. R. Soc. Victoria vol. IV, 1892, pag. 157-190). 
Wiтt O. N. - Bericht über die Untersuchung zweier Diatomaceen-Gremische. Ein Beitrag zur Kenntniss der Flora der Südsee (Journ. Mus. Godeffroy I, I873, pag. 63-70, T. VIII).

- Ueber Südsee-Diatomaceen (Journ. Mus. Godeffroy IV, 1873, pag. 111-116 [287-292], T. I5).

Wittrock V. et Nordstedt O. - Algae aquae dulcis exsiccatae Fasc. 21 ; Stockholmiae, 1889, O. L. Svanbäcks, $8^{0}$, pp. 92.

Wittrock V., Nordstedt O., Lagerheim G. - Algae aquae dulcis exsiccatae, Fasc. 30-34 (Botaniska Notiser 1903, pag. 133-141).

- Algae aquae dulcis exsiccatae, Fasc. 35; Lundae, 1903, typis Berlingianis, $8^{0}$, pp. 42.

WRIGHT C. H. - Distribution of Caloglossa Leprieurii (Mont.) J. Ag. (Journal of Botany vol. XXVII, n. 313, pag. 22, London 1889).

Wright E. P. - Fruit of Cliftonia (Proceed. Dublin Micr. Chub vol. IV, part I, r883, pag. 42).

- Tetraspores of Cliftonia (Proceed. Dublin Micr. Club vol. IV, part I, I883, pag. 46).

- Chlorocladus australiensis (Proceed. Dublin Micr, Chub. vol. IV, part I, 1885, pag. 6o).

- Pandorea Traversi J. Ag. (Journ. and Proceed of the Dubl. Micr. Club vol. IV, part I, I880, pag 3).

YENDo K. - A Revised List of Corallinae (Journ. of the College of Sc. Imper. Univ. Tokyo vol. XX, art. 12, 1905, pag. 1-46).

Zahlbruckner A. - Schedae ad "Kryptogamas exsiccatas» editae a Museo Palatino Vindobonensi (Ann. Naturhistor. Mus. Wien I9r3).

Zanardini G. - Phyceae Australasicae novae vel minus cognitae (Flora i874, pag. 486-490, 495-505).

- Phyceae papuanae novae vel minus cognitae (Nuovo Giorn. botan. ital. vol. X, 1888, pag. 34-40).

Zен W. - Neue Arten des Gattung Liagora (Notizblatt K. bot. Gart. u. Mus, Dahlem V, 1912, n. 49, pag, 268-273).

Bastow R. A. - Key to Tribes and Genera of the Florideae [Red or purple Marine Algae] (Journ. $R$. Soc. Sydney 19oo, 3 pp., 2 plates).

BORGE O. - Algologische Notizen 6. Süsswasseralgen aus Queensland; Süsswasseralgen aus Madeira (Botaniska Notiser 1911, pag. 197-207, T, 2).

Braun A. - Charae Preissianae adiectis reliquis speciebus e Nova Hollandia hucusque cognitis (Linnaea vol. XVII, I, 1843, pag. 113-I19).

Burbury F, E. - Tasmanian Diatomaceae, s. n. t., pp. 7 in $8^{\circ}$.

Clem.and J. Burton - Note on Agar-Agar Seaweed from Western Australia ( $R$. Soc, of New Wales 19r3, pag. 75\%. 


\section{INDICE}

Abrotanifolia (St.) Ag. var. (Cystoseira) 70 Abscissa $\mathrm{H}$. et $\mathrm{H}$. (Polysiphonia) 38 Acanthocarpa Harv. (Callophyllis) 19 Acanthocarpa (Harv.) J. Ag. (Rhodophyllis) 19 Acanthocarpum Kuetz. (Callithamnion) 55 Acanthophorum Kuetz. (Callithamnion) 55 Achnanthes Bory 130

Actinocyclus Ehr. 105

Actinoptychus Ehr. 103

Acuminatum var. Brebissonii Cl. (Gyrosigma) 150

Acus Kuetz (Synedra) 114

Acuta Kuetz. (Sigmatella) 122

Acuta W. Sm. (Stanroneis) 147

Acutum (W. Sm.) Rab. (Pleurostauron) 147

Adenocystis $\mathrm{H}$. et $\mathrm{H} .75$

Adnata Zanard. (Caloglossa) 35

Adriatica Zanard. (Galaxaura) 15

Adriaticum Kuetz. (Rhabdonema) 110

Advena (A. S.) Cl. var. parca (A. S.) Cl. (Navicula) 142

Aeruginea (Kuetz.) Thur. (Calothrix) 97 Aestuarii (Mert.) Liebm. (Lyngbya) 95

Afine Kuetz. (Aglaophyllum) 32

Affine Harv. (Nitophyllum) 32

Affinis Harv. (Jania) 63

Agardhii Grev. (Scaberia) 67

Alternans $V . H$. (Biddulphia) $10-$

Alternans J. Ag. (Dilophus) 74

Alternans Bail. (Triceratium) 107

Amansia Lamour, 46

Ambigua Müll. (Melosira) 98

Amboinensis Karst. (Caloglossa) 3ొ

Amphibia Grun. (Nitzschia) 12:

Amphicephala Naeg. (Cymbella) 152

Amphiceros Ehr. (Rhaphoneis) 116

Amphiceros Kuetz. (Navicula) 141

Amphioxys (Ehr.) Grun. (Hantzschia) 126

- var. intermedia Grun. 12

Amphipentas Ehr. 108

Ainphitetras Ehr. 108

Amphora Ehr. 153

Amylaceus (Fucus) 26

Amylaceus (Lichen) 26
Amylophora J. Ag. 25

Anabaena Bory 96

Anceps Ehr. (Stauroneis) 147

- var. gracilis (Rab.) Brun. 147

Anceps (Lew.) Bréb. (Stenopterobia) 129

Anceps Lewis (Surirella) 129

Angulata Lewis (Mastogloia) 150

Angulata Greg. (Orthosira) 102

Angulosa Ehr. (Grammatophora) 109

- var. hamulifera (Kuetz.) Grun. 109

Angustata Kuetz (Phycopteris) 73

Angustifolia (Harv.) J. Ag. (Lenormandia) 48

Angustifolium (Sond.) J. Ag. (Carpoglossum) 71

Angustum (J. Ag.) H. et H. (Plocamium) 32

Anomoeoneis Pfitz. 147

Antarctica (H. et H.) D. T. (Bornetia) 52

Antarctica H. et H. (Griffithsia) 52

Antarctica H. et H. (Melobesia) 59

Antarcticum Kyl. (Antithamnion) 55

Antithamnion Naeg. 54

Apiculata Grun. (Mastogloia) 150

Apiculata Greg. (Pinnularia) 137

Appendiculata Kuetz. (Navicula) 144

Appendiculata (Ag.) Cl. (Pinnularia) 144

- var. budensis Grun. 144

Arborea Harv. (Acanthophora) 42

Arborea (Harv.) Falk. (Chiracanthia) 42

Arcta Kuetz. (Corticularia) 83

Arcuata Ehr. (Grammatophora) 109

Arcus Greg. (Amphora) 153

Ardissonia De Not. 115

Arenaria Donk. (Navicula) 139

Arenicola (Grun.) Cl. var. subaequalis $\mathrm{Cl}$. (Amphora) 153

Areschougia Harv. 22

Argus Grun. (Campyloneis) 131

Articulata (J. Ag.) Schm. (Euptilota) כ̆ 4

Articulata \%. Ag. (Ptilota) 54

Aspera Ehr. (Navicula) 148

Aspera A. Schm. (Navicula) 148

Aspera (Ehr.) Cl. (Trachyneis) 148

- var. vulgaris Cleve 148

Asperum (Mert.) Grev. (Gelidium) 16 
Aterrima H. et H. (Polysiphonia) 40 Atlantica J. Ag. (Chnoospora) 80 Attenuata (Bonn.) Ag. (Crouania) 56 Aucklandicum Kuetz. (Ceramium) 56 Auliscus (Ehr.) Bail. 104 Aurita (Lyngb.) Bréb. (Biddulphia) 106 Aurita Ag. (Odontella) 106 Australasica Sond. (Corallopsis) 37 Australasica Kuetz. (Halopithys) 46 Australasica (Mont.) Falk. (Protokuetzingia) 46 Australasica Endl. (Rytiphloea) 46 Australasicus Sond. (Dictyurus) 50 Australica (A. S.) Cl. (Cymbella) 152 Australicum A.S. (Cocconema) 152 Australicum Witt. (Pleurosigma) 149 Australis \%. Ag. (Acropeltis) 29

Australis (Ag.) Schm. (Brongniartella) $\mathbf{4 4}$ Australis Sond. (Bryopsis) 90

Australis J. Ag. (Cladostephus) 82 Australis Kuetz. (Climacosphenia) 112 Australis Havv. (Corynospora) 52 Australis (Harv.) J. Ag. (Cronania) 56 Australis Sond. (Dictyurus) 50 Australis Ehr. var. gigantea (Grev.) Fricke (Entopyla) 113

Australis C. Ag. (Griffthsia) 82

Australis (Harv.) J. Ag. (Monospora) 52

Australis Sond. (Nizymenia) 25

Australis Kuetz. (Phycoseris) 84

Australis Sond. (Rhodymenia) 29

Australis Darbish. (Spencerella), 20

Australis Harv. (Stilophora) 78

Australis Petit (Trachysphenia) 117

Australis Aresch. (Ulva) 84

Axillaris (R.Br.) (Fucus) 67

Axillaris (R. Br.) Grev. (Seirococcus) 67

Bacillaria Gmel. 121

Baileyana Grun. (Navicula) 138

Bainesii F. Müll. et Harv. (Cladophora) 89

Balticum var. Brebissonii V. H. (Pleurosigma) 150

Banksii Turn. (Fucus) 71

Banksii (Turn.) Decne (Hormosira) 71

Beccarii Zanard. (Caloglossa) 34

Bellotia Harv. 76

Berkeleyi Crn. (Cylindrocarpus) 79

Bicuspidata Aresch. (Lobospira) $7 t$

Biddulphia Gray $106^{\circ}$

Binotata Grun. (Cocconeis) 135

Binotata Cleve (Mastogloia) 135

Binotata Grun. (Orthoneis) 135

- var. quadrilocularis nob. 135

Blandi Harv. (Polysiphonia) 38

Bomboides (A. Schm.) Cl. (Diploneis) 142

Bomboides A. Schm. (Navicula) 142

Bombus Ehr. (Diploneis) 142

- var. egena (A. Schm.) Cl. 142
Bombus Kuetz. (Navicula) 142

Bornetia Thur. 52

Boryi Ag. (Sargassum) 64, 65

Bostrychia Mont. 49

Botryocarpa (Harv.) (Chantransia) 14

Botryoides Turn. (Fucus) 36

Botryoides (Turn.) Gaill. (Laurencia) 36

Brebissonii Grun. (Pleurosigma) 150

Brevipes C. Ag. (Achnanthes) 130

- var. angustata Meresch. 130

- var. intermedia Cleve 131

Brongniartella Bory 44

Brownii (J. Ag.) D. T. (Chrysymenia) 31

Brownii Harv. (Gloiosaccion) 31

Bryocladia Schmitz 42

Bryopsis Lamour. 90

Bullosa Menegh. (Anabaena) 97

Cactoides (Turn.) Ag. (Caulerpa) 90

Caelata Chase (Campyloneis) 132

Caelata Arn. (Cocconeis) 132

Caelatus Bail. (Auliscus) 104

Caespitosa B. et F. (Hyella) 94

Calcicola (Ag.) Rabenh. (Hypheothrix) 96

Calcicola Gom. (Schizothrix) 96

Callicoma Kuetz. (Cladophora) 39

Callithamnion Harv. (Dasya) 51

Callithamnion (Sond.) Falk. (Heterosiphonia) 5̃

Callithamnion Sond. (Polysiphonia) 51

Caloglossa Harv, 34

Caloneis Cleve 141

Calothrix C. Ag. 97

Campylocoma Kuetz. (Blossevillea) 69

Campylodiscus Ehr. 129

Campylodiscus (Grun.) (Diploneis) 132

Campyloneis Grun. 131

Canaliculata (Grev.) Sond. (Kuetzingia) $\mathbf{4 6}$

Canaliculata Grev. (Rytiphloea) 46

Cancellata Donk. var. apiculata (Greg.) (Navicula) 137

- var. subapiculata Grun. 137.

Cancellata Harv. (Polysiphonia) 39

Candida Nees (Plocaria) 26

Capensis Kuetz. (Achnanthes) 130

Capensis Kuetz. (Porphyra) 13

Capensis Aresch. (Ulva) 85

Carconensis Grun. (Stephanodiscus) 103

Carpoglossum Kuetz. 71

Carpopeltis Schmitz 57

Carpophyllum J. Ag.' (Sargassum) 64

Caudata Kuetz. (Blossevillea) 69

Caudata Harv. (Carpomitra) 77

Caulerpa Lamour. 90

Cenomyce J. Ag. (Hypnea) 27

Cephalornithos Kuetz. (Blossevillea) 68

Cephalornithos (Lab.) J. Ag. (Cystophora) 68

Cephalornithos Labill. (Fucus) 68 
Ceramioides Kuetz. (Hypnea) 27

Ceramium Ag. 56

Cerataulus Ehr. 10 \%

Cervicornis (Kuetz.) Schm. (Bryocladia) 42

Cervicornis Kibetz. (Polysiphonia) 42

Cesatii (Rab.) Grun. (Cymbella) 152

Cesatii Rabenh. (Navicula) 15:

Chaetangium Kuetz. 15

Chaetosphaeridium Klebh. 86

Chantransia (DC.) Schmitz 14

Charoides Lamour. (Amphiroa) 61

Charoides Harv. (Erythroclonium) 21

Charoides Sond. (Hypnea) 28

Charoides (Lam.) W. v. B. (Metagoniolithon) 61

Charoides Harv. (Rhabdonia) 21

Chauvinii Harv. (Lenormandia) 48

Chauvinii var. angustifolia Harv. (Lenormandia) 48

Chiracanthia Falk, 4 ?

Chlorotica J. Ag. (Enteromorpha) 85

Chnoospora J. Ag. 80

Chondria (Ag.) Harv. 37

Chordalis H. et H. (Desmarestia) 80

Choreonema Schmitz 58

Chroococcus Naeg. 93

Chrysymenia J. Ag. 31

Cladodermum (Zan.) Hk. (Antithamnion) ó5

Cladophora Kuetz. 89

Cladosiphon Kuetz. 79

Cladostephus C. Ag. 81

Cladostephus Mont. (Polysiphonia) 44

Clavigera Kjellm. (Galaxaura) 15

Clavigera J. Ag. (Rhabdonia) 22

Cliftoni Harv. (Halymenia) 31

Cliftoni J. Ag. (Stenocladia) 24

Climacosphenia Ehr. 112

Coccinea Zanard. (Desmia) อิร

Cocconeis Ehr. 132

Codium Stackh. 91

Cohaerens (Bréb.) Naeg. (Chroococcus) 93

Cohaerens Bréb. (Pleurococcus) 93

Coleae J. Ag. (Amylophora) 25

Colpomenia Derb, et Sol, 81

Columbina Mont. (Porphyra) 13

Comosa Grun. (Griffithsia) 53

Comosa (Lab.) Ag. (Phyllospora) 70

Comosus Labill. (Fucus) 70

Comosus C. Ag. (Sporochnus) 77

Complanatus Harv. (Phacelocarpus) 23

Compressa (Reinsch) Skottsb. (Desmarestia) 80

Compressa ( $\left.L_{\circ}\right)$ Grev. (Enteromorpha) 85

Compressa C. Ag. (Ulva) 86

Conferta Harv. (Areschougia) 24, 25

Conferta var. Cliftoni J. Ag. (Stenocladia) 24

Conferta var. Harveyi $J$. Ag. (Stenocladia) 25

Conferva (L.) Lagerh. 87

Confervicola (Roth) Ag. (Calothrix) 97
Confervoides (Roth) Le Jol. (Ectocarpus) 83

Confervoides (L.) Grev. (Gracilaria) 26

Confervoides Bory (Trinitaria) 80

Confluens (R. Br.) Kuetz. (Carpoglossum) 71

Conspicuum Harv. (Callithamnion) 56

Constricta Meresch. (Licmophora) 112

Constrictum Ehr. (Gomphonema) 151

Contorta Kitt. (Surirella) 127

Contortus Pantoc. (Campylodiscus) 127

Corallina Lamour. 62

Coralloidea (J. Ag.) Kuetz. (Euptilota) 54

Coralloidea J. Ag. (Ptilota) 54

Coriacea (Sond.) Harv. (Thysanocladia) 23

Coriaceus Sond. (Sphaerococcus) 23

Corium (Ag.) Gom. (Phormidium) 94

Corneum J. Ag. (Chaetangium) 16

Corymbifera Thur. (Chantransia) 14

Corynecladia J. Ag. 37

Coscinodiscus Ehr. 101

Costata W. Sm. (Amphora) 154

Crassinervia Bréb. (Navicula) 149

Crenata J. Ag. (Zonaria) 72

Crenulata Kuetz. var. ambigua Grun. (Melosira) 98

Cribrosa Grun. (Mastogloia) 135

Cribrosa Grun. (Orthoneis) 135

Crispa Harv. (Hennedya) 17

Crispata (Roth) Kuetz. (Cladophora). 88

Crispata Lamour. (Corallina) 62

Crispata Kjellm. (Porphyra) 13

Crouania J. Ag. 56

Crozieri (Ehr.) Grun. (Rhabdonema) 111

Cruciatum (Ag.) Naeg. (Antithamnion) 55

Cruciatum Harv. (Callithamnion) 55

Cryptocephala Kuetz. (Navicula) 137

Cryptonemia J. Ag. 58

Crystallina (Ag.) Grun. (Ardissonia) 115

Curvata Ehr. (Grammatophora) 109

Curvata (Kuetz.) Grun. (Rhoicosphenia) 151

- var. marina (W. Sm.) Rabenh. 151

Cuspidata var. naviculiformis Auersw. (Cymbella) $15 \%$

Cuvieri Lamour. (Corallina) 62

Cyclotella Kuetz. 103

Cylindrica Lamour. (Galaxaura) 15

Cymbella C. Ag. 152

Cymbiformis var. parva V. H. (Cymbella) 15:

Cystophora J. Ag. 68

Cystoseira C. Ag. 70

Dactyliophora P. et Gr. (Galaxaura) 15

Dactylus Ehr. (Pinnularia) 144

Daemelii (Sond.) J. Ag. (Amansia) 46

Daemelii Sond. (Vidalia) 46

Dasya Ag. 50

Decipiens Grun. (Coscinodiscus) 102

Decipiens Mont. (Polysiphonia) 39

Decipiens (Grun.) Joerg. (Thalassiosira) 102 
Decurrens (R. Br.) Ag. (Sargassum) 64 Decurrens Kuetz. (Pterocaulon) 64 Decussata Ehr. (Gallionella) 99 Decussata Kuetz. (Melosira) 98 Debilis Harv. (Chondria) 37 Debilis J. Ag. (Chondriopsis) 37 Delisea Lamour. 36

Dendroides Harv, (Rhabdonia) 22 Denudata Kuetz. (Hypnea) 37

Dermatolithon Fosl. 60

Desmarestia Lamour. 80

Dichotoma H. et H. (Delesseria) 34

Dichotoma J.Ag. (Nemastoma) 55

Dichotoma (H. et H.) J. Ag. (Schizoneura) 34 Dictymenia Grev. 43

Dicranema Sond. 18

Didyma (Ehr.) Cl. (Diploneis) 143

Didyma Ehr. (Navicula) 143

Diesingiana J. Ag. (Zonaria) 72

Difformis (L.) Aresch. (Leathesia) 79

Digenea Ag. 41

Digito-radiata (Greg.) Ralfs (Navicula) 137

- var. Cyprinus (Ehr.) V. Hk. 137

Dilophus J. Ag. 74

Dimeregramma Ralfs 117

Diploneis Ehr. 142

Directa (IV. Sm.) Ralfs (Navicula) 137

- var. Incus (Grun.) Cleve 137

Dirupta Greg. (Cocconeis) 132

Dispar (Harv.) J. Ag. (Antithamnion) 54

Dispar Harv. (Callithamnion) 55

Distans var. heterostriata Mer. (Navicula) 138

Distans Greg. (Nitzschia) 122

Divaricata (R. Br.) Harv. (Hymenorladia) 28

Divergens Ralfs (Navicula) 144

Divergens W. Sm. (Pinnularia) 144

Dolicharthra J. Ag. (Galaxaura) 14

Dorycarpa (Turn.) Grev. (Scytothalia) 67

Dorycarpus Turn. (Fucus) 67

Draparnaldia Bory 87

D)ubia Ehr. (Navicula) 146

Dubia W. Sm. (Nitzschia) 123

Dubium (Ehr.) Cl. (Neidium) 146

Dnebenii Aresch. (Macrocystis) 76

Echionotus J. Ag. (Phacelocarpus) 24

Eckloni Suhr (Hypnea) 27

Ecklonia Hornem. 76

Ecostala J. Ag. (Dictymenia) $\$ 3$

Ectocarpus Lvngb. 83

Elata Harv. (Acropeltis) 57

Elata (Harv.) Schm. (Carpopeltis) 57

Elata J. Ag. (Cryptonemia) 57

Elegans Sond. (Amphiroa) 61

Elegans (Mart.) Ag. (Dasya) 51

Elliptica (Kuetz.) Cl. (Diploneis) 143

Flliptica Kuetz. (Navicula) 143
Elongata Bail. (Climacosphenia) 112

Elongata J. Ag. (Griffithsia) 52

Elongata (Harv.) D. T. (Monospora) 52

Elongatum Harv. (Callithamnion) 52

Encyonema Kuetz. 153

Enteromorpha Link 85

Entophytum B. et F. (Nostoc) 96

Entopyla Ehr. 113

Episcopalis H. et H. (Hypnea) 27

Epithemia Bréb. 119

Ericoides (Harv.) Schm. (Bryocladia) 42

Eriophorum Harv. (Bellotia) 76

Erosa var. obliqua Grun. (Schizymenia) 58

Erosum Kuetz. (Aglaophyllum) 33

Erosum Harv. (Nitophyllum) 33

Eruca Ehr. (Amphicampa) 118, 119

Eruca Brightw. (Eunotia) 118

Erythroclonium Harv. 21

Eucheuma J. Ag. 22

Eunotia Ehr. 118

Euptilota Kuetz. 54

Euzoniella Falk. 45

Excentricus var.catenata Grun. (Coscinodiscus) 102

Fallax Kuetz. (Blossevillea) 65

Fallax Sond. (Sargassum) 65

Farinosa Lamour. (Melobesia) 60

Fasciata Del. (Ulva) 84

Fasciculatus J. Ag. (Dilophus) 74

Fastigiata Harv. (Iania) 63

Fastigiata J. Ag. (Chnoospora) 80

Fastigiatum (Bory) J. Ag. (Chaetangium) 16

Fastuosa Ehr. (Surirella) 127

- var. abludens Grun. 128

Favus Ehr. (Triceratium) 108

Ferruginea C. Ag. (Lyngbya) 95

Filare Kuetz. (Stypocaulon) 82

Filiformis ( $\mathrm{Fl}$. Dan.) Grev. (Dumontia) 57

Filiformis $H$. et $H$. (Laurencra) 37

Filipendula (Harv.) Falk. (Herposiphonia) 45

Filipendula Harv. (Polysiphonia) 45

Fimbriata Harv. (Dictymenia) 43

Fimbriata (Ehr.) Grun. (Orthoneis) 13ã

Finbriatum Harv. (Nitophyllum) 33

Firmum Harv. (Halosaccion) 31

Flabellata Sond. (Melobesia) 61

Flabellatus Collins (Dilophus) 74

Flabelliformis Harv. (Griffithsia) 51

Flava Harv. (Zonaria) 72

Flava var. tenuior Sond. (Zonaria) 72

Flavescens Zanard. (Polysiphonia) 41

Flexella Jan. et Rabh. (Cocconeis) 132

Flexuosa (Wulf.) J. Ag. (Enteromorpha) 85

Foliacea Kuetz. (Melobesia) 61

Foliosa (Harv.) J. Ag. (Mychodea) 18

Foliosus Harv. (Gymnogongrus) 18

Forcipata Grev. (Navicula) 138 
Forcipata Grev. var. nummularia (Grev.) Cleve 138 Formosa Cleve (Amphora) 154

Formosum W. Sm. (Pleurosigma) 149 Forsteri (Mert.) Grev. (Laurencia) 36 Fragilis (Lamk.) (Galaxaura) 15 Frauenfeldianum Grun. (Pleurostauron) 148 Frawenfeldii Grun. (Bacillaria) 123 Frauenfeldii Grun. (Nitzschia) 123 Frauenfeldii Grun. (Triceratium) 107 Frustulia C. Ag. 148 Frustulia C. Ag. 122 Frutex Harv. (Polysiphonia) 39 Fulgens (Grev.) Grun. (Ardissonia) 115 Fulmen (Brightw.) Grun. (Pleurostauron) 148 Funicularis (Mont.) Sauv. (Halopteris) 82 Furcula Wor. (Dilophus) 74

Fusca (Greg.) Cl. (Diploneis) 143 Fusca Ralfs (Navicula) 143

Fuscata Kuetz. (Corlicularia) 83

Fuscescens Harv. (Polysiphonia) 39

Galaxaura Lamour. 14

Galeatum J. Ag. (Codium) 91

Gallionella Bory 99

Gallionii (Bory) Ehr. (Synedra) $11 t$

Gastrum var. Placentula V. H. (Navicula) 140

Gelatinosa Hens. (Thalassiosira) 102

Gelidium Lamour. 16

Genuina var. egena A. Schm. (Navicula) 142 Gephyria Arnott 113

Gibba Kuetz. (Epithemia) 120

- var. ventricosa Grun. 121

Gibba var. brevistriata Grun. (Navicula) 145

Gibba (Ehr.) O. Müll. (Rhopalodia) 120

Gibberula Kuetz. (Epithemia) 120

Gibberula (Ehr.) O. Müll. (Rhopalodia) 120

Gigantea Grun. (Amphora) 154

Gigantea Grev. (Gephyria) 113

Glabrata Grun. (Achnanthes) 130

Globifera (Lamour.) J. Ag. (Rhabdonia) 22

Globosa Wolle (Aphanochaete) 86

Globosa Borzi (Nordstedtia) 87

Globosum (Nordst.) Klebh. (Chaetosphaeridium) 86

Globosum Nordst. (Herposteiron) 86

Globulifera Kuetz. (Conferva) 87

Glomerata (Vauch.) Ag. (Draparnaldia) 87

Glyphodesmis Grev. 117

Glossophora J. Ag. 74

Gomontia Born. et Flah. 90

Gomphonema C. Ag. 151

Goulardi Bréb. (Synedra) $11 t$

Gracilaria Grev. 26

Gracilis H. et H. (Laurencia) 37

Gracilis Hantzsch (Nitzschia) 123

Gracilis Ehr. (Stauroneis) 147

Gracilis Grun. (Surirella) 127
Grammatophora Ehr. 109

Grande J. Ag. (Sargassum) 66

Grande Bright. f. quadrata Temp. (Triceratium) 107

Granifera Ell. et Sol, var. australis (Grun.) D. T. (Corallina) 62

Granifera Sond. (Corallina) 62

Granulata Ralfs var. (Melosira) 98

Granulata Bail. (Navicula) 138

- var. Peragallorum nob. 138

Granulata Bréb. (Navicula) 138

Granulata Ehr. (Navicula) 138

Granulata A. Schm. (Navicula) 138

Grateloupia Ag. 57

Gregaria Falk. (Vidalia) 47

Grevillei (W. Sm.) Gr. et Eul. (Campyloneis) 132

- var. Argus V. H. 131

Grevillei W. Sm. (Cocconeis) 131

Grevillei Kuetz. (Cystoclonium) 19

Grevillei Sond. (Dicranema) 18

Griffitthsia Ag. 51

Griffithsioides Sond. (Callithamnion) 53

Griffithsioides (Sond.) D. T. (Monospora) 53

Guineensis J. Ag. (Dilophus) $\mathbf{7 4}$

Gunniana J. Ag. (Enteromorpha) 86

Gunnianus J. Ag. (Dilophus) 74

Gyrosigma Hass. 150

Haloplegma Mont. 53

Halopteris Kuetz. 82

Hamulifera Grun. (Navicula) 141

Hamuliferus (Grun.) DT. (Libellus) 141

Hantzschia Grun. 126

Hapalathrix Harv. (Dasya) 50

Hapalosiphon Naeg. 96

Hartleyana H. L. Sm. (Navicula) 145

Hartleyana Grev. (Pinnularia) 145

Harveyana J. Ag. (Stenocladia) 25

Harveyi J. Ag. (Glossophora) 74

Hawkeri J. Ag. (Amansia) $4 \bar{\tau}$

Hennedya Harv. 17

Herposiphonia Naeg. 45

Heteroidea Hantzsch (Cocconeis) 132

Heteromitus Mont. 44

Heterosiphonia Mont. 51

Hieroglyphicum (Ag.) Kuetz. et var. (Rhizoclonium) 88

Hippocampus Hass. (Gyrosigma) 150

Hirsuta Harv. (Lasiothalia) 56

Holtingii (Mert.) Ag. (Alysium) 15

Hombroniana (Mont.) Kuetz. (Callophyllis) 19

Homoeocladia C. Ag. 122

Hordeacea (Harv.) Sauv. (Halopteris) 82

Hordeacea Harv. (Sphacelaria) 82

Hordeaceum Kuetz. (Stypocaulon) 82

Hormosira Endl. 71

Hormörichum sp. Kuetz. 88 
Hornemanni (Mert.) Schm. (Chondrococcus) 55

Humerosa Bréb. (Navicula) 139

Humilis Donk. (Ñavicula) 139

Hungarica Gr. var. humilis (Donk.) Grun. (Navicula) 139

Hyalodiscus Ehr. 100

Hydrophora (Harv.) J. Ag. (Gloiosaccion) 31

Hydrophora Harv. (Halosaccion) 31

Hyella Born. et Flah. 94

Hymenocladia J. Ag. 28

Hypheothrix Kuetz. 96

Hypnea Lamour. 27

Hypneoides Harv. (Delisea) 36

Hypnoides (R. Br.) Ag. (Caulerpa) 91

Hypnoides Kuetz, (Chauvinia) 91

Hystrix H. et H. (Polysiphonia) 40

Implexa var. gracilis Grun. (Chnoospora) 81 Inaequale Kuetz. (Psichohormium) 87

Incisa Greg. (Eunotia) 119

Incisa (J. Ag.) Falk. (Euzoniella) 45

Incisa J. Ag. (Polyzonia) 45

Incompta Hary. (Polysiphonia) 40

Incus Grun. (Navicula) 138

Inducta A. Schm. (Surirella) 127

Inermis Kuetz. (Carpomitra) 77

Inermis R. Br. (Fucus) Ti

Inermis ( $\mathrm{R}, \mathrm{Br}.) \mathrm{J}$. Ag. (Perithalia) $7 \%$

Inflata (Kuetz.) Grun. (Aclinanthes) 130

Inflata Heid. (Stauroneis) 148

Inflata Kuetz. (Stauroneis) 130

Intlatum (Heid.) nob. (Pleurostauron) 148

Inflexa Eréb. (Amphipleura) 155

Inflexa H. L. Sm. (Amphora) 150

Inflexa (Bréb.) Eulenst. (Okedenia) 155

- var. synedroides nob. 155

Integrifolium Harv. (Myriodesma) 72

Intercedens Grun. var. abludens Grun. (Surirella) 127

Intermedia $P$. et R. (Desmarestia) 80

Inlerposita Heydr. (Chantransia) 14

Interrupta Kuetz. (Phycopteris) 73

Interrupta Heib. (Striatella) 111

Interrupta Ehr. (Tessella) 111

Interrupta Ag. (Zonaria) 73

Iridaea Bory 17

Iridis var dubia V.H. (Navicula) 14i

Jania Lamour. 63

Japonica Okam. (Acanthopeltis) 68

Juergensii C. Ag, var. constricta Grun. (Licmophora) 112

Kallymenioides Harv. (Halymenia) 31

Kallymenioides (Harv.) D. T. (Sebdenia ?) 31

Kuetzingia Sond. 46

Kuetzingioides Harv. (Amansia) 47
Kunthii Ag. (Dictyota) 74

Kunthii Harv. (Dictyota) it

Kunthii (Ag.) J. Ag. (Glossophora) 74

Labillardieri (Mert.) J. Ag. (Phacelocarpus) 24

Lacerifolium (Turn.) Ag. (Sargassum) 65

Lacerifolius Kuetz. (Carpacanthus) 65

Laciniata Ag. (Porphyra) 13

Laciniata (Lightf.) DT. (Wildemania) 13

Lactuca (L.) Le Jol. ff. (Ulva) 83

Laetevirens Kuetz. (Cladophora) 89

Laevigata Grun. (Synedra) 114

Laevissima Kuetz. (Navicula) 139

Laminosum (Ag.) Gom. (Phormidium) 94

Laminosus (Kuetz.) Hansg. (Hapalosiphon) 96

Lamourouxii Decne (Mastophora) 60

Lanceolata (Bréb.) Grun. (Achnanthes) 130

- var. dubia Grun. 130

- var. inflata A. Mayer 131

Lanceolata Kuetz. var, arenaria (Donk.) V. Hk. (Navicula) 139

Lancifolia J. Ag. (Delesseria) 34

Lancifolium (H. et H.) J. Ag. (Paraglossum) 34

Lapidescens auct. (Galaxaura) 15

Lapidescens var. annuligera P. et G. (Galaxaura) 15

Lasiothalia Harv. 56

Latissima Hook, et Harv. (Halymenia) 17

Latissima ( $\mathrm{H}$. et $\mathrm{H}_{\text {.) }}$ Grun. (Iridaea) 17

Latissimum J. Ag. (Rhodoglossum) 17

Laurencia Lamour. 36

Laurencia (H. et H.) Harv. (Areschougia) 22

Laurencia H. et H. (Carpothamnion) 22

Leathesia Gray 79

Leclancherii Harv. (Myrionema) 78

Lenormandia Sond. 48

Lenta Kjellm. (Galaxaura) 15

Lepidoptera Greg. (Amphiprora) 136

Lepidoptera (Greg.) Cleve (Tropidoneis) 136

Leprienrii (Mont.) J. Ag. (Caloglossa) 34, 35

Lessonii $H$. et $H$. (Adenocystis) 75

Lessonii Bory (Asperococcus) 75

Lessonii minor Kuetz. (Asperococcus) $\mathbf{7 5}$

Libellus Cleve 141

Liber W. Sm. (Caloneis) 141

- var, elongata (Grun.) Cl. 142

- var. linearis (Grun.) V. Hk, 142

Elongata Grun. (Navicula) 142

Lichenoides (L,) Harv. (Gracilaria) 26

Lichenoides (Ell.) Heydr. (Lithothammion) 59

- var. antarctica (H. et. $\mathrm{H}_{\text {.) }}$ Fosl. 59

- var. Patena (H. et H.) Fosl. 59

Licmophora C. Ag. 112

Licmophorum Harv. (Callithamnion) 52

Ligula Mont. (Ulva) 84

Ligulata Lamour. (Desmarestia) 80

Linearis (Ag.) W. Sm. (Nitzschia) 123

- var, tenuis Grun. 123 
Lineata Ehr. (Cocconeis) 133

Lineatus Ehr. (Coscinodiscus) 101 Lingulata $/ . A g$. (Enteromorpha) 86 Linza (L.) J. Ag. (Enteromorpha) 86 Lithothamnion Phil. 59

Lobata (Lam.) Falk. (Pollexfenia) 43 Lobospira Aresch. 74

Longipes C. Ag. (Achnanthes) 131

- f. lata H. Perag. 131

Lophocarpum J. Ag. (Sargassum) 65 Lophurella Schmitz 38

Lorenzianus Grun. (Campylodiscus) 129

Lucasii Gepp (Gracilaria) 27

Luxurians Harv. (Cryptonemia) 58

Lyngbya C. Ag. 95

Lyra Ehr. (Navicula) 139

- var. plur. 139-140

Lyrata Ehr. (Gallionella) 99

Lyrata (Ehr.?) Kuetz. (Melosira) 99

Lysigonium Link 99

Macrocarpa Kuetz. (Cystoseira) 70

Macrocystis C. Ag. 76

Macrophyllum Zanard. (Sargassum) 64

Maculatus Gr. et St. (Actinoptychus) 104

Major Decne (Galaxaura) 14-15

Major Kuetz. (Navicula) 145

Major (Kuetz.) Rab. (Pinnularia) 144

Marginulata Grun. (Nitzschia) 123

Marina Ktz. var. tropica (Ktz.) Grun. (Grammatophora) 109

Marina (Donk.) Grun. (Hantzschia) 126

Marinum Donk. (Pleurosigma)

Martialis Hanst. (Conferva) 87

Martiana C. Ag. (Homoeocladia) 122

Martiana V. Hk. (Nitzschia) 122

Mastogloia Thwait. 150

Mastophora Decne 60

Maxima Perag. (Cocconeis) 134

Maxima Grun. (Mastogloia) 134

Media Grev. (Desmarestia) 80

Media Arnott (Gephyria) 113

Melobesia Lamour. 60

Melosira Ag. 98

Menziesii J. Ag. (Desmarestia) 80

Mertensii Kuetz. (Thamnocarpus) 32

Mesolepta Ehr. (Navicula) 145

Mesolepta (Ehr.j W. Sm (Pinnularia) 145

Metagoniolithon Web. v. Bosse 61

Micrarthrodia Lamour. (Jania) 63

Microcarpa H. et H. (Polysiphonia) 38, 40

Microspora Thur. 88

Minor Sond. (Cryptopleura) 33

Hinor var. nana V. H. (Dimeregramma) 117

Minutum Kuetz. (Rhabdonema) 11

Minus (Sond.) Harv. (Nitophyllum) 33

Mirabilis Ehr. (Amphicampa) 118

Mixta H. et H. (Bostrychia) 49
Mnioides Harv. (Caloglossa) 35

Molesta Kuetz. (Cocconeis) 133

- var. amygdalina (Bréb.) Grun. 133

- f. minor H. Perag. 133

Mollis H. et H. (Polysiphonia) 40

Monilifera J. Ag. (Cystophora) 68

Monospora Solier 52

Mucronatum (J. Ag.) Naeg. (Antithamnion) 5็

Mucronatum J.Ag. (Callithamnion) 55

Mucronatum J. Ag. var. (Codium) 92

Muelleri Sond. (Caulerpa) 91

Muelleri Kuetz. (Codium) 92

Muelleri Sond. (Erythroclonium) 21

Muelleri Sond. (Lenormandia) 49

Multipartita Harv. (Rhodophyllis) 20

Multipartitum Kuetz. (Aglaophyllum) 33

Multipartitum H. et H. (Nitophyllum) 33

Musciformis (Wulf.) Lam. (Hypnea) 27

Muscorum (Ag.) Gom. (Symploca) 95

Musculus Kuetz. (Epithemia) 121

Musculus (Kuetz.) O. M. (Rhopalodia) 121

Mychodea Harv. 18

Myriodesma Decne 72

Myrionema Grev. 78

Myrionema sp. plur. 78

Myriophyllum $f$. australiensis Grun. (Cladostephus) 82

Myriotrema Lenorm. (Phycoseris) 83

Myriotricha A. Br. (Nitella) 155

Nanum (Greg.) Ralfs (Dimeregramma) 117

Naumanni Asken. (Chantransia) 14

Naveana Rabenh. (Conferva) 87

Navicula (Bory) Cleve 137

Naviculaceum Bréb. (Pleurosigma) 150

Naviculiformis Auersw. (Cymbella) 153

Neidium Pfitz. 146

Nidifica J. Ag. (Hypnea) 28

Nidificum (Harv.) J. Ag. (Plocamium) 32

Nigrita Sond. (Polysiphonia) 39

Nitella A. Br. 1ว̄

Nitescens (Greg.) Cl. (Diploneis) 143

Nitescens Ralfs (Navicula) 143

Nitidus Greg. (Coscinodiscus) 102

Nitophyllum Grev. 32

Nitzschia (Hass.) Grun. 122

Nitzschii Kuetz. (Sigmatella) 122

Nizymenia Sond. 25

Nobile J. Ag. (Ceramium) 56

Nobilis var. dactylus $V$. Hk, (Navicula) 144

Nobilis J. Ag. (Porphyra) 13

Nostoc Vauch. 96

Novae-Zelandiae Hav. (Jania) 63

Novae-Zelandiae J. Ag. (Schizymenia) 58

Novae-Zelandiae Cl. (Stephanodiscus) 102

Nummularia Grev. (Navicula) 138

Nummuloides Bory (Gallionella) 99

Nummuloides $A g$. (Melosira) 99 
Obesa Harv. (Sarcocladia) 25 Obovata J. Ag. (Schizymenia) 58 Obovatum Sond. (Sargassum) 65 Obtusa Greg. (Amphora) 154

Obtusa W. Sm. var. scalpelliformis Grun. (Nitzschia) 123

Obtusata Harv. (Galaxaura) 15

Oceanica Ehr. (Grammatophora) 110

- var. plur. 110

Ocellata (Arn.) Grun. (Entopyla) 113

Officinalis L. (Corallina) $(22$

Ogasawaraensis Okam. (Caloglossa) 35

Okedenia Eulenst. 155.

Oligophyllus Kuetz.(Carpacanthus) 66 Opuntioides (Harv.) J. Ag. (Coeloclonium) 21 Oppositifolia (Ag.) J. Ag. (Thysanocladia) 23 Orbignyana Mont. (Macrocystis) 76

Ornata Kuetz. (Gigartina) 22

Orthoneis Grun. 135

Osmundaria Lamour. 48

Ostreobium Born. et Flah. 98

Ovalis Kuetz. var. affinis V. H. (Amphora) 15

Ovalis Bréb. (Surirella) 128

- var. angusta (Kuetz.) V. Hk, 128

- var, excelsa O. Muell, 19x

Ovata Grun. (Mastogloia) 130

Ovata Grun. (Orthoneis) 130;

Paclıycarpum J. Ag. (Sargassum) 66 Pachydictyon J. Ag. 73

Pacifica J. Ag. (Chnoospora) 80

Palea W. Sm. var, tenuirostris Grun. (Nitzschia) 124

Palmata (L.) Grev. (Rhodymenia) 29)

Panduriformis Greg. (Nitzschia) 124

Paniculata Kuetz. (Blossevillea) (i.)

Paniculata (Turn.) J. Ag. (Cystophora) 69

Paniculata ). Ag. (Dictyota) 73

Paniculata Suhr (Sphacelaria) 8:-

Paniculatum J. Ag. (Pachydictyon) 73

Paniculatum Kuetz. (Stypocaulon) 82

Paradoxa Gmel, (Bacillaria) 121

Paradoxa Kuetz. (Blossevillea) 66

Paradoxa (Lyngb.) Ag. (Licmophora) 112

Paradoxa Grun. (Nitzschia) 121

Paradoxum (R. Br.) Harv, (Sargassum) 66

Paraglossum J. Ag. 34

Paralia Heib. 10 s

Parca A. Schm. (Navicula) 14.

Parietina (Naeg.) Thur. (Calothrix) 97

Parva (IV. Sm.) Cl. (Cymbella) 153

Parva (Ehr.) Greg. (Pinnularia) 145

Parvula Kuetz. (Sphenella) 151

Parvulun (Kuetz.) Rab. (Gomphonema) 151

Parvum W. Sm. (Cocconema) 153

Patena Heydr. (Lithothamnion) 59

Patena H. et H. (Melobesia) 59

Paxillifer (O. F. M.) Heib. (Nitzschia) 121
Paxillifer O.F. Muell. (Vibrio) 121

Pectinalis (O. F. M.) Rab. (Eunotia) 119

Pectinata J. Ag. (Cystophora) 69

Pedicellata Harv. (Pollexfenia) 43

Pediculus Ehr. (Cocconeis) 133

Pellucida Grun. (Cocconeis) 133

Penicilliformis (Roth) Aresch. (Urospora) 88

Pennata A. Schm. (Navicula) var. maior H. Perag. 140

- var. maxima Cleve 140

Pentacrinus Wall. (Triceratium) 108

Perforatus Ehr. (Coscinodiscus) 102

Periclados (Sond.) Schm. (Lophurella) 38

Periclados Sond. (Rhodomela) 38

Perithalia J. Ag. 77

Pernotata Meresch. (Mastogloia) 150

Peronii Mert. (Fucus) 64, 65

Peronii (Turn.) Ag. (Sargassum) 64, 65

Phacelocarpus Endl, et Dies, 23

Phormidium Kuetz, 94

Phyllophora (H. et H.) Schm. (Carpopeltis) 57

Phyllospora C. Ag. 70

Pinnata Greg. (Cocconeis) 134

Pinnatifudum J. Ag. (Myriodesma) 72

Pinnularia Ehr. 144

Placentula Ehr. (Cocconeis) 133

- var. lineata (Ehr. V. Hk. 133

Placentula (Ehr.) Kuetz. (Navicula) 140

Plana (Sond.) Harv. (Mastophora) 61

Plana Sond. (Melobesia) 61

Platythalia Sond. 71

Pleurosigma W. Sm. 149

Pleurostauron Rabenh. 147

Plicata Donh. (Navicula) 141

Plicatus (Donk.) D. T. (Libellus) 141

Plocamium Lamour. 32

Plumula (Ell.) Thur. (Antithamnion) 55

Plumula (Ell.) Thur. (Antithamnion) 55

Plumosa (Huds.) Ag. (Bryopsis) 90

Pollexfenia Harv. 43

Polycarpa Hook. (Rhodymenia) 30

Polycarpha (Harv.) J. Ag. (Hymenocladia) 29

Polyarpha (Havv.) (Rhodymenia) 29

Polyrhiza (Harz.) (Chantransia) 14

Polyrhiza (Lag.) B. et F. (Gomontia) 90

Polyrhizum Lagerh. (Codiolum) 90

Polysiphonia Grev. 38

Polysticta Grev. (Navicula) 138

Porphyra Ag. 13

Preissianum Sond. (Plocamium) 32

Preissii var. minor Lenorm. (Dasyphila) 56

Preissii Sond. (Haloplegma) 53

Preissii Kuetz. (Plocamium) 32

Procerum nidificum Harv. (Plocamium) 32

Prolifera /. Ag. (Dumontia) 57

Prolifera J. Ag. (Grateloupia) 57

Prolifera Lamour. (Osmundaria) 48

Proliferum J. Ag. (Polyphacum) 47 
Proteus Greg. (Amphora) 154

Protokuetzingia Falk. 46

Pseudobacillum Grun. (Navicula) 139

Psendomarginata Greg. (Cocconeis) 134

Psichohormium sp. Kuetz. 87

Pterocaulon Kuntze (Sargassum) 64

Pulchella Gray (Biddulphia) 106

Pumila Grev. (Gracilaria) 19

Pumilum J. Ag. (Gloiosaccion) 31

Punctata (W. Sm.) Grun. var. elongata Grun. (Nitzschia) 124

Punctata W. Sm. (Tiryblionella) 124

Punctatissima Grev. (Cocconeis) 136

Punctatissima Lagerst. (Orthoneis) 136

Punctatum Brightw. (Triceratium) 108

Pupula Kuetz. (Navicula) 140

Pustulata Lamour. (Melobesia) 60

Pustulatum (Lam.) Fosl. (Dermatolithon) 60

Pyrifera var. (L.) Ag. (Macrocystis) 76

Quekettii B. et Fl. (Ostreobium) 93

Quercifolia Harv. (Landsburgia) $\mathbf{~} 1$

Quercifolia Sond. (Platythalia) 71

Quercifolia Decne (Thuretia) 50

Quercifolium (R. Br.) J. Ag. (Carpoglossum) 71

Quercifolium Kuetz. (Nyriodesma) 71

Quercifolius R. Br. (Fucus) 71

Racemosa Harv. (Blossevillea) 69

Racemosa Harv. (Cystophora) 69

Racenosus Kuetz. (Carpacanthus) 66

Radicata (Turn.) J. Ag. (Ecklonia) 76

Radicans (Harv.) (Chantransia) 14

Radicans Okam. (Dilophus) 74

Ralfsii var. samoënsis Grun. (Actinocyclus) 105

Ramentacea J. Ag. (Calliblepharis) 20

Ramentacea Ag. (Delesseria) 20

Ramentacea (Ag.) J. Ag. (Rhodophyllis) 20

Ramulosa (E. B.) Hook. (Enteromorpha) 86

Ramulosa Harv. (Polysiphonia) 40

Ramulosum (Reinsch) Kyl. (Antithamnion) 55

Recta Hantzsch (Nitzschia) 125

Rectangulata Greg. (Navicula) 146

rectangulata (Greg.) Rab. (Pinnularia) 146

Refractum K. (Callithamnion) 5 ã

Regalis (Grev.) (Campyloneis) 132

Reineckei Born. (Ostreobium) 93

Repens J. Ag. (Dilophus) 74

Reticulata Roper et var. trigona Grun. (Biddulphia) 106

Reticulata D. T. (Odontella) 106

Reliculata Mont. (Ulva) 84

Reticulatus Grev. (Auliscus) 105

Retroflexa Kuetz. (Blossevillea) 69

Retroflexa (Lab.) J. Ag. (Cystophora) 69

Retroflexus Turn. (Fucus) 68

Retroflexus Labill. (Fucus) 69

Retzii (Ag.) Gom. (Phormidium) 95
Retzii (Ag.) Gom. (Phormidium) 94

Revolutum (Ag.) J. Ag. (Dicranema) 19

Rhabdonema Kuetz. 110

Rhabdonia Harv. 21

Rhaphoneis Ehr. 116

Rhipis A. Schm. (Auliscus) 105

Rhizoclonium Kuetz. 88

Rhizodes Kuetz. (Spermatochnus) 78

Rhizodes C. Ag. (Sporochuus) 78

Rhizodes (Ehrh.) J. Ag. (Stilophora) 78

Rhodophyllis Kuetz. 19

Khodymenia Grev. 29

Rhoicosphenia Grun. 151

Rhomboides var. saxonica (Rab.) D. T. (Frustulia) 148

Rhomboides var. crassinervia $V . H$. (Vanheurckia) 149

Rhombus Ehr. (Rhaphoneis) 116

Rhopalodia O. Müll. 120

Rhyncocarpa Kuetz. (Scytothalia) 67

Rhyncocephala Kuetz. var. amphiceros (Kuetz.) Grun. (Navicula) 141

Rigida Kuetz. (Amphipleura) 120

Rigida (Kuetz.) Perag. (Nitzschia) 125

Rigida Kuetz. (Phycoseris) 84

Rissoana Kuetz. (Hypnea) 27

Rivularis Harv. (Bostrychia) 50

Robertsianum Grev. (Triceratium) 108

Robusta Ehr. (Surirella) 128

- var. splendida V. Hk. 128

- var. tenera $V . H k .128$

Robustum Grun. (Rhabdonema) 111

Roperiana Grev. (Biddulphia) 107

Roperiana D. T. (Odontella) 107

Rosea Batt. (Conchocelis) 93

Rubens (L.) Lamour. (Jania) 63

Rubrum (Huds.) Ag. (Ceramium) 57

Rufescens Kuetz. (Conferva) 87

Rugulosa J. Ag. (Scaberia) 68

Salicornioides A. Rich. (Castraltia) 67

Salicornioides Mart. (Castraltia) 68

Samoënsis (Grun.) nob. (Actinocyclus) 105

Samoënsis Grun. (Campylodiscus) 129

Sanguinea var. lancifolin $H$. et $H$. (Delesseria) 34

Sanguinea Harv. (Rhodymenia) 30

Sarcocaulon (Harv.) Schm. (Brongniartella) 44

Sarcocaulon Harv. (Dasya) 44

Sarcocladia Harv. 25

Sarcomenia Sond. 35

Sargassum C. Ag. 63

Saxonica Rabenh. (Frustulia) 149

Scaberia Grev. 67

Scaphulaeforme (Grev.) Grun. (Pleurostauron) 148

Schimperi Decne (Galaxaura) 15

Schizoneura J. Ag. 34

Schizymenia J. Ag. 58

Schousboei Born. (Dermocarpa) 94 
Schousboei Thur. (Xenococcus) 94 Scoliopleura A. Schm. (Navicula) 141 Scoticus (Kuetz.) Grun. (Hyalodiscus) 100 Sculptus (W. Sm.) Ralfs (Auliscus) 105 Scutellum Ehr. (Cocconeis) 134

- var. maxima (Grun.) Cleve 134

- var. minuta Grun. $13 t$

- var. parva Grun. 134

-- var. stauroneiformis Rabh. 134

Scytothalia Grev. 67

Sebdenia Berth. 31

Seirococcus Grev. 6 ;

Sergianum Borzi (Nostoc) 96

Serpentina Ehr. (Eunotia) 118

Serpentina Ehr. (Grammatophora) 110

Serrata Harv. (Kuetzingia) 47

Sessilis Harv. (Phacelocarpus) 24

Setacea (Ell.) Ag. (Griffithsia) 53

Seticulosa J. Ag. (Hypnea) 28

Sigma (Kuetz.) VV. Sm. (Nitzschia) 120

- var, rigida Grun. 125

- var. Sigmatella (Greg.) Grun. 125

Sigmatella Kuetz. 122

Siliculosus f. arctus Kuck. (Ectocarpus) 83

Simplex (Wulf.) Ag. (Digenea) 41

Simpliciuscula Harv. (Bostrychia) 50

Sinuata Greg. (Cymbella) 153

Sinuosa (Roth) D. et S. (Colpomenia) 81

Sinuosa D. et $S$. (Colpomenia) 79

Simuosus Kuetz. (Asperococcus) 81

Simosus Zanard. (Hydroclathrus) 81

Siphonocladus Schmitz 89

Smithii Thwait. (Mastogloia) $15 \mathrm{l}$

- var. conifera Br. 151

Smithii var, suborbicularis Greg. (Navicula) 143

Sol Wall. (Coscinodiscus) 102

Sonderi Harv. (Dictymenia) 43

Sonderi Harv. (Erythroclonium) 21

Sonderi A. Br. (Nitella) 156

Sonderi J.Ag. (Rhabdonia) 21

Sonderi Harv. (Rhabdonia) 21

Sonderiana J. Ag. (Griffithsia) 53

Sorex Kuntze (Cystopleura) 119

Sorex Kuetz (Epithemia) 119

Speciosa Sond. (Gigartina) 22

Speciosum (Sond.) J. Ag. (Eucheuma) 22

Spectabilis Greg. (Amphora) 15.

Spencerella Darbish. 25

Sphaerophora (Kuetz.) Pfitz. (Anomoeoneis) 147

- var. Guntheri O. Müll. 147

- var. rostrata O. Müll. 147

Sphaerophora Kuetz. (Navicula) $14 \overline{6}$

Spicigera Aresch. (Sphacelaria) 82

Spinosum (Ehr.) Bail. (Triceratium) 108

Spinuligerum Sond. (Sargassum) $6 t$

Spirale Harv. (Epineuron) 47

Spiralis Lamour. (Delesseria) 47

Spiralis Lamour. (Vidalia) 47
Spirographidis Schifn. (Antithamnion) 55 Splendens Shadb. (Actinosphoenia) 103 Splendens (Ehr.) Ralfs (Actinoptychus) 103 Splendida Greg. (Cocconeis) 136 Splendida (Greg.) Grun. (Orthoneis) 136 Splendida (Ehr.) Kuetz. (Surirella) 128 Spongiosus C. Ag. (Cladostephus) 81 Spongiosus Kuetz. (Cladostephus) 81 Sporochnus C. Ag. 77

Stagnorum Kuetz. (Conferva) 88 Stagnorum (Kuetz.) Lagerh. (Microspora) 88 Stauroneis Ehr. 147

Stauroptera Grun. (Navicula) 146

Stauroptera (Grun.) Cl. (Pinnularia) 146

Stelliger Bail. (Hyalodiscus) 101

Stelligera Decne (Amphiroa) 61

Stelligera Lamk. (Corallina) 62

Stelligera Cl. et Gr. (Cyclotella) 103

Stelligerum (Lamk.) W. v. B. (Metagoniolithon) 61

Stenocladia J. Ag. 24

Stenophyllum J. Ag. (Sargassum) 66

Stenopterobia Bréb. 129

Stephanodiscus Ehr. 102

Stephanopyxis Ehr. 99

Stilophora J. Ag. 78

Strangulans Grev. (Myrionema) 78

Striatella C. Ag. 111

Stuartii Harv. (Areschougia) 23

Subangulatus Gr. et St. (Cerataulus) 107

Suborbicularis (Greg.) Cl. (Diploneis) 143

Subsessilis Kuetz. (Achnanthes) 131

Subtilis Bail. (Hyalodiscus) 101

Subulata Sond. (Jania) 62

Sulcata Kuetz. (Melosira) 100

Sulcata (Ehr.) Cl. (Paralia) 100

Surirella Turp. 127

Surirella Grun. (Dimeregramma) 116

Surirella (Ehr.) Grun. (Rhaphoneis) 116

Symploca Kuetz. 95

Synedra Ehr. 11

Tabellaria Ehr. 111

Tahitense Witt (Pleurosigma) 149

Tasmanica Havv. (Acanthophora) 42

Tasmanica Kuetz. (Griffithsia) 51

Tenera Harv. (Dasya) 36

Tenera (Harv.) J. Ag. (Sarcomenia) 35

Tenera Greg. (Surirella) 128

Tenuissima Sond. (Jania) 63

Tenuissimum (Hauck) Schiff. (Antithamnion) 55

Tenuissimum Born. et Thur. (Nostoc) 96

Tenuissimus Cleve var. australiensis Grun. (Actinocyclus) 105

Teres Harv. (Thuretia) 50

Tessella Ehr. 111

Thalassiosira Cleve 102

Thermalis (Ehr.) Auersw. (Nitzschia) 125

Thuretia Decne 50 
Thuretii Bréb. (Campylodiscus) 129

Thuretii (Born.) Schm. (Choreonema) 58

Thuretii Born. (Nelobesia) 59

Thuyoides Harv. (Metachroma) $7 \pm$

Thysanocladia Endl. 23

Tincta (Ag.) Grun. (Licmophora) 112

Tingens Harv. (Callithamnion) 56

Tomentosum auct. (Codium) 92

Tomentosum var. australasicum Aresch.(Codium) 9:2

Tomentosus Kuetz. (Cladostephus) 81

Tongatensis Harv. (Polysiphonia) 41

Torulosa (Carm.) Lagerh. (Anabaena) 96

Torulosa Kuetz. (Blossevillea) 70

Torulosa (R. Br.) J. Ag. (Cystophora) 70

Torulosus R. Br. (Fucus) 70

Toxarium Bail. 116

Trachyneis Cleve 148

Trachysphenia Petit 117

Trevelyana Donk. (Navicula) 146

Trevelyana (Donk.) Rab. (Pinnularia) 145,146

Triceratium Ehr. 10-

Trichocarpa Kuetz. (Corallina) 62

Tridens (Mert.) Grev. (Dictymenia) 43

Tridens Sond. (Dictymenia) 43

Tridens Mert. (Fucus) 43

Tristichum (Grev. et Ag.) Sond. (Sargassum) 66

Tropica Kuetz. (Grammatophora) 110

Tropidoneis Cleve 136

Tuberiformis Gray (Leathesia) 79

Turgida V. H. (Biddulphia) 107

Turgida W.Sm. (Biddulphia) 107

Turgida Ktz. var. Westermanni (Ehr.) Grun. (Epithemia) 120

Turgidus Ehr. (Cerataulus) 107

Turgidus (Brèb.) Naeg. (Chroococcus) 93

Turneriana J. Ag. (Zonaria) 73

Turris Grev. (Creswellia) 100

Turris (Grev.) Ralfs (Stephanopyxis) 99

Ulna (Nitzsch) Ehr. (Synedra) 114

- var. lanceolata (Kuetz.) Grun. 115

- var. oxyrhynchus (Kuetz.) Grun. 115

- f. medioconstricta Forti 115

Ulva (L.) J. Ag. 83

Ulva Sond. (Phycoseris) 84

Umbellata ]. Ag. (Corynecladia) 37

Limbellata Harv. (Galaxaura) 14, 15

Umbellata var. major J. Ag. (Galaxaura) 14

Uncialis Kuetz. (Phycoseris) 8 õ

Uncialis Suhr (Ulva) 85

Uncinata Kuetz. (Laurencia) 37

Uncinatum (Ag.) Gom. (Phormidium) 95̃

Undulans Greg. (Synedra) 116

Undulata Sond. (Cryptonemia) 58

Cndulata Bail. (Synedra) 116

Undulatum Bail. (Toxarium) 116
Undulatus (Bail.) Ralfs (Actinoptychus) 103

Unipunctata (Lyngb.) Ag. (Striatella) 111

Urospora Aresch. 88

Usnea (Br.) J. Ag. (Hymenocladia) 29

Utricularis (Bory) Skottsh. (Adenocystis) 75

Utricularis Bory (Asperococcus) 75

Valida J. Ag. (Hypnea) 27

Valonioides Sond. (Cladophora) 89

Valonioides (Sond.) Reinb. (Siphonocladus) 89

Varians D. T. (Lysigonium) 99

Varians Ag. (Melosira) 99

Variolosa Mont. (Nothogenia) 15

Variolosum (Mont.) J. Ag. (Chaetangium) 15

Veneris Kuetz. (Eunotia) 119

Veneris Kuetz. (Himantidium) 119

Ventricosa (Ehr.) O. M. (Rhopalodia) 121

Ventricosum Grun. (Encyonema) 153

Versicolor (H. et H.) Falk. (Herposiphonia) 45

Versicolor H. et H. (Polysiphonia) 45

Verticillata Harv. (Rhabdonia) 21

Verticillatus (Lightf.) Lyngb. (Cladostephus) 81

Verticillatus australis f. Ag. (Cladostephus) 82

Vestita Harv. (Crouania) 56

Vidalia Lamour. 47

Vieillardi Kuetz. (Digenea) 41

Villosa Harv. (Dasya) 51

Virgata var. australis Grun. (Corallina) 62

Virgata (Rop.) Grun. (Hantzschia) 126

- var. subtilior nob. 126

Virgata Roper (Nitzschia) 126

Viridis Kuetz. (Navicula) 146

Viridis (Nitzsch) Ehr. (Pinnularia) 146

Vitrea Norm. var. recta V. Hk, (Nitzschia) 125

Vulgaris Schum. (Actinoptychus) 104

Vulgaris (Thw.) D. T. (Frustulia) 149

Vulgaris Ag. (Porphyra) 13

Vulgaris V.H. (Vanheurckia) 149

Weissflogii (A. Schm.) Cl. (Diploneis) 144

Weissflogii A. Schm. (Navicula) 144

Westermanni Kuetz. (Epithemia) 120

Williamsonii Greg. (Diadesmis) 117

Williamsonii (W. Sm.) Grun. (Glyphodesmis) 117

Williamsonii W. Sim. (Himantidium) 117

Willii Reinsch (Desmarestia) 80

Wulfeni Kuetz. (Digenea) 41

Xenococcus Thur. 94

Xiphocarpa Kuetz. (Scytothalia) 67

Zanzibariensis Goeb. (Caloglossa) 35

Zebra Kuntze (Cystopleura) 120

Zebra (Ehr.) Kuetz. (Epithemia) 120

Zonaria (Drap.) J. Ag. 72.

Zostereti Grun. (Navicula) 141

Zostericola Harv. (Cladosiphon) 79 



$$
\text { - } \therefore \text { 10) } 10:
$$

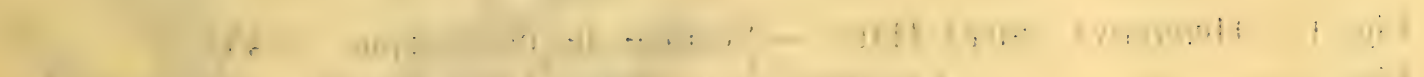

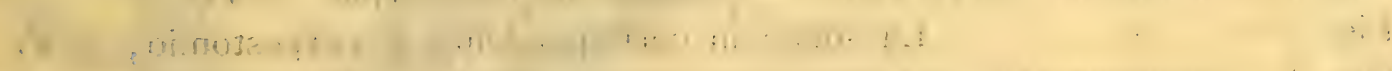

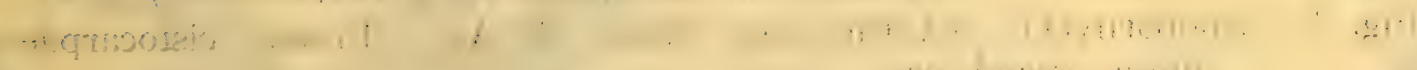

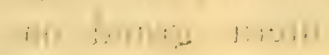


TAVOLA I.

Fig. 1 - Hennedya crispa Harv. - Sezione del cistocarpio $\times 45$.

Fig. 2 - - L L L stessa in corrispondenza al carpostomio; $\times 45$.

Fig. 3 - Rhodophyllis acanthocarpa (Harv.) J. Ag. - Fronda cistocarpifera intera; grand. nat.

Fig. 4 - - - Fronda cistocarpifera, frammento; $\times 10$.

Fig. 5 - - Cistocarpio $\times 68$. 




\section{TAVOLA II.}

Fig. 1 - Stenocladia Cliftoni J. Ag. - Fronda sterile e fronda cistocarpifera grand. nat.

Fig. 2 - Stenocladia Harveyana J. Ag. - Fronda cistocarpifera; grand. nat. Fig. 3- - Cistocarpii ; $\times 45$.

Fig. 4 e 5 - Sezione della fronda attraverso al cistocarpio in differenti posizioni; $\times 45$. 

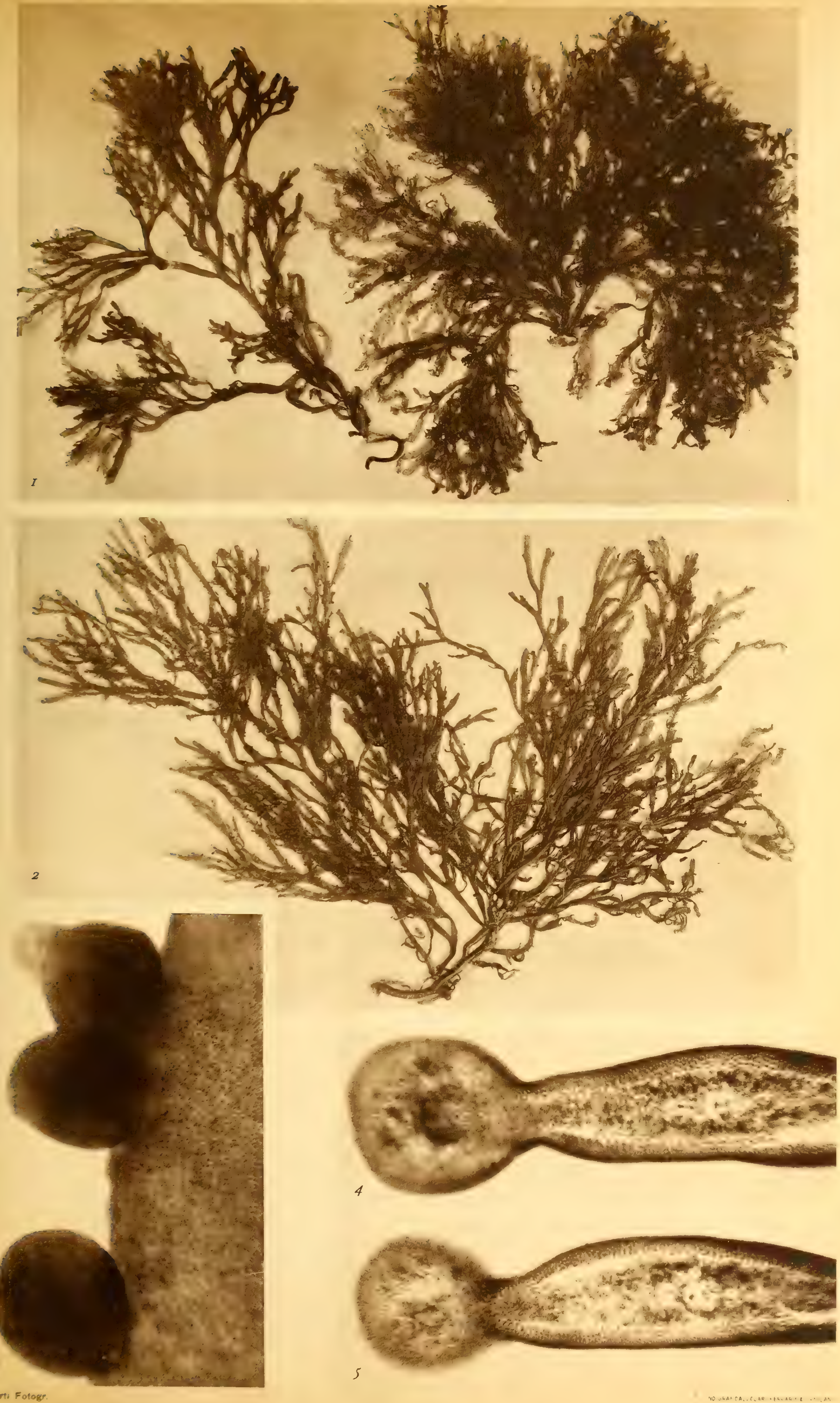



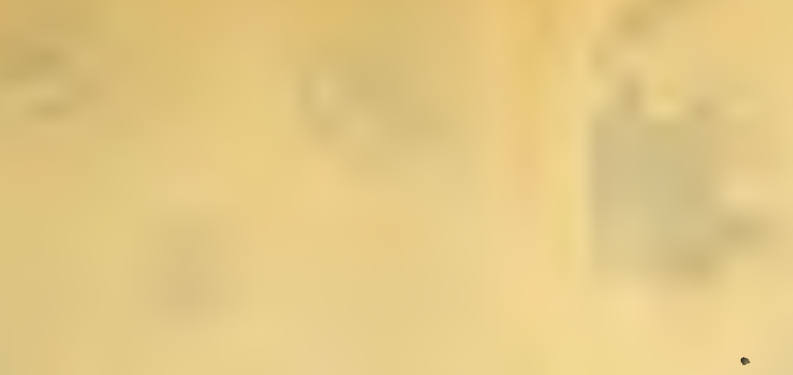

$111: 11+1$

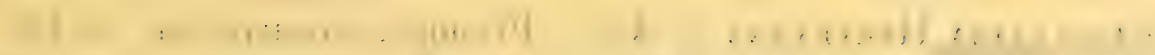

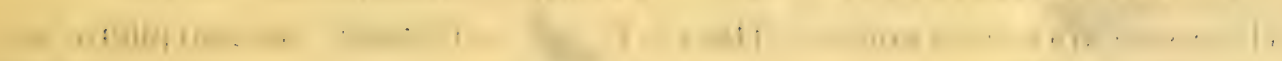

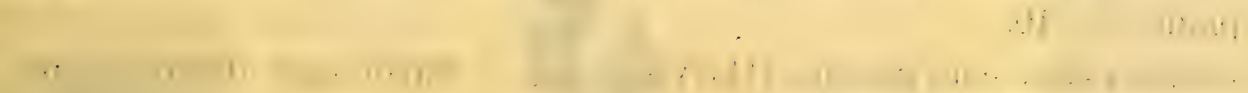

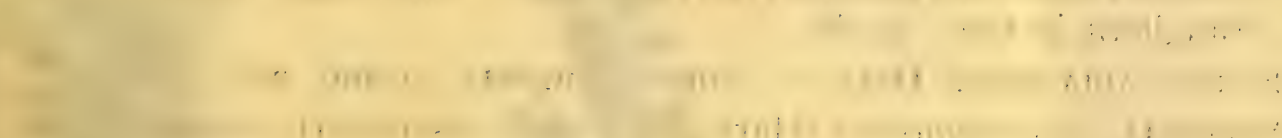

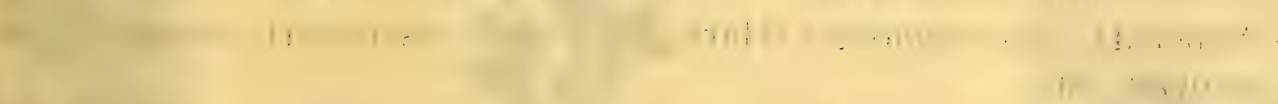

$\cdot$

.

+. $+115, \ldots$
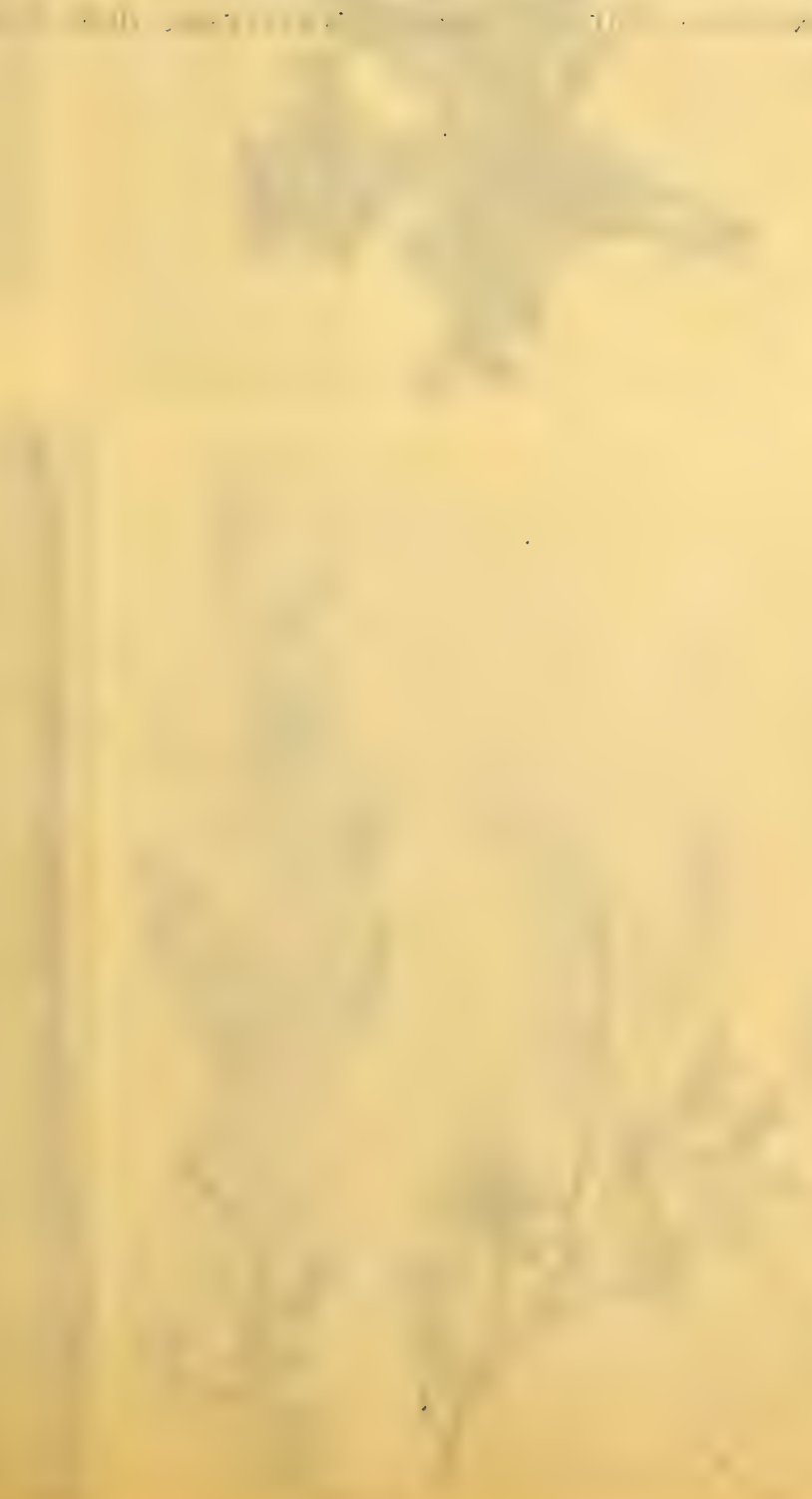

$+4$

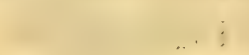

i. $\quad$ in:
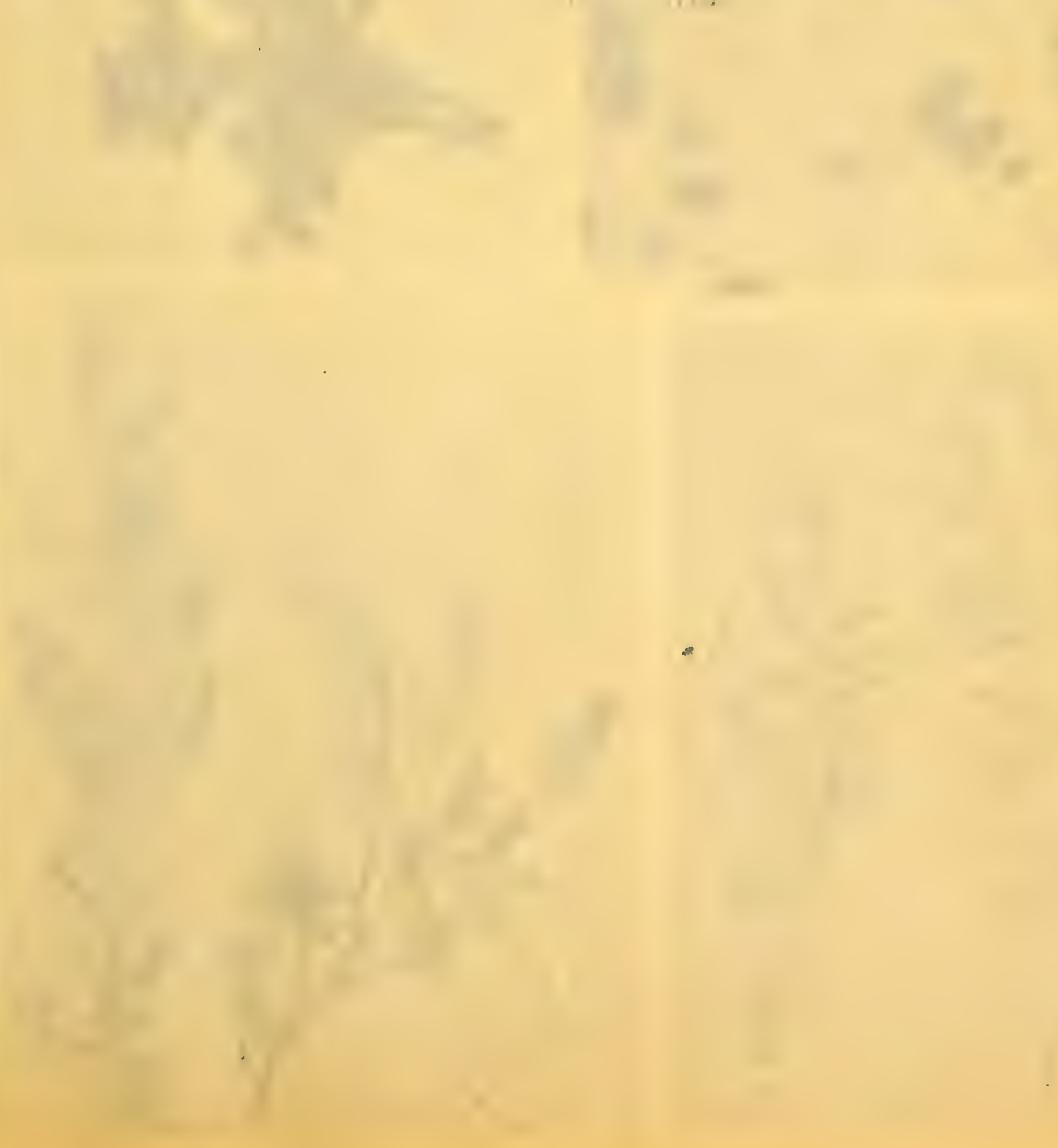
TAVOLA III.

Fig. 1 - Stenocladia Harveyana J. Ag. - Fronda cistocarpifera; $\times 10$.

Fig. 2 - Hymenocladia polymorpha (Harv.) J. Ag. - Fronda cistocarpifera in piano; $\times 10$.

Fig. 3 - Hymenocladia polymorpha (Harv.) J. Ag. - Particolare di fronda spo. rangifera in toto; $\times 45$.

Fig. 4 - Sarcocladia obesa Harv. - Fronda completa; grand. nat.

Fig. 5 - Sebdenia callymenioides (Harv.) De Toni - Esemplari giovanili; grandezza nat.

Fig. 6 - Plocamium nidificum (Harv.) J. Ag. - Pinnule sporangifere; grand. nat.

Fig. 7 - $\quad$ - Fronda sterile; grand. nat.

Fig. 8-Rhodymenia sanguinea Harv. - Sezione trasversale della fronda vegetativa; $\times 45$. 


TAVOLA IV.

Fig. 1 - Rhodymenia sanguinea Harv. - Fronda cistocarpifera ; 1/2 grand. nat. Fig. 2 - - C Cistocarpi in piano interi; $\times 10$.

Fig. 3 - - $\quad$ - Sezione trasversale del cistocarpio; $\times 45$.

Fig. 4 - Caloglossa Beccaril Zanard. - Fronda intera con sori; $\times 10$.

Fig. 5 _ _ _ Lamine sorifere; $\times 10$;

Fig. 6 - _ _ _ soro in formazione; $X 68$. 

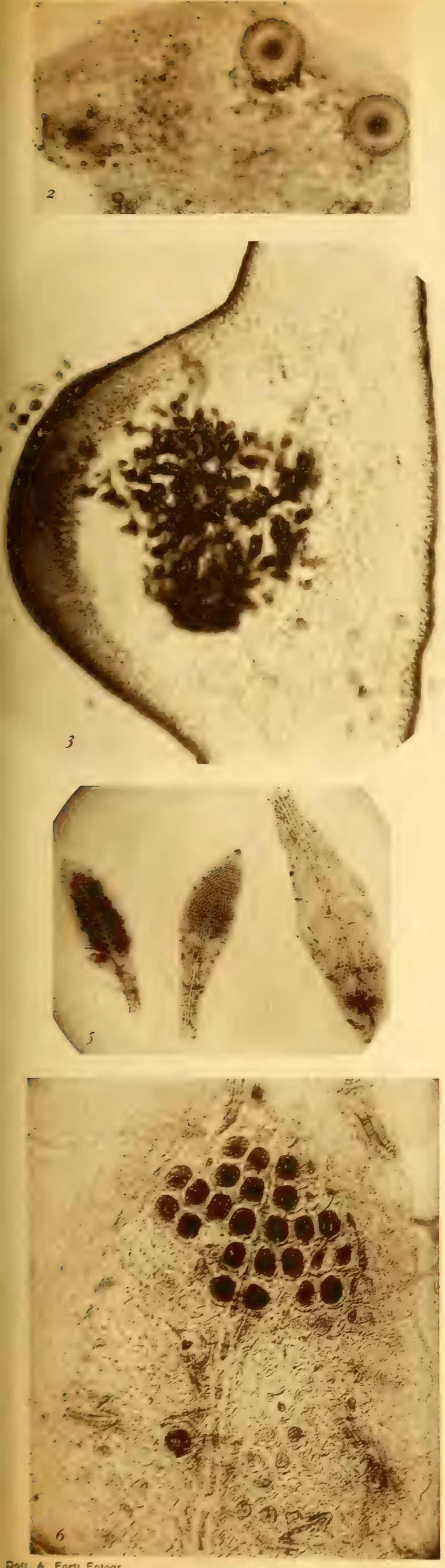
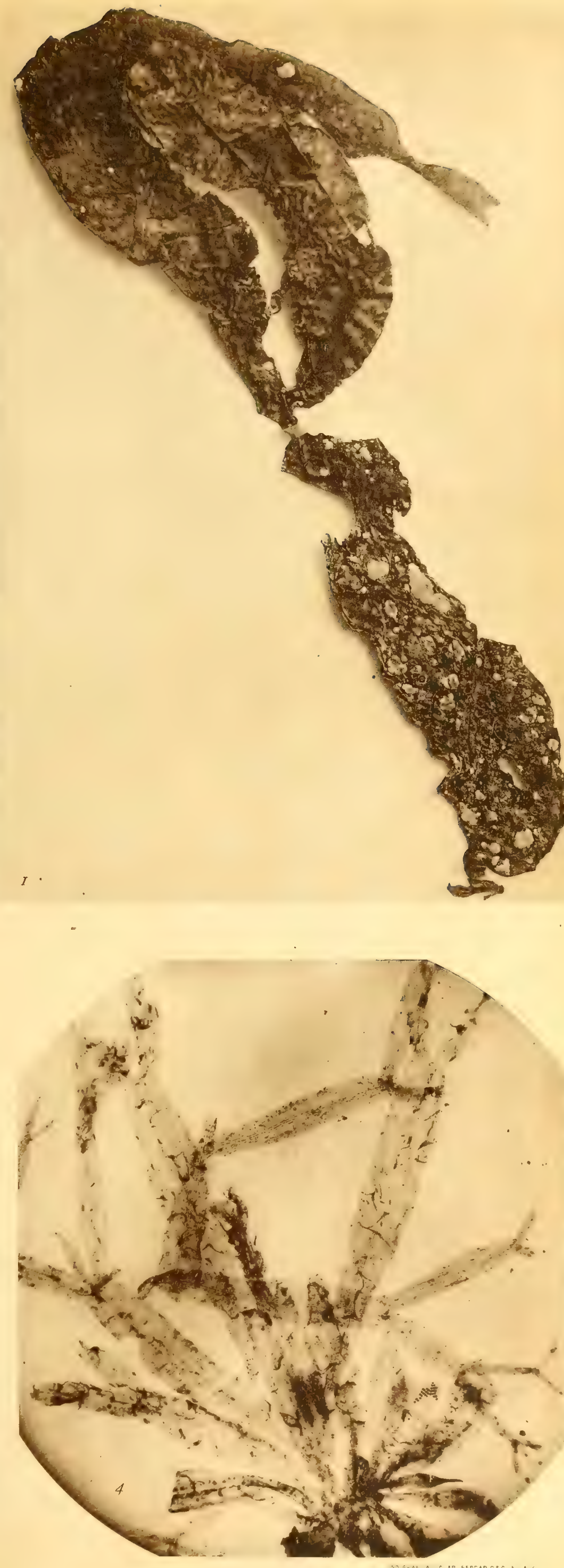


TAVOLA V.

Fig. 1 - Caloglossa Beccari Zanard. - Soro maturo; $\times 68$.

Fig. 2 - Sarcomenia tenera (Harv.) J. Ag. - Fronda completa; grand. nat.

Fig. 3 - - Frammenti di rami vegetativo e sporangifero $; \times 10$.

Fig. 4 - $\quad$ - $\quad$ Sezione trasversale di ramo sporangifero; $\times 68$.

Fig. 5 - Laurencia Gracilis Hook. et Harv, - Estremità di rametti sterili ; X 10. 

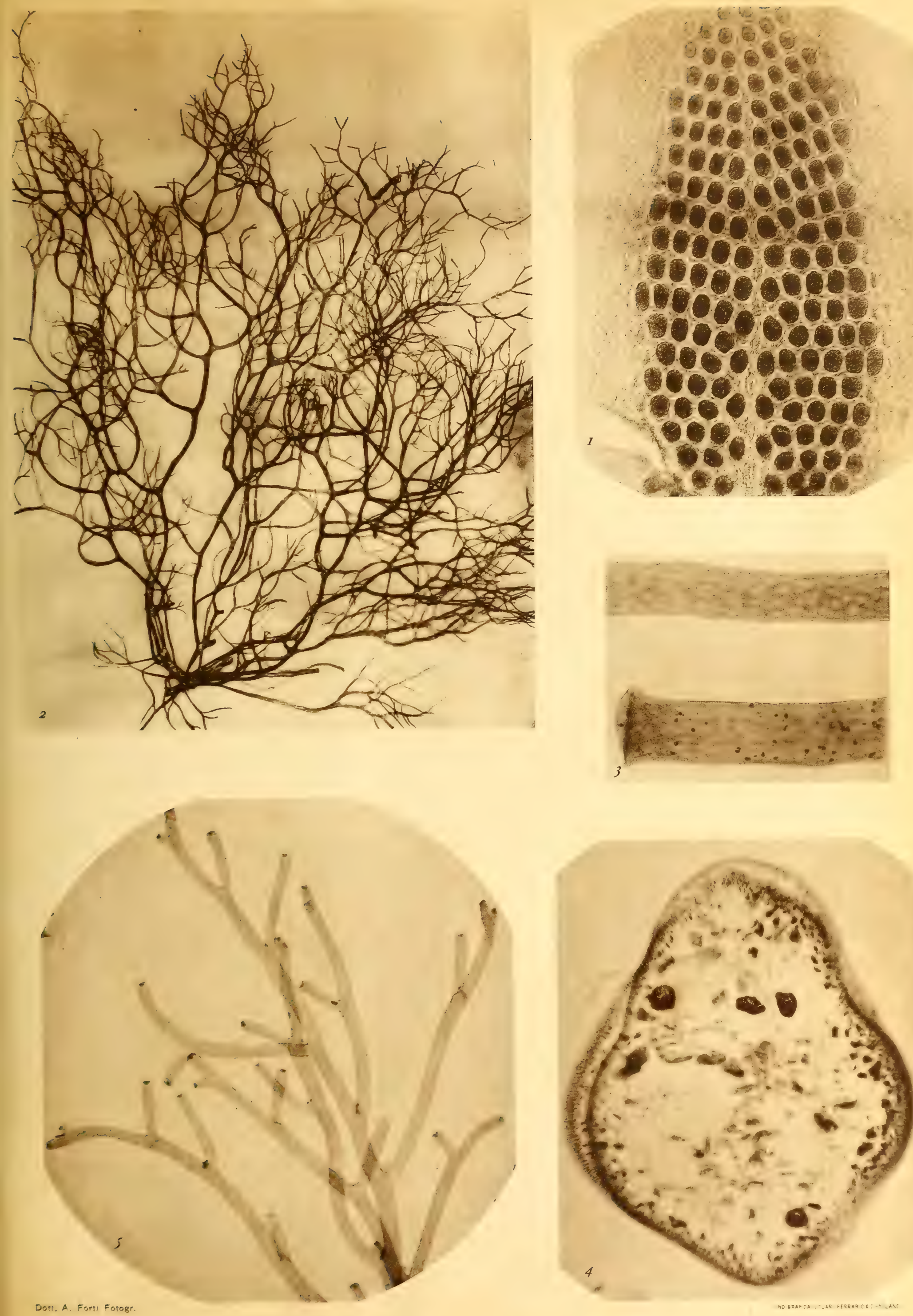



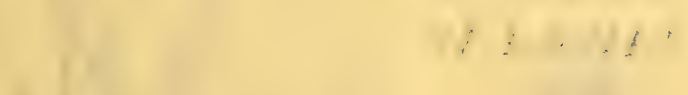

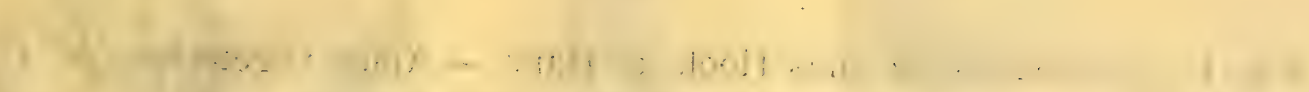

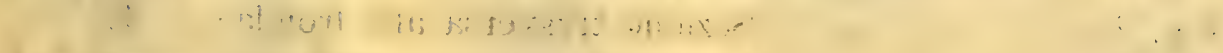

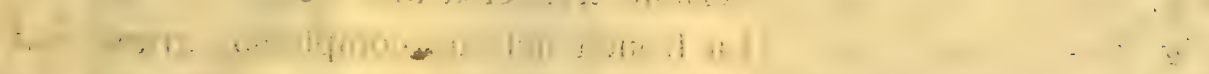

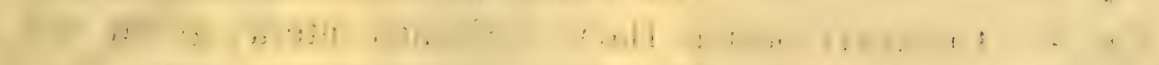

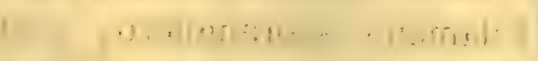

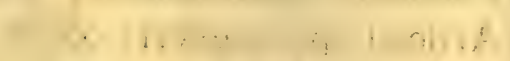

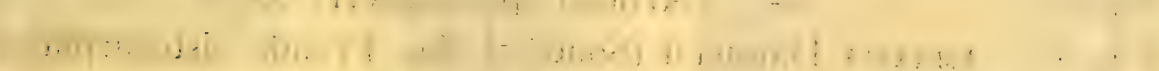$$
\text { i. } 15, \quad \therefore
$$
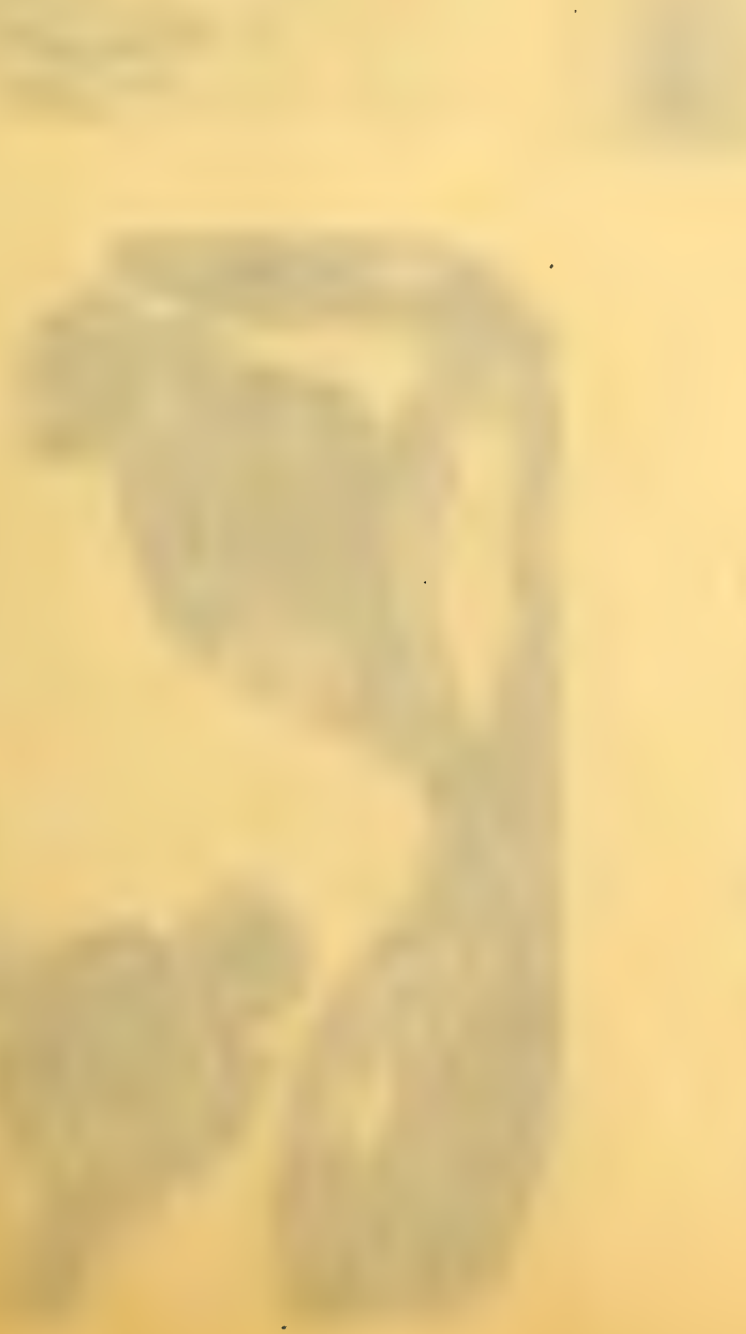


\section{TAVOLA VI.}

Fig. 1 - Laurencia GRacilis Hook. et Harv. - Apice vegetativo; $X 45$.

Fig. 2 - - - Sezione trasversa alla fronda; $\times 45$.

Fig. 3 - - - La fronda nel sua complesso; grand. nat.

Fig. 4 - ChONDRIA DEBILIs Harv. - Fronda intera; grand. nat.

Fig. 5 - $\quad-\quad$ Filamento sporangifero $\times 10$.

Fig. $6-\quad-\quad-$ Articoli sporangiferi; $\times 68$.

Fig. 7 - Amansia Dafmeli (Sond.) J. Ag. Fronda cistocarpifera; $\times 10$.

Fig. 8- - $\quad$ Cistocarpii ; $\times 54$. 

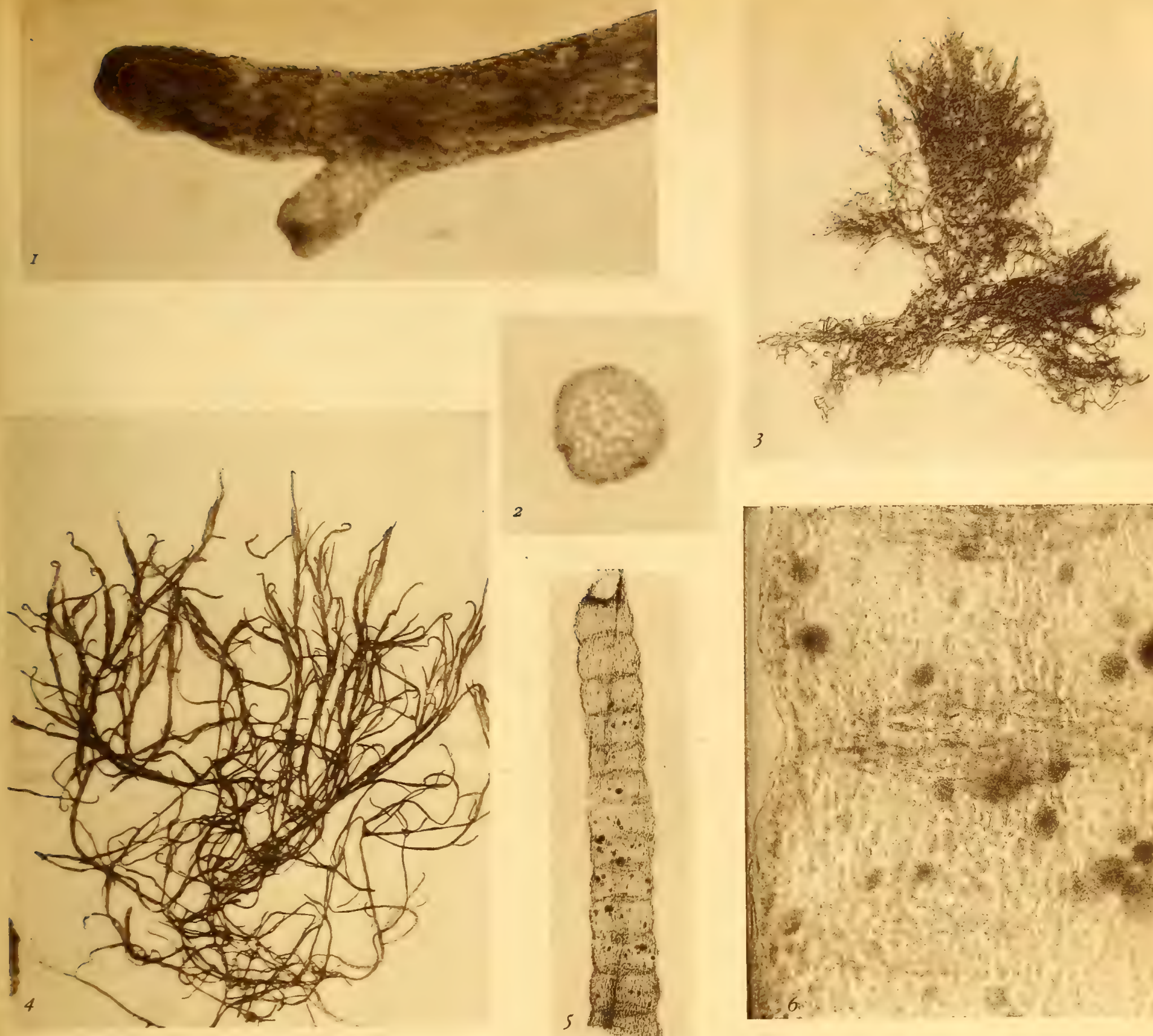

2
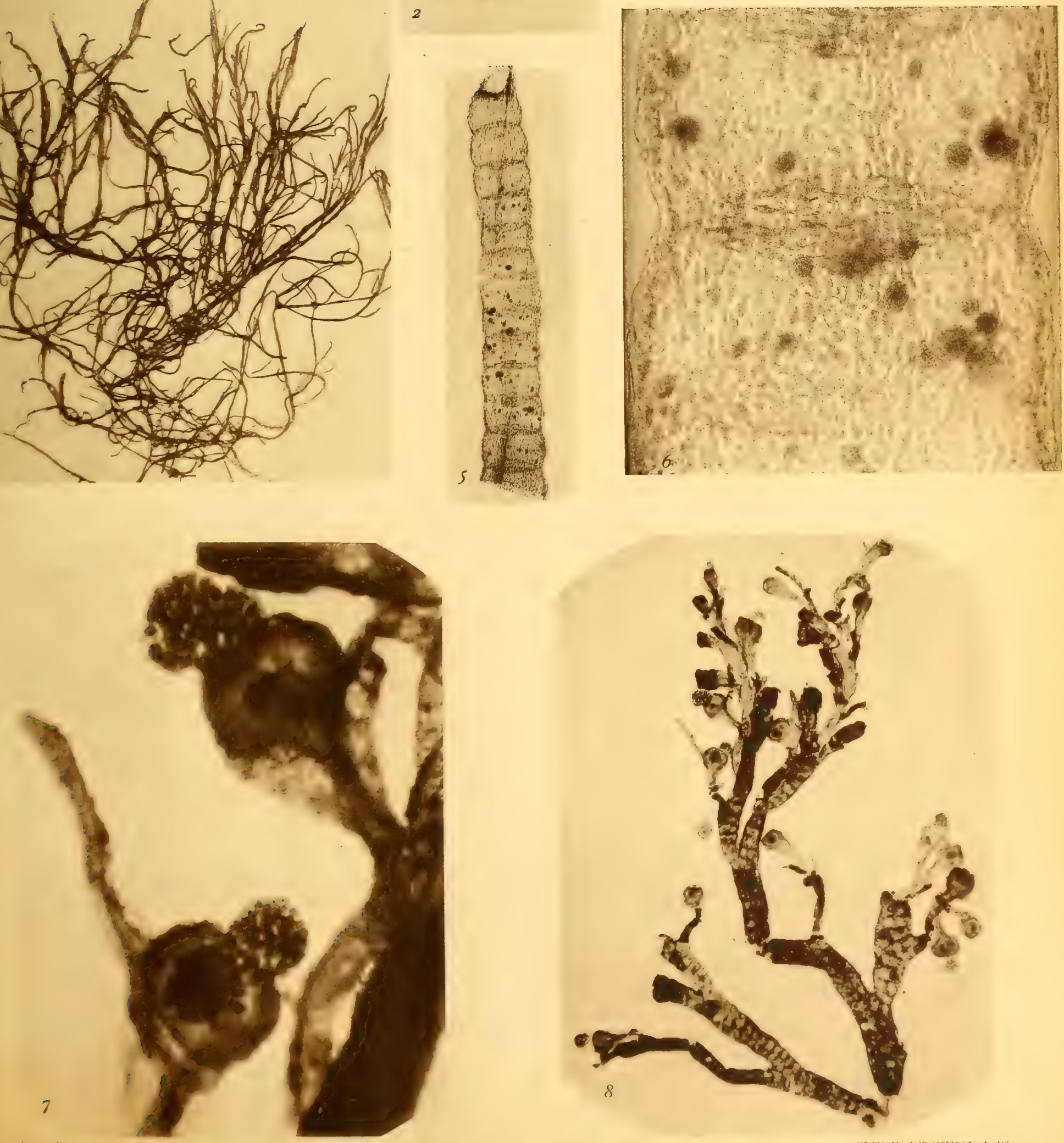



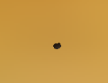


TAVOLA VII.

Fig. 1 - I enormandia Chaurini Harv. - Il complesso della fronda stichidifera; grand. natur.

Fig. 2 - Euptilota coralloidea (J. Ag.) Kuetz. - La fronda intera; gr. natur. Fig. 3 - - Pinnula cistocarpifera $; \times 15$.

Fig. 4 - Lenormanda angustrfolia (Harv.) J. Ag. - Frammento di fronda stichidifera; grand. natur.

Fig. 5 - Lasiothalia hirsuta Harv. - Fronda intera; grand. natur.

Fig. 6 - $\quad$ - Sezione di un rametto sporangifero; $\times 68$. 

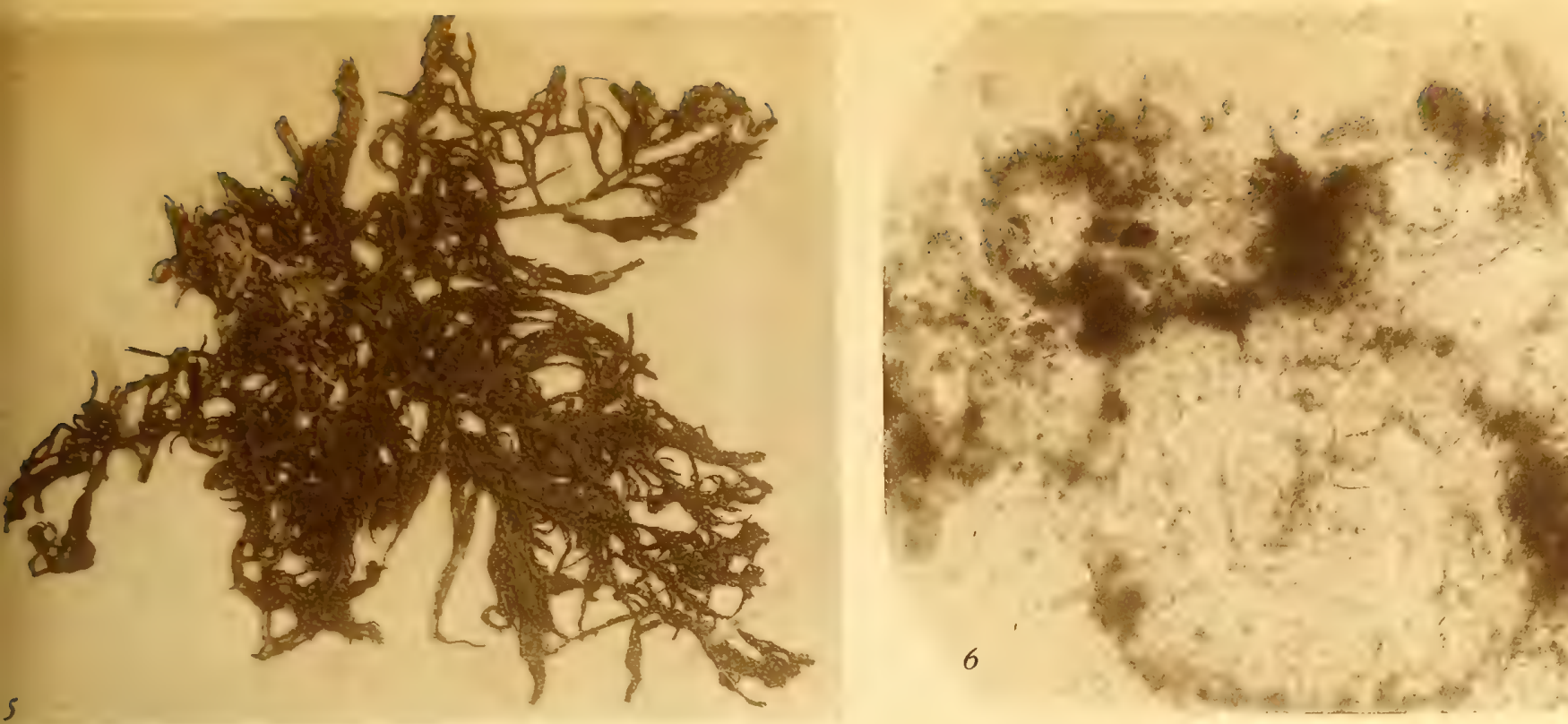

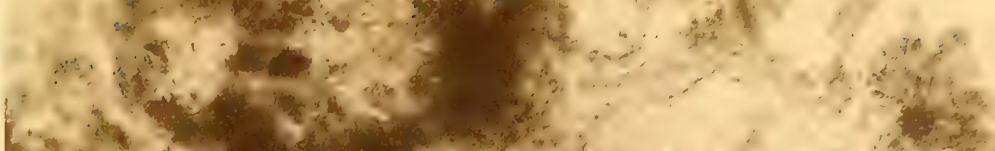

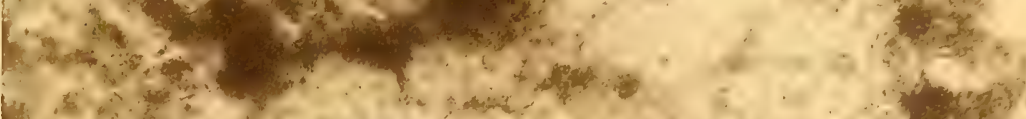

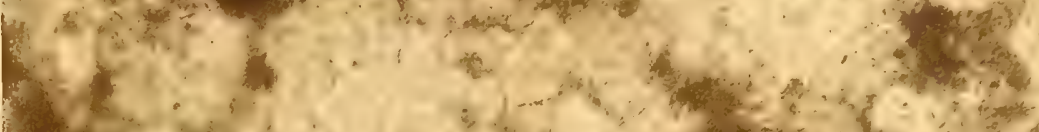

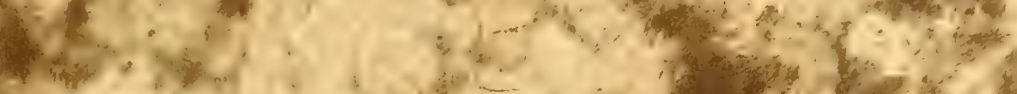

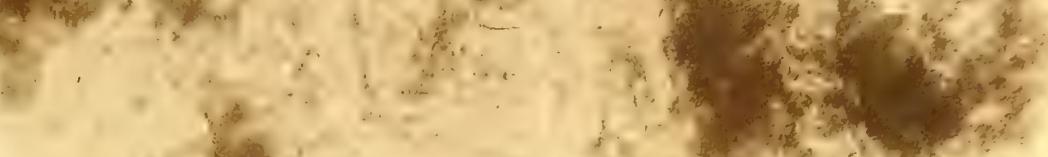

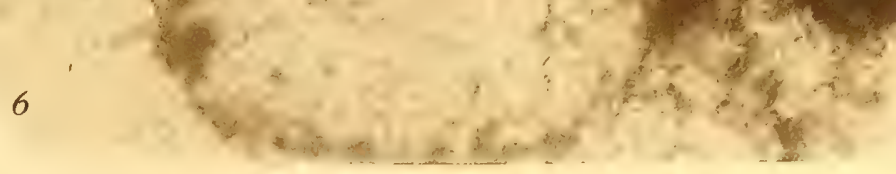
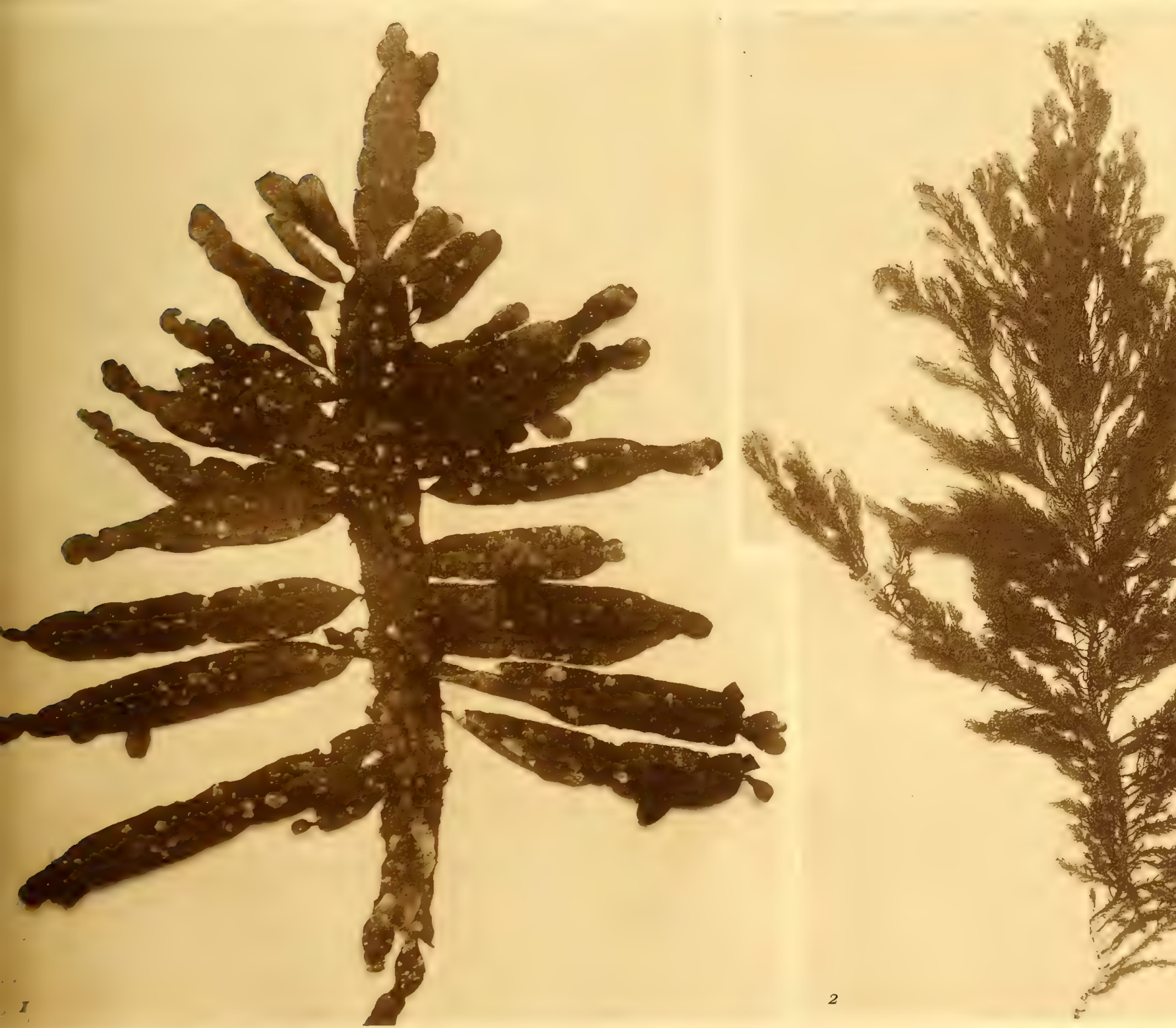

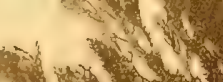

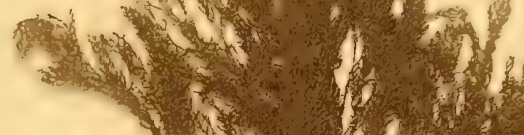

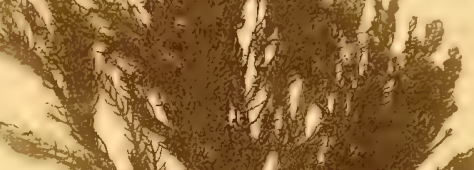
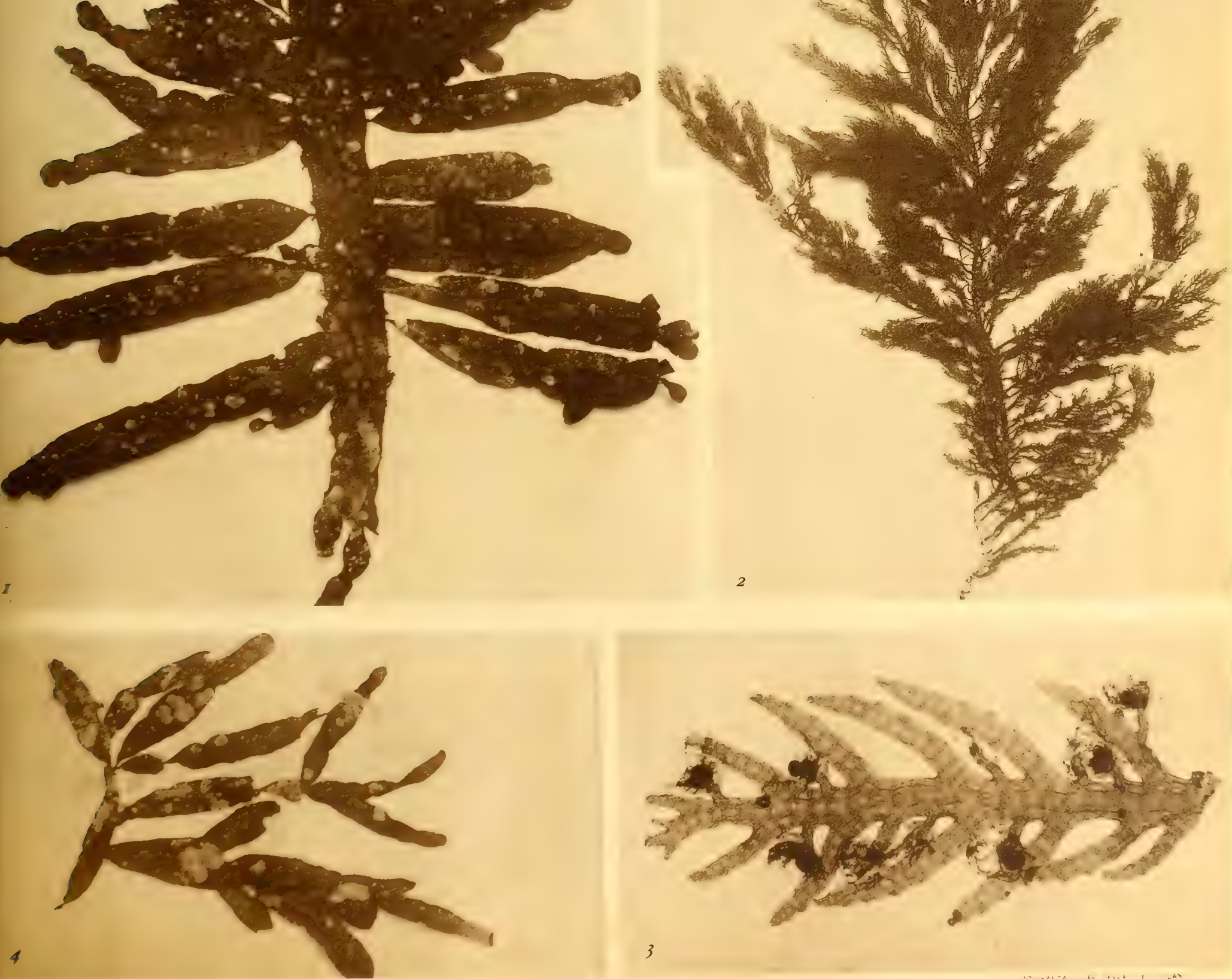



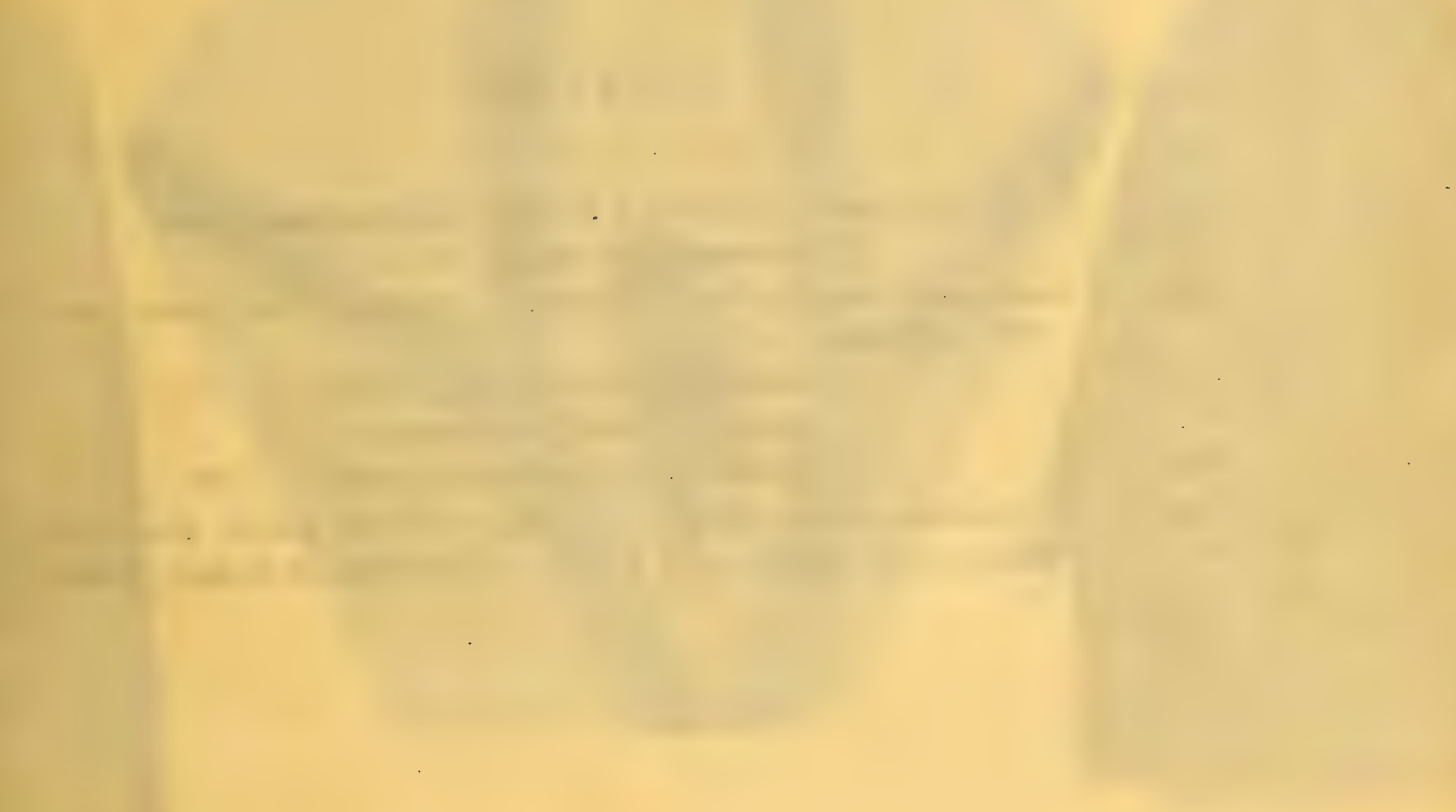


TAVOLA VIII.

Fig. 1 - Lasiothalia hirsuta Harv. - Rametto sporangifero; $\times 68$.

Fig. 2 - - - Frammento di fronda; $\times 45$.

Fig. 3 - Carpopeltis elata (Harv.) Schmitz - Sezione di un apice con giovani cistocarpi; $\times 68$.

Fig. 4 - $\quad$ - $\quad$ Estremità di fronda cistocarpifera $; \times 10$.

Fig. 5 - $\quad-\quad$ Sezione di fronda vegetativa $\times 68$.

Fig. 6 - _ - Sezione di un apice cistocarpifero; $X 68$.

Fig. 7 - Schizymenia Novae-Zelandiae J. Ag. - Fronda intera; grand. natur.

Fig. 8 - Pachydictyon paniculatum J. Ag. - Frammenti di fronda; grand. nat. 



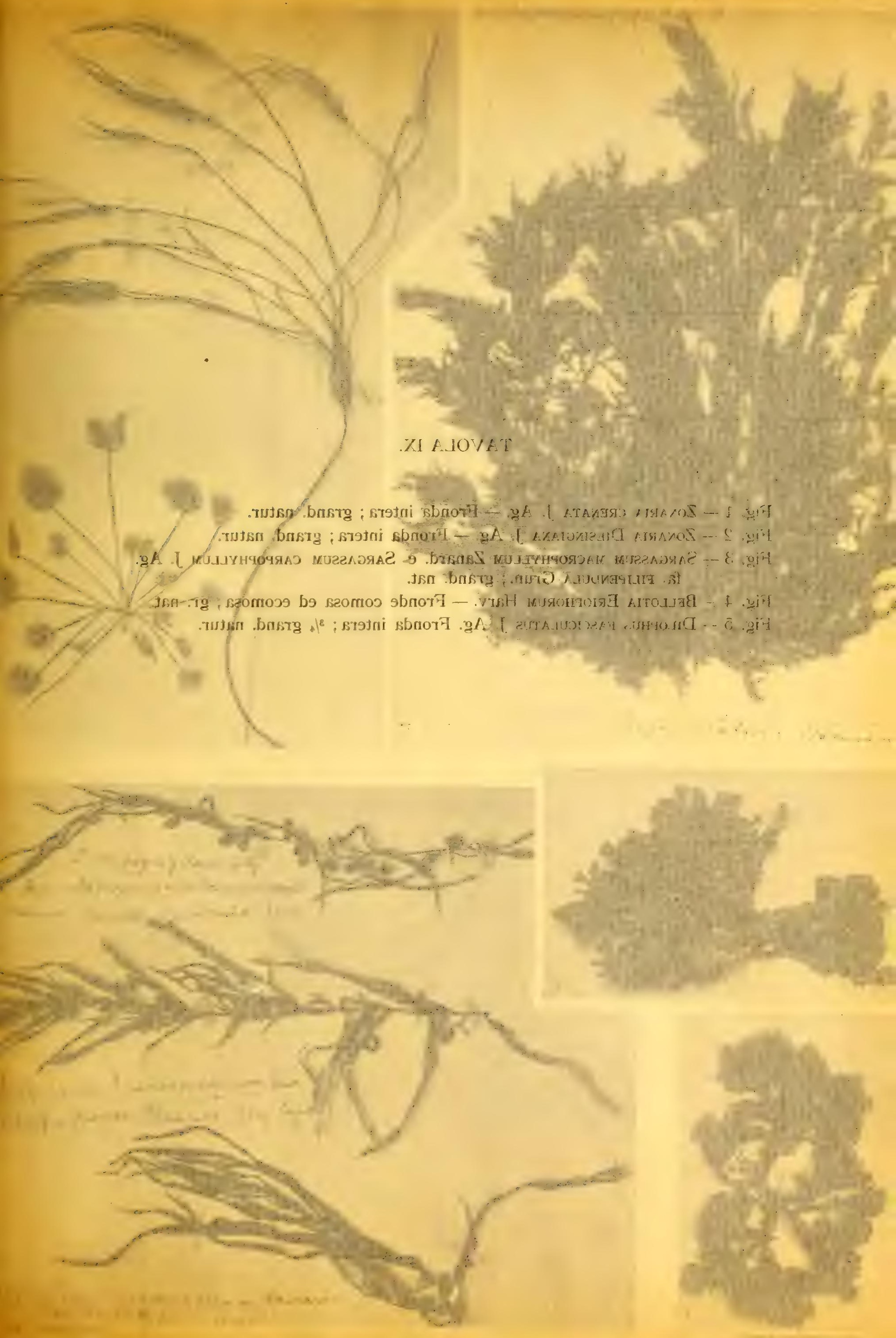


TAVOLA IX.

Fig. 1 - Zonaria CREnAta J. Ag. - Fronda intera; grand. natur.

Fig. 2 - Zonaria Diesingiana J. Ag. - Fronda intera; grand. natur.

Fig. 3 - Sargassum macrophyllum Zanard. e Sargassum carpophyllum J. Ag. fa. Filipendula Grun.; grand. nat.

Fig. 4 - Bellotia Eriophorum Harv. - Fronde comosa ed ecomosa; gr. nat.

Fig. 5 - Dilophus fasciculatus J. Ag. Fronda intera; $3 / 4$ grand. natur. 


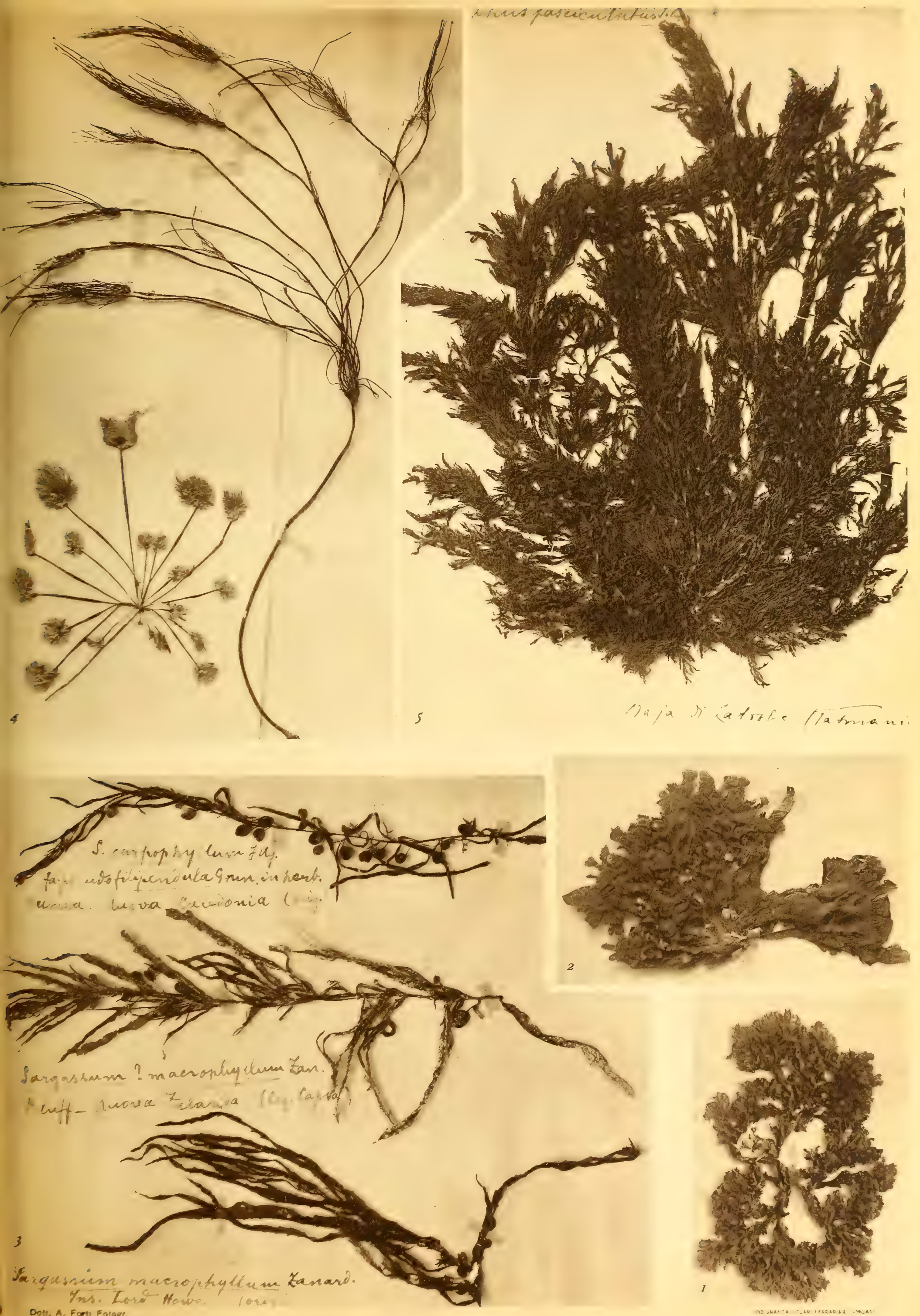




TAVOLA X.

Fig. 1 - Dilophus fasciculatus J. Ag. - Frammento di fronda $\times 10$.

Fig. 2 - Enteromorpha Gunniana J. Ag. - Tallo ingrandito; $X 10$.

Fig. 3 - - - Agglomerazioni di fronde disseccate; grand. natur.

Fig. 4 - Codium galeatum J. Ag. - Fronda intera; $1 / 2$ grand. natur.

Fig. 5 - $\quad-\quad$ Apice di una fronda ;

Fig. 6- - - Otricoli separati; $\times 45$. 

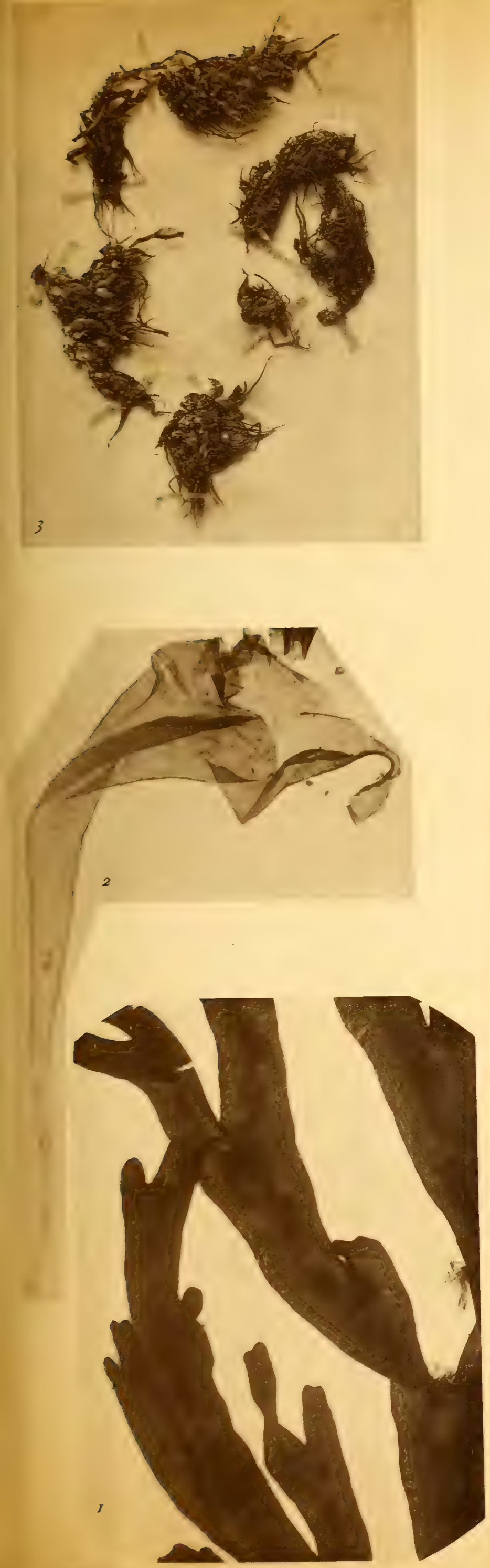
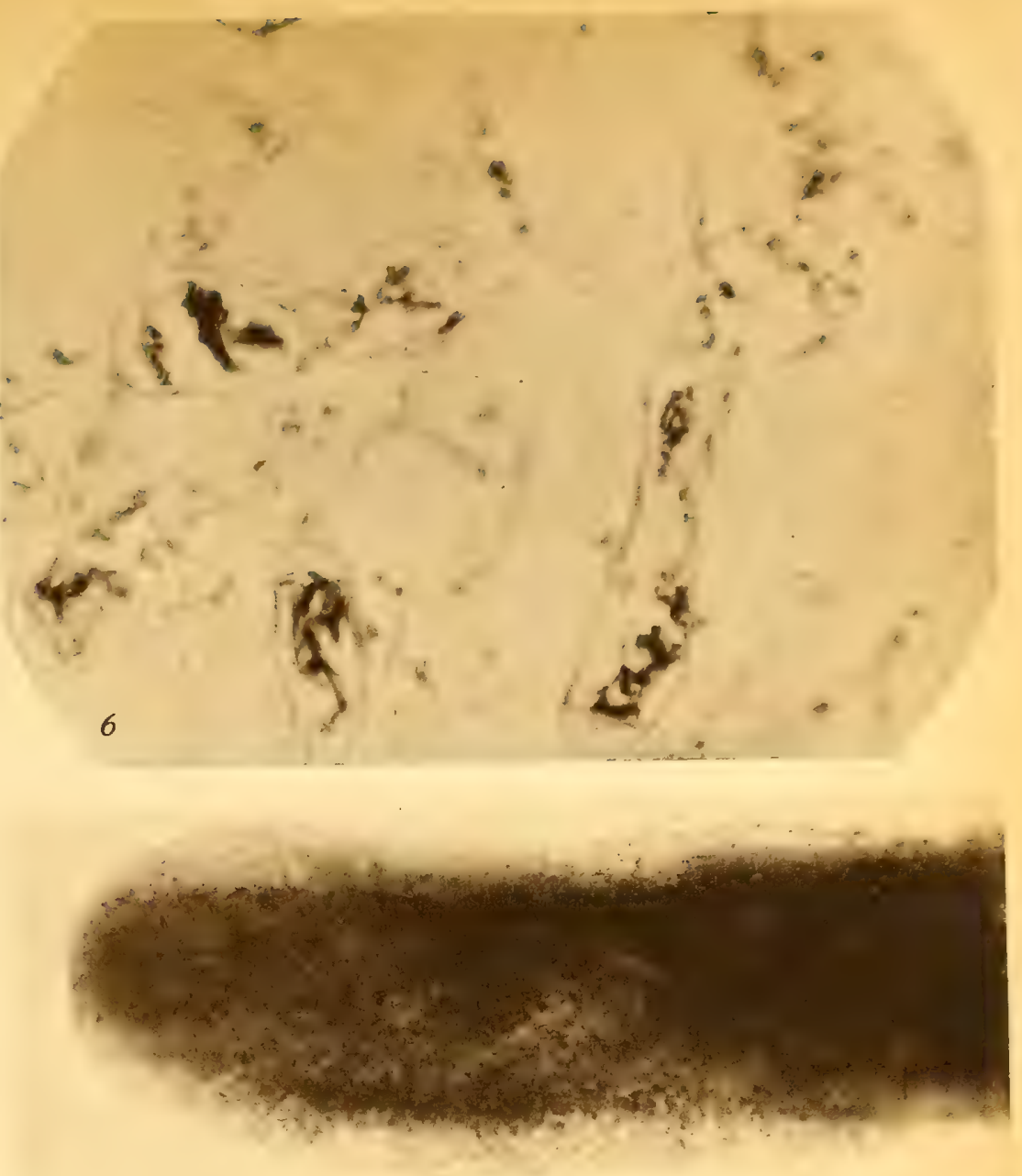

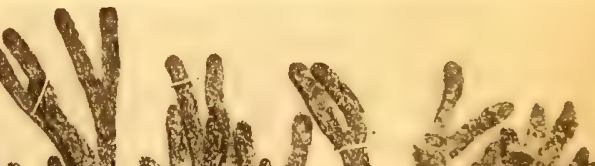
A. 1. 1.2

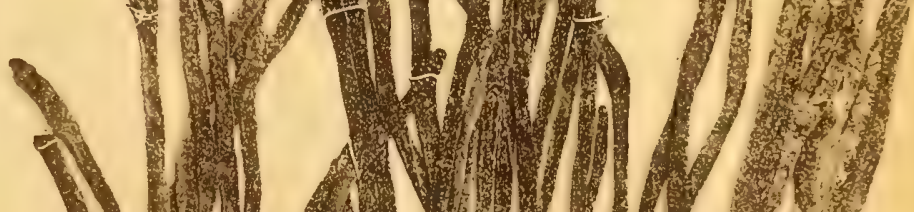
$+1$
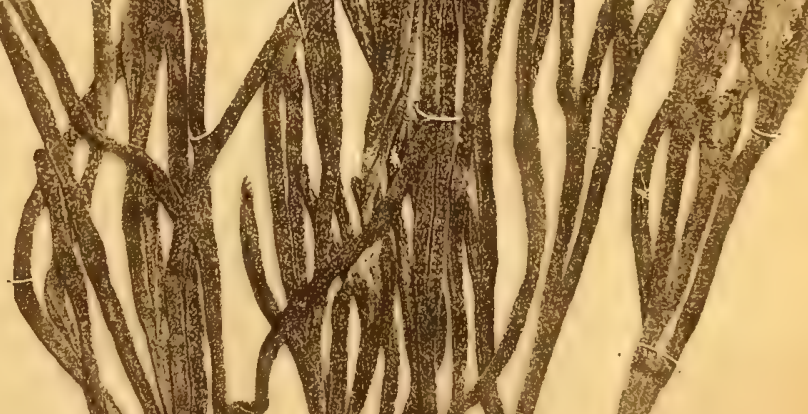

(n)

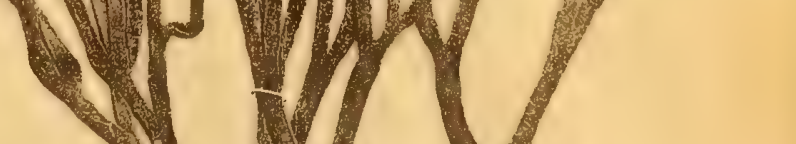


2 


\title{
Contribution à la flore algologique
}

\section{de la Tripolitaine et de la Cyrénaïque.}

\author{
PAR G.-B. DE TONI et ACH. FORTI
}

La flore marine de la Tripolitaine et de la Cyrénaïque a fait l'objet de quelques études qui ont apporté une contribution à la connaissance des Algues, à l'exception des Myxophycées et des Bacillariées.

Le premier b staniste qui dressa une liste d'une vingtaine d'Algues draguées à quelques kilomètres au nord de Tripoli durant la croisière du commandant D'Albertis au bord du Violante fut $\mathrm{M}$. Piccone ( $\mathrm{I}$ ) qui contribua par des travaux nombreux "et soignés à la phycologie méditerranéenne; d'autres additions à la même flore ont été fournies par MM. De Toni et Levi (2) qui ont déterminé les phycées rapportées de Tripoli par feu le professeur R. Spigai; F. Ardissone (3) publia plus tard l'indication de quelques espèces envoyées de Tripoli par M. Corbelli, tandis qu'un bon nombre de phycées recueillies sur le littoral de Benghazi à Derna, au printemps de I88 I, par M. G. Haimann furent illustrées par M. Piccone (4). D’un autre côté, à la connaissance de la flore algologique d'eau douce ont contribué de petites récoltes faites çà et là par Rohlfs, Taubert, Krause, Stecker, Ruhmer, Duveyrier et d'autres voyageurs, comme il résulte de la bibliographie rédigée par M. Ascherson dans l'ouvrage récent de Durand et Barratte (5).

(I) Piccone A., Risultati algologici delle crociere del Violante comandato dal capitano-armatore Enrico D'Albertis (Annali del Museo Civico di Storia Naturale di Genova, vol. XX, p. I06-142; Genova, I883).

(2) De Toni G.-B. et Levi D., Pugillo di Alghe Tripolitane (Rendiconti della Reale Accademia dei Lincei, vol. IV, fasc. 5, p. 240-250; Roma, 1888).

De Toni G.-B., Secondo pugillo di Alghe Tripolitane (Rendiconti della Reale Accademia dei Lincei, ser. 5, vol. I, P. I40-147; Roma, I892).

De Tonr G.-B., Terzo pugillo di Alghe Tripolitane (Rendiconti della Reale Accademia dei Lincei, ser, 5, vol. IV. p. 45I-457; Roma, I895).

(3) Ardissone F., Note alla Phycologia mediterranea (Rendiconti del R. Istituto Lombardo, ser. II, vol. XXVI, fasc. XVII ; Milano, 1893). $1892)$.

(4) Piccone A., Alghe della Cirenaïca (Annuavio del R. Istituto Botanico di Roma, vol. V, fasc. 2, p. 45-52; Roma,

(5) Durand E. et Barratte G., Floræ Libycæ Prodromus. (Catalogue raisonné des plantes de Tripolitaine; Genève, 1910; Imprimerie Romet, Froreisen successeur; in-4\%).

T. V. - FAsC. 7 . 
M. R. Muschler (I) profita d'une grande partie de ces documents pour dresser le Catalogue des Algues connues jusqu'à présent pour la région libyque; mais ce catalogue n'est pas complet, quelques publications lui étant échappées que M. Béguinot (2) n'a pas omis de signaler dans sa bibliographie relative aux ouvrages contenant des notices botaniques ou agraires sur la Tripolitaine et la Cyrénaïque.

Les matériaux, dont traite la présente contribution, nous ont été communiqués par M. le $D^{r}$ Antoine Vaccari, médecin de la marine italienne, qui, parmi les végétaux récoltés dans ses voyages (3), nous rapporta quelques Algues de Tobrouk et de Benghazi, et par M. le professeur Alex. Trotter qui, chargé par le Ministère d’Agriculture du Royaume d'Italie, visita la région tripolitaine et y recueillit des Algues à Homs et Tripoli et des Chara à Tadjoura (4).

A l'époque de ces explorations, les conditions de la guerre n'ont pas permis une récolte plus abondante, et nous nous bornons ici à donner les résultats de l'examen des matériaux que nous communiquèrent MM. Vaccari et Trotter, parce que ces matériaux sont intéressants en particulier pour ce qui concerne un groupe d'Algues pour lesquelles on n'avait guère de renseignements pour le littoral de Tripolitaine et Cyrénaïque, savoir les Diatomées obtenues par les lavages des Algues marines, des Éponges, des Zostéracées et des Characées.

La liste des Diatomées apporte une contribution tout à fait nouvelle, puisque M. R. Muschler, dans son travail synthétique sur la flore algologique tripolitaine, ne cite qu'une seule espèce, sans nom, appartenant au genre Campylodiscus.

Mais aussi quelques-unes des Algues supérieures, dont nous donnons l’énumération, sont nouvelles pour le littoral tripolitain, et même pour le littoral nord-africain qui est bien loin d'être suffisamment exploré.

Les connaissances sur le littoral septentrional de l'Afrique, à part la régiọ baignée par l'océan Atlantique sur laquelle existe une illustration très importante des matériaux récoltées par P. K. A. Schousboe (5), sont très limitées; seule, l'Algérie forma l'objet des études soigneuses de C. Montagne (6) et de F. Debray (7) qui rédigea un Catalogue des

(1) Muschler R. in Durand E. et Barratte G., op. cit., p. 293-313.

(2) Béguinot A., La flora, il paesaggio botanico e le piante utili della Tripolitania e Cirenaica (Padova, I9ı, Fratelli Drucker, in-8\%).

(3) Une première illustration des matériaux récoltés par M. A. VAccari est contenue dans le mémoire : Béguinot A. et VACCARI A., Contributo alla flora della Libia in base a piante raccolte dall'ottobre I9I I al luglio I9I2 (Ministero degli affari esteri. Direzione centrale degli affari coloniali. Ufficio di Studi coloniali. Monografie e rapporti coloniali, no 16 ; Roma, I912; G. Bertero e C., in-80 p. 72 , tab. I-X).

(4) M. A. Letourneux, en avril 1886, récolta à Ainzara et à Tadjoura un Chara (Ch. gymnophylla. A. Br. ?) cf. Letourneux A., Note sur un voyage botanique à Tripoli de Barbarie (Bulletin de la Société botanique de France, t. XXXVI, I889, p. 97).

(5) Bornet E., Les Algues de P. K. A., Schousboe, récoltées au Maroc et dans la Méditerranée de I8I5 à I829 (Mémoires de la Société Nationale des sciences mathématiques et naturelles de Cherbourg, t. XXVIII, I892, p. I65-373, $3 \mathrm{pl}$.).

(6) Montagne C., Cryptogames algériennes (Annales des sciences natuvelles, $2^{\mathrm{e}}$ sér., t. X; Paris, I838) ; Exploration scientifique de l'Algérie. Algues (Paris, I846).

(7) Debray F., Liste des algues marines et d'eau douce récoltées jusqu'à ce jour en Algérie (Bulletin scientifique du Nord de la France et de la Belgique, t. XXV; Paris, I 893); Catalogue des Algues du Maroc, d'Algérie et de Tunisie, (Alger, I897); dans ce dernier travail sont incluses aussi les Algues algériennes récoltées par M. le commandant E. D’Albertis pendant la croisière du Corsaro aux Açores et publiées par M. Prccone, Alghe della Crociera del Corsaro alle Azzorre (Nuovo giomale Bolanico Italiano, vol. XXI, no 2, p. I71-2I4; Firenze, I889). 
Algues du Maroc, d'Algérie et de Tunisie; à l'exception des Diatomacées qui furent énumérées par P. Petit (I) soit d'après la détermination de nouveaux matériaux diatomifères, soit sur la base d'études d'autres auteurs tels que MM. Belloc et Lanzi; aussi M. Sauvageau (2) s'occupa d'Algues d'eau douce et de quelque espèce marine de la Tunisie; au reste, le côté même égyptien, à part les anciennes contributions de Forskål, Delile et d'autres botanistes et celles plus récentes d'Areschoug (3) et surtout de R. Muschler (4), est bien loin, comme le littoral de la Tunisie, d'être suffisamment connu quant à sa richesse algologique (5).

Une exploration méthodique de tout le littoral méditerranéen de l'Afrique serait certainement très utile pour déceler les liens floristiques du littoral même avec les autres côtes baignées par cette mer, et pour fournir des renseignements sûrs à propos de certaines phycées qui jusqu'à ce jour paraissent localisées dans certains points et avoir une répartition géographique très restreinte, comme on avait pu soupçonner, par exemple, à l'égard du Galaxaura adriatica Zanard., Schimmelmannia ornata Schousb., etc. (6) dont l'aire, d'abord limitée, a été agrandie à la suite d'explorations plus soigneuses.

Nous ne possédons pas de renseignements suffisants sur les flores algologiques de l'île de Pantellaria, du littoral méridional de la Sicile et de la Grèce, de l'Archipel grec, de l'Asie mineure et de la Syrie, malgré les importants travaux de Langenbach, Mazza, Bory, Reinbold, Schroeder et autres phycologues, pour reconnaître avec sûreté s'il existe des liens floristiques entre ces régions et le littoral de Tripolitaine et Cyrénaïque.

Ces comparaisons phytogéographiques trouvent un plus grand secours si l'on tient compte des espèces plus particulières, puisque, en accordant trop de valeur aux espèces communes, on peut facilement être conduit à la même erreur par laquelle jadis on soutenait l'existence d'une relation entre la flore de la mer Baltique et celle de la mer d'Ochotsk(7).

(i) Petit P., Catalogue des Diatomacées du Maroc, d'Algérie et de Tunisie (Alger, I897).

(2) Sauvageau C., Algæ (in Catalogue raisonné des plantes cellulaires de la Tunisie, I897).

(3) Areschoug J.-E., Alger samlade vid Alexandria af framl. Dr Hedenborg (OEfuersigt af Kongl. VetenskapsAkademiens Forhandlingar; Stockholm, I870, nº IO, p. 929-939).

(4) Muschle R R. Énumération des Algues marines et d'eau douce observées jusqu'à ce jour en Égypte (Mémoires présentés à l'Institut Egyptien et publiés sous les auspices de S.A. Abbas II, khédive d'Egypte, t. V, fasc. III, p. I4I237 ; Le Caire, I908).

(5) Les ouvrages qui donnent l'illustration des herbiers importants tels que ceux de CROUAN, THURET, ZANARDINI (dont des renseignements se trouvent foumis par M. Picouenard, Mme Lemoine, MM. DE Toni et LEvi), contiennent des indications relatives aux Algues de l'Afrique méditerranéenne; aussi, dans quelques autres publications monographiques des familles ou des genres d'Algues, on rencontre naturellement des indications sur des Algues provenant du littoral africain baigné par la Méditerranée.

(6) Nous citons à propos deux espèces, savoir Galaxavira adviatica Zanard. et Schimmelmannia ornata Schousb., car on a longtemps cru qu'elle fussent respectivement cantonnées, la première dans la mer Adriatique supérieure (d'où son nom spécifique), la seconde à Tanger où la récolta P.-K.-A. SchousboE. Or, les explorations successives ont démontré l'existence du Galaxaura adriatica en plusieurs localités méditerranéennes d'Antibes à Tripoli, de Livourne, Porto-Maurizio, Albissola, Naples, Tarante à l'Asie mineure, et celle du Schimmelmannia ornata à Acireale (Sicile), où elle fut découverte en 1864, la première fois par M. ARDISSONE, et à Guéthary dans le golfe de Gascogne (océan Atlantique) où la signala récemment M. SAuvageau. On peut consulter les publications suivantes : Bentivoglio T., La Galaxaura adriatica Zanard. à Taranto e la sua area di distribuzione nel Mediterraneo (La Nuova Notarisia, XIV, 1903, P. IO9-II2); et MAzzA A., La Schimmelmannia ornata Schousb. nel Mediterraneo (La Nuova Notarisia, XIV, I903. P. 45-6I, I tab.). Les découvertes dans le bassin de la Méditerranée d'Aglaozonia melanoidea et Nemoderma tingitanum par M. SAUvageau, de Callophyllis laciniata par M. BoRzI, de Laminaria Rodriguezii Born. (espèce des iles Baléares), rencontrée aux côtes de Sicile par M. MAzzA et par MM. BEck et Schiller à l'île Pelagosa, etc. prouvent à suffisance combien de surprises pourrait nous fournir l'exploration ultérieure et méthodique du bassin méditerranéen, qu'on juge comme une des mers les mieux connues.

(7) BORy dE SAINT-VINCENT, Histoire des Hydrophytes ou plantes agames des eaux, récoltées par MM. d'Urville 
Avancer des hypothèses est toujours facile: il ne l'est pas autant de les transformer en réalités ; il est donc préférable de donner des matériaux à la connaissance de la flore tripolitaine et cyrénaïque, que de hasarder des recherches phytogéographiques qui ont besoin, vraiment, qu'une région et les régions à comparer soient minutieusement explorées et par cela bien connues.

\section{FLORIDEÆ LAMOUR.}

Famille des CORALLINACE $Æ(G r a y)$ Harv.

Corallina (Tournef.) Lamour.

I. Corallina rubens L., Systema naturæ (éd. XII, I766), I, p. I304 ; Solms-LAUbach, Die Corallinenalgen, p. 6 ; DE Toni, Sylloge Algarum IV, p. I836; PredA, Florideæ (I908), p. I2, fig. II.

Jania mbens LAmour., Histoire des polypiers coralligènes flexibles (I8I6), p. 27I, tab. 9, fig. 6-7; Exposition méthodique (I82I), p. 24, tab. 69, fig. II-I2; KuetzING, Tab. Phyc., VIII, tab. 84, fig. II-IV ; Bornet et Thuret, Études Phycologiques, p. 96, tab. 50-5I ; Ardissone, Phyc. Medit., I, p. 459.

Hab : Tripoli (A. TrotTer); Tobrouk (A. Vaccari).

Espèce atlantique et méditerranéenne, signalée aussi au Natal. Elle avait été connue de Tripoli par les récoltes de M. R. Spigar; elle croît aussi sur les côtes de la Cyrénaïque (Haimann, Ruhmer), de l'Égypte (R. Muschler, Areschoug), de la Tunisie, de l'Algérie (Debray) jusqu'à Tanger (Schousboe).

\section{Amphiroa Lamour.}

2. Amphiroa rigida LAMour., Histoire des polyp. corall. flex. (I8I6), p. 297, tab. XI, fig. I; ZANARDini, Iconographia (I873), tab. XCIX, B ; Kuetz., Tab. Phyc., VIII, tab. 42, fig. IV; ARdiss., Phyc. Medit., I, p. 456 ; De Toni, Sylloge Algarum, IV, p. I807 ; PREDA, Florideæ, p. I5, fig. III.

Hab. : Tripoli (A. Trotter).

Espèce très répandue dans la Méditerranée. K. Yendo (Corallina Japon.,p. 6), la signale aussi au Japon. Du littoral africain $A$. rigida fut rapportée de l'Algérie (DEbray, Piccone, Ardissone) et de la Cyrénaïque (G. Haimann, sec. Piccone).

\section{Lithophyllum PHIL.}

3. Lithophyllum byssoĩdes (LAMARCK) FosLIE, Revised Systematical Survey of the Melobesieæ (I90o), p. 20; DE Tonr, Sylloge Algarum, IV, p. I790 ; PREdA, Florideæ, p. 25 ; Lemorne, Struct. anat. des Mélobésiées (I9II), p. I32.

Millepora (Nullipora) byssoides LAMARck, Histoire des animaux sans vertèbres (Ire édit., I8r6, p. 203; IIe édit., I836, p. 3I2 (excl. var. B.).

Spongites byssoides KUETz., Tab. Phyc., tab. XIX, fig. $e$.

Lithothamnion byssoides PHILIPPI, in Wiegmann's Archiv, I837, p. 384; HAUck, Meeresalgen,p. 275, tab. II, fig. $I$.

et Lesson dans leur voyage autour du monde sur la corvette de Sa Majesté, la Coquille, p. 36 (Paris, I.829). Voir DE TONi G.-B., Phyceæ Japonicæ novæ, addita enumeratione Algarum in ditione maritima Japoniæ hucusque collectarum, p. I6 (Memorie del R. Istituto Veneto di scienze, lettere ed arti, vol. XXV, no 5 ; Venezia, I895). 
Hab. : Tripoli et Homs (A. Trotter).

$M^{\text {me }} \mathrm{P}$. Lemorne, dans son récent ouvrage, publié dans ces Annales, a indiqué la répartition géographique du L. byssoides: Algérie, Adriatique, Grèce, île de Rhodes, Sicile, Maroc, Corse; M. Heydrich a signalé la même espèce dans la mer Rouge.

4. Lithophyllum Racemus (LAmarck) Foslie, Rev., Syst. Surv. of the Melobesieæ (Igoo), p. I7; DE Toni, Sylloge Algarum, IV, p. I778; PrEDA, Florideæ, p. 2 I.

Millepora (Nullipora) Racemus LAMARck, Hist. des anim. sans vertèbres (I8I6), p. 203.

Lithothamnion Racemus Aresch. in J. AgARdH, Species Algarum, II (I852), p. 52 ; ARdIss., Phyc. Medit., I, p. 453 .

Hab. : Tripoli (A. Trotter).

Cette espèce est indiquée pour la mer Adriatique et pour la Méditerranée (golfe de Naples, Sicile).

Lithothamnion PHIL.

5. Lithothamnion fruticulosum (Kuetz.) Foslie, forma clavulata Foslie, Die Lithothamnien des Adriatischen Meeres und Marokkos (I904), p. 7, tab. I, fig. Io-I7, tab. III, fig. 9-I4.

Hab. : Homs (A. TrotTer).

La forme typique n'est pas nouvelle pour le littoral africain, puisqu'elle fut rapportée de Stora, cap de Garde (Debray, Piccone) et croît aussi à Tanger (d'après Foslie).

\section{Dermatolithon FosL.}

6. Dermatolithon pustulatum (Lamour.) Fost., Rev. Syst. Surv. of the Melobesieæ (Igoo), p. II, Die Lithoth. des Adriat. Meer. u. Marokkos (I904), p. 38 ; De Toni, Sylloge Algarum, IV, p. I77I.

Melobesia pustulata Lamour., Hist. polyp. corall. flex. (I8I6), p. 315, Expos. méthod. (I82I), p. 46, tab. 73, fig. I7-I8 ; KUetz., Tab. Phyc., XIX, tab. 94, fig. $a-b$; Ardiss., Phyc. Medit., I, p. 446.

Hab. : Tripoli (A. TrotTer).

Espèce à répartition géographique très ample, comme Lithothamnion membranaceum et Melobesia farinosa; elle est connue aussi sur le littoral nord-africain d'Alexandrie (Areschoug), à la Cyrénaïque (Haimann), à l'Algérie (Montagne, Debray) jusqu'à Tanger (Schousboe).

\section{Melobesia LAMOUR.}

7. Melobesia Corallina Crouan, Florule du Finistère (I867), p. I50, tab. 20, gen. I33 bis ; Solms-Laubach, Die Corallinenalgen, p. 9, tab. 2, fig. 25, tab. 3, fig. 2I-24; DE TonI, Sylloge Algarum, IV, p. I768; PREDA, Florider, p. 32.

Lithophyllum Corallina HEYdrich, Corallinæ insbes. Melobes. (1897), p. 47.

Hab.: Tobrouk (A. VACCARI).

La répartition géographique de cette jolie Melobesia va du nord de la Grande-Bretagne au Maroc et dans la Méditerranée où l'espèce ne paraît pas commune.

8. Melobesia Cystosira HAucK, Meeresalgen (I885), p. 266, tab. 3, fig. I-2,6; DE TonI, Sylloge Algarum, IV, p. I770 ; Preda, Floridex, p. 3 I ; Woronichin, Rhodophyceen des Schwarzen Meeres (Igog), p. 302, fig. II-I2.

Lithophyllum Cystosire Hevdr., Corall. insbes. Melobes. (I897), p. 47. 
Hab. : Tobrouk (A. VACCARI).

M. Cystosira croît en forme de croûtes sur les Cystoseira et sur d'autres Algues. On la connaît dans la mer Adriatique (HAUCK) et la mer Noire (Woronichin).

9. Melobesia farinosa Lamour., Hist. polyp. corall. flex. (I8I6), p. 315 , tab. XII, fig. 3 ; Kuetz., Tab. Phyc., XIX, tab. 95, fig. c-e; Solms-Laubach, Die Corallinenalgen, p. II, tab. I, fig. 4 ; tab. III, fig. II ; Ardiss., Phyc. Medit.. I, p. 445; De Toni, Sylloge Algarum, IV, p. I764; Preda, Florideæ, p. 28. fig. VII.

Hab. : Tripoli (A. Trotter).

Espèce presque cosmopolite, vivante sur les Zostéracées, les Polypiers et les Algues surtout à thalle foliacé. Elle vit aussi sur le littoral africain (Debray, Piccone).

Famille des SQUAMARIACE $Æ$ (Zanard.) J. Ag.

Peyssonnelia DeCne.

Io. Peyssonnelia polymorpha (Zanard.) Schmitz in Falkenberg, Die Meeresalgen des Golfes von Neapel (I879), p. 264; HAuck, Meeresalgen, p. 35, tab. I, fig. 6 ; MAzzA, in Nuova Notavisia, XIV (I903), p. 98, $n^{0}$ I67; De Toni, Sylloge Algarum, IV, p. I7or ; PredA, Florideæ, p. 48 ; Lemoine et Mouret, Sur une algue nouvelle pour la France (I9r2), p. 359 (avec répartition géographique très détaillée).

Nardoa polymorpha ZANARD., Rivista sulle Corallinee (1844), p. 37.

Lithymenia polymorpha ZANARD., Iconographia, I (I863), p. I27 (295), tab. XXX.

Hab. : Tripoli (A. Trotter).

Espèce de Peyssonnelia vivant à une profondeur variable (jusqu’à 60 mètres), très incrustée de substance calcaire, comme les congénères exotiques $P$. calcea et $P$. tamicnsis; d'autres espèces sont légèrement incrustées, telles que $P$. Harveyana Crouan, $P$ NovaHollandia Harv., P. Rosenvingii Schmitz, P. multifida Harv., P. involvens Zanard.

$P$. polymorpha est une espèce méditerranéenne qui vit aussi dans le littoral nort-africain aux côtes de l'Algérie (Debray, Sauvageau).

Une espèce très affine nous paraît être le Peyssonnelia? compacta Foslie (I905), vivant dans la Méditerranée et la mer Adriatique.

II. Peyssonnelia Squamaria (Gmel.) Decne, Plantes de l'Arabie heureuse (I84I), p. I68, tab. 5, fig. I6-I7; Kuetz., Tab. Phyc., XIX, tab. 87, fig. $a$ - $b$; Ardiss., Phyc. Medit., I, p. 227 ; De Toni, Sylloge Algarum, IV, p. I697; PREDA, Florideæ, p. 46, fig. XIV.

Fucus Squamarius GMelin, Historia Fucorum (I768), tab. 20, fig. $\mathrm{r}$.

Hab. : Tripoli (A. Trotter).

L'aire de répartition géographique de cette espèce très polymorphe va de Brest à Cadix et au Maroc et dans la Méditerranée de l'Afrique jusqu'à la région supérieure de la mer Adriatique. Sur le littoral africain, elle fut déjà rapportée de Tripoli (R. SPIGAI), du port d'Alexandrie (R. Muschler), de la Cyrénaïque (Haimans) ; elle fut signalée de l'Algérie (Debray) et son limite extrême est à Tanger où la recueilla M. Schousboe (BORnet). 
Famille des RHIZOPHYLLIDACEÆ (Mont.) Schmitz.

\author{
Contarinia ZANARD.
}

I2. Contarinia peyssonneliaformis ZANARD., Saggio di classificazione naturale delle ficee (r843), p. 45, Iconographia (I86I), p. 47 (I05), tab. XII ; Ardiss., Phyc. Medit., I, p. 232 ; DE Toni, Sylloge Algarum, IV, p. I680; PREDA, Florideæ, p. 56, fig. XIX.

Hab. : Tripoli, sur les Cystoseira (A. TrotTer).

Déjà rapportée de Tripoli par R. Spigai (sec. De Toni) et A. Corbelli (sec. Ardissone) et connue aussi de Saint-Eugène (DEBray), cette espèce, vivant de préférence sur les Cystoseira, n’est pas commune dans la Méditerranée (mer Adriatique, île de Gozo, etc.), peut être souvent inaperçue, puisqu'elle se trouve quelquefois incrustée par d'autres Algues ou des animaux à sécrétion calcaire, qui la rendent presque méconnaissable. Contarinia peyssonneliaformis Zanard, a beaucoup de ressemblance avec Rhododiscus pulcherrimus Crouan et avec quelques Cruoria, mais elle en diffère par la division irrégulièrement croisée (pas zonée) des sporanges. On ne peut pas la confondre avec Plagiospora gracilis Kuck.

\title{
Famille des CERAMiACE $Æ$ (Bonnem.) Naeg.
}

$$
\text { Ceramium (WIGg.) Ag. }
$$

I3. Ceramium ciliatum (Ellis) Ducluz., Essai sur l'histoire naturelle des Conferves des environs de Montpellier (I805), p. 64; LYNGBYE, Tentamen Hydrophytologiæ Danicæ (I8I9), p. I2I, tab. 37 ; ARdiss., Phyc. Medit.,I, p. II7 ; DE TonI, Sylloge Algarum, IV, p. I473; MAzzA, Saggio di Algologia Oceanica, no 436 ; PREDA, Florideæ, p. IO2.

Conferva ciliata Ellis, in Philosophical Transactions, 57, p. 425, tab. I8, f. h-H.

Echinoceras sp. plur. Kuetz., Tab. Phyc., XII.

Hab. : Homs (A. Trotter).

Espèce extrêmement commune dans la Méditerranée et répandue aussi dans l'océan Atlantique, des îles Feroẻ aux îles du Cap Vert jusqu'à Cadix et à Tanger. Du littoral nord-africain elle fut rapportée de Tripoli (R. SpIgaI), de l'Algérie (Debray), d'Alexandrie (Areschoug) et de Porto-Saïd (Ehrenberg, R. Muschler).

I4. Ceramium circinatum (KUETZ.) J. Ag., Species Algarum, II (I85I), p. I26; Epicrisis (I876), p. 99; Florideernes morphologi (I879), tab. III, fig. I4-I9; ARdiss., Phyc. Medit,, I, p. III; DE Toni, Sylloge Algarum, IV, p. I469; MAzzA, Saggio di Alg. ocean., no 435 ; PredA, Florideæ, p. Io4.

Hormoceras circinatum KUETZ., Ueber Ceramium Ag. (I84I), p. 733.

Hormoceras sp. plur. KUETz., Tab. Phyc., XII.

Hab. : Tripoli (A. TrotTER); Tobrouk (A. VACCARI).

Espèce commune à l'océan Atlantique et à la Méditerranée où elle est très répandue.

C. circinatum fut signalé sur les côtes d'Algérie (Debray, Ardissone).

\section{Spyridia Harv.}

I5. Spyridia filamentosa (WULF.) HARV., in HoOKER, British Flora, II (I853), p. 336; Phycologia britannica 
(1849), tab. 46; KUetz., Tab. Phyc., XII, tab. 42, fig. $a$-b; De TonI, Sylloge Algarum, IV, p. I427 (avec synonymie très détaillée); ARDIss., Phyc. Medit., I, p. I93.

Fucus filamentosus Wulfen, Cryptogama aquatica (1803), p. 64.

Hab. : Tripoli (A. TrotTer).

Espèce à large répartition géographique dans l'océan Atlantique, l'océan Indien, la mer Rouge et la Méditerranée, où elle est très commune, souvent rejetée sur les plages mêmes de l'Égypte (Ardissone), d'Algérie (Debray). Schousboe la distribua dans ses Algues $\left(\mathrm{n}^{\mathrm{OS}} 262,263,364,364\right.$ a) sous les noms de Ceramium friabile, pilosum et setosum.

\section{Famille des RHODOMELACEA (REIchb.) Harv.}

Halodictyon ZANARD.

I6. Halodictyon mirabile ZANARD., Saggio di classificazione naturale delle ficee (I843), p. 52; Iconographia (I860), p. I7 (6I), tab. V; Kuetz., Tab. Phyc., XII, tab. 36, fig. $a$ - $b$; DE TonI, Sylloge Algarum, IV. p.r244; Falkenberg, Die Rhodomelaceen (Igor), p. 692, tab. I5, fig. I-20; PredA, Florideæ, p. 263, fig. LXXX.

Hab. : Tobrouk (A. VACCARI).

Espèce méditerranéenne pas commune. Découverte par Zanardini en I840 à Pola (mer Adriatique), puis récoltée par SAndri et Vidovich en Dalmatie, sa présence dans le bassin de la Méditerranée fut confirmée plus tard avec des exemplaires provenant d'Antibes (Bornet et Flahault), de Ligurie (Strafforello), du golfe de Naples (Falkenberg), de Sardaigne (Piccone) et des îles Baléares (Rodriguez). M. Bornet l’indique avec doute de Tanger, car les échantillons de la collection Schousboe ne portaient aucune indication d'origine; mais, comme ils étaient mélangés de fragments de Plumaria Schousboei Schm. (Callithamnion elegans Schousboe) que Schousboe a plusieurs fois récoltée à Tanger, M. Borner croit probable que c'est de cette localité que les échantillons proviennent.

\section{Rytiphlœa AG.}

I7. Rytiphlaatinctoria (Clem.) AG., Systema Algarum (I824), p. I6o, Species Algarum, II (I828), p. 52; KUETZ., Tab. Phyc., XV, tab. I3, fig.e-i; Ardiss., Phyc. Medit., I, p. 422 ; DE Toni, Sylloge Algarum, IV, p. Io95; FAlKEnBeRG, Die Rhodomelaceen, p. 438, tab. 8, fig. 28-3I ; Preda, Florideæ, p. I89; fig. LXI.

Fucus tinctorius Clemente, Ensayo (I807), p. 3I6.

Rytiphlaa semicristata J. AG., Algæ maris Mediterranei et Adriatici (I842), p. I45 (planta procarpifera. fide Bornet et Thuret, Études Phycologiques, p. 92).

Hab. : Tripoli (A. Trotter).

L'aire de répartition de cette Rhodomélacée dans l'océan Atlantique va de Brest aux Canaries, dans la Méditerranée des côtes de Provence jusqu'à la Grèce, à Giaffa, et à l'Adriatique supérieure; elle croît aussi dans la mer Rouge (Turner, Zanardini) ; le long du littoral nord-africain, $R$. tinctoria fut signalée à Suez (FIGARI, d'après R. Muschler), à Alexandrie (Areschoug, R. Muschler), en Cyrénaïque (Hanmann), en Algérie(Montagne, Debray), à Tanger (Schousboe, Bornet); elle fut aussi extraite par la drague au nord de Tripoli (PICcone). 
Lophosiphonia FALK.

I8. Lophosiphonia cristata Falk.,Die Rhodomelaceen (I9or), p. 499, tab. 9, fig. 7-Io ; DE Toni, Sylloge Algarum, IV, p. Io6g.

Hab. : Tobrouk (A. VACCARI).

L'espèce croît dans la Méditerranée (golfe de Naples) et ressemble beaucoup au Lophosiphonia subadunca (Kuetz.) Falk. dont elle serait, d'après l'opinion de M. PREDA (Floridea, p. 200), une simple variété.

I9. Lophosiphonia obscura (AG.) FALK., Die Rhodomelaceen (Igor), p. 500 ; DE TONI, Sylloge Algarum, IV, p. 1069 ; PREDA, Florideæ, p. 200.

Hutchinsia obscura AG., Species Algarum, II (I828), p. I08.

Polysiphonia obscura J. AG., Algæ maris Mediterranei et Adriatici (1842), p. I23; Species Algarum, II (I863), p. 943 ; Kuetz., Tab. Phyc., XIII, tab. 40, fig. $a$ - $b$; Ardiss., Phyc. Medit.. I, p. 369.

Hab. : Tripoli (A. Trotter).

Espèce à large répartition géographique, soit dans la Méditerranée, soit dans l'océan Atlantique, caractérisée par le grand nombre (I0-18) de cellules péricentrales. Pour la région nord-africaine $L$ obscura a été signalée d'Alexandrie (Areschoug), à la Cyrénaïque (Haimann), à l'île Gallite (Piccone). Le Ceramium aciculare Schousboe, qui par M. Bornet est attribué au Lophosiphonia obscura, n'a pas d'indication d'origine.

\section{Herposiphonia NAEG.}

20. Herposiphonia tenella (AG.) NAEG., Ueber Polysiphonia und Herposiphonia (I846), p. VIII, fig. 2 ; AMBronN, Ueber einige Fälle von Bilateralität bei den Florideen (I880), p. I3, tab. IV, fig. 9, I3-I7 ; FALKENBERG, Die Rhodomelaceen, p. 304, tab. III, fig. I3-I7 ; DE TonI, Sylloge Algarum, IV, p. ro5I ; PREDA, Florideæ, p. I95.

Hutchinsia tenella AG., Species Algarum, II (I828), p. 105.

Polysiphonia tenella J. Ag., Algæ maris Mediterranei et Adriatici (1842), p. I23 (non DE NotaRIS, nec Erbario Crittogamico italiano).

Hab. : Tripoli (A. Trotter).

Cette petite espèce se trouve, d'après M. Bonnet, à Biarritz, aux Canaties et dans la Méditerranée, où elle n'est pas rare dans la première zone de profondeur. Sur le littoral nord-africain, elle fut signalée près Guyotville (DEBray). On la confond souvent avec Herposiphonia secunda (Ag.) Naeg.

\section{Dipterosiphonia FALK.}

21. Dipterosiphonia rigens (Schousb.) Falk., Die Rhodomelaceen (IgoI), p. 323, tab. 3, fig. 4-7; De TONI, Sylloge Algarum, IV, p. I048 ; PREDA, Florideæ, p. 197, fig. LXV.

Ceramium rigens SCHOUSBOE, Alg., $\mathrm{n}^{0} 447$.

Hutchinsia rigens SchousBoe, AG. in Bot. Zeit, I827, p. 638 ; Species Algarum, II (I828), p. III.

Polysiphonia rigens ZANARD, Synopsis algarum in mari Adriatico hucusque collectarum (I84I), p. 65; Ardiss., Phyc. Medit., I, p. 372.

Hab. : Tripoli (A. Trotter).

Petite espèce, vivant sur les feuilles des Zostéracées et sur les Algues. On la connaît T. V. - FASC. 7 . 
dans la Méditerranée où elle est suffisamment répandue sur les côtes françaises et italiennes ainsi que sur celles d'Algérie (Debray) ; D. rigens fut récoltée à Tanger (Schousboe) et à Cadix (C. Agardh); d'après Grunow et Falkenberg, elle aurait été récoltée aux Canaries et à Madère (Liebetruth).

\section{Brongniartella Bory.}

22. Brongniartella byssoides (Good. et Woodw.) Schmitz, Die Gattung Lophothalia (r893), p. 2I7 ; FALKENBERG, Die Rhodomelaceen(I9OI),p. 543, tab. I9, fig. 8-Io; DE TonI, Sylloge Algarum, IV,p. I008 (avec synonymie très détaillée); MAzzA, Saggio di Algologia oceanica, no 309 ; PredA, Florideæ, p. 203, fig. LXVII.

Fucus byssoides Goodenough et Woodward, Observations on the British Fuci (I797), p. 229.

Polysiphonia byssoides Greville, Flora Edinensis (I824), p. 309 ; Ardiss., Phyc. Medit., I, p. 382.

Hab.: Tripoli (A. Trotter), Tobrouk (A. Vaccari), Benghazi (A. Vaccari).

Espèce très élégante, avec l'habitus du Dasya elegans Ag., comme l'a figurée M. Zanardin dans son Iconographia, tab. 23 sous le nom de Polysiphonia dasyceformis; elle est commune à l'océan Atlantique et à la Méditerranée où elle est très répandue, surtout à une certaine profondeur.

Polysiphonia Grev.

23. Polysiphonia truticulosa (Wulf.) Sprengel, Systema vegetabilium, vol. IV (I827), p. 350; KuEtz., Tab. Phyc., XIV, tab. 28, fig. e-g; Ardiss., Phyc. Medit.,I, p. 393; DE Toni, Sylloge Algarum, IV,p. 950 (avec synonymie très détaillée); FALKENB., Die Rhodomelaceen,p. I33, tab. 2I, fig. I-5; PrEDA, Florideæ, p. 2I2, fig. LXIX.

Fucus fruticulosus WULFEN, in JACQUIN, Collectanea (1789), p. I59, tab. I6, fig. I ; Cryptogama aquatica (1803), p. 56.

Hab. : Tripoli (A. Trotter).

Espèce méditerranéenne et atlantique, très répandue. Sur le littoral nord-africain, elle fut signalée à partir d'Alexandrie (Areschoug) et de la Cyrénaîque (Haimann) jusqu'à Tanger (Schousboe).

\section{Laurencia LAMOUR.}

24. Laurencia obtusa (Huds.) Lamouroux, Essai sur les genres des Thalassiophytes non articulées (I8I3), p. 42 ; Kuetzing, Tabulæ Phyc., XV, tab. 54-55; Ardiss., Phyc. Medit., I, p. 326; De Toni, Sylloge Algarum, IV, p. 79I ; Falkenb., Die Rhodomelaceen, p. 246 ; Preda, Florideæ, p. 257.

Fucus obtusus Hudson, Flora anglica, p. 586.

Chondria obtusa AG., Species Algarum, I (I82I), p. 340.

Hab. : Benghazi (A. VACCARI).

Espèce très répartie dans les mers, récoltée déjà par Ruhmer dans la même localité et signalée autrefois pour Tripoli (PICCONe, DE Toni et Levi) et pour la Cyrénaïque (HaIMANN); elle est commune en Égypte (Areschoug) et le long du littoral nord-africain (Montagne, Sauvageau, etc.).

25. Laurencia paniculata J. AG., Species Algarum, II (I863), p. 755, Epicrisis (I876), p. 65I ; ARDIsS., Phyc. Medit., I, p. 328 ; De Ton, Sylloge Algarum, IV, p. 788 ; FalkenberG, Die Rhodomelaceen, p. 247 ; PREDA, Florideæ, p. 259. 
Hab. : Tripoli (A. TROTteR).

Espèce méditerranéenne (îles Baléares, Ligurie, petites îles italiennes, golfe de Naples, Sicile, mer Adriatique) ; elle est indiquée de Cadix par J. Agardh.

26. Laurencia papillosa (Forsk.) Grev., Algæ Britannicæ (I830), p. LII ; KUetz., Tab. Phyc., XV, tab. 62 ; Ardiss., Phyc. Medit., I, p. 330 ; De Toni, Sylloge Algarum, IV, p. 789 ; Falkenb., Die Rhodomelaceen, p. 248, tab. 23, fig. 3 ; MAzzA, Saggio di Algologia Oceanica, nº 264; PrEDA, Florideæ, p. 258. Fucus papillosus ForskaL, Flora ægyptiaco-arabica (I775), p. I9o.

Chondria papillosa AG., Species Algarum (I82I), p. 344.

Hab. : Tripoli (A. Trotter), Homs (A. Trotter).

Espèce à large répartition géographique dans les mers et les océans, si les déterminations faites par les différentes auteurs sont exactes. Elle paraît commune le long du littoral nord-africain, ayant été signalée déjà à Tripoli (Piccone, De Toni), dans la Cyrénaïque (Haimann), l'Algérie (Monragne, Debray) et l'Égypte (Ehrenberg, Figari, AresChoug, Muschler).

Famille des RHODYMENIACEÆ (NAEg.) J. Ag.

Gastroclonium KuETZ.

27. Gastroclonium clai'atum (Roth) ARDIss., Phycologia mediterranea, I (I883), p. 322 ; DE Toni, Sylloge Algarum, IV, p. 569 ; PREDA, Florideæ, P. 305.

Conferva clavata Rотн, Catalecta Botanica, I (I797), p. I6o, tab. I, fig. 2.

Lomentaria clavata J. AG., Species Algarum, II (I863), p. 735 ; Epicrisis (I876), p. 634.

Gastroclonium salicornia KUETZ., Phycologia generalis (I843), p. 44I, tab. 53, fig. I; Tab. Phyc., XV, tab. I00, fig. $a-c$.

Hab. : Tripoli (A. TrotTer).

Espèce méditerranéenne, signalée pour les côtes des îles Baléares, de France, d'Italie et du littoral illyrique; elle ne manque pas sur le littoral algérien (Debray, 'Ardissone).

Famille des SPHÆROCOCCACEÆ (Dum.) Schmitz.

\section{Hypnea Lamour.}

28. Hypnea musciformis (WULF.) LAMour.,Essai sur les genres de la famille des Thalassiophytes non articu-, lées (1813), p. 43 ; Kuetz., Tab. Phyc., XVIII, tab. I9, fig. a-e ; Ardiss., Phyc. Medit., I, p. 28I; DE Toni, Sylloge Algarum; IV, p. 472.

Fucus musciformis Wulfen, in JACQUin, Collectanea, III (I789), p. I54, tab. I4, fig. 3, Cryptogama aquatica (I803), p. 47, n. 23.

Hab. : Tripoli (A. Trotter).

Espèce trè̀s répandue dans les mers et les océans, extrêmement variable quant à l'habitus, ce qui explique sa synonymie. Rapportée déjà de Benghazi (Ruhmer) et de la. Cyrénaïque (Harmans), elle n'est pas rare sur le littoral nord-africain à partir d'Alexandrie (Areschoug, Muschler) jusqu'à Tanger (Schousboe). 
Sphærococcus Sтаскн.

29. Spharococcus coronopifolius (Good. et Woodw.) AG., Synopsis Algarum Scandinaviæ (I8I7), p. 29; Species Algarum, I (I82I), p. 290 ; Ardiss., Phyc. Medit., I, p. 247 ; DE Toni, Sylloge Algarum; IV, p. 395 ; PREDA, Florideæ, P. 3I4, fig. XCVII.

Fucus coronopifolius GoOdenOUgh et WoOdWaRd, Observations on the British fuci (I797), p. 185.

Rhynchococcus coronopifolius Kuetz.; Phycologia generalis (I843), p. 403, tab. 6I, fig. I; Tab. Phyc., XVIII, tab. Io, fig. $e-h$.

Hab. : Tripoli (A. TrotTER).

Espèce vivant dans l'océan Atlantique du nord de la Grande-Bretagne jusqu'à Cadix et Tanger, aux Canaries et au Brésil et dans la Méditerranée. Du littoral nord-africain Spharococcus coronopifolius a été rapporté de Tripoli (R. Spigai, De Toni), de la Cyrénaique (Haimann), d'Alexandrie (Ehrenberg, Schimper, R. Muschler) et de l'Algérie Montagne, Debray).

\section{Famille des GIGARTINACE $Æ$ (Schmitz).}

Gymnogongrus Martius.

30. Gymnogongrus Griffithsia (TUrn.) Martius, Flora Brasiliensis, I (I833),p. 27 ; KUetz., Tab. Phyc., XIX, tab. 65, fig. e-g; Ardiss., Phyc. Medit., I, p. I76 ; DE Toni, Sylloge Algarum; IV, p. 242 ; PrEDA, Florideæ, p. 347, fig. CIX.

Fucus Griffithsia TURNER, Fuci (I808), tab. 37.

Gymnogongrus Wulfeni ZANARD., Iconographia (I872), tab. 94.

Hab. : Tripoli (A. Trotter).

La répartition géographique de cette Algue est suffisamment large, puisqu'elle croît aussi dans l'océan Atlantique, de l'Angleterre aux Canaries et à l'Amérique boréale, et dans la Méditerranée où elle est très commune. Déjà rapportée de Tripoli par R. SPIGaI, l'espèce se trouve çà et là sur le littoral nord-africain à partir d'Alexandrie (Areschoug, R. Muschler), à l'Algérie (Montagne, Debray) et au Marocco (Schousboe).

\section{Actinococcus KuErz.}

3I. Actinococcus aggregatus Schmitz, Die Gattung Actinococcus (1893), p. 385, tab. VII, fig. 4-7; DE TonI, Sylloge algarum, IV, p. 259 ; PrEDA, Florideæ, p. 346, fig. CVIII.

Hab. : Tripoli, sur Gymnogongrus Griffithsia (A. Trotter).

Cette espèce d'Actinococcus n'est pas nouvelle pour le littoral nord-africain, car elle fut observée sur la même plante à Saint-Eugène, près Alger, par M. Debray.

\section{Famille des GELIDIACEÆ (Kuetz.) Schmitz.}

\section{Wrangelia AG.}

32. Wrangelia penicillata Ag., Species Algarum, II (I828), p. I38 ; Kuetz., Tab. Phyc., XII, tab. 40 ; Ardiss., Phyc. Medit., I, p. 3I2; DE TonI, Sylloge Algarum, IV, p. I35; PREDA, Florideæ, p. 368, fig. CXV. Griffithia penicillata AG., Systema Algarum (I824), p. I43.

Dasya spinella Duby, Mémoire sur le groupe des Céramiées (I832), II, p. I3, tab. II, fig. 4-5, tab. III, fig. 2 (non Dasya spinella Ag.). 
Hab. : Tripoli (A. Trotter).

Espèce commune dans la Méditerranée et propre aussi aux côtes européennes atlantiques et aux Bermudes. Schousboe la distribua dans ses Alga Schousboeanx, $\mathrm{n}^{\circ} 396$, sous le nom de Griffithsia caudata.

Famille des HELMINTHOCLADIACE E (Harv.) Schmitz.

Chantransia (D. C.) Schmitz.

33. Chantransia Saviana (Menegh.) Ardiss., Phyc. Medit., I, p. 276; De Toni, Sylloge Algarum, IV, p. 68 PREDA, Florideæ, p. 384 , fig. CXXII.

Callithamnion Savianum Meneghini, Lettera al Dott. Jacob Corinaldi (I840), no 3.

Hab. : Tobrouk, sur les Cystoseira (A. VACCARI).

Cette espèce est nouvelle pour le littoral nord-africain ; elle est commune à la Méditerranée et aux côtes européennes de la région tempérée de l'océan Atlantique.

FUCOIDEÆ (Ag.) J. Ag.

Famille des SARGASSACEÆ (Decne Kuetz.

Sargassum AG.

34. Sargassum Hornschuchii AG., Species Algarum, I (I82I), p. 40; J. AG., Species Sargassorum Australiæ (I889), p. 97, tab. IX; Ardiss., Phyc. Medit., II (I886), p. I9; DE Toni, Sylloge Algarum, III (I895), p. 68. Stichophora Hornschuchii Kuetz., Species Algarum (I849), p. 627; Tab. Phyc., X (1860), tab. 7I, fig. a-d (non bona).

Hab. : Tripoli (A. TrotTER).

Espèce exclusivement méditerranéenne, très variable; sur le littoral nord-africain, elle fut signalée à Alexandrie (R. Muschler) et en Algérie (Montagne). Le Sargassum lunense Caldesi (I865) est simplement une forme à phylloïdes très longs de S. Hornschuchii, commeil résulte de la comparaison faite par M. A DE Toni (Intorno al Sargassum lunense del Caldesi ; Modena, I907).

35. Sargassum linifolium (TURn.) AG., Species Algarum, I (I82I), p. I8; KUEtz., Tab. Phyc., XI, tab. 24; Ardiss. Phyc. Medit., II, p. I6, var. ; DE TonI, Sylloge Algarum, III, p. 90.

Fucus linifolius TURNER, Fuci (I80I), tab. İ8.

Hab. : Tripoli (A. Trotter).

Espèce très répandue dans le bassin méditerranéen, surtout dans l'Adriatique supérieur; elle paraît commune en Tripolitaine d'où des exemplaires furent rapportés de Tripoli (d'apres Piccone, Ascherson, Ardissone) et de Benghazi (Ruhmer). S. linifolium est aussi connu pour la Cyrénaïque (Haimann), l'Algérie (Montagne, Debray) et l'Égypte (Areschoug, R. Muschler). Schousboe le distribua sous le nom de Fucus natans. 
36. Cyštoseira sp.

Cystoseira AG.

Hab.: Tripoli, Homs (A. Trotter), Tobrouk (A. Vaccari).

Ce sont des fragments de plusieurs formes de Cystoseira, dont la détermination reste douteuse, vis-à-vis de la difficulté d'identifier avec sûreté les Cystoseira avec des échantillons stériles et dépourvus des parties basilaires. Quelques fragments seraient, d'après des comparaisons faites avec des matériaux d'herbier, reférables aux Cystoseira abrotanifolia Ag. et oiscors Ag., espèces pas rares le long du littoral africain.

Famille des DICTYOTACEÆ (LAmour.) Zanard.

Taonia J. AG.

37. Taonia Atomaria (Good. et Woodw.) J. AG., Species Algarum, I (I848), p. IOI ; REINKE, Entwickelungsgeschichtliche Untersuchungen über die Dictyotaceen des Golfs von Neapel (I878), p. 26, tab. IV, fig. I3-20, tab. V, fig. I-I9; Ardiss., Phyc. Medit., I, p. 483; DE TonI, Sylloge Algarum, III, p. $24 \mathrm{I}$. Ulva Atomaria Goodenough et Woodward, Observations on the British fuci (I797), p. 53.

Stypopodium Atomaria KUetz., Phycologia generalis (I843), p. 44I ; Tab. Phyc., IX, tab. 6I.

Hab. : Tripoli (A. TrotTER).

La Taonia Atomaria est commune à l'océan Atlantique et à la Méditerranée. Sur le littoral nord-africain elle fut signalée aux côtes d'Algérie (Montagne), de la Cyrénaïque (Piccone) et de l'Égypte (Areschoug). Cette espèce, d'après Schousboe, se trouve rarement à Tanger.

Dictyota Lamour.

38. Dictyota dichotoma (Huds.) LAmour., Exposition des caractères du genre Dictyota $[$ in Desvaux, Journal de Botanique, vol. II] (I809), p. I5 (42); Kuetz., Tab. Phyc., IX, tab. Io, fig. I; Ardiss., Phyc. Medit., I, p. 478 ; DE Toni, Sylloge Algarum, III, p. 263 (avec synonymie très détaillée).

Ulva dichotoma Hudson, Flora Anglica (I762), p. 476.

Zonaria dichotoma AG., Species Algarum, I (I82I), p. I33.

Hab. : Tripoli (A. Trotter), Tobrouk (A. VACCARI).

Espèce à répartition géographique très ample $(D$. dichotoma ne se trouve dans la région arctique), commune dans la Méditerranée; elle est bien représentée au littoral nord-africain, ayant été déjà récoltée à Tripoli (SpIgaI), en Cyrénaïque (HaImann), en Algérie (Montagne, Piccone) et en Égypte (Ehrenberg, Muschler, etc.). Sous plusieurs noms, elle fut distribuée de Tanger par Schousboe. M. Trotter nous a communiqué aussi des exemplaires de la var. implexa (Lamour.), caractérisée par le thalle plus étroit et les segments supérieurs très allongés.

39. Dictyota linearis (AG.) Greville, Algæ britannicæ (I833), p. XIIII; Kuetz., Tab. Phyc., IX, tab. 2I, fig. II; ARdiss., Phyc. Medit., I, p. 48 I ; DE Toni, Sylloge Algarum, III, p. 275.

Zonaria linearis AG., Species Algarum, I (I82I), p. I34 (excl. synon.).

Hab. : Tripoli (A. Trotter).

Cette espèce, déjà connue de Tripoli (PıCcone), est, comme la précédente, commune 
dans les mers et les océans de la zone tropicale et tempérée. D'après Areschoug, elle se trouve à Alexandrie. Schousboe, qui la recueillit rejetée sur la plage à Tanger en 1827 , lui donna le nom de Neurocarpus tenuissimus.

Famille des ENCELIACE E (Kuetz.) Kjellm.

Phyllitis Kuetz.

40. Phyllitis Fascia (Mueld.) Kuetz., Phycologia generalis (x843), p. 342, tab. 24, fig. III ; Tab. Phyc., VI tab. 49 ; DE Toni, Sylloge Algarum, III, p. 487 (non Le Jolis).

Fucus Fascia Mueller, Flora Danica, tab. 768 .

Hab. : Tobrouk (A. VACCARI).

Espèce à répartition géographique très ample, commune dans la Méditerranée autant comme forme typique que comme forme debilis; elle est connue aussi d'Algérie (Debray, Ardissone).

\section{CHLOROPHYCEÆ (KuETz.) Wittr.}

Famille des ULVACEÆ (Lamour.) Rabenh.

Ulva (L.) J. Ag.

4I. Ulva fasciata Delile, Flore d'Égypte (I8I3), p. 297, tab. 58, fig. 5; Montagne, Flore d'Algérie, p. I5I, tab. I4, fig. I-2; ARDiss., Phyc. Medit., II, p. I95; De Toni, Terzo Pugillo di Alghe Tripolitane (I895), p. $455, n^{0} 22$, Sylloge Algarum, I (I889), p. II4.

Phycoseris fasciata Kuetz., Tab. Phyc., VI, tab. 28.

Hab.: Tripoli (A. TROTTER).

Déjà récoltée à Tripoli par R. SPIGAI, cette espèce est très répandue dans les mers et les océans; sur le littoral nord-africain, elle fut signalée à Benghazi (RUHMER), à Alexandrie (Ehrenberg, Fraas, R. Muschler) et en Algérie (Montagne).

\section{Enteromorpha Link.}

42. Enteromorpha clathrata (Roth) J. AG., Till algernes systematik, VI (I882), p. I53; DE TonI, Sylloge Algarum, I, p. I33.

Conferva clathrata RоTн, Catalecta, III (I806), p. I75.

Ulva clathrata AG., Species Algarum, I (I82I), p. 422 partim ; Icones Algarum Europæarum, tab. I7; ARDISS., Phyc. Medit., II, p. 200 (excl. var.).

Hab. : Tobrouk (A. VACCARI).

Espèce pas rare dans la Méditerranée et vivant aussi dans les océans. Elle est connue sur le littoral nord-africain, d'Algérie (Debray) et de Tanger (Schousboe).

Famille des CHÆTOPHORACEÆ (HARv.).

Phæophila Hauck.

43. Phaophila Floridearum HAuck, Verzeichniss der im Golfe von Triest gesammelten Meeresalgen (I876), p. $56, n^{0} 245$, c. ic. ; Beiträge zur Kenntniss der Adriatischen Algen, I, (I877), p. I77, fig. I-7 ; Mee- 
resalgen, p. 464, fig. 200 ; DE Toni, Sylloge Algarum, I, p. 2 I2 ; Huber, Contributions à la connaissance des Chætophorées (I893), p. 327, pl. XVI, fig. I-II.

Ochlochate Phaophila Falkenberg, Die Meeresalgen des Golfes von Neapel (I879), p. 233.

Hab. : sur Polysiphonia, Benghazi (A. VACCARI).

Espèce vivant épi-endophytique sur plusieurs Algues et sur les Zostéracées, indiquée la première fois pour la mer Adriatique par F. HAUCK, puis signalée à Naples par FALKENBERG, au Croisic et dans le golfe du Lion par Huber. A. HANsGirg, d'après l'observation d'un exemplaire authentique (qui lui fut communiqué par M. Bornet) du Ochlochate dendroides Crouan, croit que Phoophila Floridearum Hauck ne diffère pas de l'espèce des frères Crouan; mais M. Huber a cru devoir maintenir le genre Phooophila de Hauck pour l'Ochlochate dendroides Crouan et les formes voisines, réservant le genre Ochlochate Thwait. pour l'O. Hystrix et d'autres espèces.

Ochlochaete THWAITES.

44. Ochlochate Hystrix Thwaites, in Harvey, Phycologia Britannica (I849), tab. 226; De Toni, Sylloge Algarum, I, p. $2 \mathrm{I} 3$.

Aphanochate Hystrix Rabenhorst, Flora Europæa Algarum, III (I868), p. 39I; Cooke, British freshwater Algæ, p. I97, tab. 80, fig. 2.

Hab. : sur Polysiphonia, Benghazi (A. VACCARI).

Espèce ayant un habitus coléochætoïde, vivant sur les plantes des littoraux, comme son congénère, $O$. ferox Huber, signalée par Huber dans les marais salants des environs du Croisic.

\section{Ulvella Crouan.}

45. Ulvella Lens Crouan, Notice sur quelques Algues marines (I859), p. 288, tab. XXII. fig. E, 25-28; DE ToNI, Sylloge Algarum, I, p. I48, partim; HUBER, Contributions à la connaissance des Chætophorées (I893), p. 294, tab. XI, fig. 4-6.

Phyllactidium Lens CrouAN, Florule du Finistère (I867), p. I30, gen. 67.

Hab.: sur Polysiphonia, Benghazi (A. VACCari).

D'après Huber, il paraît douteux que l'espèce, signalée par HansGirg comme Ulvella Lens dans la mer Adriatique, soit réellement l'espèce des frères Crouan.

Famille des CLADOPHORACEÆ (Hass.) Wittr.

Cladophora KuEtz.

46. Cladophora catenata (AG.) HAUck, Meeresalgen (I885), p. 45I; ARdiss., Phycologia Mediterranea, II (I887), p. 226 ; De Toni, Sylloge Algarum, I, p. 307.

Conferva catenata AG., Systema Algarum (I824), p. IIg.

Cladophora prolifera var. flaccida KUETz., Species Algarum (I849), p. 390.

Hab.: Benghazi (A. VACCARI).

Cette espèce, affine au Cladophora prolifera (Rотн) Kuetz., semble propre à la Méditerranée où elle se trouve suffisamment répandue. M. Bornet (Algues Schousb, p. 48) en a discutél'ancienne synonymie. 
47. Cladophora Hutchinsia (Dillw.) KuEtz., Phycologia germanica (I845), p. 210 ; Species Algarum, p. 39I;

Tab. Phyc., III, tab. 87, fig. I ; ARdiss., Phyc. Medit., II, p. 230; DE Toni, Sylloge Algarum, I, p. 3I4. Conferva Hutchinsice Dillwyn, British Confervæ (I809), tab. Iog.

Hab. : Tripoli (A. TRоттеR).

D’après M. Ardissone (Note alla Phycologia Mediterranea, 1893, p. 15), M. Debray aurait récolté $\mathrm{Cl}$. Hutchinsice à Alger; l'espèce est répandue sur les côtes atlantiques et méditerranéennes de l'Europe.

48. Cladophora ramulosa Meneghini, Algarum species novæ vel minus notæ (I844), p. $306, \mathrm{n}^{0} 32$; KUETZ., Species Algarum, p. 39I; Tab. Phyc., III, tab. 85, fig. II ;ZANARD., Iconographia (I862), p. 99 (48I), tab. XXIV, A (sphalmate Cladophora ramosa).

Cladophora utriculosa var. ramulosa HAUck, Meeresalgen (I885), p. 455.

Hab.: Tobrouk (A. VACCARI).

L'espèce n'est pas rare dans la Méditerranée et la mer Adriatique où elle fut récoltée la première fois en Dalmatie par M. Vidovich.

49. Cladophora utriculosa Kuetz., Phycologia generalis (1843), p. 260 ; Tab. Phyc., III, tab. 94, fig. I ; ARDIss., Phyc. Medit., II, p. 229 ; De Toni, Sylloge Algarum, I, p. 3г 2.

Hab. : Tripoli (A. Trotter).

Espèce très répandue dans la Méditerranée et dans les mers intérieurs jusqu'à Balaklava (Woronichin) et vivant aussi dans l'océan Atlantique, de la Baltique à Cadix, aux îles du Cap Vert et au Brésil. Elle n'est pas nouvelle pour le littoral nord-africain, puisque Montagne la signala en Algérie et Schousboe à Tanger.

Famille des ANADYOMENACE $Æ$ (ENDL.) Hauck.

Anadyomene Lamour.

50. Anadyomene stellata (Wulf.) AG., Species Algarum, I (I82I), p. 400 (Anadyomene); Hauck., Meeresalgen (I885), p. 468, fig. 204 ; Ardiss., Phyc. Medit., II, p. I8I ; DE Toni, Sylloge Algarum, I, p. 368.

Ulva stellata WulfeN, in JACQuin, Collectanea, I (I786), p. 35I ; Cryptogama aquatica (I803), p. 6, nº Io (bonne description!).

Anadyomene flabellata Lamouroux, Histoire des polypiers coralligènes flexibles (I8I2), p. 365, tab. x4, fig. 3; Exposition méthodique, p. 3I, tab. 69, fig. I5-I6; KuEtz., Tab. Phyc., VII, tab. 24.

Hab. : Tripoli (A. Trotter).

Déjà rapportée de Tripoli par R. Spigal, cette espèce nous a été signalée de la localité Derna par M. A. MAzzA. Anadyomene stellata est très commune dans la Méditerranée ou elle croît attachée aux grandes Algues, surtout du genre Cystoseira, et aux rhizomes des Zostéracées; elle est aussi suffisamment répandue dans l'océan Atlantique. Elle ne manque pas aux côtes algériennes (MONTAGNe).

T. V. - FASC. 7 . 


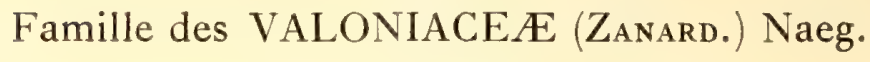

Valonta GIN.

5I. Valonia utricularis (Roth) Ag., Species Algarum, I (I82I), p. 43I; Kuetz., Tab. Phyc., VI,tab. 86, fig. II, b-e ; Ardiss., Phyc. Medit.,II, p. I63 ; DE Toni, Sylloge Algarum, I, p. 376; Kuckuck, in Botanische Zeitung, I907, p. I66, fig. II-I7.

Conferva utricularis Rотн, Catalecta, I (I797), p. I60, tab. I, fig. I.

Hab.: 'Tripoli (A. Trotter).

Récoltée jadis à Tripoli par R. SpIGAI, cette espèce ne manque pas sur le littoral de la Tunisie et de la Cyrénaíque (Piccone). Schousboe la recueillit à Tanger et à Gibraltar. Les auteurs donnent comme aire de répartition géographique du Valonia utricularis la Méditerranée, la mer Rouge et les parties voisines de l'océan Atlantique, Cadix, Madère, les îles Canaries et Bermudes.

M. le prof. A. TRотter nous a communiqué de la même localité aussi des exemplaires d’un singulier organisme animal, qui par les anciens auteurs a été classé parmi les Algues. Il s'agit de la forme figurée par Kuetzing (Tab. Phyc., VI, tab., 89j) sous le nom d'Ascothamnion intricatum Kuetz., auquel correspondent comme synonymes Ulva intricata Clemente Ensayo, p. 329 et Valonia intricata Ag., Species, I (1821) p. 430.

La nature animale de cet singulier organisme avait-elle été reconnue par Delle Chiaje Memorie sulla storiae notomia degli animali senza vertebre del Regno di Napoli, vol. III, p. 203) qui en publia la description sous le nom de Hydra verticillata, changé plus tard par lui-même (Descrizione e notomia degli animali invertebrati della Sicilia citeriore osservati vivi negli anni $1822-1830$, tomo V, p. 142, tomo VI, tab. 79, fig. 1-2 ; Napoli, 184I) en Zoobotryon verticillatum. C'est une espèce très commune dans la Méditerranée, surtout à Naples et à Trieste; Ehrenterg, qui avait des exemplaires de Suez et d'Alexandrie, donna a ce Bryozoaire le nom de Zoobotryon pellucidus (Symbolæ Physicæ, animalia evertebrata, tab. III; Berlin, 1828).

Une illustration très détaillée de cette production marine, qui par son habitus a trompé longtemps les naturalistes, se trouve donnée par Reichert, (Vergleichende anatomische Untersuchungen über Zoobotryon pellucidus Ehrenberg) dans les Abhandlungen der Königlichen Akademie der Wissenschaften ₹u Berlin, 1869, II [1870], p. 233-338, t. I-VI, ouvrage cité aussiavec d'autres publications in DE TonI, Sylloge Algarum, I, p. 38 I.

Famille des CAULERPACE $Æ$ (REIChr.) J. Ag.

Caulerpa Lamour.

52. Caulerpa prolifera (Forsk.) LAmour., in Journal de Botanique, I8og, p. 30 ; DE TonI et Levi, Pugillo di Alghe tripolitane (r888), no 28 ; DE TonI, Sylloge Algarum, I, p.450; ARdiss., Phyc. Medit., II, p. I66; Weber van Bosse, Monographie des Caulerpes (I898), p. 278 ; Reinke, Ueber Caulerpa (I899), p. I2, fig. II.

Fucus prolifer FonskaL, Flora ægyptiaco-arabica (I775), p. I93.

Phyllerpa prolifcra Kuetz, Species algarum (I849), p. 494; Tab. Phyc., VII, tab. 3. 
Hab.: Tripoli (A. Trotter).

Cette espèce, très répandue dans la Méditerranée (elle n'a pas été jusqu'ici récoltée dans la mer Adriatique) des côtes françaises et espagnoles, jusqu'à la Grèce, à Giaffa et à la mer Noire, n'y est toutefois commune qu'en certaines localités (Livourne, Naples, etc.). A Tripoli, elle ne doit pas être rare, puisqu'elle y fut récoltée jadis par R. SPIGar et par M. Corbelli et signalée aussi par M. Piccone à quelques kilomètre du port. Caulerpa prolifera croît aussi en Égypte de Suez (Muschler) à Alexandrie (Areschoug), en Cyrénaïque (Haimann), à Benghazi (Ruhmer) et en Algérie (Montagne, Debray, Weber van Bosse). L'espèce se trouve aussi dans l'océan Atlantique aux côtes américaines (HARvey, J. Agardh) et des îles indo-occidentales (Boergesen).

\section{Famille des SPONGODIACE $Æ$ LAmour.}

Codium STACKн.

53. Codium Bursa (L.) AG., Species Algarum, I (r82I),p. 437; Kuetz.,Tab. Phyc., VI, p. 99, fig. I ; ArcAngeli, Su alcune Alghe del gruppo delle Celoblastee (I874),p. I74, tab. II ; Ardiss., Phyc. Medit., II, p. r69; DE Toni, Sylloge Algarum, I, p. 490.

Alcyonium Bursa L., Systema naturæ, I, p. I295.

Fucus Bursa Turner, Fuci (I8Ir), tab. I36.

Lamarkia Bursa OLIVI, Zoologia Adriatica (I792), p. 258.

Hab. : Tripoli (A. Trotter).

Cette espèce, commune dans la Méditerranée, se trouve aussi dans l'océan Atlantıque depuis l'Angleterre jusqu'aux îles Canaries et à Tanger. Rapportée déjà de Tripoli par M. Corbelli, elle fut signalée aussi çà et là dans le littoral nord-africain du port d'Alexandrie (Muschler) à l'Algérie (Montagne).

Famille des UDOTEACE $Æ($ Endl.) J. Ag.

Halimeda LAmour.

54. Halimeda Tuna (Ell. et Soland.) Lamour., Histoire des polypiers coralligènes flexibles (I8I6), p. 309, tab. XI, fig. 8 ; Kuetz., Tab. Phyc., VII, tab. 2I, fig. 4 ; ZAnard., Iconographia (I876), p. I29 (54I), tab. II2 ; ARdiss., Phyc. Medit., II, p. I74; De Toni, Sylloge Algarum, I, p. 5 I 8 ; Barton-GEPP, The Genus Halimeda (Igor), p. II (avec synonymie très détaillée et distribution géographique). Corallina Tuna Ellis et SolANDER, The Natural History of many curious Zoophytes (I 786 ), p. III, tab. 20, fig. $e$.

Halimeda sertolara ZANARD., Synopsis Algarum in mari adriatico hucusque cognitarum (I84I), p. I24, tab. IV, fig. I.

Hab.: Tripoli (A. Trotter), Tobrouk (A. VACCARI).

Espèce assez répandue dans la Méditerranée, l'océan Atlantique boréal, l'océan Indien, la mer Rouge et l'océan Pacifique; elle est communeà Tripoli (R. Spigai, Piccone, Ardissone), en Cyrénaïque (Haimann), à Benghazi, (Ruhmer), en Algérie (Montagne), en Égypte (Areschoug, R. Muschler). 


\section{CHARACE五 RICH。}

Chara VAILL.

55. Chara aspera (Deth.) Willdenow, Magazin der naturf. Freunde, III (I809), p. 298 ; Migula, Synopsis Characearum europæarum (I898), p. I40; Die Characeen, p. 653.

Hab.: Tadjoura (A. Trotter).

L'espèce est déjà connue pour la flore africaine.

56. Chara crinita Wallroth, Annus botanicus, I8I5, p. I90; A. Braun, Die Characeen Afrikas (I867), p. 829 ; Migula, Synopsis Characearum Europæarum (I898), p. 83; Die Characeen, p. 348.

Hab. : Tadjoura (A. TROTteR).

Comme l'espèce précédente, Chara crinita Wallr. est déjà connue pour le continent africain, dans les eaux douces et légèrement salées.

\section{GLAUCOPHYCEÆ HIER.}

Goniotrichum KUETZ.

57. Goniotrichum elegans (Chauv.) Zanardini, Notizie sulle Cellulari marine delle Lagune venete (I842), p. 69 ; Iconographia phycologica, III, p. 67, tab. 46, A; A. ForTi, Myxophyceæ, in DE Toni, Sylloge Algarum, V, p. 687 .

Bangia elegans Chauvin, Algues de Normandie, n⿳0 I59; HaRvey, Phycologia Britannica (I849), tab. 246.

Hab.: Benghazi (A. VACCARI).

Cetteespèce épiphytique sur les Algues et les Zostéracées n'est pas rare; pour le littoral africain, elle fut signalée de Tanger(Schousboe, Bornet) et de Raz-Aknatar près Guyotville, Bab-el-Oued (Debray).

\section{MYXOPHYCEÆ (WALLr.) Stiz.}

Famille des RIVULARIACE E (MEN.) Kirchn.

Calothrix Ag.

58. Calothrix aruginea (Kuetz.) Thuret, Essai de classification des Nostochinées (I875), p. 382 ; BoRnet et Thuret, Notes algologiques, p. I57, pl. 37; A. Forri, Myxophyceæ, in DE Toni, Sylloge Algarum, V, p. $6 \mathrm{I} 2$.

Leibleinia aruginea Kuetzing, Phycologia generalis (I843), p. 22I; Species Algarum (I849), p. 276; Tab. Phyc., I, p. 46, tab. 83, fig. I.

Hab. : sur Polysiphonia, Benghazi (A. VACCARI).

Cette espèce a une large répartition géographique. Pour le littoral nord-africain, M. Debray l'a indiquée sur les Zostères, à Tipaja, Kouali. 


\section{PERIDINIE⿸ (EHR.)}

Famille des PROROCENTRACEA (SchuetT).

\section{Exuviaella Cienk.}

59. Exuviaella Lima (EHr.) Buetschli, Dinoflagellata [Bronns Klassen und Ordnungen des Thier-Reichs] (I885) tab. 5I, fig. 2 ; Paulsen [Peridiniales K. Brandt et C. Apstein, Nordisches Plankton] (Igo8), p. 5, fig. I.

Cryptomonas Lima EHRENBERG, Ueber das Leuchten und über neue mikroskopische Leuchthiere des Mittelmeeres (I859), p. 793; Ueber das Funkeln und Aufblitzen des Mittelmeeres bewirkenden unsichtbar kleinen Lebensformen (I873), fig. 24-25.

Hab.: Benghazi (A. VACCARI).

Espèce néritique, connue pour le bassin méditerranéen et pour la mer Adriatique (Br. Schroeder).

\section{BACILLARIEÆ (NITZSCH).}

\section{IMMOBILES ( $\mathrm{I})$.}

\section{Famille des MELOSIRACE E (Kuetz.).}

\section{Melosira AG.}

6o. Melosira Roeseana RABEHн., Algen, $\mathrm{n}^{\mathrm{0s}} 382$ (I852), et 504 ; Süsswasser Diatomaceen, p. I3, tab. IO, suppl., fig. 5; Flora Europæa Algarum. I, p. 42 ; VAN HEURck, Synopsis, p. I99, tab. 89, fig. I-6; DE Toni, Sylloge Algarum, II, p. I337; DE Toni-Forti, Alghe spediz. Ruwenzori, p. 30. Orthosira spinosa Greville, in Ann. Sc. Nat., sér, 2, vol. XV, tab. 4, fig. I4-I7.

Hab.: Lavages des Chara, Tadjoura (A. Trotter).

Cette espèce caractéristique, appartenant à la section Lyparogyra du genre Melosira, n’a pas été récoltée jusqu'à ce jour dans l'Afrique du Nord ; ellefut rapportée par l'expédition du Ruwenzori de S. A. R. le duc des Abruzzi et est adaptée aussi à la vie aérienne, c'est-à-dire à se développer sur les rochers humides.

\section{Hyalodiscus EHR.}

6r. Hyalodiscus radiatus (O'MEARA) Grunow, in Cleve et Grunow, Beiträge zur Kenntniss der arktischen Diatomeen (I880), p. II7; DE ToNi, Sylloge Algarum, II, p. I367.

Pyxidicula radiata O'MEARA, in Linnean Society Journal, Botany, vol. XV, p. 58, tab. I, fig. 9.

Hab.: Lavages des Algues, Tripoli (A. Trotter).

Pour le bassin de la Méditerranée, l'espèce est indiquée du golfe de Naples (sondages). Comp. Tempére et Peragallo, Diatomées du monde entier, p. 4o, n. 73-75.

(1) Pour la disposition des Bacillariées immobiles, nous avons adopté la classification proposée par M. FoRTI (Contribızioni Diatomologiche XII. Metodo di classificazione delle Bacillariee immobili fondato sull'affinità morfologica dei frustoli ed in relazione con l'evoluzione dell'auxospora; Atti $R$. Ist. Veneto, t. LXXI, part. II, I9I2, p. 677731 ). 
62. Hyalodiscus stelliger BaIley, Notes on new Species and Localities of microscopical organisms (I853), p. 10 ; VAn HeUrck, Synopsis, p. 213, tab. 84, fig. I-2 ; DE Toni, Sylloge Algarum, II, p. I367.

Hab. : Lavages des Algues, Zostéracées et Éponges, Tripoli (A. TrotTER); Benghazi (A. VACCARI).

Comme l'espèce précédente, Hyalodiscus stelliger Bail. n'était pas connu pour le littoral nord-africain.

63. Hyalodiscus subtilis BAILEY, Notes on new Species and Localities of microscopical organisms (I853), p. ro, fig. I2 ; VAn Heurck, Traité des Diatomées, p. 449 ; De Ton, Sylloge Algarum, II, p. I366.

Hab.: Lavages des Algues, Benghazi (A. Vaccari).

Espèce nouvelle pour le littoral nord-africain.

Pyxidicula EHr.

64. Pyxidicula mediterranea Grunow, in VAN HeUrck, Synopsis, tab. 95, fig. I5-I6; Traité des Diatomées (I899), p. 510, fig. 258.

Hab.: Lavages des Algues, Zostéracées et Éponges, Tripoli (A. Trotter); entre les Cystoseira, Tobrouk (A. VACCARI), Benghazi (A. VACCARI).

L'espèce fut observée la première fois dans des récoltes de Diatomées provenant de l'Adriatique supérieure; et son aire de répartition géographique a été augmentée (golfe de Juan, etc.).

\section{Endictya EHr.}

65. Endictya oceanica EHr., in Berichte d. Berliner Akad., 1845, p. 76; Mikrogeologie, tab. 35 A, fig. XVIII, 6-7 ; A. Schmidr, Atlas, tab. 65, fig. IO-I5; VAN Heurck, Traité des Diatomées, p. 445, fig. I69; DE Toni, Sylloge Algarum, II, p. II 89.

Orthosiva oceanica BRIGHTwell, On some of the rarer or undescribed species of Diatomaceae (I860), p. 96, tab. 6, fig. I6.

Hab.: Lavages des Algues, Zostéracées et Éponges, Tripoli (A. TrotTer); Benghazi (A. VACCARI).

Ehrenberg a décrit son espèce sur des exemplaires observés dans le guano de la baie de Saldanha (Afrique du Sud); puis l'espèce même fut rencontrée fossile dans les dépôts de Bory, Nagy-Kürtös en Hongrie, par Pantocsek. Brébisson (1872) signala la présence d'Endictya oceanica dans la mousse de Corse ; puis on l'observa dans plusieurs localités de la Méditerranée et de la mer Adriatique (Nice, Villefranche, Cannes, golfe Juan, à l'embouchure du Var, Lissa, etc.).

Famille des RHIZOSOLENIACE E P. Petit.

Dactyliosolen CASTR.

66. Dactyliosolen mediterraneus H. PERAG., Monographie du genre Rhizosolenia et de quelques genres voisins, p. I04, tab. I, fig. 8-9.

Landeria mediterranea H. Peragallo, Diatomées de la baie de Villefranche (r888), p. 8I, tab. 6, fig. 45 ; DE TONI, Sylloge Algarum, II, p. 77 I. 
Hah. : Benghazi (A. VACCARI).

Nous avons rencontré seulement des fragments de ce Dactyliosolen, qui, étant planctonique, est sans doute accidentel dans les matériaux rapportés de Benghazi.

L'espèce est suffisamment répandue dans la Méditerranée (Peragallo, B. Schroeder).

Famille des COSCINODISCACE E (Kuetz.).

Coscinodiscus EHR.

67. Coscinodiscus excentricus EHR., in Abhandlungen d. Berlin. Akad., I839, p. I46; Mikrogeologie, tab. I8, fig. 32, tab. 2I, fig. 6 ; A. Schmidt, Atlas, tab. 58, fig. 46-49; VAN Heurck, Synopsis, p. 2I7, tab. I30, fig. 4, 7-8 ; RATTRAY, A Revision of the genus Coscinodiscus (I890), p. I4 (avec répartition géographique très détaillée); DE ToNI, Sylloge Algarum, II, p. I2IO.

Hab.: Lavages des Algues, Zostéracées et Éponges, Tripoli (A. Trotter).

Espèce à large répartitıon géographique, connue aussi à l'état fossile dans plusieurs dépôts, par exemple dans le Tripoli d'Oran (P. PETIT); on l'a trouvée dans les sondages du port d'Alger(Tempère, Peragallo).

68. Coscinodiscus nitidus GRÉGORY, On new Forms of marine Diatomaceæ, found in the Firth of Clyde and in Loch Fine (I857), p. 499, no 45, tab. X, fig. 45 ; A. Schmidt, Atlas, tab. 58, fig. I8 ; Rattray, Op. cit., p. 30 (id.) ; DE TONI, Sylloge Algarum, II, p. I22I.

Hab.: Lavages des Algues, Tripoli (A. Trotter), Benghazi (A. Vaccari).

Comme l'espèce précédente, Coscinodiscus nitidus Greg. a été rapporté de plusieurs localités les plus éloignées aussi dans le bassin de la Méditerranée; Tempére et Peragallo l’ont signalé dans les sondages du port d'Alger.

Fainille des HELIOPELTACE E H. L. Sмith.

Actinoptychus EHR.

69. Actinoptychus vulgaris Schumann, Die Preussischen Diatomeen (I867), p. 64; DE TonI, Sylloge Algarum, II, p. I384.

Actinoptychus sp. EHrenberg, Mikrogeologie, tab. I8, fig. 22-30, etc.

Hab. : Benghazi (A. VACCARI).

Espèce vivant dans les eaux marines, dont on a distingué plusieurs variétés.

Famille des EUPODISCACE正 (Kuetz.).

Actinocyclus EHr.

70. Actinocyclus crassus VAN HeUrck, Synopsis, p. 215, tab. I24, fig. 6,8 (vix Eupodiscus crassus W. Sm.) ; Van Heurck, Traité des Diatomées (I909), p. 524, tab. 34, fig. 897 ; DE Toni, Sylloge Algarum, II, p. II69.

Hab. : Lavages des Algues, des Zostéracées et des Éponges, Tripoli (A. Trotter).

M. Tempere el Peragallo (Diatomées du monde entier, p. 23o, n 438) ont signalé Actinocyclus Ehrenbergii Ralfs dans les sondages du port d'Alger. 
7I. Actinocyclus subtilis (Greg.) Ralfs, in Pritchard History of Infusoria, p. 835 ; Van Heurck, Synopsis, p. 216, tab. 124, fig. 7; Traité des Diatomées, p. 524, tab. 23, fig.66r; DE TonI, Sylloge Algarum, II, p. II 83 .

Eupodiscus subtilis Gregory; On new Forms of Marine Diatomaceae, found in the Firth of Clyde and in Loch Fine (1857), p. 50I, no 50, tab. XI, fig. 50.

Hab.: Lavages des Algues, des Zostéracées et des Éponges, Tripoli (A. Trotter); lavages des Cystoseira, Tobrouk (A. VAcCari), Benghazi (A. VAccari).

Espèce connue à l'état vivant (marine) et fossile; elle n'est pas rare dans le bassin de la Méditerrannée à partir de l'Adriatique supérieure et jusqu'aux littoraux de la Sicile et de l'Espagne; on l'a signalée dans la Mousse de Corse.

\section{Aulacodiscus EHR.}

72. Aulacodiscus Petersii Ehr., in Abhandl. d. Berlin. Akad., 1845, p. 36r ; A. Schmidr, Atlas, tab. 35, fig. 4, tab. 4I, fig. I-2 ; RatTray, A Revision of the genus Aulacodiscus (I888), p. 365 ; DE TonI, Sylloge Algarum, II, p. III5.

Hab. : Lavages d'Algues, Homs (A. Trotter).

Notre forme est tétramère (on peut comparer la figure donnée par Schmid, Atlas, tab. 162, fig. 8: exemplaire de Naples), tandis que celle illustrée par Peragallo (Diatomées de France, pl. CXII, fig. I : exemplaire de Menton) est pentamère. L'espèce n'est pas nouvelle pour la Méditerranée, puisqu'elle y fut rencontrée par M. Castracane (Atti dell' Accademia Pontificia dei Nuovi Lincei, t. XLII, I889) parmi d'autres Diatomées contenues dans l'appareil digestif d'un Holothuria provenant du golfe de Naples, où Aulacodiscus Petersii Ehr. paraît suffisamment commun, sa présence dans ce golfe ayant été confirmée parle diatomologue M. Barbò (qui en prépara des Typenplattes), par M. Schroeder et par MM. Tempére et Peragallo qui l'ont indiqué dans la même région, outrè qu'à Menton sur les côtes françaises (Tempère et Peragallo, Diatomées du monde entier, p. 39, nos $73-75)$.

Famille des BIDDULPHIACE $Æ$ (Kuetz.) Heiberg.

Biddulphia GraY

73. Biddulphia pulchella Gray, A Natural Arrangement of British Plants, I, p. 294 ; Grev.,Descriptions of New and Rare Diatoms, series V (I862), p. 25, tab. 3, fig. 3-4 ; VAN Heurck, Synopsis, p. 204, tab. 97, fig. I-3; Traité des Diatomées, p. 470, fig. I97, tab. 20, fig. 630 ; DE. TonI, Sylloge Algarum, II, p. 870 (avec synonymie très détaillée).

Hab. : Lavages des Algues, des Zostéracées et des Éponges, Tripoli, Homs (A. TrotTER), Benghazi (A. VACCARI).

Espèce très commune aussi dans la Méditerranée; sur le littoral nord-africain (elle vit aussi à Tanger), on la signala de plusieurs localités: Cap Bon (Lanzi), Alger (Debray, Tempère et Peragallo), Cherchell (Montagne), Arzew (Leuduger-Fortmorel).

74. Biddulphia aurita (LyngB.) Bréb., Considérations sur les Diatomées (I838), p. I2 ; Van Heurck, Synopsis, p. 205, tab. 98, fig. 4-9; Traité des Diatomées, p. 47I, tab. 20, fig. 63I. 
Diatoma auritum LyNGByE, Hydrophytologia (I8I9), p. I82, tab. 62, fig. D.

Odontella aurita AG., Conspectus (I832), p. 56 ; DE TonI, Sylloge Algarum, II, p. 862.

Hab.: Benghazi (A. VAcCARI).

Espèce nouvelle pour le littoral nord-africain, signalée déjà pour la Méditerranée (côtes de Sicile); on peut rappeler ici que MM. Tempère et Peragallo ont distribué B. aurita dans les numéros r 52 - 53 (Village Nègre, Oran) des Diatomées du monde entier.

\section{Triceratium (EHR).}

75. Triceratium antediluvianum (EHR.) GRUNOw, Algen Novara (I867), p. 24.

Biddulphia antediluviana VAN HEURCK, Synopsis, p. 207, tab. rog, fig. 4-5 ; Traité des Diatomées, p. 475, tab. 2I, fig. 642 .

Amphitetras antediluviana EHR., Lebende Thierarten der Kreidebildung (I840), p. 62 ; RALFs, in Annals and Magazines of natural History, vol. XII, tab. VIII, fig. 5 ; KuetzInG, Die Kieselschaligen Bacillarien (I844), p. I35, tab. I9, fig. III, tab. I9, fig. 86; A. Schмidt, Atlas, tab. 9o, fig. I-4, 6-9; DE TonI, Sylloge Algarum, II, p. 899.

Hab.: Lavages des Algues, Zostéracées et Éponges, Tripoli (A. TrotTER); lavages des Cystoseira, Tobrouk (A. VACCARI), Benghazi (A. VACCARI).

L’espèce, pas rare dans la Méditerranée (golfe de Naples, golfe Juan, embouchure du Var, Menton, Cannes, Villefranche, Catane, etc.), fut rencontrée aussi dans le littoral nordafricain au golfe de Gabès (LAnzi). Elle vit aussi à Tanger (Schousboe).

76. Triceratium balearicum Cleve et Grunow, in Cleve et Moeller, Diat., no I54-I55; Cleve, On some new or little known Diatoms (I88I), p. 25.

Amphitetras balearica DE ToNI, Sylloge Algarum, II, p. 902.

Hab. : Benghazi (A. VACCARI).

Cette belle espèce se trouve dans la Méditerrannée aux côtes des îles Baléares où la récolta M. F. Soederlund.

\section{Isthmia AG.}

77. Isthmia nervosa KuETZ., Die Kieselschaligen Bacillarien (I844), p. I37, tab. XIX, fig. 5; A. SchmidT, Atlas, tab. 135, fig. I-6, tab. I45, fig. II; De Toni, Sylloge Algarum, II, p. 833; VAN HeURck, Traité des Diatomées, p. 452, tab. 34, fig. 891.

Hab. : Lavages des Algues, Tripoli (A. TROTTER).

Isthmia nervosa Ag. est une espèce connue tant à l'étant vivant qu'à l'état fossile; elle n’avait pas jusqu’ici été signalée pour le littoral nord-africain.

Famille des STRIATELLACEÆ (Kuetz.) Heiberg.

Grammatophora EHR.

78. Grammatophora angulosa EHR., Kreidethiere (1840), p. 73, no 44; Mikrogeologie, tab. XVIII, plur. fig.; VAN Heurck, Synopsis, p. I64; Traité des Diatomées, p. 355, tab. 3I, fig. 862.

Hab. : Benghazi (A. VACCARI).

T. V. - FAsC. 7 . 
Var. hamulifera (Kuetz.) Grunow, Die Oesterreichischen Diatomaceen (I862), p. 4I9; VAN Heurck, Synopsis, tab. 53, fig. 4; Traité des Diatomées, p. 355, tab. XI, fig. 48I $a$; DE Toni, Sylloge Algarum, II, p. 757 .

Grammatophora hamulifera Kuetzing, Die Kieselschaligen Bacillarien (1844), p. I28, tab. I7, fig. 23.

Hab. : Lavages des Cystoseira, Tobrouk (A. VACCARI).

Grammatophora angulosa Ehr. est surtout commune dans la mer Adriatique; la variété hamulifera serait plus rare; la forme typique fut déjà signalée pour le littoral nordafricain par plusieurs auteurs (DEBRAY, L.ANZI, etc.); on la trouve aussi à l'état fossile dans le Tripoli d'Oran.

79. Grammatophora gibberula KuETz., Die Kieselschaligen Bacillarien (I844), p. I29, tab. 30, fig. 8I; DE TONI, Sylloge Algarum, II, p. 75I.

Hab. : Benghazi (A. VAccari).

Cette espèce, pas rare dans la Méditerranée (Naples, Dalmatie, etc.) représente avec toute probabilité une simple variété du Grammatophora marina (Lyngb.) Kuetz.

80. Grammatophora longissima Petit, Catalogue des Diatomées de l'île Campbell et de la Nouvelle-Zélande (I877), p. 34, tab. 5, fig. 2I ; DE TONI, Sylloge Algarum, II, p. 758.

Hab.: Lavages des Algues, Tripoli (A. Trotter).

M. l'abbé F. Castracane proposa (I880) une variété italica, qu'il observa dans quelques récoltes faites à l'île de Lesina et à Piombino.

81. Grammatophora marina (Lyngb.) Kuetzing, Die Kieselschaligen Bacillarien (I844), p. I28, tab. XVII, fig. XXVI, I-6; VAn Heurck, Synopsis, p. I63, tab. 53, fig. Io-II; Traité des Diatomées, p. 354, tab. XI, fig, 479; DE TonI, Sylloge Algarum, II, p. 752.

Diatoma marimum LyngBye, Tentamen Hydrophytologiæ Danicæ (I8I9), p. I8o, tab. 62 A.

Hab. : Lavages d'Algues, de Zostéracées et d'Éponges, Tripoli, Homs (A. Trotter); lavages des Cystoseira, Tobrouk (A. VACCARI).

Espèce à large répartition géographique, très commune dans le bassin de la Méditerranée, où elle fut déjà signalée à plusieurs reprises le long du littoral africain comme à Arzew (Leuduger-Fortmorel), Tipaza, Alger (Debray), La Calle (Montagne), Cap Bon et golfe de Gabès (Lanzi). La Grammatophora marina se trouve aussi à Tanger (Sçrousboe). Entremêlées à la forme typique, on rencontre dans les lavages d'Algues de Tripoli, Tobrouk et Benghazi les variétés tropica (Kuetz.), adriatica et undulata (Ehr.).

82. Grammatophora oceanica EHR., Mikrogeologie (I854), tab. XIX, fig. 36 , et al. icon.; DE TonI, Sylloge Algarum, II, P. 755 .

Grammatophora marina KuETz., Die Kieselschaligen Bacillarien (I844), tab. I8, fig. I, I-5 (nec al. icon.).

Hab.: Lavages d'Algues, de Zostéracées et d'Éponges, Tripoli, Homs, Tobrouk, Benghazi (A. Trotter, A. VACCARI).

Avec la forme genuina, sont entremêlées les variétés communis Grun., macilenta (W. Sm.) Grun., subtilissima Bail. et minutissima. L'espèce a été déjà signalée pour le littoral nord-africain. 
83. Grammatophora serpentina (RALFs) EHR., in Berichte der Berlin. Akad., I844; W. Smith, Synopsis of the British Diatomaceæ, II (I856), p. 43, tab. XLII, fig. 315; VAN HeURck, Traité des Diatomées, p. 355, tab. XI, fig. 482 ; DE TONI, Sylloge Algarum, II, p. 757.

Striatella taniaformis et serpentina RALFs, in Annals and Magazines of Natural History, XI, tab. IX, fig. $5 \beta$.

Hab. : Lavages d'Algues, Zostéracées et Éponges, Tripoli, Homs (A. Trotter).

Espèce très largement distribuée dans les mers et les océans, trouvée aussi à l'état fossile; elle fut déjà rencontrée sur le littoral nord-africain à Tipasa (Debray), à Alger (Tempère, Peragallo), au Cap Bon et au golfe de Gabès (Lanzi).

\section{Rhabdonema KuETz.}

84. Rhabdonema adriaticum Kuetzing, Die Kieselschaligen Bacillarien (I844), p. I26, tab. I8, thg. , , W. SMith, Synopsis of the British Diatomaceæ, II (I856), p. 35, tab. 38, fig. 305 ; VAN HeUrck, Synopsis, p. I66, tab. 54, fig. II-I3; A. Schmidt, Atlas, tab. 2I7, fig, I7-26; De Toni, Sylloge Algarum, II. p. 764 .

Hab. : Lavages des Algues, des Zostéracées et des Éponges, Tripoli, Homs (A. TrotTER) ; lavages des Cystoseira, Tobrouk (A. VACCARI); Benghazi (A. VACCARI).

Espèce très commune, aussi dans la Méditerranće; sur le littoral nord-africain on la signala d'Algérie (Montagne), de Oued-Raha, Cap-Bon, golfe de Gabès (Lanzi). Le Tripoli d'Oran contient bon nombre de frustules du Rhabdonema adriaticum Kuetz.

Striatella AG.

85. Striatella delicatula (Kuetz.) Grunow, in VAN Heurck, Synopsis, tab. 54, fig. 5-6; Traité des Diatomées, p. 363, tab. I2, fig. $483 a$; DE Toni, Sylloge Algarum, II, p. 769.

Hyalosira delicatula KUETz., Die Kieselschaligen Bacillarien (I844), p. I25, tab. I8, fig. III, I.

Hyalosira minutissima, obtusangula, rectangula, KuEtz., ibidem.

Hab. : Lavages des Zostéracées et d'Éponges, Tripoli (A. Trotter).

Espèce qui ordinairement croît sur les Algues filamenteuses, très répandue dans le bassin de la Méditerranée.

86. Striatella unipunctata (LyngB.) AG., Conspectus criticus Diatomacearum (I832), p. 6I; KUETZING, Die Kieselschaligen Bacillarien (I844), p. I25, tab. I8, fig. V, I-4, tab. 24, fig. VI, fig. $3 a-b$; VAN HEURCK, Synopsis, p. I65, tab. 54, fig. 9-I0; Traité des Diatomées, p. 363, tab. I2, fig. 485 a; DE Tonr; Sylloge Algarum, II, p. 766.

Fragilaria unipunctata LYNGBYE, Tentamen Hydrophytologiæ Danicæ (I8I9), tab. 62 G.

Tessella pedicellata DuJARDin, Histoire naturelle des Zoophytes Infusoires (I84I), p. 670, tab. XX, fig. I4,

Hab. : Lavages d'Algues, Tripoli(Trotter); Benghazi (A. VAccari).

Espèce commune dans la Méditerranée; sur le littoral nord-africain, ellea été signalée à Alger (DEBRAY).

\section{Tessella EHr.}

87. Tessella interrupta EHR., Die Infusionsthierchen (1838), p. 202; KuETzING, Die Kieselschaligen Bacillarien (I844), p. I25, tab. I8, fig. IV, I-2 ;

Striatella interrupta HEIBERG, Conspectus criticus Diatomacearüm Danicarum (I864), p. 73, tab. 5, 
fig. I5 ; VAN Heurck, Synopsis, p. I65, tab. 54, fig. 8 ; Traité des Diatomées, p. 363, fig. II5 (Tessella interrupta), tab. I2, fig. $484 a$; DE TONI, Sylloge Algarum, II, p. 768.

Hab.: Lavages d'Algues, Tripoli (A. Trotter); lavages des Cystoseira, Tobrouk (A. VACCARI), Benghazi (A. VACCARI).

Tessella interrupta Ehr. a une répartition géographique suffisamment étendue, même dans les mers extra-européennes. Sur le littoral africain de la Méditerranée, elle n'est connue jusqu'ici que d'Alger (Debray) et du Cap Bon (Lanzi).

\section{Famille des LICMOPHORACEÆ Kuetz.}

\section{Licmophora AG.}

88. Licmophora Ehrenbergii (Kuetz.) Grunow, in Hedwigia, I867, p. 36; Van Heurck, Synopsis, tab. 470, fig. I0-II; Traité des Diatomées, p. 344, tab. 3I, fig. 853; DE TonI, Sylloge Algarum, II, p. 734. Podosphenia Ehrenbergii Kuetzing, Die Kieselschaligen Bacillarien (I844), p. I2I, tab. 9, fig. XIII, tab. 24, fig. I.

Podosphenia cuneata EHr., Infusionsthierchen (1838), tab. XVII, fig. VIII (non Echinella cuneata LYNGBYe, I8r9).

Hab. : Lavages des Algues, des Zostéracées et des Éponges, Tripoli (A. Trotter); lavages des Cystoseira, Tobrouk (A. VACCARI).

Espèce commune à l'océan Atlantique et à la Méditerranée.

Var. ovata (W. Sm.) Van Heurck, Traité des Diatomées (I899) p. 344, tab. 3I, fig. 855.

Licmophora ovata GRun., in Hedwigia, I867, p. 36 ; VAN HeurcK, Synopsis, tab. 47, fig. I2 ; De Toni, Sylloge Algarum, II, P. 734.

Podosphenia ovata W. SMITH, Synopsis of the British Diatomaceae, I (I853) p. 83, tab. 24, fig. 226.

Hab. : Lavages des Algues, Tripoli (A. TRотTER).

Cette variété n'a pas été signalée jusqu'à ce jour sur le littoral méditerranéen de l'Afrique; elle est commune dans la mer Adriatique et sur les côtes de Sicile.

89. Licmophora flabellata (CARM.) AG., Conspectus criticus Diatomacearum (I83I), p.4I; VAN HeURCK, Synopsis, tab. 46, fig. 2-3; Traité des Diatomées, p. 342, tab. 3I, f. 852; DE TonI, Sylloge Algarum, II, p. 73 I.

Echinella flabellata Carmichael (sec. Greville), Ehrenberg, Die Infusionsthierchen (I838), p. 220, tab. 9, fig. I.

Hab. : Lavages des Cystoseira, Tobrouk (A. VACCARI).

Licmophor a flabellata (Carm). Ag. est une espèce commune dans la Méditerranée. Elle a été signalée, sur le littoral africain, à Alger (Debray).

90. Licmophora Lyngbyei (Kuetz.) Grunow, in Hedwigia, I867, p. 35; Van Heurck, Synopsis, p. 158, tab. 46, fig. I, tab. 47, fig. I6-I9; Traité des Diatomées, p. 344, tab. XI, fig. 460; DE TonI, Sylloge Algarum, II, p. 734 .

Podosphenia Lyngbyei KuETz., Die Kieselschaligen Bacillarien (I844), p. I2I, tab. 9, fig. X, 3, tab. I0, fig. I-2.

Echinella cuneata Lyngbye, Tentamen Hydrophytologiæ Danicæ (I8I9), tab. 70, F (non Podosphenia cuneata EHr.). 
Hab. : Lavages d'Algues, Homs (A. Trotter), Benghazi (A. Vaccari).

Espèce commune dans l'océan Atlantique et dans la Méditerranée ; sur le littoral nord-africain, elle est connue des environs d'Alger (Debray).

9I. Licmophora tincta (AG.) Grunow, in Hedwigia, I867, p. 35 ; VAN Heurck, Synopsis, tab. 48, fig. I3-I5 ; Traité des Diatomées, p. 344, tab. 3x, fig. 586 ; DE Tonı, Sylloge Algarum, II, p. 736.

Gomphonema tinctum AG., Conspectus, p. 35 .

Hab. : Benghazi (A. VACCARI).

Espèce épiphyte sur plusieurs Algues, très répanđue dans la Méditerranée et dans l'océan Atlantique.

Climacosphenia EHr.

92. Climacosphenia elongata BAILEY, Notes on new Species and Localities of Microscopical Organisms (I853), p. 8, tab. I, fig. Io-II ; GRUNow, Die Oesterreichischen Diatomaceen, I (I862), p. 35I, tab.6, fig. 22, a-d; De TonI, Sylloge Algarum, II, p. 739.

Hab. : Lavages des Algues, Tripoli (A. Trotter), Benghazi (A. Vaccari).

Bailey, qui avait distribué cette espèce sous le nom de Climacosphenia ramosa, reconnut que le caractère du pédicelle rameux est tout à fait secondaire et changea le nom en Climacosphenia elongata.

L'espèce est commune dans la mer Rouge et l'océan Atlantique; elle paraît nouvelle pour le littoral nord-africain.

Famille des FRAGilariacE E (Kuetz.) V. Hk.

Fragilaria LyngB.

93. Fragilaria construens (EHr.) Grunow. Var. Venter Grunow, in VAN Heurck, Synopsis (r880), p. I56, tab. 45, fig. 2r B, 22-23, $24 \mathrm{~B}, 26$ (fig. superior et inferior); Traité des Diatomées, p. 325, tab. II, fig. 45 I ; DE TonI, Sylloge Algarum, II, p. 689.

Hab. : Eaux douces, à Tadjoura(A. Trotter).

Grunow décrivit cette petite variété sur des exemplaires récoltés à Bruxelles par M. Delogne.

94. Fragilaria hyalina (KuEtz.) Grunow, Die Oesterreichischen Diatomaceen, I (r862), p. 374 (var. major); VAN Heurck, Synopsis, p. I55, tab. 44, fig. I4-I5; Traité des Diatomées, p. 324, tab. II, fig. 443 ; DE TonI, Sylloge Algarum, II, p. 682.

Diatoma hyalinum Kuetzing, Die Kieselschaligen Bacillarien (I844), p. 47, tab. I7, fig. 20.

Hab. : Lavages d'Algues, Homs (A. Trotter).

Espèce commune dans la Méditerranée; dans les mêmes préparations microscopiques des lavages d'Algues provenant de Homs, on trouve des frustules plus petites qu'on peut rapporter à la variété vitrea (Kuetz.).

\section{Cymatosira Grun.}

95. Cymatosira Lorenziana GRunow, Die Oesterreichischen Diatomaceen, I (I862), p. 378, tab. 7, fig.25; VAN HeURCK, Synopsis, tab. 45, fig. 42 ; DE TonI, Sylloge Algarum, II, p. 697. 
Hab. : Lavages d'Algues, Tripoli, Homs (Trotter), Benghazi (A. Vaccari).

Cette Cymatosira fut récoltée la première fois benthonique dans le golfe du Quarnero (Adriatique supérieure), pendant l'hiver de i 858, par M. le Dr Lorenz.

\section{Famille des SYNEDRACEA (Rabenh.).}

\section{Synedra EHr.}

96. Synedra affinis Kuetz., Die Kieselschaligen Bacillarien (I844), p. 68, tab. I5, fig. VI, XI, tab. 24, fig. I, 5 ; VAn Heurck, Synopsis, p. I53, tab. 4I, fig. I3; Traité des Diatomées, p. 324, tab. Io, fig. 430 ; DE Toni, Sylloge Algarum, II, p. 66I.

Hab. : Lavages d'Algues, Tripoli, Homs (A. Trotter); lavages des Cystoseira, Tobrouk (A. VACCARI).

Espèce très répandue surtout dans les eaux saumâtres et littorales. Pour la flore nordafricaine, Synedra affinis a été signalé à Oued-Tiout, à Oued-Taria (Belloc) et à Bou-Saâda, Hodna, oasis de Tougourt (Belloc). Dans les lavages d'Algues de Tripoli, et Benghazi, on trouve quelques exemplaires dont les frustules sont plus petites (var. parva Van Heurck, à laquelle on peut rapporter la Synedra gracilis Kuetz.).

97. Synedra pulchella KuEtz., Die Kieselschaligen Bacillarien (I844) p. 68, tab. 29, fig. 37; VAN HEURCK, Synopsis, P. I49, tab. 40, fig. 27 ; tab. 4I, fig. I (forma major!) ; Traité des Diatomées, p. 309, tab. Io, fig. 403 (forma major); DE TonI, Sylloge Algarum, II, p. 45I.

Hab. : Lavages d'Algues, Homs (A. Trotter).

Il paraît que cette espèce est adaptée à la vie promisque, car on l'a signalée dans les eaux marines, saumâtres et douces. La forme typique du Synedra pulchella fut rencontrée sur les plantes aquatiuues à Oued-Massine (Debray), à Sidi Yahia (Belloc), à Oued Rahâ (Lanzi), à Constantine (Tempère et Peragallo).

98. Synedra Ulna (Nitzsch) EHr., Var. subcqualis Grunow, in VAN Heurck, Synopsis, p. I5I, tab. 38, fig. I3 ; Traité des Diatomées, p. 3Io, tab. I0, fig. 4II ; DE TonI, Sylloge Algarum, II, p. 654.

Hab. : Tadjoura (A. TROTTER).

Var. longissima (W. SM.) BRUn, Diatomées des Alpes et du Jura, p. I20, tab. 5, fig. 8; VAN HeUrck, Synopsis, p. I5I, tab. 38, fig. 3 ; Traité des Diatomées, p. 3I0, tab. I0, fig. 4I2 ; DE ToNI, Sylloge Algarum, II, p. 654 .

Hab. : Benghazi (A. VACCARI).

Cette variété est adaptée aux eaux douces et saumâtres et n'est pas rare le long des littoraux; elle a été signalée à Sidi-M' Sid, Chott-el-Fedjedi (Belloc, Petit).

Ardissonia De Not.

99. Ardissonia Baculus (GREg.) Grunow, in Cleve et Grunow, Beiträge zur Kenntniss der arktischen Diatomeen (I880), p. I08; VAN HEurck, Synopsis, tab. 42, fig. 9; Traité des Diatomées, p. 3I6, tab. 30, fig. 835 (Synedra); DE Toni, Sylloge Algarum, II, p. 676.

Synedra Baculus Gregory, in Trans. Microsc. Soc., I867, p. 88, tab. I, fig. 54. 
Hab. : Lavages des Cystoseira, Tobrouk, Benghazi (A. VACCARI).

Espèce exclusivement marine, pas rare dans les sondages (France, Dalmatie, etc.), connue du litoral nord-africain sur les côtes d'Algérie (Debray); en dehors de la Méditerranée, Ardissonia Baculus se trouve dans l'océan Atlantique.

I00. Ardissonia crystallina (AG.) GRUNow, in ClEve et GRunow, Beiträge zur Kenntniss der arktischen Diatomeen (I880), p. IO8; VAN HEURCK, Synopsis, tab. 42, fig. Io ; DE ToNi, Sylloge Algarum, II, p. 673.

Diatoma crystallinum AG., Conspectus criticus Diatomacearum (I83I), p. 52.

Diatoma crystallinum Kuetz., Die Kieselschaligen Bacillarien (I844), p. 69, tab. I6, fig. I; VAN HEuRck, Traité des Diatomées, p. 3I5, tab. Io, fig. 435.

Hab. : Lavages des Algues, Tripoli (A. Trotter), Benghazi (A. Vaccari).

Espèce vivant sur les Algues, sur lesquelles elle est fixée au moyen d'un coussinet; connue de la Méditerranée et la mer Adriatique, Ardissonia crystallina se trouve aussi dans l'océan Atlantique.

IOI. Ardissonia gigantea (LoBARz.).

Synedra gigantea LobARzewski, Einige neue Diatomaceen (I840), p. 276, tab. VI ; KuetzING, Die Kieselschaligen Bacillarien (I844), p. 69 (non Synedra gigantea EHR., I84I).

Synedra fulgens (GREV.) SM., var. gigantea RABEnHoRST, Flora Europæa Algarum, I (I864), p. I40.

Hab. : Lavages d'Algues, Tripoli (A, Trotter).

M. Lobarzewskı a découvert cette Synedracée sur le Sargassum Hornschuchii Ag. à Zare (Dalmatie); c'est une forme intermédiaire entre Ardissonia fulgens (Grev.) Sm. et Ardissonia superba (Kuetz.) Grun.

I02. Ardissonia robusta (RALFs) DE NotaRIs, in Erbario Crittogamico italiano ser. II (I87o), n. 334, Nuovo Giornale Botanico italiano, vol. III (I87I), p. 95 ; DE TonI, Sylloge Algarum, II, p. 675.

Synedra robusta Ralfs, in Pritchard History of Infusoria, p. 789, tab. 8, fig. 3 ; VAN Heurck, Traité des Diatomées, p. 316, tab. 30, fig. 836-837.

Hab.: Lavages d'Algues, de Zostéracées et d'Éponges, Tripoli, Homs (A. Trotter); lavages des Cystoseira, Tobrouk, Benghazi (A. VACCARI).

Cette magnifique espèce se trouve souvent sur les Algues dans la Méditerranée et la mer Adriatique. M. BréBisson a représenté une figure singulière d'un fragment de valve de cette espèce (Diatomacées renfermées dans le médicament vermifuge connu sous le nom de Mousse de Corse, 1872, pl. VII, fig. 7).

\section{Toxarium BAIL.}

I03. Toxarium undulatum BAILEY, Notes on New Species and Localities of microscopical Organisms (I853), p. I5, fig. 24-25; DE TONI, Sylloge Algarum, II, p. 677.

Synedra undulata BAILEY mss., W. Smith Synopsis of the British Diatomaceæ, II (I856), p. 97 ; GREGoRY, On new Forms of Marine Diatomaceæ (I857), p. 53I, tab. XIV, fig. I07 ; VAN HEURCK, Synopsis, p. I54, tab. 42, fig. 2 ; Traité des Diatomées, p. 317, tab. 10, fig. 437.

Hab. : Lavages des Algues, des Zostéracées et des Éponges, Tripoli (A. Trotter); lavages des Cystoseira, Tobrouk, Benghazi (A. VACCARI), 
Cette remarquable espèce a été signalée sur le littoral nord-africain au golfe de Gabès (LANZI); elle n'est pas rare dans la Méditerranée(Villefranche, Sicile, Mousse de Corse, etc.).

\section{Famille des RAPHONEIDACEÆ H. VAN Heurck.}

\section{Raphoneis EHR.}

I04. Raphoneis amphiceros EHR., in Berichte d. Berlin. Akad., I844, p. 87 ; Mikrogeologie, tab. I8, fig. 82 ; VAN Heurck, Synopsis, p. I47, tab. 36, fig. 22-23; Traité des Diatomées, p. 330, tab. Io, fig. 394; De TonI, Sylloge Algarum, II, p. 699.

Cocconeis amphiceros EHr., in Berichte d. Berlin. Akad., I840.

Doryphora amphiceros KUETz., Die Kieselschaligen Bacillarien (I844), p. 74, tab. 2I, II, fig. I, tab. 5, fig. X.

Hab. : Lavages des Algues, Tripoli (A. Trotter).

Espèce épiphyte relativement commune sur les Algues surtout filamenteuses.

Var. rhombica Grunow, in Van Heurck, Synopsis, p. I47, tab. 36, fig. 20-2I ; Traitédes Diatomées, p. 330, tab. Io, fig. 395 .

Hab. : Lavages des Cystoseira, Tobrouk (A. VACCARI).

105. Raphoneis nitida (Greg.) Grunow, Alg. Novara (I868), p. 99 ; DE Tonr, Sylloge Algarum, II, p. 700. forma major Peragallo, in Le Micrographe préparateur, LXXXIII, fig. 3I-32.

Hab. : Benghazi (A. VACCARI).

Cette forme n'a pas été signalée jusqu'à ce jour sur le littoral nord-africain.

\section{MOBILES.}

Famille des EUNOTIACEÆ (Kuetz.).

Pseudoeunotia Grun.

ro6. Pseudoeunotia Doliolus (Wall.) Grunow, in Van Heurck, Synopsis(r88I), tab. 35, fig. 22; De Toni, Sylloge Algarum, II, p. 8II.

Synedra Doliolus Wallich, On the siliceous Organisms found in the digestive Cavities of the Salpœ (I860), p. I3, tab. II, fig. I9.

Himanthidium? Doliolus Grunow, Die Oesterreichischen Diatomaceen, I (I862), p. 342, tab. 8, fig. 8.

Hab. : Lavages d'Algues, Homs (A. T'rotter).

Espèce dont la répartition géographique paraît jusqu'ici très limitée. M. WALLich l'a considérée comme un Synedra, l'ayant observée dans le contenu de l'appareil digestif des Salpes des océans Indien et Atlantique; elle a été rencontrée fossile à Mondaino en Italie (Castracane) etse retrouve dans le guano du Pérou (Grunow).

\section{Eunotia EHr.}

I07. Eunotia Arcus Ehr. Var. minor, VAn Heurck, Synopsis, p. I42, tab. 34, fig. 3 ; Traité des Diatomées, p. 299, tab. 9, fig. 363; DE Toni, Sylloge Algarum, II, p. 79 I. 
Hab. : Tadjoura (A. TROTTER).

L'espèce est très commune dans les eaux douces, stagnantes; on la connaît aussi à l'état fossile dans plusieurs dépôts.

\section{Rhopalodia O. Muelz.}

I08. Rhopalodia gibba (EHR.) O. Mueller, Rhopalodia, ein neues Genus der Bacillariaceen (I895), p. 65, tab. I, fig. I5-I7 ; A. SCHмidT, Atlas, tab. 253, fig. I-II.

Epithemia gibba KuETz., Die Kieselschaligen Bacillarien (1844), p. 35, tab. 4, fig. XXII ; VAN Heurck; Synopsis, P. I39, tab. 32, fig. I-2.

Navicula gibba EHR., in Abhandl. d. Berlin. Akad., I830 ; Infusionsthierchen(I838), p. I84, tab. XIII, fig. I9.

Cystopleura gibba O. Kuntze, Revisio, II (I89I), p. 89I,; De Toni, Sylloge Algarum, II, p. 780.

Hab. : Tadjoura (A. Trotter).

Espèce d'eau douce, d'après M. Petrt commune sur les plantes aquatiques de l'Afrique septentrionale (Algérie, Tunisie, Maroc).

T09. Rhopalodia gibberula (EHR.) O. MUELlER, Rhopalodia, ein neues Genus der Bacillariaceen, (I895); p. 58 ; Bacillariaceen aus den Natronthälern von Elkab (I899), p. 286 ; DE ToNi et ForTi; Alghe Ruwenzori, p. 29 .

Epithemia gibberula Kuetz., Die Kieselschaligen Bacillarien (I844), p. 35, tab. 30, fig. 3 ; VAN Heurck, Traité des Diatomées, p. 297, tab. 30, fig. 825 .

Ennotia gibberula EHR, in Abhandl. Berlin. Akad., I84I, p. 4r4.

Cystopleura gibberula O. Kuntze, Revisio, II (I89I), p. 89I ; DE ToNI, Sylloge Algarum, II, p. I88.

Hab. : Tadjoura (A. TrotTer).

Cette espèce, avec ses nombreuses variétés, est très répandue dans les eaux douces et sur les rochers humides de l'Afrique.

IIo. Rhopalotia Musculus (Kuetz.) O. Mueller, Bacillariaceen aus den Natronthälern von El Kab (I899), p. 294. Epithemia Musculus Kuetz., Die Kieselschaligen Bacillarien (I844), p. 33, tab. 30, fig. 6 ; VAN HEURCK, Synopsis, p. I40, tab. 32, fig. I4-I5 ; Traité des Diatomées, p. 297, tab. 9, fig. 359.

Cystopleura Musculus O. Kuntze, Revisio, II (I89I), p. 89I ; DE TONI, Sylloge Algarum, II, p. 785.

Hab. : Benghazi (A. VACCARI).

Espèce très répandue dans la Méditerranée et l'océan Atlantique.

\section{Famille des NITZSCHIACEA Grun.}

\section{Hantzschia Grun.}

Irr. Hantzschia Amphioxys (EHr.) Grunow, in Cleve et Grunow, Beiträge zur Kenntniss der arktischen Diatomeen (I880), p. I03; VAN Heurck, Synopsis, p. I68, tab. 56, fig. I-2 ; Traité des Diatomées, p. 38I, tab. I5, fig. $483 b$; DE TONI, Sylloge Algarum, II, p. 56I.

Eunotia Ampirioxys EHR., Verbreitung (I843), p. I25, plur. fig.

Nitzschia Amphioxys W. Smith, Synopsis of the British Diatomaceæ, I (1853), p. 40, tab. I3, fig. I05.

Hab. : Lavages des Cystoseira, Tobrouk (A. VACCARI).

Espèce ałaptée à la vie promisque. Dans l'Afrique du Nord, Hantzschia Amphioxys

Ehr. Grun. a été signalée jusqu'à ce jour à Hammam-bou-Hadjar, Hammam-el-Salahin, 'Tougourt, Er-Rahel (Belloc), au cap Bon (Lanzi) et dans le lac Ogoulmine (Debray).

T. V. - FASC. 7 . 
II2. Hantzschia marina (DoNk.) GRunow, in Cleve et Grunow, Beiträge zur Kenntniss der arktischen Diatomen (I880), p. I05; VAN Heurck, Traité des Diatomées, p. 382, tab. I5, fig. 489 b; De Toni, Sylloge Algarum, II, p. 564 .

Epithemia marina Donkin, On the marine Diatomaceæ of Northumberland (I858), p. 29, tab. 3, fig. I4.

Hab. : Lavages des Algues, Tripoli (A. Trotter).

Cette espèce n'a pas été signalée jusqu'ici sur le littoral méditerranéen de l'Afrique.

\section{Nitzschia Hassall.}

II3. Nitzschia apiculata (Greg.) Grunow, in Cieve et Grunow, Beiträge zur Kenntniss der arktischen Diatomeen (I880), p. 73 ; VAN HEURCK, Synopsis, p. I73, tab. 58, fig. 26-27 ; Traitédes Diatomées, p. 387 , tab. I5, fig. 505 ; DE Toni, Sylloge Algarum, II, P. 505.

Tryblionella apiculata GREGORY, in Micr. Joum., V (I857), p. 79, tab. I, fig. 43.

Hab. : Lavages d'Algues, Homs (A. Trotter).

Espèce adaptée aux milieux les plus différents, comme d'autres Nitzschia.

II4. Nitzschia constricta (GREg.) Grunow, in Cleve et GRunow, Beiträge zur Kenntniss der arktischen Diatomeen (I880), p. $7 x$; VAN Heurck, Synopsis, p. I72, tab. 58, fig. 8 ; Traité des Diatomées, p. 386 , tab. I5, fig. 50I; DE TONI, Sylloge Algarum, II, p. 502.

Tryblionella constricta GREGoRY, in Micr. Journ., III (I855), p. 40, tab. I, fig. I3.

Hab. : Lavages d'Algues, Homs (A. Trotter).

Dans le bassin de la Méditerranée, Nitzschia constricta a été rencontré par Castracane parmi les matériaux contenus dans le tube digestif d'un Salpa de Messine ; l'espèce est également connue de la mer Adriatique et des mers extra-européennes. D’après M. PAul Petit (Catalogue des Diatomacées du Maroc, d'Algérie et de Tunisie, p. 34) elle est très commune dans toute l'Algérie et la Tunisie, mais elle ne doit pas être confondue, comme a voulu le faire M Belloc, avec Nilzschia dubia W.Sm.

II5. Nitzschia Denticula Grunow, in Cleve et Moeller, Diatoms (I878), n. 224; Cleve et Grunow, Beiträge zur Kenntniss der arktischen Diatomeen (I880), p. 492, fig. 68; VAN HEURCK, Synopsis, p. I75, tab. 60, fig. Io ; Traité des Diatomées, p. 390, tab. I5, fig. 5I4; DE TonI, Sylloge Algarum, II, p. 5 I8.

Denticula Kuetzingii Grunow, Die Oesterreichischen Diatomaceen, II (I862), p. 548, tab. I2, fig. I5 $a-c$, $27 a-b$.

Hab. : Tadjoura (A. TrotTer).

L'espèce est très répandue dans les eaux douces d'Europe.

II6. Nitzschia linearis (AG.) W. Smith, Var. salinarum Grun., in Peragallo, Diatomées de France, tab. LXXIII, fig. I3.

Hab. : Lavages d'Algues, Homs (A. Trotter), Benghazi (A. Vaccari).

La forme typique n'est pas rare le long du littoral nord-africain depuis Tanger (Schousboe) jusqu'à l'Algérie (Tempère, Peragallo), à Biskra, etc. (Belloc, Debray).

II7. Nitzschia littoralis Grunow, in Cleve et Grunow, Beiträge zur Kenntniss der arktischen Diatomeen (I880), p. 75 ; De Toni, Sylloge Algarum, II, p. 508. 
Nitzschia Tryblionella, var. littoralis VAN HEURCK, Synopsis, p. I72, tab. 59, fig. I-3; Traité des Diatomées, p. 385 , tab. I5, fig. 496 .

Hab. : Lavages d'Algues, Homs (A. TrotTer).

Espèce promisque aux eaux douces et saumâtres vivant surtout au voisinage des embouchures des fleuves et le long du littoral. On l'a signalée dans la vase marine, à Arzew (Leuduger-Fortmorel, d'après P. Petit).

II8. Nitzschia macilenta Gregory, in Micr. Journ.; VII (I859), p. 83, tab. 6, fig. 8-9; VAN Heurck, Synopsis, tab. 64, fig. 6-7 ; DE TonI, Sylloge Algarum, II, p. 527.

Hab. : Lavages d'Algues, de Zostéracées et d'Éponges, Tripoli (A. Trotter).

L'espèce ne manque pas dans le bassin de la Méditerranée (Côtes de la Sicile, mer Adriatique).

II9. Nitzschia obtusa W. SMItH, Synopsis of the British Diatomaceæ, I (I853), p. 39, tab. I3, fig. I09; VAN Heurck, Synopsis, p. I80, tab. 67, fig. I; Traité des Diatomées, p. 397, tab. I6, fig. 537 ; DE TonI, Sylloge Algarum, II, p. 533.

Hab. : Lavages de Zostéracées et d'Éponges, Tripoli (A. Trotter).

Comme d'autres Nitzschia, cette espèce est adaptée à la vie promisque et on la rencontre souvent aux environs des deltas desfleuves; pour la région nord-africaine, elle a été signalée de l'Oued Terrouch (Peragallo), bassin du Hodna, Biskra, oasis de Tougourt (BELLOC).

I20. Nitzschia marginulata Grunow, Var. didyma Grunow, in Cleve et Grunow, Beiträge zur Kenntniss der arktischen Diatomeen (I880), p. 72 ; De TonI, Sylloge Algarum, II, p. 504; Van Heurck, Synopsis, tab. 58, fig. I4-I5.

Hab : Lavages des Zostéracées et des Éponges, Tripoli (A. TrotTer). L'espèce avec ses variétés est propre aux eaux marines.

I2I. Nitzschia marina GRunow, in CLEVE et GRUnow, Beiträge zur Kenntniss der arktischen Diatomeen (I880), p. 70 ; Van Heurck, Synopsis, tab. 57, fig. 26-27; De Toni, Sylloge Algarum, II, p. 500.

Nitzschia angustata var. marina GRUNow, in CLEVE et MOELLER, Diatoms (I878), n ${ }^{\circ}$ I54-I55.

Hab. : Lavages de Zostéracées et d'Éponges, Tripo!i (A. Trotter).

M. GRunow n'a donné aucune indication précise de localité méditerranéenne pour son espèce, qui se trouve aussi dans les mers extra-européennes.

I22. Nitzschia panduriformis GREGORY, On new Forms of Marine Diatomaceæ found in the Firth of Clyde and in Loch Fyne (I857), p. 57, tab. 6, fig. I02 ; CLEVE et GRUnOw, Beiträge zur Kenntniss der arktischen Diatomeen (I880), p. 7r, tab. 5, fig. 92 ; VAN HEURCK, Synopsis, p. I72, tab. 58, fig. I-3; Traité des Diatomées, p. 386, tab. 15, fig. 500 ; DE Toni, Sylloge Algarum, II, p. 501.

Hab. : Lavages d'Algues, Tripoli, Homs (A. Trotter); lavages des Cystoseira, Tobrouk, Benghazi (A. VACCARI).

Espèce à large répartition géographique. Sur le littoral nord-africain, elle a été signa- 
lée dans la vase marine à Arzew (Leuduger-Fortmorel) et dans les sondages du port d'Alger (Tempère, Peragallo). Le premier qui a décrit cette Nitzschia fut Gregory en I 857 , non Grunow, comme on trouve indiqué par erreur dans le Traité des Diatomées de M. VAN HEURCK.

Dans nos matériaux, la forme typique se trouvait mêlée aux variétés continua Grunow et delicatula Grunow.

I23. Nitzschia punctata (W. Sm.) Grunow, in Cleve et Grunow, Beiträge zur Kenntniss der arktischen Diatomeen (I880), p. 69; VAN Heurck, Synopsis, p. I7I, tab. 57, fig. 2 ; Traitédes Diatomées, p.384, tab. I5, fig. 49I; DE Toni, Sylloge Algarum, II, p. 496.

Tryblionella punctata W. Smith, Synopsis of the British Diatomaceæ, I (I853), p. 36, tab. ro, fig. $76 a$, tab. XXX, fig. 26r.

Hab. : Lavages d'Algues, Tripoli (A. Trotter); Benghazi (A. Vaccari).

Espèce promisque aux eaux douces et salées; on l'a récoltée çà et là dans le bassin de la Méditerranée. La forme rencontrée à Benghazi correspond à la forme coarctata.

I24. Nitzschia Sigma (Kuetz.) W. Smith, Synopsis of the British Diatomaceæ, I (I853), p. 39, tab. I3, fig. I08;

VAN Heurck, Synopsis, p. I79, tab. 65, fig. 7-8; Traité des Diatomées, p. 396, tab. I6, fig. 53I ; DE TonI, Sylloge Algarum, II, p. 530.

Synedra Sigma KuEtz., Die Kieselschaligen Bacillarien (1844), p. 67, tab. 30, fig. II4.

Hab. : Benghazi (A. VACCARI).

Espèce à large répartition géographique dans les mers intérieures et les océans ; on l'a indiquée pour l'Afrique du Nord de Aïn-d'Or (Belloc), Hammam-Henna (Peragallo), golfe de Gabès (Lanzi).

Nitzschiella RABENH.

I25. Nitzschiella longissima (BréB.) Rabenh., Flora Europæa Algarum, I (I864), p. I64; VAn HeurCK, Traité des Diatomées, P. 404, tab. I7, fig. 568.

Nitzschia longissima RALFs, in CLEVE et GRUNow, Beiträge zur Kenntniss der arktischen Diatomeen (I880), p. I00; DE Toni, Sylloge Algarum, II, p. 547.

Ceratoneis longissima BréB., in KuetzIng, Species Algarum (ז849), p. 89.

Hab. : Benghazi (A. VACCARI).

Cette espèce n'est pas signalée du littoral méditerranéen de l'Afrique.

Famille des SURIRELLACE E (Kuetz.) Grun.

\section{Podocystis KuETz.}

I26. Podocystis spathulata (Shadb.) H. Van Heurck, Traité des Diatomées (I899), p. 365 ; Peragallo, Les Diatomées marines de France, tab.'58, fig. I2; OEstrup, Marine Diatoms (of Koh Chang), p. 278, tab. I, fig. 3 .

Euphyllodium spathulatum SHADBolT, in Transactions of the microscopical Society, II, (I854), p. II, tab. 4, fig. 4 .

Hab. : Lavages d'Algues, d'Éponges et des Zostères, Tripoli (A. Trotter), Tobrouk, Benghazi (A. VACCARI). 
Cette espèce se trouve souvent sur les Algues filamenteuses, comme son congénère $P$. adriatica Kuetz., dans le bassin de la Méditerranée (Saint-Nazaire, Villefranche, d'après M. Peragallo). H. Peragallo a distingué avec précision cette espèce du Podocystis adriatica Kuetz.; elle est plus grande, plus ovale et montre une structure tout à fait différente. Ces deux espèces sont, au reste, reliées entre elles par des formes intermédiaires telles que Doryphora elegans Roper, Podocystis americana Bail., Podocystis australica Witt, et Podocystis africana Leud. Fortm.

\section{Suriraya TURPIN.}

I27. Suriraya fastuosa Ehr., in Berichte d. Berlin. Akad., I84I, p. I9; KuetzInG, Die Kieselschaligen Bacillarien (I844), tab. 28, fig. I9 $a$ - $d$; VAN HEURCK, Synopsis, p. I88, tab. 73, fig. I8 ; Traité des Diatomées, p. 372 , tab. I3, fig. 583 ; DE Toni, Sylloge Algarum, II, p. 582.

Hab. : Lavages d’Algues, Tobrouk, Benghazi (A. VACCARI); à Tripoli, dans les lavages d'Algues, la variété minor et la forme suborbicularis Grev.

La forme typique et les autres formes de cette élégante Suriraya sont communes dans la Méditerranée; sur le littoral africain on les a signalées dans la vase marine, à Arzew (Leuduger-Fortmorel, Petit).

\section{Campylodiscus EHR.}

I28. Campylodiscus adriaticus Grunow, in Wien. Verhandlungen, I862, p. 440.

Var. massiliensis Grunow, in Peragallo, Diatomées de France, tab. 53, fig. 5 (massilensis).

Hab. : Benghazi (A. VACCARI).

Cette variété est indiquée par M. H. Peragallo, à Villefranche et à Naples.

I29. Cambulodiscus bicostatus W. SmItH, Synopsis of the British Diatomaceæ, II (I856), p. 88; VAN HEURCK, synopsis, tab. 75, fig. 2; Traité des Diatomées, p. 379, tab. I4, fig. 599; DEBY, Analysis of the Diatomaceous Genus Campylodiscus (I89I), tab. 7, fig. 38 a DE TonI, Sylloge Algarum, II, p. 626 ; Peragallo, Diatomées marines de France, tab. 5I, fig. 4-5.

Hab. : Lavages des Algues, Tripoli (A. Trotter).

Espèce singulièrement adaptée à la région littorale et aussi à l'eau saumâtre; elle est très répandue en Algérie et Tunisie, où elle a été signalée par MM. Belloc, Debray, Petit et Peragallo.

130. Campylodiscus Horologium Williams, in Annals and Magaz. of Nat. History, x848; Deby, Campylodiscus tab.6, fig. 29 a; VAN Heurck, Traité des Diatomées, p. 377, tab. 32, fig. 870; De Toni, Sylloge Algarum, II, p. 6i6 ; Peragallo, Diatomées de France, tab. 57, fig. 2-3.

Hab. : Benghazi (A. VACCARI).

Cette espèce, rare dans l'Océan, se trouve fréquemment dans la Méditerranée; elle n'est pas connue sur le littoral nord-africain.

13I. Campylodiscus impressus Grunow, in A. Schmid, Atlas, tab. 5I, fig. Io; Peragallo, Diatomées marines de France, tab. 55, fig. Io. 
Hab. : Lavages des Algues, Tripoli (A. Trotter).

Dans la Méditerranée, on connaît cette espèce aux îles Baléares (Grunow) et à Naples (Peragallo).

I32. Campylodiscus parvulus W. Smith, in Annals and Magaz. of Nat. History, I85I, p. 7, tab. I, fig. I4 ; Synopsis of the British Diatomaceæ, I (I863), p. 30, tab. 6, fig. 56 ; VAN HeURCK, Synopsis, p. I9I, tab. 77, fig. 2 ; Peragallo, Diatomées de France, tab. 54, fig. 9, tab. 55, fig. 5-6.

Hab. : Benghazi (A. VACCARI).

Espèce très répandue ; confondue quelquefois avec le Campylodiscus Thuretii Bréb.

Famille des HETEROIDE E.

Amphiprora (Eнr.) Cleve.

I33. Amphiprora alata KUETz., Die Kieselschaligen Bacillarien (1844), p. I07, tab. III, fig. 63 ; VAN HEURCK, Synopsis, p. I2I, tab. XXII, fig. II-I2; Traité des Diatomées, p. 262, fig. 52, tab. 5, fig. 289; ClEVE, Synopsis of the Naviculoid Diatoms, I, p. I5; DE TonI, Sylloge Algarum, II, p. 33I.

Hab. : Lavages des Zostéracées et des Éponges, Tripoli (A. Trotter).

L'espèce est connue de la Méditerranée (Ligurie, côtes de Sicile, etc.) où elle n'est pas rare.

\section{Amphora EHr.}

134. Amphora arenaria Donkin, On the marine Diatomaceæ of Northumberland (I858), p. 3I, tab. 3, fig. I6; H. L. Sмiтh, in The Lens, II (I873), p. 78 , tab. 2 , fig. I4 ; A. Schmidt, Atlas, tab. 40, fig. ro ; Van Heurck, Traité des Diatomées, p. I30, tab. 24, fig. 673; De TonI, Sylloge Algarum, II, p. 408.

Hab. : Lavages des Algues, Homs (A. Trotter).

Amphora arenaria Donk. vit surtout dans les estuaires; elle n'a pas été indiquée jusqu'ici dans l'Afrique méditerranéenne.

135. Amphora cymbifera GREgory, On new Forms of marine Diatomaceæ found in the Firth of Clyde and in Loch Fyne (I857), p. 526, tab. I4, fig. 97 ; H. L. SмITH, in The Lens, II (I873), p. 85, tab. 3, fig. 26 ;

A. Schmid, Atlas, tab. 25, fig. I3, I7-I9, tab. 26, fig. 33; VAN Heurck, Traité des Diatomées, p. I33, tab. 24, fig. 658 .

Amphora Erebi EHR., in Berichte d. Berlin. Akad., I856, p. 526; Mikrogeologie (I854), tab. XXXV, A 23, fig. 2?

Amphora Terroris ClEVE, Synopsis of the Naviculoid Diatoms, II, p. I22.

Hab. : Lavages d'Algues, Zostéracées et Éponges, Tripoli, Homs (A. Trotter), Tobrouk (A. VACCARI).

Espèce marine, méditerranéenne et océanique, signalée sur le littoral nord-africain à Tipaza où elle se rencontre sur les Algues marines (Debray) et en Tunisie (Belloc).

I36. Amphora Eunotia CLEvE, On Diatoms from the arctic Sea (I873), p. 2I, tab. 3, fig. I7; Diatoms collected during the expedition of the Vega (I883), p. 462; Synopsis of the Naviculoid Diatoms, II, p. I22, tab. 4, fig. 2-3; Van Heurck, Traité des Diatomées, p. I36, tab. 24, fig. 684; De TonI, Sylloge Algarum, II, p. 489 .

Amphora cymbifera GREG., in A. ScHMId, Atlas, tab. 25, fig. 35 (non alibi). 
Hab. : Lavages d'Algues, Homs (A. Trotter).

L'espèce est indiquée par M. Cleve du Gronland, Spitzberg, Bahusie, Bal-el-Mandeb et Labuan. L'aire de répartition géographique du Amphora Eunotia est donc très large, car elle va de la zone arctique à l'île de Bornéo.

I37. Amphora formosa CleVE, in A. Schmidt, Atlas (I875), tab. 28, fig. 34; tab. 39, fig. 2 [nomen]; Synopsis of the Naviculoid Diatoms, II, p. I38 [diagnosis] ; DE TonI, Sylloge Algarum, II, p. 397.

Hab. : Lavages d'Algues, Tripoli (A. Trotter), Tobrouk (A. VAcCari).

Espèce connue des mers chaudes et tempérées. M.Cleve l'indique, pour la Méditerranée, aux îles Baléares et dans le golfe de Naples.

Amphora gracilis Ehrenberg, Verbreitung, p. I22; forma parva Grunow, in VAn Heurck, Synopsis, tab. I, fig. 3 .

Hab. : Entre les Chara, Tadjoura (A. Trotter).

Cette forme rentre, d'après quelques auteurs, dans la série des formes créées pour le Amphora ovalis Kuetz. On l'a signalée déjà dans les eaux douces de l'Afrique du Nord à Tougourt, Kebilli, El-Faouara (BELloc).

I39. Amphora Grevilliana GREGoRY, On the post-tertiary Diatomaceous Sand of Glenshira, part. II (I857), p. 7, n. 36, tab. 2, fig. 36; H. L. Sмiтн, in The Lens, II (I873), p. 77, tab. 2, fig. 9; A. Schмidt, Atlas, tab. 35, fig. 4I; Van Heurck, Traité des Diatomées, p. I3I, tab. 24, fig. 676; DE Toni, Sylloge Algarum, II, p. 395 .

Hab. : Lavages d'Algues, Tripoli (A. Trotter).

Espèce exclusivement marine, également répandue dans les mers froides et dans les mers chaudes, puisqu'elle va du Spitzberg au détroit de Magellan. Dans la Méditerranée, elle a été indiquée par Cleve dans le golfe de Naples. Amphora Grevilliana a été rencontrée à l'état fossile dans le dépôt de S. Monica (Californie), etc.

I40. Amphora incequistriata DE TONI et FORTI, n. sp. (I9I3) (Fig. I).

Frustulum secus axem paravalvarem ad instar tegulæ incurvum ita ut distincte singula valva tantum simul conspicienda sit, ex latere prono zona connectiva e facie convexa, ex supino binæ valvæ obliquæ zonam connectivam e facie concava amplectentes observandæ maneant.

Zona connectiva lata, hyalina, zonulis pluribus corstituta, ex frustuli latere convexo amplioribus, ex altero angustioribus.

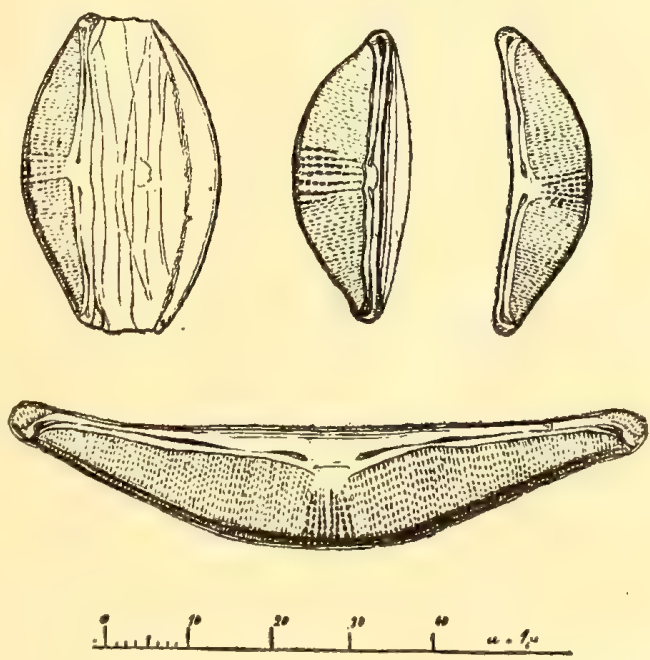

Fig. 1. - Amphora incequistriata n. sp.

Valvæ cymbitormes, ventricosulæ, convexæ, regione media, inter rhaphes nodulos centrales intercedenti, laxius evidentiusque striato-punctata quam apices versus. 
Striæ centrales grosse punctatæ satis irregulariter radiantes I $\mu$ circ. distantes, laterales lœvissimæ subparallelæ punctulis oblongis delicatissimis consertæ, o,5 $\mu$ circ. distantes.

Rhaphe parcissime curvata, nodulos hamatos centrales inter se satis distantes leniter inflexos (uno alteroque versus marginem externum vergente) ostendens.

Longitudo valvarum, 33-38

Var. elongata n. var. (I9I3).

Valvæ quam in forma typica longiores et acutiores; ceterum ut in forma typica.

Longitudo valvarum, $70-76 \mu$; latitudo valvarum, 9- Io $\mu$.

Hab. : Characeis adfixa, in aquis stagnantibus prope (" Tadjoura » Libyæ italicæ (legit Dr Alexander Trotter).

Cette espèce peut être aisément distinguée de ses congénères d'eau douce par la structure du frustule et par ses stries hétéromorphes. Notre espèce diffère par ses caractères généraux (structure spéciale de la zone connectivale, forme de la valve, dimensions, etc.) de l'Amphora venusta, espèce marine récemment décrite et figurée par M. E. Oestrup (Diatoms from North-East Greenland, p.212, tab. XIII, fig. I6; Kobenhavn, I9 Io).

I4I. Amphora lineolata Ehrenberg, Die Infusionsthierchen (I838), p. I88, tab. XIV, fig. 4; KuEtzIng, Die Kieselschaligen Bacillarien (I8I4), p. I07, tab. V, fig. 36; VAN Heurck, Synopsis des Diatomées de Belgique, P. 57, tab. I, fig. I3, 23 ; Traité des Diatomées, p. I38, tab. I, f. I0 ; A. Schmidt, Atlas, tab. 26, fig. 5I; Cleve, Synopsis of the Naviculoid Diatoms, II, p. x26; DE Toni, Sylloge Algarum, II, p. 394 .

Hab. : Lavages d'Algues, Tripoli (A. Trotter).

Cette espèce paraît également adaptée pour vivre dans les eaux douces et saumâtres et dans les eaux marines. Elle possède une large répartition géographique.

L'espèce désignée par Donkin sous le même nom est tout à fait différente. M. Rabenhorst lui donne le nom de Amphora Donkinii.

142. Amphora libyca Ehrenberg, in Berichte der Berl. Akademie, I840, p. II ; Kuetzing, Die Kieselschaligen Bacillarien (I844), P. I07, tab. 29, fig. 28; A. Sснмidt, Atlas, tab. 26, fig. I02-I05; DE Toni, Sylloge Algarum, II, p. 384

Amphora ovalis var. libyca (EHR.) Cleve, Synopsis of Naviculoid Diatoms, II (I895), p. I04 (avec synonymie).

Hab. : Entre les Chara, Tadjoura (A. Trotтer).

Amphora libyca Ehr., comme l'indique son nom spécifique, fut récoltée la première fois dans la Libye; elle a une large répartition géographique dans les eaux douces et légèrement salées. On l'a confondue avec les nombreuses formes de Amphora ovalis (Bréb.) Kuetz., dont la synonymie très complexe devra à l'avenir être contrôlée en séparant les formes ayant une aire stauriforme de celles qui ne possèdent aucune trace de stauros.

I43. Amphora Normanii Rabenhorst, Flora Europæa Algarum, I (I864), p. 88; CLEvE, Synopsis of the Naviculoid Diatoms, II, p. II9; De Toni, Sylloge Algarum, II, p. 384; VAn Heurck, Traité des Diatomées, p. I28, tab. I, fig. 4 .

Amphora humicola Grunow, in A. Schmid, Atlas der Diatomaceenkunde (1875), tab. 26, fig. 90-92; VAN HEURCK, Synopsis, p. 56, tab. I, fig. I2. 
Hab. : Lavages des Chara, Tadjoura (A. TrotTer).

Espèce d'eau douce, qui vit ordinairement sur la terre humide (d'où le nom que lui donna M. Grunow). M. Cleve l'indique de Suède, de la Belgique (où la rencontra M. Delogne), du Harz et de l'île de Java. M. Norman l'a recueillie sur les parois des calidaires à Hull en Angleterre. Dans l'Afrique du Nord, A. Normanii Rabenh. a été signalée à Oued-Terrouch par M. Peragallo.

I44. Amphora obtusa Gregory, in Microsc. Journ., V (I857), tab. I, fig. 34; H. L. Smith, in The Lens, II (I873), p. 70, tab. I, fig. 5; VAn Heurck, Traité des Diatomées, p. I37, tab. 24, fig. 688; Cleve, Synopsis of the Naviculoid Diatoms, II, p. I3I; DE TonI, Sylloge Algarum, II, p. 404.

Hab. : Benghazi (A. VACCARI).

Cette espèce paraît nouvelle pour le littoral nord-africain.

D’après M. Cleve, Amphora oblusa Greg. est très répandue dans les mers intérieures et les Océans.

I45. Amphora ocellata Donkin, in Microsc. Journ. (I86I), p. II, tab. I, fig. II $b$; H. L. Smixh, in The Lens, II, (I873), p. 70, tab. I, fig. 2 ; VAN HEURCK, Synopsis, p. 56, tab. I, fig. 26; Traité des Diatomées, p. I30, tab. I, fig. 3 ; Cleve, Synopsis of the Naviculoid Diatoms, II, p. I33; De Toni, Sylloge Algarum, II, p. 377 .

Hab.: Benghazi (A. VAcCARI).

Signalée dans la mer Adriatique et dans l'océan Atlantique (côtes de Suède, Angleterre et Belgique), cette espèce est nouvelle pour le littoral nord-africain.

I46. Amphora ostrearia Brébisson, in KuetzIng, Species Algarum (I849), p. 94 ; H. L. Smith, in The Lens, II (I873), p. I2, tab. I, fig. I6; A. Schmid, Atlas, tab. 26, fig. 23; VAN Heurck, Synopsis, p. 55, tab. I, fig. 25; Traité des Diatomées, p. I39, tab. I, fig. I; Cleve, Synopsis of Naviculoid Diatoms, II, p. I29 (var. typica); DE Toni, Sylloge Algarum, II, p. 376.

Hab. Lavages d'Éponges et des Zostéracées, Tripoli (A. Trotrer).

Espèce très variable et à large répartition géographique, elle a été indiquée dans la Méditerranée par M. Cleve.

I47. Amphora proboscidea (GREG. ?) CLEVE, Synopsis of Naviculoid Diatoms, II (I895), p. II3, tab. 3, fig. I9-2I; an GREGORY, Diatoms of Clyde, p. 526, tab. XIV, fig. $9^{8}$ (?)

Amphora commutata forma proboscidea (GREG.) DE Toni, Sylloge Algarum, II, p. 402.

Hab. : lavages d'Éponges et feuilles des Zostéracées, Tripoli (A 'TRotTER); lavages d'Algues, Homs (A. Trotter), Benghazi (A. Vaccari).

Espèce marine, voisine de l'Amphora Graeffii Grun.) Cleve, qui a été récoltée par M. JACQues Brun dans le golfe de Naples; elle doit être absolument séparée de Amphora commutata Grun. (Amphora affinis W. Smith, non Kuetzing) avec laquelle on l'a toujours confondue.

I48. Amphora Proteus Gregorx, Diatomaceæ found in the Firth of Clyde and in Loch Fyne (I857), p. 5I8, tab. I3, fig. 8I ; H. L. Sмiтн, in The Lens, II (1873), p. 79, tab. III, fig. I; A. Schmidt, Atlas, tab.27, fig. 3 ; Van Heurck, Traité des Diatomées, p. I29, tab. 24, fig. 67 I Cleve, Synopsis of Naviculoid Diatoms, II, p. I03; DE TONI, Sylloge Algarum, II, p. 403.

T. V. - FASC. 7 . 
Hab.: Lavages d'Algues, Zostéracées et Éponges, Tripoli (A. Trotter), Benghızi (A. VACCARI).

Espèce marine, avec large répartition géographique; elle est bien connue de la Méditerranée (côtes de l'île de la Sardaigne, etc.) ; sur le littoral nord-africain, M. Lanzı l'a signalée au Cap Bon en Tunisie.

I49. Amphora robusta Gregory, Diatomaceæ found in the Firth of Clyde and in Loch Fyne (I857), p. 5I6, tab. I3, fig. 79; H. L. Sмiтh, in The Lens, II (I873), p. 80, tab. 3, fig. 2; A. Schmid, Atlas, tab. 27, fig. 40-4I ; Van Heurck, Traité des Diatomées, p. I29, tab. 24, fig. 670; Cleve, Synopsis of Naviculoid Diatoms, II, p. I03; DE ToNI, Sylloge Algarum, II, p. 403.

Hab.: Lavages d'Algues, Homs (A. Trotter).

Espèce marine et fossile, connue déjà de la Méditerranée et de la mer Adriatique; elle est commune dans les dépôts de Misserghin, Village nègre et Saint-Eugène (Oran), d'après MM. Tempère et Peragallo, Diatomées du Monde entier, nos i 5 I-I 53, i 55 (deuxième édition).

I50. Amphora salina W. Smitr, Synopsis of the British Diatomaceæ, I (I853), p. I9, tab. 30, fig. 25I ; H. L. Sмith, in The Lens, II (I873), p. 84, tab. 3, fig. 29; VAN HeURCK, Synopsis, p. 57, tab. I, fig. I9; Traité des Diatomées, p. I34, tab. I, fig. 6; DE TonI, Sylloge Algarum, II, p. 386.

Hab.: Lavages d'Algues, Tripoli (A. 'TROTIER).

Espèce assez répandue dans les eaux salées et saumâtres; signalée déjà dans les eaux saumâtres de l'Afrique boréale, à Sidi M'Sid, Hammam-el-Meskoutin, oasis de Sidi-Yahia Tougourt, El-Faouara, Chott d'Ayata (M. E. Bel.LOC). Quelques auteurs ont attribué A. salina comme synonyme au Amphora coffeaformis (Ag.) Kuetz., qui a l'aspect général de $A$. salina, mais des frustules diminuant insensiblement jusqu'aux extrémités qui sont généralement très peu rétrécies; tandis que M. CLeve, dans son Synopsis, II, p. I 2o, réunit A. salina W. Sm. au A. coffeaformis (Ag.) Kuetz., M. Henri VAn Heurck maintient les deux espèces.

\section{Cymbella C. Ag.}

I5I. Cymbella cymbiformis (AG. ?) Brebisson et Godey, Algues des environs de Falaise (I836), p. 49, tab. VII ; VAN Heurck, Synopsis, p. 64, tab. 2, fig. II; Traité des Diatomées, p. I47, tab. I, fig. 38 ; CLEVE, Synopsis of Naviculoid Diatoms, I (I894), p. I72 ; DE TonI, Sylloge Algarum, II, p. 363.

Cocconema cymbiforme EHR., in Abhandlungen der Berlin. Akademie, I835; Infusionsthierchen (I838), p. 225 , tab. XIX, fig. VIII.

Frustulia cymbiformis KuetzING, Synopsis Diatomearum (I833), tab. I, fig. Io.

Hab. : Entre les Chara, Tadjoura (A. Trotter).

Espèce d'eau douce très commune; pour l'Afrique boréale, elle a été signalée déjà de plusieurs localités, notamment à Oued-Taria, Oued-Fedrahman, Tunnel d'Adélia, affluent de l'Oued-Merdja, canal de la minoterie de Blida, Saint-Eugène, près Alger (Debray), Le Rummel, Constantine (Tempère et Peragallo). Il n'est pas absolument certain que le Cymbella cymbiformis, décrit par C. A. AGardh (Conspectus criticus Diatomacearum, p. ro; Lundæe, I83o), soit la même forme décrite quelques ans après par M. BRÉBIsson, car les descriptions sont très abrégées. 
I52. Cymbella lanceolata (EHr.) -Krrchner, Die Algen Schlesiens (I878), p. I88; VAN Heurck, Synopsis, p. 63 , tab. 2, fig. 7 ; Traité des Diatomées, p. I46, tab. I, fig. 37 ; Cleve, Synopsis of Naviculoid Diatoms, I (I894), p. I74; DE ToNI, Sylloge Algarum, II, p. 362.

Cocconema lanceolatum EHRENBERG, Die Infusionsthierchen (I838), p. 224, tab. I9, fig. 6.

Hah. : Entre les Chara, Tadjoura(A. Trotter), Benghazi(A. Vaccari).

Espèce vivant dans les eaux douces ou légèrement salées. Pour l'Afrique du Nord, elle est connue de l'Oued-Taria (Debray) et de Sidi-M'Sid, Hammam-el-Meskoutin(Belloc). Les individus de Benghazi sont peut-être emportés par les eaux douces du littoral.

La diagnose donnée par C. Agardh (Conspectus criticus Diatomacearum, p. 9; Lundæ, I83o) pour son Cymbella lanceolata n'a rien à faire avec les caractères des vrais Cymbella, et sa description concorde mieux avec celle des Navicula, ainsi que Kuftzing (Die Kieselschaligen Bacillarien, P. 94; Nordhausen, I 844) l'a soupconné.

I53. Cymbella subaqualis GRunow, in VAN Heurck, Synopsis des Diatomées de Belgique (I885), p. 6r, tab. 3, fig. 2 ; suppl., fig. I ; Traité des Diatomées, p. I43, tab. I, fig. 26 ; DE TonI, Sylloge Algarum, II, p. 35 I (excl. synon.).

Hab. : Entre les Chara, Tadjoura (A. Trotter).

Cette espèce d'eau douce, d'après M. Cleve (Synopsis of the Naviculoid Diatoms, I, p. I70; Stockholm, I894) constituerait réellement le Cymbella aqualis W. Smith; d'ailleurs, A. Schmid, dans son Atlas (tab. 9, fig. 69) figure, sous le nom de Cymbellacequalis W. Sm. une forme que Cleve juge identique à Cymbella angustata W. Sm. Nous avons classé notre matériel d'après les figures données par M. VAN Heurck.

\section{Famille des NAVICULOIDE E (Kuetz.) Cl.}

Trachyneis Cleve.

I54. Trachyneis aspera (Ehr.) Cleve, Synopsis of the Naviculoid Diatoms, I (I894), p. I9I ; Peragallo, in Micrographe préparateur, VII (I899), p. 240, tab. 30, fig. I.

Stauroptera aspera EHRENBERG, Amer. (I843), p. I34, tab. I, fig. I-2.

Navicula aspera Ehrenberg, Mikrogeologie (I856), tab. 38, A fig. 5 ; De Toni, Sylloge Algarum, II, p. Io9; VAn Heurck, Traité des Diatomées p. 205, tab. 4, fig. I65.

Hab.: Lavages d'Algues, Éponges, Zostéracées, Tripoli et Homs (A. TrotTer), Tobrouk et Benghazi (A. VACCARI).

Trachyneis aspera Cl. n'est pas rare dans la Méditerranée. Dans nos matériaux, on trouve la forme typique mêlée aux variétés vulgaris Cleve et intermedia Grun.

\section{Stauroneis EHR.}

I55. Stauroneis balearica Cleve, On fome new or little Known Diatoms (I88I), p. I4, tab. 3, fig. 4I; PERAGallo, in Micrographe préparateur, V (I897), p. 247, tab. 7, fig. 35; DE ToNI, Sylloge Algarum, II, p. 2 Io. Navicula balearica CLEvE, Synopsis of the Naviculoid Diatoms, I (I894), p. III.

Hab. : Lavages d'Algues, Tripoli (A. Trotter).

L'espèce a été signalée dans les îles Baléares où elle a été récoltée par M. F. SoEdER- 
Lund. Placée par Cleve dans le genre Stauroneis, puisque son nodule central est dilaté en stauros, elle a été plus tard rapportée par son auteur aux Naviculae orthostichae, dans la revision du groupe entier des Naviculoïdées.

\section{Pleurosigma W. SM.}

I56. Pleurosigma formosum W. Smitr, in Annals and Magazines of Natural History, 2 ser., IX (I852), p. 5, tab. I, fig. I; Synopsis of the British Diatomaceæ, I (I853), p. 63, tab. 20, fig. I95 ; VAN HEURCK, Synopsis, p. II6, tab. I9, fig. 4; Traité des Diatomées, p. 254, tab. 6, fig. 268; Peragallo, Monographie du genre Pleurosigma, tab. I, fig. 3-5 ; ClEve, Synopsis of the Naviculoid Diatoms, I (I894), p. 45 ; DE TonI, Sylloge Algarum, II, p. 243.

Hab.: Lavages d'Algues, Tripoli (A. Trotter), Benghazi (A. Vaccari).

Var. balearica Grunow, in Peragallo, Monographie du genre Pleurosigma (I89I), p. 4, tab. I, fig. 6-7; Cleve, op. cit., p. 45 ; De Tons, op. cit., p. 243.

Hab.: Lavages des Cystoseira, Tobrouk et Benghazi (A. VACCARI).

Le type de l'espèce est très répandu dans la Méditerranée; sur le littoral nord-africain, il a été signalé parmi les Diatomées de la vase marine à Arzew (Leuduger, d'après P. Petit) et dans les sondages du port d'Alger (Tempère, Peragallo); la variété est indiquée aux îles Baléares et à Singapour.

I57. Pleurosigma longum Cleve, Diatoms of the Arctis Sea (1873), p. I9.

Var. lanceolatum Peragallo, Diatomées de France, tab. XXXI, fig. 7-8.

Hab.: Benghazi (A. VACCARI).

Cette variété est indiquée par M. Peragallo à Villefranche, Naples, Baléares.

58. Pleurosigma marinum Donkin, Var. italicum Peragallo, Monographie du genre Pleurosigma (I89r, p. 8, tab. 3, fig. Io (Pl. italicum); DE Toni, Sylloge Algarum, II, p. 236 ; Cleve, Synopsis of the Naviculoid Diatoms, I (I894), p. 37.

Hab. : Lavages des Cystoseira, Tobrouk (A. VACCARI).

Cette variété du très répandu Pleurosigma marinum Donk. a été jusqu'à ce jour rencontrée dans le golfe de Naples et à Lissa dans la mer Adriatique.

159. Pleurosigma rigidum W. Sмiтh, Synopsis of the British Diatomaceæ, I (1853), p. 64, tab. 20, fig. I98; VAN Heurck, Synopsis, tab. I9, fig. 3 ; Traité des Diatomées, p. 25I, tab. 6, fig. 265 ; Peragallo, Monographie du genre Pleurosigma, tab. 6, fig. 4-6 ; CLEVE, Synopsis of the Naviculoid Diatoms, I (I894), p. 39 ; DE TONI, Sylloge Algarum, II, p. 237.

Hab. : Lavages d'Algues, Tripoli (A. Trotter), Tobrouk et Benghazi (A. Vaccari).

Espèce très répandue dans les mers intérieures et les Océans; MM. Tempère et Peragallo l'ont aussi signalée dans les sondages du port d'Alger.

Rhoicosigma Grun.

I60. Rhoicosigma oceanicum PERAgallo, Monographie du genre Pleurosigma, tab. 50, fig. 5-6; VAN HEURCK, Traité des Diatomées, p. 260 ; fig. 5I ; DE TonI, Sylloge Algarum, II, p. 268. 
Hab.: Benghazi (A. VACCARI).

Espèce nouvelle pour le littoral méditerranéen de l'Afrique. M. Cleve (Synopsis of the Naviculoid Diatoms, I, p. I2O), necroit pas $R h$. oceanicum Perag. suffisamment distinct du Rh. compactum (Grev.) Grun.; mais M. Peragallo n'est pas du même avis et a figuré à part son espèce et celle de Greville dans ses Diatomées de France, tab. VI, fig. 3-7 (Rh. oceanicum), fig. 8 ( $R$. compactum).

\section{Navicula (BORY).}

I6r.Navicula abrupta (GREg.) Donkin, The Natural History of the British Diatomaceæ (I87r), p. I3, tab. 2, fig. 6; A. Schmid, Atlas, tab. 3, fig. I-2; Van Heurck, Synopsis, p. 94, tab. Io, fig. 4 ; Traité des Diatomées, p. 203, tab. 4, fig. I62 ; CLEVE, Synopsis of the Naviculoid Diatoms, II (I895), p. 6I ; DE Toni, Sylloge Algarum, II, p. 96.

Navicula Lyra var. abrupta GREGORY, Diatoms of the Clyde (I857), p. 486, tab. 9, fig. I4.

Hab. : Lavages d'Algues, Homs (A. Trotter).

Espèce marine, répandue dans les mers intérieures et les Océans; sur le littoral nordafricain, on la connaît à Arzew (Leuduger, d'après P. Petrit).

C'est Donkin qui a élevé Navicula abrupta au rang d'espèce, non Gregory qui l'a décrite seulement comme variété du Navicula Lyra Ehr.

I62. Navicula advena A. Schmidt, Atlas der Diatomaceenkunde (I875), tab. 8, fig. 29 (sans description); PERAGALlo, in Micrographe préparateur, VII (I899), p. 46, tab. 5, fig. II ; DE TonI, Sylloge Algarum, II, p. 88 ; VAN Heurck, Traité des Diatomées, p. 200, tab. 26, fig. 752.

Diploneis advena CLEVE, Synopsis of the Naviculoid Diatoms, I (I894), p. 8 I.

Hab. : Lavages d'Eponges et des Zostéracées, Tripoli (A. Trotter); lavages d'Algues, Homs (A. Trotter).

Espèce trouvée jusqu'ici dans les mers de la région océanique ; elle n'est pas rare dans la Méditerranée.

I63. Navicula arenaria Donkrn, in Microscopical Journal, I (I86I), p. Io, tab. I, fig. 9; The Natural History of the British Diatomaceæ, p. 56, tab. 8, fig. 5 .

Navicula lanceolata (AG.?) KUETz., var, arenaria (DONK.) VAN HeURck, Synopsis (I885), p. 88, tab. 8, fig. I8 ; Traité des Diatomées, p. I86, tab. 3, fig. I42 ; DE Toni, Sylloge Algarum, II, p. 58.

Hab. : Lavages des Cystoseira, Tobrouk (A. VACCARI).

Cette espèce parait nouvelle pour le littoral nord-africain.

I64. Navicula Baileyana GRunow, in A. Schmid, Die in den Grundproben der Nordseefahrt enthaltenen Diatomaceen (I874), tab. I, fig. 3I; Atlas, tab. 6, fig. 26.

Navicula granulata BAILEY, Notes on new species (I854), p. Io, fig. I6 (non Navicula granulata BRÉB.).

Navicula polysticta GREville, Descriptions of some new species and varieties of Navicula observed in Californian Guano (I859), p. 28, tab. 4, fig. I2.

Hab.: Lavages des Cystoseira, Tobrouk (A. VACCARI).

L'espèce est déjà indiquée dans le bassin de la Méditerranée, où elle fut récoltée par M. Peragallo. 
165. Navicula bomboides A. Schmidr, Die in den Grundproben der Nordseefahrt enthaltenen Diatomaceen (I874), tab. I, fig. 2 ; Atlas, tab. I3, fig. 36 (non tab. I3, fig. 38) ; VAN Heurck, Synopsis, p. 89, tab. suppl. B, fig. I9 ; Traité des Diatomées, p. I93, tab. 3, fig. I46; DE TonI, Sylloge Algarum, II, P. 7 I. Diploneis bomboides CLEVE, Synopsis of the Naviculoid Diatoms, I (I894), p. 88.

Hab. : Lavages des Cystoseira, Tobrouk(A. VACCARI).

Espèce à large répartition géographique. Sur le littoral méditerranéen de l'Afrique, elle est indiquée par CLEVE comme provenant d'Alexandrie (d'après des préparations de la collection DeBY).

166. Navicula Bombus (Ehr.) Kuetzing, Species Algarum (I849), p. 83; A. Schмidt, Atlas, tab. 69, fig. 28-29 Van Heurck, Synopsis, p. 90, tab. suppl. B, fig. 22 ; Traité des Diatomées, p. I94, tab. 3, fig. I49; De Toni, Sylloge Algarum, II, p. 75 .

Diploneis Bombus Ehrenberg, in Berichte der Berlin. Akad., I844, p. 84; Cleve, Synopsis of the Naviculoid Diatoms, I (I894), p. 90.

Hab. : Lavages d'Éponges et Zostéracées, Tripoli (A. TrotTER).

Espèce à large répartition géographique dans les océans et dans la Méditerranée et ses bassins intérieurs. Sur le littoral nord-africain, à part la région marocaine baignée par l'océan Atlantique, elle a été recueillie au Cap Bon, dans le golfe de Gabès (L.ANZI) et dans le port d'Alger (Tempere, Peragallo).

I67. Navicula brachysira BRÉBIsson, in RABEnHORST, Die Süsswasser-Diatomaceen (I853), p. 39, tab. 5, fig. IIc-e; VAN HEURCK, Atlas suppl., fig. I26.

Navicula serians BréB., var, brachysira VAN HEURCK, Synopsis, p. IoI; De TonI, Sylloge Algarum, II, p. I4I.

Navicula serians, var. minor Grunow, in VAN HeUrck, Synopsis, tab. I2, fig. 8-9.

Anomoeoneis? brachysira CLEVE, Synopsis of the Naviculoid Diatoms, II (I895), p. 7 .

Hab. : Tadjoura (A. Trotter).

Espèce d'eau douce, qui n'est, selon toute probabilité, qu'une simple forme à valves plus petites et plus finement striées du Navicula serians Bréb.

I68. Navicula brevis Gregory, Var, elliptica Grunow, in Van Heurck, Synopsis des Diatomées de Belgique, p. 97, tab. II, fig. I8; Traité des Diatomées, p. 209, tab. 4, fig. I8I ; DE TonI, Sylloge Algarum, II, p. I23.

Caloneis brevis var. vexans (GRUNow) CLEVE, Synopsis of the Naviculoid Diatoms, I (I894), p. 6I.

Navicula brevis var. vexans GRunow, in Cleve et GRunow, Beiträge zur Kenntniss der Arktischen Diatomeen (I880), p. 30.

Hab. : Lavages d'Éponges et de Zostéracées, Tripoli (A. Trotter).

169. Navicula coffeiformis A. Sснмid, Atlas der Diatomaceenkunde, tab. 8, fig. 7 ; Nordsee Diatomaceæ, tab. I, fig. 22 ; tab. II, fig. I3; DE TONI, Sylloge Algarum, II, p. 68.

Diploneis coffeiformis ClEVE, Synopsis of the Naviculoid Diatoms, I (I894), p. 8I.

Hab. : Benghazi (A. VACCARI). Naples.

Dans le bassin de la Méditerranée, on a rencontré cette espèce dans le golfe de I70. Navicula Crabro (EHr.) KuetzIng, Species Algarum (I849), p. 83; VAN Heurck; Synopsis, p. 89, tab. 9, fig. I-2; Traité des Diatomées, p. I92, tab. 3, fig. I44; DE TonI, Sylloge Algarum, II, p. 68. 
Pinnularia (Diploneis) Crabro EHrenderg, in Berichte der Berlin. Akad., I844, p. 85 ; Cleve. Synopsis of the Naviculoid Diatoms, I (1894), p. Ioo (Diploneis).

Hab. : Lavages d'Algues, Homs (A. Trotter), Tobrouk et Benghazi (A. Vaccari); Tripoli et Benghazi, la forme minuta Cleve.

Espèce extrêmement variable sous le rapport de la forme générale et de la sculpture des valves, ce qui explique le nombre de variétés signalées par les auteurs et surtout par M. Cleve; elle est très répandue dans les mers intérieures et les océans; elle est également à l'état fossile. Sur le littoral méditerranéen nord-africain, elle a été signalée dans la vase marine à Arzew (Leuduger d'après P. Petit) et dans les sondages du port d'Alger (Tempère, Peragallo).

I7T. Navicula directa RALFs, in PRITChaRd, Infusoria, p. 906; W. SMITH, Synopsis of the British Diato maceæ, I (I853), p. 56, tab. I8, fig. I72 (sub Pinnularia); VAN Heurck, Traité des Diatomées, p. I89, tab. 25, fig. 722; DE TONI, Sylloge Algarum, II, p. 22.

Hab. : Benghazi (A. VACCARI).

Var. Incus A. SchмпD, Atlas der Diatomaceenkunde, tab. 47, fig. 7 (Navicula Incus).

Hab. : Benghazi (A. VACCARI).

L'espèce et la variété ont été signalées, pour la première fois, sur le littoral méditerranéen de l'Afrique, dans la région de la Cyrénaïque; mais la forme typique est indiquée des côtes de la Tunisie (Lanzi).

I72. Navicula forcipata GREvILle, Var. punctata CLEVE, Synopsis of the Naviculoid Diatoms, II (I895), p. 65.

Hab. : Lavages des Cystoseira, Tobrouk (A. VACCARI).

On connaît cette variété pour le littoral océanique de l'Afrique au Maroc (Cleve). M. Leuduger communiqua à M. P. Petit des matériaux récoltées à Arzew, contenant la forme figurée dans A. Schmid (Nordseefahrt Diatomaceæ, t. 2, fig. I6, I8).

I73. Navicula granulata BréBISson in DonkIN, On the marine Diatomaceæ of Northumberland (I858), p. I7, tab.3, fig. I9 $a-b$; The Natural History of the British Diatomaceæ (I87I), p. I7, tab. 3, fig. I ; VAN Heurck, Synopsis, p. 98, tab. II, fig. I5 ; Traité des Diatomées, p. 2II, tab. 4, fig. I83 ; DE TonI, Sylloge Algarum, II, p. I27 (non Navicula granulata BAILEx nec EHRENBERG).

Navicula monilifera Creve, Synopsis of the Naviculoid Diatoms, II (I895), p. 43.

Hab. : Lavages d'Éponges et Zostéracées, Tripoli (A. Trotter).

Espèce marine, plus grossièrement ponctuée que le Navicula humerosa Bréb. qui en est voisine; elle a déjà été signalée sur le littoral nord-africain dans le golfe de Gabès (M. Lanzi).

I74. Navicula Hennedyi W. Sмттн, Synopsis of the British Diatomaceæ, II (I856), p. 93 ; A. Schmidt, Atlas, tab. 3, fig. I8; VAN Heurck, Synopsis, p. 93, tab. 9, fig. I4; Traité des Diatomées, p. 204, tab. 4 , fig. I60; CleVE, Synopsis of the Naviculoid Diatoms, II, p. 57; DE TonI, Sylloge Algarum, II, p. I03.

Hab. : Benghazi (A. VACCARI).

Cette espèce paraît nouvelle pour le littoral méditerranéen de l'Afrique. 
I75. Navicula lanceolata Kuetzing, Die Kieselschaligen Bacillarien (I844), p. 94, tab. 30, fig. 48 ; A. Schmidr, Atlas, tab. 47, fig. 49; Van Heurck, Synopsis, p. 88, tab. 8, fig. I6; Traité des Diatomées, p. I86, tab. 3, fig. I39; DE TonI, Sylloge Algarum, II, p. 57 ; CLEvE, Synopsis of the Naviculoid Diatoms, II (I895), P. 2I.

Hab.: Entre les Chara, Tadjoura (A. Trotтеr). Espèce très répandue dans les eaux douces.

I76. Navicula Libellus Gregory, Diatomaceæ of Clyde (I857), p. 528, tab. I4, fig. Ior.

Libellus Grevillei CLEVE, Diatoms of the Arctic Sea, I873, p. 18 partim.

Hab. : Benghazi (A. VACCARI).

Cette espèce est inconnue sur le littoral méditerranéen de l'Afrique.

I77. Navicula Liber W. Smith, Synopsis of the British Diatomaceæ, I (1853), p. 48, tab. I6, fig. I33; De TonI, Sylloge Algarum, II, p. I57 a; VAN HEURCk, Traité des Diatomées, p. 222, tab. 5, fig. 2 Ig.

Caloneis Liber CLEVE, Synopsis of the Naviculoid Diatoms, I (I894), p. 54.

Hab. : Lavages d'Algues, Tripoli [les variétés tenuistriata Cleve et var. umbilicata Grunow] (A. Trotter); lavages d'Algues, Homs (A. Trotter), Tobrouk (A. Vaccari) [à Homs et Tobrouk la variété linearis Grunow?

Espèce marine, très répandue dans les mers intérieures et les océans. M. Cleve a réuni, sous le nom de Caloneis Liber, de nombreuses formes et variétés qui devront peut-être conserver leur autonomie.

I78. Navicula Lyra Ehrenberg, Verbreitung Amer. (I843), p. I3I, tab. I, I, fig. 9 ; A. Schmidt, Atlas, tab. 2, fig. II, I6, 25; VAN HEURCK, Synopsis, p. 93, tab. Io, fig. I; Traité des Diatomées, p. 202, tab. 4, fig. I6I; CleVE, Synopsis of the Naviculoid Diatoms, II (I895), p. 63 ; De Toni, Sylloge Algarum, II, p. 95

Hab. : Lavages d'Algues, d'Éponges et de Zostéracées, Tripoli (A. Trotтer); lavages des Cystoseira, Tobrouk (A. VACCARI); Benghazi (A. VACCARI); dans les lavages d'Algues de Homs, quelques frustules correspondent aux variétés intermedia Perag., forma elliptica Perag.; à Benghazi, en dehors de la forme typique, on trouve : forma sublypica A. S., var. subelliptica, var. recta f. subelliptica Perag.

L'espèce est très répandue dans les eaux marines. Elle est relativement variable. Sur le littoral nord-africain, elle est connue à Arzew (Leuduger, d'après P. PetiT) et au Cap Bon (LANZI).

I79. Navicula nobilis (Ehr.) KUetzing, Die Kieselschaligen Bacillarien (I844), p. 98, tab. 4, fig. 24; VAn Heurck, Traité des Diatomées, p. I64, tab. 2, fig. 67 ; DE Toni, Sylloge Algarum, II, p. 9.

Pinnularia nobilis Ehrenberg, in Abhandlungen Berl. Akademie, 1840, p. 20 ; CLeve, Synopsis of the Naviculoid Diatoms, II (I895), p. 92.

Hab. : Benghazi (A. VACCARI).

Espèce exclusivement d'eau douce, avec toute probabilité accidentelle dans la récolte, comme Gomphonema acuminatum Ehr. et Cymbella lanceolata Ehr. 
I80. Navicula notabilis Greville, Descriptions of new and Rare Diatoms, series VIII (I863), p. I8, tab. I, fig. 9 ; A. Schmidt, Atlas, tab. 8, fig. 46-48; Van Heurck, Traité des Diatomées, p. 200, tab. 26 , fig. 750 (non Navicula notabilis PANTOCSEK).

Diploneis notabilis CLEVE, Synopsis of the Naviculoid Diatoms, I (I894), p. 93 (forma genuina Cleve).

Hab. : Lavages d'Algues, Tripoli (A. Trotter).

Espèce exclusivement marine, indiquée aussi pour la Méditerranée, mais non pour le littoral nord-africain. Elle préfère les mers chaudes et tempérées où elle est très répandue dans des localités fort éloignées les unes des autres.

I8I. Navicula oblonga Kuetzing, Die Kieselschaligen Bacillarien (I844), p. 97, tab. 4, fig. 2I; A. SchmidT, Atlas, tab. 47, fig. 63-68; Van Heurck, Synopsis, p. 8I, tab. 7, fig. I; Traité des Diatomées, p. I76, tab. 3, fig. IO0; Cleve, Synopsis of the Naviculoid Diatoms, II (I895), p. 2I; DE Toni, Sylloge Algarum, II, p. 37 .

Frustulia oblonga Kuetzing, Algarum aquæ dulcis Germanicarum Decades (I833), n. 7I (fide LAGERSTEDT)

Hab. : Entre les Chara, Tadjoura (A. Trotter).

Espèce vivant dans les eaux douces de l'Europe et de l'Amérique, mais adaptée aussi aux milieux légèrement salés. On la connaît également à l'état fossile dans plusieurs dépôts, et M. Petrit l'a signalée des formations de Philippeville (Afrique).

182. Navicula palpebralis BréBIsSON, Var. angulosa (GREG.) VAN Heurck, Synopsis des Diatomées de Belgique (I885), p. 97, tab. II, fig. Io; Traité des Diatomées, p. 209, tab. 4, fig. I77; Cleve, Synopsis of the Naviculoid Diatoms, II (I895), p. 70 ; DE TonI, Sylloge Algarum, II, p. II 8.

Navicula angulosa Gregory, in Trans. Microscopical Society, IV (I856), p. 42, tab. 5, fig. 8; A. Schmidt, Nordseefahrt Diatomaceæ, tab. 2, fig. Ig.

Hab. : Lavages d'Éponges et des Zostéracées, Tripoli (A. Trotter).

La forme typique est très répandue dans la Méditerranée et a été signalée déjà sur le littoral nord-africain (golfe de Gabès, etc.); la variété angulosa est indiquée par M. Cleve dans le golfe de Naples.

183. Navicula pennata A. Schmidt, Atlas der Diatomaceenkunde (I876), tab. 48, fig. 41-43; Cleve, Synopsis of the Naviculoid Diatoms, II (I895) p. 32 .

Hab. : Benghazi (A. VACCARI).

Espèce nouvelle pour le littoral méditerranéen de l'Afrique, ayant été indiquée sur le littoral du Maroc occidental (Cleve); elle ne paraît pas rare dans la Méditerranée et l'Adriatique.

I84. Navicula peregrina (Ehr.) KuetzIng, Die Kieselschaligen Bacillarien (I844), p. 97, tab. 28, fig. 53; A. Schmid, Atlas, tab. 47, fig. 57-60; VAn Heurck, Synopsis, tab. 7, fig. 2; Traité des Diatomées, p. I77, tab. 3, fig. IOI; Cleve, Synopsis of the Naviculoid Diatoms, II (I895), p. I8; DE Toni, Sylloge Algarum, II, p. 38.

Pinnularia peregrina Ehrenberg, Verbreitung Amer. (1843), p. I33 cum icon.; W. Smith, Synopsis of the Britich Diatomaceæ, I (I853), p. 56, tab. I8, fig. I70.

Hab. : Lavages des Cystoseira, Tobrouk (A. VACCARI).

L'espèce et les nombreuses variétés sont adaptées aux eaux douces et saumâtres; elle a été signalée dans le bassin de la Méditerranée.

T. V. - FASC. 7 . 
I85. Navicula radiosa Kuetzing, Var. acuta (W. Sm.) Grunow, Die Oesterreichischen Diatomaceen (I860). p. 524; VAN Heurck, Synopsis, p. 83, tab. 7, fig. I9; Traité des Diatomées, p. I80, tab. 3, fig. II3; DE Toni, Sylloge Algarum, II, p. 42.

Pinnularia acuta W. Smith, Synopsis of the British Diatomacex, I (I853), p. 56, tab. I8, fig. I7I.

Hab. : Entre les Chara, Tadjoura (A. Trotter).

L'espèce typique et sa variété sont très répandues dans les eaux douces.

I86. Navicula Smithii Brébisson, in W. SMith, Synopsis of the British Diatomaceæ, II (I856), p. 92; VAN Heurck, Synopsis, p. 9I, tab. 9, fig. I2; Traité des Diatomées, p. I97, tab. 4, fig. I5I $a-b ;$ DE TonI, Sylloge Algarum, II, p. 86.

Navicula elliptica W. Sмiтн, Synopsis of the British Diatomaceæ, I (I853), p. 48, tab. I7, fig. I52 (non $\mathrm{Na-}$ vicula elliptica KuEtzING).

Diploneis Smithii Cleve, Synopsis of the Naviculoid Diatoms, I (I894), p. 96.

Hab. : Lavages d'Algues, Tripoli (A. Trotter), Tobrouk (A. Vaccari).

Espèce à large répartition géographique, qui se retrouve dans la Méditerranée; elle est connue sur le littoral nord-africain, dans les sondages du port d'Alger (Tempère, PerAGALLO).

I87. Navicula vacillans A. Schmidt, Atlas der Diatomaceenkunde (I875), tab 8, fig. 6I; VAN HEURCK, Synopsis, tab. 9, fig. 9; Traité des Diatomées p. I94, tab. 3, fig. I50 ; DE Tonr, Sylloge Algarum, II, p. 76

Diploneis vacillans Cleve, Synopsis of the Naviculoid Diatoms, I (1894), p. 95.

Hab. : Lavages d'Algues, Homs (A. Trotter).

Espèce exclusivement marine, largement répartie dans les mers et les océans; quelques formes ont été signalées aussi dans la Méditerranée (îles Baléares, etc.)

188. Navicula viridis (Nitzsch) KuetzING, Die Kieselschaligen Bacillarien (I844), p. 97, tab. 30, fig. I2; A. Schmidt, Atlas, tab. 42, fig. II-I4 ; VAN Heurck, Synopsis, p. 73, tab. 5, fig. 5 (Pinnularia viridis) : Traité des Diatomées, p. I65, tab. 2, fig. 70 ; DE TONI, Sylloge Algarum, II, p. II.

Bacillaria viridis NiтzScH, Beitrag zur Infusorienkunde (I8I7), p. 97, tab. 6, fig. I-3.

Pinnularia viridis EHREnberG, Die Infusionsthierchen (I838), p. I82; CLEve, Synopsis of the Naviculoid Diatoms, II (1895), P. 9 I

Hab. : Entre les Chara, Tadjoura (A. Гrotтer).

C'est une des espèces de Navicula la plus anciennement connue. Elle est très répandue dans les eaux douces, même sur le continent africain. Nous en avons déjà donné dans un autre Mémoire (Alghe del Ruwenzori, p. I4) la répartition géographique dans la région africaine, d'après les matériaux rapportés par S. A. R. le duc des Abruzzi et les indications des auteurs (Castracane, O. Mueller, Ehrenberg, Gutwinski et ChmieLEWski, Petit).

I89. Navicula Zanardiniana GRunow, Die Oesterreichischen Diatomaceen (I860), p. 525, tab. III, fig. I2 ;

DE TonI, Sylloge Algarum, II, p. I74.

Caloneis Zanardiniana CLEVE, Synopsis of the Naviculoid Diatoms, I (I894), p. 63.

Hab. : Benghazi (A. VACCARI).

Seulement signalée jusqu'à ce jour entre les Cystoseira recueillies près de Fiụme, dans la mer Adriatique. 
I90. Navicula Zostereti Grunow, Die Oesterreichischen Diatomaceen (I860), p. 528, tab. 4, fig. 23; A. ScHmidT, Atlas, tab. 47, fig. 42-44 (non Nordseef. Diatomaceen, tab. 3, fig. 3); Cleve, Synopsis of the Naviculoid Diatoms, II (I895), p. 3I ; DE Toni, Sylloge Algarum, II, p. 43.

Hab. : Lavages d'Algues, Tripoli (A. TrotTer).

C'est avec doute que nous ajoutons cette espèce aux autres, trouvées dans les lavages d'Algues de Tripoli, n'en ayant observé qu'un seul exemplaire. Navicula Zostereti Grun. a été découverte dans le benthos de la mer Adriatique, à Fiume, où elle fut récoltée par M. Lorenz. Pour le littoral nord-africain, on l'a signalée au Cap Bon (LAnzi), à Arzew dans la vase marine (Leuduger, d'après P. Petit).

\section{Mastogloia ThWAIT.}

I9I. Mastogloia angulata LewIS, On some new and rarer Species of Diatomaceæ (I86I), p. 65, tab. 2, fig. 4; CLEVE, Synopsis of the Naviculoid Diatoms, II (r895), p. I47.

Mastogloia apiculata Grun., Die Oesterreichischen Diatomaceen (r860), p. 577, tab. 7, fig. 9 (non Mastogloia, apiculata W. Sмiтн, I856).

Hab. : Benghazi (A. VACCARI).

Espèce répandue dans la Méditerranée jusqu'à la mer Noire et à la mer Adriatique. En Tunisie, on a signalé le Mastogloia apiculata W. Sm. ( $\boldsymbol{M}$. angulata Perag., non Lewis) avec laquelle on confond souvent le Mastogloia angulata Lewis.

I92. Mastogloia Danseii Thwattes, in W. Sмith, Synopsis of the British Diatomaceæ, II (I856), p. 64, tab. 62, fig. 388; VAn Heurck, Synopsis, p. 70, tab. 4, fig. I8 ; Traité des Diatomées, p. I55, tab. 2, fig. 64; DE TONI, Sylloge Algarum, II, p. 3I5.

Dickieia Danseii ThwaITes, in Ann. Mar., I848, $2^{\mathbf{e}}$ sér., vol. I, tab. XII, K.

Mastogloia elliptica (AG.) CLEVE, var. Danseii Cleve, Synopsis of the Naviculoid Diatoms, II (I895), p. I52.

Hab. : Lavages d'Algues, Tripoli (A. 'Trotter), Homs (A. Trotter).

Espèce préférant les eaux saumâtres, surtout littoranées. M. BELloc la signala pour l'Afrique du Nord, à Chott El-Hodna, lac Fetzara, Chott El-Djerid, Tunis; elle n'est pas rare dans la Méditerranée. Selon M. CLeve, le Frustulia elliptica Ag. serait la forme typique, et Mastogloia Danseii n'en serait qu'une simple variété; mais l'attribution du Frustulia elliptica Ag., qui nous paraît une espèce collective et mal définie, au type du Mastogloia Danseii Thw., n'est pas certaine, puisqueM. W. Smith a référé, lui aussi, avec doute l'espèce de C. Agardh au Mastogloia Smithii Thwait.

193. Mastogloia erythrøa GRunow, Die Oesterreichischen Diatomaceen (I860), p. 577, tab. 5, fig. 4; A. Schmidt, Atlas, tab. I86, fig. 25-26; CLEVE, Synopsis of the Naviculoid Diatoms, II (I895), p. I54; DE TonI, Sylloge Algarum, II, p. 316.

Hab. : Lavages des Cystoseira, Tobrouk (A. VACCARI).

Espèce marine, vivant dans tout le bassin de la Méditerranée jusqu'à la mer Adriatique et à la mer Noire. Elle vit également dans la mer Rouge et dans l'océan Atlantique, où, d'après M. Schmid, elle a été récoltée au Maroc.

I94. Mastogloia Grevillei W. Smith, Synopsis of the British Diatomaceæ, II (I856), p. 64, tab. 62, fig. 389 ; Van Heurck, Synopsis, tab. 4, fig. 20; Traité des Diatomées, p. I55, tab. 2, fig. 65; Cleve, Synopsis of the Naviculoid Diatoms, II (I895), p. r46; DE TonI, Sylloge Algarum, II, p. 3I5. 
Hab. : Benghazi (A. VACCARI).

Espèce propre aux eaux douces et saumâtres, comme d'autres Mastogloia; elle paraît nouvelle pour le littoral méditerranéen de l'Afrique.

I95. Mastogloia Meleagris (Kuetz.) GRUnow, Ueber einige neue und ungenügend bekannte Arten und Gattungen von Diatomaceen (I863), p. I56; DE Toni, Sylloge Algarum, II, p. 3 I4.

Navicula Meleagris Kuetzing, Die Kieselschaligen Bacillarien (I844), p. 92, tab. 30, fig. 37.

Mastogloia lanceolata Thwartes, in W. Smith, Synopsis of the British Diatomaceæ, II (I856), p. 64; VAN Heurck, Synopsis, p. 70, tab. 4, fig. I5-I7 ; Traité des Diatomées, p. I54, tab. 2, fig. 62 ; Cleve, Synopsis of the Naviculoid Diatoms, II (I895), p. I53.

Hab : Lavages d'Éponges et de Zostéracées, Tripoli (A. Trotter), Benghazi (A. VACCARI).

Mastogloia Meleagris (Kuetz.) Grun. préfère les eaux marines, quoiqu'clle soit aussi adaptée aux milieux saumâtres; sur le littoral africain baigné par la Méditerranée, on la connaît en plusieurs localités : Aïn-Hammam-Biban (Peragallo), Sidi-Yahia, Aïnd'Or, oasis d'Ourlana, etc. (Belloc), golfe de Gabès (LanzI). Nous suivons ici l'opinion de M. Grunow qui, en admettant lidentité du Mastogloia lanceolata Thwait. et du Navicula Meleagris Kuetz., a conservé le nom spécifique Meleagris qui est le plus ancien.

I96. Mastogloia minuta Greville, Descriptions of some new Diatomaceous forms from the West Indie(I857) p. I2, tab. 3, fig. I0 ; A. Schmidt, Atlas, tab. I87, fig. 22 ; Cleve, Synopsis of the Navicus loid Diatoms, II (I895), p. I5I, tab. 2, fig. 7 ; DE TONI, Sylloge Algarum, II, p. 3 I7.

Hab. : Lavages d'Algues, Homs (A. Trotter).

Selon les auteurs (Grunow, Cleve, Leuduger, etc.), cette espèce possède une large distribution dans les océans Atlantique et Pacifique. Elle est bien différente du Mastogloia pusilla Grun.

I97. Mastogloia Smithii ThwaItes, Var. lacustris Grunow, Algen und Diatomaceen aus dem Kaspischen Meere (I878), p. III; VAN HEURCK, Traité des Diatomées, p. I54, tab. 2, fig. 6r ; Cleve, Synopsis of the Naviculoid Diatoms, II (I895), p. I52 ; De TonI, Sylloge Algarum, II, p. 3I4.

Hab. : Entre les Chara, Tadjoura (A. Trotter).

Var. intermedia Grunow, Ibidem, p. IIo; Cleve, Ibidem, p. I52; De Toni, Ibidem

Hah. : Lavages d'Algues, Homs (A. Trotter), Benghazi (A. Vaccari).

Var, amphicephala Grunow, in Cleve et Moeller, Diatom., n. I6I ; VAn Heurck, Synopsis, tab. 4, fig. 27 ; CLEVE, Ibidem, P. I52.

Hab. : Benghazi (A. VAcCARI).

Mastogloia Smithii Thwait. est une espèce promisque aux eaux douces et saumâtres et qui se trouve aussi dans les sources thermales (BELloc, LANZI). 
Famille des GOMPHONEMECEÆ (Kuetz.) Grun.

Gomphonema C. AG.

I98. Gomphonema acuminatum Ehrenb., Die Infusionsthierchen (I838), p. 2I7, tab. I8, fig. 4; VAN HEURCK, Synopsis, tab. 23, fig. I6; Traité des Diatomées, p. 270, tab. 7, fig. 299 ; CLEVE, Synopsis of the Naviculoid Diatoms, I (I894), p. I84; DE TonI, Sylloge Algarum, II, p. 423.

Hab. : Benghazi (A. VACCARi).

Espèce exclusivement d'eau douce, signalée déjà dans l'Afrique septentrionale (Belloc, Petit); elle paraît, comme Navicula borealis Ehr. et quelques autres espèces, accidentelle dans la récolte, peut-être emportée par les eaux douces avec des fragments d'autres Algues.

199. Gomphonema exiguum Kuntzing, Die Kieselschaligen Bacillarien (I844), p. 84, tab. 30, fig. 58 (pas exacte) ; Van Heurck, Synopsis, p. I26, tab. 25, fig. 34; Traité des Diatomées, p. 275, tab. 7, fig. 3I7; Cleve, Synopsis of the Naviculoid Diatoms, I (I894), p. I88; DE Toni, Sylloge Algarum, II, p. 430.

Hab. : Lavages d'Algues, Homs (A. Trotter).

Espèce marine, probablement très répandue, mais souvent inaperçue, par suite de son extrême petitesse.

200. Gomphonema montanum Schumann, Die Diatomeen der Hohen Tatra (1867), p. 67, tab. 3, fig. 35 b; VAN Heurck, Synopsis, p. I24, tab. 23, fig. 33 et 36; Traité des Diatomées, p. 27I, tab. 7, fig. 303; DE Toni, Sylloge Algarum, vol. II, p. 425.

Gomphonema sulclavatum Grun., var. montana (Schum.) Cleve, Synopsis of the Naviculoid Diatoms, I (I894), p. I84.

Hab. : Entre les Chara, Tadjoura (A. Trotter).

Espèce d'eau douce, intermédiaire entre les Gomphonema lanceolatum Ehr. et Gomphonema acuminatum Ehr. Sur la répartition géographique du Gomphonema subclavatum Grun (duquel, d'après M. Cleve, G. montanum Schum. représente une simple variété), on peut consulter, à l'égard de la région africaine, notre Mémoire sur les Algues du Ruwenzori.

20I. Gomphonema olivaceum (LyngB.) KuEtzING, Algarum aquæ dulcis Germanicarum Decades (I833), n. I3; Die Kieselschaligen Bacillarien (I844), p. 85, tab. 7, fig. I3 et I5 ; VAN HEURCK, Synopsis, p. I26, tab. 25, fig. 20 $a-b$; Traité des Diatomées, p. 274, tab. 7, fig. 3I5 $a-b$; CLEvE, Synopsis of the Naviculoid Diatoms, I (I894), p. I87; DE TonI, Sylloge Algarum, II, p. 433.

Echinella olivacea LyngBye, Tentamen Hydrophytologiæ Danicæ (I8I9), p. 209, tab. 70, fig. C I-3.

Hab. : Lavages d'Algues, Tripoli (A. Trotter).

Espèce très commune dans les eaux douces, où elle croît, attachée aux cailloux, mais adaptée aussi à la vie dans des milieux saumâtres, comme d'autres espèces de Gomphonema qui ne sont jamais exclusivement marines. Notre Gomphonema olivaceum (Lyngb.) Kuetz. est accidentellement mêlé aux matériaux d'Algues marines. L'espèce a été signalée, pour l'Afrique boréale, par M. Bory de SAint-Vincent.

Rhoicosphenia Grunow.

202. Rhoicosphenia curvata (KuEtz.) Grunow, Algen Novara (I867), p. 8; VAN Heurck, Synopsis, p. I27, tab. 26, fig. I-3; Traité des Diatomées, P. 275, tab. 7, fig. 3I9; Cleve, Synopsis of the Naviculoid Diatoms, II (1895), p. I65 ; DE ToNr, Sylloge Algarum, p. 437. 
Gomphonema curvatum Kuetzing, Synopsis Diatomearum (I833), p. 39, fig. 5I; Die Kieselschaligen Bacillarien (I844), p. 85, tab. 8, fig. I-3 (incl. var.).

Gomphonema marinum W. Sмith, Synopsis of the British Diatomaceæ, I (I853), p. 8I, tab. 29, fig. 246.

Hab. : Lavages d'Algues, Tripoli, la forme marina (A. Trotter); entre les Chara, Tadjoura, la forme typica (A. Trotter).

Rhoicosphenia curvata (Kuetz.) Grun. est une forme très répandue dans les eaux douces et marines. On la connaît dans plusieurs localités de l'Afrique du Nord, par cxemple dans le lac près Oran (Montagne), à Biskra (Belloc), dans l'Oued-Raha (Lanzi) et dans Le Rummel à Constantine (Tempère, Peragallo).

Famille des ACHNANTHACE $Æ$ (Kuetz.) Grun.

Achnanthes Bory.

203. Achnanthes coarctata (BréB.) Grunow, in Cleve et Grunow, Beiträge zur Kenntniss der Arktischen Diatomeen (I880), p. 20 ; VAN HEURCK, Synopsis, p. I30, tab. 26, fig. I7-20 ; Traité des Diatomées, p. 280, tab. 8, fig. 327 ; CLEVE, Synopsis of the Naviculoid Diatoms, II (I895), p. I92; De ToNI, Sylloge Algarum, II, p. 476 .

Achnanthidium coarctatum BréBisson, in W. SMith, Notes of an Excursion in the South of France and the Auvergne (I855), tab. I, fig. Io; Synopsis of the British Diatomaceæ, II (I856), p. 3r, tab. 6I, fig. 379.

Hab. : Lavages d'Algues, Tobrouk (A. VAcCari).

Espèce très adaptée aux milieux les plus divers. Bien que préférant les eaux douces, la terre humide, les mousses, on la trouve aussi dans les eaux saumâtres ou tout à fait marines (port de Nice, etc.) ; en effet, d'après M. Belloc, Achnanthes coarctata a été rencontrée dans les eaux douces ou légèrement salées à Tougourt et à Biskra.

204. Achnanthes longipes C. AgArdh, Systema Algarum (I824), p. I; VAN Heurck, Synopsis, p. I29, tab. 26, fig. I3-I6; Traité des Diatomées, p. 279, tab. 8, fig. 323; Cleve, Synopsis of the Naviculoid Diatoms, II (I895), p. Ig6; DE TonI, Sylloge Algarum, II, P. 470.

Hab. : Lavages d'Algues, d'Éponges et de Zostéracées, Tripoli (A. Trotter); lavages des Cystoseira, Tobrouk (A. VACCARI).

Achnanthes longipes est une espèce commune dans la Méditerranée; pour la côte nord de l'Afrique, elle a été signalée dans le golfe de Gabès par M. Lanzi.

205. Achnanthes parvula KUETZING, Die Kieselschaligen Bacillarien (I844), p. 76, tab. 2I, fig. 5 ; VAN HEURCK, Synopsis, p. I29, tab. 26, fig. 25-28; Traité des Diatomées, p. 280, tab. 8, fig. 326 ; DE TonI, Sylloge Algarum, II, p. 474.

Achnanthes brevipes var. parvula ClEve, Synopsis of the Naviculoid Diatoms, II (I895), p. I93.

Hab. : Lavages d'Algues, Tripoli (A. Trotter).

206. Achnanthes subsessilis Kuetzing, Algarum aquæ dulcis Germanicarum Decades (r833), n. 42; Die Kieselschaligen Bacillarien (I844) p. 76, tab. 20, fig. 4 ; VAN HeURck, Synopsis, p. I29, tab. 26, fig. 2I-24;

Traité des Diatomées, p. 279, tab. 8, fig. 325; DE Toni, Sylloge Algarum, II, p. 473.

Achnanthes brevipes var. intermedia CLEVE, Synopsis of the Naviculoid Diatoms, II (I895), p. I93.

Hab. : Lavages d'Algues, Tripoli (A. Trotter), Homs (A. Trotter). 
Comme plusieurs espèces du genre Achnanthes, cette forme vit dans les eaux saumâtres et marines. Elle est très commune dans la Méditerranée. Elle ne manque pas sur le littoral nord-africain, puisqu'elle a été signalée dans le Chott-el-Hodna, à Biskra, dans l'oasis de Tougourt (Belloc) et dans l'Oued-Raha au Cap Bon (Lanzi).

Famille des COCCONEIDACEÆ (Kuetz.) Grun.

Cocconeis Ehrenb.

207. Cocconeis britannica NAEgeli, in KuetzING, Species Algarum (I849), p. 890 ; VAn Heurck, Synopsis, tab. 30, fig. I-2 [valva superior]; CLEve, Synopsis of the Naviculoid Diatoms, II (I895), p. I8I ; DE Toni, Sylloge Algarum, II, p. 45I.

Cocconeis scutelliformis Grunow, in CL. et MoELL., Diat. n. I48-I54, 208-2Io.

Hab. : Benghazi (A. VACCARI).

Espèce répandue dans la Méditerranée, mais non rencontrée jusqu'ici sur les côtes africaines.

208. Cocconeis dirupta Gregory, On new Forms of marine Diatomaceæ found in the Firth of Clyde ( $\mathrm{I} 857$ ), p. I9, tab. I, fig. 25 ; VAN Heurck, Synopsis p. I33, tab. 29, fig. I3-I5 ; Traité des Diatomées, p. 290, tab. 8, fig. 343 ; Cleve, Synopsis of the Naviculoid Diatoms, II (I895), P. I75 ; DE Toni, Sylloge Algarum, II, p. 453.

Hab. : Lavages d'Algues, Tripoli (A. Trotтеr).

Espèce à large répartition géographique : elle s'étend des côtes du Groenland jusqu'à la Chine, au cap de Bonne-Espérance, à Haïti, au Brésil, etc.; elle a déjà été signalée dans le bassin de la Méditerranée (CLeve).

209. Cocconeis maxima Grunow, in H. Peragallo, Diatomées marines de France (I897), tab. 3, fig. I-4.

Hab. : Benghazi (A. VACCARI).

On connaît, dans le bassin de la Méditerranée, cette belle espèce de Villefranche et de Naples.

2I0. Cocconeis molesta Kuetzing, Die Kieselschaligen Bacillarien (I844), p.7I, tab. 5, fig. VII, I-2 ; VAN HEURCK, Synopsis, tab. 30, fig. I8-I9; Traité des Diatomées, p.290, tab.29, fig. 823 ; Cleve, Synopsis of the Naviculoid Diatoms, II (I895), p. I74; DE ToNi, Sylloge Algarum, II, p. 459.

Hab. : Lavages d'Algues, etc., Tripoli, Homs (A. Trotter). Plusieurs formes $\mid$ f. minor Perag., crucifera Grun., amygdalina Grun.].

Espèce très variable, exclusivement marine, commune dans la Méditerranée où elle se rencontre souvent sur les Algues filamenteuses.

2II. Cocconeis Scutellum Ehrenberg, Die Infusionsthierchen (I838), p. I94, tab. I4, fig. 8; VAN HEURCK, Synopsis, p. I32, tab. 29, fig. I-3 ; Traité des Diatomées, p. 287, tab. 8, fig. 338 ; Cleve, Synopsis of the Naviculoid Diatoms, II (I895), p. I70; DE' TonI, Sylloge Algarum, II, p. 444.

Hab. : Lavages d'Algues, etc., Tripoli, Homs (A. Trotter); Tobrouk, Benghazi (A. VACCARI). 
Espèce épiphyte, très commune dans les mers; sur le littoral nord-africain, elle a été rapportée déjà de Tipaza, d'Alger (Debray) et du golfe de Gabès (Lanzi).

\section{Orthoneis GRUN.}

- 212. Orthoneis aspera Peragallo, Diatomées marines de France (I897), tab. 5, fig. I3.

Hab. : Benghazi (A. VAcCari).

.M. H. Peragalio a trouvé son espèce à Villefranche et l'a indiquée comme très rare. Il pense que le Mastogioia asperula Grun. (Cleve, in Diatomiste, I, p. 6I, tab. 23, fig. 12) n'est peut-être qu'une forme plus petite et apiculée de la même espèce.

2I3. Orthoneis binotata Grunow, Algen Novara (I868), p. I5; VAn Heurck, Synopsis, tab. 28, fig. 7 ; Traité des Diatomées, p. 284, tab. 29, fig. 815; De Toni, Sylloge Algarum, II, p. 466.

Cocconeis binotata GRunow, Ueber einige neue und ungenugend bekannte Arten und Gattungen von Diatomaceen (I863), P. I45, tab. 4, fig. I3 $a-b$.

Mastoglsia binotata CLEVE, Synopsis of the Naviculoid Diatoms, II (I895), p. I48.

Hab. : Lavages d'Algues, Tripoli, Homs (A. Trotrer); Tobrouk, Benghazi (A. VACCARI).

Espèce à large répartition géographique dans les mers et les océans; pour le littoral nord-africain, elle a été signalée à Arzew (Lecduger, d'après P. Petit); à Cartagène (LANZi) et à Alger (Debray).

214. Orthoneis fimbriata (EHr.) Grunow, Algen Novara (I867), p. I5; VAN HeurCK, Synopsis, tab. 28, fig. 3 ; De Toni, Sylloge Algarum, II, p. 465.

Maslogloia fimbriata (BRW.) Cleve, Synopsis of the Naviculoid Diatoms, II (I895), p. I48.

Cocconeis fumbriata EHRENBerG, in Abhandlungen der Berlin. Akademie, I858, p. I3; Brightwell, in Microscopical Joumal, VII (I859), p. I79, tab. 9, fig. 43.

Hab. : Lavages d'Algues, etc., Tripoli, Homs, (A. Trotter); Benghazi, Tobrouk (A. VACCARI).

Espèce épiphyte sur les grandes Algues des mers européennes et extra-européennes, signalée déjà sur le littoral nord-africain dans le golfe de Gabès (LANZI).

215. Orthoneis splendida (Greg.) Grunow, Algen Novara (I867), p. I5; VAn Heurck, Synopsis, tab. 28, fig. I-2 ;

Traité des Diatomées p. 284, tab. 29, fig. 3I4.

Cocconeis splendida Gregory, On new Forms of marine Diatomaceæ found in the Firth of Clyde (1857), p. 2I, tab. I, fig. 29 .

Mastogloia splendida CLEVE, Synopsis of the Naviculoid Diatoms, II (I895), p. I48.

Cocconeis punctatissima GreviLle in Microscopical Journal, I857, tab. I, fig. I.

Orthoneis punctatissima LAGersTedt, Sötvattens-Diatomaceer fran Bohuslän (I876), p. 57; DE TuNi, Sylloge Algarum, II, p. 466.

Hab. : Lavages d'Algues, Tripoli (A. Trotter); lavages des Cystoseira, Tobrouk, Benghazi (A. VACCARI).

Cette espèce vient souvent sur les Algues filamenteuses, principalement sur les Cladophora de la Méditerranée. Du littoral nord-africain, elle a été rapportée de Tipaza [Algérie] (Debray) et du port d'Alger (Tempère, Peragallo). 




